WOLFGANG BERGEM,

PAULA DIEHL,

HANS J. LIETZMANN (HG.)

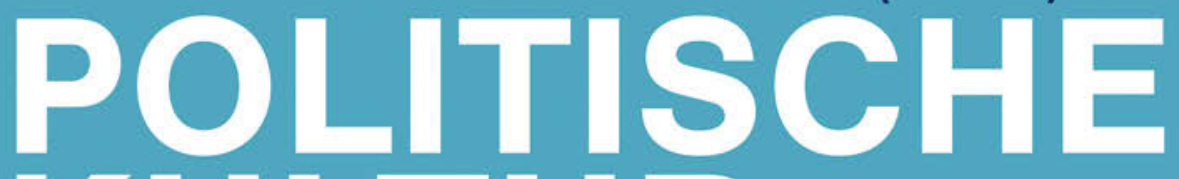

KULTUR-
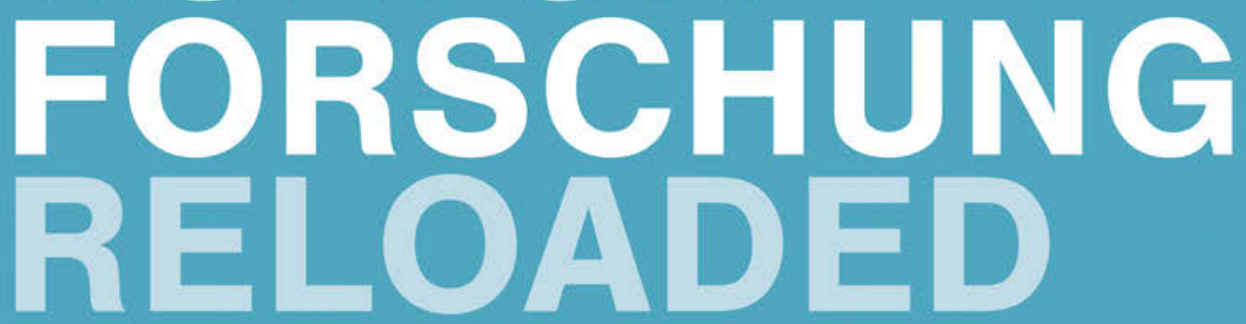

NEUE THEORIEN, METHODEN UND ERGEBNISSE

[transcript] Edition Politik 
Wolfgang Bergem, Paula Diehl, Hans J. Lietzmann (Hg.)

Politische Kulturforschung reloaded

Edition Politik | Band 76 
Die freie Verfügbarkeit der E-Book-Ausgabe dieser Publikation wurde ermöglicht durch den Fachinformationsdienst Politikwissenschaft POLLUX

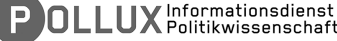

und ein Netzwerk wissenschaftlicher Bibliotheken zur Förderung von Open Access in den Sozial- und Geisteswissenschaften (transcript, Politikwissenschaft 2019)

Bundesministerium der Verteidigung|Gottfried Wilhelm Leibniz Bibliothek -Niedersächsische Landesbibliothek|Harvard University|Kommunikations-, Informations-, Medienzentrum (KIM) der Universität Konstanz|Landesbibliothek Oldenburg|Max Planck Digital Library (MPDL)|Saarländische Universitäts- und Landesbibliothek|Sächsische Landesbibliothek Staats- und Universitätsbibliothek Dresden | Staats- und Universitätsbibliothek Bremen (POLLUX - Informationsdienst Politikwissenschaft)| Staats- und Universitätsbibliothek Carl von Ossietzky, Hamburg | Staatsbibliothek zu Berlin | Technische Informationsbibliothek Hannover | Thüringer Universitäts- und Landesbibliothek Jena (ThULB) | ULB Düsseldorf Universitäts- und Landesbibliothek Düsseldorf | Universitätsbibliothek Erfurt | Universitäts- und Landesbibliothek der Technischen Universität Darmstadt | Universitäts- und Landesbibliothek Münster | Universitäts- und Stadtbibliothek Köln | Universitätsbibliothek Bayreuth | Universitätsbibliothek Bielefeld | Universitätsbibliothek der Bauhaus-Universität Weimar | Universitätsbibliothek der FernUniversität Hagen | Universitätsbibliothek der Humboldt-Universität zu Berlin | Universitätsbibliothek der Justus-Liebig-Universität Gießen | Universitätsbibliothek der Ruhr-Universität Bochum | Universitätsbibliothek der Technischen Universität Braunschweig|Universitätsbibliothek der Universität Koblenz Landau | Universitätsbibliothek der Universität Potsdam | Universitätsbibliothek Duisburg-Essen | Universitätsbibliothek Erlangen-Nürnberg| Universitätsbibliothek Freiburg | Universitätsbibliothek Graz| Universitätsbibliothek J. C. Senckenberg an der Goethe-Universität Frankfurt|Universitätsbibliothek Kassel|Universitätsbibliothek Leipzig|Universitätsbibliothek der LMU München|Universitätsbibliothek Mainz | Universitätsbibliothek Marburg | Universitätsbibliothek Oldenburg| Universitätsbibliothek Osnabrück | Universitätsbibliothek Siegen | Universitätsbibliothek Vechta | Universitätsbibliothek Wien | Universitätsbibliothek Wuppertal | Zentral- und Hochschulbibliothek Luzern | Zentralbibliothek Zürich

Die Publikation beachtet die Qualitätsstandards für die Open-Access-Publikation von Büchern (Nationaler Open-Access-Kontaktpunkt et al. 2018), Phase 1 https://oa2020-de.org/blog/2018/o7/31/empfehlungen_qualitätsstandards_oabücher/

$$
* * *
$$

Wolfgang Bergem (Prof. Dr.), geb. 1962, lehrt Politikwissenschaft an der Universität Siegen.

Paula Diehl (Prof. Dr.), geb. 1970, hat den Lehrstuhl für Politische Theorie und Ideengeschichte an der Christian-Albrechts-Universität Kiel inne.

Hans J. Lietzmann (Prof. Dr.), geb. 1952, hat den Lehrstuhl für Politikwissenschaft an der Bergischen Universität Wuppertal inne.

Die Herausgeber*innen bilden zugleich das Sprecherteam des Arbeitskreises »Politik und Kultur« in der Deutschen Vereinigung für Politikwissenschaft. 
Wolfgang Bergem, Paula Diehl, Hans J. Lietzmann (Hg.)

\section{Politische Kulturforschung reloaded}

Neue Theorien, Methoden und Ergebnisse

[transcript $]$ 


\section{Bibliografische Information der Deutschen Nationalbibliothek}

Die Deutsche Nationalbibliothek verzeichnet diese Publikation in der Deutschen Nationalbibliografie; detaillierte bibliografische Daten sind im Internet über http://dnb.d-nb.de abrufbar.

\section{(c) $(1) \Theta \Theta$}

Dieses Werk ist lizenziert unter der Creative Commons Attribution-NonCommercial-NoDerivs 4.0 Lizenz (BY-NC-ND). Diese Lizenz erlaubt die private Nutzung, gestattet aber keine Bearbeitung und keine kommerzielle Nutzung. Weitere Informationen finden Sie unter https://creativecommons.org/licenses/by-nc-nd/4.०/deed.de Um Genehmigungen für Adaptionen, Übersetzungen, Derivate oder Wiederverwendung zu kommerziellen Zwecken einzuholen, wenden Sie sich bitte an rights@ transcript-verlag.de

Die Bedingungen der Creative-Commons-Lizenz gelten nur für Originalmaterial. Die Wiederverwendung von Material aus anderen Quellen (gekennzeichnet mit Quellenangabe) wie z.B. Schaubilder, Abbildungen, Fotos und Textauszüge erfordert ggf. weitere Nutzungsgenehmigungen durch den jeweiligen Rechteinhaber.

\section{(C) 2019 transcript Verlag, Bielefeld}

Umschlaggestaltung: Kordula Röckenhaus, Bielefeld Druck: Majuskel Medienproduktion GmbH, Wetzlar Print-ISBN 978-3-8376-4747-1 PDF-ISBN 978-3-8394-4747-5 https://doi.org/10.14361/9783839447475

Gedruckt auf alterungsbeständigem Papier mit chlorfrei gebleichtem Zellstoff. Besuchen Sie uns im Internet: https://www.transcript-verlag.de Bitte fordern Sie unser Gesamtverzeichnis und andere Broschüren an unter: info@transcript-verlag.de 


\section{Inhalt}

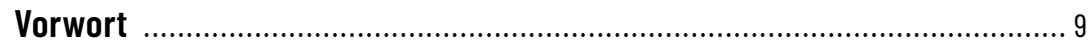

\section{Kulturkonzepte}

Kulturen politischer Partizipation

Hermeneutische und historische Perspektiven

Hans J. Lietzmann

Interdisziplinarität, Politische Repräsentation

und das Imaginäre

Plädoyer für eine neue Perspektive

der politischen Kulturforschung

Paula Diehl

39

\section{Herangehensweisen}

Deutungsmacht als machtsensible Perspektive politischer

Kulturforschung

Dennis Bastian Rudolf

61

,Doing political culture in Alltagspraktiken

der Politisierung von Konsum

Theoretische und methodische Herausforderungen eines neuen

Forschungsfelds der politischen Kulturforschung

Sigrid Baringhorst / Mundo Yang / Katharina Witterhold

Politische Reartikulationen kultureller Topoi in populistischen Verstetigungsprozessen 


\section{Medien und Unterhaltung}

Politische Kultur und Komik

Perspektiven für vergleichende Analysen

Ludgera Vogt

\section{Politische TV-Serien und Politische Kultur}

Ein Forschungsprogramm und Betrachtungen zu einem deutschen Sonderweg

Andreas Dörner

\section{Good Night, and Good Luck}

Über das Verhältnis von Narration, Film und Politik

Sandra Nuy 185

Alltagskulturelle Selbstdisziplinierung

Über die Macht der Ohnmacht in Fernsehserien

Samuel Salzborn 203

\section{Kulturkämpfe und Identität}

Die Identitäten des Populismus

Die Politisierung der Kultur

und der neue Kampf der Kulturen

Jörn Knobloch

ıIdentität ‘ in politischer Kultur, Demokratietheorie und der Identitären Bewegung

Wolfgang Bergem

\section{"Wir sind das Volk"}

Zur Herausbildung eines Diskurses

der identitären Demokratie im Umfeld der Partei

Alternative für Deutschland (AfD) 


\section{Identitätserzählungen im Konflikt}

Der Wettstreit der Narrative im israelischen Kulturkampf

Jan Christoph Suntrup 



\section{Vorwort}

Von Alexis de Tocqueville stammt das klassische Zitat, dass zum Verständnis einer »neuen Welt« eine »neue politische Wissenschaft« benötigt werde. Auch in unserer Gegenwart haben sich die politischen Dynamiken grundlegend noch einmal verändert. Die Politikwissenschaft und besonders die politische Kulturforschung sind herausgefordert, auf diese neuen Konstellationen zu reagieren. Sie benötigen für ihre Analysen neue Instrumente.

Traditionelle politische Systeme haben an Macht deutlich eingebüßt und bilden nicht mehr die weitgehend autarken »Institutionen « in einer gesellschaftlichen »Umwelt«. Die »politische Gesellschaft« selbst hat wesentlich an Gestaltungsmacht und Selbstbewusstsein gewonnen und treibt immer häufiger die institutionellen Akteure vor sich her. Dabei bilden Zivilgesellschaft, Medien, Unterhaltung und Robotik ganz eigene Machtkerne heraus. Neue Formen der Repräsentation und Beteiligung, direkt-demokratische Designs, politische Bewegungen anstelle von Parteien, Occoupy oder Pegida, Trumpismus oder Gelbwesten, aber auch »echo chamber«, »fake news « und »alternative facts« - das sind erste Stichworte in dieser Entwicklung. Angesichts dessen ist die klassische politische Kulturforschung, die sich stark an Fragen der Einstellungsforschung und des Behaviorismus orientierte, überfordert. Ihre Instrumentarien und Zugänge reichen nicht mehr aus, um die komplexen handlungsleitenden und strukturbildenden Mentalitäten, Imaginationen und Narrative in ihrer ganzen symbolischen Kraft zu ermitteln. Die vorherrschende Vielfalt des Politischen und die Vielzahl der Vor- und Darstellungen politisch legitimer Einflussnahme sind in ihrer Komplexität mit den traditionellen Hermeneutiken nicht mehr zu erfassen. Die Verfügung über eine gänzlich neue Generation medialer Kommunikationsmöglichkeiten verstärkt diese Dynamik in einem bislang noch kaum absehbaren Maß. Ferner setzt der neue Grad an Individualität, der die politische Gesellschaft prägt, und die damit nachlassende Bindekraft der Institutionen, eine Pluralität und Heterogenität in politischen Prozessen frei, zu deren Verständnis ebenfalls die Herausbildung neuer Ansätze unerlässlich ist.

Ein solcher »Reset « in der politischen Kulturforschung und eine solches »Reloading « bedeutet keineswegs, dass wichtige Stimmen der politischen Kulturfor- 
schung irrelevant geworden wären. Vielmehr müssen diese mit Ansätzen aus Philosophie, Kultursoziologie oder aus kulturphilosophisch inspirierten Politiktheorien erweitert werden. Schon Karl Rohe hob (in der 1980ern) neben der institutionellen politischen Kultur den hohen Einfluss sozialer Mentalitäten hervor; und Eric Voegelin (1952) oder Cornelius Castoriadis (1975) wiesen - jeder auf seine Weise auf die grundlegende Bedeutung gesellschaftlicher Selbstbilder und Imaginationen hin. Diese Hinweise sind freilich in der deutschen Politikwissenschaft noch nicht stilbildend und erkenntnisleitend geworden. Der jetzige Neustart verknüpft nun diese Ansätze mit aktuellen interdisziplinären, besonders kulturwissenschaftlichen, sozial-kulturellen und medienwissenschaftlichen Hermeneutiken. Er führt die politische Kulturforschung wieder an den jetzigen Stand der gesellschaftlichen Urteilsbildung heran.

Der vorliegende Band entstand aus diesem Vorhaben des Arbeitskreises »Politik und Kultur« der Deutschen Vereinigung für Politikwissenschaft (DVPW) und versammelt Befunde neuerer Forschung zum Zusammenhang von Politik und Kultur. Der Band verbindet diese Befunde mit innovativen theoretischen, programmatischen und methodischen Ansätzen. Das Ergebnis ist ein erweitertes Verständnis von politischer Kultur, das sowohl die politischen Dimensionen von Kultur als auch die kulturellen Dimensionen von Politik zu ihrem Gegenstand macht.

Im ersten Teil des Bandes stehen zwei konzeptuelle Zugänge im Mittelpunkt, die beide theoretische und methodischen Antworten auf die Diagnose einer nicht hinreichenden Ausstattung des traditionellen Instrumentariums der (deutschen) Politikwissenschaft zur Analyse der gegenwärtigen politischen Ereignisse und Prozesse ausgehen.

Hans J. Lietzmann geht den Grundlagen einer Theorie der politischen Kultur der Partizipation nach und beleuchtet die Politisierung der Gesellschaft anhand des Zusammenhangs von politischer Kultur, Repräsentation und Partizipation in sieben politisch-kulturellen Zyklen der Partizipation seit Beginn des 19. Jahrhunderts in Deutschland.

Paula Diehl plädiert für mehr Interdisziplinarität in der politischen Kulturforschung, auf deren Grundlage sie die Beziehungen zwischen symbolischer Repräsentation und dem politischen Imaginären als dem gesellschaftlich geteilten Repertoire von politischen Vorstellungen, Bildern, Symbolen, Emotionen, Mythen und anderen Narrativen ins Auge fasst.

Der zweite Teil ist neueren Zugangsweisen zur politischen Kulturforschung gewidmet.

Dennis Bastian Rudolf stellt die Potentiale des Konzepts der Deutungsmacht als einer auf die Durchsetzung von Leitideen und Geltungsansprüchen konkurrierender Sinnproduzenten gerichteten weichen Form von Macht in den Vordergrund, mit dem die politische Kulturforschung machtförmige Prozesse analysieren kann. 
Sigrid Baringhorst, Mundo Yang und Katharina Witterhold rücken Alltagskulturen in den Fokus politischer Kulturforschung und sprechen sich für eine Überwindung der herkömmlichen Trennung zwischen Kultur und Materialität aus. Sie untersuchen die politische Dimension von individuellen konsumbezogenen Alltagspraktiken vor allem im Blick auf Boykott und >Buykott`als neuen Formen politischer Partizipation.

Bernadette Goldberger schließlich zielt auf Grundlage einer Auseinandersetzung mit der Populismustheorie Laclaus darauf, empirische Populismusforschung als politische Kulturforschung zu konzipieren. Anhand der Artikulationen des Popularen im argentinischen Kirchnerismus zeigt sie den Rekurs populistischer Symbolisierungsstrategien auf etablierte kulturelle Topoi auf.

Der dritte Teil stellt Medien und Unterhaltung auf das Untersuchungsfeld der politischen Kulturforschung.

Ludgera Vogt geht politischer Komik und politischem Humor als Gegenständen der politischen Kulturforschung nach, indem sie anhand der Modulierung von Witz und Komik, Parodie und Satire sowie Humor und Lachen in der politischen Kommunikation der Medienkulturen aufzeigt. Dafür wählt sie eine vergleichende Perspektive zwischen den USA und Deutschland, bei der die Unterschiede zwischen den politischen Kulturen der beiden Länder analysiert werden.

Andreas Dörner untersucht politische Fernsehserien als fiktionale Konstruktionen des Politischen. Diese medialen Unterhaltungsformate werden als einem Bestandteil politischer Kulturen analysiert. Auch Dörner plädiert für eine Verbindung der qualitativen politischen Kulturforschung mit dem Begriff des politischen Imaginären.

Sandra Nuy zeigt die Notwenigkeit einer Öffnung der politischen Kulturforschung für die Analyse von Narrationen auf und entwirft eine Methode zur Analyse von Narrativierungen des Politischen. Dafür verknüpft sie die Konturen einer Dramaturgie als Theorie, die Aspekte des Ästhetischen, des Materialen und des Kommunikativen. Als Beispiel für die Anwendung dieser Methode wählt sie den Film Good Night, and Good Luck.

Samuel Salzborn erfasst Fernsehserien als massenwirksamen Ort, an denen das Verhältnis zwischen objektiven Strukturen und subjektiver Dimension des Politischen als Machtkonstellation hervorgebracht und vermittelt wird. Salzborn stellt eine hierbei sichtbar werdende Tendenz der optimierten Selbstüberwachung ohne Gewalt in der Alltagskultur heraus.

Der vierte Teil des Buches geht verschiedenen Aspekten des kulturellen Konflikts und der Identität als Gegenstand politischer Kulturforschung nach.

Jörn Knobloch erkennt in den aktuellen Auseinandersetzungen um Populismus, Migration und den angemessenen Umgang mit dem fundamentalistischen Islam einen neuen, gesellschaftsinternen Kampf der Kulturen und stellt die Unterschei- 
dung von zwei verschiedenen normativen Leitideen von Identität im neuen Populismus ins Zentrum seines Beitrags.

Wolfgang Bergem zeigt die Spannbreite der mit dem Begriff der Identität bezeichneten Erscheinungen auf, indem er Identität als Kategorie in Konzepten der politischen Kultur, als grundlegende Vorstellung der Theorie der identitären Demokratie, als zentraler Topos der zunehmend einflussreich werdenden Identitären Bewegung und auch als Legitimitätsgenerator in repräsentativen Demokratien untersucht.

Jasmin Siri und Marcel Lewandowsky erkunden in einer wissenssoziologisch-systemtheoretischen Analyse anhand der Konstruktion homogenisierender Kollektivitätsbeschreibungen im Trägermilieu und medialen Resonanzraum der AfD, wie neue rechtspopulistische Bewegungen in pluralisierten Öffentlichkeiten entstehen und sich stabilisieren.

Jan Christoph Suntrup schließlich erhellt die konflikthafte Konkurrenz verschiedener Narrative über Identität im israelischen Kulturkampf zwischen säkularen und religiösen bzw. fundamentalistischen Kräften und plädiert für eine kulturwissenschaftliche Vertiefung der politischen Kulturforschung.

Zusammenfassend ist das hier vorliegende »Reloading « von der Gewissheit geprägt, dass die politische Kulturforschung innerhalb der Politikwissenschaft aufgrund neuer Tendenzen in Kultur und Gesellschaft eine wesentlich zentralere Rolle als bisher und einen für politische Analysen unverzichtbaren Ort einnehmen wird.

Natalie Hoost an der Bergischen Universität Wuppertal danken wir für ihre wertvolle Unterstützung in der Vorbereitung der Drucklegung des Bandes.

Siegen, Bielefeld und Wuppertal, im Februar 2019

Wolfgang Bergem, Paula Diehl, Hans J. Lietzmann 


\section{Kulturkonzepte}





\section{Kulturen politischer Partizipation Hermeneutische und historische Perspektiven}

Hans J. Lietzmann

Die »politische Kulturforschung « hat in der deutschen Politikwissenschaft traditionell einen schweren Stand. Sie verfolgt ihre Ziele und Anliegen jenseits des mainstreams des Faches. Die Auseinandersetzung mit den Motiven, den Gefühlen, den Inszenierungen, den Bildern und den Kommunikationen, die den politischen Institutionen zugrunde liegen und die sie im politischen Alltag befeuern, führt in der Diskussion über die Analyse des politischen Geschehens eine randständige Existenz. Im Fokus stehen stattdessen vordringlich die institutionellen und strukturellen Aspekte des politischen Systems sowie die normativen und programmatischen Analysen politischer Vorhaben und Akteurinnen und Akteure. Im Kontext der traditionellen Differenzierung zwischen polity, policy und politics stehen die strukturellen Polities und die programmatischen Policies im Vordergrund. Die Analyse der politischen Dynamik und ihrer Triebkräfte, ihrer kulturellen und emotionalen Motivationen oder Befindlichkeiten - also der Politics - treten in den Hintergrund.

Dieses Ungleichgewicht der Perspektiven betrifft auch in besonderer Weise die Auseinandersetzung mit den Formen politischer Partizipation. Auch hier sind die strategischen Instrumente und die systematischen Institutionen sowie auch ihr Wandel im Wechsel der politischen Epochen breit untersucht. Hingegen bleiben die Beweggründe, die zu den jeweiligen Veränderungen, Erweiterungen und Ergänzungen geführt haben, erkennbar unterbelichtet. So bekommt der strukturelle Wandel den Anschein eines fast naturwüchsigen, aus sich selbst heraus bestärkten Wachstumsprozesses. Die Gesellschaften und die Menschen, die sich in ihnen bewegen und als Zuschauer, Begleiter und Rezipienten das politische Geschehen erleben und kommentieren, scheinen sich in den Institutionen kaum abzubilden. Das politische System scheint gegenüber den Alltags- und Lebensprozessen eine weitgehend abgekapselte Existenz zu führen. Dieses Eigenleben des politischen Systems scheint sich fast ausschließlich aufgrund von Regierungserfordernissen oder von Strategien der politischen Eliten fortzubilden und zu wandeln. Die jeweilige Form der politischen Repräsentation oder Partizipation erhält den Charakter eines aus sich selbst heraus erweiternden und vervollständigenden Ereignisses. Politische Kontingenzen, historische Wahrnehmungsmuster und Konventio- 
nen, gesellschaftliche Verschiebungen und machtpolitische Einschränkungen oder Erweiterungen der Beteiligung an der politischen Gemeinschaftsbildung werden aus der Erörterung ausgespart.

\section{Die Kultur der politischen Gesellschaft}

Dieser Befund ist angesichts der zentralen Wichtigkeit und auch der zunehmenden Relevanz politisch-kultureller Prägungen in den pluralen und heterogenen Gesellschaften der Gegenwart irritierend und befremdlich; doch diese eindimensionale und eingeschränkte Sicht der politischen Analyse hat systematische Gründe. Sie liegen der Gesamtorientierung der Politikwissenschaft - und besonders in Deutschland - zugrunde. Denn diese unterscheidet sich beispielsweise noch einmal grundsätzlich von anderen, z.B. den romanischen Politikwissenschaften Frankreichs oder Italiens, die bereits im Begriff von diversen, unterscheidbaren Politikwissenschaftën« ausgehen (Le scienze politiche: Comparato u.a. 2011; Nucci 2011. Les sciences politiques: Grunberg 2011; Jobard/Laborier 2002). Diese unterscheiden damit wenigstens in ihrem Gesamtaufbau eine mehr regierungssystemische Orientierung von einer ganz anderen, nämlich mehr gesellschaftlichen Orientierung, eine mehr institutionell-systematische von einer eher sozialwissenschaftlichen Orientierung. In der deutschen Politikwissenschaft bildete sich hingegen über lange Jahre allein eine deutlich regierungszentrierte und entsoziologisierte Sichtweise auf politische Prozesse heraus; dort, wo sie - etwa ab den 7oer Jahren des 20. Jahrhunderts - die Gesellschaft in den Blick nimmt, erfolgt dies vordringlich unter einer empirisch-phänomenologischen Perspektive. In ihr werden in großer methodischer Genauigkeit Erhebungen der politischen Realgeschehen erhoben, die über die empirische Registrierung des (sich wandelnden) Status quo allerdings nur selten hinausblicken. Eine theoriegeleitete Perspektive auf diese Realphänomene deutscher Politik fehlt in den meisten Fällen und wird auch kaum vermisst. Dieser Verzicht auf eine gesellschaftliche Perspektive unterschlägt aber einen entscheidenden Aspekt der politischen Dynamik. Die sich immer mehr herausbildende "politische Gesellschaft« nimmt wesentlichen Einfluss auf politische Strukturen und Entscheidungen. Diese bleiben aber mit der alleinigen Konzentration auf die "positiven «, rechtlich fixierten oder formal errichteten Institutionen außer Betracht. Deren praktische, gesellschaftliche Existenz - ihr Vollzug in der politischen Gesellschaft - bleibt neben ihrer formalen Existenz unterkomplex. So dominiert auch weiterhin das Bemühen, die Institutionen auch der "politischen Gesellschaft «(Castoriadis 1990; Greven 1999; Lietzmann 2012; Hildebrand 2001) allein aus ihrem institutionellen Setting, quasi »aus sich selbst heraus « und ohne ihren gesellschaftlichen Unterbau zu interpretieren. Die Institutionengeschichte wird dadurch zu einer blutleeren, gegenüber der praktischen gesellschaftlichen 
Politik abstrakten »Naturgeschichte«. Sie verliert ihre lebendigen Beweggründe, ihre praktische Grundlage im Leben und im Vollzug der »politischen Gesellschaft» selbst.

Für die verschärfte Situation der Politikwissenschaft in Deutschland hat dies seine Ursache in einer annähernd einhundertjährigen Unterdrückung und Auslöschung politikwissenschaftlicher Forschung und Lehre zwischen der Mitte des 19. und des 20. Jahrhunderts (Lietzmann 1996; 2002; 2011); bekanntlich wurden die letzten Vertreter der »Lehre von der Politik« Mitte des 19. Jahrhunderts von ihren Lehrstühlen vertrieben und in die Emigration getrieben (Bleek 2001). In einer Epoche der europäischen soziologischen Orientierung auf Motive und Kulturen gesellschaftlicher Entwicklungen, massenemotionaler und individueller Gefühlslagen stand so die deutsche politikwissenschaftliche Forschung ganz im Schatten einerseits der Soziologie (Simmel, Tönnies, Kracauer, Max und Alfred Weber u.a.), der Psychologie (Freud, De Man, Reich, Bettelheim) sowie andererseits der juristisch orientierten Staatsrechtslehre (Kelsen, Schmitt, Heller, Forsthoff, Neumann, Kirchheimer e tutti quanti). Die Staatsrechtslehre mit ihrer legalistisch-herrschaftsorientierten Perspektive verdrängte in dieser Phase die soziologischen und gesellschaftsbezogenen Ansätze fast gänzlich aus der politikorientierten Forschung; ein Verdrängungsprozess, der dauerhafte Desorientierung in der Politikwissenschaft in Deutschland auch nach ihrer Wiederbegründung in der Folge des 2. Weltkrieges nach sich zog (Lietzmann 2011).

Das, was sich hier in der deutschen Politikwissenschaft so klar auch institutionell voneinander schied, blieb allerdings auch insgesamt in der Politikwissenschaft weitgehend ausgeklammert. Pierre Rosanvallon hat dies erst kürzlich wieder in einer fulminanten Studie über die Ausklammerung der »unsichtbaren Institutionen «, d.h. der bestimmenden gesellschaftlich-politischen »Haltungen« und der "gegendemokratischen « Strategien, aus der Tradition der politischen Theorie und Reflexion aufgezeigt (Rosanvallon 2017). Diese unsichtbaren Institutionen funktionieren wie ein imaginäres Schattenbild des politischen Systems auf der Oberfläche der Gesellschaft; sie sind der imaginäre Ausdruck der politischen Gesamtordnung im Wahrnehmungshorizont der politischen Gesellschaft. Sie sind aber nicht bloß "Abbild« der Formalstruktur und des Regierungshandelns, sondern sie entwickeln eine eigene Strahlkraft und Wirkung, ohne die die Regierungsstruktur, die jeweilige Form der Gewaltenteilung und Repräsentation, überhaupt nicht zustande käme oder sich erhielte. Letztlich sind die rechtlichen oder formalen politischen Institutionen nur existent und wirksam, weil die unsichtbaren Institutionen sie existent und wirksam sein lassen; und sie sind existent und wirksam auch nur so lange und in dem Maße, wie sie über die Unterstützung der unsichtbaren Institutionen, ihr symbolisches Kapital, verfügen. Im politikwissenschaftlichen Diskurs aber bleibt diese Parallelwelt zur formalen Struktur der Gewaltenteilung und Repräsentation ausgespart. Diese unsichtbare, aber unabdingbare Existenzvoraussetzung der 
politischen Ordnung wird nicht nur nicht thematisiert, sondern sie wird auf erstaunliche Weise regelrecht de-thematisiert. Dass sich die politischen Gesellschaften jeweils »ihre« politischen Ordnungen und Regelwerke erschaffen und einzig durch ihre Zustimmung am Leben halten, findet nur rudimentär Beachtung; der Wesensgehalt dieser kognitiven, emotionalen und imaginären Dimension nicht nur der Gesellschaft, sondern eben auch substantiell des politischen Systems, wird strukturell aus dem Blick genommen. Es mag dahingestellt sein, ob (wie Rosanvallon annimmt) die politikwissenschaftlichen Autorinnen und Autoren dabei »eine bestimmte Dimension oder Form von Demokratie bewusst ignorieren oder kaschieren wollen, um sich mit bequemeren Theorien [...] oder simplifizierenden Beschwörungen [...] zufrieden« geben zu können (ebd.: 135); fest steht, dass es sich um eine signifikante Ignoranz und auch um ein signifikantes Außer-AchtLassen einer entscheidenden Dimension politischer Prozesse handelt. Denn das tiefgreifende Erleben der politischen Prozesse durch die Gesellschaft, deren Amalgamierung mit den Erfahrungen, die reale Verarbeitung von Chancen und Risiken der politischen Existenz im Rahmen der gesellschaftlichen Routinen, die Beurteilung der subjektiven Führungsqualität der Regierungen, der Angemessenheit der politischen Institutionen gegenüber den eigenen Erwartungen, aber auch der objektiven Gestaltungshemmnisse, die sich den politischen Eliten in den Weg stellen, kurz: Der gesellschaftliche Verarbeitungsprozess des politischen Alltags im Erleben der politischen Gesellschaft bleibt in der politischen Analyse über weite Strecken außer Betracht.

\section{Die Politische Kultur in der politischen Gesellschaft}

Diese gesellschaftliche Wahrnehmung des Politischen analytisch einzufordern und sie angesichts einer zunehmenden Pluralisierung der Gesellschaften auch einer differenzierten Analyse zuzuführen, ist einer der Hauptantriebe der politischen Kulturforschung. Tatsächlich handelt es sich ja bei den emotionalen, mentalen, regionalen, ethnischen Wahrnehmungsmustern und den Habitus gegenüber den jeweiligen politischen Institutionen um die tragende Kraft der jeweiligen politischen Gesellschaften (Rohe 1987; 1990). Die lebenspraktischen Erwartungen gegenüber dem politischen System und die geronnenen Erfahrungen mit dem Regierungssystem und seiner Praxis bestimmen zugleich auch die politische Handlungsstruktur, die Absichten und die Möglichkeiten der Regierenden. Keine politische Elite kann in den politischen Gesellschaften der Gegenwart gegen die Erwartungshaltung der Bevölkerung erfolgreich sein. Keine politische Elite und keine politische Institution kann sich unabhängig machen von der Gesellschaft und sich in einem gesellschaftlichen Vakuum selbst gestalten. 
Schon in dem Konzept von Karl Rohe (1990) wird zwischen der »Deutungskultur « und der verfestigten "Soziokultur « unterschieden. Und bereits Rohe stellte fest, dass er mit seinem (gesellschaftlich orientierten) Verständnis der politischen Kultur in den 1980er Jahren politikwissenschaftliches Neuland betrat. Mir scheint es nun darüber hinaus wichtig, noch einmal die »Deutungskultur auch als eine von den politischen Eliten gestaltete und von deren eigenen regierungspraktischen und handlungsopportunen Gesichtspunkten angeleitete kulturalistische Praxis zu sehen. Deutungskultur in diesem Sinne ist dann (auch) ein Rezeptionsangebot an die politische Gesellschaft; diese erfolgt in strategischer Absicht, wenngleich nicht immer im vollen Bewusstsein ihrer Eigenarten und Spezifizitäten. Sie bewegt sich im Rahmen der gesellschaftlichen Kommunikation und bietet Handlungsmuster und Handlungsverständnisse für den politischen Prozess an. Sie kann sich hingegen nicht gewiss sein, mit diesem Angebot politisch zu reüssieren. Ob die politische Gesellschaft das Angebot akzeptiert und die Deutungskultur zu einer tragfähigen Soziokultur werden lässt, ist kontingent. Die politischen Eliten hantieren mit einem »symbolischen Kapital«; sie bemühen sich, ihre Absichten und ihr Handeln gegenüber der politischen Gesellschaft plausibel und nachvollziehbar zu machen. Sie werben um Gefolgschaft und Akzeptanz. Sie arbeiten an und bemühen sich um Reputation in ihren Handlungen. Und sie bestätigen ganz und gar die Webersche These, dass diese Reputation, diese politische Macht und der politische Machtgebrauch lediglich »die Chance«, nicht die Gewissheit der gesellschaftlichen Gefolgschaft bedeute (Weber 1922: 122). Es prägt vielmehr die modernen Gesellschaften, dass die Regierungspraxis und die Alltagspraxis der politischen Gesellschaft miteinander ein Amalgam eingehen. Wie dieses Amalgam sich gestaltet und was es beinhaltet, ist eine in jeder Epoche offene Frage. Sein Inhalt ist kontingent, sein Bestand in hohem Maße prekär (Palonen 1998).

Gleichzeitig ist es so, dass die jeweilige Wahrnehmung von Politik und die gesellschaftlich bestimmenden Vorstellungen politischer Gestaltung - die »unsichtbaren Institutionen « (Rosanvallon) - nicht nur die Praxis der politischen Eliten und der Akteurinnen und Akteure der Regierungen bestimmen. Sondern es sind gerade diese wandlungsfähigen kulturellen Dispositionen und symbolischen Präferenzen der politischen Gesellschaft, die die formalen, also die »sichtbaren«, Institutionen in ihrer Praxis nachhaltig gestalten. Erst die politisch-kulturelle `Software bestimmt die tatsächliche Reichweite der juristischen Kompetenzen und die Handlungsmöglichkeiten der politischen Institutionen. Sei es, dass die institutionellen Akteurinnen und Akteure ihr Handeln auf die Präferenzen der politischen Gesellschaft ausrichten, sei es, dass durch Interventionen von Gerichten, Aufsichtsbehörden oder zivilgesellschaftlichen Akteurinnen und Akteuren die Handlungsweisen und Handlungsformen der Institutionen umgebildet und an den neuen politisch-kulturellen Standard angepasst werden. Es handelt sich um »öffentliche Narrative« der gesellschaftlichen Kommunikation, die diesen Einfluss ausüben; aber 
auch um »strategische Narrative« politischer Akteurinnen und Akteure, die sich im Feld der öffentlichen Kommunikation um Durchsetzung bemühen (Roselle et al. 2014). Es ist ein kontinuierlicher, kaum einmal bruchartiger, sondern eher sukzessiver Prozess der gegenseitigen Angleichung und Adaption. "Sichtbare und "unsichtbare Institutionen befruchten und beeinflussen sich dabei gegenseitig und gestalten gemeinsam die politische Dynamik der politischen Gesellschaft.

Das, um was es bei dieser Adaption geht, beschreibt die - notabene individualistische - Darstellung der französischen Schriftstellerin Annie Ernaux über ihre Form der Darstellung gesellschaftlicher Ereignisse:

»Es geht nicht darum, mein Leben zu beschreiben, sondern das, was durch mich hindurch gegangen ist. Ich gehe also von mir aus, schreibe aber vor allem außerhalb von mir. Ich bin nur ein Durchgangsort«. Und ich habe die »ehrgeizige Absicht«, an diesem Durchgangsort »die Dinge zu erfassen, die das Leben im Allgemeinen betreffen, so wie es jedem gegeben ist zwischen seiner Ceburt und seinem Tod« (Ernaux, zit.n. Vormweg 2018: 3).

Gesellschaftlich gesprochen geht es darum, die »unsichtbaren Institutionen« der politischen Kultur darin zu identifizieren, worin sich eine Gesellschaft als ihrer selbst sichtbar und gewiss wird - dort, wo sich die Intentionen und die Selbstwahrnehmung mit den äußeren politischen Institutionen verbinden und sich gegenseitig befruchten. Dieser Prozess der zunehmenden Sichtbarkeit und Lesbarkeit der Gesellschaft in den politischen Institutionen und damit die »Produktion einer lesbaren Welt « (Rosanvallon 2017: 278) machen den Kern dessen aus, wie sich die politische Gesellschaft in die Gestaltung der positiven, äußeren, sichtbaren Institutionen »einmischt«. Es handelt sich um eine grundlegend »kognitive Dimension der Politik« (ebd.), in dem Sinne, dass sie ein Gemeinwesen dadurch erzeugt, dass sie diesem dabei hilft, sich selbst und vor seinen eigenen Augen darzustellen.

\section{Partizipation und Demokratie in der politischen Gesellschaft}

Die politische Gesellschaft der Gegenwart entwickelt in ihrem Selbstverständnis immer weitergehende Ansprüche an die Wiedererkennbarkeit ihrer selbst in den politischen Institutionen. Die kulturelle Lesbarkeit des politischen Systems soll in immer größerem Maße eine unmittelbare Abbildung des eigenen, subjektiven Habitus und der eigenen subjektiven Befindlichkeit erkennen lassen. Im Gegensatz zu früheren Gesellschaften, die eine gewisse (unterschiedlich große) Unterwerfungsbereitschaft und eine Stellvertretung der eigenen Interessen erlaubten, werden in der Gegenwart der politischen Gesellschaft die Handlungsformen einer elitären, oligarchischen und traditionell repräsentativen Wahrnehmung politischer Interessen skeptisch gesehen. Bewegungen des Populismus, aber auch die empirisch 
feststellbare allgemeine Skepsis gegenüber den Parteien und ihrem über lange Zeit fraglosen Vertretungsanspruch in politischen Fragen sind hier deutliche Fingerzeige.

Dieser Wandel in den Erwartungen und die mit ihm verbundene Radikalisierung der Wiedererkennbarkeit der Gesellschaft in den politischen Institutionen weist auf einen wesentlichen Wandel der politischen Vertretungskultur hin. Er geht einher mit deutlich veränderten Erwartungen der Gesellschaft an die politische Partizipation. Zunächst mit einer ausgeprägten Distanz zu den hergebrachten Formen politischer Partizipation: Den traditionellen Repräsentanten misstrauen die Bürger kulturell, die »Chemie« gegenüber den repräsentativen Institutionen verändert sich deutlich zum Negativen. Die Bürgerschaft sieht sich durch sie zunehmend weniger vertreten, d.h. sie erkennen sich in ihnen nicht wieder und sie anerkennen sie nicht als kompetente und adäquate Vertreter ihrer Interessen. Weder der Bundestag (46 Prozent) noch die Bundesregierung (43 Prozent) erreicht in letzten Umfragen das Vertrauen wenigstens der Hälfte der Befragten, geschweige denn das EU-Parlament (28 Prozent). Das geringste Vertrauen gilt schließlich den politischen Parteien (22 Prozent). Lediglich die ortsnahen, kommunalen Stadträte (die ja formal nicht einmal Parlamente sind) erreichen eine wenigstens noch 57-prozentige Zustimmung der politischen Gesellschaft in Deutschland (alle Werte: Bertelsmann 2014). Jugendliche bekennen sich zwar zunehmend und auf breiter Basis zu den Ansprüchen und Werten der Demokratie - wie die neueste ShellStudie erweist (Albert et al. 2015) - doch stimmen knapp 70 Prozent der Aussage $z u$, dass sich die von ihnen gewählten »Politiker [...] nicht darum [kümmerten], was Leute wie [sie selbst] denken« (ebd.). Sie fühlen sich also von der Praxis der repräsentativen Demokratie nicht beachtet, können sich in ihr kulturell nicht aufgehoben finden und wiedererkennen.

Von der Gesamtbürgerschaft der politischen Gesellschaft gehen drei Viertel der Menschen davon aus, auf die gegenwärtige Politik keinerlei Einfluss nehmen zu können (infratest dimap 2013); gleichwohl äußern ebenso viele Menschen den Wunsch, selbst Einfluss nehmen zu wollen. Die kulturelle Wende, die sich darin erkennen lässt, betrifft nun eher nicht die Politikerinnen und Politiker, die politischen Institutionen und die politischen Entscheidungen. Denn es ist ja nicht etwa so, dass in vergangenen Epochen und unter Bedingungen einer hohen kulturellen Zustimmung zu dem traditionell repräsentativen Modell die Bürgerschaft einen intensiveren Einblick in die politischen Abläufe oder eine größere Nähe zu den politischen Eliten gehabt hätte. Das, was sich hingegen deutlich verschoben hat, ist der Blick auf die eigene Kompetenz und auch auf die eigene Bereitschaft und Fähigkeit, die Richtigkeit politischer Entscheidungen und politischen Handelns selbst $\mathrm{zu}$ bestimmen; die empirische Abfrage erweist eine hohe Selbstzuschreibung von Kompetenz und politischem Beurteilungsvermögen der politischen Gesellschaft: Die Menschen fühlen sich im Stande und sind bereit, die politischen Entscheidun- 
gen weitgehend selbst vorzunehmen (Lietzmann 2012; Bertelsmann Stiftung 2014: 49, 56).

Hier hat sich eine deutliche Neuausrichtung der kulturellen Erwartungen an den politischen Prozess, die Politikerinnen und Politiker und die politischen Institutionen ergeben. Die politische Gesellschaft »liest« den Bereich der Politik auf ganz neue Art und Weise. Sie »konstruiert sich ihre Vorstellung von Politik ganz neu. Sie stellt neue Ansprüche und verweigert den traditionellen »Konstruktionen « politischer Repräsentation und Partizipation in Deutschland kulturell und habituell die Gefolgschaft.

Bedeutsam ist zugleich, dass dieser Vertrauensverlust in die Repräsentativität der politischen Institutionen - wie eine große Parlamentarier-Befragung der letzten Jahre zeigt (Alemann et al. 2011) - nicht einseitig nur auf der Seite der Regierten zu finden ist. Auch die Repräsentanten selbst operieren zunehmend skeptisch in der dünnen Luft der politischen Institutionen; sie können den Anspruch des politischen Systems, das in ihnen die personale Vertretung der politischen Gesellschaft als Ganzer sieht, nicht nur vor der Bürgerschaft, sondern auch vor sich selbst kaum mehr glaubhaft aufrecht erhalten. Hinzu kommt, dass auch sie selbst ihre sachliche Vertretungsmacht als weitgehend unwirksam erachten: So glauben auch die Parlamentsabgeordneten in Bund, Ländern und Kommunen nur in einem verschwindend geringen Maße, auf den Kerninhalt der politischen Entscheidungen, über die sie abstimmen sollen, wahrhaft Einfluss nehmen zu können.

In den kommunalen politischen Arenen zeigt sich zudem, dass auch die kommunalen Repräsentanten und Verwaltungseliten in einem hohen Maße bereit sind, die urbanen und regionalen Gesellschaften verstärkt in die deliberative Aushandlung und die partizipative, gemeinsame Entscheidung von Planungsentscheidungen mit einzubeziehen (Bertelsmann Stiftung 2014: 63). So scheint es in der politischen Gesellschaft der Gegenwart einen aufwachsenden Konsens darüber zu geben, dass sich ein neues Verständnis von Repräsentation und Partizipation den Weg bahnt. Konsensuale, weniger hierarchische Entscheidungsund Aushandlungsformen treten ergänzend $\mathrm{zu}$ den bisherigen Verfahrensformen und - darum geht es hier im Verständnis der politischen Handlungs- und Verwaltungskultur - werden als praktikable und angemessene Formen des politischen Gesamtsystems und als eine neue Form der Lesbarkeit und der Selbstwirksamkeit der politischen Gesellschaft in die politische Kultur integriert.

Das hat reale Hintergründe in den Entmachtungsprozessen, die mit der politischen Europäisierung und der Vernetzung globaler Entscheidungsstrukturen zu tun haben. Die Gleichzeitigkeit dieses politisch-kulturellen Wandels sowohl in der Bürgerschaft als auch in den politischen Handlungseliten deutet aber zugleich darauf hin, dass der Sinneswandel und die politisch-kulturelle Neuorientierung einen gesamtgesellschaftlichen Charakter angenommen haben. Die Neuorientierung der politischen Kultur, die sich hier zeigt, ist keine Frage von tagespolitischer oder be- 
fristeter Herrschaft und Opposition; sondern es kommt zu einer Neuorientierung über die Gesamtheit der politischen Organisation, zu einer politisch-kulturellen Neudefinition von Regierung und Partizipation.

\section{Die Theorie einer politischen Kultur der Partizipation}

In den kulturellen und symbolischen Formen, in denen Gesellschaften sich die ihnen gemäße Gestaltung der politischen Entscheidungen organisieren, erweist sich das Selbstbild, das sie von sich selbst als Gemeinschaft entwerfen. Selbst der Verzicht auf die Ausgestaltung eines politischen Entscheidungssystems, wie wir es für familiale Nomadenvölker weitgehend unterstellen, erweist sich als eine signifikante Lesart des Gemeinschaftsverständnisses; es zeigt den Verzicht auf politische Gestaltung. Seit jeher wird daher die Debatte um die Formen der politischen Partizipation und der Repräsentation als eine zentrale Fragestellung der Politikwissenschaft und der politischen Theorie behandelt. Denn Partizipation und Repräsentation bezeichnen und konfigurieren die Nahtstelle zwischen den einzelnen Mitgliedern oder Gruppen einer Gemeinschaft einerseits und anderseits dem gemeinschaftlichen Verbund mit seinen mal mehr, mal weniger dominanten Aufgaben. Es geht um Autonomie, geteilte Wertorientierungen, Handlungsmacht, Weltbetrachtung und die Vorstellungen gemeinschaftlicher Verbundenheit. Die kulturelle Differenz, die sich in all diesen Fragen feststellen lässt, weist hin auf die epochalen und gesellschaftlichen Unterschiede in Fragen der Akzeptanz und der Organisation von Herrschaft. Es ist dies die Frage nach der Selbstwirksamkeit und dem Selbstbild von politischen Gesellschaften, nach der Reputation von Handlungspartnern und dem Legitimationsglauben an Formen der Aufgaben- und Arbeitsteilung.

Stilbildend ist daher die Aussage des ebenso konservativen wie klugen Politikwissenschaftlers Eric Voegelin, dass in der Frage der politischen Repräsentation und der Partizipation die unterschiedlichsten Stränge der politischen Praxis zusammenlaufen. Im amerikanischen Exil der 1950er Jahre stellt dieser deutschösterreichische Theoretiker auf, wie »eine Gesellschaft« in dieser Debatte um die Organisation des politischen Miteinanders ganz grundlegend ihre politische und symbolische Form, vor allem aber die substantielle »Existenz für ihr Handeln gewinnt« (Voegelin 1952: 19). Das, was hier in der traditionellen Redeweise Voegelins als »Existenz« benannt ist, meint ausdrücklich den Gegenstand, den wir späterhin als "politische Kultur « und als "symbolisches Kapital « diskutieren. Voegelin weist nach, dass sich in der politischen Partizipation die je spezifische Grundform der Selbstinterpretation einer Gesellschaft als ein »politischer« Handlungszusammenhang manifestiert.

Gesellschaften konstituieren und symbolisieren sich in besonderer Weise darin, wie sich in ihnen die Teilhabe an der politischen Herrschaft organisiert, dar- 
stellt und wahrgenommen wird. Zwar wird die historisch reale Teilhabe erst in der konkreten Gestalt, in der sie institutionell realisiert wird, erfahrbar; aber hinter den Institutionen stehen eine symbolische Ordnung und ein kulturelles Selbstbild der politischen Gesellschaft. Dieses ist für die jeweiligen Gesellschaften sehr spezifisch; und es unterliegt einem ebenso gesellschaftsspezifischen konzeptionellen Wandel. Die politische Gesellschaft »artikuliert« in den Formen ihrer politischen Teilhabe und Partizipation ihr politisch-symbolisches Selbstverständnis (Voegelin 1952: 78); man kann mit Voegelin deshalb auch normativ sagen: Gesellschaften beginnen überhaupt erst (wahrhaftig) zu existieren, wenn und insoweit sie dieses symbolische Selbstbild artikulieren. Wird die Artikulation dieses Selbstbildes verunmöglicht, so sind sie substantiell nicht existent, ihrer selbst entfremdet und sich selbst fremd. Die ihnen gemäße technische Form der Partizipation ist der symbolische Kernbereich oder - vice versa - der Kern der symbolischen Gestalt ihrer politischen Sozio-Kultur. In ihm werden sie für sich selbst und andere existentiell sichtbar und lesbar (vgl. auch Göhler 2016: 26).

Mit großer zeitlicher und auch lebensweltlicher Distanz finden wir ähnliche Erkenntnisse bei Cornelius Castoriadis, dem griechischstämmigen französischen Soziologen in den 1970er Jahren. Auch Castoriadis fragt nach dem Zusammenhang zwischen den institutionellen Formen der politischen Gesellschaft und den ihnen zugrunde liegenden kulturellen und emotionalen Dispositionen und Wahrnehmungen. Wie entstehen - so fragt Castoriadis - Vorstellungen einer Gesellschaft von sich selbst? Wie manifestieren sie sich in der technisch institutionellen Realität? Und wie lassen sich der Zusammenhang und das Wechselspiel zwischen den politisch-kulturellen Erwartungen und der institutionellen Realisierung beschreiben?

Für Castoriadis steht im Zentrum die »capacité imaginaire« (Castoriadis 1975: 218); so bezeichnet er die Fähigkeit einer Gesellschaft, eine technisch-institutionelle Realität - z.B. die Institutionen der Repräsentation - unter dem Vorzeichen einer spezifischen Interpretation und Sichtweise zu betrachten. Den Institutionen werden quasi die Vorstellungen, die eine politische Gesellschaft sich von sich selbst und ihrer spezifischen Repräsentationspraxis macht, zugeschrieben; die Gesellschaft eignet sich die Institutionen damit symbolisch an. Die politische Gesellschaft verschafft sich so die "sinnliche Wahrnehmbarkeit" (Castoriadis) oder die »Artikulation« (Voegelin) ihres politischen Selbstverständnisses. In den imaginär definierten Institutionen »drückt sich« die Wirklichkeit der politischen Gesellschaft kulturell real »aus « (Castoriadis 1984: 218); in den konzeptionellen Formen der Repräsentation gewinnt die jeweilige historische politische Praxis eine symbolische Gestalt, die »nicht mehr bloß virtuell« erscheint (Castoriadis 1975: 218).

Wenn dann von Repräsentation oder Partizipation die Rede ist, so geschieht das immer im Kollektiv geteilten Sinn dieser symbolischen Lesart. Was gemeint ist, wird »automatisch« richtig, d.h. politisch korrekt, verstanden. Für die politi- 
sche Gesellschaft wird politische Repräsentation und Partizipation nur in dieser Lesart sichtbar; sie ist - nur in Form dieser symbolischen Imagination - Teil ihrer politischen Kultur. Insofern liegt in der Repräsentation ein keineswegs festgelegtes, sondern ein sehr dynamisches und wandlungsfähiges Konzept. Es ist an die "gesellschaftliche Imagination « gebunden. Und es simuliert quasi einen Raum gesellschaftlicher Partizipation, der mit den imaginären Vorstellungen der Gesellschaft kompatibel sein muss (Nassehi 2002: 46). Wird die institutionelle Form der Partizipation symbolisch erlebt, so wird sie erst dadurch praktisch realisierbar. Es ist dies allerdings ein höchst wechselvoller, äußerst prekärer und dynamischer Prozess gesellschaftlich-kultureller Anerkennung. Und dieser Erfolg ist politisch nicht selbstverständlich, nicht dauerhaft, sondern kontingent.

Der Erfolg politischer Institutionen hängt also von der Lesbarkeit und auch der Vereinbarkeit mit den Vorstellungen der politischen Gesellschaft ab. So wie die politische Gesellschaft die Praxis der politischen Partizipation beständig mit den Parametern ihrer "gesellschaftlichen Imagination« abgleicht, so wird es den Institutionen und den politischen Akteurinnen und Akteuren darauf ankommen, kontinuierlich die Kompatibilität ihres Handelns mit den Vorstellungen der politischen Gesellschaft zu verdeutlichen.

Also gilt: »Jede Macht muss inszeniert werden« (Rosanvallon 2017: 214). Denn das politische System muss inszeniert werden, um seinem Machtanspruch Sichtbarkeit und fühlbare Gestalt zu verleihen. »(Letztlich erreicht) jede politische Macht [...] Unterwerfung [...] auf dem Weg der Theatralität« (Balandier 1980, zit.n. Rosanvallon 2017: 214); sie ist dabei freilich Schauspieler und Publikum, insofern sie ja auch selber an der Selbstdarstellung ihrer selbst teilnimmt. Sie realisiert im besten Fall die eigene Imagination "guter Partizipation«.

Doch die Rede von der Theatralität und die Feststellung einer grundsätzlichen »theatralischen Dimension« (Rosanvallon 2017: 281) des Politischen darf deshalb nicht pejorativ verstanden werden. Denn alle Gesellschaften greifen auf Inszenierungen, Ritualisierungen und die damit verbundenen - teils durchaus metaphysischen - Mystifikationen zurück (Stollberg-Rilinger 2008; Rüther 2008). Diese sind selbstverständlicher Bestandteil unserer Gegenwartsgesellschaften; sie zeigen den »hybriden Charakter der Moderne« und ihres Vernunftglaubens (Latour 2008). Solange Menschen politisch handeln, werden Alltagsmythen, Inszenierungen und Rituale Teil dieser alltäglichen Praxis. Sie sind Teil der politischen Kultur und keine Politik kommt ohne Rückgriff auf metaphysische Vorverständnisse und symbolische Formen aus. Der Legitimitätsglauben der Menschen an die jeweilige politische Herrschaft (Weber 1922) gründet als »soziale Ordnung [...] auf regulativen Fiktionen«, auf Bildern und Vorstellungen (Koschorke 2002: 77). Das gilt selbstverständlich auch für alle Formen der Repräsentativität - von der absolutistischen Monarchie, die ihrer Epoche als repräsentativ galt, bis hin zur Demokratie: Ihre 
jeweilige institutionelle Form muss von den Menschen "geglaubt«, dass heißt als glaubhaft, als effektiv und als angemessen imaginiert werden (Weber 1922).

Alle Formen der Partizipation sind wie jede Form politischen Handelns auf gesellschaftliche "Anerkennungsdynamiken« angewiesen, wie sie schon Max Weber beispielhaft mit seinen »Formen legitimer Herrschaft« beschrieben hat. Damit unterliegen aber alle politischen Systeme auch entsprechenden »Aberkennungszyklen« (Koschorke 2002: 77). An dieser Grenze zwischen politischer Theorie und gesellschaftspolitischer Realität wird deshalb immer wieder die Unabhängigkeit des Wirklichen von der Vernünftigkeit deutlich und zugleich die Abhängigkeit der Wirklichkeit der Partizipation von der gesellschaftlichen Imagination und der politischen Kultur. Die Theoriedebatte hat dies bislang jedoch eher verdunkelt.

Nicht ausgeschlossen ist also, dass sich das gesellschaftlich Imaginäre und die reale Praxis der Partizipation und Repräsentation auseinander entwickeln und voneinander entfremden. Die Gesellschaften erkennen sich dann in der partizipativen Praxis nicht mehr wieder. Im Zuge solcher »Aberkennungszyklen« kommt es zur politischen Legitimationskrise der Institutionen, zum Verlust der Reputation der politischen Eliten und zum Schwund der politischen Identifikation der politischen Gesellschaft mit den partizipativen Institutionen. Das aber ist der Befund der politischen Gegenwart in Deutschland. Es ist zugleich der Hintergrund der aufgeregten Debatte um Politische Kultur, Repräsentation und Partizipation in der Politikwissenschaft.

\section{Politischkulturelle Zyklen der Partizipation}

1808 - 1849 - 1919 - 1945 - 1975 - 1989 - 2000

Die politische Kultur der Partizipation lässt sich natürlich bereits in den Formen der klassischen Vorbilder Athens und Roms aufzeigen. Sie lässt sich in unterschiedlicher Form auch in der Politik der mittelalterlichen Stände und der städtischen Zünfte finden (Stollberg-Rilinger 2008; Rüther 2008). Mit der Herausbildung moderner Gesellschaften in Deutschland und mit der politisch-kulturellen Adaption der Herausforderungen, die sich gesellschaftlich aus der Revolution in Frankreich und der (vorübergehenden) Dominanz Napoleons in Europa ergab, wurde freilich noch einmal ein ganz neues Kapitel politischer Repräsentation aufgeschlagen. Eines, in dem die Sichtbarkeit der Politik als Herrschaft und ihre noch deutlichere Wahrnehmung als übertragene und mandatierte Ausübung politischer Herrschaft eine verstärkte Rolle spielen. Die selbstbewusster werdenden Klientele fordern eine kulturell adaptiertere Form der Repräsentation, dass heißt eine ihnen selbst mehr (im Wortsinne) an-ge-messene, eine sie selbst mehr berücksichtigende und ihnen abgeschaute Form der Repräsentation ein. 


\section{8: Die Preußische Städteordnung}

Zwar wird der unmittelbare politische Kontext der Französischen Revolution in der historisch-akademischen Forschung bisweilen in Frage gestellt, doch angesichts des militärischen und wirtschaftlichen Zusammenbruchs steht es außer Frage, dass die preußische Reformpolitik, die sich darauf richtete, eine neue »Verbindung von Administration und Nation « zu stiften, als eine politisch-kulturelle Reaktion auf die politischen Umbrüche in Frankreich und die Forderung der Aufklärung zu verstehen ist (vgl. Cancik 2007). Die Reformpolitik, die darauf zielte, »eine innigere Teilnahme der Nation an der Administration« zu gestalten, um das »Missverstehen « und den »Widerwillen « gegenüber den Verwaltungsabsichten einzugrenzen (Altenstein 1808, zit.n. Cancik 2007: 2f.), liest sich kulturell als eine zeitgenössische Debatte um Politikverdrossenheit und Bürgerbeteiligung.

Wenn Karl August von Hardenberg seine - mit Freiherr vom und zum Stein entwickelten - Reformen als die »demokratischen Grundsätze in einer monarchischen Regierung« entwickelt, wird deren grundlegender Charakter und ihre über die bloße »Partizipation« der Bürger in der preußischen Gemeindeordnung kulturell hinausweisende Perspektive deutlich. Die Annahme, dass sich in den punktuellen Reformschritten eine kulturelle Neuausrichtung des politischen Systems und eine Reaktion auf einen fundamentalen Mentalitätswandel der Bürgerschaft verstehen lässt, liegt auf der Hand (Koselleck 1967: 259ff.).

Anders als in Frankreich blieb die Partizipation zwar noch als "Ehrenamt organisiert, doch die mit ihr beabsichtigte »Amalgamisierung von Repräsentanten mit den Verwaltungsbehörden « war die Reaktion auf eine kulturelle Krise des politischen Systems (Hardenberg 1808, zit.n. Cancik 2007: 39). Sie sollte eine Diffusion der sich entwickelnden >Wissensgesellschaft mit der Risikokommunikation der öffentlichen Hand bewirken: eine Kommunikation in beiden Richtungen. So zeigte sich einerseits das Drängen der Öffentlichkeit auf Transparenz und auf Mitentscheidung bei der Verausgabung der (von ihr beigebrachten) öffentlichen Mittel; andererseits entwickelte sich aber auch das Bedürfnis der Verwaltung auf Teilhabe an dem zivilgesellschaftlichen Wissen und eine Vermehrung des "Wissens des Staates« (Stein 1807, zit.n. Cancik 2007: 43) bei der anstehenden Organisation der sich entwickelnden Industrialisierung. Insgesamt werde »das öffentliche Zutrauen und die Opinion [...] mehr gewonnen und dadurch wird jedes Geschäft erleichtert « (Hardenberg 1807, zit.n. Cancik 20017: 39). Oder wie die Preußische Städteordnung dies in ihrem $\$ 169$ formuliert:

»Die ganze Geschäftsführung in allen das Cemeinwesen betreffenden Angelegenheiten soll sich zwar zur Begründung der Einheit in dem Magistrat konzentrieren und von demselben geleitet werden. Der Bürgerschaft wird indessen zur lebendi- 
gen Teilnahme an diesen Angelegenheiten die kräftigste Mitwirkung dabei zugestanden«.

Im Zuge dieser historischen Neugestaltung der Partizipationskultur ist also diese Reform der Preußischen Städteordnung von 1808 einer der wenigen - aber für unseren Zusammenhang entscheidenden - Schritte zur Schaffung einer »sichtbaren Institution« des neuen Paradigmas. Bei aller Staatsorientierung sollen doch bei allen Entscheidungen die Vorhaben transparent gemacht und die Meinung der Bürgerinnen und Bürger eingeholt werden.

\section{8: Die Preußische Nationalversammlung}

Die Entfesselung der bürgerlichen Selbsttätigkeit und die Einbindung des bürgerlichen Bereicherungswillens in die politische Kultur und die Verwaltung der Städte durch die Preußische Städteordnung war das hauptsächliche Ziel der preußischen Reformanstrengungen gewesen. Es entsprach der sich herausbildenden politischen Kultur der Partizipation und der realen Angewiesenheiten der verschiedenen politischen Lager aufeinander. Zugleich war die neue Ordnung für alle Beteiligten in ihren teils demokratischen Ansprüchen und in ihrer Praxis ebenso modern wie ungewohnt.

Gleichzeitig entwickelte sich aber auf der Folie der städtischen Partizipation ein allgemeiner gesamtpreußischer Anspruch der Bürger auf Beteiligung am Gemeinwesen (Koselleck 1967: 560ff.). Der Pfad wies deutlich in Richtung auf Gewaltenteilung und Konstitutionalisierung. Der wichtigste Treiber dieser mentalen und kulturellen Dynamik war natürlich das sich herausbildende Primat des Ökonomischen. Der autoritäre Verwaltungsstaat »erlag gleichsam seiner eigenen Schöpfung: der modernen bürgerlichen Gesellschaft« (ebd.: 587). Die rasante Industrialisierung, deren infrastruktureller Erneuerungsbedarf, die finanziell klamme monarchische Staatsverwaltung und das selbstbewusste »enrichissez-vous « des bürgerlichen juste milieu gingen miteinander ein Bündnis ein, das in der DreiklassenPartizipation der Preußischen Verfassung von 1848 mündete.

Wie in allen Zyklen der Partizipationskultur werden auch in dieser, von Friedrich Wilhelm IV. oktroyierten Verfassung strategisch-hinhaltend Zugeständnisse mit Versuchen des monarchischen Machterhaltes verbunden. Die Reservierung der Exekutive und der Ministerverantwortlichkeit, die Teilhabe an der Gesetzesinitiative und das Verkündigungsrecht der Gesetze beim König werden gewährt im Tausch gegen das (Dreiklassen-)Wahlrecht des gehobenen Bürgertums. Die politische Partizipation wird um das Wahlrecht erweitert, das die vorherige ständische Struktur ersetzt, aber zugleich auf die soziale Teilhabe der führenden Wirtschaftsbürger eingegrenzt. Der sich »unsichtbar « herausbildende Anspruch des Bürgertums auf Mitregentschaft, der sich bereits im Vormärz und in den Städten positiviert hatte, 
manifestierte und materialisierte sich in der Verfassung von 1848 nun auf Ebene des Gesamtstaates. Die drei »Klassen«, nach denen der Zensus je nach Einkommen bzw. Steuerlast eingeteilt war, waren jeweils berechtigt, ein Drittel der Abgeordneten zu bestimmen; auf diese Weise bestimmten in der ersten »Klasse« ca. 4 Prozent, in der zweiten »Klasse« ca. 11 Prozent und in der dritten »Klasse« ca. 85 Prozent der Bürger die parlamentarische Partizipation. Im Ergebnis verfügten also ca. 15 Prozent der Wähler über annähernd 67 Prozent der Partizipationsmöglichkeiten.

\section{9: Die Republik}

Mit der fortschreitenden Industrialisierung und der Vergrößerung der Arbeiterschaft wandelte sich notabene auch der institutionell »unsichtbare«, aber in den sozialen Kämpfen deutlich erkennbare Anspruch auf breitere politische Partizipation von Männern und Frauen in der Gesamtbevölkerung. In Preußen waren aber nicht nur der politisch-kulturelle Alltag und die Lage der Industriearbeiter politisch mangelhaft vertreten; auch die katholische Bevölkerung war in Parlamenten und Verwaltungen dramatisch benachteiligt. Auch der - mit 10 Prozent der Einwohner - große Anteil der polnischen Bevölkerung unterlag gravierenden Nachteilen (Hohorst et al. 1975).

Wie bereits in der Preußischen Städteordnung lösten sich die ständischen und bündischen Privilegien der politischen Ordnung unter dem kulturellen Druck der gesellschaftlichen Nivellierung und der Gleichbetroffenheit von Kriegsfolgen, politischem Missmanagement und Autoritarismus auf. Die gewachsene sozialstrukturelle Bedeutung, die sowohl die Arbeiter, aber auch das gebildete nicht-agrarische Bürgertum und auch die Frauen in der Gesellschaft einnahmen, bildete sich mit Beginn der Weimarer Republik deutlich in einem neuen Partizipationsmodus ab: So waren weniger die prinzipiellen diskursiven demokratischen Argumente, sondern vielmehr die kulturelle Gestaltungsbereitschaft und der soziale Durchsetzungswille der in ihren politischen Ambitionen behinderten Gruppen entscheidend für die Einführung eines allgemeinen und gleichen Wahlrechts (Gerhards/Rössel 2000). Es folgte dem Anspruch, die lebenspraktischen Anliegen von Männern und Frauen, Agrariern und Städtern, Protestanten und Katholiken, Polen und Deutsche in ihren unterschiedlichen kulturellen Orientierungen gleichmäßig zu berücksichtigen. Alle deprivilegierten Akteure klagten erfolgreich ihre kulturelle Selbstwirksamkeit in der neuen Republik ein.

\section{5: Professionalisierte Parteienherrschaft als Partizipationskultur}

Es ist unter dem Gesichtspunkt der politischen Kultur äußerst interessant, dass die Parteien, die nach dem 2. Weltkrieg zu den hauptsächlichen Akteuren der Vertretung der politischen und lebensweltlichen Orientierungen der Menschen wurden, 
auf kaum einschlägige Traditionen hierfür und auf keinerlei grundsätzliche Reflexion über ihre Rolle und Aufgabe zurückgreifen konnten (Landshut 1959; Greven 1977; Alemann 1995; 2003). Ihre neue Rolle als umfassende kulturelle Gesamtvertretung ihrer Klientele fanden sie in einer längeren Formierungsphase, in der sie sich zu einem differenzierten Kartell verdichteten (Alemann 2003).

Politisch unterwarfen sich die soziokulturellen Lager diesem Vertretungsoligopol und überantworteten ihm die Vertretung in allen Lebenslagen. Konzeptionell entwickelte sich dies im Rahmen des "Standardmodells« parlamentarischer »Repräsentation «, in dem die parteigebundenen Abgeordneten anstelle und im $\mathrm{Na}$ men ihrer Wähler unabhängig handeln (»Standing for« und »Acting for «; Pitkin 1967; Lietzmann 2016). Aus dieser Fremdermächtigung, die an die obrigkeitsstaatlichen und autoritativen Traditionen der deutschen Politik vor 1945 und auch vor 1933 schlüssig anschloss, entfaltete sich eine Professionalisierung der politischen Vertretung. Schlechte Erfahrungen und beredtes Schweigen im gesellschaftlichen politischen Engagement prägten die Entstehung dieses gesellschaftlich isolierten, obrigkeitsstaatlichen Parteiensystems. Mit dieser bedeutsamen Professionalisierung verband sich deshalb eine starre Trennung von politischen und privaten Arenen. Das Agreement zwischen einer Überantwortung der soziokulturellen Verantwortlichkeit durch die Bürgerinnen und Bürger und der Machtaneignung durch die sich professionalisierenden Parteiorganisationen sah sich dennoch über lange Jahre heftigen Angriffen von Seiten konservativer, verwaltungsorientierter Eliten gegen den sich etablierenden »Parteienstaat« ausgesetzt (Stolleis et al. 1986). Die strukturelle, »sichtbare « Institutionalisierung entfaltete sich im Rahmen auch dieser Debatte in der Rahmengesetzgebung durch das deutsche Parteiengesetz, das die Partizipationsvoraussetzungen einer - dann auch legitimen - Parteiendemokratie technokratisch eingrenzte; die wichtigen Festlegungen der Parteienfinanzierung wiederum - die angesichts eines selbstinteressierten parteilichen Gesetzgebers immer umstritten waren - strukturierte das Bundesverfassungsgericht in dramatischen Urteilen.

Weniger also der 1848 und 1919 durchgreifende Wille zur Beteiligung prägte diesen Partizipationszyklus als vielmehr der Wunsch nach einem gewissen Rückzug aus der politischen Verantwortung, Verantwortungstransfer sowie die Bereitschaft, sich einer Professionalisierung des politischen »Geschäftsverkehrs« auszusetzen. Als eine Konsequenz aus der Eigenerfahrung einer grandios gescheiterten politischen Emphase im Nationalsozialismus und aus der Konzentration auf den privaten Neustart mag diese »Geschäftsübertragung « auf Parteien nachgerade verständlich sein. 


\section{5: Neokorporatismus als Partizipationskultur}

Die Diskussion über "Parteienherrschaft « und den »Parteienstaat « in den 1950er Jahren hatte in den 1970er Jahren ihren Wiedergänger in der Angst vor einem »Verbändestaat«. Denn zunehmend agierten die wichtigsten Berufsverbände und Lobbygruppen als institutionelle Teilhaben an der politischen Partizipation. Gewerkschaften und Unternehmerverbände, Kirchen und Sozialverbände nahmen in politischen Verteilungs- und Organisationsfragen entscheidend an den partizipativen Prozessen teil. Und die politische Kultur löst sich zunehmend von den (Volks-)Parteien und wendet sich in der verschärften ökonomischen Krise mit einem hohen verbandlichen Organisationsgrad einer unmittelbareren Interessenvertretung $\mathrm{zu}$. Die Verbände des Neokorporatismus rücken an die Stelle der Parteien und versinnbildlichen - wie Otwin Massing das früh umreißt - die neuen »Vorstellungssyndrome der eigenen politischen Kultur « (Massing 1965: 271), die sich in der Gesellschaft breit machen. Sie sind Kern einer neuerlichen Schleife der Partizipationskultur.

Die Partizipation, die sich von ständischer zu besitzbürgerlicher Vertretung und von besitz- auf gesamtbürgerliches Wahlrecht verbreitert hatte, sie erweitert nun ihre allgemeinpolitische Professionalisierung um einen sozial spezifischen und sozialkulturell segmentierten, politikfeldspezifischen Professionalisierungsschub. Die Verbände mit ihrer segmentierten Professionalisierung »überspielen« (Stolleis et al.t.1986: 20) die Parteien und treten als neue Stellvertreter der Gesellschaft auf. Die damit verbundenen Ängste und Strukturierungsbedürfnisse kristallisieren sich - wie bei der Debatte um den »Parteienstaat « - in dem Versuch zu einem neuen »Verbändegesetz«: dieses Mal allerdings erfolglos (Alemann/Heinze 1981). Die »Soziokultur«, also die sozialen Akteurinnen und Akteure in ihrer politisch-kulturellen Prägung, sind weiterhin bereit, die eigenen Interessen in die Hände politischer Stellvertreter zu legen; sie bestehen allerdings auf höherer Prägnanz in der Stellvertretung und größere Zielgenauigkeit. Das Bedürfnis nach Selbstwirksamkeit wird heftiger; es entschließt sich freilich noch nicht dazu, auch tatsächlich eigenständig aktiv zu werden.

\section{9: Direktdemokratische Selbstwirksamkeit}

Die 1980er Jahre sind in West- und prominent in Ostdeutschland durch eine Radikalisierung der Selbstorganisation der Partizipation geprägt. NGOs und Bürgerinitiativen und der Ruf nach Referenden und direktdemokratischen Abstimmungen bestimmen die politische Kultur des gesamten deutschsprachigen (und auch des europäischen) Raums (Lietzmann 2000).

Die Anti-Atomkraft-Bewegung und die Frauenbewegung, bürgerinitiierte Mobilisierung quer durch alle Bevölkerungsschichten und kulminierend in der breiten, vielgesichtigen »Wir sind das Volk!«-Bewegung in den ostdeutschen Regio- 
nen und Städten wenden sich von der traditionellen Stellvertretung durch Stände, Parteien, Verbände und selbst- wie fremdernannten Führern ab. Verblüffender Weise ist diese Entwicklung sowohl in West-, als in Ostdeutschland - wenn auch in gänzlich unterschiedlichen Orchestrierungen - sozial als ein überraschendes, in seiner Spezifik bislang kaum thematisiertes Parallelgeschehen, als eine Gleichzeitigkeit des Ungleichzeitigen zu erleben. »Subpolitik« (Beck), »Schattenpolitik« (Alemann), »Kampagnenpolitik« (Leggewie, Greven) lauten die Zuschreibungen der professionellen Beobachter im Westen. Diese informellen, »unsichtbaren« Institutionen treten in die Öffentlichkeit und finden keine traditionelle begriffliche $\mathrm{Zu}$ ordnung. Dabei etablieren sie sich als ganz gegenwärtige, ur-politische Partizipationsmodelle. Sie treten aus der soziokulturellen Fremdheit der vergangenen Epochen in die Sphäre der sichtbaren Institutionen; tatsächlich sind sie die neue Form gesellschaftlich kultureller Partizipation und sie sind wirkmächtig - bis hin zum Zusammenbruch des ostdeutschen Institutionensystems. Ein Merkmal dieses partizipatorischen »Aufbruchs « ist freilich, dass sich die politischen Forderungen als »Interessensbekundungen« nach wie vor an die politischen Vertreter als ausführende Akteurinnen und Akteure richtet; kaum einmal tritt in diesen Bewegungen der Ruf nach innerbürgerschaftlichem Dialog oder nach bürgerschaftlich selbstverwalteten Arealen auf den Plan. Selbstverwaltung bezieht sich zumeist auf die Organisation der Privatheit: z.B. selbstverwaltete Betriebe, Werkstätten oder Kindergärten.

Zugleich mit der bürgerinitiierten Selbstwirksamkeit entwickeln sich in allen Bundesländern, Gemeinden und auch den Parteien und Verbänden die Bewegungen und auch die erfolgreichen Kodifizierungen direktdemokratischer Verfahren. Mit bis heute anhaltender, kontinuierlich ansteigender Unterstützung werden die rechtlichen Institutionen in Ländern und Gemeinden (und auch in der Europäischen Union) durch neue - zum Teil noch informelle - Formate und Performanzen unmittelbarer Bürgerbeteiligung, Bürgerintervention und bürgerschaftliche Entscheidungsverfahren ergänzt (Lietzmann 2011b).

\section{0: Dialogische Verfahren als politischkulturelle Praxis}

Wenn wir dieses Wissen nun auf die neuesten Entwicklungen der politischen Gesellschaft in Deutschland (und auch Europa) übertragen, so lässt sich leicht ein kultureller Wandel konstatieren. Die gesellschaftliche Praxis der Politik und der politischen Beteiligung an der Demokratie ist in Distanz zu den etablierten Formen der repräsentativen Demokratie gegangen und sucht nach neuen Wegen. Auch diese ruhen auf der Basis eines stabilen Demokratieverständnisses und sie verkörpern eine Form der Partizipationskultur. Diese neuen Ausdrucksformen erweitern und verändern allerdings das traditionelle Standard-Modell der repräsentativen Demokratie, das nach wie vor in der politischen Theorie und auch in den Vor- 
stellungen eines Großteils der politischen Eliten vorherrscht. Sie fordern dieses Standard-Modell gesellschaftlich heraus, da sie seine Legitimation in Frage stellen und eine grundlegende Erweiterung verlangen (Lietzmann 2011b; Sippel 2015). Die gesellschaftlichen Ansprüche an politische Mitsprache haben sich mit dem zunehmenden Bildungsstand der Bürgerinnen und Bürger erheblich verändert.

So wie den Menschen in allen anderen Lebenslagen ihres Alltags abverlangt und zugemutet wird, dass sie sich weitgehend in Eigeninitiative um die Besorgung ihrer Interessen kümmern, so fordern sie dies nun auch in Fragen der Politik ein: Denn wenn sich das bürgerliche und gesellschaftliche, also das subjektive wie das allgemeine Selbstbild, immer weiter dem eines »unternehmerischen Selbst« (Bröckling) annähert, ist es weder der Sache nach noch auch nur symbolisch erträglich, dass >in politices $<$ die politischen Akteurinnen und Akteure als vormundschaftlicher >Vater Staat auftreten und die Gesellschaft in einen vorpubertären $\mathrm{Zu}$ stand zurückdrängen wollen.

Sowohl die positiven Ergebnisse der ausgebildeten Wissensgesellschaft als auch die Informationsmöglichkeiten der verfügbaren Medien passen nicht länger in einen Arbeitsmodus politischer Entscheidungen, der den Menschen ihre dialogische und deliberative Beteiligung selbst dann vorenthält, wenn sie diese lautstark einfordern (Bächtiger et al. 2014; Bertelsmann 2014). Anders als das Standard-Modell der politischen Repräsentation berücksichtigen die dialogischen und deliberativen Institutionen, dass nicht länger von einer quasi-homogenen Staatlichkeit als politischem Akteur ausgegangen werden kann. Pluralität der Lebenszuschnitte und die Heterogenität ihrer Ausdrucksformen prägen den Alltag der politischen Gesellschaft. Zwar war auch früher bereits die politische Pluralität größer als sie sich in den politischen Institutionen ausdrückte. Im Gegensatz $\mathrm{zu}$ heutigen Auffassungen wurde sie allerdings homogener wahrgenommen; die Menschen ließen sich auf ein höheres Maß an Vereinheitlichung und Stellvertretung (durch Kirchen, Verbände, Parteien u.v.m.) ein. Diese Bindungen sind im Laufe der Zeit erodiert; sie prägen nicht mehr die Mentalitäten. Die politischen Gesellschaften der Gegenwart bestimmen eher mit einer ausgeprägt konflikthaften Gestalt und einem individuell-leidenschaftlichen Gestus die Debatten. Diese sind daher weit weniger als zuvor auf rationalen Konsens, Folgebereitschaft und Übereinstimmung als vielmehr auf Aushandlung, Kompensation und Koordination hin angelegt.

Wenn all dies zum Selbstbild der modernen Gesellschaften gehört, so sind die geschlossenen Container-Modelle der Repräsentation definitiv überholt. Es braucht dann neue Formen politischer Repräsentation, in denen gerade auch diese Vielfältigkeit der Meinungen und Interpretationen sich darstellen und zur Mitsprache und Mitentscheidung kommen kann. So wie es bei der Parlamentarisierung der Regierungsbildung, bei der Herausbildung der Parteien und auch der Verbände um eine gesellschaftliche Pluralisierung der politischen Repräsentation 
ging, so ist diese auch gegenwärtig in erweiterter Form erforderlich. Dialogische Institutionen erweisen sich als die geeigneten Formate und werden auch symbolisch in diesem Sinne verstanden.

Hinzu kommt der wichtige Faktor der Verantwortungsteilung auch bei Misserfolgen. Das (empirisch vorfindliche) Misstrauen in die Einflussmacht der gewählten Repräsentanten (Rosanvallon 2017) sowie die von diesen selbst empfundene Machtlosigkeit erfordern auch eine institutionelle Performance politischer Repräsentation, die anstelle von Führung und Dezision auf Kontingenzbewältigung und Risikoadäquanz umstellt. Diese Debatte ist in den Rechts- und Sozialwissenschaften weit fortgeschritten. In den politischen Debatten sind die Darstellungsprobleme der politischen Akteurinnen und Akteure allenthalben $\mathrm{zu}$ beobachten: Bei der Flüchtlingspolitik, bei der Energiepolitik, bei der Umwelt- und bei der Industriepolitik sind diese Dimensionen unmittelbar erkennbar. Aber auch im kommunalen Rahmen wird eine Vielzahl von Entscheidungen weit außerhalb der Reichweite der klassischen politischen Strukturen getroffen. Das ist längst in die Vorstellungen der Menschen eingeschrieben. Pseudo-vormundschaftliche und autoritative Attitüden wirken in diesem Zusammenhang deshalb politisch und kulturell völlig unangemessen. Politische »Groß-Kopferte« werden allenthalben als »Scheinriesen« entlarvt, die kleiner werden, je näher man ihnen kommt.

An dieser Stelle kommen nun die dialogischen und deliberativen Dynamiken ins Spiel. Ihr Sinn und ihr Mehrwert liegen in der Verflüssigung der Strategien. Die politischen Problemlagen der Gegenwart erfordern einen hybriden Modus der Steuerung aus Stabilität einerseits, aber auch aus Diskursivität und Flexibilität andererseits, insgesamt aus einer breiten Debatte der Entscheidungsmöglichkeiten und der risikobehaftet getroffenen Entscheidungen. Um die politische Gesellschaft in die Kontingenz der Entscheidungsverläufe einzubeziehen, braucht die Repräsentation eine flexible Vielfalt, einen multiplen, reaktionsfähigen »Misch-Masch « aus institutionellen Darstellungen. Es ist das paradoxe Ergebnis dieser Entwicklung, dass aus der Vielfalt der Wege die Stabilität und Solidität des Ergebnisses erwächst. Repräsentanz und Akzeptanz können politische Entscheidungen in dieser risikohaften Welt nur durch ausdrückliche und umfassende Entscheidungsbeteiligung der Betroffenen erzeugen; diese folgen strikt einem soziokulturellen Impuls nach Selbstwirksamkeit. So gilt in der politischen Gesellschaft derzeit der Grundsatz: »Own the process, own the result « (Shapiro 2002: 198). Nur durch Mitentscheidung entstehen in der politischen Gesellschaft erwartete und geforderte politische Verantwortungsteilung und die kulturelle Akzeptanz für die in der Kontingenz der Gegenwart (zwangsläufig auch) eintretenden Misserfolge. 


\section{Literatur}

Albert, Matthias et al. 2015: Ders./Hurrelmann, Martin/Quenzel, Gudrun und TNS Infratest. Jugend 2015: 17. Shell Jugendstudie. www.shell.de/aboutshell/our-commitment/shell-youthstudy-2015.html. Zugegriffen: 15.Oktober 2015.

Alemann, Ulrich von 1995: Parteien. Reinbek: Rowohlt.

Alemann, Ulrich von 2003: Das Parteiensystem der Bundesrepublik Deutschland. (3.Aufl.). Opladen: Leske+Budrich.

Alemann, Ulrich von/Heinze, Rolf G. 1981: Verbände und Staat: Vom Pluralismus zum Korporatismus. Analysen, Positionen, Dokumente. Opladen: Westdeutscher Verlag.

Alemann, Ulrich von/Rauh, Joachim/Klewes, Christina 2011: Die Bürger sollen es richten. Aus Politik und Zeitgeschehen 61 (44-45): S. 25-32.

Bächtiger, André/Setala, Maija/Grönlund, Kimmo 2014: Towards a New Era of Deliberative Mini-Publics. In: Ders./Kimmo Grönlund/Maija Setala, Deliberative Mini-Publics. Involving citizens in the democratic process Colcester: ECPR, S. 225-247.

Bertelsmann Stiftung 2014. Partizipation im Wandel - Unsere Demokratie zwischen Wählen, Mitmachen und Entscheiden. Gütersloh: Bertelsmann Stiftung.

Bleek, Wilhelm 2001: Geschichte der Politikwissenschaft in Deutschland. München: Beck.

Cancik, Pascal 2007: Verwaltung und Öffentlichkeit in Preussen. Kommunikation durch Publikation und Beteiligungsverfahren im Recht der Reformzeit. Tübingen: Mohr Siebeck.

Castoriadis, Cornelius 1975: L'institution imaginaire de la société. Paris [Zit. n. d. dt. Ausgabe: Gesellschaft als imaginäre Institution. Frankfurt a.M.: Suhrkamp 1984].

Castoriadis, Cornelius 1984: Gesellschaft als imaginäre Institution/. Frankfurt: Suhrkamp 1984./[franz.: L'institution imaginaire de la société./Paris: Gallimard 1975.]

Gerhards, Jürgen/Rössel, Jörg 2000: Akteure, Interessen und Deutungsmuster im politischen Diskurs: Eine Analyse derr Debatte über das gleiche Wahlrecht in Preußen (1890-1918). In: Sociologica Internationalis Bd. 38, H. 2, S. 181-218.

Göhler, Gerhard, 2016: Symbolische Repräsentation aus deutscher und französischer Sicht. In: Paula Diehl/Felix Steilen (Hg.), Politische Repräsentation und das Symbolische, Historische, politische und soziologische Perspektiven, Wiesbaden: Springer VS. S. 23-50.

Greven, Michael Th. 1977: Parteien und politische Herrschaft. Meisenheim a.G.: Hain, Anton.

Greven, Michael Th. 1999: Die politische Gesellschaft. Opladen: Leske+Budrich. 
Grunberg, Gérard 2011: Des sciences politiques à la science politique. Le rôle de Sciences Po dans développement de la science politique en France. In: Le science politiche. Modelli Contemporanei Vittor Ivo Comparato/Regina Lupi/Giorgio E. Montanari. Milano, FrancoAngeli, 2011. S. 33-46

Hildebrand, Mathias 2001: Politik aus der Erfahrung des Totalitarismus: Cornelius Castoriadis, Claude Lefort und Marcel Gauchet. In Hans J. Lietzmann (Hg.), Moderne Politik, Opladen: Leske+Budrich S. 311-330.

Hohorst, Gerd et al.t. 1975: In: Gerd Hohorst/Jürgen Kocka/Gerhard A. Ritter, Sozialgeschichtliches Arbeitsbuch, Materialien zur Statistik des Kaiserreichs 1870-1914. München: Beck.

Jobard, Fabien/Laborier, Pascale 2002: Editorial. In: Sciences politiques allemandes. Politix, Vol. 15, H. 59, Paris: Hermes-Sciences. S. 9-14.

Koschorke, Albrecht 2002: Macht und Fiktion. In: Ders./Thomas Frank/Susanne Lüdemann/Ethel Matala de Maza, Des Kaisers neue Kleider, Über das Imaginäre politischer Herrschaft, Frankfurt a.M.: Fischer. S. 73-84.

Koselleck, Reinhart 1967: Preußen zwischen Reform und Revolution. Stuttgart: Ernst Klett [zit.n. d. Ausgabe 3. Auflage, 1981].

Landshut, Siegfried 1959: Vorwort zu Maurice Duverger, Die politischen Parteien. Tübingen: Mohr (Siebeck), S. VII-VIII.

Latour, Bruno 2014: Sollten wir nicht mal über Politik reden?. In: Trivium, Heft 16. Lietzmann, Hans J. 1996: Politikwissenschaft in der Bundesrepublik Deutschland. In: Hans J. Lietzmann/Wilhelm Bleek, Politikwissenschaft Geschichte und Entwicklung in Deutschland und Europa, München: Oldenbourg, S. 38-76.

Lietzmann, Hans J. 2000: „Greenpeace« als politischer Akteur. Eine neue Generation der Interessenvertretung betritt die politische Bühne. In: Ulrich Willems/Thomas von Winter (Hg.), Politische Repräsentation schwacher Interessen, Opladen: Leske+Budrich, S. 261-285.

Lietzmann, Hans J. 2002: La retour de la science politique allemande. Une continuité paradoxale dans la période de l'après guerre. In: Politix. Revue des Sciences Sociales du Politique. Paris: Hermes-Science. Vol. 15, H. 59, S. 15-33.

Lietzmann, Hans J. 2011a: German Political Science. Traditions and genealogical steps. In: Vittor Ivo Comparato/Regina Lupi/Giorgio E. Montanari, Le science politiche. Modelli Contemporanei. Milano: FrancoAngeli. S. 59-71.

Lietzmann, Hans J. 2011b: Auf zum nächsten Level! Bürgerbeteiligung im Wandel der Zeit. In: »Bürgerbeteiligung 3.0. Vom Volksbegehren zur OccupyBewegung«. Politische Ökologie. 29.Jg., 1, Heft 127, S. 28ff.

Lietzmann, Hans J. 2012: Kontingenz der Repräsentation: Bürgerbeteiligung. In: Olaf Asbach/Rieke Schäfer/Veit Selk/Alexander Weiß (Hg.), Zur kritischen Theorie der politischen Gesellschaft, Wiesbaden: Springer VS. S. 165-188.

Lietzmann, Hans J. 2016: Die Demokratisierung der Repräsentation. Dialogische Politik als neue Form der repräsentativen Demokratie. In: Manuela Glaab (Hg.), 
Politik mit Bürgern - Politik für Bürger, Praxis und Perspektiven einer neuen Beteiligungskultur, Wiesbaden: Springer VS, S. 41-59.

Lietzmann, Hans J. 2018: Citizenship, Democracy and the Iconology of Politics: A Plea for an Iconological Turn in democratic Theory. In: A. Björk/Claudia Wiesner/Hanna-Mari Kivistö/Katja Mäkinen (Ed.), Shaping Citizenship, A Political Concept in Theory, Debate, and Practice, New York/London: Routledge, S. 55-70.

Massing, Otwin 1965: Parteien und Verbände als Faktoren des politischen Prozesses, Aspekte politischer Soziologie. In: Gisela Kress/Dieter Senghaas, Politikwissenschaft, Frankfurt a.M.: Fischer.

Nassehi, Armin 2002: Politik des Staates oder Politik der Gesellschaft? Kollektivität als Problemformel des Politischen. In: Kai- Uwe Hellmann/Rainer SchmalzBruns (Hg.), Theorie der Politik, Niklas Luhmanns politische Soziologie, Frankfurt a.M.: Suhrkamp. S. 38-59.

Nucci, Loreto Di 2011: Le facolta di Scienze politiche in Italia. In: V. I. Comparato/R. Lupi/G. E. Montanari (Hg.), Le science politiche, Modelli Contemporanei (Political Sciences, Contemporary Modells) Perugia: FrancoAngeli. S. 71-84.

Palonen, Kari 1998: Das »Webersche Moment«: Zur Kontingenz des Politischen. Opladen: Westdeutscher Verlag.

Pitkin, Hannah Fenichel 1967: The Concept of Representation. Berkeley/Los Angeles/London: University of California Press.

Preußische Städteordnung 1808: Ordnung für sämtliche Städte der preußischen Monarchie mit dazu gehöriger Instruktion, Behuf der Geschäftsführung der Stadtverordneten bei ihrem ordnungsgemäßen Versammlungen. Vom 19. November 1808, PrGS 1808, S. $324 \mathrm{ff}$.

Rohe, Karl 1987: Politische Kultur und der kulturelle Aspekt von politischer Wirklichkeit. Konzeptionelle und typologische Überlegungen $\mathrm{zu}$ Gegenstand und Fragestellung Politischer Kulturforschung. In: Dirk Berg-Schlosser/Jakob Schissler (Hg.), Politische Kultur in Deutschland, Bilanz und Perspektiven der Forschung, Politische Vierteljahreshefte/Sonderband Vol. 18, S. 39-48.

Rohe, Karl 1990: Politische Kultur und ihre Analyse. Probleme und Perspektiven der politischen Kulturforschung. In: Historische Zeitschrift, Band 250, Heft 1, S. 321-346.

Rosanvallon, Pierre 2017: Die Gegen-Demokratie. Politik im Zeitalter des Misstrauens. Hamburg: Hamburger Edition (Franz. Le Seuil: Paris 2006/2014).

Roselle, Laura et al.t. 2014: 'Strategic narrative: A new means to understand soft power . In: Dies./Alister Miskimmon/Ben O'Loughlin, Media, War \& Conflict, Vol. 7, No. 1, S. 70-84.

Rüther, Stephanie 2008: Rituale der Ratswahl in der vormodernen Stadt. In: Dies./Matthias Puhle/Jutta Götzmann/Gerd Althoff, Spektakel der Macht, Rituale im Alten Europa 800-1800, Darmstadt: Primus. S. 33-37. 
Shapiro, Ian, 2002: Optimal Deliberation. In Journal of political Philosophy. 10 (2): S. 196-211.

Sippel, Hanns-Jörg 2015: Auf dem Weg zu einer (neuen) Kultur der Bürgerbeteiligung. In: Ders.(Hg.), Kursbuch Bürgerbeteiligung, Berlin, Dt. Umweltstiftung.

S. 22-47.

Stein, Rokkan 1961: Mass Suffrage, Secret Voting, and Political Participation. In: Europäisches Archiv für Soziologie 2, S. 132-152.

Stein, Rokkan 2000: Staat, Nation und Demokratie in Europa. In: Peter Flora (Hg), Frankfurt a.M.

Stollberg-Rilinger, Barbara 2008: Des Kaisers alte Kleider. Verfassungsgeschichte und Symbolsprache im alten Reich. München: Beck.

Stolleis, Michael/Schäffer, Heinz/Rhinow, René A./Lange, Klaus/Breuer, Rüdiger 1986: Parteienstaatlichkeit: Krisensymptome des demokratischen Verfassungsstaats? VVDStrL 44, Berlin: De Gruyter.

Vormweg, Christoph 2018: »Wenn ich schreibe, sage ich alles«. Ernaux, Annie/Louis, Èduard/Eribon Didier. In: Frankreichs Schriftsteller mit Soziologenbrille. Deutschlandfunk Kultur. 07.01.2018. www.deutschlandfunkkultur.de/Schriftsteller-mit-soziologenbrille-wenn-ich-schreibe-sage.974.de.html?dram:article_id=407714.

Voegelin, Eric 1952: The New Science of Politics. An Introduction. (zgl. Walgreen Lectures/University of Chicago 1951: Truth and Representation) Chicago. [Zit. n. d. dt. Ausgabe: Die neue Wissenschaft von der Politik. München: Anton Pustet 1959].

Weber, Max 1922: Wirtschaft und Gesellschaft. (zit. i. d. F. Tübingen: J.C.B. Mohr. 1980)

\section{Online}

Infratest-Dimap. 2013. Bürger und Parlamente - Bürger gegen Parlamente. www.infratest-dimap.de/fileadmin/_migrated/content_uploads/Parlamentarismus_1302.pdf. Zugegriffen: 15. Oktober 2015. 


\section{Interdisziplinarität, Politische Repräsentation und das Imaginäre \\ Plädoyer für eine neue Perspektive der politischen Kulturforschung}

Paula Diehl

\section{Die kulturelle Wende}

Mit der Wahl von Donald Trump zum Präsidenten der Vereinigten Staaten von Amerika hat sich Grundsätzliches in der Politik verändert. Seitdem ist deutlich geworden, dass neue Parameter für die Beurteilung von Aussagen und Handlungen in die politische Arena eingezogen sind. Hat es im Fall des ehemaligen italienischen Premiers Silvio Berlusconi Jahre gedauert, bis die Wissenschaft die kulturellen Transformationen der Politik durch und mit Berlusconi reflektiert hat - wie etwa die interdisziplinäre Arbeit aus der Soziologie, Kommunikationswissenschaft und Linguistik zeigt (Bolasco et.alt. 2006) -, ist kurze Zeit nach dem Amtsantritt des neuen US-amerikanischen Präsidenten die Beunruhigung nicht nur der Politik, sondern auch der Politikwissenschaft sichtbar geworden. Sogar MainstreamZeitschriften wie Perspectives on Politics der American Political Science Association haben sich dem Problem gewidmet (siehe die Ausgabe von Oktober 2018). Es sieht allerdings so aus, als ob der disziplinäre politikwissenschaftliche Zugang zu dieser neuen Form der Politik nicht mehr ausreicht, um Phänomene wie Trump und Berlusconi $\mathrm{zu}$ analysieren und $\mathrm{zu}$ verstehen. Vielmehr muss davon ausgegangen werden, dass sich die Politikwissenschaft und auch die Politische Theorie für Impulse aus Nachbardisziplinen öffnen müssen.

Von Berlusconis Fall wissen wir bereits, dass seine neue Art der politischen Kommunikation und Organisation das gesamte politische Spektrum in Italien geprägt hat. Seine saloppe Sprache, die Bedeutungsverschiebungen grundlegender demokratischer Begriffe wie Freiheit sowie die Einführung eines apolitischen Vokabulars in das politische Repertoire haben auch den politischen Diskurs seiner Gegner beeinflusst (Bolasco u.a. 2006). Ferner färbte der dekonstruierende Inszenierungsstil des »Cavaliere«, wie sich Berlusconi von der Presse nennen ließ, den Zugang nicht nur der Parteien, sondern auch der Wählerinnen und Wähler 
zur Politik und zu den politischen Institutionen (Diehl 2008). Manche Politikwissenschaftlerinnen und Politikwissenschaftler schätzen sogar, dass ohne Berlusconi Beppe Grillo und die 5-Sterne-Bewegung nicht möglich gewesen wären (Diamanti 2014). Dies wird sowohl im Sinne einer Übernahme von Berlusconis Brüchen mit dem politischen Code als auch als eine Reaktion auf seinen apolitischen Stil gedeutet.

Bei Trump zeichnet sich mit der Popularisierung des Argumentations- und Denkmusters von "alternativen Fakten« und »Fake News« eine Wende in der Wahrnehmung der Politik und im politischen Handeln ab, die noch zu reflektieren ist. Trump bezieht Elemente der Reality-Shows in die Politik mit ein. In RealityShows wie The Apprentice, in der Trump sich selbst spielte, konnte er jahrelang ein Celebrity-Image konstruieren, das zwischen Realität und Fiktion liegt. Vergleicht man die Show mit Trumps Selbstdarstellung als Präsident und sogar mit seinen politischen Entscheidungen, werden Elemente der Reality-Shows in der Politik deutlich. Ein gutes Beispiel dafür liefern seine Interviews. Sie sind wie eine Soap-Opera aufgebaut, bei der die Zuschauerinnen und Zuschauer bei der Stange gehalten werden, weil sie nicht alles auf einmal erfahren, sondern immer wieder auf die nächste Folge verwiesen werden. Der oft von Trump ausgesprochene Satz »We'll see what happens", um ein Interview mit Reportern abzuschließen, ist nicht nur bloßer Verlegenheitsgestus, um heiklen Fragen auszuweichen, er sichert sich die Aufmerksamkeit der Zuschauerinnen und Zuschauer, indem er verspricht, beim nächsten Mal die gewünschte Information zu geben bzw. die weitere Entwicklung der Geschichte zu erzählen. Schaut man sich seine so genannten »rallies« an, wird eine andere bekannte Struktur aus der Unterhaltungsbranche deutlich: die postmoderne Ringkampf-Unterhaltung aus den Wrestling-Shows. Auch hier ist semi-fiktionale Unterhaltung das grundlegende Element seiner Auftritte, hinzu kommt der Konsum von Gewaltinszenierung und -sprache als Gelegenheit zur Entladung von Emotionen (Diehl 2017). Ferner nutzt Trump Tweets gezielt, um Skandale und Provokationen zu schaffen, womit er sein Publikum aufmerksam hält. Schließlich bildet sich die Struktur der Reality-Shows auf das politische Handeln des Präsidenten ab, etwa, wenn man die Entlassungsdynamiken der Mitglieder seines Kabinetts beobachtet. Zwei Drittel der Mitglieder wurden entlassen oder gezwungen, zurückzutreten. ${ }^{1}$ Ironischerweise gründete sich der Spannungswert der Sendung The Apprentice genau auf die Ausscheidung der Kandidaten aus der Show. Der Satz »You are fired!« bildete den Höhepunkt jeder Folge.

1 Siehe: Denise Lu and Karen Yourish: You're Hired! You're Fired! Yes, the Turnover at the Top of the Trump Administration Is ... »Unprecedented.«; in: The New York Times, update 20. Dezember 2018. https://www.nytimes.com/interactive/2018/03/16/us/politics/all-themajor-firings-and-resignations-in-trump-administration.html Letzter Abruf, 26.12.2018, 15.00 Uhr. 
Berlusconi und Trump haben ihre jeweiligen nationalen politischen Kulturen geprägt und prägen sie weiterhin, insofern sie Produzenten eines neuen politischen Stils sind und eine neue Logik des politischen Handelns durchsetzen, die durchaus Einfluss auf andere politische Akteurinnen und Akteure ausüben. Aber sie sind zugleich Produkte politisch-kultureller Transformationen, ja, Manifestationen tiefgehender Veränderungen des politischen Imaginären. Ihr Erfolg war nur möglich, weil sie sich an der Spitze neuer Tendenzen der politischen Kultur positionieren konnten. Deswegen müssen beide Fälle sowohl als Symptom als auch als Ursache eines umfassenden politisch-kulturellen Wandels reflektiert werden. $\mathrm{Zu}$ diesem Wandel gehören ebenso post-demokratische Erscheinungen (Crouch 2008) und die damit verbundene Ablehnung politischer Institutionen und Parteien wie technologische, massenmediale, popkulturelle und Life-Style Transformationen.

Solche Veränderungen sind nicht erst mit dem Web 2.0 erschienen. Tiefgehende Transformationen der Politik reichen bis in die 1990er Jahre zurück. Genau mit Blick auf das Material aus den 1990er Jahren machte Andreas Dörner auf das Phänomen des Politainments (Dörner 2001) aufmerksam und zeigte, dass in massenmedialen Demokratien Politik stärker mit Unterhaltung verwoben ist, als den Politikwissenschaftlerinnen und Politikwissenschaftlern lieb wäre. Fast 20 Jahre nach Dörners Diagnose bleibt der Trend zum Politainment weiterhin bestehen, aber er ist angesichts zunehmender Fragmentierung des Publikums, Pluralisierung von Lifestyles, neuer digitaler Produktionsbedingungen sowie neuer Interaktionsmöglichkeiten durch soziale Medien und Smartphones komplexer geworden. Sicher ist: Nicht nur haben sich die Kommunikationsstrategien der politischen Akteurinnen und Akteure verändert, auch die Repräsentation und Wahrnehmung von Politik ist komplexer geworden, denn politische Information, Auseinandersetzungen und sogar die Politikerinnen und Politikern selbst präsentieren sich zunehmend in einem unsicheren Status, der sich irgendwo zwischen Realität und Fiktion bzw. zwischen Politik und Unterhaltung bewegt. Wenn also Barack Obama in einer fiktiven Serie als Präsident der USA erscheint oder die ehemalige Präsidentin der Schweiz, Micheline Calmy-Rey, als Präsidentin in einem TV-Gesangwettbewerb auftritt, sind die Zuschauerinnen und Zuschauer nicht sicher, ob diese Auftritte als fiktiv oder real zu betrachten sind bzw. ob sie zur Politik oder Unterhaltung gehören (Diehl 2010). Vor diesem Hintergrund müssen Trump und Berlusconi als Radikalisierung eines umfassenden politisch-kulturellen Wandels reflektiert werden. Sie geben Hinweise auf eine strukturelle Transformation des politischen Imaginären (Diehl 2015), die die Vorstellungen von Politik und dem Politischen prägen.

Doch die Transformationen des politischen Imaginären durch die Unsicherheit des Status politischer Repräsentation ist nicht das einzige Phänomen, das die Politische Kulturforschung in ihr Analysefeld miteinbeziehen muss. Neben dieser strukturellen Veränderung ist auch eine konjunkturelle Entwicklung zu beachten: 
die Wende zu politischen Kulturkämpfen ${ }^{2}$. Rechtspopulistische und rechtsextremistische Strömungen in Europa, USA, Lateinamerika und Indien gestalten die politische Auseinandersetzung als Kampf um die Kultur, kulturelle Identität, Ethnizität und sogar um die Bestimmung von Sexualität und Gender. Hier werden kulturelle Praktiken, Narrative, Ästhetik und Symbolik im Sinne einer anti-pluralistischen Identität politisiert. Man beobachtet eine Politisierung nicht nur des Privaten - wie es etwa die Anti-Gender Bewegungen zeigen -, sondern auch eine Politisierung des Präpolitischen (Kogl 2009).

Die Identitäre Bewegung kann als Paradebeispiel dieser politischen Besetzung des Präpolitischen und des Privaten gesehen werden. Sie ist europaweit vernetzt und bewegt sich im Rahmen »metapolitischer « Aktionen. Diese zielen auf Themen wie »Ablehnung der multikulturellen Gesellschaft, militanter Gemeinschaftssinn, Widerstand gegen außereuropäische Einwanderung, Ethno-Differentialismus, Ablehnung des jakobinischen Nationalismus zugunsten einer Aufwertung der >patries charnelles`, Bindung an ein Europa der Ethnien und nicht an den antieuropäischen Souveränismus« (Camus 2017: 233). Auffallend ist auch die professionelle Nutzung des Internets und der sozialen Netzwerke. So konnte der »Bloc Identitaire« aus Frankreich dazu beitragen, »die Vorstellungen wie die vom >großen Austausch $<$ [der autochthonen Bevölkerung durch Migranten] oder Ideen wie die einer $>$ Remigration [ [er Migranten] « zu verbreiten (ebd.). Aber auch rechtspopulistische Bewegungen, Parteien und Internetforen operieren im Feld der Kulturkämpfe. Parteien wie die AfD in Deutschland, die FPÖ in Österreich oder die Freiheitspartei von Geert Wilders in den Niederlanden profilieren sich hauptsächlich mit dem politischen Kampf um die kulturelle Hegemonie. Ihre Kulturpolitik bildet Synergieeffekte mit rechtsradikalen Foren und Gruppierungen, schafft aber auch graue Zonen zwischen rechtsextremistischen Ideologemen und demokratischen Prinzipien. Solche Diskurse bleiben nicht isoliert, sondern wirken auf die politische Kultur insgesamt. Inzwischen ist die Zirkulation rechtsextremistischer Ideologeme in der demokratischen Öffentlichkeit und sogar ihre Übernahme durch Mainstream-Politikerinnen und -Politiker - teilweise in gemilderter Form - gut dokumentiert (Wodak 2016).

Die aktuelle kulturelle Wende der Politik kann in zwei Dimensionen verstanden werden: Während die Vermengung von Politik und massenmedialer Unterhaltung ein strukturelles Phänomen ist, zeigen sich die Kulturkämpfe in rechtsextremistischen und rechtspopulistischen Spektren als konjunkturelle Tendenz. Man könnte denken, dass beide Dimensionen wenig miteinander zu tun haben, doch ein Blick auf Donald Trumps spielerischen Umgang mit rechtsextremistischen Topoi

2 Zum Thema diskutierte der Arbeitskreis Politik und Kultur der DVPW im März 2019 im Rahmen der von Jörn Knobloch, Jan Christoph Suntrup und Odila Triebel organisierten Tagung Die neuen Kulturkämpfe an der Universität Potsdam. 
wie Anti-Muslime-Ressentiments, Diskriminierung von Mexikanerinnen und Mexikanern und sogar Rassismus zeigt, dass man von einer Überlagerung von Politainment und Kulturkampf ausgehen muss. Wie sehr sich die zwei Dimensionen der kulturellen Wende der Politik im Westen mischen, zeigt das Symbol der Identitären Bewegung, das Lambda (vgl. auch den Beitrag von Wolfgang Bergem in diesem Band). Das Symbol hat seinen Ursprung in der Geschichte Spartas, wurde aber durch den Blockbuster Film 300 des US-amerikanischen Regisseurs Zack Snyders von 2006 als Sinnbild des Kampfes um die eigene Identität der Spartaner bekannt. Die Identitäre Bewegung in Europa appropriierte dieses Symbol, um ihren Kulturkampf gegen Muslime, Gender-Politik und linke Diskurse einzuleiten (Mrozek 2017).

Zusammengefasst: Die aktuelle kulturelle Wende der Politik vollzieht sich in zwei Dimensionen. Die erste Dimension ist strukturell und mit neuen Technologien und neuen Nutzungspraktiken der Massenmedien verbunden, die die Gesellschaft tief prägen. Die zweite Dimension ist ideologisch und konjunkturell. Die hier beschriebenen Transformationen aktueller Politik sind nur zwei Beispiele für die Herausforderungen an die Politikwissenschaft. Doch sie zeigen bereits, wie Politik und Kultur mehr denn je miteinander verwoben sind. Diese Verwobenheit zwingt die Politikwissenschaft und die Politische Theorie, die Demarkation ihres Feldes zu überdenken und die Grenzen ihrer Gegenstände, Methoden und Theorien zu erweitern.

\section{Politische Kultur »reloaded «}

Angesichts der aktuellen kulturellen Wende der Politik scheint das traditionelle politikwissenschaftliche Instrumentarium nicht auszureichen, um die gegenwärtige politische Situation und die aktuellen Ereignisse zu analysieren. Ist die Politikwissenschaft deswegen in der Krise? Zumindest gibt es Indizien dafür. Zwei davon sind die methodologische Krise der Umfragen und das Bedürfnis nach einer theoretischen Reformulierung des Konzepts der politischen Repräsentation.

Die immer wieder beschriebene »Volatilität des Wählerverhaltens« erreichte ein Niveau, das die Effektivität von Umfragen etwa beim Brexit oder bei Trumps Wahl in Frage stellte. Nach beiden Ereignissen gerieten die politischen Umfragen in die Krise und mit ihnen auch die Prognosekraft ihrer Methoden. Inzwischen wird dieses Manko durch die Einbeziehung anderer Parameter, etwa durch die Berücksichtigung von Aktivitäten in sozialen Medien, ergänzt. Ob diese Ergänzung tatsächlich eine wirksame Korrektur bedeutet, scheint noch umstritten zu sein (Mellon/Prosser 2017).

Die zweite Krisenerscheinung kommt aus der englischsprachigen politischen Theorie und betrifft das Verständnis von politischer Repräsentation. Nach dem 
»standard account « wird politische Repräsentation als Vertretung verstanden. Dazu gehören die Handlungen politischer Repräsentanten. Hanna Pitkin nannte sie »representation as acting for « (Pitkin 1967). Zu diesem Verständnis von Repräsentation gehören weder Symbole, Bilder und Inszenierungen noch kulturelle Praktiken oder kollektive Vorstellungen. In der englischsprachigen Politikwissenschaft entdeckten aber Politiktheoretikerinnen und Politiktheoretiker im ersten Jahrzehnt des neuen Milleniums den so genannten. »representative turn« (Näsström 2011). Damit verbunden ist eine konstruktivistische Sicht auf die politische Repräsentation und mit ihr das Bewusstsein, dass politische Repräsentation von politisch-kulturellen Faktoren abhängig ist. In beiden Krisenerscheinungen wird deutlich, dass Politik und Kultur verwoben sind und dass die Theorien und Methoden der Disziplin diese Verwobenheit berücksichtigen müssen.

Die kulturelle Wende der Politik zeigt auf etwas, das seit langem ein Problem ist: Politik ist nie abgekoppelt von Gesellschaft, sondern entsteht in ihr und muss entsprechend auf die gesellschaftlich-kulturellen Praktiken, Interaktionen, Produktionen und Vorstellungen verweisen. In zunehmend komplexeren Gesellschaften wird diese Verwobenheit zwischen Politik, Gesellschaft und Kultur immer schwerer zu entzerren. Traditionelle Forschungsdesigns, die Politik nur institutionell definieren und eine starke Trennungslinie zwischen Politik und Kultur ziehen, sind in ihren Erklärungsmöglichkeiten eingeschränkt. Oft vernachlässigen sie grundlegende Bereiche der Politischen Kultur, wie die politische Unterhaltung. Diese werden an die Medien- und Kulturwissenschaften abgegeben, obwohl sie genuin zum Politischen gehören. Diese Gegenstände als nicht-politikwissenschaftlich zu deklarieren, löst aber das Problem nicht, sondern verschiebt es $\mathrm{zu}$ den benachbarten Disziplinen. Für die Politische Kulturforschung hat das Bewusstwerden dieses Sachverhalts eine Öffnung ihres Feldes gegenüber kulturellen Praktiken, online- und offline-Interaktionsformen, Life-Styles, massenmedialen Produktionen und Unterhaltungsformen zur Konsequenz. Dabei sind die Transformationen der politischen Kultur durch politische Unterhaltung, Aktivitäten in sozialen Medien oder präpolitische kulturelle Praktiken grundlegend, um die Machbarkeit, Sagbarkeit und Vorstellbarkeit des Politischen zu verstehen, ohne die politische Handlungen, Diskurse und Normen nicht nachvollziehbar sind.

Die Politische Kulturforschung scheint deswegen dafür prädestiniert zu sein, wichtige Impulse für die gesamte Disziplin zu geben. Denn sie richtet ihren Blick auf den Intersektionsbereich von Politik, Kultur und sozialer Praxis. Angesichts der kulturellen Wende der Politik muss die Politische Kulturforschung aktualisiert und revitalisiert werden - der Titel dieses Sammelbandes schlägt dafür das englische Wort »reloaded« vor. Der vorliegende Text versteht sich als Beitrag zu dieser Revitalisierung. Er bietet einen Ansatz, der auf die interdisziplinäre Öffnung der Politischen Kulturforschung und der Politischen Theorie zielt, den Begriff der politischen Repräsentation erweitert und das politische Imaginäre konzeptualisiert. 
Aus der Perspektive der Politischen Kultur »reloaded« ist Politische Kultur mehr als die Orientierung der Bürgerinnen und Bürger gegenüber dem politischen System (Almond/Verba 1989; Greiffenhagen 2009). Sie ist vielmehr auch das Zusammenwirken von kulturellen Praktiken, Diskursen, Symbolen, Bildern und Inszenierungen, mit denen Politik vorgestellt und gelebt wird. Politik und politische Kultur haben eine symbolische und eine imaginäre Dimension; sie zu berücksichtigen scheint angesichts der kulturellen Wende notwendiger denn je. Deshalb muss eine erneuerte Politische Kultur Methoden und Theorien integrieren, die die Untersuchung der symbolischen und imaginären Dimensionen der Politik ermöglichen. Allerdings bedeutet diese Erneuerung nicht, dass die traditionellen Herangehensweisen der Politischen Kulturforschung wie die Erhebung von politischen Präferenzen, die Untersuchung von politischem Verhalten oder Sozialisationsmustern überflüssig geworden wären. Im Gegenteil, diese sind nach wie vor grundlegend, doch sie sollen mit neu zu explorierenden Feldern verknüpft werden. Die Frage ist nur: Wie soll dies gehen? Hierfür werden hier drei Vorschläge gemacht: Erstens sollen sich die Politische Kulturforschung und die Politische Theorie für mehr Interdisziplinarität öffnen. Zweitens wird das Konzept der politischen Repräsentation auf das Symbolische erweitert. Drittens wird das Konzept des politischen Imaginären sowohl für die Politische Theorie als auch für die Politische Kulturforschung produktiv gemacht.

\section{Mehr Interdisziplinarität wagen!}

Konzeptionelle Überlegungen, die mehr Interdisziplinarität ermöglichen, sind allerdings rar und werden oft nicht systematisch verfolgt. Zwei Versuche sind für die Revitalisierung der Politischen Kulturforschung von besonderem Interesse: der Appell von Wendy Brown für mehr Interdisziplinarität in der Politischen Theorie und der Paradigmenwechsel der Politischen Kulturforschung von Birgit Schwelling.

Vor 15 Jahren hatte Wendy Brown dafür plädiert, die Grenzen der politischen Theorie zu erweitern. Nach Brown riskierte die politische Theorie, die wichtigsten Phänomene ihres Forschungsfeldes aufgrund eines mangelhaften Instrumentariums nicht mehr behandeln zu können. Sie schlug deswegen vor, mehr Interdisziplinarität zu wagen und Inspirationen von benachbarten Disziplinen zu holen. Browns Begründung war: "Questions about the nature of the political cannot presume its radical independence from the cultural, the economic, and, above all the technological« (Brown 2002: 571). Es gibt keine »radikale Unabhängigkeit des Politischen«. Politiktheoretikerinnen und Politiktheoretiker, so Brown, müssten lernen, sich innerhalb eines weniger abgegrenzten disziplinären Bereiches zu bewegen. Sie müssten andere disziplinäre Felder befragen, um neue Impulse für die eigene Theoriebildung zu gewinnen. Dazu zählt Brown Literatur- und Rhetoriktheori- 
en sowie die Theorien der Visual Studies. All diese Disziplinen haben gemeinsam, dass sie Symbolisierungsprozesse untersuchen und kollektive Vorstellungen berücksichtigen. Und sie melden seit Jahren ein stärkeres Interesse am Politischen an.

Zum gleichen Zeitpunkt wurde in Deutschland ein zweiter Vorschlag zur Interdisziplinarität der Politikwissenschaft gemacht. Birgit Schwelling plädierte für einen kulturellen Blick auf die Politikwissenschaft und sammelte anschließend in einem Band Ansätze, die dies möglich machen konnten (Schwelling 2001; 2004). Sie reflektierte die Auswirkung des »cultural turn« auf die Politische Kulturforschung und bot einen Ansatz an, der kulturanthropologische und wissenssoziologische Herangehensweisen integrierte (Schwelling 2001). Das Politische betrachtet Schwelling unter dem Aspekt der Kultur (Schwelling 2004: 12). Ihr Artikel von 2001 setzte sich zum einen mit der traditionellen Herangehensweise der »civic culture« (Almond/Verba) und zum anderen mit den »symbolzentrierten Ansätzen «, die politische Kultur als Symbolsystem betrachten, kritisch auseinander (Schwelling 2001: 610). Schwelling invalidiert diese Perspektiven nicht, aber sie zielt auf »ein alternatives Programm zur Erforschung politischer Kulturen «, das den Gegenstand der Politischen Kulturforschung erweitern und zugleich präzisieren kann (Schwelling 2001: 613). Die bestehenden Herangehensweisen der Politischen Kulturforschung ergänzt Schwelling mit einem Ansatz, »in dessen Rahmen politische Kultur nicht als gesonderter Bereich neben anderen Kulturen (ökonomische, religiöse usw.) und nicht im Sinn eines nur einen Teilausschnitt politisch relevanter Phänomene betreffenden Konzepts verstanden wird, sondern vielmehr im Sinn eines kulturellen Blicks auf politisch relevante Phänomene konzeptualisiert ist (ebd.). So verstanden sei Kultur ein »Sinn stiftendes Muster«, deswegen könne sich die Anwendung dieses Kulturbegriffs auf die Politische Kulturforschung »nicht durch einen spezifischen, nur auf den Bereich des Politischen zugeschnittenen Kulturbegriff" auszeichnen, sondern müsse breiter angelegt werden (ebd.: 617). Im Zentrum stehen die »kulturellen Grundlagen politisch relevanter Phänomene«, auf deren Basis Wertesysteme, Alltagsbewältigung, institutionelle Logiken sowie offizielle Symbole entstehen. Die Autorin machte auf die Bedeutung von kollektiv geteiltem Wissen und Wissensvorräten aufmerksam. Bei den Wissenssoziologen Peter Berger und Thomas Luckmann schließt Wissen nicht nur kognitives Wissen, sondern auch internalisiertes Wissen ein. Schwelling betont den sozial und historisch kontextualisierten Zugang zu kulturell dimensionierten Fragen und kann auf die »expliziten, materialen Produkte menschlichen Handelns« eingehen sowie diese mit symbolzentrierten Ansätzen verbinden (ebd.: 613).

Browns und Schwellings Vorschläge sind bekannt, doch eine systematische Neuorientierung der Politischen Theorie und Politischen Kulturforschung lösten sie nicht aus. Der hier vorgeschlagene Revitalisierungsansatz der Politischen Kulturforschung greift diese beiden Empfehlungen zur Interdisziplinarität auf 
und wendet sie zugleich auf die Mechanismen der politischen Repräsentation an. Der vertretene Ansatz vollzieht einen Paradigmenwechsel. Anstatt einen institutionellen Blick auf die Politik zu werfen, vom rationalistischen Verständnis der politischen Repräsentation auszugehen oder Einstellungen und Präferenzen in der politischen Kultur zu privilegieren, werden die fluiden Konstruktionsmechanismen von Vorstellungen, Ideen und Auffassungen untersucht, die Verwobenheit von affektiven und rationalen Vorgängen des Politischen berücksichtigt sowie die kulturellen Praktiken und politische Wahrnehmungsmuster analysiert. Dafür wird der Begriff der politischen Repräsentation nicht im engen Sinne als Vertretung verstanden, sondern entfaltet seine Bedeutung für die Politik und für die politische Kultur, wenn er auf symbolische, ästhetische und Inszenierungspraktiken erweitert und mit dem politischen Imaginären verbunden wird. Da der Begriff des politischen Imaginären in der Politischen Theorie eine seltene Erscheinung ist (vgl. Diehl 2019) und in der Politischen Kulturforschung bisher nicht verwendet wurde, soll das politische Imaginäre konzeptualisiert werden. Am Ende präsentiert der Text einige der wichtigsten theoretischen und methodologischen Konsequenzen dieses Paradigmenwechsels.

\section{Die Erweiterung des politischen Repräsentationsbegriffs}

In den 1990er Jahren entwickelte Gerhard Göhler ein Konzept der symbolischen Repräsentation, das er als komplementär zu Pitkins Verständnis von Repräsentation als »acting for « konzipierte. Dafür bezog er Kenntnisse aus der Kultursoziologie, Kulturanthropologie und Kulturphilosophie mit ein. Für Göhler hat politische Repräsentation eine Handlungsdimension, die als Vertretung verstanden wird, und eine symbolische Dimension. Beide Dimensionen sind komplementär (Göhler 1997) und bestimmen die Beziehung zwischen Repräsentierten und Repräsentanten. Repräsentation als Handlung bzw. Vertretung gehört zur Willensbeziehung, also dem Bereich der Entscheidungsfindung, des Unterzeichnens von Verträgen, der Gesetzgebungsverfahren etc. Symbolische Repräsentation dagegen ist mit der Symbolbeziehung verbunden und bringt grundlegende politische Wertvorstellungen und Ordnungsprinzipien eines Gemeinwesens zum Ausdruck. Göhler ist sich dessen bewusst, dass Repräsentation ein Verfahren ist, das sich mit Symbolen und symbolischen Handlungen verbindet. Sein Blick auf die politische Repräsentation ist aber institutionell. Er betrachtet die Aktivität der politischen Repräsentanten sowie die Symbolkraft politischer Institutionen, ihrer Gebäude, Bilder und ihres Zeremoniells und konnte deswegen von symbolzentrierten Ansätzen der Politischen Kulturforschung rezipiert werden (u.a. Brodocz 2002, Diehl 2005 u. 2015, Lietzmann 2015, siehe auch den Beitrag von Hans J. Lietzmann in diesem Band). Obwohl Göhlers Arbeiten in Deutschland gut bekannt sind, gibt es kaum Überset- 
zungen in andere Sprachen (Ausnahmen Göhler 2014a; 2014b). Dies erklärt auch, warum der »representative turn« die Erweiterung des Repräsentationsbegriffs auf das Symbolische ganz ohne den Einfluss der deutschen Politikwissenschaft zu entwickeln versuchte und dabei teilweise wichtige Orientierungspunkte aus der deutschen Kulturphilosophie und Soziologie ignoriert. Die Ausblendung dieser Tradition hat Vor- und Nachteile für die Erweiterung des Verständnisses der politischen Repräsentation.

Das »representative turn« versuchte, das Konzept der politischen Repräsentation außerhalb der traditionellen Grenzen der Politischen Theorie zu entwickeln. Politische Repräsentation wird dabei zunehmend als performativer und symbolischer Prozess verstanden. Mit Göhler könnte man sagen, dass diese englischsprachigen Autorinnen und Autoren Repräsentation in ihrer symbolischen Dimension verstehen. Zum einen zeigen sie, dass Repräsentieren als symbolischer Akt zu begreifen ist und performative Bedeutung hat. Damit vollziehen sie einen Paradigmenwechsel, denn sie begreifen die symbolische Repräsentation nicht als komplementär zur Vertretung, wie Gerhard Göhler, sondern sprechen der symbolischen Dimension eine höhere Bedeutung zu. Damit verschieben sie die Aufmerksamkeit auf die Symbolisierung kollektiver Identität, die Rolle von Emotionen, die performative Bedeutung von Präsenz, die jetzt als Aktivitäten der politischen Repräsentation berücksichtigt werden können.

Zum anderen aber - und das ist der Hauptunterschied zu Göhlers Konzept erweitern die Vertreterinnen und Vertreter des »representative turns « die Kategorie des Repräsentanten auf politische Akteure, die keine formalisierte Repräsentationsrolle haben und auch nicht unbedingt als Repräsentantinnen und Repräsentanten autorisiert wurden - der Erfolg des Repräsentanten hängt hauptsächlich von seiner Akzeptanz durch die Repräsentierten ab. Repräsentation erscheint als symbolischer Konstruktionsprozess, der Sinn produziert, unabhängig von ihrer institutionellen Verankerung. In diesem Sinne findet politische Repräsentation sowohl in institutioneller als auch in nicht-institutioneller Form statt, wie Lisa Disch (2012), Suzanne Dovi (2007) oder Michael Saward (2006; 2010) argumentieren. Sie wird nicht nur als Aufgabe für Amtsinhaber verstanden, sondern kann ebenso von informellen und nicht-gewählten Repräsentanten beansprucht werden - Beispiele dafür sind Führer einer Bewegung, Akteure der Zivilgesellschaft oder Prominente, die sich für eine Sache engagieren.

In seinem Buch The Representative Claim von 2010 definiert Michael Saward Repräsentation als »to make a claim«. Repräsentieren ist ein performativer Akt, mit dem man einen Anspruch bzw. eine Forderung stellt. Die Bedeutung des Wortes »to make« ist hier wichtig. Sie zeigt auf die Gestaltung und Performativität im Akt des Repräsentierens. Politische Repräsentanten sind performative Hersteller von Ansprüchen (»claim makers«), sie treten für eine Idee, eine Gruppe bzw. eine Forderung ein und drücken somit mehrere Ansprüche aus: den Anspruch, Repräsen- 
tant zu sein, im Namen einer Gruppe aufzutreten und eine politische Forderung zum Ausdruck zu bringen. Sie setzen Forderungen, Ideen und Vorstellungen in die Welt und insofern tragen sie zur Gestaltung des politischen Imaginären bei. Doch einen »Claim« zu machen, ist noch keine Garantie dafür, dass die Repräsentation gelingt. Repräsentation ist deshalb auch immer eine Beziehung, die instabil bleibt. Sie kann potentiell immer scheitern. Während Saward die Herstellung von Ansprüchen in den Vordergrund stellt, betont Lisa Disch die diskursive Herstellung von Identität im Moment der Repräsentation (2012). Durch symbolische Vorgänge ist der politische Repräsentant demnach in der Lage, einen kollektiven Willen zu formen und eine allgemeine politische Identität herzustellen. Eine amorphe Wählerschaft (»amorphous constituency«) wird in ein »Volk« transformiert. Es handelt sich um einen symbolischen Prozess, der, wenn erfolgreich, die Vorstellungen über Forderungen, Interessen, Repräsentierte und Repräsentanten prägt.

Die meisten Autorinnen und Autoren des »representative turn « konzentrieren sich auf die diskursive Ebene der Repräsentation. Bilder, Ästhetik und Inszenierungen werden zwar nicht ausgeschlossen, doch methodisch finden sie kaum Beachtung. Eine Ausnahme ist die Untersuchung von Emanuela Lombardo und Petra Meier. Beide Autorinnen analysieren die Bedeutung von Gender in der symbolischen Repräsentation im Allgemeinen und die Symbolisierung politischer Institutionen im Besonderen. In ihrem Buch The Symbolic Representation of Gender zeigen sie, dass männliche und weibliche Körper unterschiedlichen kulturellen Blicken unterworfen sind, wenn es um die politische Repräsentation geht (Lombardo/Meier 2014). In Deutschland wurde die Performativität des Körpers in der politischen Repräsentation vor allem von der Soziologie betrachtet (z.B. Hitzler 2002; Soeffner 1994) und ist in der Politikwissenschaft ein neues Untersuchungsfeld (Diehl 2008; 2010; 2014, 2015).

Denkt man die deutschen und die englischsprachigen Auslegungen des Begriffs weiter, ergeben sich wichtige Konsequenzen für die Politische Theorie und für die Politische Kulturforschung. Was die neuen deutsch- und englischsprachigen Ansätze zur politischen Repräsentation verbindet, ohne es jedoch explizit zu machen, ist das Bewusstsein, dass politische Repräsentation mit den kulturellen Bedingungen der Politik verbunden ist und zugleich zur politischen Kultur beiträgt. Politische Repräsentation muss deshalb als symbolischer Prozess verstanden werden, der mit kulturellen Codes, kollektiven Praktiken und Vorstellungen aus dem politischen Imaginären verbunden ist. Denn politische Repräsentation ist ein performativer Akt, der das kulturelle Repertoire mobilisiert und zugleich darauf wirkt.

Ein solcher performativer Akt wird verständlicher, wenn man die wissenssoziologische Perspektive berücksichtigt. Für Peter Berger und Thomas Luckmann ist die Gesellschaft eine Konstruktion, die auf der »fundamentalen Dialektik der Gesellschaft « beruht. Menschen benutzen immer Symbole, um miteinander zu kommunizieren, sie inszenieren sich und schaffen Bilder. Dadurch werden sie zu Sinn- 
produzenten. Das tun sie aber nicht allein, sondern im Austausch miteinander. So gesehen, ist Gesellschaft an sich schon ein Produkt der symbolischen Praxis und zugleich Erzeuger von Symbolen. Symbolisierung erzeugt Sinn, indem sie Vorstellungen der Wirklichkeit mobilisiert. »Zusammen produzieren die Menschen eine menschliche Welt mit einer ganzen Fülle ihrer sozio-kulturellen und psychologischen Gebilde«, die dann objektive Faktizität gewinnen (Berger/Luckmann 2001: 54). Repräsentation ist in dem Sinne performativ, in dem sie Vorstellungen des Politischen erzeugt, tradiert oder verändert (Diehl 2015). Bilder, Symbole und Inszenierungen sind kein bloßes Beiwerk des politischen Handelns, sondern ihre konstitutiven Mittel (Lietzmann 2018). Durch symbolisch-performative und imaginäre Vorgänge wird Politik gesellschaftlich konstruiert. So gesehen wirkt politische Repräsentation auf die Wahrnehmung, Auffassung und Vorstellung von Politik, also auf das politische Imaginäre.

\section{Das politische Imaginäre}

Das politische Imaginäre kann sowohl als kollektives Repertoire von Vorstellungen, Bildern, Symbolen, Emotionen, Diskursen, Narrativen, Mythen etc. als auch als Vorstellungskraft betrachtet werden, die dieses kollektive Repertoire erzeugt. Es ermöglicht die Herstellung derjenigen Wahrnehmungs- und Vorstellungsmuster, mithilfe derer Gesellschaft und Politik gedeutet werden und woran sich politisches Handeln orientiert und bestimmt (Diehl 2015). Der Philosoph Cornelius Castoriadis beschreibt es als »unaufhörliche und (gesellschaftlich-geschichtlich und psychisch) wesentlich indeterminierte Schöpfung von Gestalten/Formen/Bildern, die jeder Rede von setwas« zugrunde liegen« (Castoriadis 1990: 12). Dies ist für Castoriadis die Basis für die Herstellung einer gemeinsamen Realität innerhalb der Gesellschaft. Deswegen kann das Imaginäre als eine soziale Instanz charakterisiert werden, die Vorstellungen der Gesellschaft über sich selbst entstehen lässt. Mit dem Begriff des Imaginären will Castoriadis Institutionalisierungsprozesse, Dynamiken und Transformationen der Gesellschaft und zugleich ihre Strukturen sowie Bedingungen erfassen. Die Imagination und das Imaginäre werden als gesellschaftliche und geschichtliche Gegenstände verstanden, die für die Entstehung von Gesellschaft grundlegend sind (Diehl 2019). Nach dieser Perspektive kann politische Kultur bzw. Kultur überhaupt ohne die Berücksichtigung des Imaginären nicht verstanden werden. Denn die Realität ist für den Menschen nicht unmittelbar verständlich. Damit sie »Sinn machen« kann, braucht der Mensch Vorstellungen, Wahrnehmungs- und Deutungsmuster, die die unzähligen phänomenologischen Eindrücke einordnen können. Solche Vorstellungen, Wahrnehmungs- und Deutungsmuster werden in der Gesellschaft konstruiert. Damit diese Konstruktionen gelingen können, ist eine kollektiv geteilte Imagination notwendig. Für den Men- 
schen gibt es keine Realität außer derjenigen, »in der die Gesellschaft mit ihren Institutionen ’herrscht«". Realität gibt es deswegen immer nur »als gesellschaftlich instituiert (Castoriadis 1990: 516).

Hier ergeben sich Parallelen von Castoriadis' Philosophie des sozialen Imaginären und dem wissenssoziologischen Zugang von Berger und Luckmann zur sozialen Konstruktion der Wirklichkeit, die für das Verstehen der performativen Wirkung von politischer Repräsentation grundlegend sind. Für Berger und Luckmann produziert der Mensch eine Welt, die er nicht als ein menschliches Produkt, sondern als Wirklichkeit wahrnimmt (Berger/Luckmann 2001: 54, 65). In diesem Sinne sind Tätigkeiten und Wissensproduktionen performative Akte, die zu dieser Wirklichkeitskonstruktion beitragen. Diskursive, symbolische, ästhetische und Inszenierungshandlungen der Politik fallen in diese Kategorie und helfen, das, was man unter Politik versteht, $\mathrm{zu}$ formen. Auch für Castoriadis ist das, was man unter Realität versteht, ein menschliches Produkt. Aber es gibt einen Unterschied zwischen beiden Theorien, der hier von Bedeutung ist. Für Castoriadis können symbolische Handlungen nur wirken, weil sie vom Imaginären strukturiert werden. Die Vorstellungskraft gehört zu einer Gesellschaftsdimension, die es ermöglicht, dass Symbole kollektiv wirken und sich verändern. Verknüpft man die wissenssoziologische Perspektive mit Castoriadis' Ansatz, können dann symbolische Praktiken mit der Vorstellungskraft in Verbindung gesetzt werden.

\section{Symbolische Repräsentation als Indikator politischer Kultur und der Veränderung des politischen Imaginären}

Es klingt fast trivial zu sagen, dass die Art und Weise, wie man sich Politik vorstellt, wie man Politik wahrnimmt und erlebt, vom politischen Imaginären abhängt. Schaut man sich aber die Verbindung zwischen Politik und Imaginärem unter dem Aspekt der politischen Repräsentation näher an, werden die Ergebnisse dieser Beobachtung interessanter. Die Erweiterung der politischen Repräsentation auf das Symbolische ermöglicht es, auf eine tiefere Schicht der politischen Kultur einzugehen sowie kulturelle Prozesse und Praktiken hinter der politischen Repräsentation zu betrachten. Wie gesehen wurde, wirken Symbole und symbolische Praktiken der politischen Repräsentation performativ auf die Politik, insofern sie etwas in die Welt setzen, das Existenz beansprucht. Die Beziehung der politischen Repräsentation zum politischen Imaginären ist aber eine doppelte: Zum einen bringt die politische Repräsentation kollektive Normen und Vorstellungen des politischen Imaginären zum Ausdruck. Zum anderen aber sind ihre Symbole und symbolischen Vorgänge selbst performative Elemente der Veränderung dieser Normen und Vorstellungen. 
Das ist der Grund, warum Symbole und symbolische Praktiken der politischen Repräsentation umkämpft sind. Repräsentation als symbolische Tätigkeit hat eine strategische Bedeutung, die man mit Pierre Bourdieu als »symbolischen Kampf« bezeichnen kann (1997). Politische Akteurinnen und Akteure versuchen in diesem Kampf, das politische Imaginäre zu beeinflussen. Wenn institutionelle und informelle Repräsentanten »claim makers«, also Hersteller von Ansprüchen, sind (Saward 2010), ist ihre Tätigkeit nie nur rational, sondern schließt Vorstellungen, Visualisierungsvorgänge und Emotionen mit ein. Kollektive Vorstellungen, Bilder und Gefühle mitzugestalten ist das Ziel von politischen Akteurinnen und Akteuren, um die Politik beeinflussen zu können. Kulturkämpfe wie die zurzeit beobachtete Offensive von Rechtspopulisten sind geeignete Anschauungsobjekte, um solche Prozesse beobachten zu können. Wenn Repräsentation aber auch der diskursiven und symbolischen Herstellung von Identität (Disch 2012) und somit zugleich auch der Definition der Gruppe bzw. des Volkes dient, dann sind die Kulturkämpfe genau die Momente, in denen die etablierten Normen des politischen Imaginären in die Krise geraten sind.

Versteht man Repräsentation auch als symbolische Aktivität, wird ihre Brisanz für das Politische deutlich. In Symbolen, Bildern und Inszenierungen werden sowohl demokratisierende als auch antidemokratische Konzepte "getestet«. Der Kampf um die Re-Etablierung bzw. Abschaffung der »Confederate Flag« in den USA, der Versuch der AfD, das Wort »völkisch« zu normalisieren bzw. den Genozid an den Juden und Minderheiten während des Nationalsozialismus zu relativieren, oder auch die strategische Einbindung des Wortes »Heimat« als ein Dach für multikulturelle Identität durch den grünen österreichischen Präsidenten Alexander Van der Bellen auf seinen Wahlplakaten von 2016 sind deutliche Beispiele dafür. So verschieden, wie diese Akteurinnen und Akteure sind, so sehr erheben sie mittels der Repräsentation alle gleichermaßen performative Ansprüche, die auf die Veränderung der Wahrnehmung und des Verständnisses der Politik zielen: Der affirmative Anspruch auf die weiße Hegemonie bzw. den Anspruch auf die Anerkennung des Leids der schwarzen Bevölkerung in den Südstaaten der USA im ersten Fall, die positive Anlehnung an die nationalsozialistische Vergangenheit bzw. ihre Bagatellisierung im zweiten Fall oder die Demokratisierung eines meist konservativen und exklusivistischen Zugehörigkeitsbegriffs (Heimat) als zugänglich für alle im letzten Fall. Diese Fälle machen deutlich, dass Symbolisierung nicht nur Ansprüche, sondern auch politische Vorstellungen und Selbstverständnisse ausdrückt. Will man verstehen, wie sich die politische Kultur angesichts der konjunkturellen ideologischen Kämpfe verändert, ist die Untersuchung der politischen Repräsentation im erweiterten Sinne grundlegend, denn sie verrät ideologische Verschiebungen des politischen Imaginären.

Aber die Untersuchung der politischen Repräsentation zeigt auch auf die Transformationen der politischen Kultur auf struktureller Ebene. Diese werden 
an der Veränderung von Codes, Wahrnehmungsmustern und normativen Parametern, die im symbolischen Gebrauch zum Ausdruck kommen, sichtbar. Diese Transformationen manifestieren sich ebenfalls in Abweichungen und Störungen gewöhnlicher Formen, Ästhetik und Selbstverständnisse. Auf dieser Ebene wird der Status der politischen Repräsentation selbst instabil. Die Legitimationsinstanz der Politik wird in Frage gestellt oder verliert ihre Relevanz. Das ist der Moment der strukturellen Transformation des politischen Imaginären. Der labile Status der politischen Repräsentation in Bezug auf die Unterscheidung zwischen Realität und Fiktion beim Fall von Donald Trump ist ein gutes Indiz dafür. Vergrößert sich aber die Zahl der Akteurinnen und Akteure, bei denen dieses Phänomen auftritt, wird auch in Umfragen eine Irrelevanz der Unterscheidung zwischen Politik und Unterhaltung bei der Wahrnehmung von Politik durch die Bürgerinnen und Bürger erkennbar; so spricht einiges dafür, dass man es hier mit einem radikalen Wandel der politischen Kultur zu tun hat.

Symbolisierungsprozesse $\mathrm{zu}$ berücksichtigen gibt Aufschluss darüber, wie symbolische Praktiken mit dem politischen Imaginären verbunden sind, wie politische Legitimation hergestellt und wie das Politische vorgestellt wird. Symbolische Repräsentation ist deswegen ein wichtiger Indikator für die Untersuchung politischer Kultur und auch darüber, wie sich das politische Imaginäre verändert. Die symbolische Dimension der politischen Repräsentation muss daher in die Politische Kulturforschung inkorporiert werden. Dies impliziert wiederum die Reflexion über die Prozesse der Sinnkonstruktion anhand der Analyse eines breiten Materials, das Diskurse, Symbole, Praktiken, Bilder, Inszenierungen, Narrative, Mythen und ästhetische Formen mit einschließt. Das Konzept der politischen Repräsentation muss daher um ihre symbolische Dimension erweitert werden und in Bezug zum politischen Imaginären gesetzt werden. In den Fokus geraten dann die vielfältigen Symbolisierungsprozesse, die die Vorstellungen des Politischen prägen. Es handelt sich um einen Paradigmenwechsel der Politischen Kulturforschung von der Untersuchung von politischen Präferenzen und Sozialisierungsmustern hin zum Ausdruck und zu den Veränderungen des politischen Imaginären nicht zuletzt durch die politische Repräsentation. Es geht um die Untersuchung von dem, was Präferenzen und Sozialisierung erst möglich macht.

\section{Theoretische und methodologische Konsequenzen des Paradigmenwechsels}

Ein solcher Paradigmenwechsel hat theoretische und methodologische Konsequenzen für die Politikwissenschaft, für die Politische Theorie, für die Politische Ideengeschichte und natürlich auch für die Politische Kulturforschung. Der vorliegende Text schließt mit einer kurzen Skizze einiger dieser Konsequenzen. 
- Politikwissenschaft: Der Gegenstand der Politikwissenschaft wird von der Politik auf das Politische erweitert. Dadurch werden neue Forschungsgegenstände sichtbar, die an der Grenze zu anderen Disziplinen stehen, wie etwa die Beziehung zwischen Politik und Medien, die Bedeutung von Performativität für politische Prozesse, politische Psychologie, politische Kommunikation und politische Kulturwissenschaft.

- Politische Theorie: Die Politische Theorie bekommt neue Aufgaben, sie muss neue Konzepte, Begriffe und theoretische Modelle generieren, Orientierung für die Untersuchung der neuen Intersektionsbereiche geben und das Symbolische stärker berücksichtigen. Auch die Transformationen des politischen Imaginären müssen zum Gegenstand der theoretischen Auseinandersetzung werden.

- Politische Ideengeschichte: Mit der Berücksichtigung des Imaginären kann die politische Ideengeschichte nicht mehr ausschließlich als Untersuchung und historische Rekonstruktion von Begriffen und Denkmustern verstanden werden. Auch die kanonische Zentrierung auf bestimmte Autorinnen und Autoren - meistens europäische Männer - reicht nicht mehr aus, um die Geschichte des politischen Denkens zu rekonstruieren. Gefragt wird eine Ideengeschichte, die sich als Geschichte des politischen Imaginären versteht. Der zu analysierende Korpus wächst. Nicht nur Texte, Symbole, Praktiken und Bilder geraten in den Fokus, ${ }^{3}$ auch der Zugang zu den Texten verändert sich. Es gilt jetzt, nicht nur die Konzepte zu analysieren, sondern auch die latenten Vorstellungen, die nicht im Vordergrund des Textes stehen. Die Verknüpfung mit anderen Formen der symbolischen Materialisierung wie etwa Bildern und kulturellen Praktiken ist hierfür notwendig.

- Politische Kulturforschung: Damit steigt die politische Kulturforschung von einer marginalen Subdisziplin in den Kern der Politikwissenschaft auf. Sie muss aber auch eine stärkere Wendung zu neuen Phänomenen, Fragestellungen und Methoden vollziehen. Wie bereits angedeutet, muss die Politische Kulturforschung auf die expressive Seite von Kultur eingehen, Ästhetik, Symbolik, Rituale- und Inszenierungspraktiken, kollektive Narrative und Mythen in ihren Analysen miteinbeziehen.

- Die hier genannten Konsequenzen für die Politikwissenschaft und ihre Subdisziplinen bilden keine abgeschlossene Liste. Vielmehr ist zu erwarten, dass je tiefer die Forschungen der politischen Repräsentation und des politischen Imaginären bohren, desto diverser und komplexer die Untersuchungsfelder werden.

3 Pierre Rosanvallon hat mit seiner interdisziplinären Geschichte des Politischen einen wertvollen methodischen Vorschlag gemacht (Rosanvallon 2012). 


\section{Literatur}

Almond, Gabriel A. 1989: The Intellectual History of the Civic Culture Concept. In: Gabriel A. Almond/Sidney Verba (Hg.), The Civic Culture Revisited, London und New Delhi, S. 1-36.

Berger, Peter/Thomas Luckmann 2001: Die gesellschaftliche Konstruktion der Wirklichkeit. Eine Theorie der Wissenssoziologie, Frankfurt a.M.

Bolasco, Sergio/Galli deく Paratesi, Nora/Giuliano, Lucca 2006: Parole in Libertà. Un'analisi statistica e linguistica, Roma.

Bourdieu, Pierre 1997: Sur le pouvoir symbolique. In: Annales. Économies, Sociétés, Civilizations, Heft 3, S. 405-411.

Brodocz, André 2002: Institution als symbolische Form. In: Berliner Journal für Soziologie, Heft 2, S. 211-226.

Brown, Wendy 2002: At the Edge; in: Political Theory, Vol. 30: What is Political Theory? Special Issue, S. 556-576.

Camus, Jean-Yves 2017: Die Identitäre Bewegung oder die Konstruktion eines Mythos europäischer Ursprünge. In: Gudrun Hentges/Kristina Nottbohm/HansWolfgang Platzer (Hg.), Europäische Identität in der Krise? Europäische Identitätsforschung und Rechtspopulismusforschung im Dialog, Wiesbaden, S. 233-247.

Crouch, Collin 2008: Postdemokratie, Frankfurt a.M.

Diamanti, Ilvo 2014: The Five Star Movement: A Political Laboratory. In: Contemporary Italian Politics, Heft 1, S. 4-15.

Diehl, Paula 2005: Macht - Mythos - Utopie. Die Körperbilder der SS-Männer, Berlin.

Diehl, Paula 2008: Dekonstruktion als Inszenierungsmethode - von Berlusconi bis zu den Grünen. In: Andreas Dörner/Christian Schicha (Hg.), Politik im Spot-Format, Zur Semantik, Pragmatik und Ästhetik politischer Werbung in Deutschland, Wiesbaden, S. 313-335.

Diehl, Paula 2010: Zwischen dem Privaten und dem Politischen - Die neue Körperinszenierung der Politiker. In: Sandra Seubert/Peter Niesen (Hg.), Die Grenzen des Privaten, Baden-Baden, S. 251-265.

Diehl, Paula 2014: Die repräsentative Funktion des Körpers in der Demokratie. In: André Brodocz et al. (Hg.), Die Verfassung des Politischen, Hans Vorländer zum 60. Geburtstag, Wiesbaden, S. 115-131.

Diehl, Paula 2015: Das Symbolische, das Imaginäre und die Demokratie. Eine Theorie der politischen Repräsentation. Baden-Baden.

Diehl, Paula 2017: Antipolitik und postmoderne Ringkampf-Unterhaltung. In: APuZ, Heft 44-45, S. 25-30.

Diehl, Paula 2019: Das politische Imaginäre und die politische Repräsentation, in: Österreichische Zeitschrift für Soziologie (i.E.). 
Disch, Lisa 2012: Die Impurity of Representation and the Validity of Democracy. In: Cultural Studies, Heft 2-3, S. 207-222.

Dörner, Andreas 2001: Politainment. Politik in der medialen Erlebnisgesellschaft, Frankfurt a.M.

Dovi, Suzanne 2007: The Good Representative, Malden.

Göhler, Gerhard 1997: Der Zusammenhang von Institution, Macht und Repräsentation. In: Gerhard Göhler u.a. (Hg.), Institution - Macht - Repräsentation, Wofür politische Institutionen stehen und wie sie wirken, Baden-Baden, S. 11-62.

Göhler, Gerhard 2014a: La représentation politique dans la démocratie (Übersetzung von: »Politische Repräsentation in der Demokratie«, 1992). In: Paula Diehl/Yves Syntomer/Samuel Hayat (Hg.), Trivium, 16 | 2014. www.trivium.revues.org/4803.

Göhler, Gerhard 2014b: Political Representation - Reconsidered. In: Paula Diehl/Alexandre Escudier (Hg.), La »représentation« du politique: histoire, concepts, symboles, Cahier du CEVIPOF n ${ }^{\circ}$ 57, S. 15-26. www.cevipof.com/fr/ les-publications/les-cahiers-du-cevipof/bdd/publication/1166.

Greiffenhagen, Sylvia 2009: Theorie(n) der Politischen Kultur. In: Samuel Salzborn (Hg.), Politische Kultur, Forschungsstand und Forschungsperspektiven, Frankfurt a.M., S. 11-29.

Hitzler, Ronald 2002: Inszenierung und Repräsentation. Bemerkungen zur Politikdarstellung in der Gegenwart. In: Hans-Georg Soeffner/Dirk Tänzler (Hg.), Figurative Politik, Zur Performanz in der modernen Gesellschaft, Opladen, S. 35-49.

Kogl, Alexandra 2009: A Hundred Ways of Beginning: The Politics of Everyday Life. In: Polity, Heft 4, S. 514-535.

Lietzmann, Hans J. 2015: Die Demokratisierung der Repräsentation. Dialogische Politik als neue Form der repräsentativen Demokratie. In: Manuela Glaab (Hg.), Politik mit den Bürgern - Politik für Bürger, Praxis und Perspektiven einer neuen Beteiligungskultur, Wiesbaden, S. 41-58.

Lietzmann, Hans J. 2018: Citizenship, Democracy and the Iconology of Political Representation: A Plea for an Iconological Turn in Democratic Theory. In: Claudia Wiesner u.a. (Hg.), Shaping Citizenship, A Political Concept in Theory, Debate and Practice, New York, S. 55-70.

Lombardo, Emanuela/Meier, Petra 2014: The Symbolic Representation of Gender. A discursive Approach, Burlington.

Mellon, Jonathan/Prosser, Christopher 2017: Twitter and Facebook are not representative of the general population: Political attitudes and demographics of British social media users. In: Research and Politics, Heft 3, S. 1-9. 
Mrozek, Bodo 2017: Unter falscher Flagge. Rechte »Identitäre« setzen auf AntikenPop. Die Geschichte ihrer Symbole dürfte ihnen kaum gefallen. In: PopHistory, https://pophistory.hypotheses.org/2561\#more-2561, letzter Abruf, 21.12.2018, 14.17 Uhr.

Müller, Marion 1997: Politische Bildstrategien im amerikanischen Präsidentschaftswahlkampf 1828 - 1996, Berlin.

Näsström, Sofia 2011: Where is the representative turn going? In: European Journal of Political Theory Heft 10, S. 501-510.

Rohe, Karl 1994: Politische Kultur: Zum Verständnis eines theoretischen Konzepts. In: Oskar Niedermayer/Klaus von Beyme (Hg.), Politische Kultur in Ost- und Westdeutschland, Berlin, S. 1-21.

Pitkin, Hanna F. 1967: The Concept of Political Representation, Los Angeles.

Rosanvallon, Pierre 2012: Für eine Begriffs- und Problemgeschichte des Politischen. Antrittsvorlesung am Collège de France. 28. März 2002. In: Mittelweg 36, Heft 6, S. 43-66.

Saward, Michael 2006: Representative Claims. In: Contemporary Political Theory, Heft 5, S. 297-318.

Saward, Michael 2010: The Representative Claim, Oxford.

Schwelling, Birgit 2001: Politische Kulturforschung als kultureller Blick auf das Politische. Überlegungen zu einer Neuorientierung der Politischen Kulturforschung nach dem »Cultural Turn«. In: Zeitschrift für Politikwissenschaft, Heft 2, S. 601-629.

Schwelling, Birgit 2004: Politikwissenschaft als Kulturwissenschaft: Theorien, Methoden, Problemstellungen. Wiesbaden.

Soeffner, Hans-Georg 1994: Populisten: Profiteure, Handelsagenten und Schausteller ihrer Gesellschaften. In: Helmut Berking/Roland Hitzler/Sighard Neckel (Hg.), Politikertypen in Europa, Frankfurt a.M., S. 259-279.

Wodak, Ruth, 2016: Politik mit der Angst, Wien.

\section{Internetquellen}

$\mathrm{Lu}$, Denise/Karen Yourish: You're Hired! You're Fired! Yes, the Turnover at the Top of the Trump Administration Is ... »Unprecedented.«; in: The New York Times, update 20. Dezember 2018. https:/www.nytimes.com/interactive/ 2018/03/16/us/politics/all-the-major-firings-and-resignations-in-trumpadministration.html. Letzter Abruf, 26.12.2018, 15.00 Uhr. 

Herangehensweisen 



\section{Deutungsmacht als machtsensible Perspektive politischer Kulturforschung}

Dennis Bastian Rudolf

\section{Einleitung}

»Jeder hätte sie gern, viele kämpfen darum, manche scheinen sie zu shaben « aber bisher ist weitestgehend ungeklärt, was das ist« (2014: V). Mit diesem Satz umreißt Philipp Stoellger zu Beginn seines Vorwortes zur Deutungsmachtanalyse den Horizont eines Konzeptes, welches mit seiner Komposition auf den ersten Blick eingängig, gar intuitiv plausibel erscheint, auf den zweiten Blick aber ebenso schnell (er-)klärungsbedürftig wird. Dass die Verbindung zwischen Macht, als zentralem Begriff der Sozial- und Politikwissenschaften, und Deutung, einem hermeneutischen Prozess des Konstruierens, Erkennens und Verstehens von (Be-)Deutungen und Sinnzusammenhängen innerhalb der politischen Lebenswelt, jedoch eine enorme Anziehungskraft auf die politischen Akteurinnen und Akteure ausübt, zeigt sich nicht nur in der gegenwärtigen politischen Diskussion rund um Fake-News, alternative Fakten und dem Postfaktischen. Die soziale Konstruktion der politischen Wirklichkeit ist immer nur in Deutungen gegeben und findet als alltagschöpferischer Prozess weder im luftleeren, noch im machtfreien Raum statt. Die Art und Weise wie Deutungen entstehen variieren allerdings enorm. Ebenso wie die Faktoren, die darüber Aufschluss geben, warum bestimmte Deutungen akzeptiert und unhinterfragt gültig bleiben, während andere von vorne herein $a b$ gelehnt oder im Laufe der Zeit fragil werden. Nähert man sich diesen Fragen von Seiten der politischen Kulturforschung aus an, werden unmittelbare Assoziationen zu Karl Rohe geweckt, welcher mit dem Wechselspiel zwischen deutungskulturellen Angeboten und deren soziokultureller Verankerung die Handlungs- und Prozessaspekte politischer Kultur in den Fokus gerückt hat:

»[...] wer in einer politischen Cesellschaft für wen auf welche Weise was für politische Deutungsangebote macht und machen kann, oder noch grundlegender: $o b$ überhaupt eine hinreichende symbolische Verdeutlichung der politischen Basiskonzepte und Basisregeln eines politischen Cemeinwesens erfolgt« (1987: 42, Hervorheb. im Original) 
zählt für ihn zu den kardinalen Fragen der politischen Kulturforschung. Die damit implizierte Kritik an der klassischen Umfrage- und Einstellungsforschung in der Tradition von Gabriel Almond und Sidney Verba, verstand er jedoch nie als prinzipiellen Einwand gegen den methodischen Zugang, sondern als Anregung für eine notwendige Ergänzung (1994: 5).

Hier möchte der Beitrag ansetzen und über den Begriff der Deutungsmacht (Vorländer 2006; Stoellger 2014) eine machtsensible Perspektive für die politische Kulturforschung aufzeigen, welche versucht, die nach wie vor verhärteten Fronten unterschiedlicher Ansätze zu klären und Möglichkeiten der gegenseitigen, konzeptionellen wie methodischen Weiterentwicklung der Forschung aufzuzeigen. (2) In einem ersten Schritt erfordert dies eine Annäherung an den Begriff der Deutungsmacht. Inwiefern besteht im Kontext der politischen Kultur Deutungsbedarf und warum erscheint das Konzept der Deutungsmacht dazu geeignet, die politische Kulturforschung eine machtsensible Perspektive zu bereichern (Schubert/Kosow 2007: 45) bzw. ihr eine explizite Machtdimension einzuschreiben (Schulz 2006: 68). (3) Vor dem Hintergrund eines umfassenden Verständnisses politischen Kultur, wie es sich beispielsweise bei Samuel Salzborn findet (2009: 46f.), sollen vier konzeptionelle bzw. methodische Probleme eines klassischen Ansatzes adressiert werden, welche Anknüpfungspunkt für eine konstruktive Ergänzung durch das Deutungsmachtkonzept bieten. Dazu zählen neben dem Problem einer »Arithmetik der Macht« (Westle 2009: 51), die Komplexität politischer Unterstützungsobjekte, die historischen Eigenarten politischer Kulturen sowie die Schwierigkeiten des Instruments der Umfrageforschung bezüglich der Anwendungsmöglichkeiten in nicht-demokratischen Systemen. (4) Ausgehend davon, dient vor dem Hintergrund der Deutungsmachtperspektive David Eastons Konzept der politischen Unterstützung als zentraler Ankerpunkt für den gegenseitigen Austausch unterschiedlicher Ansätze. (5) Fazit und Ausblick widmen sich den Möglichkeiten für die Weiterentwicklung der politischen Kulturforschung bezüglich einer machtsensiblen Perspektive sowie weiteren potentiellen Forschungsfeldern für Deutungsmachtanalysen.

\section{Was ist Deutungsmacht?}

Mit Blick auf den Machtbegriff innerhalb der Politischen Theorie, handelt es sich bei Deutungsmacht terminologisch um ein recht junges Konzept, welches erstmals 2006 von einer Forschergruppe um Hans Vorländer an der TU Dresden in die Debatte eingebracht wurde. Im Sinne einer Bestimmungsmacht oder Verfü- 
gungsmacht, versuchen die Autoren jene spezifische Macht ${ }^{1} \mathrm{zu}$ konzeptualisieren, die sich in der Akzeptanz und Befolgung einer verfassungsgerichtlichen Interpretation zeigt und bezüglich ihrer Durchsetzung weder auf umfangreiche exekutive Verfügungs- oder Verhinderungs-, noch auf legislative Gestaltungsmacht zurückgreifen kann. Als eine weiche Form der Macht, manifestiert sich Deutungsmacht in der erfolgreichen Durchsetzung von Leitideen und Geltungsansprüchen, die, ausgehend von der Deutung komplexer und abstrakter Rechtsnormen der Verfassung, den grundlegenden Ordnungsvorstellungen des politischen Gemeinwesens Ausdruck verleihen (Vorländer 2006: 15-17). Machttheoretisch genügt es für ein solches Machtkonzept freilich nicht, sich allein auf einen intentionalistischen Machtbegriff zu stützen. Zwar bleibt im Sinne Max Webers sowohl personal wie strukturell von zentralem Interesse, worin jene Chance besteht, die eigene Deutung innerhalb einer sozialen Beziehung durchzusetzen, da einem Machtunterworfenem eine Deutung jedoch kaum gegen seinen Willen aufgezwungen werden kann, bleibt ein solcher Machtbegriff zwangsläufig limitiert, wenn es um die Frage der Anerkennung geht. ${ }^{2}$ Deutungsmacht zeichnet sich für Daniel Schulz deshalb gerade dadurch aus, dass sie nicht direkt über den Willen oder das Handeln Anderer verfügt, sondern mittelbar auf diese einwirkt, indem sie Geltung hinsichtlich der Definition und Relevanz zentraler gesellschaftlicher Werte, Ziele, Überzeugungen und Ordnungsvorstellungen beansprucht und entfaltet (Schulz 2006: 67). Damit beziehen sich die Autoren zum einen auf mediale Machtbegriffe, wie sie beispielsweise in Arbeiten Pierre Bourdieus oder Michel Foucaults angelegt sind, und zum anderen auf die Unterscheidung zwischen transitiver und intransitiver Macht, die Gerhard Göhler im Kontext institutionentheoretischer Überlegungen erörtert hat (1997, 2013). Deutungsmacht wird dann als eine besondere Modalität von Macht verstanden, die sich bei der Erzeugung legitimer Deutungen zwar auf die Bereitstellung und Mobilisierung unterschiedlicher Sinn- und Geltungsressourcen stützen muss, sich aber nicht exklusiv, sondern komplementär zu anderen Modi der Macht verhält. Mit Blick auf die Unterscheidung zwischen transitiver und intransitiver Macht gilt, dass einerseits harte Formen der Macht, wie ökonomische, politische oder militärische Verfügungs- oder Verhinderungsmacht, stets in symbolisch-institutionelle Sinn- und Geltungskontexte eingebettet sind, und andererseits auch die Deutungsmacht von Akteurinnen und Akteuren immer auf sozialen, ökonomischen

1 Philipp Stoellger stellt infrage, dass es sich bei Deutungsmacht tatsächlich um eine spezifische Machtform handelt. Er plädiert stattdessen dafür, die Deutungsdimension von Macht nicht als einen eigenen Typus zu verstehen, sondern als eine Dimension aller Machtverhältnisse (2014: 49).

2 Zwar mag es kurzfristig, durch Sanktionierung oder Androhung von Cewalt, durchaus möglich sein, bestimmte Deutungen autoritativ durchzusetzen bzw. konformes Verhalten zu erzwingen, wie politische Ordnung selbst bedürfen jedoch auch Deutungen der Anerkennung, um auf dauerhaft legitimierend und stabilisierend wirken zu können. 
und politischen Strukturen beruht, denen hierarchische Macht- und Herrschaftsbeziehungen eingeschrieben sind (Vorländer 2006: 17).

Im Versuch der Überwindung einer materialistischen bzw. idealistischen Verkürzung sowie in der Vermittlung des Gegensatzes von Akteurin und Akteur und Struktur, sehen Sophia Schubert und Hannah Kosow über das Konzept der Deutungsmacht die Möglichkeit, die aktuelle Machtdebatte innerhalb der Politischen Theorie der Gegenwart sowohl konzeptionell, wie auch normativ und empirisch bereichern zu können (2007: 43-45). Konzeptionell, weil über den Begriff der Deutungsmacht eine symbolischen Dimensionierung institutionalisierter Macht erreicht werden kann, die bezüglich des Sinn- und Bedeutungshaften nicht nur komplementär, sondern geradezu konstitutiv für andere Dimensionen institutionalisierter Macht erscheint. Normativ, weil mit dem Deutungsmachtkonzept der Versuch unternommen wird, sich von einem negativ-kritischen Machtverständnis zu lösen. ${ }^{3}$ Und letztlich empirisch, sofern eine Abstraktion des Konzeptes vom spezifischen Kontext des Bundesverfassungsgerichtes gelingt, welche Deutungsmacht nicht nur bezüglich einer konkreten Institution erörtert, sondern hinsichtlich des Symbolischen als konstitutive Dimension von institutionalisierter Macht im Allgemeinen. ${ }^{4}$

\section{Ein Abstraktionsversuch von Deutungsmacht innerhalb der politischen Kulturforschung}

Aus Sicht der politischen Kulturforschung ergeben sich für eine solche Abstraktion offensichtliche Anknüpfungspunkte. So betont Vorländer, dass eine jede politische Ordnung in sozial konstituierten Sinnbezügen steht, die den geteilten Bedeutungen auf mannigfaltige Weise Ausdruck und Geltung verleihen muss. Die Symbolisierung grundlegender politischer Weltbilder und Leitideen in Institutionen, Praktiken, Diskursen, Sprache, Texten und Ritualen stellt eine notwendige

3 Indem die Dichotomie der Agent-Struktur-Debatte aufgebrochen wird, kann die Institutionalisierung von Macht sowohl aus einer Akteurs- und/oder Strukturperspektive gesehen und damit sowohl positiv-produktiv wie auch negativ-repressiv analysiert werden.

4 Politische Institutionen stehen demnach für mehr als einen einzuhaltenden Regelkatalog, der Verhaltensweisen und Handlungsalternativen durch das Zusammenspiel aus Norm, Anreiz und Sanktion ermöglicht oder verunmöglicht. Da politische Institutionen nicht dauerhaft auf Cewalt beruhen können, zeigt sich ihre eigentliche Macht gerade dann, wenn sie normativ »in den Bürgern selbst verwurzelt« (Göhler 2007: 302f.) sind, weshalb niemand aus dem kulturellen Rahm fällt und dafür sanktioniert werden muss. Um auf Dauer stabilisierend zu wirken, müssen politische Institutionen allerdings bereits im Vorhinein orientierend wirken, indem sie ihre grundlegenden Werte und Sinnvorstellungen zum Zweck der normativen Integration und symbolischen Repräsentation zum Ausdruck bringen (Cöhler 1997; Brodocz 2006; Rehberg 2014). 
Legitimitätsleistung für die politische Ordnung dar, welche nur in der Arena der politischen Kultur erbracht werden kann (Vorländer 2006: 17): »Zugespitzt«, kommentiert Daniel Schulz, »ließe sich so behaupten, dass Geltung immer auf Deutung beruht und dass jede Deutung ein soziales Substrat besitzt« (2006: 67). Damit ist die Konstruktion von als legitim akzeptierten Deutungen essentiell für die Stabilität bzw. Persistenz einer jeden politischen Ordnung. Gerade ein Ansatz, »der solche machtförmigen Prozesse innerhalb der deutungs- und soziokulturellen Spannungsverhältnisse verortet« (ebd.: 68), steht jedoch außerhalb des Kontexts des Bundesverfassungsgerichts bzw. des Rechts noch aus.

Philipp Stoellger hat mit seinem Deutungsmachtkonzept zwischen Hermeneutik und Diskursanalyse zudem auf einen wichtigen Punkt aufmerksam gemacht, der für einen solchen Abstraktionsversuch weiterführend erscheint. Er weist darauf hin, dass allein durch die personale wie strukturelle Macht zur Ermöglichung und Verwirklichung einer Deutung "noch nicht verständlich [wird], wie eine Deutung selber mächtig werden kann« (Stoellger 2014: 37). Neben der Macht zur Deutung, die als personales Vermögen immer rollentheoretisch in die vor- und nachgängigen, historisch-kulturellen Deutungskontexte und -prozesse eingebettet ist und damit stets akteurs- wie strukturlogisch gedacht werden muss, ist es für das Verständnis von Deutungsmacht essentiell, die Deutung selbst, d.h. ihre Medialität, Kommunikation und Rezeption, in die Analyse miteinzubeziehen. ${ }^{5}$ Als genitivus obiectivus kommt nicht nur den Adressaten der Deutung eine herausragende Rolle hinsichtlich ihrer Anerkennung zu, sondern im Sinne eines genitivus subiectivus auch der Deutung selbst, welche mächtig werden und sich verselbstständigen kann. ${ }^{6}$ Deutungsmacht gilt es deshalb prinzipiell als eine vierstellige Relation aus Akteur, Struktur, Medium und Rezipient zu modellieren, die nicht allein von Seiten des Akteurs oder der Struktur, sondern erst durch deren Kommunikation in media-

5 Damit unterscheiden sich beide Deutungsmachtkonzepte grundlegend von anderen Ansätzen, die in ähnlicher Weise auf den machtförmigen Einfluss von Medien bzw. der medialen Vermittlung aufmerksam machen. Daniel Lambach und Christian Göbel verweisen mit ihrem Konzept einer diskursiven Macht beispielsweise auf die infrastrukturellen Kapazitäten eines autokratischen Regimes, seine Untertanen durch geschicktes Top-Down-Framing »das glauben zu machen und wollen zu lassen, was es will« (2010: 85). Als autokratisches Wesensmerkmal gerät die Seite der Adressaten bzw. deren politisch-kulturelle Einbettung bei ausreichender Diskurshoheit des Regimes jedoch aus dem Blick. Zudem wird trotz Anlehnung an einen kritisch-marxistischen Machtbegriffs bei Steven Lukes und Antonio Gramsci ausgeblendet, dass auch in Demokratien, trotz des pluralen Spielraums, diskursive Hierarchien bestehen.

6 Paula Diehl betont in ihren Überlegungen zum Verhältnis von Symbolizität und Performativität, das Texte, Bilder und Rituale selbst die Kraft von Handlungen besitzen, weil sie wirklichkeitskonstituierend bzw. -verändernd wirken (Diehl 2016: 14). Dem Legitimationsglauben des Individuums in das Medium steht somit ein Handeln des Mediums bzw. das Ergriffenwerden von demselben gegenüber. 
len Formen zu verstehen ist (ebd.: 38). ${ }^{7}$ Stoellgers mediale Erweiterung lässt sich so für eine Abstraktion des vorländerschen Deutungsmachtkonzeptes nutzen, um der Frage auf die Spur zu kommen, wann Deutungen im Kontext der politischen Kultur mächtig und für politisches Handeln relevant werden. Dafür scheint jedoch eine Verknüpfung mit den kardinalen Forschungsfragen Rohes, dem Wechselspiel aus deutungs- und soziokultureller Praxis sowie dem konstitutiven Doppelcharakter politischer Kultur notwendig.

\section{Forschungsfragen der Politischen Kulturforschung zur Deutungsmacht}

(a) Bezüglich der Frage, wer innerhalb einer Gesellschaft politische Deutungsangebote machen kann, erscheint es zunächst wenig überraschend, dass die Macht bzw. die Möglichkeit zur Deutung nur bestimmten, professionalisierten Sinn- und Symbolproduzenten aus Politik, Kultur, Wirtschaft etc. zukommen kann. Sie verfügen aufgrund personeller Autorität und/oder ermächtigender Strukturen und Ressourcen über »institutionell abgestützte Deutungskulturen« (Rohe 1994: 9), um politische Sinn- und Deutungsangebote für andere zu produzieren. Im alltagschöpferischen Prozess der politischen Kulturpflege ist es - unabhängig davon, ob es sich um bloße Anpassungsleistung oder grundlegende Innovationen handelt deren Aufgabe, politischen Sinn zu generieren, zu aktualisieren und symbolisch zu erneuern. Die Kontrolle über die politische Kultur bzw. der Einfluss auf die Kulturpolitik beschränkt sich damit jedoch nicht auf das Kulturmonopol des Nationalstaates, sondern zielt gerade auf die kommunikativen Machtverhältnisse innerhalb eines kulturellen Pluralismus bzw. dessen Vorhandensein innerhalb der Gesellschaft ab (Pelinka 2009: 226). Damit sind Deutungsmachtkonflikte zwischen den Sinnproduzenten jedoch vorprogrammiert. ${ }^{8}$

(b) Die Frage für wen diese gemacht werden bzw. »wer diese anderen sind, ob sie sich primär transnational, national, konfessionell, regional, ethnisch, sozial, sektoral oder funktional bestimmen lassen« (Rohe 1987: 40), bleibt zunächst offen.

7 Deutungsmacht definiert Stoellger entsprechend als »personal das Vermögen zur Deutung oder dazu, mit Deutung Macht auszuüben, nicht-personal die [...] Möglichkeit zur Deutung oder zur Macht in Form von Deutung, näherhin medial die Möglichkeit und das Wirkungspotential einer Deutung und strukturell die Macht zur Ermöglichung bzw. Verwirklichung einer Deutung (reps. deren Negation analog zum Machtbegriff)« (2014: 36-37, Herv. i. O.).

8 Stoellger unterscheidet zwischen einem Normalfall von Deutungsmacht, in dem diese »bereits anerkannt, daher >selbstverständlich « und nicht als solche thematisch oder problematisch [ist] « und einem Konfliktfall, in dem diese strittig wird, »nicht mehr (oder noch nicht) selbstverständlich, sondern im Vergehen (oder Entstehen) begriffen [ist] (Stoellger 2014: 41f.). Die implizite These erkennt eine Deutung demnach gerade dann als besonders mächtig an, wenn ihr unhinterfragt gefolgt wird. 
Politische Kultur hat das Individuum nicht allein, sondern immer nur mit anderen zusammen. Träger sind letztendlich nicht die Individuen selbst, sondern die gesellschaftlichen Kollektive. Deshalb ist es essentiell, sich darüber klar zu werden, »wer der kollektive Träger der von ihr zu untersuchenden politischen Kultur ist « (Rohe 1987: 40; Gabriel 1994: 25). Dies erscheint jedoch mitunter höchst problematisch, da die Nation bzw. die politische Gemeinschaft, als primäre Untersuchungsebene der politischen Kulturforschung, selbst auf einen abstrakten, unverfügbaren ${ }^{9}$ und damit deutungsbedürftigen Begriff verweist. ${ }^{10}$ Die üblicherweise gewählten Kriterien für eine inhaltliche Definition, bleiben wie Eric Hobsbawm resümiert, »fuzzy, shifting and ambiguous, and as useless for purposes of the traveller's orientation as cloud-shapes are compared to landmarks« (1990: 6). Nun sind Deutungsangebote gleichwohl nicht in der Lage, unverfügbare Begriffe wie die Nation abschließend zu definieren oder die Diskussion über sie zu beenden. Im Kontext politischer Kultur kommt ihnen - beispielsweise über die Vermittlung von mit politischem Sinn gefüllten Weltbildern - aber die Aufgabe zu, diese zu erschließen, sie sozial wie symbolisch zu bewältigen und ruhigzustellen. Was die Nation bzw. die politische Gemeinschaft ist und wer bzw. wer nicht ein Teil von ihr ist oder sein kann, lässt sich schwerlich in Form von Merkmalszuschreibungen erfassen, sondern ist das Ergebnis einer gemeinsamen Lebensweise. Unabhängig von der Untersuchungsebene (Nation, Bundesland, Stadt, Dorf etc.) stößt man jedoch auf eine weitere Schwierigkeit bezüglich der Fragen, für wen Deutungsangebote gemacht werden. Da es sich bei politischer Deutungskultur um einen, von der politischen wie sozialen Alltagswelt, abgehobenen Prozess handelt, »der vorrangig von Leuten inszeniert und verwaltet wird, die an der politischen Soziokultur, die sie thematisieren und mit Deutungsangeboten versehen, selbst nur bedingt teilhaben « (Rohe 1987: 43), besitzen Deutungen stets eine gewisse Eigenlogik. $\mathrm{Zu}$ einem nicht unerheblichen Teil kommen in politischen Deutungsangeboten die Vorstellungen einer politisch-kulturellen Elite zum Ausdruck, weshalb die in einer jeden politischen Kommunikation angelegte Möglichkeit zu berücksichtigen ist, »daß das Deutungsangebot nicht sankommt<, weil der >Empfänger sich in den offerierten Interpretationsangeboten nicht >wiederfinden und >wiedererkennen kann« (ebd.: 43). Daher muss weiterhin gefragt werden, welchen Anspruch (umfassend vs. partikular) Deutungsangebote

9 Für Hans Vorländer gelten solche Sachverhalte als unverfügbar, »die in der Perspektive von Akteuren der unmittelbaren, alläglichen Lebenswelt entzogen sind und deshalb quasi entrückt erscheinen, die gleichwohl aber auf sie zurückwirken und ihr Sinn und Geltung verleihen« (2013: 20).

10 Die Definition Almonds und Verbas verweist hier zwar auf die spezifischen Verteilungsmuster der politischen Orientierungen der Mitglieder einer Nation (Almond/Verba 1963: 13). Ziel ist dabei jedoch eher die territoriale Eingrenzung der Erhebung als der Versuch einer substantiellen Definition des Nationenbegriffs. 
innerhalb des Kollektivs vertreten bzw. von welchen gesellschaftlichen Gruppen sie Geltung einfordern sollen und können.

(c) Mit Blick auf die Gruppenbezogenheit politischer Ideen entwickelt die Differenzierung zwischen Deutungs- und Soziokultur weiterhin Relevanz bezüglich der Frage, was innerhalb einer politischen Kultur überhaupt gedeutet wird und welchen Einfluss die Produzenten politischer Deutungsangebote auf die für eine soziale Gruppe maßgebenden, Grundannahmen über die politische Welt besitzen. Der zentrale Ansatzpunkt für Rohes Überlegungen findet sich hier in Max Webers Wirtschaftsethik der Weltreligionen. Demnach sind es zwar Interessen und nicht Ideen, die das unmittelbare Handeln der Menschen beherrschen, jedoch bestimmen durch Ideen geschaffenen Weltbilder stets mittelbar ${ }^{11}$ die Bahnen, in denen die Dynamik der Interessen das Handeln fortbewegt (Weber 1920: 252). Im Kern besteht politische Kultur somit aus nichts Anderes als aus jenen

»in die politische und gesellschaftliche Wirklichkeit eingelassene(n) Ideen, die Politikhorizonte abstecken, Sinnbezüge stiften und von ihren jeweiligen gesellschaftlichen Trägern als Maßstäbe zur Auswahl, Organisation, Interpretation, Sinngebung und Beurteilung politischer Phänomene benutzt werden« (Rohe 1994: 3).

Innerhalb politischer wie gesellschaftlicher Ordnungskonzepte verstehen sich Ideen aber nicht von selbst. Sie werden in entsprechenden Sozialisations- und Kommunikationsprozessen gedeutet, vermittelt und stabilisiert. Die Idee der Demokratie, im Sinne eines Essentially Contested Concepts (Gallie 1955), ist so vielfältig wie ihre begriffs- und ideengeschichtlichen bzw. systematischen Entwürfe und Definitionen von der Antike bis in die Gegenwart. ${ }^{12}$ Sie erlaubt nicht nur einen weiten Spielraum hinsichtlich der zentralen, demokratischen Prinzipien und Werte, sondern gleichfalls konkurrierende bzw. konfligierende Deutungen über ein und dieselbe Idee. Für ein tiefergehendes Verständnis politischer Kultur ist es unerlässlich jene kulturellen Eigenarten herauszuarbeiten, in denen sich politische Prinzipien, Werte, Normen, Strukturen, und Wissensbestände über Deutungsmachtprozesse konstituieren und als evaluative Maßstäbe für die Bewertung von Politik eingebettet sind.

(d) Im Wechselspiel aus deutungskultureller Reflexion und soziokultureller Verankerung stützt sich die Anerkennung eines politischen Weltbildes jedoch nicht allein auf die kognitive Verinnerlichung eines politischen Ideen- und Wertesystems,

11 Eben hinsichtlich der Definition und Relevanz zentraler gesellschaftlicher Werte, Ziele, Überzeugungen und Ordnungsvorstellungen (Schulz 2006: 67).

12 Das gilt beim Vergleich politischer Kultur besonders für Demokratievorstellungen jenseits des Westens (Schubert/Weiß 2016). 
sondern stets auch auf dessen emotionale Veräußerlichung. Aufgrund ihres prinzipiellen Doppelcharakters, ist politischer Kultur daher stets beides »innerlich und äußerlich, [...] objektiv und subjektiv« (Rohe 1990:337). Bezüglich der Frage, aufwelche Weise politische Deutungsangebote gemacht werden, ist daher die Feststellung zentral, dass neben einer auf die kognitive und normative Dimension bezogenen Inhaltsseite, eine affektive und ästhetische Ausdrucksseite, ein konstitutives Wesenselement politischer Kultur bildet. Ob politische Weltbilder und Ideen in Wort, Schrift, Bild oder Tat zeichenhaft vermittelt werden, ist aus einer Deutungsmachtperspektive nicht nur deshalb relevant, weil Deutungsangebote unterschiedliche Formen annehmen können, sondern weil Namen, Zeichen, Gesten, Standbilder, Fahnen, Orte, Mythen und Rituale eine eigene Geschichte besitzen und deshalb mehr sind als der bloße Ausdruck des Inhalts (ebd.: 338). Abseits rationaler $\mathrm{Zu-}$ stimmung rufen sie affektive Empfindungen hervor, stellen emotionale Bindungen her und tragen dazu bei, dass Deutungen selbst mächtig werden können. Die erfolgreiche, weil als legitim anerkannte Institutionalisierung von Deutungen über Erzählungen, Ikonographien, Rituale und Symbole, ist für unseren politischen Alltag relevant, weil diese als undiskutierte Selbstverständlichkeiten in die unbewussten Denk-, Rede- und Handlungsgewohnheiten der politischen Soziokultur eingehen. ${ }^{13}$ Gerade angesichts dieser Vielfalt, in der politische Weltbilder medial vermittelt werden, bedarf es deshalb eines breiten kulturwissenschaftlichen Blicks auf politische Phänomene.

Für eine deutungsmachtsensible Perspektive politischer Kulturforschung bleibt somit festzuhalten, dass es sich bei jenen kardinalen Forschungsfragen unweigerlich auch immer um Deutungsmachtfragen handelt. Jedoch ist nicht allein von Interesse, wer für wen, auf welche Weise, was für politische Deutungsangebote macht, sondern vor allem unter welchen Bedingungen diese erfolgreich sind. Wie werden neue Deutungen institutionalisiert? Können bereits institutionalisierte Deutungen eine Umdeutung erfahren? Warum werden bestimmte Deutungsangebote angenommen, während andere abgelehnt werden? Wieso bleiben bestimmte Deutungen unhinterfragt anerkannt, während andere Deutungen fragil werden und an Geltung verlieren. Kurzum, worin liegen die institutionellen wie sozialpsychologischen Bedingungen für erfolgreiche Deutungsangebote? Als machtsensible Perspektive zielt eine entsprechende Konzeptualisierung von Deutungsmacht auf die Aufdeckung und Dechiffrierung der politischen Weltbilder sozialer Verbände $\mathrm{ab}$, die sich den Möglichkeiten, Voraussetzungen, Inhalten und Folgen politischer Kulturpolitik widmen und die nur mit Hilfe verschiedener methodischen Zugänge, gelingen kann. Eine solche Perspektive kann allerdings nicht nur im Rahmen

13 Übereinstimmend mit Rohe treten politische Weltbilder in Normalzeiten kaum in Erscheinung, da sie »so etwas wie einen selbstverständlichen Hintergrundrahmen des politischen Lebens darstellen« (Rohe 1994: 15). 
qualitativer, kulturwissenschaftlicher Ansätze als aufschlussreich gelten, sondern könnte sich besonders in der Ergänzung klassischer Ansätze als fruchtbar erweisen.

\section{Anknüpfungspunkte der klassischen Politischen Kulturforschung}

Wie eingangs bereits angesprochen, muss bis heute konstatiert werden, dass sich klassische Ansätze, wie sie in der Tradition der Almond/Verba-Studien überwiegend im Bereich der quantitativen empirischen Sozialforschung zur Anwendung kommen, und umfassende Ansätze, die vor allem im Bereich der qualitativen Kultur-, Medien- und Sozialwissenschaft prominent rezipiert werden, während des langjährigen Stillstands des DVPW-Arbeitskreises »Politische Kultur-Forschung " weitestgehend unvermittelt bzw. sogar antagonistisch gegenüberstehen. Die gegenseitige Skepsis ergibt sich aber nicht allein aus den unterschiedlichen Antworten auf die theoretisch-konzeptionelle Frage, welchen Gegenstandsbereich das Konzept politischer Kultur umfassen sollte ${ }^{14}$ bzw. mit welchen Methoden der Analyse politischer Kultur empirisch zu begegnen sei, sondern ist auch Ausdruck grundlegender Missverständnisse, die im Laufe der Jahre mitunter $\mathrm{zu}$ einer hermetischen Abschottung und Immunisierung der jeweils eigenen Ansätze geführt haben.

Sylvia Greiffenhagen betont vor diesem Hintergrund, dass es für eine konstruktive Debatte zur Weiterentwicklung der politischen Kulturforschung nun aber wichtig sei, die alten Grabenkämpfe zu überwinden und die unterschiedlichen Ansätze miteinander zu verbinden:

»Empirische Methoden liefern repräsentative und, soweit möglich, objektive Ergebnisse; hermeneutische Forschungsmethoden helfen dabei, die richtigen Fragen zu stellen und objektiv messbare Phänomene überhaupt zu verstehen « (2009: 24).

Es geht darum, die konzeptionellen und methodischen Möglichkeiten, die der Begriff der politischen Kultur eröffnet, nicht als prinzipielle Schwäche oder Unschär$\mathrm{fe}$, sondern als eine grundlegende Stärke in der empirischen Erforschung zu verstehen (Salzborn 2009: 53). Eine intelligente Koppelung zielt weder darauf ab, »die einstellungsorientierte Analyse politischer Kultur zu Gunsten eines anderen Vorgehens aufzugeben« (Gabriel 1994: 29), noch sieht sie in einstellungszentrierten

14 Prominent wird hier immer wieder Max Kaases Kritik an der Beliebigkeit der Verwendung des Begriff politischer Kultur zitiert, die dazu geführt hat, dass die wissenschaftliche Auseinandersetzung mit dem Konzept dem Versuch gleiche, »einen Pudding an die Wand zu nageln« (1983: 144). 
Ansätzen einen negativen Referenzpunkt von dem es sich mit allen Mitteln abzusetzen gilt (Schwelling 2004: 21). Mit Hilfe einer machtsensiblen Perspektive politischer Kulturforschung, sollen deshalb vielmehr die Anknüpfungspunkte für eine gegenseitige Ergänzung aufgezeigt werden, um »gleichermaßen normative wie historische, qualitative wie quantitative Ansätze zu integrieren und nutzbar machen zu können « (Salzborn 2009: 53). Zu diesem Zweck sollen in der Folge vier konzeptuelle Schwierigkeiten und Probleme klassischer Ansätze diskutiert werden.

\section{Das Problem einer "Arithmetik der Macht»}

Einer der Kerngedanken der klassischen politischen Kulturforschung besteht gerade darin, politische Kulturen im Sinne eines methodologischen Individualismus über die Beziehung zwischen Mikro- und Makroebene zu erfassen. Als überindividuelles Phänomen werden für die Analyse einer politischen Kultur die Orientierungen $^{15}$ von Individuen erfasst und anschließend deren Verteilung im Aggregat der Gesamtbevölkerung beurteilt. Vor dem Hintergrund eines »demokratische[n] Argument[s] des Gleichgewichts aller abgefragten Meinungen«(Greiffenhagen/Greiffenhagen 2003: 516) können deshalb unterschiedliche Macht- und Entscheidungspositionen innerhalb des politischen Systems nicht entsprechend in die Analyse miteinbezogen werden. Zwar erscheint

»selbstverständlich allen Forschenden deutlich, dass die Orientierungen und Handlungen von Angehörigen der politischen Elite [...] mehr Gewicht für den politischen Prozess oder die politische Stabilität und Weiterentwicklung eines Systems haben als die Orientierung eines individuellen Bürgers« (Westle 2009: 51),

das Problem einer solchen Arithmetik der Macht lässt sich jedoch durch die methodologisch bedingte Konzentration auf die repräsentative Gesamtheit der Bevölkerung nicht entsprechend abbilden. Forschungsgeschichtlich ist im Zuge der unmittelbaren Rezeption der Almond/Verba-Studie jedoch die notwendige und folgenreichenreiche Differenz zwischen einer politischen Eliten- und Massenkultur sowie die methodische Berücksichtigung bei deren Analyse verbrieft: »The fact that the two cultures of the elites and the mass exist in all political systems means that systems can be readily classified according to the character of the relationship between the two (Pye 1965: 16). Die Untersuchung einer solchen politischen Elitenkultur muss jedoch mehr leisten als die Beschreibung eines repräsentativen Fragments bestimmter Einstellungen und Werthaltungen innerhalb eines breiten

15 Der Beitrag versteht politische Orientierungen als Überbegriff zur Unterscheidung zwischen politischen Einstellungen und politischen Vorstellungen. 
gesellschaftlichen Kontexts. Dazu erfordert es aber eine grundlegende "methodological innovation which will allow us to do justice to the subtleties of the belief systems of sophisticated political leaders [...] « (Putnam 1971: 651; Pye 1965: 16). ${ }^{16}$ Das Problem einer Arithmetik der Macht geht somit gleichwohl über die Frage der Gewichtung politischer Einstellungen im Aggregat der Gesamtbevölkerung hinaus. Wenn politische Kultur stets an die systematisierte Form von Herrschaft gebunden ist, spielen die gesellschaftlichen Machtverhältnisse und Herrschaftsstrukturen eine maßgebliche Rolle bei der Vermittlung und Akzentuierung von Inhalten und Sozialisationsformen (Pelinka 2006: 225f.). Da politische Kultur nicht zu einem bestimmten Erhebungszeitpunkt einsozialisiert wird, sondern als lebenslanger Prozess politischer Sozialisation der steten Aktualisierung, Anpassung und Wandlung unterliegt, müssen die Orientierungen und Handlungen der politischer Elite bzw. jener "values and norms regarding politics and the political system held by those closest to the centers of political power, including elected officials, bureaucrats, and business leaders « (Hague et al. 2016: 205) und deren Einfluss auf die Orientierungen der Bürgerinnen und Bürger ${ }^{17}$ eine tiefergehende Berücksichtigung bei der Erschließung politischer Kultur finden.

\section{Die Komplexität der politischen Unterstützungsobjekte}

Einen grundlegenden Eckpfeiler bei der Erfassung und Systematisierung politischer Orientierungen bildet in klassischen Ansätzen politischer Kultur David Eastons Konzept der politischen Unterstützung $(1965,1975)$ bzw. dessen Weiterentwicklungen und Verfeinerungen im Kontext der empirischen Sozialforschung (Fuchs 1989; Gabriel 1994, 1999; Westle 1989, 2009). Politische Unterstützung wird in diesen Ansätzen als eine Einstellung verstanden, mit welcher sich ein Individuum evaluativ gegenüber einem politischen Unterstützungsobjekt orientiert. Während sich die spezifische Unterstützung, als Leistungsbewertung der politischen Herrschaftsträger, nur auf dieses eine Unterstützungsobjekt richtet, wird diffuse Unterstützung allen Unterstützungsobjekten entgegengebracht:

16 Dazu stehen mittlerweile auch der Politikwissenschaft eine ganze Bandbreite an Instrumenten zur Verfügung, die im Kontext eines cultural turn erarbeitet und weiter verbessert wurden.

17 Das gilt gerade, wenn aus sozialpsychologischer Sicht davon ausgegangen werden muss, dass politische Eliten ausgeprägtere und konsistentere politische belief systems besitzen als der Rest der Bevölkerung (Converse 1964, Sartori 1967, Rokeach 1960). Eine zentrale Erkenntnis von Converse' berühmter Studie, die bis heute zu den Grundlagentexten einstellungsorientierte Forschung zählt, war es deshalb, dass der Grad an politischer Teilhabe, der Bildungsstand und die Menge an politisch relevanten Informationen innerhalb einer »Arithmetik der Macht «nicht nur nach unten hin immer weiter abnimmt, sondern dass im gleichen Zug auch die politischen belief systems der Individuen immer diffuser und inkonsistenter werden. 
»The briefest way of describing the primary meaning of diffuse support is to say that it refers to evaluations of what an object is or represents - to the general meaning it has for a person - not of what it does « (Easton 1975: 444).

Diese prinzipielle Komplexität bzw. Unbestimmtheit der politischen Unterstützungsobjekte (Gemeinschaft, Ordnung, Herrschaftsträger) und ihrer Elemente (Gemeinschaftssinn, Identität - Prinzipien, Ideen, Werte, Normen, Herrschaftsstruktur - Rollen, Befugnisse, Verantwortlichkeiten) stellt für die Messung der politischen Unterstützung jedoch ein prinzipielles Problem dar. Einstellungen beziehen sich stets auf komplizierte und abstrakte Objekte, die sich dem Individuum als vom eigenen Leben relativ weit entfernt, als undurchschaubar und deshalb oft als unwichtig darstellen (Gabriel 1994: 27). ${ }^{18}$ Begriffe und Konzepte wie Nation, Staat, Demokratie, Freiheit oder Gerechtigkeit sind nicht nur in wissenschaftlichen Diskurs hochgradig umstritten und umkämpft ${ }^{19}$, sondern rufen auch bei den Bürgerinnen und Bürgern unterschiedliche Vorstellungen, Definitionen und Interpretationen hervor. Klassische Ansätze reflektieren diese Schwierigkeiten zwar entsprechend, müssen aus Gründen der Forschungsökonomie und aus theoretisch-konzeptionellen Erfordernissen jedoch auf eine mehrstufige Messung, welche unterschiedliche Interpretationen für die Unterstützungsobjekte und deren Elemente anbieten würde, verzichten. Stattdessen werden den Befragten die ausgewählten Ordnungsvorstellungen und Werte in bewusst uninterpretierter Weise vorgelegt. Dadurch bleibt jedoch im Unklaren, was die Befragten unter den einzelnen Ordnungsvorstellungen und Werten verstehen, weil »ihre Realitätsbewertung der Demokratie auf der Grundlage ihres eigenen (unbekannten) Demokratieverständnisses « (Westle 1989: 241) beruht. Egal ob für die Bewertung der abstrakten Ordnungsvorstellung einer interpretierten politischen Philosophie oder für die materielle politische Ordnung eines konkreten Staates, die Maßstäbe bleiben als »Vorstellung des Befragten vom Wünschenswerten« bzw. als »subjektive Definition des Bewertenden« (ebd.: 191) der Analyse politischer Einstellungen entzogen.

Die Bevölkerung wird mit ihren Vorstellungen jedoch keineswegs allein gelassen. Im Zuge der politischen Sozialisation versuchen relevante politische Akteurinnen und Akteure ganz gezielt ihre Deutungen komplizierter und abstrakter politischer Konzepte und Begriffe zu vermitteln. Schließlich bestimmt aus Sicht

18 Philip E. Converse führt diesen Umstand darauf zurück, dass »educated elites in general, and political elites in particular, think about< elements involved in political belief systems with a frequency far greater than that characteristic of mass publics« (2006: 6).

19 Beispielhaft dazu die Sammelbände von Gerhard Göhler, Mattias Iser und Ina Kerner zu Politische Theorie - 25 umkämpfte Begriffe zur Einführung (2012) sowie von Sophia Schubert und Alexander Weiß zur ,Demokratie< jenseits des Westens - Theorien, Diskurse, Einstellungen (2016). 
der Befragten nicht der (politik-)wissenschaftliche Diskurs, was unter Demokratie $\mathrm{zu}$ verstehen ist oder wer und wer nicht Teil der politischen Gemeinschaft sein kann. Die Maßstäbe für die Ablehnung oder Zustimmung politischer Unterstützungsobjekte und ihrer Elemente bleiben das Ergebnis jenes alltagsschöpferischen Prozesses, in dem sich politische Eliten und Bevölkerung, im Sinne der diffusen Unterstützung, über das universelle Verhältnis von Kultur, Gesellschaft und Staat austauschen (Hildebrandt 1996). Durch politische Sinnkonstruktionen wird in diesem Wechselspiel überhaupt erst verhandelt, was die Unterstützungsobjekte und -elemente repräsentieren und welche grundlegende Bedeutung sie für das politische System und deren Mitglieder besitzen. Welche relevanten Deutungen innerhalb einer Gesellschaft zirkulieren, wie diese Deutungen entstehen, funktionieren und vergehen, ist deshalb von zentralem Interesse für ein besseres Verständnis der diffusen politischen Unterstützung.

\section{Die historischen Eigenarten politischer Kulturen}

Die Notwendigkeit der Deutung politischer Unterstützungsobjekte und deren Elemente zielt insofern direkt auf die historischen Eigenarten politischer Kulturen $a b$, als dass von Seiten der empirische Sozialforschung selbst eine Vernachlässigung hinsichtlich der Erfassung von Prinzipien, Werten, Normen und Weltbildern konstatiert wird (Westle 2009: 31). ${ }^{20}$ Politische Kultur als »particular distribution of patterns of orientation toward political objects among the members of the nation« (Almond/Verba 1963: 13) betont schließlich nicht allein das Ergebnis kollektiver Sozialisation, welches zu einem bestimmten Zeitpunkt unter der Bevölkerung erhoben wird, sondern auch die längerfristigen historischen Prozesse, in denen sie sich herausbildet und verändert. So konstatiert Gabriel Almond in The Civic Culture Revisited, dass "attitudes and values seem to be significantly affected by national and group historical and life experience (1989: 24). Den Versuch, »die historische Dimension als eine kontrollierte systematische Variable« (Rohe 1994:3) in die politisch-kulturelle Analyse einzubringen, erkennt Gert Pickel demnach zwar auch durchaus als ein schlagkräftiges Argument gegen die Verengung des politischen Kulturbegriffs an, plädiert angesichts der »erhebliche[n] Umsetzungsprobleme in der Praxis« dennoch dafür, »eine bewusste - und auch reflektierte - Verengung des politischen Kulturbegriffes beizubehalten« (2010: 10). Diese Einschätzung erscheint widersprüchlich und immunisierend, wenn Rohes Einwand prinzipiell als

20 Zwar betont Westle, dass »dieses Defizit weder dem Konzept noch dem methodischen Zugang der Befragung inhärent« (2009: 31) sei, die weitere Systematisierung und Verfeinerung der Messung bleibt hier jedoch eher ein konzeptuelles Problem als eine mögliche Lösung (ebd.: 50). 
gerechtfertigte Kritik anerkannt wird, letztendlich aber trotz der aufgezeigte Probleme keinen Niederschlag in möglichen Ergänzungen findet. ${ }^{21}$ Mit Blick auf die historische Entwicklungsdimension politischer Kultur besteht somit weiterhin die Notwendigkeit einer »enzyklopädischen Dokumentation institutioneller und kultureller Details«, um zu jener »die historische Genese reflektierenden Gesamtinterpretation « (Salzborn 2009: 55) zu gelangen, welche »die politischen und gesellschaftliche Totalität der Moderne« (ebd.: 53) überhaupt erst fassbar macht. Ein solches Vorgehen kann klassische Ansätze aber nicht nur hinsichtlich jener historisch-kulturellen Eigenarten bereichern, die in politischen Deutungsangeboten transportiert werden, sondern eröffnet eine wichtige Vergleichsperspektive für das Verständnis politischer Unterstützung in unterschiedlichen politischen Kulturen.

\section{Politische Kulturforschung in nichtdemokratischen Systemen}

Kulturellen Eigenarten und historische Tiefendimensionen sind allerdings nicht nur aufgrund der zeitlichen Beschränkung von Umfrageergebnissen von Interesse, sondern vor allem deshalb, weil sich aus der engen Verbindung klassischer Ansätze der politischen Kulturforschung mit dem Instrument der Umfrageforschung gerade in nicht-demokratischen Systemen erhebliche Anwendungsprobleme ergeben. So betont Gert Pickel, dass in Autokratien, neben der begrenzten Datenbasis für vergleichende empirische Untersuchungen, ein prinzipielles Problem bezüglich der Reliabilität und Validität der Messung von politischer Unterstützung besteht (Pickel 2013: 186f; 2009). Zur Schwierigkeiten führt dies einerseits, weil Umfrageprojekte auch in nicht-demokratischen Systemen ein normatives, demokratisches Grundgerüst für die Fragenkataloge zugrundlegen und andererseits, weil zu berücksichtigen bleibt, dass die Antworten der Befragten, aufgrund von (potentiellen) Repressionsmechanismen, stark von sozialen Konformitäts- und ErwünschtheitsEffekten beeinflusst werden. Der implizite Wunsch klassischer Ansätze, Autokratien - trotz der Anfälligkeit des Instruments der Umfrageforschung - auch weiterhin "von der Demokratie her denken« (ders. 2013: 198) zu wollen, ist letztendlich Ausdruck der Entwicklung hin zu einer kulturellen Demokratieforschung, welche keine Abkehr vom Transitionsparadigma (Carothers 2002) verfolgt, sondern die Unausweichlichkeit der Demokratisierung (Pickel 2009) weiter voraussetzt. Ein solcher democracy bias bzw. solch eine Demokratisierungs-Teleologie geht trotz neue-

21 Entgegen dem Vorwurf verkennt Rohes Kritik in dieser Beziehung gerade nicht die Potentiale der Befragung hinsichtlich Erschließung, Analyse und Interpretation (Westle 2006: 275), sondern zeigt sich, unter der Voraussetzung, dass die Umfrageforschung richtig gehandhabt wird, sogar offen für einen solchen Königsweg. In diesem Verständnis müssten klassische Ansätze gerade ein eigenes Interesse daran entwickeln, ihre systematischen Erkenntnisgrenzen mit Hilfe von historischen Sprach-, Verhaltens- und Symbolanalysen zu ergänzen (Rohe 1994: 5). 
rer Erkenntnisse der Autokratieforschung davon aus, »dass genuine Legitimität sich nur aus demokratischer Legitimität speisen kann« (Holbig 2010: 38), weshalb die Beziehungen zwischen Struktur und Kultur »eigentlich fast nur für demokratische oder teildemokratische System sinnvoll zu bestimmten sind « (Pickel 2010: 620). ${ }^{22} \mathrm{Zu}$ den Leistungen neuerer Forschungsarbeiten im Bereich der Autokratieforschung gehört jedoch gerade die Einsicht, dass sich die Persistenz autokratischer Regime nicht ex negativo, sondern nur über die jeweils eigenen Funktionsweisen und -logiken verstehen und erklären lässt. ${ }^{23}$ Im Zentrum der Diskussion um die Legitimation autokratischer Regime steht somit nicht die normativ-demokratische Legitimität, sondern der von Max Weber als Schlüsselkriterium für das Verständnis politischer Herrschaft erörterte Legitimitätsglauben. Auch Autokratien sind darauf angewiesen, »ein substantielles Maß an politischer Legitimation und Unterstützung aus der eigenen Bevölkerung zu generieren« (Albrecht/Frankenberger 2010: 8). Entgegen der Annahme, dass Tendenzen einer kulturellen Demokratieforschung bereits implizit in den Arbeiten von David Easton angelegt seien (Pickel 2010: 620) ${ }^{24}$, gilt es angesichts der Universalität des Legitimitätsglaubens für die Unterstützung politischer Systeme (Easton 1965: 281), den ideologischen, strukturalen und personalen Quellen bzw. die ihm zugrunde liegenden Prinzipien und Werten für die politischen Unterstützungsobjekte genauer nachzuspüren. Eine Ergänzung der klassischen Umfrageforschung gewinnt hier vor allem deshalb an Gewicht, weil sich die Erklärungsversuche autokratischer Regimestabilität auf ein komplexes Phänomen beziehen, welches »eine breite und gleichermaßen tiefe Analyse notwendig macht« (Bank 2009: 30). Während in der bisherigen Debatte vor allem institutionalistische und politökonomische Ansätze dominierten, werden neuerdings explizit akteurszentrierte und kulturalistische Variablen eingefordert (Kailitz 2009: 471). Die Frage nach der Dauerhaftigkeit autokratischer Regime muss bezüglich ihrer politisch-kulturellen Verankerung dafür jedoch über »die Konzentration auf die shelle Seite < der Macht « (Albrecht/Frankenberger 2010: 4) hinausgehen. Eine machtsensible Perspektive politischer Kulturforschung bietet

22 Diese Argumentation zäumt das Pferd vielmehr von hinten auf, wenn sie darauf beruht, dass es nur in Demokratien ein normatives Grundgerüst gebe, zu denen sich die Bürgerinnen und Bürger klar bekennen können und welches sich mit dem Instrument der Umfrageforschung erfassen lässt.

23 Schließlich wird auch für eine politische Kulturforschung, die sich auf das Objekt Demokratie hin ausrichtet argumentiert, dass es »grundsätzlich wenig plausibel [ist], die Essenz oder den Kern eines Phänomens in etwas zu begreifen, das außerhalb dieses Phänomens liegt« (Fuchs 2004: 97, 2002).

24 Dieser Lesart wird hier vehement widersprochen, schließlich war es von Beginn an Eastons Ziel, eine möglichst allgemeine Theorie der Politik zu entwickeln, »[that] helps us to prevent research from remaining exclusively and narrowly preoccupied, at least implicitly, with one type of system, namely, democracy as it has developed in the West« (Easton 1965: 15). 
daher in nicht-demokratischen Regimen die Möglichkeit, der Frage nachzugehen, wie Autokratien versuchen die politische Kultur zu prägen und inwieweit sie da$\mathrm{zu}$ in der Lage sind, eine Kongruenz zwischen Struktur und Kultur herzustellen, welche den Legitimationsglauben in das Regime zu stimulieren vermag.

\section{Deutungsmacht in David Eastons Konzept politischer Unterstützung}

Eine machtsensible Perspektive politischer Kulturforschung bietet somit bedeutende Vorzüge und Potentiale für die Analyse politischer Kulturen unter der Berücksichtigung institutioneller, medialer wie symbolischer Dimensionen von Macht. Einen zentralen Ankerpunk für die gegenseitige Ergänzung quantitativer wie qualitativer Ansätze bietet dafür David Eastons Konzept der politischen Unterstützung. Mit Blick auf die diffuse Unterstützung der politischen Objekte und ihrer Elemente ist dafür zunächst festzuhalten, dass Eastons Konzept in Ansätzen der empirischen Sozialforschung als striktes behaviouralistisches Programm verstanden und rezipiert wird. Die »Aufnahme der Methode der Umfrageforschung als klassischem Instrument des Behaviorismus « (Pickel/Pickel 2006: 60) führt jedoch dazu, dass wichtige institutionelle, mediale, symbolische Aspekte von Macht in A Systems Theory of Politcal Life schlichtweg ausgeblendet werden. ${ }^{25}$ Diese sind jedoch von grundlegender Bedeutung, weil auch Easton zu dem Schluss kommt, dass

»equal expressions of supportive attitudes cannot automatically be given equal weight with respect to their consequences for the persistence of a system. A few powerful members, such as an active political elite, [...] may be able to make their positive or negative support count for more than high levels of support from unorganized millions« (1965: 167).

Deshalb ist es notwendig, die effektive instrumentelle Macht einer Gruppe als integralen Teil des unterstützenden Inputs zu spezifizieren. Bei Easton werden zudem aus allgemeinen Machtfragen spezifische Deutungsmachtfragen, wenn politische Systeme sich unterschiedlich gegenüber verschiedenen politischen Prinzipien und Werten orientieren, weil die Frage der Bedingungen "under which some political values instead of others become dominant for a system is a moot question « (ebd.:

25 Zudem weist Easton selbst auf die Gefahr einer Überbetonung behavioralistischer Forschungsmethoden und -techniken in der Analyse von Politik hin. Er plädiert sogar im Zuge einer PostBehavioralistischen Revolution für ein sogennantes Credo of Relevance, »because it is more important to be relevant and meaningful for contemporary urgent social problems than to be sophisticated in the tools of investigation. For the aphorism of science that it is better to be wrong than vague, post-behavioralism would substitute a new dictum, that it is better to be vague than non-relevantly precise« (Easton 1969: 1051). 
198). Eine machtsensible Perspektive politischer Kulturforschung setzt an dieser elementaren Stelle an.

\section{Deutungsmacht über gemeinschaftliche und legitimierende Aspekte von Ideologie}

Obwohl Easton keine eigene Teiltheorie über Ideologie entwirft, stellt er deren herausragenden Charakter "as a major response mechanisms for initiating and bolstering the input of diffuse support « (1965: 335) ins Zentrum seiner Diskussion. ${ }^{26}$ Er konzipiert eine sogenannte omnibus ideology, die als »the most inclusive set of ideological beliefs held by a member", in Bezug auf die politischen Unterstützungsobjekte jeweils verschiedene Aspekte abbildet und deshalb nach drei Typen unterschieden werden kann. ${ }^{27}$ Innerhalb einer communal ideology finden sich laut Easton jene auf die Persistenz und den Wandel der politischen Gemeinschaft bezogenen Aspekte, "that express, as well as reinforce, the sense of political unity among the members as a group of persons sharing a common set of structures, norms, and values for political purposes« (ebd.: 336), während legitimating ideologies,

»relate to those aspects of an omnibus set of beliefs that are oriented to supporting or challenging the regime and the right of the authorities to rule. They consist of those principles and values validating a structure, its norms, and occupants in terms of images of the future, interpretations of the present, and conceptions of the past« (ebd.: 336).

Zwei Aspekte sind hier mit Blick auf die Potentiale der Ergänzung relevant. Erstens, dass Easton instrumentelle Deutungsmachtansprüche über die politischen Unterstützungsobjekte impliziert, wenn er betont, dass »categories of belief and conviction are too inescapable and useful [as] a tool in power relationships to be neglected by men anywhere (ebd.: 291; 296f.). Und zweitens, dass die historisch-kulturelle Einbettung ideologischer belief systems eine expressive Bedingung für deren Effektivität »to capture the imagination" (ebd.: 290) darstellt. Bezüglich der diffusen Unterstützung bzw. der Maßstäbe für den Legitimitätsglauben in die politischen Unterstützungsobjekte liegt somit auch bei Easton ein Fokus auf der Imagination

26 Für die politische Gemeinschaft betont Easton die Bedeutung politischer Symbole und Rituale für die Stimulation des politischen Gemeinschaftssinns (1965: 332). Darin zeigt sich ein weiteres Anwendungsfeld für Deutungsmachtmachtanalyse, welches hier jedoch nicht genauer ausgearbeitet werden kann.

27 Der dritte Typ einer partisan ideology, die sich auf die Organisation und den Wettbewerb alltäglicher Politik durch die politischen Herrschaftsträger richtet, findet hier zunächst keine Berücksichtigung. 
politisch relevanter Weltbilder bzw. den "assumptions about the political world « (Elkins/Simeon 1979: 127).

Übereinstimmend betont Martin Greiffenhagen, dass das Legitimitätsproblem prinzipiell mehr Dimensionen besitze als in der empirischen Sozialforschung berücksichtigt werden. Wie bereits angesprochen, ist es für die Erklärung politischer Unterstützung bzw. den Legitimitätsglauben in die politischen Objekte sowie für den Vergleich politischer Kulturen essentiell, überhaupt erst zu verstehen, was diese repräsentieren und welchen grundlegenden Bedeutungsgehalt sie innerhalb einer bestimmten politischen Kultur besitzen. Das gilt besonders für die politische Gemeinschaft, in welcher Easton den zentralen Ansatzpunkt für die Idee von Persistenz und Wandel erkennt (1965: 188), die jedoch aufgrund einer Vielzahl von theoretischen Probleme bis heute am wenigsten elaboriert ist und vor allem für klassische Ansätze bis heute methodisch sehr schwer greifbar bleibt (Greiffenhagen M. 1997: 47; Westle 1989). Gängige Indikatoren zur Operationalisierung der politischen Unterstützung, im Sinne einer positiven emotionalen Bewertung, beziehen sich für die politische Gemeinschaft beispielsweise auf den Nationalstolz oder die Bereitschaft, in Kriegszeiten für das eigene Land zu kämpfen (Klingemann 1998: 10). Damit bleiben sie jedoch eine substantielle Vorstellung dessen schuldig, was die Nation bzw. die politische Gemeinschaft ist, was sie ausmacht und wer Teil von ihr ist. ${ }^{28}$ Das Argument, dass beispielsweise Nationalstolz als Indikator sowohl innergesellschaftlich als auch interkulturell weitgehend ähnlich verstanden werde (Gaber 2007: 177), erscheint mit Blick auf Eastons Konzept haltlos, weil communal ideologies "as responses that fortify sentiments of mutual political identification among the members of a political system [...] differ vastly in the content they transmit « (Easton 1965: 333). Das gilt jedoch nicht nur interkulturell, sondern eben auch innergesellschaftlich, weil communal ideologies "may also be divisive rather than integrative. They may serve as a vehicle for expressing and intensifying cleavages in the community « (ebd.). Die politische Identifikation der Mitglieder mit der politischen Gemeinschaft kann vor dem Hintergrund gemeinsamer historischer Erfahrungen sowie geteilter Traditionen und Erwartungen nur dann gelingen, wenn diese entsprechend interpretiert, kodifiziert und für zukünftige Generationen gut sichtbar, zugänglich und übertragbar gemacht werden. Der politische Gemeinschaftssinn und dessen Entwicklung verstehen sich schließlich gerade deshalb nicht von selbst, weil »each system seeks in its own way, but always using some kind of communal ideology, to generate diffuse support behind the political community« (ebd.: 334).

In ähnlicher Form gilt dies auch für die politische Ordnung und die politischen Herrschaftsträger. Jeder politischen Ordnung liegt laut Easton ein bestimmtes Set an artikulierten wie implizierten politischen Werten und Prinzipien zugrunde,

28 Auch qualitative Nacherhebungen können darüber keine ausreichende Auskunft geben. 
welches die operativen Handlungsräume des politischen Systems absteckt. Diese unterscheiden sich $\mathrm{zu}$ bestimmten Zeitpunkten sowie aus historische Perspektive jedoch grundlegend und sind mit divergierenden Lebensweisen verbunden. Für die Unterscheidung zwischen Demokratien und Autokratien scheint dies offensichtlich, aber - wie zuvor betont - berücksichtigt Easton ausdrücklich den innerdemokratischen Konflikt um die Deutung politischer Werte:

»There will usually be subsets of members who will even interpret the same goal symbols quite differently. Freedom does not bear the same content for all persons. We can expect to find members who apparently hold the same political ideals but who place substantially different emphases and priorities upon their constituent parts. This is especially true among the most articulate interpreters [...] (ebd.: 197, 297).

Die Deutung solcher Begriffe und Konzepte als Antwort auf die Frage, was ein Unterstützungsobjekt bzw. -element repräsentiert oder welche Bedeutung es innerhalb einer politischen Kultur besitzt, ist daher folgenreich für die Maßstäbe bzw. den Legitimitätsglauben ihrer Mitglieder. Analog zu Rohes Begriff des politischen Weltbildes, finden sich in legitimating ideologies jene Werte der politischen Ordnung "consisting of articulated ethical interpretations and principles that set forth the purposes, organization, and boundaries of political life« (ebd.: 290). Werte, Normen, Herrschaftsstrukturen der politischen Ordnung, aber auch Rollen der politischen Herrschaftsträger, müssen auf eine Art und Weise artikuliert und gedeutet werden, die es den Mitgliedern ermöglicht, die Vergangenheit zu interpretieren, die Gegenwart zu erklären und eine Vision für die Zukunft zu entwerfen (ebd.), welches ihr Potential, diffuse Unterstützung zu generieren, »will depend upon its success in capturing the imagination of most of the members in the system and in thereby fostering in them sentiments of legitimacy toward the authorities and regime« (ebd.: 294).

\section{Expressive und instrumentelleAspekte}

Mit der Frage nach den Erfolgsbedingungen für die positive Rezeption von Ideologie, rücken weiterhin expressive und instrumentelle Aspekte in den Fokus. Es ist offensichtlich, dass nicht jede Deutung auf der Grundlage einer Ideologie bzw. eines ideologischen beliefsystem die gleiche Wahrscheinlichkeit besitzt, als Rechtfertigung für die politische Ordnung und die Herrschaftsträger akzeptiert zu werden. Einerseits müssen die Mitglieder expressiv davon überzeugt sein, dass ihre Vorstellungen und Gefühle bezüglich ihrer eigenen Bedürfnisse und Interessen, aber auch hinsichtlich der gemeinsamen Geschichte und ihres Platzes innerhalb der Gesellschaft von einer Ideologie richtig und wahrheitsgemäß wiedergegeben werden. 
Andererseits liegen die instrumentellen oder gar manipulativen Voraussetzungen »in utilizing the beliefs « (ebd.: 296) gerade in den Händen der gesellschaftlichen Eliten. Die Artikulation, Förderung und Weiterentwicklung bestimmter ideologischer Positionen muss, unter Bedingungen eines pluralen Wettbewerbs, bei den Systemmitgliedern sowohl hinsichtlich Inhalts als auch des Ausdrucks "appeal to a prior set of predispositions « (ebd.: 295). Diese sind vielfältig, müssen aber neben den kognitiven, affektiven und evaluativen Dimensionen gerade auch die historischen, ästhetischen und symbolischen Aspekte politischer Kultur berücksichtigen, um zu einem umfassenden Verständnis politischer Unterstützung zu kommen. Deutungsprozesse der politischen Unterstützungsobjekte sind damit prinzipiell keine Einbahnstraße, sondern basieren auf den Fähigkeiten politischer Eliten »in inventing and interpreting a set of values for the politically relevant membership and defining its relationship to their presumed needs and motivations (ebd.: 297).

\section{Fazit und Ausblick}

Wenn das zentrale Ziel der klassischen politischen Kulturforschung darin liegt, jene subjektiven Rahmenbedingungen von Politik zu erfassen, welche die Stabilität eines politischen Systems beeinflussen, stehen die Wertegerüste und Einstellungen der Bürgerinnen und Bürger sowie deren Verhältnis zur politischen Struktur in Zentrum des Forschungsinteresses. Wie der Beitrag gezeigt hat, sehen sich Ansätze der empirischen Sozialforschung in der der Tradition der Almond/VerbaStudie jedoch konzeptimmanenten Problemen und Grenzen gegenüber. Von einer machtsensiblen Perspektive auf das Verständnis politischer Kultur könnten sie deshalb besonders profitieren, weil mit dieser »gerechtfertigte[n] Kritik an der empirischen Forschungspraxis« (Gabriel 1994: 24) ein grundsätzlicher Austausch zwischen zwei konkurrierenden Forschungstraditionen - und nicht etwa die Aufgabe des einen oder des anderes - angestrebt wird. Wenn sich, wie Gabriel weiter ausführt, ein vorstellungszentrierter Ansatz »ohne Probleme in die von Almond und Verba begründete Forschungstradition einbinden« (ebd.) ließe, müsste vice versa gelten, dass kulturwissenschaftliche Forschungsansätze und -ergebnisse ebenso dazu in der Lage sind, eine entsprechende Ergänzung zu leisten. Mit dem Unterstützungskonzept von David Easton existiert insofern ein zentraler Ankerpunkt, auf den sich empirische wie hermeneutische Forschungsmethoden beziehen können, um »die richtigen Fragen zu stellen« (Greiffenhagen S. 2009: 24), damit die repräsentative Umfrage- und Einstellungsforschung "mehr leisten kann als sie bislang tatsächlich geleistet hat « (Rohe 1994: 5).

Bezüglich der empirischen Forschungspraxis erweist sich eine machtsensible Perspektive nicht nur für die Ergänzung im Sinne der Deutung und Verfügbarstellung der politischen Unterstützungsobjekte und ihrer Elemente als fruchtbar, um 
jene »kulturelle Sachverhalte erfassen [zu] können, die den jeweiligen Fragern gar nicht voll bewusst sind « (ders. 1987: 46), sondern sie dient allgemein zur Analyse machtförmiger Prozesse zwischen politischer Deutungs- und Soziokultur bzw. politischer Eliten- und Massenkultur. Mit ihrer Konzeptualisierung tragen Deutungsmachtkonzepte gerade jenem Umstand Rechnung, dass es sich bei politischer Kultur um einen komplexen Forschungsgegenstand handelt, der es, sofern man Fragen und Probleme nicht willkürlich kappen will, erfordert, alle PolitikDimensionen (Polity, Politcs, Policy) in die Analyse miteinzubeziehen (ders. 1994: 15). Text-, Sprach-, Symbol- und Begriffsanalyse erhalten durch die vierstellige Deutungsmachtrelation aus Akteur - Struktur - Medium - Rezipient zudem forschungspraktische Relevanz, wenn man die Messung der individuellen sozialpsychologischen Einstellungen im Inneren der Köpfe, in den Raum öffentlicher Diskurse und Symbole verlagern kann, was besonders in autokratischen Kontexten fruchtbar erscheint (Holbig 2010: 39f.).

In der jüngeren Vergangenheit bieten sich für Deutungsmachtanalysen innerhalb der politischen Kulturforschung beispielsweise Ansätze der (politikwissenschaftlichen oder diskursiven) Narrativ- bzw. Narrationsforschung an (Arnold et al. 2012; Gadinger et al. 2014a, 2014b; Hofmann et al. 2014; Koschorke 2012; MüllerFunk 2007; Viehöver 2011), wenn dort davon ausgegangen wird, dass »machtvermittelte Erzählungen mit dem Anspruch eines natürlichen Leitdiskurses operieren « (Franke-Schwenk 2014: 364) und im Rahmen narrativer, szenischer, ikonischer und symbolischer Vermittlungsstrategien politische Weltbilder mit Werten, Prinzipien, Normen und Argumentationsstrukturen historisch-kulturell konfigurieren und verknüpfen. Nicht für die politische Ordnung, sondern auch für die politische Gemeinschaft wird somit der kognitive Inhalt und der affektiv-ästhetische Ausdruck der Vergangenheit zur einer zentraler Ressource politischer Legitimität (Bergem 2014: 45; 2009), die vor dem Hintergrund eines potentiellen Wettbewerbs um Deutungsmacht jedoch nicht automatisch dominant bleibt, sondern ständig aktualisiert und verteidigt werden muss. Deutungsmachtfragen beschränken sich aber freilich nicht auf politische Diskurse oder Erzählungen, sondern bieten sich gerade für die kulturelle Symbol-, Bild- oder Filmforschung an, weshalb es deren Spielräume es innerhalb der politischen Kulturforschung mit Hilfe benachbarter Disziplinen zu erweitern gilt (Brown 2002).

\section{Literatur}

Albrecht, Holger/Frankenberg, Rolf 2010: Die »dunkle Seite« der Macht: Stabilität und Wandel autoritärer Systeme. Der Bürger im Staat, Heft 1, S. 4-13.

Almond, Gabriel A./Verba Sidney 1963: The Civic Culture. Political Attitudes and Democracy in Five nations, Princenton. 
Almond, Gabriel A. 1989: The Intellectual History of the Civic Culture Concept. In: Gabriel A Almond./Sidney Verba (Hg.), The Civic Culture Revisited, Thousand Oaks.

Arnold, Markus/Dressel, Gert/Viehöver, Willy (Hg.) 2012: Erzählungen im Öffentlichen. Über die Wirkung narrativer Diskurse, Wiesbaden.

Bank, André 2009: Die Renaissance des Autoritarismus. Erkenntnisse und Grenzen neuerer Beiträge der Comparative Politics und Nahostforschung. In: Hamburg Review of Social Science, Heft 1, S. 10-31.

Bergem, Wolfgang 2009: Politische Kultur und Geschichte. In: Samuel Salzborn (Hg.), Politische Kultur, Forschungsstand und Forschungsperspektiven, Frankfurt a.M., S. 201-227.

Bergem, Wolfgang 2014: Narrative Formen in Geschichtspolitik und Erinnerungskultur. In: Wilhelm Hofmann/Judith Renner/Katja Teich (Hg.), Narrative Formen der Politik, Wiesbaden, S. 31-48.

Brodocz, André 2006: Die souveränen Deuter. Symbolische Voraussetzungen - instrumentelle Rahmenbedingungen - praktische Auswirkungen. In: Hans Vorländer (Hg.), Die Deutungsmacht der Verfassungsgerichtsbarkeit, Wiesbaden, S. 95-120.

Brown, Wendy 2002: At the Edge; in: Political Theory, Vol. 30: What is Political Theory? Special Issue, S. 556-576.

Carothers, Thomas 2002: The End of the Transition Paradigm. In: Journal of Democracy, Heft 1, S. 5-21.

Converse, Philip E. 2006: The Nature of Belief Systems in Mass Publics (1964). In: Critical Review, Heft 1-3, S. 1-74.

Diehl, Paula/Steilen, Felix (Hg.) 2016: Politische Repräsentation und das Symbolische, Historische, politische und soziologische Perspektiven, Wiesbaden: Springer VS.

Easton, David 1965: A Systems Analysis of Political Life, New York.

Easton, David 1969: The New Revolution in Political Science. In: The American Political Science Review, Heft 4, S. 1051-1061.

Easton, David 1975: A Re-assessment of the Concept of Political Support, in: British Journal of Political Science, Heft 4, S. 435-457.

Elkins, David J./Simeon, Richard E. 1979: A Cause in Search of Its Effect, or What Does Political Culture Explain. In: Comparative Politics, Heft 2, S. 127-145.

Franke-Schwenk, Anja 2014: Politische Narrative in autoritären Herrschaftskontexten. In: Frank Gadinger/Sebastian Jarzebski/Taylan Yildiz (Hg.), Politische Narrative, Konzepte - Analysen - Forschungspraxis, Wiesbaden, S. 363-386.

Fuchs, Dieter 1989: Die Unterstützung des politischen Systems der Bundesrepublik Deutschland, Opladen.

Fuchs, Dieter 2004: Konzept und Messung von Demokratie. Eine Replik auf Heidrun Abromeit. In: Politische Vierteljahresschrift, Heft 1, S. 94-106. 
Gaber, Rosanna 2007: Politische Gemeinschaft in Deutschland und Polen. Zum Einfluss der Geschichte auf die politische Kultur. Wiesbaden.

Gabriel, Oscar W. 1994: Politische Kultur aus Sicht der empirischen Sozialforschung. In: Oskar Niedermayer/Klaus von Beyme (Hg.), Politische Kultur in Ost- und Westdeutschland, Berlin, S. 22-42.

Gabriel, Oscar W. 1999: Bürger und Politik in Deutschland. In: Oscar W. Gabriel/Everhard Holtmann (Hg.), Handbuch Politisches System der Bundesrepublik Deutschland, München, S. 379-497.

Gadinger, Frank/Jarzebski, Sebastian/Yildiz, Taylan 2014a: Vom Diskurs zur Erzählung. Möglichkeiten einer politikwissenschaftlichen Narrativanalyse. In: Politische Vierteljahresschrift, Heft 1, S. 67-93.

Gadinger, Frank/Jarzebski, Sebastian/Yildiz, Taylan (Hg.) 2014b: Politische Narrative. Konzepte - Analysen - Forschungspraxis, Wiesbaden.

Gallie, Walter B. 1955: Essentially Contested Concepts. In: Proceedings of the Aristotelian Society, Heft 1, S. 167-198.

Göhler, Gerhard 1997: Der Zusammenhang von Institution, Macht und Repräsentation. In: Gerhard Göhler (Hg.), Institution - Macht - Repräsentation: Wofür politische Institutionen stehen und wie sie wirken, Variationen der Macht, Baden-Baden, S. 11-64.

Göhler, Gerhard 2007: Politische Institutionen als Symbolsysteme. In: Heinrich Schmidinger/Clemens Sedmak (Hg.), Der Mensch - ein »aninmal symbolicum«? Sprache - Dialog - Ritual, Darmstadt, S. 301-321.

Göhler, Gerhard 2004: Macht. In: Ders./Mattias Iser/Ina Kerner (Hg.): Politische Theorie. 22 umkämpfte Begriffe zur Einführung, Wiesbaden, S. 244-261.

Göhler, Gerhard 2013: Transitive und intransitive Macht. In: André Brodocz/Stefanie Hammer (Hg.), Variationen der Macht, Baden-Baden, S. 225-242.

Greiffenhagen, Martin: 1997: Politische Legitimität in Deutschland. Güterloh.

Greiffenhagen, Sylvia 2009: Theorie(n) der Politischen Kultur. In: Samuel Salzborn (Hg.), Politische Kultur, Forschungsstand und Forschungsperspektiven, Frankfurt a.M., S. 11-30.

Greiffenhagen, Martin/Greiffenhagen, Sylvia 2003: Politische Kultur. In: Uwe Andersen/Wichard Woyke (Hg.), Handwörterbuch des politischen Systems der Bundesrepublik Deutschland, Opladen, S. 515-520.

Hague, Rod/Harrop, Martin/McCormick, John 2016: Comparative Government and Politics: An Introduction, Basingstoke.

Hildebrandt, Mathias 1996: Politische Kultur und Zivilreligion. Würzburg.

Hobsbawm, Eric J., 1990: Nations and nationalism since 1780. Programme, myth, reality, Cambridge.

Hofmann, Wilhelm/Renner, Judith/Teich, Katja (Hg.) 2014: Narrative Formen der Politik. Wiesbaden. 
Holbig, Heike 2010: Die Finanzkrise in China: Auswirkungen auf die Legitimität der Parteiherrschaft. In: Der Bürger im Staat, Heft 1, S. 38-43.

Kaase, Max 1983: Sinn oder Unsinn des Konzepts »Politische Kultur« für die Vergleichende Politikforschung, oder auch: Der Versuch, einen Pudding an die Wand zu nageln. In: Max Kaase/Hans-Dieter Klingemann (Hg.), Wahlen und politisches System, Analysen aus Anlaß der Bundestagswahl 1980, Opladen, S. 144-172.

Kailitz, Steffen 2009: Stand und Perspektiven der Autokratieforschung. In: Zeitschrift für Politikwissenschaft, Heft 3, S. 476-488.

Koschorke, Albrecht 2012: Wahrheit und Erfindung: Grundzüge einer Allgemeinen Erzähltheorie. Berlin.

Lambach, Daniel/Göbel, Christian 2010: Die Responsivität autoritärer Regime. In: Holger Albrecht/Rolf Frankenberger (Hg.), Autoritarismus reloaded, Neuere Ansätze und Erkenntnisse der Autokratieforschung, Baden-Baden, S. 79-92.

Müller-Funk, Wolfgang 2007: Die Kultur und ihre Narrative. Eine methodologische Einführung, Wien.

Pelinka, Anton 2006: Die Politik der politischen Kultur. In: Österreichische Zeitschrift für Politikwissenschaft, Heft 3, S. 225-235.

Pickel, Gert 2009: Unausweichlichkeit der Demokratisierung oder »Democracy Bias«? Kulturelle Rahmenbedingungen und Faktoren der Stabilität und Instabilität politischer Regime. In: Totalitarismus und Demokratie, Heft 2, S. 293-322 Pickel, Gert 2010: Politische Kultur und Demokratieforschung. In: Klemens H Schrenk/Markus Soldner (Hg.), Analyse demokratischer Regierungssysteme, Wiesbaden, S. 611-626.

Pickel, Gert 2013: Die kulturelle Verankerung von Autokratien - Bestandserhalt durch ideologische Legitimationsstrategien und ökonomische Legitimität oder Demokratisierung?. In: Steffen Kailitz/Patrick Köllner (Hg.), Autokratien im Vergleich, Baden-Baden, S. 176-177.

Pickel, Susanne/Pickel, Gert 2006: Politische Kultur- und Demokratieforschung. Grundbegriffe, Theorien, Methoden. Eine Einführung, Wiesbaden.

Putnam, Robert D. 1971: Studying Elite Political Culture: The Case of `Ideology «. In: American Political Science Review, Heft 3, S. 651-681.

Pye, Lucian W. 1965: Introduction: Political Culture and Political Development. In: Ders./Sidney Verba (Hg.), Political Culture and Political Development, Princeton, S. 3-26.

Rehberg, Karl-Siegbert 2014: Institutionen als symbolische Ordnungen. Leitfragen und Grundkategorien zur Theorie Analyse institutioneller Mechanismen. In: Karl-Siegbert Rehberg (Hg.), Symbolische Ordnungen. Beiträge zu einer soziologischen Theorie der Institutionen, Baden-Baden, S. 43-84.

Rohe, Karl 1987: Politische Kultur und der kulturelle Aspekt von politischer Wirklichkeit. Konzeptionelle und typologische Überlegungen $\mathrm{zu}$ Gegenstand 
und Fragestellung Politischer Kultur-Forschung. In: Dirk Berg-Schlosser/Jakob Schissler (Hg.), Politische Kultur in Deutschland, Bilanz und Perspektiven der Forschung, Opladen, S. 39-49.

Rohe, Karl 1990: Politische Kultur und ihre Analyse: Probleme und Perspektiven der politischen Kulturforschung. In: Historische Zeitschrift, Band 250, Heft 1, S. 321-346.

Rohe, Karl 1994: Politische Kultur: Zum Verständnis eines theoretischen Konzepts. In: Oskar Niedermayer/Klaus von Beyme (Hg.), Politische Kultur in Ost- und Westdeutschland, S. 1-21.

Rokeach, Milton 1960: The Open and Closed Mind, New York.

Salzborn, Samuel 2009: Der Vergleich politischer Kulturen. Theorien, Konzepte und Methoden. In: Ders. (Hg.), Politische Kultur. Forschungsstand und Forschungsperspektiven, Frankfurt a.M., S. 45-60.

Sartori, Giovanni 1967: Politics, Ideologie, and Belief Systems. In: The American Political Science Review, Heft 2, S. 398-411.

Schubert, Sophia/Kosow, Hannah 2007: Das Konzept der Deutungsmacht. Ein Beitrag zur gegenwärtigen Machtdebatte in der Politischen Theorie?. In: Österreichische Zeitschrift für Politikwissenschaft, Heft 1, S. 39-48.

Schubert, Sophia/Weiß, Alexander 2016: »Demokratie« jenseits des Westens : Theorien, Diskurse, Einstellungen.

Schulz, Daniel 2006: Theorien der Deutungsmacht. Ein Konzeptualisierungsversuch im Kontext des Rechts. In: Vorländer, Hans (Hg.): Die Deutungsmacht der Verfassungsgerichtsbarkeit, Wiesbaden, S. 67-94.

Schwelling, Birte 2008: Der kulturelle Blick auf politische Phänomene. Theorien, Methoden, Problemstellungen. In: Dies. (Hg.), Politikwissenschaft als Kulturwissenschaft, Wiesbaden, S. 11-32.

Stoellger, Philipp 2014: Deutungsmachtanalyse. Zur Einleitung in ein Konzept zwischen Hermeneutik und Diskursanalyse. In: Philipp Stoellger (Hg.), Deutungsmacht. Religion und belief systems in Deutungsmachtkonflikten, Tübingen, S. 1-85.

Viehöver, Willy 2011: Diskurse als Narrationen. In: Handbuch Sozialwissenschaftliche Diskursanalyse. Band 1: Theorien und Methoden, Wiesbaden, S. 193-224. Vorländer, Hans 2006: Deutungsmacht - Die Macht der Verfassungsgerichtsbarkeit. In: Ders. (Hg.), Die Deutungsmacht der Verfassungsgerichtsbarkeit, Wiesbaden, S. 9-36.

Vorländer, Hans 2013: Transzendenz und die Konstitution von Ordnungen: Eine Einführung in systematischer Absicht. In: Ders. (Hg.), Transzendenz und die Konstitution von Ordnungen, Berlin, S. 1-42.

Weber, Max 1920: Gesammelte Aufsätze zur Religionssoziologie, Band 1, Tübingen. Westle, Bettina 1989: Politische Legitimität - Theorien, Konzepte, empirische Befunde, Baden-Baden. 
Westle, Bettina 2009a: Rezeptionsgeschichte des Konzepts der Politischen Kultur. In: Dies./Oscar W. Gabriel (Hg.), Politische Kultur, Eine Einführung, BadenBaden, S. 23-39.

Westle, Bettina 2006: Politische Kultur. In: Hans-Joachim Lauth (Hg.), Vergleichende Regierungslehre, Eine Einführung, Wiesbaden, S. 270-290.

Westle, Bettina 2009b: Weiterentwicklungen des Konzepts der Politischen Kultur in der empirischen Sozialforschung. In: Dies./Oscar W. Gabriel (Hg.), Politische Kultur, Eine Einführung, Baden-Baden, S. 40-55. 



\section{>Doing political culturer in Alltagspraktiken der Politisierung von Konsum Theoretische und methodische Herausforderungen eines neuen Forschungsfelds der politischen Kulturforschung}

Sigrid Baringhorst / Mundo Yang / Katharina Witterhold

\section{Einleitung: Drei Perspektiven der politischen Kulturforschung nach Raymond Williams}

Das die folgenden Überlegungen prägende Verständnis politischer Kultur weicht in zweifacher Hinsicht von gängigen Verständnissen ab: Erstens plädieren wir für eine stärkere Einbeziehung von Alltagskulturen in den Untersuchungshorizont der politischen Kulturforschung und zweitens für die Überwindung der klassischen Dualität von Kultur und Materialität im Sinne der Entwicklung einer praxeologischen Erforschung von Prozessen der Politisierung von Alltagshandeln. Dabei bilden Praktiken der Politisierung von Konsumhandeln den konkreten Forschungsgegenstand, an dem das Plädoyer für eine zweifache Öffnung der klassischen politischen Kulturforschung exemplarisch erläutert werden soll.

Die Öffnung der politischen Kulturforschung für Prozesse der Politisierung von Alltagskulturen ist allerdings prinzipiell nicht neu und schon in den Arbeiten der Cultural Studies angelegt. Folgt man etwa dem Kulturverständnis von Raymond Williams, wie er es in seinem Buch »The Long Revolution« entfaltet hat (1961: 57-70), so sind bei der Analyse von Kultur drei Begriffsverständnisse zu unterscheiden: Erstens ein normatives Verständnis, nach dem »culture is a state or process of perfection, in terms of certain absolute or universal values « (ebd.: 57). Bezogen auf die politische Kultur fallen darunter die politische Ideengeschichte, vor allem die normative politische Theorietradition mit ihrer Suche nach den guten politischen Ordnungen und Vorstellungen von guten Bürgerinnen und Bürgern. Von diesem normativen Verständnis unterscheidet Williams eine dokumentarische Begriffsdimension, nach der Kultur verstanden wird als Korpus intellektueller und künstlerischer Arbeit, in der menschliche Ideen und Erfahrungen aufgezeichnet, beschrieben und aus einer Perspektive der Kritik bewertet werden. Während die meisten Beiträge zur politischen Kulturforschung dieser Analyseperspektive folgen, ist für 
die im Folgenden entfaltete Untersuchungsperspektive die dritte von Williams vorgeschlagene Definition von Kultur maßgeblich: Von der »ideal« und »documentary definition of culture unterscheidet er eine »social definiton« (ebd.). Folgt man einem sozialen Begriffsverständnis, so wird Kultur sehr weit gefasst und als spezifischer Lebensstil verstanden, als

»description of a particular way of life, which expresses certain meanings and values not only in art and learning, but also in institutions and ordinary behaviour. The analysis of culture, from such a definition, is the clarification of the meanings and values implicit and explicit in a particular way of life, a particular culture« (ebd.: 59).

In der politikwissenschaftlichen Kulturforschung finden wir eine solche Ausweitung des Forschungsgegenstands auf das alltägliche Handeln der Bürgerinnen und Bürgern, ihr »ordinary behaviour«, bisher nur selten, und wenn überhaupt, dann primär im Sinne der Einbeziehung von Phänomenen der »popular culture«. Wegweisend für eine solche Öffnung der internationalen politikwissenschaftlichen Kulturforschung für Gegenstände der Populärkultur waren insbesondere die Arbeiten des britischen Politikwissenschaftlers John Street zur Bedeutung von Popmusik für Wahlkämpfe und Protestbewegungen und zur gegenseitigen Beeinflussung von Massenmedien und Politik (z.B. Street 1997).

Im Fokus der politikwissenschaftlichen Populärkulturforschung standen bisher Unterhaltungsmedien wie TV und Film und deren Instrumentalisierung für Zwecke der Legitimation und Absicherung von Herrschaft bzw. ihre Bedeutung für die Mobilisierung von Herrschaftskritik in sozialen Bewegungen und Protestaktionen. Zum "particular way of life« gehören in spätmodernen Gesellschaften insbesondere die fortschreitende Kommerzialisierung aller Lebensbereiche und damit die gewachsene Bedeutung kommerzieller Werbung und konsumbezogenen Handelns für die Herausbildung individueller und kollektiver Wertvorstellungen und Identitäten von Konsumentinnen und Konsumenten als Bürgerinnen und Bürger. In neueren Debatten zur »Postdemokratie« (Crouch 2008) oder »Simulative(n) Demokratie« (Blühdorn 2013) wird diese Kommerzialisierung aller Lebensbereiche primär unter dem Aspekt der Erosion demokratischer bürgerschaftlicher Orientierungen und des Endes einer emanzipatorischen, auf Systemtransformation gerichteten politischen Partizipationskultur betrachtet (so auch Barber 2007).

Postdemokratische Zeitdiagnosen weisen zu Recht auf einen grundlegenden Wandel der Kulturen der politischen Partizipation, gemeint sind die vorherrschenden Normen und Praktiken der Partizipation, hin. Doch sind ihre Beiträge in der Regel durch zwei wesentliche Defizite geprägt: Zum einen fehlt oft eine empirisch solide Analyse gegenwärtiger Partizipationsnormen und -praxen, so dass zwischen unterstellten Legitimationsverlusten politischer Institutionen und tatsächlicher Datenbasis eine große Kluft besteht. Zum anderen werden Prozesse 
der Entdifferenzierung zwischen konsumbezogenem und politischem Handeln ausschließlich aus einer Verlustperspektive betrachtet; mögliche positive Effekte einer Politisierung von Konsumhandeln etwa im Sinne der Verbreitung nachhaltiger Lebensstile oder der Skandalisierung unfairer Handelsbedingungen werden außer Acht gelassen oder als unzureichend oder gar schädlich gewertet.

Demgegenüber sollen im Folgenden ein Forschungsprogramm und erste Ergebnisse zur Untersuchung der Politisierung von Alltagshandeln vorgestellt werden, die rein pessimistische Annahmen zum Wandel politischer Partizipationskulturen mit empirischem Datenmaterial konfrontieren, das im Rahmen eines von der Deutschen Forschungsgemeinschaft geförderten Forschungsprojekts $\mathrm{zu}$ »Consumer Netizens. Neue Formen der Bürgerschaft an der Schnittstelle von politischem Konsum und Social Web « an der Universität Siegen durchgeführt wurde. Entsprechend der ersten und dritten von Raymond Williams unterschiedenen Untersuchungsperspektiven der Kulturforschung soll die Politisierung von Konsum zum einen aus der Perspektive eines normativen Kulturverständnisses und zum anderen aus der Perspektive einer empirischen Erforschung von politisiertem Konsum als »way of life« westlicher Gesellschaften beleuchtet werden. Nach kurzen Erläuterungen zur Verbreitung des Phänomens folgt bezogen auf die normative Betrachtungsperspektive eine Auseinandersetzung mit der Frage, inwiefern Konsumhandeln als »politisches Handeln« verstanden werden kann und welche Vorstellungen eines guten Bürgers bzw. einer guten Bürgerin mit der Politisierung von konsumbezogenem Alltagshandeln verbunden werden.

Konsumhandeln, so die These, ist zwar nicht an sich "politisch«, doch können individuelle konsumbezogene Alltagspraktiken unter bestimmten Bedingungen eine politische Dimension entfalten. Partizipationspraktiken bieten sich für eine empirische Erforschung des Politisch-Werdens von Alltagshandeln als kleinste Einheiten der politischen Kulturanalyse an. Wie eine solche auf Partizipationspraktiken fokussierende politische Kulturanalyse methodisch durchgeführt werden kann, wird exemplarisch am Beispiel des erwähnten Forschungsprojekts zu »Consumer Netizens« beschrieben. Im letzten Teil des Beitrags werden ausgewählte Ergebnisse dieser Studie vorgestellt.

\section{Zur statistischen Relevanz der Thematik: Wie verbreitet ist politisiertes Konsumhandeln in Deutschland?}

Untersucht man die Fachliteratur zum sog. »Political Consumerism«(Stolle/Micheletti 2013), ethischem Konsum, kritischem Konsum oder strategischem Konsum, so gibt es zahlreiche Belege dafür, dass die politische Aufladung von Konsumhandeln vor allem, wenn auch nicht ausschließlich ein Phänomen der Alltagskultur in westlichen Gesellschaften ist. In Deutschland zählen nach dem European Social Survey 
von 2002 rund 50 Prozent der Frauen und 40 Prozent der Männer zu den sogenannten. "political consumers«, in der Schweiz sind es 54 Prozent der Frauen und 47 Prozent der Männer. In Österreich scheint die Beteiligung am politischen bzw. ethisch motivierten Konsum mit 35 Prozent der Frauen und 34 Prozent der Männer insgesamt etwas geringer verbreitet zu sein (Stolle/Micheletti 2013: 67). Nach dem Ökobarometer im Auftrag des Bundesministeriums für Ernährung, Landwirtschaft und Verbraucherschutz von 2013 kaufen etwa 74 Prozent der Deutschen gelegentlich Bio-Produkte (BMELV 2013: 3) ${ }^{1}$; eine aktuellere Online-Befragung vom des Bundesumweltministeriums und Umweltbundesamts geht von 83 Prozent aus, die 2016 zumindest gelegentlich Bio-Produkte erwerben (BMUB/UBA 2017: 55).

Arbeiten zur Jugendforschung belegen, dass unter deutschen Jugendlichen die Bereitschaft hoch ist, durch bewusste Konsumentscheidungen politisch Position $\mathrm{zu}$ beziehen und ein sozial-ökologisches Verantwortungsbewusstsein auszudrücken. 51 Prozent der 18- bis 29-Jährigen geben in einem Survey des Deutschen Jugendinstituts aus dem Jahr 2009 (AID:A-DJI Survey) an, dass es für sie in Frage kommt, "aus politischen, ethischen oder Umweltgründen Waren (zu) boykottieren und kaufen«. 37 Prozent antworten, dass sie dies bereits getan haben. Mit 39 bzw. 22 Prozent ist im Vergleich dazu ihre Bereitschaft, in einer Bürgerinitiative oder Partei mitzuarbeiten, eher gering, wobei die Angaben für eine faktische Umsetzung dieser Bereitschaften noch deutlich geringer sind (5 bzw. 4 Prozent zitiert nach Gaiser/Gille 2012: 15).

In einer Online-Befragung, ${ }^{2}$ die in Kooperation mit Carolin Zorell im Rahmen des oben genannten Siegener Forschungsprojekts 2014 durchgeführt wurde, liegen die Werte im Vergleich zu den Befunden von Stolle und Micheletti und des Umweltbundesamts etwas niedriger: Datenbasis ist eine quotierte Stichprobe entlang der Verteilung von Altersgruppen, Bildung und Geschlecht für die Gesamtbevölkerung in Deutschland (nach Mikrozensus 2012). Gefragt wurde in dieser Befragung nach dezidiert intendiertem politisch-nachhaltigen Konsum: »Haben Sie in den letzten 12 Monaten aus sozialen, ökologischen oder politischen Gründen den Kauf bestimmter Produkte bewusst VERWEIGERT?« (Boycott) und »Haben Sie in den letzten 12 Monaten Produkte bewusst aus sozialen, ökologischen oder politischen Gründen gekauft?« (Buycott) Tabelle I zeigt, dass von den Befragten, die zwischen 14 und 69 Jahre alt sind, 49,5 Prozent wenigstens ein paar Mal im letzten Jahr aus

1 Vgl. BMEL

2 Die im Rahmen des oben genannten Siegener Forschungsprojekts im März 2014 durchgeführte Umfrage wurde als Online-Umfrage (Online-Panel Respondi) durchgeführt. Dabei verlief die Quotierung insgesamt erfolgreich, doch blieb eine Gruppe, Erwachsene ohne Schulabschluss, stark unterrepräsentiert: Mit nur 12 Personen haben sich nur 0,9 Prozent dieser Gruppe an der Umfrage beteiligt. Auch die Einbeziehung von Personen 70 plus wurde aufgrund mangelnder Beteiligung aufgegeben, sodass die abgegebenen 1.350 Fragebögen nur die 14-69 Jahre alte Wohnbevölkerung in Deutschland abbilden. 
sozialen, ökologischen oder politischen Gründen Produkte boykottiert haben und dass 39,5 auf diesen Gründen gezielt im letzten Jahr eingekauft haben. Tabelle 1 zeigt ferner, dass die Geschlechterverteilung stärker ausgeglichen ist, als nach den Daten von Stolle und Micheletti zu erwarten gewesen wäre:

Tabelle 1: Boykott und Buykott nach Geschlecht

\begin{tabular}{|c|c|c|c|c|c|c|c|}
\hline & \multicolumn{2}{|l|}{ Frauen } & \multicolumn{2}{|l|}{ Männer } & \multicolumn{2}{|l|}{ Gesamt } \\
\hline & & Anzahl & $\%$ & Anzahl & $\%$ & Anzahl & $\%$ \\
\hline \multirow[t]{4}{*}{ Boy-kott } & $\begin{array}{l}\text { Nein, } \\
\text { habe ich } \\
\text { nicht }\end{array}$ & 327 & 48,7 & 354 & 52,1 & 681 & 50,4 \\
\hline & $\begin{array}{l}\text { Ja, ein } \\
\text { oder ein } \\
\text { paar Mal }\end{array}$ & 266 & 39,6 & 257 & 37,8 & 523 & 38,7 \\
\hline & $\begin{array}{l}\text { Ja, regel- } \\
\text { mäßig }\end{array}$ & 78 & 11,6 & 68 & 10,0 & 146 & 10,8 \\
\hline & Gesamt & 671 & 100 & 679 & 100 & 1350 & 100 \\
\hline \multirow[t]{4}{*}{ Buy-kott } & $\begin{array}{l}\text { Nein, } \\
\text { habe ich } \\
\text { nicht }\end{array}$ & 380 & 56,6 & 437 & 64,4 & 817 & 60,5 \\
\hline & $\begin{array}{l}\text { Ja, ein } \\
\text { oder ein } \\
\text { paar Mal }\end{array}$ & 228 & 34,0 & 198 & 29,2 & 426 & 31,6 \\
\hline & $\begin{array}{l}\text { Ja, regel- } \\
\text { mäßig }\end{array}$ & 63 & 9,4 & 44 & 6,5 & 107 & 7,9 \\
\hline & Gesamt & 671 & 100 & 679 & 100 & 1350 & 100 \\
\hline
\end{tabular}

Viele Studien, die Boykott und Buykott als neue Formen der Partizipation in ihre Fragebatterien aufnehmen, verkennen die Komplexität der konsumbezogenen Aufladung des Alltagshandelns. Die Zunahme einer Politisierung des Konsums ist nur ein Aspekt einer viel umfassenderen politischen Aufladung von Alltagspraktiken wie etwa die Verbreitung neuer Engagementformen im Kontext der CommonsBewegung, die Resonanz auf webgestützte Plattformen des Teilens und Tauschens (Sharing Economy), die zunehmende Verbreitung vegetarischer und veganer Lebensstile oder der Einsatz für Tierrechte belegen. In der Siegener Online-Umfrage zeigt sich folgende Verbreitung unterschiedlicher Partizipationspraktiken basierend auf Angaben zum Fragebogen-Item: »Über den Einkauf hinaus kann man verschiedene Dinge tun, damit unser Konsum weniger soziale oder ökologische 
Probleme zur Folge hat. Welche der folgenden Dinge machen Sie regelmäßig und bewusst zur Vermeidung sozialer oder ökologischer Probleme?«

Tabelle 2: Unterschiedliche Aspekte politischen Konsums (Mehrfachantworten)

\begin{tabular}{|l|l|l|l|l|l|l|}
\hline \multicolumn{2}{|l|}{} & \multicolumn{2}{l|}{ Frauen } & \multicolumn{2}{l|}{ Männer } & \multicolumn{2}{l|}{ Gesamt } \\
\hline $\begin{array}{l}\text { Möglichst viel wie- } \\
\text { derverwenden, } \\
\text { wie z.B. Plastik- } \\
\text { tüten, Flaschen } \\
\text { usw. }\end{array}$ & 548 & 81,7 & 498 & 73,3 & 1046 & 77,5 \\
\hline $\begin{array}{l}\text { Zu Fuß gehen oder } \\
\text { Fahrrad fahren } \\
\text { statt mit dem } \\
\text { Auto fahren }\end{array}$ & 447 & 66,6 & 432 & 63,6 & 879 & 65,1 \\
\hline $\begin{array}{l}\text { Mit öffentlichen } \\
\text { Verkehrsmitteln } \\
\text { statt mit dem } \\
\text { Auto fahren }\end{array}$ & 294 & 43,8 & 299 & 44,0 & 593 & 43,9 \\
\hline $\begin{array}{l}\text { Second-Hand } \\
\text { Sachen kaufen }\end{array}$ & 258 & 38,5 & 183 & 27,0 & 441 & 32,7 \\
\hline $\begin{array}{l}\text { Insgesamt weni- } \\
\text { ger konsumieren }\end{array}$ & 207 & 30,8 & 197 & 29,0 & 404 & 29,9 \\
\hline $\begin{array}{l}\text { Auf Fleisch ver- } \\
\text { zichten }\end{array}$ & 180 & 26,8 & 113 & 16,6 & 293 & 21,7 \\
\hline $\begin{array}{l}\text { Dinge, die ich } \\
\text { besitze, von ande- } \\
\text { ren mitbenutzen } \\
\text { lassen }\end{array}$ & 164 & 24,4 & 131 & 19,3 & 295 & 21,9 \\
\hline $\begin{array}{l}\text { Freunden oder } \\
\text { Bekannten Tipps } \\
\text { zu sozialem oder } \\
\text { ökologischem } \\
\text { Konsum geben }\end{array}$ & 107 & 15,9 & 92 & 13,5 & 199 & 14,7 \\
\hline Nichts davon & 37 & 100,0 & 679 & 100,0 & 1350 & 100,0 \\
\hline Sonstiges & 8,5 & 60 & 8,8 & 97 & 7,2 \\
\hline Gesamt & Ang & 11 & 1,6 & 19 & 1,4 \\
\hline
\end{tabular}

Auch hier sieht man geschlechterspezifische Unterschiede. Frauen neigen eher dazu, Second-Hand-Sachen zu kaufen, auf Fleisch zu verzichten oder vorhandenes Hab und Gut in der Familie oder mit Freunden und Bekannten zu teilen. Generell 
sind die meisten bereit, vorhandene Güter anders zu nutzen oder Infrastrukturen wie den ÖPNV als Angebot wahrzunehmen. Demgegenüber kommt dem kommunikativen Einwirken auf andere, das in der Literatur zu ethischem Konsum stets betont wird, empirisch betrachtet eher geringer Stellenwert zu.

\section{Politisierter Konsum aus der Perspektive eines normativen Kulturverständnisses}

Was würde es nun bedeuten, dieses mit wenigen statistischen Zahlen skizzierte Phänomen der Politisierung von Konsumhandeln als Gegenstand einer »reloaded «, neu aufgeladenen politischen Kulturforschung zu verstehen? Rekurrierend auf das, was Willams als ein »ideal« oder normatives Verständnis von Kultur bezeichnet, wäre zu klären, inwiefern die diversen alltagsbezogenen Formen eines lebensstilbezogenen und in Alltagspraktiken verankerten »civic engagement « überhaupt als Ausdruck eines Wandels politischer Partizipationskulturen, d.h. eines Wandels von Normen guter Bürgerschaft wie eines Wandels von "politischer« Partizipationspraktiken, zu verstehen sind. Dazu ist zunächst unser Verständnis des »Politischen« zu klären. Unter welchen Bedingungen können Alltagspraktiken eines ethischen, strategischen oder kritischen Konsums überhaupt als "politische« Partizipationspraktiken gelten? Inwiefern wird hier der Begriff des Politischen überdehnt und wie ließe sich eine solche Überdehnung vermeiden?

Maßgeblich für die Konzeptionalisierung unserer Forschung zur Politisierung von Konsumhandeln ist ein an Konflikt und sozialem Wandel orientiertes Verständnis politischen Konsums, das im Wesentlichen anschließt an ein Verständnis des Politischen, wie es von Oliver Marchart in seinem Buch über »Die politische Differenz« (2011) entwickelt wurde. Dreh- und Angelpunkt des Politischen und damit auch des politischen Konsums ist das Streben nach Erzeugung veränderter gesellschaftlicher Mehrheitsverhältnisse mit der Absicht, auch grundlegenden sozialen Wandel herbeizuführen. Damit folgen wir nicht den gängigen Definitionen politischer Partizipation aus der empirisch-analytischen Forschung: Einflussreich für die empirische Partizipationsforschung ist bis in die Gegenwart die Definition von Max Kaase. Danach umfasst politische Partizipation all die Tätigkeiten, »die Bürger freiwillig mit dem Ziel unternehmen, Entscheidungen auf den verschiedenen Ebenen des politischen Systems zu beeinflussen « (Kaase 1995: 521). Neue Formen konsumbezogenen Engagements offline wie online erfüllen dieses strenge Kriterium der politischen Entscheidungsorientierung nur im Ausnahmefall, so etwa kollektive Boykottaktionen, zu denen im Rahmen der Proteste gegen den Ölkonzern Shell Mitte der 1990er Jahre mobilisiert wurde, um die Revision eines Parlamentsbeschlusses des britischen Unterhauses, das die Versenkung einer Ölplattform in der Nordsee erlaubt hatte, zu bewirken. 
Um der rapiden Ausdifferenzierung des politischen Engagements in eine Vielzahl neuer, oft alltagsbezogener Partizipationsformen gerecht zu werden, hat Jan van Deth einen Ansatz zu ihrer Operationalisierung vorgeschlagen. Er unterscheidet zwischen vier Typen politischer Partizipation: Aktivitäten, die in den Räumen institutionalisierter Politik verortet sind, Aktivitäten, die auf die Akteure institutionalisierter Politik gerichtet sind, Aktivitäten, die darauf zielen, kollektive oder Gemeinschaftsprobleme zu lösen, sowie Aktivitäten, die zwar nach den genannten Kriterien nicht politisch sind, die aber "are used to express political aims and intentions of participants « (van Deth 2014: 358). Die letzte Kategorie ermöglicht es, auch hoch individualisierte und expressive Handlungen etwa in sozialen Netzwerken, aber auch individuelle Akte des Buy- und Boykott als politische Aktivitäten einzustufen. Doch wird damit die politische Qualität des Handelns letztendlich allein aus der subjektiven Intention der Handelnden abgeleitet. Eine ähnliche Problematik zeigt sich auch in dem von Micheletti für das Untersuchungsfeld des politischen Konsumerismus vorgeschlagenen Definitionsvorschlag:

»[P]olitical consumerism is politics when people knowingly target market actors to express their opinions on [...] non-economic issues [...] using consumer choice as an ethical or political assessment of favourable or unfavorable business and government practice« (2003: 14).

Denn auch in den Begriffsbestimmungen von Micheletti ist der politische Charakter einer Kaufhandlung schon allein aus den Intentionen der damit verbundenen Meinungsäußerung zu nicht-ökonomischen Angelegenheiten abzuleiten.

Van Deths Versuch einer Operationalisierung des Konzepts politischer Partizipation bildet einen wichtigen Beitrag zur Anpassung der empirisch-analytischen Partizipationsforschung an ein sich schnell veränderndes und ausdehnendes Untersuchungsfeld. Mit der Entwicklung von Regeln der Operationalisierung der $\mathrm{Zu}$ ordnung einzelner empirischer Fälle zu Typen politischer Partizipation werden die wesentlichen empirischen Herausforderungen der Partizipationsforschung jedoch nicht gelöst. Denn will man die Bestimmungsmerkmale politischen Handelns definieren, so erscheint es wenig sinnvoll, politisches Handeln als auf politische Ziele gerichtetes Handeln zu bestimmen, solange der Begriff des Politischen selbst unklar bleibt.

Folgt man demgegenüber zeitgenössischen Beobachterinnen und Beobachtern im Umfeld der Postdemokratie-Debatte, so liegen ihrer Kritik an unpolitischer und postdemokratischer Partizipation häufig normative Annahmen zu politischer Partizipation zugrunde, die auf Vorstellungen republikanischer oder agonaler Demokratie zurückgehen, wie sie von Hannah Arendt, Chantal Mouffe oder in den letzten Jahren von Oliver Marchart in Deutschland entwickelt wurden. Nach Arendt ist die Charakterisierung einer Handlung als politisches Handeln an die Erfüllung unverzichtbarer Bedingungen gebunden. Politisches Handeln wird nicht instrumentalis- 
tisch, nicht als Mittel zu einem Zweck verstanden, sondern als Selbstzweck (Arendt 1981: 223f.). Zudem ist es stets ein Handeln in der öffentlichen Sphäre. Normativ handlungsleitend ist ein Streben nach Verwirklichung der dem politischen Leben inhärenten Prinzipien, wie Freiheit, Gleichheit, Gerechtigkeit und Solidarität.

In seinem Buch »Die politische Differenz« (2011) greift Marchart Arendts Vorstellungen von politischem Handeln als assoziatives und öffentliches Handeln auf und verbindet sie mit agonistischen Konzepten des Politischen, wie sie in kritischer Reflektion von Carl Schmitt bei Chantal Mouffe oder Jacques Rancière vertreten werden. »Wie sähe Politik aus, kurz bevor sie am Horizont verschwindet und nicht mehr als Politik beschreibbar ist? D.h., was macht jene Handlungsformen aus, die wir als politisch bezeichnen würden? Oder kürzer: Was ist oder wäre minimale Politik« (Marchart 2011: 293)? In seiner für das oben genannte Siegener Forschungsprojekt leitenden Antwort hebt er folgende Merkmale hervor:

Von politischem Handeln ist danach schon dann zu sprechen, wenn Handelnde

- an die Öffentlichkeit treten, nach Sichtbarkeit streben und nicht nur unter sich bleiben,

- streitförmig Position beziehen - wo keine Meinungsverschiedenheit, da keine Politik -

- und wenn ihr Handeln eine »Tendenz zur Verallgemeinerung und Majorisierung der eigenen Position « (ebd.: 324) aufweist.

Ausgehend von Marcharts Annahmen wird davon ausgegangen, dass Konsumpraktiken, als Kernpraktiken alltagspolitischen Engagements, dann als politische bzw. politisierte Praktiken verstanden werden können, wenn sie ein Streben nach Sichtbarmachung und Majoritär-Werden der mit dem konsumbezogenen Handeln verbundenen Position erkennen lassen. Mit Arendt und damit gegen die früher dominante normative Orientierung auf das Minoritär-Werden im Diskurs der politischen Differenz betont Marchart damit, dass das Politische nach Wandel durch Schaffung von Mehrheiten strebt. In dieser Richtung mehren sich die Stimmen, die die Substanzlosigkeit reiner Differenzpolitik überwinden wollen. So kritisiert z.B. Žižek (2017: 304) an Laclaus Konzeption einer bloß ganz anders sein wollenden Vielfalt von agonalen Akteuren, die nichts außer der fundamentalen Differenz zum Bestehenden eint, dass es letztlich eine rein formale Perspektive bleibt. Sie unterbietet die von uns vorgefundene empirisch bestehende Kreativität und die vor allem materiellen, d.h. substantiellen Praktiken politischer Konsumenten. 


\section{Offene Heuristik statt deduktiver Ableitung von Bürgerschaftsmodellen}

Insbesondere in Reaktion auf die zunehmende Politisierung des Konsums, verstanden als die Kopplung ökonomischen Konsumhandelns mit Motiven sozialer Verantwortungsübernahme, hat sich inzwischen eine kontroverse demokratietheoretische Debatte über die normative Bewertung einer moralischen und politischen Aufladung von Alltagspraktiken entwickelt. Als neues Forschungsprogramm der politischen Kulturforschung sollte die Analyse der politischen Aufladung von Alltagspraktiken des Konsums auch Aufschluss darüber geben, wie die Politisierung von Konsum demokratietheoretisch einzuschätzen ist, ob mit Ingolfur Blühdorn (2013) als postdemokratische Partizipation, mit Michael Th. Greven (1999) als Partizipation politisierter Nicht-Bürger, mit Michele Micheletti und Andrew McFarland (2012) oder Jan van Deth (2012) als »kreative Partizipation", mit der Bürgerinnen und Bürger in spätmodernen Gesellschaften ihre gesellschaftliche Verantwortungsübernahme ausdrücken.

Folgt man postdemokratischen Zeitdiagnosen, so erscheint die Vorstellung von Bürgerinnen und Bürgern als Konsumentinnen und Konsumenten und die damit verbundene Vorstellung von Politik als Lifestyle geradezu als Dystopie der Postmoderne (Baringhorst 2015b). Demokratieschädlich sind demnach vor allem eine drohende Fragmentierung und Hybridisierung ethischer und politischer Forderungen: Die politische Aufladung von Konsum- und Lebensstilpraktiken lenke ab, so die Annahme, von den eigentlich wichtigen Machtauseinandersetzungen um die Gestaltung rechtlich-institutioneller Strukturen der Gesellschaft. Blühdorn beschreibt diesen Wandel in etwa so: Während die sozialen Bewegungen der 1960er bis 1990er Jahre dem einzelnen noch ein zeitraubendes öffentliches Engagement für emanzipatorische Ziele grundlegender Gesellschaftsveränderung abforderten, äußere sich die wachsende Präferenz der Bürgerinnen und Bürger für Praktiken einer Lebensstilpolitik in einer systemkonformen Ökonomisierung sowie einer Issue-, Spaßund Event-Orientierung der politischen Beteiligung.

Dagegen setzt Jörn Lamla eine optimistischere Interpretation der demokratiefördernden Funktion von Verbraucherengagement: »Verbraucherdemokratie« könne »als durchaus sehr fortschrittliche Idee angesehen werden« (Lamla 2009: 137; 2013), sofern die weltgesellschaftlichen Interdependenzen zwischen Wirtschaft und Konsumgesellschaft thematisiert und politisches Handeln jenseits nationalstaatlicher Handlungsräume ermöglicht werde. Auch Micheletti deutet die politische Aufladung von Konsumhandlungen tendenziell als demokratieförderliches bürgerschaftliches Handeln, durch das Bürgerinnen und Bürger ihre politischen und ethischen Vorstellungen jenseits des Institutionengefüges der repräsentativen Demokratie zum Ausdruck bringen können. 
Wie immer man die demokratische Qualität der politischen Aufladung von Konsum- und allgemein Alltagshandeln bewertet, ob als Gefahr oder als Chance für die Entwicklung politischer Partizipationskulturen, problematisch erscheint vor allem die deduktive Herangehensweise an die Bewertung dieser mehr oder weniger neuen, aber weit verbreiteten Partizipationspraxis: Denn stets gehen die Autorinnen und Autoren von bestimmten normativen Verständnissen eines guten Bürgers aus und legen diese dann an die Partizipationspraxis der Subjekte an. Demgegenüber gehen wir in Anlehnung an Gedankengänge bei Autoren wie Marchart oder Dahlgren von einem "postfundamentalistischen« Verständnis von Bürgerschaftlichkeit aus. Weniger Zugehörigkeit zu einer sozialen Gruppe noch ein voll und ganz verinnerlichtes Staatsbürgerschaftsverständnis, sondern vielmehr einzelne "Akte« bürgerschaftlichen Engagements sind demnach als Citizenship $\mathrm{zu}$ verstehen (vgl. auch Isin 2009). Entsprechend dem Konzept des »Doing Citizenship« (Dahlgren 2009) gilt es zu erforschen, wie Citizenship erst durch Praktiken erzeugt wird und wie unterschiedlich das von Individuum zu Individuum ausfällt.

Diesem Verständnis von Bürgerschaftlichkeit folgend plädieren wir für eine offene Heuristik im empirischen Forschungsprozess, die Bürgerschaftsverständnisse nicht normativ vorgibt und schlicht auf ihre Verbreitung hin untersucht. Neue Formen informeller Partizipation im Alltag erfordern die Entwicklung neuer Modelle von Citizenship. Eine offene Heuristik leitet Bürgerschaftsvorstellungen nicht deduktiv aus normativen Prinzipien, sondern induktiv aus der komplexen Alltagspraxis der Bürgerinnen und Bürger $a b$. Heuristik meint dabei ein offen gehaltenes Set von Anhaltspunkten für alltagspolitisches Handeln, deren Bestandteile jeweils nicht schon eine bestimmte theoretische Denkfigur als Interpretationsergebnis nahelegen. Eine offene heuristische Bestimmung von Lebensstilpolitik hat demgegenüber den Vorteil, dass nicht von vorgegebenen, per Survey einfach abfragbaren fixen Wertorientierungen und bestehenden Interessen ausgegangen und nicht präjudiziert wird, welche Formen des Politischen im Material vorgefunden werden können. Wir setzen also nicht schon das Bild eines in bestimmter Weise politisch orientierten Verbrauchers voraus. Vielmehr startet die Interpretation in ihrer Orientierung an Marcharts Konzept minimaler Politik mit einem sehr basalen Verständnis des Politischen, das einen Spezialfall sozialen Handelns und sozialer Konflikte beschreibt. Das Politische liegt überall dort vor, wo Konflikte um die öffentlichen Angelegenheiten geführt werden mit dem stets Änderungen unterworfenen Ziel, majoritär zu werden. Lebensstilpolitik bedeutet, eingefahrene Routinen durch deviantes Verhalten darauf hin wzu überprüfen, ob sie nach wie vor ihre Gültigkeit durchzusetzen vermögen. So können Änderungen des Konsumverhaltens wie der Verzicht auf Fleisch oder auf mit Palmöl hergestellte Lebensmittel durchaus auch dann einen politischen, auf Gesellschaftsveränderung gerichteten und sich in konflikthaften Alltagssituationen manifestierenden Charakter bekom- 
men, wenn den Subjekten selbst rational kaum zugänglich ist, wie die bestehende Gesellschaftsordnung strukturiert ist und wie bestimmte Wertschöpfungsketten etwa bei der Produktion und Verwendung von Palmöl beschaffen sind.

\section{Politisierter Konsum als "way of life " spätmoderner Gesellschaften - Partizipationspraktiken als kleinste Einheiten der Kulturanalyse}

Die normative Untersuchungsperspektive der politischen Kulturforschung, die Frage nach der guten Ordnung und der guten Bürgerin und dem guten Bürger, ist, so unsere Forderung, enger mit der dritten von Williams vorgeschlagenen Untersuchungsperspektive, der Untersuchung der "social dimension of culture«, d.h. den lebensweltlichen Alltagspraktiken, zu verbinden. Mit Rekurs auf die »social« »definition of culture«, die Kultur als umfassenden »way of life« fasst, wäre besonders zu klären, wie sich die normativen Orientierungen, d.h. vor allem die mit der Politisierung von Alltagshandeln verbundenen Vorstellungen von guter Bürgerschaft und die mit ihnen verbundenen präferierten Partizipationsformen angemessen empirisch untersuchen lassen. Aus den theoretisch-normativen Überlegungen der Bestimmung des Politischen im politisierten Konsum stellen sich insbesondere folgende empirisch $\mathrm{zu}$ beantwortende Forschungsfragen: Wie und unter welchen Bedingungen entfalten individuelle konsumbezogene Alltagspraktiken eine politische Dimension, d.h. öffentliche Sichtbarkeit, einen auf Mehrheitsgewinnung gerichteten kollektiven Charakter sowie auf Problemlösung gerichtete konfliktive und/oder kooperativ-präfigurative Dynamiken? Dabei ist davon auszugehen, dass in digitalisierten Gesellschaften solche Prozesse der Kollektivierung und Erzeugung von Sichtbarkeit alltagssituierter Engagementpraktiken stark mit digitalen Praktiken der Selbstdarstellung, Interaktion und Vernetzung über soziale Netzwerke wie Facebook verbunden sind.

Richtet man bei der Erforschung des Wandels politischer Partizipationskulturen den Blick auf die Politisierung von Alltagskulturen, in unserem Falle auf das Politisch-Werden von Konsumhandeln und versteht Politisierung als Sichtbarmachen und Kollektivieren individueller Alltagspraktiken, so zeigt sich, dass politischer Konsum mehr ist als rein marktorientiertes ökonomisches Handeln im Sinne der binären Unterscheidung von Kaufen oder Nicht-Kaufen. Die Politisierung von Alltagshandeln/Konsumhandeln erfolgt in einer komplexen, unterschiedliche soziale Handlungsbereiche verbindenden Partizipationspraxis, die eine Umorientierung der bisherigen Partizipations- wie auch der Bewegungsforschung hin $\mathrm{zu}$ praxeologischen Analyseperspektiven erfordert: Entgegen der in der politischen Kulturforschung bisher dominanten Annahme einer Dualität von Kultur und Materialität wird hierin Reckwitz folgend davon ausgegangen, dass politische Partizipationspraktiken als politische Kulturtechniken, als Ausdruck eines >doing political 
culture $\mathrm{zu}$ verstehen sind, in dem stets materielle Praktiken vorkommen und dies nicht nur bei interobjektiven (ding- oder artefaktbezogenen), sondern auch intersubjektiven und selbstreferentiellen, d.h. auf die Kultivierung von Subjekten bezogene Praktiken (Reckwitz 2016: 36ff.). Die Untersuchung des Politisch-Werdens von Alltagshandeln stellt, so die These, ein besonders geeignetes Untersuchungsfeld dar, um einen Perspektiven-, ja im Grunde Paradigmenwechsel der inhaltlichen wie methodischen Ausrichtung der politischen Kulturforschung hin zu praxeologischen Perspektiven zu begründen und explizieren.

Was ist gemeint? Fokus einer praxeologischen politischen Kulturforschung sind politisch-kulturelle Praktiken. Aus einer praxeologischen Perspektive »sind nicht Weltbilder oder Regeln der Bedeutungsproduktion die kleinste Einheit der Kulturanalyse, sondern die Praktik, im Plural die Praktiken. Kultur ist ein Ensemble von >Kulturtechniken« (ebd.: 34). Solche Praktiken sind ökonomische Praktiken wie Kaufen, Wegwerfen, Tauschen, aber auch Säen und Ernten, ebenso wie diskursive Praktiken des Streitens, des Demonstrierens, in der digitalen Welt des Erstellens, Postens, Likens, Sharens, des Verfremdens wie des Löschens von Webartefakten, des Sich-Vernetzens und Sich-Organisierens. Theodore Schatzki (2012: 14) spricht von "an open-ended, spatially-temporally dispersed nexus of doings and sayings «, Reckwitz von typisierten Formen des "Sich-Verhaltens«, die durch zwei Merkmale charakterisiert sind: »ihre Ermöglichung und Regulierung durch implizierte Wissensordnungen und ihre materiale Verankerung in den Körpern sowie in den Artefakten « (Reckwitz 2016: 35). Diskursive Praktiken, die in der traditionellen politischen Kulturforschung nahezu ausschließlich untersucht werden, sind aus einer praxeologischen Sicht anderen, nicht-diskursiven Praktiken nicht grundsätzlich über- oder untergeordnet. Eine Analyse der Politisierung von Konsum, die sich primär auf den Wandel öffentlicher Diskurse richtet, verkennt vor allem die im Prozess der Politisierung von Konsum ebenso relevanten interobjektiven (ding- oder artefaktbezogenen) Dimensionen des Handelns.

So lassen sich auf der Mikroebene einer sozial-ökologischen Alltagspolitik folgende intersubjektiven und interobjektiven Dimensionen von Praktiken unterscheiden: Erstens materielle Praktiken in materiellen Kontexten, die zu differenzieren sind in technische Praktiken wie das Messen der Umweltbilanz eines Apfels, das Dämmen eines Hauses, das Reparieren von Gebrauchsgegenständen und wirtschaftliche Praktiken wie der Buykott oder Boykott (Konsumverzicht) bestimmter Waren und Dienstleistungen, Praktiken des Prosuming im Sinne einer Kopplung von Konsum mit Produktion oder Zirkulation von Gütern und Dienstleistungen. Zweitens politische kollektive bzw. individualisiert-kollektive Praktiken, die anders als häufig angenommen stets körpergebunden und materiell kontextualisiert bleiben, so z.B. die Mitgliedschaft in einer UmweltNRO, die Beteiligung an Protestaktionen gegen konsumrelevante politische oder ökonomische Entscheidungen oder das Mitzeichnen von Petitionen. Und drit- 
tens politisch-kulturelle bzw. diskursive Praktiken wie die Thematisierung von Aspekten konsumbezogener gesellschaftlicher Verantwortungsübernahme in Alltagsgesprächen, in Briefen an Firmen und Politikerinnen undPolitiker oder im Rahmen von Demonstrationen vor Geschäften. Einzubeziehen sind in dem $\mathrm{Zu}$ sammenhang auch digitale Praktiken wie das Liken, Sharen, Posten und Bloggen von Texten, Bildern und Videos in sozialen Netzwerken wie Facebook oder Twitter.

Versteht man die Politisierung von Konsum im Alltag als Ausdruck eines >doing political culture Ensemble unterschiedlicher Kulturtechniken, das weder allein auf ökonomische noch auf diskursive Praktiken reduziert werden sollte. Auch Dinge und Artefakte sind konstitutiv für diese Praxis. Dies schließt Medientechnologien der Produktion, Zirkulation und Konsumption von Medieninhalten ebenso ein wie etwa Technologien des Hausbaus - Stichwort Internet der Dinge - oder Technologien des landwirtschaftlichen Anbaus in Urbanen Gärten oder Höfen der solidarischen Landwirtschaft.

Damit ist eine Erforschung der Politisierung von Alltagspraktiken empirisch weitaus anspruchsvoller als die gängigen Umfragen zu Biokonsum, Label-Wissen oder Boykottverhalten in nationalen oder international vergleichenden Surveys suggerieren. Auch in der Politik- und Kommunikationswissenschaft dominieren bisher schlichte und zumeist recht eindimensionale Erhebungen von quantitativen Daten zu Motiven von Buykott- und Boykott-Verhalten oder zum Zusammenhang von medialem Informations- und Kommunikationsverhalten und politischem Konsum. Demgegenüber haben wir in einem komplex angelegten Untersuchungsdesign versucht, die Vielschichtigkeit von Praktiken der Politisierung von Konsumhandeln durch ein zugegebenermaßen aufwendiges Forschungsdesign explorativ zu erfassen.

\section{Forschungsprojekt "Consumer Netizens" - methodisches Design und ausgewählte Forschungsergebnisse}

Erkenntnisleitende Forschungsfragen des Projektes waren: Wie wird Konsumhandeln politisch? Inwiefern lassen sich Typen von Konsumbürgerinnen und Konsumbürger unterscheiden? Wie sind sozial-ökologische konsumbezogene Partizipationspraktiken hinsichtlich ihres politischen und demokratischen Charakters einzuschätzen? Welche Rolle spielt das Social Web für Prozesse der Politisierung von Konsum?

Den Ausgangspunkt der empirischen Untersuchung bildete eine Erhebung von Projekten im deutschsprachigen Internet, die sich thematisch mit kritischem oder nachhaltigem Konsum befassten. Im Ergebnis konnten ca. 320 Web-Projekte erfasst und hinsichtlich ihrer Strategien, Vernetzung und Beteiligungsangebote ana- 
lysiert werden (vgl. Baringhorst/Yang 2014). Etwa 30 einschlägige internetbasierte Projekte wurden anschließend als Multiplikatoren zur Ansprache potentieller Untersuchungsteilnehmerinnen und Untersuchungsteilnehmer gewonnen. Mit Hilfe eines Online-Kurz-Fragebogens konnten verschiedene Merkmale systematisch erfasst und miteinander abgeglichen werden. So gelang es durch Abfrage von Wohnort (Postleitzahlenbereich), Alter, Geschlecht, Bildung, politischem Engagement, Mediennutzung und Konsumaktivität, Teilnehmerinnen und Teilnehmer im Sinne eines theoretischen Samplings, also im Sinne theoretisch relevanter, möglichst unterschiedlicher Merkmale zu bestimmen. Diese potentiellen Teilnehmerinnen und Teilnehmer wurden per Email und telefonisch kontaktiert, um im persönlichen Gespräch weitere, für das Theoretical Sampling relevante Informationen abzufragen. Es folgten die ersten persönlichen Treffen mit ihnen. Bei diesen Gesprächen wurden weitere biographische Daten erhoben und es fand eine Instruktion in das Schreiben von über acht Wochen zu führenden Partizipationstagebüchern statt. Gleichzeitig wurde eine zweite Rekrutierungswelle initiiert, um auch Personen, die sich nicht über das Internet ansprechen ließen, als Untersuchungsteilnehmerinnen und Untersuchungsteilnehmer zu gewinnen. Am Ende der Erhebung lagen 26 verwertbare Partizipationstagebücher vor. Im Anschluss an die Abfassung der Tagebücher wurden mit den Teilnehmerinnen und Teilnehmern Interviews geführt. Der Aufbau der Interviews sah zu Beginn einen narrativen Impuls vor, darauf folgten gezieltere Fragen zu der persönlichen Motivation des politischen Konsums, der Mediennutzung, dem Verhältnis zu politischen Organisationen und Netzwerken, dem Wissensmanagement sowie dem Demokratie- und Bürgerverständnis, um bei der Interpretation des Tagebuchs offen gebliebene Fragen zu klären.

Gingen wir zum Zeitpunkt der Antragsstellung gemäß dem damaligen Forschungsstand davon aus, dass unterschiedliche, voneinander abgrenzbare Milieus politischer Konsumentinnen und Konsumenten im Netz existieren, so zeigten die qualitativen Befunde deutlich, dass die mittels Umfragedaten erstellten MilieuTypen wie LOHAS (Lifestyle of Health and Sustainability) eher quantitative Modellierungen sind (z.B. Müller-Friemauth u.a. 2009), die nur bedingt im Rahmen qualitativer Forschung bestätigt werden können. Mit anderen Worten: Es fand sich eine deutlich höhere Vielfalt und milieuübergreifende Verbreitung von Praktiken des politischen Konsums und der Netznutzung als bisher angenommen.

Anhand der Partizipationstagebücher rekonstruierte vor allem Katharina Witterhold in ihrer Dissertation die individuellen Sets von Praktiken und bündelte diese im Zuge der weiteren Interpretationsarbeit zu einer Typologie der bürgerschaftlichen Medien- und Konsumpraktiken (2015; 2017). Darauf aufbauend und rekurrierend auf ein theoretisches Verständnis von Consumer Netizens als kreativen, problemlösungsorientiert kollaborierenden Bürgerinnen und Bürgern, die vor allem lebenspolitisch engagiert sind, wurde von Mundo Yang eine zweiteilige Typologie neuer und alter Formen der Citizenship rekonstruiert (Yang/Baring- 
horst 2016). In Anlehnung an Überlegungen von Jörn Lamla zur Aktualität der von Riesman et al. (1950) vorgelegten Unterscheidung zwischen innen- und außengeleitetem Sozialcharakter wird vorgeschlagen, von einem innengeleitetem Typ der Konsumbürgerinnen und Konsumbürgern auszugehen, die vor allem ethisch konsumieren und rational-diskursiv versuchen, an Öffentlichkeit teilzuhaben. Diesen ethischen Konsumbürgerinnen und Konsumbürgern geht es in erster Linie darum, prinzipientreu zu allgemeinem Wandel (z.B. in Richtung Nachhaltigkeit) beizutragen. Sie organisieren und beteiligen sich dabei auf herkömmliche Art und Weise politisch (in NROs oder sozialen Bewegungen) und sehen das Internet vor allem als technischen Zugang zu einer diskursiven Öffentlichkeit. Demgegenüber lässt sich mit einem um die Konfliktivität des Politischen erweiterten Verständnis von Riesmans Typ des außengeleiteten Sozialcharakters verständlich machen, wie die neueren kritisch-kreativen Konsumbürgerinnen und Konsumbürger mit ihrer Neigung $\mathrm{zu}$ ästhetisch-expressiven Praktiken und zur Teilnahme an ProdusageProzessen als Teil einer von verfassten Institutionen eher losgelösten Demokratisierung der Konsumgesellschaft (Lamla 2013) verstanden werden können.

Mit dieser Fassung der kreativen außengeleiteten Konsumbürgerin und des kreativen außengeleiteten Konsumbürgers lässt sich auch klären, wie der politisch-soziologisch identifizierte Trend zur Individualisierung sich gleichzeitig mit den empirischen Entwicklungen neuer Formen der Kollektivierung vereint, ohne deshalb schon zwangsläufig von einer individualistischen Fragmentierung, Entpolitisierung und privatistischen Haltung in Hinsicht auf die Demokratiefrage ausgehen zu müssen. Die empirisch gesättigte Vorstellung des neuen demokratischen Charakters bestimmter Consumer Netizens als kreative außengeleitete Sozialcharaktere lässt sich bezogen auf die Aspekte bürgerschaftliches Ethos, Praktiken und Organisationsformen folgendermaßen präzisieren:

\section{Bürgerschaftliches Ethos}

Wenn sich politischer Konsum im Netz überhaupt als politisch denken lässt, so im Sinne einer stark individualisierten Sorge um die öffentlichen Angelegenheiten (res publica). Ausgangspunkt ist zum einen ein starker Nonkonformismus, der mit einer Distinktion zur Gesellschaft des Massenkonsums einhergeht, und zum anderen motiviert eine starke Hoffnung auf Veränderung dazu, aus den bestehenden Bahnen politischer Beteiligung auszubrechen. Die kreativen Consumer Netizens sehen im Netz vor allem die Möglichkeit, unabhängiger von Parteien, aber auch nunmehr konventionell erscheinenden reinen Protestbewegungen nach persönlich ansprechenden Problemlösungen zu suchen. Der daraus entstehende durchaus kritisch zu sehende Zwang, kreativ zu sein (Reckwitz 2012), führt zu einer fortschreitenden Politisierung der Gesellschaft. Das Private ist öffentlich nicht nur im Sinne einer verstärkten Inklusion von Frauen und ihren Anliegen in diese Formen 
bürgerschaftlichen Engagements, sondern auch hinsichtlich der Anerkennung von Praktiken als relevante Beiträge zur Lösung globaler Probleme, die jenseits der Logiken von Geldverkehr und staatlicher Steuerungsmacht liegen, die also z.B. auch die lebensstilpolitische Bildung von Nachbarschaftsgärten umfassen. Zwar verstehen sich kreative Consumer Netizens selten selbst als politisch. Dennoch fassen sie sich häufig als Teil umfassender sozial-ökologischer Wandlungsprozesse auf, die sie zu ihrer Privatangelegenheit erklären.

\section{Praktiken}

Dementsprechend umfassen die Praktiken der kreativen Consumer Netizens deutlich mehr als die Abstimmung mit dem Einkaufswagen (Boykott, Buykott), sondern sind als Teil pragmatischer Suchbewegungen und Erprobungsprozesse aufzufassen. Es werden mit Hilfe des Netzes Lösungen für private Konsumbedürfnisse gesucht und schließlich im Sinne des Produsage (Bruns 2008) mit anderen geteilt. Gegenüber ihrem sozialen Umfeld erfüllen diese Bürgerinnen und Bürger auch Aufgaben des Majoritär-Werdens (Marchart 2011), z.B. des Mainstreamings nachhaltiger Konsummuster. Dabei legen sie Wert auf politische Abgrenzung und soziale Distinktion mit Blick auf die bislang dominanten Alltags- und Konsumpraktiken. Im Rahmen kollektiver Peer-to-peer-Prozesse werden z.B. neue nachhaltige Konsummuster erprobt, getestet, bewertet und zum Teil sozialunternehmerisch gefördert (per Crowdfunding). Auch ohne starke politische Programmatiken werden zivilgesellschaftliche Selbstlösungskapazitäten im Sinne der Verbreitung neuer Konsumpraktiken erarbeitet (z.B. Nahverkehrslösungen, car-sharing) und im eigenen Lebensstil verankert. Gerade die starke Situierung der Praktiken in semi-öffentlichen Räumen (z.B. im Freundes- und Bekanntenkreis) kommt der aktiven Teilnahme von Frauen entgegen. $\mathrm{Zu}$ betonen ist, dass zwar Utopien einer Produsage-Demokratie oder einer Bewegungsgesellschaft, also der protestförmigen Politisierung aller Alltagsbereiche, nicht Hauptzweck dieser Praktiken sind, aber dennoch stärker mit ihnen verbunden werden als z.B. die Mitarbeit in Parteien.

\section{Organisationsformen}

Auch kreative Consumer Netizens organisieren sich. Dementsprechend können überschwängliche Hoffnungen auf intelligente Schwärme als überholt gelten (Baringhorst 2015a). Die dabei entstehenden neuen kollektiven Formationen (Dolata/Schrape 2014) sind im Netz wie vor Ort situiert. Sie nehmen weder die Form reiner Protest- oder Diskursorganisationen oder repräsentativer Verbände an, sondern strukturieren sich ähnlich wie populärkulturelle Flash Mobs zwischen öffentlichen und privaten Handlungsräumen. Neue Verbrauchernetzwerke folgen 
dem Muster konnektiven Handelns (Bennett/Segerberg 2012) nicht nur im Bereich von unternehmenskritischen Kampagnen (Yang/Voss 2015), sondern vor allem bei der Schaffung von experimentellen Räumen für die Erprobung neuer politisch geforderter Lebensstile (Baringhorst 2016). Gerade hier zeigen sich aber auch Schwächen. Zum einen fehlt es noch an politisch schlagkräftigen Organisationen (Baringhorst 2011). Zum anderen unterminieren Tendenzen der Kommerzialisierung, Kontrolle und Manipulation durch Medienunternehmen den Anspruch einer problem- und nicht profitbezogenen Lösung öffentlicher Angelegenheiten (Yang/Baringhorst 2016). Und schließlich besteht für außengeleitete Konsumentinnen und Konsumenten auch kaum Anreiz, sich weitergehend politisch zu organisieren. Damit bleibt die Frage offen, ob es sich bei den vielen Ansätzen für eine andere Konsumgesellschaft, wie Carrot Mob, sharing-economy, Do-It-together, re-use, community supported agriculture, um kurzlebige kulturelle Hypes oder ernstzunehmende Trends handelt.

Die Befunde bestätigen die verbreitete Kritik an einer Fragmentierung und Popularisierung öffentlicher Diskurse im Netz kaum (Baringhorst/Witterhold 2015). Demgegenüber ist eine hohe Integration der vielfältigen Wissenspraktiken in zentralen Diskussionen über verbraucher-, umwelt- und sozialpolitische Anliegen zu beobachten. Die Vielfalt der zum Teil höchst privaten Lösungsangebote, die von Laien über das Netz distribuiert werden, integriert eine hohe Anzahl individueller Problemlagen in die Bearbeitung allgemeiner Herausforderungen wie konsuminduzierter Umweltschäden. Dennoch zeigen sich bei den Recherche-, Informationsund Validierungspraktiken der Consumer Netizens Probleme moralischer und kognitiver Überforderung. Wissen ist in dieser Hinsicht in der Alltagspraxis kein objektives Gut, sondern Gegenstand der konfliktiven Aushandlung sozialer Beziehungen (z.B. Paar-Beziehungen zwischen Vegetariern und Nicht-Vegetariern). Diese Probleme können einerseits durch Verbraucherbildung und lösungsorientierte Weitergabe von Tipps bearbeitet werden. Sie werden allerdings im Rahmen praktischer Einübung und Nachahmung von Vorbildern (z.B. Idolen im Netz) häufig ohne Reflektion niedrigschwellig gelöst. In dieser Hinsicht zeichnet sich noch ein großer Forschungsbedarf $a b$, wenn es um einen pragmatischen Begriff von Öffentlichkeit (Dewey 1927) geht, der auch unabhängig von staatlicher Anleitung für den Kontext einer sowohl global entgrenzten als auch immer umfassender politisierten Gesellschaft gilt. Wie sich problemlösendes Know-how auch im Rahmen medialer Infrastrukturen sammeln und politisch wenden lässt, wird bisher nur ansatzweise in der Politikwissenschaft bearbeitet. Deutlich wird in den Befunden des Projekts, dass Wissen im Netz stark visuell (Yang 2014) und expressiv (Baringhorst 2015b) vermittelt wird, wobei z.B. Online-Map-Anwendungen verbraucherpolitisches Wissen bereitstellen, das sich nicht mehr auf sprachlich vermittelte Kommunikation reduzieren lässt. 


\section{Literatur}

Arendt, Hannah 1981: Vita activa oder Vom tätigen Leben, München und Zürich.

Barber, Benjamin 2007: Consumed: How Markets Corrupt Children, Infantilize Adults, and Swallow Citizens Whole, New York.

Baringhorst, Sigrid 2011: Die Grenzen der webbasierten Konsumentenmacht. In: Ökologisches Wirtschaften 4, S. 15-17.

Baringhorst, Sigrid 2015a: Mehr Demokratie durch Online-Aktivismus? Zum Wandel politischer Partizipation im Netz. In: Lothar Harles/Dirk Lange (Hg.), Zeitalter der Partizipation. Paradigmenwechsel in Politik und politischer Bildung, Schwalbach/Ts., S. 76-85.

Baringhorst, Sigrid 2015b: Konsum und Lebensstile als politische Praxis - Systematisierende und historisch kontextualisierende Annährungen, in: Forschungsjournal Soziale Bewegungen, Heft 2, S. 17-27.

Baringhorst, Sigrid 2016: Nachhaltigkeit durch politischen Konsum und Internetaktivismus? In: Gertraud Diendorfer/Manfried Welan (Hg.), Demokratie und Nachhaltigkeit, Verbindungslinien, Potentiale und Reformansätze, Bd 2 der Schriftenreihe des Demokratiezentrums Wien, Innsbruck u.a., S. 43-60.

Baringhorst, Sigrid/Witterhold, Katharina 2015: Verbraucherinformation - Top down oder bottom up? Neue Formen netzbasierter Generierung einer kritischen Verbraucheröffentlichkeit. In: Christian Bala/Klaus Müller (Hg.), Abschied vom Otto Normalverbraucher, Moderne Verbraucherforschung - Leitbilder, Information, Demokratie, Essen, S. 145-166.

Baringhorst, Sigrid/Yang, Mundo 2014: Kreative politische Handlungen im Social Web - Beiträge zur Demokratie? In: Michael Schröder (Hg.), Politik und politische Bildung in der digitalen Welt, Chancen und Herausforderungen, Reinbek und München, S. 37-58.

Baringhorst, Sigrid/Mundo, Yang/Quednau, Tobias (Hg.) 2015: Das Private ist politisch: Konsum und Lebensstile, Sonderheft des Forschungsjournal Soziale Bewegungen, Heft 2.

Bennett, Lance W./Segerberg, Alexandra 2012: The Logic of Connective Action. Digital Media and the Personalization of Contentious Politics, Cambridge.

Blühdorn, Ingolfur 2013: Simulative Demokratie. Neue Politik nach der postdemokratischen Wende, Frankfurt a.M.

BMELV (2013): Ökobarometer 2013. Repräsentative Bevölkerungsbefragung im Auftrag des Bundesministeriums für Ernährung, Landwirtschaft und Verbraucherschutz (BMELV). Online unter: www.bmel.de/SharedDocs/Downloads/Ernaehrung/Oekobarometer_2013.pdf?_blob=publicationFile

Bruns, Axel 2008: Blogs, Wikipedia, Second Life, and beyond. From production to produsage, New York u.a. 
BMUB, Bundesministerium für Umwelt, Naturschutz, Bau und Reaktorsicherheit/UBA, Umweltbundesamt (2017): Umweltbewusstsein und Umweltverhalten in Deutschland 2016. Ergebnisse einer repräsentativen Bevölkerungsumfrage. Berlin, Dessau-Roßlau: BMUB/UBA. Online unter: https://www. bmub.bund.de/fileadmin/Daten_BMU/Pools/Broschueren/umweltbewusstsein_deutschland_2016_bf.pdf

Crouch, Colin 2008: Postdemokratie, Frankfurt a.M.

Dahlgren, Peter 2009: Media and political engagement. Citizens, communication, and democracy, Cambridge.

Dewey, John 1927: The Public and Its Problems, New York.

Dolata, Ulrich/Schrape, Jan-Felix 2014: Kollektives Handeln im Internet. Eine akteurtheoretische Fundierung. In: Berliner Journal für Soziologie, Heft 1, S. 5-30.

Gaiser, Wolfgang/Gille, Martina 2012: Politische Partizipation junger Menschen, in: Polis, Heft 1, S. 15-17.

Greven, Michael Th. 1999: Die politische Gesellschaft. Kontingenz und Dezision als Probleme des Regierens und der Demokratie, Wiesbaden.

Isin, Engin F. 2009: Citizenship in Flux: The Figure of the Activist Citizen, in: Subjectivity, Heft 29, S. 367-388.

Kaase, Max 1995: Partizipation. In: Dieter Nohlen (Hg.), Wörterbuch Staat und Politik, Bonn, S. 521-527.

Lamla, Jörn 2009: Konsumpraktiken in der virtuellen Alltagsökonomie. In: Renate Buber/Hartmut H. Holzmüller (Hg.), Qualitative Marktforschung, Wiesbaden, S. 779-803.

Lamla, Jörn 2013: Verbraucherdemokratie, Frankfurt a.M.

Marchart, Oliver 2011: Die politische Differenz, Frankfurt a.M.

McFarland, Andrew S. 2012: Why Creative Participation Today? In: Michele Micheletti/Andrew S. McFarland (Hg.), Creative Participation, Responsibility - Taking in the Political World, Boulder, S. 15-33.

Micheletti, Michele 2003: Political virtue and shopping. Individuals, consumerism, and collective action, New York.

Micheletti, Michele/McFarland, Andrew S. (Hg.) 2012: Creative Participation. Responsibility-Taking in the Political World, Boulder.

Müller-Friemauth, Friederike/Sinus Sociovision/KarmaKonsum (2009): LOHAS: Mehr als Green-Glamour. Eine sozio-kulturelle Segmentierung. Frankfurt a.M./Heidelberg: KarmaKonsum, Sinus Sociovision.

Reckwitz, Andreas 2012: Die Erfindung der Kreativität. Zum Prozess gesellschaftlicher Ästhetisierung, Bielefeld.

Reckwitz, Andreas 2016: Kreativität und soziale Praxis. Studien zur Sozial- und Gesellschaftstheorie, Bielefeld.

Riesman, David/Glazer, Nathan/Denney, Reuel 1950: The Lonely Crowd. A Study of the Changing American Character, New Haven. 
Schatzki, Theodore R. 2012: A Primer on Practices. Theory and Research, in: J. Higgs et al. (Hg.), Practice-Based Education, Perspectives and Strategies, Rotterdam, S. 13-26.

Stolle Dietlind/Micheletti, Michele 2013: Political Consumerism. Global Responsibility in Action, Cambridge.

Street, John 1997: Politics \& Popular Culture, Cambridge.

Van Deth, Jan W. 2014: A Conceptual Map of Political Participation, in: Acta Politica, Heft 3, S. 349-367.

Van Deth, Jan W. 2012: Is Creative Participation Good for Democracy? In: Michele Micheletti/Andrew S. McFarland (Hg.), Creative Participation, Responsibility Taking in the Political World, Boulder, S. 148-172.

Williams, Raymond 1961: The Long Revolution, London.

Witterhold, Katharina 2015: Politik des Lebensstils als eher weiblicher Partizipationsstil? Beteiligungspraktiken politischer Konsumentinnen und Konsumenten on/offline. In: Forschungsjournal Soziale Bewegungen, Heft 2, S. 46-56.

Witterhold, Katharina 2017: Politische Konsumentinnen im Social Web. Praktiken der Vermittlung zwischen Bürger- und Verbraucheridentität, Bielefeld.

Yang, Mundo 2014: Mobilisierende Internetvideos zwischen Culture Jamming und Framing. In: Caja Thimm/Mark Dang-Anh/Jessica Einspänner-Pflock (Hg.), Digitale Gesellschaft - Partizipationskulturen im Netz, Münster, S. 128-147.

Yang, Mundo/Baringhorst, Sigrid 2014: Reintermediation durch Social-Web? Eine Analyse von Social-Web-Projekten im Bereich des politischen Konsums. In: Franziska Oehmer (Hg.), Politische Interessenvermittlung und Medien, Funktionen, Formen und Folgen medialer Kommunikation von Parteien, Verbänden und sozialen Bewegungen, Wiesbaden, S. 399-423.

Yang, Mundo/Baringhorst, Sigrid 2016: Politischer Konsum im Netz als Ausdruck des Wandels politischer Partizipation. In: Melanie Jaeger-Erben/Jana RückertJohn/Martina Schäfer (Hg.), Nachhaltiger Konsum durch soziale Innovationen - Wissenschaftliche Perspektiven, Strategien der Förderung und gelebte Praxis, Wiesbaden, S. 191-216.

Yang, Mundo/Voss, Kathrin 2015: Four Types of Online Grassroots Campaigning in Germany. Paper presented at the International Conference »Protest Participation in Variable Communication Ecologies. Meanings, Modalities and Implications«, 24-26 June 2015, Alghero, Sardinia (Italy).

Žižek, Slavoj 2017: Die populistische Versuchung. In: Heinrich Geiselberger (Hg.), Die große Regression, Berlin, S. 293-313. 



\section{Politische Reartikulationen kultureller Topoi in populistischen Verstetigungsprozessen}

Bernadette Goldberger

\section{Einleitung}

Der Begriff des Populismus wird in der gegenwärtigen politischen Konjunktur zunehmend inflationär gebraucht, dabei allerdings ungleich seltener klar definiert. Im Alltagsgebrauch transportiert die Charakterisierung politischer Parteien oder Akteurinnen und Akteure als »populistisch« häufig den Vorwurf, »einfache Antworten auf komplexe Fragen « anzubieten. Dagegen meint ein sozialwissenschaftliches Verständnis von Populismus - bei aller Uneinigkeit, ob dieser als politische Strategie, Ideologie oder diskursive Praxis zu konzeptualisieren sei (Priester 2012) - eine spezifische Form politischer Mobilisierung, die sich durch ihre Referenz auf ein homogen vorgestelltes »Volk« auszeichnet, welches in dichotomer Kontrastierung mit nicht-zugehörigen Teilen der Gesellschaft als legitimer Ausdruck eines authentischen Willens der gesamten politischen Gemeinschaft moralisch überhöht wird (Mudde 2004; Müller 2016; Taggart 2000).

Ausgehend von der zunehmenden Präsenz populistischer Politikangebote in den Parlamenten und Regierungen der europäischen Länder stellt sich die Frage, wie populistische Projekte sich langfristig stabilisieren und die affektive Mobilisierung der Anrufung des »Volkes« gegenüber illegitimen Eliten oder unrechtmäßig Privilegierten aufrechterhalten, wenn sie selbst an der Regierungsmacht sind. Wie kann die antagonistische Spaltung, auf der populistische Identitätsentwürfe beruhen, in einer dauerhaften symbolischen Ordnung konsolidiert werden und wie können diese Konsolidierungsprozesse empirisch nachvollzogen und theoretisch gefasst werden?

In kritischer Auseinandersetzung mit der Populismustheorie Ernesto Laclaus soll im Folgenden versucht werden, empirische Populismusforschung als politische Kulturforschung zu konzipieren, welche den Rekurs populistischer Symbolisierungsstrategien auf etablierte kulturelle Topoi in den Blick nimmt und damit einen Beitrag zur Theoretisierung der Funktion politisch-kultureller Artikulationen für die Verstetigung popularer Subjektivitäten leistet. Die Ambiguität »zwischen kulturwissenschaftlicher Analytik und normativer Politischer Theorie« (Reckwitz 
2006: 348) in Laclaus Ansatz bedingt seine Vernachlässigung der Rolle symbolischer Inszenierungen in der populistischen Praxis. Sein diskurstheoretischen $\mathrm{Zu}$ gang wird daher um eine kultursoziologische Perspektive erweitert, die ein Verständnis des metaphorischen Funktionierens populistischer Verstetigungsprozesse ermöglicht.

Dazu wird anhand einer Studie zu politisch-kulturellen Artikulationen des Popularen im argentinischen Kirchnerismus (Goldberger 2018) exemplifiziert, wie populistische Symbolpolitik ein Modell des »Volkes« inszeniert, das der antagonistischen Spaltungsrhetorik vorgängig scheint und dieser als langfristig stabilisierende Legitimitätsbasis dient. Am konkreten Fall der regierungsunterstützenden Mediendebatten zur Fußballweltmeisterschaft 2010 und ihrer narrativen Verknüpfung der popularkulturellen Symbolik des argentinischen Fußballs mit der diskursiven Konstruktion des Kirchnerismus wird gezeigt, wie historisch verankerte Bilder des Popularen so in die interpretative Struktur der antagonistischen Konstellation integriert werden, dass sie einerseits zu Sinnbildern der kirchneristischen Identitätserzählung werden und diese assoziativ aktualisieren, andererseits die politischen Konfliktlinien durch ihre Reinszenierung in einem lebensweltlichen Erfahrungszusammenhang selbst kulturell überformt und als Konfrontation zweier soziokulturell distinkter Gesellschaftsblöcke rekonfiguriert werden. Die illustrativen Beispiele sind dem analysierten Medienmaterial während des WM-Zeitraums sowie ergänzenden Interviews entnommen, die 2011 und 2012 mit Vertretern des pro- wie anti-kirchneristischen Diskurspektrums als Protagonisten und Beobachter dieser kulturellen Deutungskämpfe geführt wurden.

\section{Die diskurstheoretische Populismustheorie Ernesto Laclaus und ihre Leerstellen}

Laclau konzeptualisiert Populismus als diskursive Logik mit der Zielsetzung, ein politisch mobilisierbares »populares Subjekt « zu konstruieren. Neben dieser Stoßrichtung, das »Volk« als Identitätskategorie eines kollektiven politischen Akteurs zu etablieren, erhellt sein Ansatz die spezifische Form der populistischen Konstruktionslogik als zweites zentrales Charakteristikum. Die Anrufung des »Volkes« greift das soziale Unbehagen mit der etablierten institutionellen Ordnung auf und artikuliert es über die Einführung einer gesellschaftlichen Spaltungslinie zu einem konsistenten politischen Diskurs. Dieser kanalisiert heterogene politische Forderungen und frustrierte soziale Ansprüche gegen einen diskursiv konstruierten Machtblock, dem die Verantwortung für den Ausschluss ersterer aus dem politischen Repräsentationsraum zugeschrieben wird. Die Konstruktion eines Antagonismus zwischen "power and the underdog " (Laclau 2005b: 38) schafft zwei dichotome und intern homogene Lager. So entsteht ein Kollektiv der Außenvorgelasse- 
nen, dessen interne Kohäsion nicht auf gemeinsamen positiven Merkmalen, sondern auf der Äquivalenz seiner Mitglieder gegenüber einer die eigenen Ansprüche missachtenden oder gar bedrohenden anti-popularen Kraft beruht (Laclau 2005a: 77-86).

Antagonistische Identitäten bezeichnen in Ernesto Laclaus - aus seiner gemeinsamen Arbeit mit Chantal Mouffe übernommenen - Definition ein Verhältnis zwischen sozialen Kräften, die das volle Sein des jeweils anderen durch ihre eigene Präsenz untergraben und sich wechselseitig in ihrer Konstitution als in sich geschlossene Totalitäten blockieren (Laclau/Mouffe 2006: 161-167). Das antagonistische Gegenüber bietet so allerdings gerade die ermöglichende Kontrastfolie für das Versprechen einer vollständigen Identität in der populistischen Rhetorik. Die innere Unmöglichkeit einer spannungsfreien Gemeinschaft kann auf einen äußeren Feind projiziert werden, der als konstitutives Außen die Illusion ebendieses "Einheitsvolkes à venir« stützt und damit den affektiv mobilisierenden Horizont der populistischen Konstruktion darstellt (ebd. : 185-187). Populismus erweist sich mithin als Identifikationsmodus, der seine Subjekte in ihrer Erfahrung eines geteilten Mangels anruft und über das Benennen eines externen Verursachers eine Gemeinschaft des Anspruchs auf die Entfaltung des von der Macht Verhinderten initiiert.

Allerdings bleibt die Allianz heterogener Proteste fragil, solange sie auf die Referenz auf dasselbe feindliche Außen und damit auf eine negative bestimmte Identität beschränkt ist. Ziel der populistischen Konstruktion ist es, der Figur des Volkes Existenz aus sich selbst heraus zu verleihen, sodass dieses selbst konstitutive Wirkung erlangt und zum vorgängigen Fundament und Antrieb der Kämpfe wird, als deren Folge es eigentlich entstanden ist. Die langfristige Konsolidierung der popularen Subjektivität durch ihre Verankerung in einem von der antagonistischen Konstellation unabhängig erscheinenden Grund leistet nach Laclau ein leerer Signifikant, der als hegemonialer Repräsentant der imaginären Einheit des Volkes symbolische Präsenz verleiht (Laclau 2002). Der leere Signifikant ist gerade nicht als Ausdruck einer allen »popularen Subjekten « zugrundeliegenden positiven Essenz $\mathrm{zu}$ verstehen. Der Begriff weist vielmehr darauf hin, dass ein partikularer Vertreter des Anti-Status-Quo-Diskurses nicht die gesamte Bandbreite der versammelten Inhalte abstrahierend abbildet, sondern in Zurücknahme seiner eigenen Bedeutung zur privilegierten Projektionsfläche eines "popularen Willens« auf Realisierung eines reinen Seins avanciert, das vom Antagonismus blockiert wird. Der Name einer Partei, Person oder Losung an der Spitze der populistischen Konstruktion wird identifikatorischer Ausdruck der gesamten politischen Bewegung, in seiner Verkörperung kristallisiert das Kollektiv der Marginalisierten zu einem singulären Akteur. Insofern konstituiert er das »Volk« als geschlossene Totalität, indem die imaginäre Vorstellung einer versöhnten Selbstidentität in seiner Gestalt 
Existenz erlangt und in der Folge zum umfassenden Ganzen des politischen Repräsentationsraums erklärt wird (Laclau 2005a: 93-100):

»[T] he people can be conceived as populus, the body of all citizens; or as plebs, the underprivileged. [...] In order to have the >people < of populism, we need something more: we need a plebs who claims to be the only legitimate populus - that is, a partiality which wants to function as the totality of the community (ebd: 81, Herv. i. O.).

Zwar muss das »wahre Volk«, das gleichzeitig das "ganze Volk« sein will, notwendigerweise immer wieder scheitern, da der verhindernde Antagonismus konstitutiv für die imaginäre Einheit bleibt (Laclau/Mouffe 2006: 177-179). Diese realisiert sich nur im homogenisierenden Effekt der Äquivalenz »gegenüber dem vom System zu Selbstbezeichnungszwecken Dämonisierten« (Laclau 2002: 68). Die Persistenz der antagonistischen Bedrohung dient insofern als Beweis für die Existenz des Volkes und erneuert die Mobilisierungskraft der populistischen Rhetorik (Aboy Carlés 2005: 131-135). Indem die Subjekte auf die Anrufung des hegemonialen Repräsentanten antworten, der das Begehren nach der blockierten »Fülle« des zu sich selbst gekommenen Volkes ausdrückt, erkennen sie sich als Teil dieser popularen Gemeinschaft und verleihen ihr im Akt der Identifikation performativ Realität.

Die Konzeptualisierung von Populismus als formale Logik der Konstruktion politischer Identitäten ermöglicht es, die abstrakte Struktur populistischer Diskurse und ihre subjektkonstituierende Wirkungsdynamik in der empirischen Forschung in analytische Kategorien zu fassen. Demgegenüber bleiben Definitionsversuche auf Basis inhaltlicher Charakteristika häufig deskriptive Typologien ohne konzeptuellen Kern. Warum bestimmte populistische Identitätsangebote Anerkennung als hegemoniale Verkörperungen des popularen Lagers finden und wie sie ihre Anrufungskraft konkret entfalten, kann mit dem diskurstheoretischen Werkzeug dagegen nur unbefriedigend erklärt werden. Laclau begründet die Identifikation mit ganzheitsversprechenden politischen Konstruktionen mit dem in das diskursiv verfasste Subjekt eingeschriebenen Begehren nach Wiederherstellung des vorsymbolischen Genießens (Laclau 2005a: 110-117). Seine politiktheoretische Übersetzung psychoanalytischer Konzepte blendet aber die Historizität triebstruktureller Dispositionen als Verarbeitungsmuster gesellschaftlicher Bedingungsfaktoren aus und mündet in der Ontologisierung von Populismus als grundlegender Logik des Politischen (ebd.: 154) und schließlich in seiner normativen Überhöhung zum Paradigma demokratischer Vergesellschaftung schlechthin (ebd.169-171).

Die spezifische Bezugnahme auf das »Volk « als in sich geschlossene und homogene Entität und das mobilisierende Versprechen der Rückkehr zu einem Goldenem Zeitalter stellt aber sehr wohl eine ideologische Weichenstellung mit strukturierende Effekten auf die postulierten Inhalte und damit mehr als eine formale Logik der Identitätskonstruktion dar. Bei Laclau werden populistische Konstruktionsmodi 
ausgehend von ihrer ideologischen Variabilität und ihrer Kompatibilität mit gegensätzlichen politischen Programmatiken dagegen zu einer ideologisch neutralen Konfigurationsform der diskursiven Praxis erklärt, deren Zielvorstellungen einzig von den artikulierten Inhalten bestimmt würden (Laclau 2010: 46). Dies erklärt seine Vernachlässigung der konkreten Mobilisierungsstrategien zur Konsolidierung der populistischen Volkskonstruktion, die für ihn Teil der »kontingenten « Praxis sind.

Dieses praktische Funktionieren populistischer Projekte soll im Folgenden in den Blick gerückt werden. Woher bezieht eine spezifische politische Bewegung, Partei oder Führerfigur die Fähigkeit, erfolgreich als Kristallisationspunkt der popularen Einheit zu fungieren? Die sprachtheoretische Abstraktion in der Rede vom leeren Signifikanten lässt die aktiven kulturellen Deutungskämpfe in den Hintergrund treten, in denen die diskursiv instituierte Volkskonstruktion von konkreten Akteurinnen und Akteure zum objektiven Grund der politischen Gemeinschaft "sedimentiert « (Laclau 1990: 34f.) wird. Ihre Konsolidierung in einem stabilen Bedeutungssystem ist nicht alleinige Konsequenz eines performativen Benennungsaktes durch selbsttätig wirkende Repräsentationen, die kraft ihrer »Entleerung« zum Platzhalter aller popularen Wünsche werden. Politische Subjektivitäten werden nicht ex nihilo geschaffen, die hegemonialen Akteure beziehen sich vielmehr auf bestehende Bedeutungen aus dem verfügbaren kulturellen Symbolrepertoire und verknüpfen diese mit den politischen Konfliktlinien.

\section{Kulturelle Topoi und die "metaphorische Reichweite" der populistischen Konstruktion}

Kulturelle Praxis macht die soziale Wirklichkeit verstehbar, sie inszeniert gesellschaftliche Vorstellungen und produziert auf diese Weise kollektive Wahrnehmungs- und Deutungsroutinen. Kulturelle Topoi sind symbolische Kondensate solcher überindividueller Interpretationsschemata, die chiffrenhaft auf bestimmte Normvorstellungen und Weltsichten verweisen (Knoblauch 2001). »Sie haben mir die Beine abgeschnitten«, die legendäre Aussage Diego Maradonas nach seinem Dopingausschluss von der WM 1994, wurde etwa zum Topos einer argentinischen Opfererzählung, die der Historiker Luis Alberto Romero als die Vorstellung beschreibt, »dass Argentinien eine Bestimmung zur Großartigkeit hat und es aus irgendeinem Grund eine Verschwörung gibt, um das zu verhindern « (Interview Romero, 15.6.2012). Als 2010 die argentinische WM-Teilnahme mit einer o:4-Niederlage im Viertelfinale endet und bekannt wird, dass Maradonas Vertrag als Nationaltrainer nicht verlängert wird, wird sein Kollege Fernando Signorini aus dem Coachingteam im Interview gefragt, ob dem ehemaligen Spielerstar damit erneut die Beine abgeschnitten worden seien (6, 7, 8, 28.7.2010: 2:54-3:42). Das Bild 
aktualisiert zunächst die Vorstellung, Maradona werde als Ikone der »Argentinität« - verstanden als »typisch argentinisches« Ambivalenzverhältnis zu Disziplin und Regeln - erneut für seinen transgressives Wesen und sein unehrerbietiges Aufbegehren gegen die Eliten verfolgt (Alabarces 2010: 181-192). Signorinis Antwort, »Ja, aber sie werden ihm wieder nachwachsen« $(6,7,8$, 28.7.2010: 2:54-3:42), bestätigt die Lesart vom »Komplott der Mächtigen«, evoziert aber zusätzlich die Deutung, Maradona sei gerade aufgrund seiner (national-)charakterlichen Attribute letztlich selbst von den überlegenen Gegnern nicht $\mathrm{zu}$ Fall zu bringen. Der Interviewausschnitt wird in der kirchneristischen TV-Sendung 6, 7, 8 in einen Videoclip integriert, in dem unter anderem Staatspräsidentin Fernández de Kirchner Maradona für seine »Verteidigung der Nationalfarben« lobt und dieser selbst die Festigkeit seiner Werte betont, die »sie« nicht hätten. Für den politischen Zusammenhang wird so die metaphorische Aussage transportiert, das destino de grandeza (»Bestimmung zur historischen Größe«) der - in der Verkörperung Maradonas popularen - Nation sei nicht zu verhindern, die authentisch patriotische Haltung daher die der Treue und Loyalität zum eigenen kulturellen Sein, was auch auf das national-populare Projekt des Kirchnerismus bezogen werden kann.

Topoi sind unmittelbar schlüssig wirkende, im Common sense verankerte kulturelle Bilder. Nur deshalb kann im Jahr 2009 ein argentinischer TV-Werbespot funktionieren, in der drei Freunde ein Paar sich bewegender Beine im Wald finden und sofort richtig erkennen: »Das sind die Beine, die sie Diego >94 abgeschnitten haben!« (Gran DT, Clausura 2009). Der Konsens über diese symbolischen Motive endet allerdings bei dem Wissen um ihren Referenzcharakter. Darüber hinaus lassen sich aus ihnen »verschiedenartige und sogar völlig gegensätzliche Argumente gewinnen, derselbe Topos kann bei derselben Problemfrage beiden Kontrahenten nützlich sein« (Bornscheuer 1976: 98). Diese »interpretatorische Polyvalenz« (ebd.: 99) topischer Bedeutungen macht sie zu kollektiv geteilten narrativen Ankerpunkten der gesellschaftlichen Selbstverständigung, die über ideologische Grenzen hinweg anschlussfähig sind. So beziehen sich die Vertreterinnen und Vertreter der regierungskritischen Seite auf dieselben Topoi, erklären die Inszenierung Maradonas als Opfer und Ankläger aber zum perfekten Verdichtungspunkt einer als "peronistisch« abgelehnten politischen Kultur paranoider Allmachtsphantasien und ihrer Prägekraft auf Argentinien als »Land mit epischer Berufung und skandalösen Misserfolgen, um unser Epos aufrechterhalten zu können«, wie der konservative Essayist Santiago Kovadloff urteilt (Interview Kovadloff, 12.6.2012). Das unrühmliche WM-Ausscheiden unter Maradonas Führung wird entsprechend als Bestätigung dafür gedeutet, »dass Argentinien von seinen eigenen Exzessen geschlagen wurde, von seinem maßlosen Selbstwertgefühl, seiner Angeberei, seiner Blindheit « (ebd.). Aus der kulturellen Vertrautheit und interpretativen Offenheit der habitualisierten Topik ergibt sich ihr legitimatorisches Potential für Auseinandersetzungen um politische Deutungsmacht. Die konkurrierenden Akteursgruppen des po- 
litischen Feldes versuchen daher, an diese symbolisch verdichteten Erzählungen anzuknüpfen und sie als »Transportmittel« zur narrativen Strukturierung der eigenen Botschaften zu nutzen (Schwab-Trapp 2001: 273).

Die Reartikulation historisch wirkmächtiger Vorstellungsbilder aus dem kollektiven Bedeutungsfundus zur sinnstiftenden Erzählung aktueller Entwicklungen parallelisiert die politischen Konflikte mit lebensweltlichen kulturellen Repräsentationen und etabliert so ein Problemverständnis, das im bemühten Verweiszusammenhang angelegt ist (Hall 1986: 99-104). Kulturelle Topoi funktionieren damit als konventionalisierte Metaphern, die $\mathrm{zu}$ »metaphorischen Konzepten« (Lakoff/Johnson 1998) werden. Als solche transportieren sie eine bestimmte Logik aus ihrem ursprünglichen Kontext in den politischen Raum, der dadurch im Lichte dieser Logik anschaulich gemacht und modelliert wird. Metaphorische Bedeutungskonstitution in populistischen Diskursen ist nicht an sprachliche Wendungen gebunden, sondern meint analogisierende Argumentationen, die komplexe politische Sachverhalte in Anlehnung an einen anderen Erfahrungsbereich in stereotype Szenarien gießen (Musolff 2004: 32-61). Dies legt bestimmte Schlussfolgerungen nahe, die handlungsanleitend wirken und die unmittelbare Schlüssigkeit der Metapher erneut bestätigen (Lakoff/Johnson 1998: 179).

Entgegen Lakoff und Johnsons kognitivistischem Metaphernverständnis soll allerdings betont werden, dass die Inszenierung politischer Werte und Identitäten im Rückgriff auf etablierte Topoi diese selbst entlang der politischen Konfliktlinien restrukturiert. Politisch-kulturelle Artikulationen sind nicht einfach »Besetzungen« vorgegebener Deutungsrahmen. Letztere erfahren vielmehr in ihrer Neuzusammensetzung eine Neueinschreibung, die mit Willy Viehöver als »Prozess der Narrativisierung « (Viehöver 2012: 178, Herv. i. O.) verstanden werden kann und die diskursiven Einzelbestandteile kreativ rekonfiguriert (Viehöver 2014: 71-74). Das relationale Gefüge ist mehr als die Summe seiner Teile und lässt eine kohärente Gesamtstruktur entstehen, welche auf die integrierten Elemente der narrativen Konfiguration zurückwirkt (Somers 1994: 616f.). Letztere werden durch den homogenisierenden Effekt der erzählerischen Matrix zu Sinnbildern der gesamten Konfiguration und drücken diese metaphorisch aus. Als Beispiel: Die Verstaatlichung der TV-Übertragungsrechte für die argentinischen Fußballspiele der ersten Division im Jahr 2009 wurde vom Kirchnerismus unter dem Namen Fútbol para Todos (»Fußball für alle«) als Symbol seiner inkludierenden Wendepolitik inszeniert und narrativ mit anderen anti-neoliberalen Maßnahmen der Regierung verknüpft. Fútbol para Todos wird dadurch zu einem Baustein des antagonistischen Konflikts, der auch für alle anderen Errungenschaften des national-popularen Projekts steht. Er weist über sich selbst hinaus auf die interpretative Struktur der umfassenden dichotomen Teilung hin und transportiert diese auch in den spezifisch fußballerischen Erfahrungsbereich. Gleichzeitig bleibt die historische Formung seines partikularen Ursprungskontextes - des Fußballs als sportlicher Praxis der popularen 
Massen - präsent und wirkt prägend auf die Bedeutungsstruktur auch im politischen Zusammenhang, wo sie den popularen Charakter der kirchneristischen Bewegung bestätigt.

Der Verstetigungsprozess populistischer Politikkonstruktionen kann damit in der Praxis als Erhöhung ihrer metaphorischen Reichweite konzeptualisiert werden. Durch die »konnotativen Resonanzen« (Hall 1986: 95), die zwischen der hegemonialen Repräsentation des populistischen Projekts und vielfältigen kulturellen Verdichtungspunkten hergestellt werden, wird die populare Identitätserzählung in alltagsnahe Szenarien übersetzt sowie zugleich in die kulturellen Symboliken eingeschrieben, sodass diese sie auch abseits des politischen Kontextes evozieren. Diese beiden Stoßrichtungen politisch-kultureller Artikulationen sollen im Folgenden anhand ausgewählter Beispiele aus dem empirischen Material verdeutlicht werden.

\section{Maradona und die kirchneristische Rückeroberung des argentinischen Selbstwerts}

Die Reinszenierung der antagonistischen Konfrontation in ihrer narrativen Verknüpfung mit historisch verankerten Topoi aus dem kulturellen Bedeutungsfeld des Fußballs bringt die populare Gemeinschaft symbolisch auf die Bühne. Die Veranschaulichung des politischen Konflikts in lebensweltlichen Kategorien verleiht der vorgestellten Einheit konkrete Gestalt und macht ihre politischen Ziele, Wertvorstellungen und moralischen Prinzipien alltagsnah verstehbar.

Der Politikwissenschaftler Edgardo Mocca, Moderator der Fernsehshow 6, 7, 8 und damit selbst Teil der regierungsunterstützenden Diskursproduktion, beschreibt im Interview die argentinische WM-Teilnahme 2010 als symbolisches Kampffeld um hegemoniale Deutungsmacht, auf dem von kirchneristischer Seite die Vorstellung propagiert wurde, dass

»[...] dieses Land, das 2001 [...] auf die Höhe seiner Identitätskrise gelangt war, zu seiner Auflösung als politische Gemeinschaft und als politisches Subjekt, sich erholte dank der Ersetzung des Neoliberalismus durch ein national-populares Projekt, das den Selbstwert wiedererlangte, indem es auch das Konsumniveau wiedererlangte, das Niveau der Befriedigung von Rechten, von Forderungen etc. und heute können wir [...] uns als Argentinier wünschen, die WM zu gewinnen, um der Welt zu zeigen, dass wir nicht nur ein großes Land sind, [...] sondern dass wir außerdem den besten Fußball der Welt haben« (Interview Mocca, 15.5.2012). 
Die Aussage resümiert prägnant die kirchneristische Erzählung der recuperación, ${ }^{1}$ die in der narrativen Konfiguration der WM-Debatten das zentrale politisch-kulturell artikulierte Motiv darstellt. Dass die verlorene Größe der argentinischen $\mathrm{Na}$ tion durch die anti-neoliberale Wende des Kirchnerismus nach dem Finanzkollaps von 2001 zurückgewonnen wurde, wird im Bild der (erhofften) erfolgreichen WMPerformance ausgedrückt, weil auch die Überlegenheit des argentinischen Fußballs Teil einer idealisierten Vergangenheit ist, die mit dem Abschied des Ausnahmespielers Maradona in den (politisch neoliberal bestimmten) 1990er Jahren endgültig verloren ging (Alabarces/Rodríguez 2000: 124-129). Mit seiner Rückkehr als Nationaltrainer bei der WM 2010 scheint die Möglichkeit des Anschlusses an ein imaginiertes Goldenes Zeitalter der Vergangenheit wieder in greifbarer Nähe (Alabarces 2010: 197f.). "Dass wir ein großes Land sind und den besten Fußball haben« kondensiert den wirtschaftlichen Aufschwung, die Überwindung der politischen Anomie und den kulturellen Wandel hin zu einer Aufwertung des Eigenen seit dem Regierungsantritt Néstor Kirchners 2003 als weitere Bestandteile der kirchneristischen recuperación. Die WM-Inszenierung symbolisiert so die »Wiedergewinnung des argentinischen Selbstwerts«, die sich der Kirchnerismus mit seinem national-popularen Kurs auf seine Fahnen geschrieben hat und die von Mocca als dahingehender Bewusstseinswandel beschrieben wird, dass

»Argentinien, das sich selbst als ein Land in absolutem Verfall dachte, [...] versucht, diese Perspektive durch einen Perspektive der Selbstaufwertung unserer Ressourcen, unserer Geschichte, selbst unserer Tragödien zu ersetzen« (Interview Mocca, 15.5.2012).

Die Figur Maradonas bildet das Zentrum der politisch-kulturellen Artikulationen im regierungsnahen WM-Diskurs 2010, das die sinnstiftende Strukturierung des sozialen Raumes organisiert und den innenpolitischen Antagonismus über die Konfliktachse des argentinischen Selbstwerts in die Debatten um den sportlichen Bewerb trägt. Maradona steht für die Verteidigung einer ambivalent konnotierten national-popularen Argentinität. Durch seine Politisierung als Repräsentant der authentischen Nation wird die Bewertung seiner Trainerschaft zum Scheidepunkt, der die antagonistische Lagerbildung zwischen Kirchnerismus und Anti-Kirchnerismus reproduziert und diese als konträre Haltungen zum eigenen politisch-kulturellen Erbe inszeniert. Auch wenn Maradona selbst parteipolitisch nicht $\mathrm{zu}$ vereinnahmen ist, bestätigt der kirchneristische Funktionär Sebastián Etchemendy aus seiner Alltagserfahrung:

»Maradona fügt sich in die ideologische Spaltung ein, in einer Weise, dass die eher national-popularen Schichten ihn verteidigen, wir verteidigen ihn. Und die eher

1 Der Begriff trägt den Bedeutungsgehalt der Erholung, Wiedergewinnung sowie Rückeroberung. 
mittelständischen, gehoben mittelständischen Schichten greifen ihn an « (Interview Etchemendy, 4.9.2012).

Seine Präsenz als Teamchef macht die WM $2010 \mathrm{zu}$ einem Teil des »kulturellen Kampfes « ${ }^{2}$ gegen die anti-popularen Sektoren um die hegemoniale Definition des argentinischen »Volkes« und seines legitimen Nationalcharakters.

Die Inszenierung eines Kampfes zwischen einer minderbewerteten popularen Kultur und einem von Verachtung für das Populare geprägten antagonistischen Außen, dem Überidentifikation mit Europa und die Disqualifizierung des autochthon Argentinischen vorgeworfen wird, dient dazu, die politische Polarisierung der argentinischen Gesellschaft seit den intensiven Protesten gegen die geplante Anhebung der Agrarexportsteuern im Jahr 2008 in kulturellen Begriffen als Konflikt zwischen einem "popularen« Kirchnerismus und einem »elitären« AntiKirchnerismus durchzuspielen. Sie beschränkt sich allerdings nicht darauf, den politischen Antagonismus in andere Erfahrungszusammenhänge zu tragen, sondern vermittelt darüber hinaus ein Modell des »Popularen«, das dem »national-popularen Charakter « mithilfe fußballerischer Repräsentationen inhaltliche Substanz verleiht und die Kernkategorie der kirchneristischen Identitätskonstruktion mit positiv bestimmtem Bedeutungsgehalt füllt. Zentraler Bestandteil der symbolischen Produktivität Maradonas für das national-populare Narrativ ist seine fehlbare und widersprüchliche Persönlichkeit (Archetti 2008: 270-277). Der Schriftsteller Eduardo Galeano beschreibt den argentinischen Nationaltrainer in einem Zeitungsinterview im Vorfeld der Weltmeisterschaft folgendermaßen:

»Er ist der menschlichste der Cötter, weil er wie ein jeder von uns ist. [...] Er ist kein Cott, der uns vom Himmel herab seine Reinheit zeigt und uns straft. [...] Das erklärt sein Ansehen. Wir erkennen uns in ihm, in seinen Tugenden, aber auch in seinen Fehlern«(Galeano, Página/12, 7.6.2010).

Maradona ist ein Idol mit Makeln, dessen identifikatorische Besetzung eine Rehabilitation der negativ konnotierten argentinischen Autostereotype ermöglicht. Mit seiner wechselhaften Lebensgeschichte, seinen Skandalen und seinem exzessiven Charakter vermittelt er das Motiv der Gleichzeitigkeit von Genie und Scheitern, das auf den argentinischen Identitätsdiskurs übertragen dem Selbstbild als »Land der Extreme« entspricht. Der Verlauf der eigenen Geschichte wird als quasi schicksalshafte Pendelbewegung zwischen Aufstieg und Fall gezeichnet, die das Land trotz seiner verheißungsvollen Voraussetzungen immer wieder in die Katastrophe

2 Der Begriff der »batalla cultural«steht für den kirchneristischen Kampf um den Common sense im Sinne einer Wiedergewinnung des popularen Selbstwerts. Staatspräsidentin Cristina Fernández de Kirchner selbst bezog sich in ihren Reden häufig darauf, z.B. http://edant.clarin.com/ diario/2008/04/16/elpais/p-00502.htm oder www.pagina12.com.ar/diario/economia/2-1958362012-06-07.html. 
schlittern lasse (Armony/Kessler 2004: 91f.), und mit der Widersprüchlichkeit des nationalen Charakters erklärt, dessen paradigmatische Verkörperung Maradona darstellt.

Das »Tor gegen die Engländer« per Handspiel bei der Fußballweltmeisterschaft 1986 in Mexiko spielt aufgrund seiner Bedeutung als symbolische Revanche für die Niederlage im Krieg um die Malvinasinseln 1982 eine wichtige Rolle im nationalen Mythenhaushalt und wurde zum Synonym für die als kulturell typisch erachtete viveza criolla (»kreolische Gerissenheit«) der Argentinierinnen und Argentinier, die im Wesentlichen die Subversion herrschender Normen durch gewitzten Schwindel und kreatives Talent meint. Maradonas Performance inszeniert die ambivalent besetzte viveza criolla als moralisch legitime Waffe der Subalternen im Kampf gegen den überlegenen Feind und verleiht ihr widerständige Bedeutung im Sinne einer popularen Transgression (Alabarces 2010: 173-176). Damit ist er

»ein Held für einen Gutteil der Bevölkerung, aber [ein Held] einer bestimmten Argentinität, einer national-popularen, auch das Schlechteste der Argentinität. Das heißt, der gewitzte Betrüger, der ein Tor mit der Hand macht, er repräsentiert all das« (Interview Etchemendy, 4.9.2012).

Maradonas symbolische Konstruktion als Repräsentant »des Besten und des Schlechtesten« der Argentinität macht ihn zu einem Träger der politisch-kulturellen Bedeutungen des Popularen, mit denen die antagonistische Auseinandersetzung überformt ist. In seiner Figur kann die Widersprüchlichkeit der national-popularen Argentinität ins Anerkennenswerte gewendet werden: Indem die "typisch argentinische« Undiszipliniertheit $\mathrm{zu}$ rebellischer Unangepasstheit umgedeutet wird, wird auch das Scheitern $\mathrm{zu}$ einem positiv konnotierten popularen Wert. Dementsprechend attestiert der Leiter der argentinischen Nationalbibliothek Horacio González fußballerischen Heldenfiguren populare Repräsentativität »vor allem, wenn sie gescheitert und schon zurückgetreten sind, und alles verloren haben, nachdem sie den Ruhm hatten. Das sind Figuren des Popularen« (Interview González, 6.9.2012). Ricardo Forster, Gründungsfigur des kirchneristischen Intellektuellenzirkels Carta Abierta, vergleicht Maradona indes mit der brasilianischen Spielerlegende Mané Garrincha, der »spielte wie er lebte, frei und gleichzeitig verprassend, was er verdient hatte, [und] in Armut starb« (Interview Forster, 8.5.2012). Die Unfähigkeit zu einem maßvollen Leben und das »Hängenbleiben« in der sozialen Herkunftsklasse werden als Treue zu den eigenen Wurzeln positiv umgedeutet und als populare Qualitäten romantisch überhöht. Maradonas Festhalten an seinen einfachen Ursprüngen wird insbesondere in seinem Geburtsviertel Villa Fiorito als Topos einer affektiv verbundenen Solidargemeinschaft auf Basis kommunitärer Werte ausgedrückt und mit dem rational und pragmatisch geprägten Wertehorizont der modernisierungsorientierten und individualisierten Mittelschichten kontrastiert. 
In den Erzählungen der regierungsunterstützenden Vertreter geht die $\mathrm{Zu-}$ schreibung der Herkunftsloyalität mit gleichförmigen Charakterisierungen Maradonas als personifiziertem Widerspruch gegen die »Mächtigen« einher, die ihn als Symbol des populistischen Bruchs mit dem Status quo konstruieren. Der Journalist Hernán Brienza bezeichnet Maradona als "Anti-System-Symbol« und führt aus: "Auch wenn er keine revolutionäre diskursive Konstruktion hat, ist seine symbolische Aufladung an sich revulsiv in dem Sinn, dass er das Etablierte durcheinanderbringt« (Interview Brienza, 10.9.2012). Die Verfolgung Maradonas durch das »Establishment « untermauert im Anschluss daran seine Rolle als Repräsentant des verachteten Popularen.

»Die Rechten mögen Diego nicht besonders. Sie sagen, er ist ein ungebildeter negro. Einige sagen, seinetwegen halten sie uns Argentinier in der Welt für Drogensüchtige. Er ist eine widersprüchliche Persönlichkeit« (Interview Ortiz, 3.11.2011),

erklärt ein Aktivist der kirchneristischen Jugendorganisation La Cámpora, und folgert daraus:

»Die Sache ist die, dass Diego die Stimme derer ohne Stimme ist. Diego ist die Stimme des Volkes, das keinen Zugang zum Sprechen hat. Er ist der, der sagt, was er will, weil er Diego ist. [...] Ich glaube, dass Diego die argentinische Ikone ist, mehr als sonst irgendjemand. Ich würde sagen, dass Perón auf dieser Höhe ist« (ebd.).

Maradona wird über seine erlittene Abwertung durch den Elitendiskurs zum Sprecher der Subalternen stilisiert, der unverfroren gegen die von den Mächtigen vorgesehene Unterordnung der »einfachen Leute« rebelliert, und mit dem ehemaligen Staatspräsidenten Juan Domingo Perón assoziiert, dessen politische Bewegung in der Folge ebenfalls als Inbegriff der nunmehr transgressiv konnotierten »Argentinität« erscheint. Diese Form der diskursiven Verknüpfung, die sich in den untersuchten Narrationen wiederkehrend findet, dient der Inszenierung des Kirchnerismus als authentischem Vertreter eines orthodoxen Peronismus. Maradona hatte sich im Vorfeld der WM 2010 offen unterstützend für die Regierung ausgesprochen und diese auch in der Streitfrage der Fußballübertragungsrechte unterstützt, deren Verstaatlichung die oppositionelle Mediengruppe Clarín ökonomisch schwächen sollte. Angesichts der internen Fragmentierung des Peronismus in einen kirchneristischen und einen »dissidenten« Flügel ist es für die regierungsnahen Diskursproduzentinnen und Diskursproduzenten wichtig, Maradona nicht auf eine kirchneristische Ikone zu reduzieren oder als reine Marionette der Regierung darzustellen. Página/12-Kommentator Luis Bruschtein präzisiert daher, Maradona sei 
»mehr als Kirchnerist, Peronist. Auch wenn er das nie gesagt hat und immer den Eindruck erweckt hat, dass er sich eher mit irgendeiner Form der Linken identifiziert, machen ihn seine Stimmungsschwankungen, sein explosives Verhalten und Maradonas politische Inkorrektheit dem Peronismus ähnlicher« (Bruschtein, Pági$n a / 12,31.7 .2010)$.

In der Figur Maradonas konvergieren politische und kulturelle Bedeutungen, die ihn als metaphorischen Ausdruck des peronistischen Wertehorizonts funktionieren lassen. Seine Berufung zum Teamchef der selección nach Jahren der Drogenabhängigkeit, der persönlichen Krisen und des körperlichen Verfalls ist Symbol für den »Triumph des Popularen«, das erneut als hegemoniale Repräsentation des $\mathrm{Na-}$ tionalen eingesetzt wird. Die Rückkehr des vom Schicksal gebeutelten underdog an die Spitze der (Fußball-)Nation übersetzt in einer symbolischen Inszenierung die kirchneristische Erzählung der recuperación und veranschaulicht die politische Erfahrung des Kirchnerismus als Erneuerung des klassisch peronistischen Versprechens auf Inklusion der popularen Schichten. Der Journalist Eduardo Anguita beschreibt dies so:

»Maradona [...] hat etwas Außergewöhnliches in der menschlichen Verfasstheit, dass er ein Typ ist, der es schaffte, ganz unten zu sein, weil er vom Drogenkonsum kaputtgemacht wurde, weil er sich mit seiner Familie stritt, [...] und er erholte sich [se recuperó] auf eine außergewöhnliche Weise. Daher ist das, dass Maradona es schaffte, als Trainer des Nationalteams an der Spitze zu sein, ich glaube, im sozialen Spiegel, im sozialen Imaginären heißt das für den Armen, für den einfachsten Arbeiter: >Wenn Maradona konnte, wie werde ich nicht können? Wenn mein Cott konnte« (Interview Anguita, 8.6.2012).

Der »menschliche und fehlbare Gott« Maradona, der dennoch nach jedem seiner persönlichen und sportlichen Rückschläge wieder »auferstanden« ist, ermöglicht die Hoffnung auf Ermächtigung der Marginalisierten, weil das populare Kollektiv sich in ihm wiedererkennen und an seinen topischen Bedeutungen teilhaben kann. In einer extrem ungleichen Gesellschaft ohne systemische Möglichkeiten sozialer Mobilität ist Maradona als "cabecita negra, der in der Welt triumphiert (Interview Ortiz, 3.11.2011) $)^{3}$ zumindest ein Beweis für die Handlungsspielräume, in denen die scheinbar unabänderlichen Hierarchien durchkreuzt werden können. Damit funktioniert er in einem übertragenen Sinn auch als Symbol für den historischen Bruch

3 »Cabecita negra« ist ein Schimpfwort für Angehörige der popularen Schichten. Der Begriff ist klassistisch konnotiert, wenngleich er mit seiner Anspielung auf die dunkle Hautfarbe auch eine rassistische Komponente enthält, und zielt in einem breiteren Sinn auf die Bezeichnung einer minderwertig vorgestellten »popularen Kultur«. Seine Verwendung hat weiter eine antiperonistische Konnotation, da er historisch als abwertende Bezeichnung für die Anhängerinnen und Anhänger des Peronismus verwendet wurde. 
mit der neoliberalen Ideologie der Alternativlosigkeit nach der Finanz- und Wirtschaftskrise von 2001/2002 und für die national-populare Politik der Selbstbestimmung, die der Kirchnerismus für sich beansprucht.

»Nachdem er die irredente Leidenschaft ausdrückt, ist Maradona im Fußball letztlich Kirchner in der Politik«, heißt es in Página/12 (Bruschtein, Página/12, 31.7.2010). Maradonas charakterliche Attribute entsprechen dem »kulturellen Wesen « und dem konfrontativen Politikstil des Kirchnerismus, folglich werden beide auch aus denselben Gründen von den oppositionellen Kräften abgelehnt: »Die schlimmsten Attribute, die ihnen die Opposition anhängt, und die Wunder, die ihnen die oficialistas [Vertreterinnen und Vertreter des Regierungsblocks, Anm.] zuschreiben, fließen im Kirchnerismus und in Maradona zusammen" (ebd.). Die Artikulation Maradonas mit der Regierung in der WM-Erzählung der pro-kirchneristischen Medienlandschaft schafft analoge Konfigurationen in der politischen und in der sportlichen Debatte. Dadurch werden erstens die Positionen auch in der antagonistischen Konstellation klar verteilt: Über die gemeinsame Mentalitätsprägung wird der Kirchnerismus trotz der zum damaligen Zeitpunkt knapp siebenjährigen Regierungsverantwortung nicht mit der Macht identifiziert, sondern ist nun ebenso wie Maradona auf der Seite des Volkes und mit den »wirklich mächtigen« Gruppen des Landes konfrontiert. Als diese werden die sogenannten »hegemonialen Medien« identifiziert, die nicht nur während der Weltmeisterschaft als pars pro toto für das anti-kirchneristische Lager eingesetzt werden. Ricardo Forster spricht in einer 6, 7, 8-Diskussion von

»einem gewissen argentinischen Journalismus, der ihn [Maradona, Anm.] degradierte, erniedrigte, die Niederlage Maradonas suchte, und gemeinsam mit der Niederlage Maradonas die Niederlage des Nationalteams und [...] natürlich die des Mediengesetzes, der Regierung, alles in einen Topf geworfen« $(6,7,8$, 20.6.2010b: 1:04-1:25).

Der Analogieschluss zwischen dem "popularen Helden« Maradona und der kirchneristischen Regierung, der von der gemeinsamen Verfolgung durch den antagonistischen Feind bestätigt wird, führt zweitens dazu, dass das Konfliktmotiv in beiden Konstellationen ident erscheint. Pablo Llonto, Chefredakteur der Fußballzeitschrift Un Caño, beschreibt anlässlich der WM 2010 den anti-popularen Idealtypus des 21. Jahrhunderts anhand elitärer Freizeitpraktiken und Werthaltungen und schließt: »Kurz gesagt, er hasst die Regierung, er hasst die piqueteros, ${ }^{4}$ er hasst Chávez und er hasst Maradona (Llonto, Un Caño, Juni 2010). Kritik an der Regierungspolitik wird ebenso wie die Kritik an Maradona systematisch mit einer allgemeinen

4 Organisationen der Arbeitslosenbewegung, die ausgehend von einem Aufstand in der Provinz Neuquén im Jahr 1996 Straßensperren (piquetes) als neuartiges Mobilisierungsformat verbreiteten und zentrale Protagonisten der Proteste von 2001 waren. 
Verachtung des "popularen Charakters« in Bezug gesetzt. Damit erscheint erstere nun ebenso wie letztere als ungerechtfertigtes Ressentiment einer elitären Oberund Mittelschicht und Folge ihres Alterzentrismus, der »das Europäische« als Norm setzt und die eigene periphere Herkunft demgegenüber als unterentwickelt und minderwertig betrachtet. Dies hat zur Folge, dass der Kirchnerismus im Umkehrschluss als die politische Kraft herausgestellt wird, welche die Widersprüchlichkeit des Argentinischen affirmiert und in der Verteidigung der lateinamerikanischen Wurzeln und des kreolischen Erbes die populare Würde wiederherstellt.

\section{Die metaphorische Vervielfachung des antagonistischen Konfliktszenarios}

Neben der popularkulturellen Veranschaulichung der antagonistischen Konfliktkonstellation dient die diskursive Reartikulation kultureller Topoi in ihrer umgekehrten Stoßrichtung der antagonistischen Aufladung der symbolischen Motive selbst, die im Prozess der Narrativisierung von der Logik der politischen Polarisierung affiziert werden und nun auch in ihrer isolierten Verwendung den Sinngehalt der übergreifenden innenpolitischen Konfliktlinien und ihrer zugehörigen Diskursmuster transportieren. Die topischen Bedeutungsträger werden durch ihre Einschreibung in die populistische Rhetorik zu Elementen der dichotomen Teilung und fungieren als Ausdruck der gesamten narrativen Struktur der populistischen Konstruktion: werden sie in medialen oder Alltagsdiskursen eingesetzt, rufen sie alle anderen Aspekte ihres diskursiven Umfelds mit auf und aktualisieren das Gesamtbild der narrativen Konfiguration.

In der kirchneristischen Diskussionssendung 6, 7, 8 verortet Gabriel Mariotto, Leiter der 2009 im Zuge des umstrittenen Mediengesetzes geschaffenen staatlichen Medienregulationsbehörde AFSCA, den Kampf gegen die sogenannten hegemonialen Medien auch in der "popularen Kultur« und nennt als Beispiel

»[...] diese rebellische Haltung, die Diego hat, der darauf hinwies, als sie ihm das Nationalteam exzessiv kritisierten, dass sie das Ziel verfolgten, dass der Fußball zu Torneos y Competencias zurückkehrt und dass er nicht mehr für alle ist « $(6,7,8$, 15.6.2010: 6:14-7:04).

Mariotto zufolge entlarvt Maradona die Kritik an ihm und seinen Spielentscheidungen richtigerweise als versteckte Kritik an Fútbol para Todos. Die Verstaatlichung der Fußballübertragungslizenzen wurde von Regierungsseite wie erwähnt als Vorzeigeprojekt ihrer anti-neoliberalen Inklusionspolitik präsentiert. Kritik an Maradona ist damit in dieser Lesart eigentlich Kritik an der Politik der popularen Ermächtigung. Bemerkenswert ist nun die Schlussfolgerung, die Mariotto im folgenden Satz daraus zieht: »Deswegen hat er [Maradona, Anm.] diese Entgleisung 
begangen, die er begangen hat, Produkt der Sensibilität und der Rebellionsfähigkeit, die die populare Kultur besitzt« (ebd.: 6:14-7:04). Gemeint ist ein Vorfall bei der Pressekonferenz nach dem Vorrundenspiel gegen Uruguay im Oktober 2009. Argentinien hatte sich mit diesem Match in letzter Sekunde für die WM-Teilnahme qualifiziert. Maradona reagierte bei der Pressekonferenz auf die dementsprechend vorangegangene Kritik an der mangelhaften Performance des Nationalteams, indem er die anwesenden Journalistinnen und Journalisten und speziell Toti Pasman von der Mediengruppe Clarín, der eine Frage an ihn richtete, als "Schwanzlutscher « beschimpfte. ${ }^{5}$ Die aufsehenerregende Episode, die Maradona eine zweimonatige Suspendierung von der Trainerschaft eintrug, wird hier politisch aufgeladen und $\mathrm{zu}$ einem Statement gegen die »hegemonialen Medien« erklärt. Der »popularen Kultur« wird »Rebellionsfähigkeit« attestiert, sie ist nunmehr, ob sie sich in schwulenfeindlichen Beschimpfungen oder in schlechten Englischkenntnissen manifestiert, ${ }^{6}$ per se transgressiv und pro-kirchneristisch konnotiert.

Selbst eine schlicht homophobe Beleidigung verweist nun auf den Antagonismus zwischen »Volk« und »Machtblock«, entsprechend wird ihre ablehnende oder anerkennende Bewertung in der pro-kirchneristischen Medienlandschaft zu einem Gradmesser für die Positionierung im Rahmen der innenpolitischen Auseinandersetzungen gemacht und mit einer popularen bzw. anti-popularen Haltung parallelisiert. Der bereits zitierte Pablo Llonto schlägt in diese Kerbe, wenn er in seiner Beschreibung des anti-popularen Idealtypus vermutet, dass dieser "gekränkt war, und nicht wenig, als Diego präzise erwähnte, wo der Journalist Pasman seine Lippen hinwenden solle« (Llonto, Un Caño, Juni 2010), und im Anschluss diverse Oppositionspolitikerinnen und Oppositionspolitiker des Hoffens auf eine WMNiederlage bezichtigt, weil sie diese zur Stimmungsmache gegen Maradona einsetzen und auf dessen Verfasstheit als »negro villero « ${ }^{7}$ zurückführen wollten. Popular ist, wer Maradonas Ausfälle gegen das mediale Establishment solidarisch unterstützt, anti-popular dagegen, wer ihn wegen seiner verbalen Entgleisungen

5 Die genaue Aussage, die legendär wurde und auch in den für diese Studie durchgeführten Interviews anerkennend zitiert wurde, lautete: »Vos también Pasman, vos también la tenés adentro«. (»La«, weil der Slangbegriff für Penis - den Maradona Pasman zuschrieb, »drinnen« zu haben im Argentinischen weiblich ist.)

6 Carlos Tévez ist einer der wenigen Protagonisten der WM 2010 abseits von Maradona, an dessen Figur das populare Herkunftsnarrativ in Szene gesetzt wird. Seine habituelle Verwurzelung in den sozialen Bezügen seines bescheidenen Ursprungsmilieus wird dabei unter anderem in der anerkennenden Darstellung seiner rudimentären Englischkenntnisse bei einer Pressekonferenz veranschaulicht (6, 7, 8, 20.6.2010a: 40:39-41:54).

7 Villero ist die Bezeichnung für den Bewohner einer villa miseria, also eines Elendsviertels. »Negro« wird im argentinischen Alltagsdiskurs der politischen Rechten als Schimpfwort für dieselbe Personengruppe verwendet. Die Bezeichnung bezieht sich nicht unbedingt auf die ethnische Zugehörigkeit, sondern die soziale Schicht und transportiert implizit erhöhte Delinquenz und soziales »Schmarotzertum«. 
einer repräsentativen Funktion unwürdig befindet. In beiden Fällen steht hinter der jeweiligen Positionierung ein bestimmtes Verhältnis zur "popularen Kultur« im Allgemeinen, welches analog zur Verortung innerhalb der dichotomen politischen Bruchlinie gesetzt wird.

Wurde einerseits der politische Konflikt über die Verknüpfung mit popularkulturellen Topoi in eine Auseinandersetzung zwischen einem als rückständig und defizitär verachteten »Volk« und einem elitistischen und europaorientierten Machtblock erweitert, sind nun umgekehrt alle weiteren Indizien dieses kulturellen Konflikts austauschbare Episoden der immergleichen antagonistischen Konfrontation zwischen Kirchnerismus und Anti-Kirchnerismus bzw. popularer Nation und anti-pueblo und bestätigen deren Existenz permanent. Dazu ein abschließendes Beispiel aus der WM-Berichterstattung: Der argentinische Stürmerstar Carlos Tévez wird in einem Interview daraufhin befragt, wie er angesichts seiner eigenen Hässlichkeit $\mathrm{zu}$ einer so attraktiven Freundin kommen konnte. Tévez kontert schlagfertig und entschärft damit das blamable Potential der Situation, was in der 6, 7, 8-Diskussionsrunde nach Einspielung des Videoclips euphorisch beklatscht und unmittelbar auf die politische Situation umgemünzt wird. Der Vorfall lasse hoffnungsvoll erkennen, »dass die Gesellschaft gelernt hat, sich gegen die schlechten Journalisten zu verteidigen «, andererseits bestätige er erneut die Verfolgung all derer, die auch nur entfernt Nähe zur Regierung zeigten (6, 7, 8, 15.6.2010: 15:50-19:35). Der oben erwähnte Studiogast Gabriel Mariotto liest daraus eine allgemeine Tendenz ab:

»Es gibt eine Art Verachtung für die populare Kultur. Jegliche Manifestation der popularen Kultur - und die Spieler sind ein vollständiger Ausdruck dieser Manifestation - wird immer vom ästhetischen Vorurteil einer Schicht beurteilt. Also erscheint ein Zusammentreffen zweier Kulturen. Ein genuiner Ausdruck, der rational und sensibel argumentiert, gegenüber einem Stereotyp und einem Wertvorurteil einer sozialen Schicht« (ebd.: 15:50-19:35).

Die kulturelle Überformung der populistischen Spaltungslinie restrukturiert den Antagonismus zu einer Auseinandersetzung zwischen einem authentischen popularen Kollektiv, das die negativen Autostereotype stolz affirmiert, und einer ressentimentgetriebenen Mittel- und Oberschicht, die das genuin Argentinische ablehnt. Die widerstreitenden politischen Positionen werden in ambivalenzfreie Klischeebilder gegossen, die dem Konflikt seine unmittelbare Evidenz verleihen und ihn in eine Konfrontation zweier homogener soziokultureller Gesellschaftsblöcke mit konträren nationalen Selbstbildern übersetzen. Sämtliche Polemiken des WMZeitraums, von Maradonas Auftreten in Jogginghose bis hin zur Ausweisung argentinischer Fußballdelinquenten aus Südafrika, werden in derselben Konfliktlogik konfiguriert und stellen Nebenschauplätze eines größeren Kampfes um die Rehabilitierung des Popularen als legitimer Repräsentation des Nationalen dar. 
Der Konflikt zwischen Kirchnerismus und Anti-Kirchnerismus erscheint damit als Kampf, der sich nicht nur im politischen Feld ereignet, sondern permanent überall präsent und den politischen Oppositionen vorgängig ist.

\section{Schlusswort}

Die vorangegangenen Ausführungen zeigen, dass populare Subjektivitäten in populistischen Konstruktionsmodi nicht einfach durch ihre Kristallisierung in einem hegemonialen Repräsentanten konsolidiert werden. Die analytische Berücksichtigung der Reartikulation historisch wirkmächtiger Symbolrepertoires in populistischen Schließungsprozessen kann das statische Modell des leeren Signifikanten dynamisieren. Dieser stellt kein singuläres Element dar, das die Identität der popularen Einheit performativ aus sich selbst heraus generiert. Er ist vielmehr als hegemoniales Wirkprinzip zu verstehen, das in der artikulatorischen Praxis auf die beständige Mobilisierung vielfältiger privilegierter Knotenpunkte aus unterschiedlichen Bedeutungszusammenhängen angewiesen ist, um die metaphorische Reichweite seiner identifikatorischen Funktion zu erhöhen. Dazu wird der antagonistische Bruch in immer neue soziale Kontexte getragen und dort mit feldspezifischen Ausdrucksmitteln reproduziert. Dies meint keine einfache Wiederholung, sondern eine »Vervielfachung« des Konfliktszenarios, die den Antagonismus mit den im jeweiligen Erfahrungsbereich verfügbaren kollektiven Vorstellungsbildern des sozialen Imaginären reinszeniert und ihn dadurch erneut entstehen lässt. Die politische Frontenbildung wird quasi in unterschiedlichen Räumen in deren respektiver symbolisch-kulturellen Sprache mehrfach ausgedrückt. So entsteht das Bild einer Gesellschaft, in der sich auf allen Ebenen ständig dieselbe Problematik ereignet.

Die politischen Wirkfolgen dieser symbolischen Inszenierungen gehen über die momentane Veranschaulichung der populistischen Identitätserzählung nicht hinaus. Der ephemere Charakter der politisch-kulturellen Artikulationen tut ihrem identitätsstabilisierenden Potential allerdings keinen Abbruch, da ihr Effekt gerade auf der metaphorischen Übertragbarkeit mannigfacher ähnlich gearteter Konfliktkonstellationen in verschiedenartigen Kontexten beruht. Diese scheinen sich wechselseitig zu entsprechen und schaffen in ihrem Zusammenspiel ein diskursives Netz von Repräsentationen, das sich flächendeckend durch die gesellschaftliche Debatte zieht und von vielfältigen Stützpfeilern getragen wird. Wird einer dieser Einzelkonflikte thematisiert, wird aufgrund der konnotativen Resonanzen zwischen dem populistischen Projekt und den artikulierten kulturellen Symboliken immer auch das ganze Bild aktualisiert. Die Vielfalt und Komplementarität der antagonistischen Szenarien schafft ein Räderwerk "popularer Bilder«, das den kulturellen Konflikt um die Wiedergewinnung des argentinischen Selbstwerts pri- 
mordial erscheinen lässt und die Bruchlinie zwischen Kirchnerismus und AntiKirchnerismus unhintergehbar macht.

Staatspräsidentin Cristina Fernández de Kirchner hatte schon in ihrer Rede zum Amtsantritt vor dem Kongress Ende 2007 »eine andere Erzählung von uns selbst« proklamiert, welche »die notwendige Anerkennung des Erreichten« ermögliche (Fernández de Kirchner 2007). Die diskursive Einschreibung der innenpolitischen Auseinandersetzungen in eine historische Matrix popularer Kämpfe und staatliche Interventionen wie etwa das Programm Fútbol para Todos bildeten offizielle Hinweisreize von Regierungsseite zur narrativen Strukturierung des kirchneristischen Zyklus als national-popularer Renaissance und Möglichkeitsbedingung eines erneuerten Selbstwerts. Die offiziell lancierte Zielsetzung, »die Erzählung zu konstruieren« (»construir el relato«), wurde aber nicht allein »von oben« durchgesetzt, sie fand Unterstützung bei vielfältigen Akteurinnen und Akteuren im Medien-, Kunst- und Unterhaltungsbereich und in intellektuellen und jugendlichen Milieus der progressiven Mittelschichten, die im Zuge der polarisierten Debatten um die Agrarexportsteuern 2008 und das darauffolgende Mediengesetz 2009 mobilisiert wurden und als Aktivistinnen und Aktivisten im »kulturellen Kampf « die politischen Botschaften in alltagsnahe Narrationen übersetzten (Kitzberger 2011; Novaro 2011). Die sogenannte batalla cultural des Kirchnerismus wurde »nicht vom Ideologiebüro der Exekutive«, sondern von einem »kirchneristischen kulturellen Dispositiv« getragen (Sarlo 2013: 30, Herv. i. O.), das eine dezentral organisierte und institutionell schwach formalisierte kulturelle Infrastruktur zur Verbreitung konvergenter national-popularer Inhalte bildete. Die symbolische Inszenierung des Popularen im regierungsunterstützenden Mediendiskurs zur Fußballweltmeisterschaft 2010 stellte dabei einen Baustein unter vielen dar, dessen öffentliche Resonanz angesichts des frühen Ausscheidens der argentinischen Nationalmannschaft beschränkt blieb. Die politische Aufladung des Fußballs als Kampffeld in einem innenpolitischen Konflikt bietet gleichwohl einen exemplarischen Fall politisch-kultureller Verstetigungsprozesse in der Konsolidierungsphase populistischer Politikmodelle. Dieser macht anschaulich, wie die Verankerung der kirchneristischen Identitätserzählung in historisch wirkmächtigen kulturellen Topoi dazu beiträgt, die diskursive Konstruktion der popularen Einheit als legitime Repräsentation des Nationalen zu etablieren und gleichzeitig in der narrativen Restrukturierung der politischen Konfliktlinien den antisystemischen und "subalternen« Charakter eines populistischen Modells »an der Macht« zu wahren. 


\section{Literatur}

Aboy Carlés, Gerardo 2005: Populismo y democracia en la Argentina contemporánea. Entre el hegemonismo y la refundación In: Estudios Sociales, Heft 28, S. 125-149.

Alabarces, Pablo 2010: Für Messi sterben? Der Fußball und die Erfindung der argentinischen Nation, Frankfurt a.M.

Alabarces, Pablo/Rodríguez, María Graciela 2000: Football and Fatherland. The Crisis of National Representation in Argentinian Soccer. In: Gerry Finn/Richard Giulianotti (Hg.), Football Culture. Local Contests, Global Visions, London/Portland, S. 118-133.

Archetti, Eduardo 2008: El potrero y el pibe. Territorio y pertencencia en el imaginario del fútbol argentino. In: Horizontes Antropológicos, Heft 14(30), S. 259-282.

Armony, Victor/Kessler, Gabriel 2004: Imágenes de una sociedad en crisis. Cuestión social, pobreza y desempleo. In: Marcos Novaro/Vicente Palermo (Hg.), Argentina en democracia. Buenos Aires, S. 91-113.

Bornscheuer, Lothar 1976: Topik. Zur Struktur der gesellschaftlichen Einbildungskraft, Frankfurt a.M.

Fernández de Kirchner, Cristina 2007: Discurso de la Presidenta de la Nación, Cristina Fernández de Kirchner, en el acto de asunción del mando en el Congreso de la Nación ante la Asamblea Legislativa, 10.12.2007.

www.casarosada.gob.ar/informacion/archivo/16462-blank-35472369

Goldberger, Bernadette 2018: Populismus an der Macht. Symbolische Inszenierungen im argentinischen Kirchnerismus am Beispiel der Fußball-WM 2010. Weilerswist: Velbrück.

Hall, Stuart 1986: Popular-demokratischer oder autoritärer Populismus. In: Helmut Dubiel (Hg.), Populismus und Aufklärung, Frankfurt a.M., S. 84-105.

Kitzberger, Philip 2011: »La madre de todas las batallas «: el kirchnerismo y los medios de comunicación. In: Andrés Malamud/Miguel De Luca (Hg.), La política en tiempos de los Kirchner, Buenos Aires, S. 179-189.

Knoblauch, Hubert, 2001: Diskurs, Kommunikation und Wissenssoziologie, in: Reiner Keller/Andreas Hirseland/Werner Schneider/Willy Viehöver (Hg.): Handbuch sozialwissenschaftliche Diskursanalyse. Band 1: Theorien und Methoden, Opladen, S. 207-223.

Laclau, Ernesto 1990: New Reflections on the Revolution of Our Time, London/New York.

Laclau, Ernesto 2002: Was haben leere Signifikanten mit Politik zu tun?. In: Ders., Emanzipation und Differenz, Wien, S. 65-78.

Laclau, Ernesto 2005a: On Populist Reason. London: Verso. 
Laclau, Ernesto 2005b: Populism. What's in a Name?. In: Panizza, Francisco (Hg.), Populism and the Mirror of Democracy, London, S. 32-49.

Laclau, Ernesto 2010: Discurso, antagonismo y hegemonía en la construcción de identidades políticas. Conferencia de Ernesto Laclau en la Facultad de Ciencias Sociales de la Universidad de Buenos Aires, 10 de mayo de 2010. In: Alvaro García Linera/Ernesto Laclau/Guillermo O’Donnell, Tres pensamientos políticos: conferencias organizadas por las Falcultades de Ciencias Sociales y de Filosofía y Letras de la UBA, Buenos Aires, S. 41-70.

Laclau, Ernesto/Mouffe, Chantal 2006: Hegemonie und radikale Demokratie. Zur Dekonstruktion des Marxismus, Wien.

Lakoff, George/Johnson, Mark 1998: Leben in Metaphern. Konstruktion und Gebrauch von Sprachbildern, Heidelberg.

Mudde, Cas 2004: The Populist Zeitgeist. In: Government and Opposition, Heft 39(4), S. 541-563.

Müller, Jan-Werner 2016: Was ist Populismus? Ein Essay, Berlin.

Musolff, Andreas 2004: Metaphor and Political Discourse. Analogical Reasoning in Debates about Europe, Basingstoke.

Novaro, Marcos 2011: La cultura política y el sentido común bajo el kirchnerismo. In: Andrés Malamud/Miguel De Luca (Hg.), La política en tiempos de los Kirchner, Buenos Aires, S. 129-140.

Priester, Karin 2012: Rechter und linker Populismus. Annäherung an ein Chamäleon, Frankfurt a.M.

Reckwitz, Andreas 2006: Ernesto Laclau. Diskurse, Hegemonie, Antagonismen. In: Stephan Moebius/Dirk Quadflieg (Hg.), Kultur. Theorien der Gegenwart, Wiesbaden, S. 339-349.

Sarlo, Beatriz 2013: Los intelectuales, la tierra fértil del kirchnerismo. In: Cuadernos de Literatura, Heft 17(33), S. 18-33.

Schwab-Trapp, Michael 2001: Diskurs als soziologisches Konstrukt. Bausteine für eine soziologisch orientierte Diskursanalyse. In: Reiner Keller/Andreas Hirseland/Werner Schneider/Willy Viehöver (Hg.), Handbuch sozialwissenschaftliche Diskursanalyse, Band 1: Theorien und Methoden, Opladen, S. 261-283.

Somers, Margaret R. 1994: The Narrative Constitution of Identity: A Relational and Network Approach. In: Theory and Society, Heft 23(5), S. 605-649.

Taggart, Paul 2000: Populism, Buckingham.

Viehöver, Willy 2012: Öffentliche Erzählungen und der globale Wandel des Klimas. In: Markus Arnold/Gert Dressel/Willy Viehöver (Hg.), Erzählungen im Öffentlichen, Über die Wirkung narrativer Diskurse, Wiesbaden, S. 173-215.

Viehöver, Willy 2014: Erzählungen im Feld der Politik, Politik durch Erzählungen. Überlegungen zur Rolle der Narrationen in den politischen Wissenschaften. In: Frank Gadinger/Sebastian Jarzebski/Taylan Yildiz (Hg.), Politische Narrative, Konzepte - Analysen - Forschungspraxis, Wiesbaden, S. 67-91. 


\section{Print- und TV-Quellen}

Bruschtein, Luis, 2010: Casi mitos, in: Página/12, 31.7. www.pagina12.com.ar/diario/elpais/1-150478-2010-07-31.html

Galeano, Eduardo, 2010: Messi es el mejor del mundo porque sigue jugando como un chiquilín en su barrio. Interview mit Julio Boccalatte und Marcos González. in: Página/12, Suplemento Líbrero, 7.6. www.pagina12.com.ar/diario/ suplementos/libero/10-5288-2010-06-07.html

Gran DT, Clausura 2009: https://www.youtube.com/watch?v=TNfP4TZ3jGk

Llonto, Pablo, 2010: Deben ser los gorilas, deben ser... In: Un Caño, Juni, 32f. https:// issuu.com/revistauncanio/docs/n26

6, 7, 8, 28.7.2010 https://www.youtube.com/watch?v=WbhxXBiMJzA

$6,7,8,15.6 .2010$ https://www.youtube.com/watch?v=pPue9NMOEO4

$6,7,8,20.6 .2010$ a https:/www.youtube.com/watch?v=SebmoerqfRk

$6,7,8,20.6 .2010 b$ https://www.youtube.com/watch?v=2j-mNXZmpzo

\section{Interviews}

Anguita, Eduardo: Herausgeber der kirchneristischen Wochenzeitschrift Miradas al Sur, 8.6.2012.

Brienza, Hernán: Journalist der kirchneristischen Tageszeitung Tiempo Argentino, 10.9.2012.

Etchemendy, Sebastián: Politikwissenschaftler und Regierungsberater, 4.9.2012.

Forster, Ricardo: Initiator des kirchneristischen Intellektuellenzirkels Espacio Carta Abierta, 8.5.2012.

González, Horacio: Leiter der argentinischen Nationalbibliothek, 6.9.2012.

Kovadloff, Santiago: Philosoph, Schriftsteller und Übersetzer; Kolumnist für die konservative Tageszeitung La Nación, 12.6.2012.

Mocca, Edgardo: Politologe, Herausgeber der popular-progressiven Zeitschrift Umbrales de América del Sur und Kolumnist in Página/12, 15.5.2012.

Ortiz Maldonado, Pablo: Leiter des Kultursekretariats der kirchneristischen Jugendorganisation La Cámpora für die Stadt Buenos Aires; 3.11.2011.

Romero, Luis Alberto: Historiker, 15.6.2012. 
Medien und Unterhaltung 



\section{Politische Kultur und Komik Perspektiven für vergleichende Analysen}

Ludgera Vogt

Die politische Kulturforschung hat sich bislang noch nicht eingehend mit den Themen der politischen Komik und des politischen Humors beschäftigt. Das ist erstaunlich, weil zum einen die komische Dimensionierung politischer Kommunikation in den unterhaltungsorientierten Medienkulturen moderner Gegenwartsgesellschaften durchaus relevant erscheint und zum anderen schon beim ersten Hinsehen deutliche Unterschiede zwischen den verschiedenen politischen Kulturen sichtbar sind.

Der folgende Beitrag behandelt die Thematik daher explorativ und speist einige erste Gesichtspunkte in die aktuelle Forschungsdiskussion ein. Die hier entwickelten Überlegungen sind erstmals entstanden im Kontext eines von der Deutschen Forschungsgemeinschaft geförderten empirischen Projekts, das sich mit politischer Komik im Bundestagswahlkampf 2013 beschäftigt hat. Die wichtigsten Ergebnisse dieses Forschungsprojekts sind kürzlich publiziert worden (Dörner/Vogt 2017). Die im Projekt gewonnenen Perspektiven werden im vorliegenden Beitrag mit Überlegungen der qualitativen politischen Kulturforschung verknüpft und anhand konkreter Beispiele zur US-amerikanischen und deutschen Medienkultur verdeutlicht. Im ersten Abschnitt werden einige allgemeine Überlegungen zum Zusammenhang von politischer Komik und politischer Kultur vorgetragen. Der zweite Abschnitt zeigt grundlegende Unterschiede der Verwendung von Komik und Satire in den politischen Kulturen der USA und Deutschlands auf. Der dritte Abschnitt schließlich behandelt Fallbeispiele, die den Unterschied noch einmal konkret deutlich werden lassen.

\section{Politische Kultur und politische Komik}

Politische Kulturen im Sinne der qualitativen, kulturalistisch ausgerichteten Forschung enthalten Grundannahmen über die politische Welt: Elementare Vorstellungen davon, was die politische Welt bewegt, welche individuellen oder kollektiven Akteure entscheidend sind und was normativ jeweils eine sgute Politik 
(vgl. Rohe 1994: 162). In diesem Spektrum sind auch Vorstellungen darüber enthalten, wie politisch richtig kommuniziert wird, was gesagt werden kann und wie es gesagt werden soll. Politische Kulturen definieren das Sagbare in einer Gesellschaft (Dörner 2003). Abweichungen von diesen Normalitätsvorstellungen werden mitunter heftig sanktioniert, d.h. wer erfolgreich kommunizieren will, der muss sich an die Normalitätsvorstellungen halten.

An dieser Stelle kommt nun die komische Modulierung politischer Kommunikation ins Spiel. Ob, in welchem Ausmaß und in welcher Weise komisch kommuniziert werden darf, hängt von den jeweiligen Vorstellungswelten einer politischen Kultur bzw. einer Teilkultur ab. Ist es politischen Akteuren jeweils allgemein erlaubt oder wird von ihnen sogar erwartet, witzig und humorvoll aufzutreten? Haben sich in den politischen Kulturen jeweils bedeutsame kommunikative Institutionen und mediale Formate etabliert, in denen politische Komik einen spezifischen Raum hat? Und welche Formen der politischen Komik, welche Ebenen des politischen Humors werden hier primär gepflegt? Geht es ums Mitlachen oder Verlachen, funktioniert das Lachen eher inkludierend oder exkludierend, wird es eher selbstironisierend zum Distanzabbau eingesetzt oder fremdironisierend, um politische Gegner anzugreifen? Solche Fragen eröffnen den Zugang zur politischen Kultur von Komik und Humor in einer Gesellschaft.

Warum aber kann der komischen Modulierung in der politischen Kommunikation überhaupt ein größerer Stellenwert zukommen? Das wird klar, wenn man einen genaueren Blick auf die möglichen Funktionen von Lachen und Komik in der Politik wirft (siehe dazu auch schon Hoinle 2003). ${ }^{1}$

Das Besondere liegt hier darin, dass die möglichen Effekte teilweise diametral entgegengesetzt sind. Genau hierin begründet sich auch das hohe Risiko, das mit dem Einsatz des Humors als kommunikativem Instrument verbunden ist. So kann das gemeinsame Lachen zunächst einmal gemeinschaftsbildend wirken. Man fühlt sich in der Gruppe wohl, man versichert sich gegenseitig gemeinsamer Werte, Haltungen und Sichtweisen und man kann sich durch das gemeinsame Lachen über Dritte sehr gut nach außen abgrenzen. Inklusion und Exklusion, Zusammenbinden und Ausschließen sind hier jeweils engstens verknüpft. Die Sozialdemokraten können beispielsweise gut über die Konservativen lachen, die Linken über die AfDMitglieder und die Grünen über die Anhänger der FDP.So wie die durch Lachen verbundene Gemeinschaft jeweils die anderen ausschließt, wohnt dem Humor immer auch eine hochgradig aggressive Dimension inne. Mit Witz und Komik lässt sich der jeweilige Gegner im politischen Kampf bloßstellen und klein machen: Gelingt der Witz, nimmt man dem anderen die Stärke und gibt ihn der Lächerlichkeit

$1 \mathrm{Zu}$ den grundlegenden Funktionen von Komik und Lachen siehe Morreal (1983) und Knop (2007: 61), zu politischen Funktionen Hoinle (2003) und Yarwood (2004). 
preis. Nichts kann einen Akteur kommunikativ so sehr treffen wie Hohn und Häme. Schon Sigmund Freud (1905/1986: 116-118) hat daher den Witz als ein Medium des menschlichen Aggressionstriebs herausgestellt.

Diese aggressive Dimension findet sich jedoch nicht nur in der Auseinandersetzung zwischen politischen Kontrahenten, sondern auch in der satirischen Kommentierung von politischen Vorgängen durch mediale Beobachter. Das Spektrum reicht hier vom leicht spöttischen Blick bis zur massiven Verhöhnung, die auch vor der Thematisierung körperlicher Merkmale und charakterlicher Schwächen nicht Halt macht. Eine solche Degradationskomik, wie es die Humortheoretiker nennen, kann durchaus gesellschaftliche Folgen zeitigen. Dies hat der amerikanische Kommunikationswissenschaftler Russell Peterson (2008) in seiner Studie zu den Effekten von Late-Night-Shows herausgearbeitet. Das Verlachen von Politik und Politikerinnen und Politikern, so der Befund, »turns democracy into a joke«. Das satirische Dauerfeuer kann demnach politische Institutionen und die Menschen, die in ihnen tätig sind, zur Lachnummer degradieren und somit Politikverdrossenheit fördern.

Die gerade beschriebene aggressive Dimension des Humors steht in direkter Nachbarschaft zum Wohlfühlfaktor der Spaßgesellschaft, in der die gute Stimmung der gemeinsam lachenden Menschen auch Wohlwollen gegenüber der politischen Klasse produzieren kann. Komik ist unterhaltsam, bereitet Spaß und erzielt deshalb da, wo sie gelingt, auch hervorragende Einschaltquoten.

Schauen wir uns diesen Zusammenhang noch einmal genauer an: Witz und Komik waren und sind noch immer scharfe Waffen der Kritik. Umberto Eco hat es in seinem Mittelalterkrimi Der Name der Rose (1985) vorgeführt, in dem ein kirchentreuer Mönch das Buch des Aristoteles über die Komödie mit Gift versetzt, damit dessen Inhalt sich nicht verbreiten möge. Denn das Lachen sei gefährlich für Autoritäten und untergrabe ihre Herrschaft. Entsprechend wird Herrschaftskritik auch heute noch gern humorig vorgetragen, im Kabarett beispielsweise, im Karneval oder auch im politischen Witz, der ja gerade in nichtdemokratischen Systemen oft Hochkonjunktur hat. Und doch können kritische Humorformate auch das genaue Gegenteil von dem leisten, was die Kritik intendiert. Dem Lachen kommt hier eine Art Ventilfunktion zu: Die Akteure können `Dampf ablassen «. Die Aggressionen, die sich beispielsweise gegenüber Politikerinnen und Politikern aufgebaut haben, werden so entschärft. Der Bürger kann Distanz gewinnen, kann sich die Politikerinnen und Politiker kleinreden, indem er über sie lacht, und so sein eigenes Unwohlsein reduzieren. Sigmund Freud spricht hier von einer Triebabfuhr, die in zivilisierten Bahnen verläuft und verhindert, dass es Brüche im System gibt. Und das Lachen entlastet. Man nimmt die Dinge und mitunter dann auch sich selber nicht so ernst und kann sich lachend zurücklehnen (Knop 2007: 65f.). Schon William Shakespeare schickte regelmäßig einen Clown auf die Bühne, um einen >comic relief‘ gegenüber den höchst tragischen Wendungen seiner Königsdramen zu platzieren. So ähnlich 
funktioniert es auch noch heute: Der Kabarettist hat zwar gerade einige solide recherchierte Befunde zur Unaufhaltsamkeit des Klimawandels präsentiert, aber da dies mit wohlgesetzten Gags und Pointen gewürzt war, kann das Publikum sein Vergnügen in den Vordergrund stellen.

Schließlich sei noch eine letzte, nicht unwichtige Funktion des Lachens genannt, wie sie schon vom philosophierenden Dritten Earl of Shaftesbury zu Beginn des 18. Jahrhunderts erkannt wurde (Cooper 1709/1992: 342): die Erkenntnisfunktion. Genauer gesagt vermag das positive Erlebnis des Lachens bestimmte Einsichten nachhaltiger in unserem Gedächtnis zu verankern, da sie von einer positiven Emotion getragen werden. Gerade die Satire hebt ja sehr stark auf diese Erkenntnisfunktion des Lachens $a b$, wenn sie sich als aufklärerisch versteht.

Für politische Akteure ergeben sich aus den Spezifika der Humorkommunikation spezifische Chancen. Die erste Chance ist ganz generell darin begründet, dass Humorkommunikation in der Regel Unterhaltungskommunikation darstellt. Unterhaltung aber ist etwas, was von den Menschen meist als angenehm empfunden wird. Die Forschung spricht hier vom »Feel-Good-Faktor», den man sich als politischer Akteur nutzbar machen kann (Dörner 2001: 57). Politik im Unterhaltungsformat erreicht deshalb auch weit mehr Zuschauer und Zuhörer als das herkömmliche politische Kommunizieren, denn das Publikum hat hier primär nicht das Gefühl, belehrt, sondern unterhalten zu werden und dabei auch noch etwas über Politik zu erfahren.

Hinzu kommen spezifische Vorteile von Komik und Lachen in der politischen Kommunikation: Man kann den politischen Gegner angreifen, ohne dass es beim Publikum als zu aggressiv ankommt. Gerade in der deutschen politischen Kultur, die grundsätzlich eher konsens- und mitteorientiert ist, kommt ein zu aggressives Auftreten meist nicht gut an, weshalb auch Formen des >negative campaigning im Wahlkampf hierzulande viel weniger gebräuchlich sind als beispielsweise in den Vereinigten Staaten. Wird der Angriff aber humorvoll vorgetragen, nimmt das ein wenig die Schärfe und lässt den jeweiligen Akteur freundlicher wirken als er wirklich ist.

Der entscheidende Aspekt des Humoreinsatzes ist ohne jeden Zweifel die Sympathiegenerierung. Humorvolle Menschen wirken oftmals sympathischer im Vergleich mit ernsthaften oder gar verbissen agierenden Personen. Mit Humor verbindet sich der Eindruck des >Menschlichen und des Geerdeten. Humor baut Distanz ab, d.h. die politischen Akteure erscheinen nicht mehr als abgehoben, sondern als Menschen wie du und ich. Vor allem die Fähigkeit zur Selbstironie wird vom Publikum als großer Pluspunkt für Akteure gesehen. Selbstironie ist in den Augen der meisten Beobachter das genaue Gegenteil zur Arroganz, sie hebt die Stimmung und signalisiert eine gewisse Gelassenheit gegenüber dem Wichtigkeitsempfinden der eigenen Person (vgl. Tsakona/Popa 2011: 7; Yarwood 2004: 359). Einschlägige Studien zeigen insgesamt, dass witzige Kommentare, Wortspiele und Scherze 
die Aufmerksamkeit und das Interesse am Redner sowie die Glaubwürdigkeit der Quelle verstärken (vgl. Lyttle 2001). Man ist grundsätzlich geneigt, den jeweiligen Akteuren Glauben zu schenken und kritische Nachfragen zu unterlassen.

\section{Politische Komik in den politischen Kulturen der USA und Deutschlands}

\section{Die USA: Komik als 'Muss in der politischen Kommunikation}

Der große Stellenwert, der komischen Modulationen in der politischen Kommunikation der USA zukommt, wird deutlich, wenn man einen Blick auf die umfangreiche Forschungsliteratur zum Thema wirft (vgl. Dörner/Vogt 2017). Im amerikanischen Raum gibt es eine längere Tradition der Beschäftigung mit dem $\mathrm{Zu}$ sammenhang von Politik und Komik, die den Gegenstand vor allem unter dem Begriff "political humor« behandelt hat (vgl. Yarwood 2004, Tsakona/Popa 2011). Der Bedeutungsaufschwung von Satire- und Parodieformaten ist laut Jones (2005; 2010) Teil des Phänomens der »Entertaining Politics«. In den USA präsentieren schon seit geraumer Zeit Formate jenseits von Nachrichten und Dokumentationen politische Akteure und Positionen. $\mathrm{Zu}$ nennen sind Spielfilme, Talkshows, TVSerien, Sitcoms, Fake-News-Shows, Sketch-Comedy-Shows und auch Sendungen des Reality-TV. Eine besondere Rolle kommt dabei den sogenannten Fake-NewsShows wie der Daily Show zu. Angesichts der weitreichenden Öffnung der Populärkultur gegenüber der Politik wurden diese Sendungen $\mathrm{zu}$ »free-flowing arenas for communication about politics« (Jones 2010: 5). Gerade die hybriden Formate beschleunigen die Erosion der Trennung von >Ernst< und 〉Unterhaltungく.

TV-Satire besitzt in den USA eine enorme Popularität (Jones 2005). Zudem gipfelt die crossmediale Verbreitung und Vermarktung der Sendungen (hier ist die Verfügbarkeit im Internet zu nennen) in einem Kult um Formate und Moderatoren, der auch umfangreiche Anschlusskommunikation unter den Nutzern der Sendungen anregt. Die Forschung konnte zudem herausarbeiten, dass Satireformate zunehmend Funktionen des traditionellen Journalismus übernehmen (vgl. Harrington 2011). In diesem Kontext wird auch auf das "political culture jamming « der Satire-Formate verwiesen, bei welchem durch den Einsatz von alternativen komischen Weltbildern auf mögliche Unstimmigkeiten in den herrschenden Sichtweisen hingewiesen werde. Aus dieser Perspektive erscheint etwa die Nachrichtensatire The Daily Show with Jon Stewart (Comedy Central) nicht primär als »fake news «, sondern als »oppositional news«, die über den Einsatz von Humor ein kritisches Hinterfragen realisiere, welches in den »real news « kaum noch vorkomme (Baym 2009: 126f.). Somit regt TV-Satire die politische Debatte und die Meinungsbildung bei jenen an, die nicht den traditionellen politischen Informationsjournalismus verfolgen (Jones 2010: 21). 
Genese und Erfolg von Formaten wie der The Daily Show und The Colbert Report erklären sich nach Ansicht von Jones (ebd.: 48) zum einen durch Veränderungen auf dem US-amerikanischen Fernsehmarkt - insbesondere das Aufkommen des Kabelfernsehens und die damit verbundenen Programminnovationen - und zum anderen durch die Tradition des talk radio als meinungsstarke Diskussion über Politik zwischen Moderatoren und Bürgern (vgl. auch Tryon 2016: 71). In den neuen Formaten tauschen nicht mehr nur Experten und Mitglieder des politischen Establishments ihr »Insiderwissen« aus, sondern auch »Outsider« sprächen und diskutierten über Politik, stimmten über Entscheidungen und politische Akteure ab (Jones 2010: 43). So bietet etwa Jon Stewart seriösen Gästen (z.B. Autoren und Wissenschaftlern) eine breite öffentliche Bühne für ihre Themen und bekommt bisweilen den Status eines öffentlichen Intellektuellen zugeschrieben, der »freies Denken« förderte (Parsi 2011).

Eine Sonderstellung in der US-amerikanischen Medienkultur nehmen schon seit vielen Jahrzehnten die Late-Night-Shows ein, in denen die gastgebenden Moderatoren einesteils längere Passagen mit Stand-Up-Kommentaren zum Tagesgeschehen und Game-Show-Einlagen gestalten und im zweiten Teil der Sendung jeweils prominente Gäste aus diversen Bereichen des öffentlichen Lebens zum Interview begrüßen. Diese Formate haben sich in den USA aus den Variety Shows der 1940er- und 1950er-Jahre entwickelt und gewannen dann große Popularität, so etwa in der Tonight Show mit Johnny Carson, die von 1962 bis 1992 auf NBC ausgestrahlt wurde (vgl. Timberg 2002 und Halper 2009). Zahlreiche Produktionen mit Moderatoren wie David Letterman, Jay Leno und Conan O'Brien setzten den Erfolg fort. Neuere umfangreiche Studien haben den enormen Stellenwert aufzeigen können, den diese Formate mittlerweile für die politische Kultur der Vereinigten Staaten gewonnen haben. Zentral ist dabei die Studie von Michael Parkin (2014), der ein großes Sample von 205 Auftritten amerikanischer Präsidentschaftskandidaten in »entertainment talk shows« in den Jahren 1992 bis 2012 untersucht hat.

Parkin legt dar, dass zwar schon in den 1960er-Jahren Politiker dann und wann einmal in Unterhaltungstalk gegangen sind. Erst mit Bill Clintons erstem Präsidentschaftswahlkampf zu Beginn der 1990er-Jahre sei jedoch eine regelrechte »entertainment talk show strategy« erkennbar geworden, die dann von Clinton selbst und anderen Kandidaten weiterentwickelt und perfektioniert wurde. Mit der Wahlkampagne 2000 wurden Auftritte in Late-Night-Talkshows zur nicht hintergehbaren Bedingung eines erfolgreichen Wahlkampfs, zur Kampagnenroutine. Beobachter fassten das Phänomen so zusammen: »The rule today, you can't sit in the Oval Office still you've sat with Regis, Rosie, Dave, Oprah and, of course, Jay« (Parkin 2014: 31f.). An den großen Hosts der Late-Night-Szene ging also gleichsam kein Weg ins Weiße Haus mehr vorbei. Das Fazit der Entwicklung lautet: 
»The entertainment talk show strategy has grown from the last resort of desperate candidates to a standard campaign practice employed by nearly everyone pursuing the presidency. Candidates have embraced this strategy, assuming it provided a unique opportunity to gain exposure and engage with voters who might be following the campaign with limited interest. «(Parkin 2014: 52).

Parkin zeigt dann in genauen Analysen auf, warum die Auftritte in solchen Formaten vorteilhaft sind. Die Kandidaten können sich humorvoll in informeller Tonlage als sympathische Menschen präsentieren und werden bei ihren Darlegungen, anders als bei journalistischen Interviews, kaum unterbrochen oder mit kritischen Nachfragen konfrontiert (ebd.: 120).

Die Zuschauer bekommen durch die Interviews politische Informationen, vor allem können sie beeinflusst werden in ihren Urteilskriterien (priming effects) und sie können zu einer positiveren Beurteilung von Kandidaten gebracht werden (ebd.: 142). In jedem Fall erhalten die Kandidaten zusätzliche öffentliche Aufmerksamkeit und können ihre Prominenz erhöhen. Parkins Untersuchung zeigt also, dass LateNight-Shows, die in den Interviews oft humorvolle Elemente einsetzen, ein höchst relevantes Mittel der Kampagnenführung in den USA geworden sind.

Während Parkin jedoch auf den Aspekt von Komik, Lachen und Humor relativ wenig systematisches Augenmerk richtet, sind zwei weitere amerikanische Studien genauer in den spezifischen Zusammenhang von Politik und Komik eingedrungen. So untersucht Alison Dagnes, inwiefern die politische Satire in den USA tatsächlich, wie oft behauptet wird, einen ideologischen Bias aufweist: Sind die meisten Satiren eher linksliberal orientiert und richtet sich folglich die meiste satirische Kritik eher gegen konservative Positionen und Akteure als gegen liberale? Auf der Grundlage von Inhaltsanalysen, Interviews und auch einer Analyse von Gästelisten gelangt die Studie am Ende zu dem Befund, der schon zu Beginn als Vermutung formuliert wurde:

»Ideology clearly plays a role here because liberalism serves as a better foundation for satire than conservatism does, simply by virtue of its philosophy. Put another way: conservatives want to maintain the status quo and liberals want to change it. Satire aims at questioning the power structure - so why would conservatives want to do that? The short answer is: they don't.« (Dagnes 2012: xiv).

Satire ist meist von Links aus formuliert. Konservative haben gar keinen Grund, Satire im Sinne einer humorvollen Kritik der gegebenen Verhältnisse zu formulieren, weil sie mit den etablierten Machtstrukturen einverstanden sind. Interessant für den vorliegenden Zusammenhang sind dann die Aussagen, die Dagnes auf der Grundlage ihrer Analysen für die politische Kommunikation in den USA insgesamt trifft: 
"Satire has historically been a parallel protest using laughter to underscore the nation's social wrongs. It has never worked particularly well in times of national sorrow, but it works beautifully when it focuses on the power hierarchy and serves as a check on the establishment. [...]. It will continue to play an important role in American culture because once you give permission to mock the power structure, this permission is impossible to take away.« (ebd.: 212).

Satire und humorvoll modulierte Kommunikation werden somit für die USA als ein Kernelement einer demokratischen Öffentlichkeit gesehen, dass die politische Debatte belebt und für ein breiteres Publikum interessant erscheinen lässt.

Die oben aufgezeigte starke Tendenz US-amerikanischer Politikerinnen und Politiker in satirischen Formaten (Late-Night-Shows, Fake-News-Shows) aufzutreten, kann vor diesem Hintergrund auch als eine Strategie gelesen werden, dem Trend zur Politikerverdrossenheit entgegenzutreten und durch selbstironisch-humorvolles Auftreten Sympathiepunkte beim Publikum zu sammeln.

\section{Politik und Komik in Deutschland: Die starke Tradition des kritischen Kabaretts}

In der politischen Kultur Deutschlands spielt der Zusammenhang von Politik und Komik auch nicht annähernd so eine wichtige Rolle wie in den Vereinigten Staaten. Dies zeigt sich schon daran, dass in der deutschen Forschung das Interesse an dieser Thematik über lange Zeit hinweg ausgesprochen gering ist. Einzig das Kabarett und die damit verbundene Erscheinungsweise der Satire erfährt eine gewisse Beachtung. Mit ihren Komponenten Angriff, Indirektheit und Normrückbindung zielt Satire demzufolge auf eine Entlarvung und Abschaffung von gesellschaftlichen Missständen (vgl. schon Brummack 1971 und Behrmann 2002: 9). Der Schärfegrad der Kritik kann dabei variieren. Ursprünglich aus der Literatur kommend, tritt Satire in diversen Medien auf, wobei seit dem 20. Jahrhundert das Kabarett als die häufigste Ausdrucksform in Deutschland erscheint (Behrmann 2002: 17).

Ästhetik, Poetik und Geschichte des Kabaretts sind mittlerweile umfangreich untersucht (siehe etwa Budzinski/Hippen 1996; Fleischer 1989; Vogel 1993).

Comedy - auch als Begriff und Forschungsgegenstand - etablierte sich in Deutschland auf breiter Linie erst in den frühen 1990er-Jahren und ist untrennbar verknüpft mit der Programmoffensive der privaten Anbieter auf diesem Gebiet. Zwar hat es beispielsweise mit Heinz Erhardt oder Otto Waalkes Vorläufer auch im öffentlich-rechtlichen Fernsehen gegeben, aber die Forschung widmet sich der Comedy erst spät. Es handelt sich um Arbeiten, die die Programmentwicklung beleuchten (Nagel 2003; Kosthorst 2010). Comedy erscheint als amerikanisch inspiriertes, popkulturelles Phänomen; ihre Genese und Ausbreitung in Deutschland gilt als Beleg für eine neue Spaßkultur (Strasser/Graf 2004: 74). 
Charakteristisch für die deutsche Debatte ist die Diskussion eines Gegensatzes zwischen Kabarett und Comedy, meist mit einem normativen Votum für das sanspruchsvollere< Kabarett (vgl. allg. Glodek et.al. 2007). Während Kabarett einen wertvollen Beitrag für die Demokratie leiste, sei Comedy rein kommerziell ausgerichtet (Nagel 2003). Letztlich könne Comedy politisches Kabarett nicht substituieren (Fleischer 2003: 275f.; Wellstein 2007: 165). Neue Formate gerade der öffentlich-rechtlichen Anbieter in Deutschland versuchen jedoch offenbar, alte Grenzziehungen zu überwinden und die Unterhaltungspotentiale der Comedy mit Bildungspotentialen der Satire gerade im Hinblick auf politische Gegenstände zu verbinden und dabei den "public value« ihrer Sender zu vermehren (Kleinen-von Königslöw/Keel 2012: 70). Überschneidungen und Crossover-Phänomene sind inzwischen breit verankert. Künstler wie Dieter Nuhr, der als etablierter Kabarettist sowohl in Formaten wie der Comedy Woche (RTL) als auch dem Satire Gipfel (ARD) - die Nachfolgesendung des von Dieter Hildebrand geleiteten Scheibenwischer - auftritt, sowie Formate wie die heute-show (ZDF) deuten auf eine Konvergenzbewegung hin, die Comedy mit satirischen Elementen und kabarettistischem Anspruch verbindet.

Politisch-kulturell interessant ist in diesem Zusammenhang das Phänomen der sogenannten Ethno-Comedy. Das sind Comedy-Formate, die im Zeitalter verstärkter Migrationsprozesse das Zusammenleben der verschiedenen, ethnisch definierten Bevölkerungsgruppen thematisieren und ihren Witz vor allem aus dem Spiel mit Stereotypen schlagen (siehe etwa Keding/Struppert 2006; Kotthoff 2013; Leontyi 2017). Die gesellschaftliche Problemlage eines multikulturellen Zusammenlebens wird in einer komisch modulierten Tonlage mit großer Publikumsresonanz behandelt und vermag damit Spannungen abzubauen. Ethno-Comedy bietet offenbar Möglichkeiten, die Migrationsthematik in einer unverkrampften Art und Weise zu reflektieren, die im sonstigen Mainstream-Diskurs mit seinen Tabuzonen und Political Correctness-Normen kaum denkbar wären.

Konkret zu Politikerinnen- und Politikerauftritten in humorvoll modulierten Talkformaten wurde in Deutschland bislang fast gar nicht geforscht. Kleinen-von Königslöw und Keel (2012) widmen sich in einer Arbeit zwar der konkreten Analyse der heute-show und vergleichen diese explizit mit der US-amerikanischen Daily Show, auf die besondere Funktionslogik der Studiointerviews mit politischen Akteuren wird dabei jedoch nicht weiter eingegangen. Der grundsätzlich sehr nützliche Überblick zu Formen des Zusammenspiels von Unterhaltung, Humor und Politik bei Kleinen-von Königslöw (2014) erwähnt, dass systematische Untersuchungen von Politikerinnen- und Politikerauftritten in Unterhaltungsshows unter dem Aspekt der Komik und Humorverwendung nicht vorliegen, sondern sich bislang auf gelegentliche Einzelfallbetrachtungen beschränken. Lediglich zu der Late Night Comedy von Harald Schmidt liegen einige Studien vor, diese fokussieren aber den Humoreinsatz des Moderators und betrachten kaum das modulierte Interaktionsgeschehen vor den Kameras (siehe u.a. Nitsch/Lichtenstein 2013). Das 
gleiche schließlich gilt auch für die Studie von Bernd Gäbler (2016), der Satireformate im deutschen Fernsehen betrachtet und auch normativ evaluiert, auf die Studiointerviews in der heute-show jedoch nicht eingeht.

Allerdings lässt sich sowohl im Hinblick auf Harald Schmidt wie auch im Hinblick auf die heute-show klar die Beobachtung formulieren, dass in Deutschland insgesamt nur ein vergleichsweise kleiner Teil der politischen Klasse willens ist, in satirisch modulierten Talkformaten aufzutreten (vgl. Dörner 2006, Dörner/Vogt 2016 und Peter 2015). In Deutschland ist es, ganz anders als in den USA, keineswegs zwingend, in solchen Formaten aufzutreten, wenn man aussichtsreich für ein Amt oder Mandat kandidieren will. Im Gegenteil, hierzulande scheint die Gefahr, durch derartige Auftritte starke Seriositätsverluste und damit Imageschäden zu erleiden, besonders präsent. Es scheint dem Publikum in Deutschland schwer vorstellbar, das Amtscharisma einer Politikerin oder eines Politikers mit dem ausgelassenen Blödeln in einem Comedyformat zu verbinden. Selbst Gerhard Schröder, der »Medienkanzler«, war zwar im legendären Bundestagswahlkampf 1998 in einer Kampagne nach amerikanischem Vorbild durchaus in Entertainment-Sendungen gegangen; Comedy und Satire sparte er jedoch ganz klar aus.

Hier zeigt sich also, bei allen »Amerikanisierungs«-Phänomenen in der deutschen politischen Kultur, ein deutlicher Unterschied: Während das amerikanische Elektorat von den politischen Protagonisten die Fähigkeit und auch die Bereitschaft erwartet, sich humorvoll und selbstironisch zu präsentieren, stellt dies in Deutschland keine politisch-kulturelle Norm dar. Politische Akteure können den Weg in die Komik suchen, wie dies auch einige im Wahljahr 2013 gemacht haben (Dörner/Vogt 2017). Sie müssen es aber nicht tun. Und es bedarf eines besonderen Humortalents, sich in Deutschland komisch zu präsentieren. Die Akteure müssen ein feines Gespür haben für die Balance zwischen Komik und Seriosität, Spaßmacher und Amtsinhaber. Da nur wenige Personen in Deutschland über eine solche Fähigkeit verfügen, passiert es durchaus öfters, dass die Balance misslingt. Und aus solchen Erfahrungen heraus halten die meisten Politikerinnen und Politiker lieber Abstand zur Komik.

\section{Fallbeispiele}

An dieser Stelle soll zur Konkretisierung der Unterschiede auf zwei Fallbeispiele aus der jüngsten Zeit eingegangen werden. Zum einen auf Barack Obama, der wie kaum ein anderer das Spiel von Komik und Selbstironie beherrscht, und zum anderen auf die gescheiterte Kampagne von Peer Steinbrück, der 2013 mit »Humor und guten Bildern« punkten wollte. 


\section{Barack Obama: Selbstironie im fortgeschrittenen Stadium}

Barack Obama hatte seine Wahlkämpfe vor allem durch den Einsatz neuer Kommunikationsstrategien so erfolgreich bestreiten können. Das betrifft zum einen den gezielten, hochprofessionellen Einsatz des Internets, mit dem u.a. auch die traditionellen Filtermechanismen der etablierten Massenmedien umgangen werden konnten. Zum anderen hat er mehr noch als die vorangehenden Wahlkämpfer humorbezogene Medienformate in die eigene Kommunikationspraxis integriert. So war Obama nicht weniger als fünf Mal in der satirisch geprägten Talkreihe The Late Show with David Letterman (ausgestrahlt von CBS) zu Gast. Und er trat mehrfach ohne Berührungsängste bei Jon Stewart in dessen bissiger Nachrichtensatire The Daily Show (Comedy Central) auf. In der Wahlkampagne zur zweiten Amtszeit 2012 konnte Obama durch einen ausgesprochen gelungenen Auftritt bei Stewart die vorher nur begrenzt erfolgreich verlaufenen >Duelle gegen seinen Konkurrenten Mitt Romney vor allem beim jüngeren, gebildeten Publikum ausbügeln.

Im März 2015 hatte Obama sich an der Rubrik Mean Tweets in der Late-Night Show Jimmy Kimmel Live (ausgestrahlt vom Network ABC) beteiligt. Prominente verlesen und kommentieren dort Twitter-Kommentare, die sich über die jeweilige Person lustig machen oder mitunter auch aggressive Wendungen enthalten. Die Herausforderung besteht darin, Negatives über die eigene Person vorzulesen und so zu kommentieren, dass das Publikum Spaß hat. Man darf nicht beleidigt und kein Spielverderber sein. Mitlachen macht sympathisch.

Abbildung 1: Barack Obama lacht über einen Witz, der über ihn getwittert wurde.

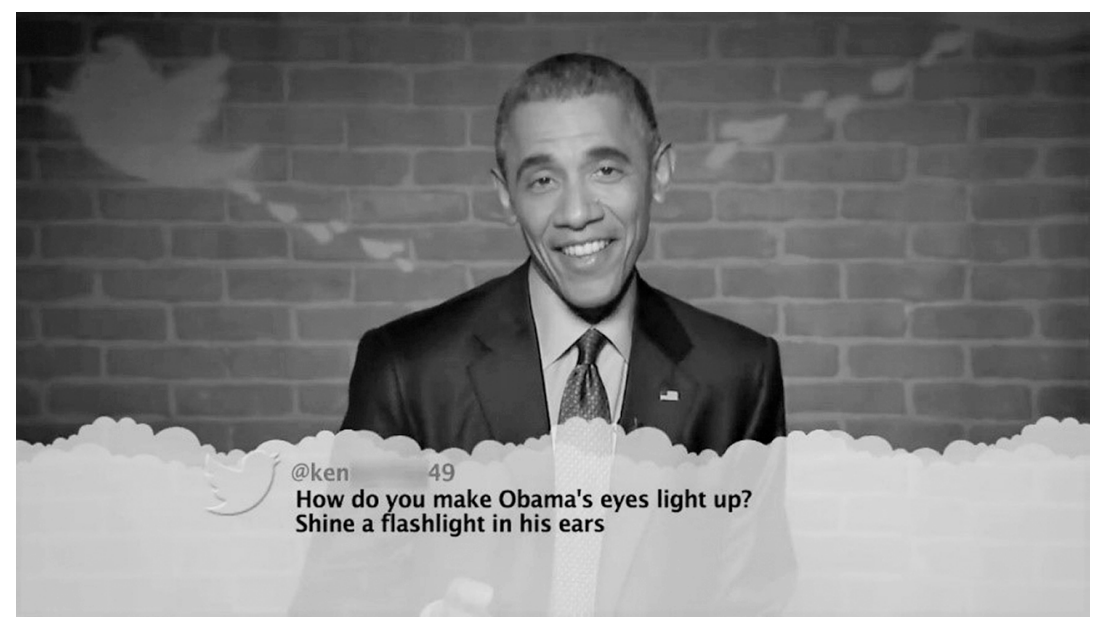

So musste sich Obama u.a. mit Kritik an seinen Jeans auseinandersetzen und mit dem Wunsch, man möge den passionierten Golfer um die halbe Welt zu einem 
Golfkurs fliegen und den Rückflug streichen. Einer der getwitterten Witze lautete (siehe Abb. 1): „Wie bringt man Obamas Augen zum Leuchten? Scheine mit einer Taschenlampe in seine Ohren. «Der Präsident quittierte den Tweet - der immerhin unterstellt, der Kopf des Amtsträgers sei leer - lachend mit dem Kommentar: »Ich finde, der ist ziemlich gut «. Obama konnte in dieser Sendung zum einen nochmals seine hohe Internetaffinität unter Beweis stellen, die vor allem für das jüngere $\mathrm{Pu}$ blikum durchaus wichtig ist. Und zum anderen konnte er sich als humorvoller, selbstironischer Mensch präsentieren, der mit Spott gelassen umgehen kann. Diesen Eindruck konnte er dann beim Live-Auftritt in Kimmels Talkshow (die Mean Tweets sind vorproduzierte Clips) noch vertiefen.

Einen Höhepunkt der humorkommunikativen Strategien stellt aber ohne Zweifel der Auftritt Obamas in der populären Nachrichtensatire The Colbert Report (Comedy Central) dar. Der Auftritt markiert einen radikalen Schritt in der politischen Humorkommunikation insofern, als der Präsident hier nicht mehr nur als Gast in einer Talksequenz auftritt, sondern sogar einen Rollentausch mit dem Moderator vornimmt und tatsächlich ganz offensiv die Tätigkeit eines Satirikers ausübt.

Der Colbert Report ist ein Spin off, ein Ableger der Daily Show with Jon Stewart. Moderator Steven Colbert war früher Ensemblemitglied in der erfolgreichen Nachrichtensatire und machte sich 2005 mit einem eigenen Format selbständig. The Colbert Report wurde seither bis Ende 2014 erfolgreich bei Comedy Central ausgestrahlt, bevor Colbert dann die Nachfolge David Lettermans in dessen Late-NightTalkshow bei CBS übernahm. Im Zentrum des Colbert Report stand die Figur eines Journalisten, der als Korrespondent in stramm konservativer Manier das Tagesgeschehen kommentierte. Durch die satirische Überspitzung wurden so rechtspopulistische Meinungen, Wertungen und Sprechweisen karikiert.

In der Sendung am 9. Dezember $2014^{2}$ tritt nun zunächst Colbert in seiner gewohnten Rolle in abfällig-überheblichem Ton auf, leitet das sendungstypische Segment The Wørd ein, indem er behauptet, er könne problemlos die Aufgaben eines Präsidenten übernehmen, aber der Präsident sei im Gegenzug nicht in der Lage, eine Moderatorentätigkeit wahrzunehmen. Um seine Kompetenz zu beweisen, kündigt Colbert also an, im Folgenden eine Lösung für die umstrittene gesetzliche Regelung der Krankenkassen (»Obamacare«) vorschlagen zu wollen.

In diesem Moment tritt, unter lautem Schreien und tosendem Applaus des Saalpublikums, der amtierende Präsident in offizieller Kleidung (dunkelblauer Anzug, weißes Hemd, hellblaue Krawatte) auf die Bühne. Dieser möchte nun die Rolle des Moderators übernehmen mit der Begründung, Colbert habe seinen Beruf attackiert und er wolle diese Attacke nun mit einem Angriff auf Colberts Beruf beantworten. Es sei überhaupt nicht schwer, Colberts Moderationstext vorzutragen.

2 Eine Videoaufzeichnung der Sendung ist abrufbar auf youtube: https://www.youtube.com/ watch?v=95KTrtzOY-g 
Colbert verschwindet daraufhin von der Bühne und überlässt dem >neuen Moderator das Wort, der nun auch am üblichen Moderationstisch Platz nimmt. Als erste >Amtshandlungく nimmt er eine Modulation (im Sinne von Goffman 1977) vor: er benennt die eigentlich angekündigte Kolumne The Wørd um in The Decree, da dies "more presidential« klinge und angemessener sei. Hier nimmt Obama schon ironische Distanz zu seinem Amt, indem er die präsidentielle Kommunikationsform des Dekretierens zum Bestandteil eines komödiantischen Auftritts macht. Er beginnt dann, den Moderationstext mit Blick in die Kamera vorzutragen.

Damit ist eine neue Qualität des politischen Humors erreicht. Indem der amtierende Präsident der Vereinigten Staaten in die Rolle eines Comedians schlüpft und dort Texte vorträgt, die Satireautoren des Fernsehformats zuvor geschrieben haben, löst sich die klassische Rollenverteilung zwischen Subjekten und Objekten der politischen Satire fast vollständig auf. Die Politikerin oder der Politiker nutzt die professionellen Texte, um sich in besonders brillanter Weise als selbstironischer Akteur zu inszenieren und damit die Sympathien des Publikums zu erheischen, was ihm beim Saalpublikum auch problemlos gelingt. Obama in der Rolle des Stephen Colbert liest also vom Teleprompter Colberts Text ab: „Nation, as you know, I, Stephen Colbert, have never cared for the president. The guy is so arrogant, I bet he talks about himself in the third person." [lautes Lachen und Beifall].

Obama kann also, indem er die Rolle Colberts einnimmt, heftiger und radikaler Selbstironie aussprechen als ihm dies in seiner normalen Sprechrolle möglich wäre. Die Kunstfigur »Colbert« eröffnet in Verbindung mit dem Rollentausch neue Räume des Sagbaren.

Obama in der Rolle des Moderators fährt dann fort, indem er über die Gesundheitsreform lästert. Nun scheint hier aufgrund des vorbereiteten Textes gleichwohl eine Situation definiert, die relativ vorhersehbar und damit für den Akteur Obama berechenbar ist. Die Redaktion der Sendung hat jedoch einen zusätzlichen Kontingenzfaktor eingebaut, der situative Unberechenbarkeit hineinbringt. Das gesendete Bild stellt nun einen split screen dar, einen geteilten Bildschirm, auf dem im linken Bildteil der vortragende Präsident zu sehen ist und auf dem rechten Bildteil ein Textinsert, der fortlaufende redaktionelle Kommentare zu Obama/Colberts Text sichtbar macht. 
Abbildung 2: Barack Obama fungiert als Moderator und Komiker

in der Sendung The Colbert Report.

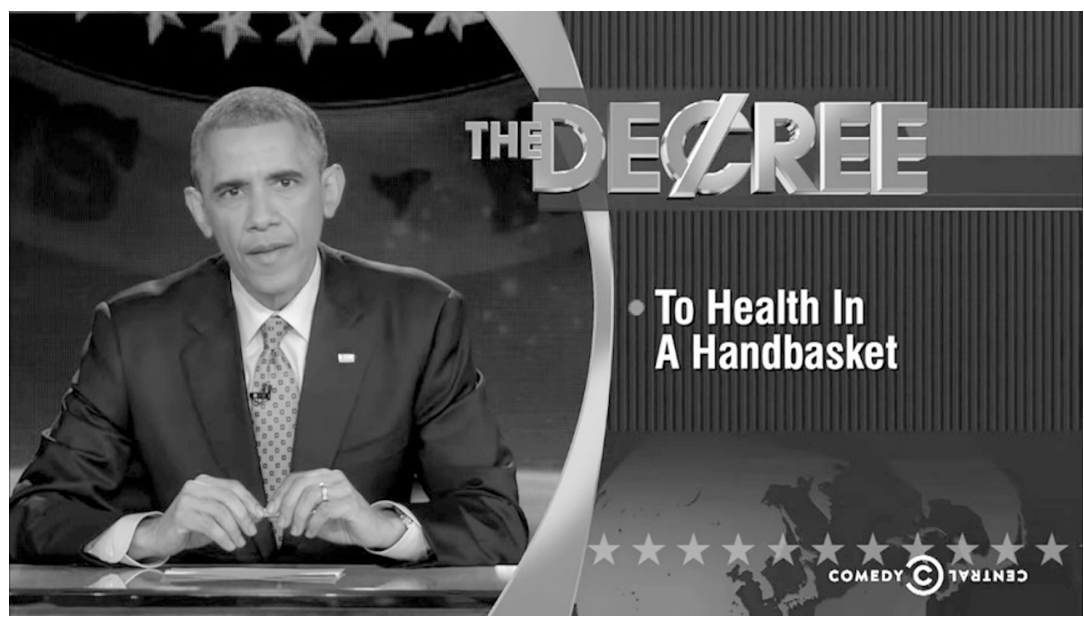

Diese Kommentare eröffnen gleichsam eine zusätzliche Ebene der Ironisierung, auf die der Akteur Obama keinerlei Einfluss hat. Diese zusätzliche Ebene sorgt im weiteren Verlauf der Sequenz immer wieder für Heiterkeit beim Saalpublikum. So wird in diesem Kommentar Obamas Sprechweise kommentiert und auch der Inhalt. Als beispielsweise der vorgetragene Text die Gesundheitsreform lobt, weil diese den Krankenversicherungsschutz der Kinder bis zu deren 26. Lebensjahr über die Krankenversicherung der Eltern garantiere, erscheint als Insert »\#aka Double Puberty«. Die Verlängerung der Versicherung wird somit als Verlängerung der Pubertät und damit sozusagen als verlängerte Abhängigkeit der Kinder von ihren Eltern - und verlängerte Leidenszeit der unter der Pubertät ihrer Kinder leidenden Eltern - verlacht.

Auch schlichte Comedy-Pointen werden auf diese Weise platziert, als Obama den großen Erfolg seiner Gesundheitsreform daran festmacht, dass schon 8 Millionen Menschen auf diese Weise versichert werden konnten. Die Kommentarleiste vermerkt in diesem Moment: "So, half as popular as Grumpy Cat Video«. Die sogenannte »Grumpy Cat « ist eine Katze, die sich wegen ihres grimmigen Gesichtsausdrucks im Internet großer Beliebtheit erfreut und deren Abbildungen viral das Netz durchfluten. Indem der Kommentar eines der wichtigsten politischen Anliegen von Obamas Präsidentschaft, das die Existenz vieler Menschen sehr elementar betrifft, mit einem sinnfrei-komischen Internet-Phänomen auf eine Ebene stellt, wird die Relevanz von »Obamacare« heruntergespielt und die Versicherungsreform der Lächerlichkeit preisgegeben. 
Es folgt noch eine ganze Reihe weiterer Pointen, in denen immer wieder auch der Bezug zu politischen Fakten und Zusammenhängen hergestellt wird: etwa zu einem Alternativprojekt der Republikaner, das sprachlich umständlich und somit spöttisch in Anlehnung an den republikanischen Majority Leader »Mitch-McConnell-Care« genannt wird. Oder der Verweis auf das präsidentielle Vetorecht im gesundheitspolitischen Bereich wird mit dem Insert »POTUS interruptus « kommentiert und auch hier wieder veralbert, denn der Präsident (POTUS ist das gebräuchliche Akronym für »President of the United States«) wird hier in einen Kontext mit einer veralteten Verhütungsmaßnahme gestellt. Bei aller Albernheit geht es jedoch immer auch um politische Inhalte und nicht um reine Gag-Kommunikation.

Den Höhepunkt stellt schließlich eine selbstreferentielle Schleife in der Kommunikation dar. Das heißt, der Diskurs thematisiert hier sich selbst und verkompliziert die Kommunikationskonstellation dadurch noch einmal. Es wird mit den Rahmungen (Goffman 1977), die das Publikum aus seinem alltäglichen Medienkonsum kennt, gespielt, um daraus neuen Witz zu generieren. Als Obama im Vortragstext die Schwierigkeiten thematisiert, die Gesundheitsreform speziell bei jungen Menschen bekannt und populär zu machen, sagt er:

»But young people don't watch real news shows like this one. (Insert: Or fake ones like Fox) [extrem lautes und langes Gejohle und Pfeifen des Saalpublikums] They watch comedy shows. And I just don't see the president go on one of those. They're beneath his dignity. (Insert: But above his approval rating). [lautes Lachen und Johlen].«

Hier platziert zum einen die Sendung einen selbstreferentiellen Kommentar, indem sie sich selbst als »real news show u und die ernst gemeinte (konservative) Nachrichtensendung Fox News als »fake news show« bezeichnet. Das enthält eine lustige Spitze gegen den Konkurrenzkanal Fox, aber zugleich auch eine sehr ernste Aussage. Denn der humoristisch gerahmten Satire wird dadurch letztlich mehr Wahrheit und mehr Erkenntnisgewinn zugeschrieben als den >richtigen Nachrichten. Erst die satirische Zuspitzung, so die zugrundeliegende Botschaft, macht den wahren Kern der Dinge sichtbar.

Zum anderen bezieht sich Obama ironisch auf sich selbst, indem er verneint, jemals in eine comedy show gehen zu wollen, da dies unter der Würde des Präsidenten liege. Diese Aussage erfolgt aber just während seines Auftritts in einer comedy show. Er widerlegt sich selber und macht so im Scherz sichtbar, was den politischen Diskurs oft im Ernst betrifft: Politikerinnen und Politiker widersprechen sich, lügen die Menschen an, behaupten im gleichen Moment das eine und tun das andere. Und es ist kein linksradikaler Fundamentalist, der dies sichtbar macht, sondern der Präsident selbst. Das Saalpublikum mag diese Art der selbstironischen Selbstthematisierung und gibt seiner Begeisterung lautstarken Ausdruck. 
Politischer Akteur und Zuschauer sind hier vereint im Gelächter über eine vielfach anzutreffende und zu kritisierende politische Alltagspraxis.

Auch das Publikum aber wird schließlich explizit thematisiert, als Obama konstatiert, junge Leute sähen nur comedy shows und würden keine seriösen Nachrichtensendungen einschalten. Diese Behauptung, die durch die neuere amerikanische Forschung in der Tendenz durchaus bestätigt wird, drängt die Zuschauer ebenfalls in eine Position der Selbstbezüglichkeit und regt dazu an, das eigene Mediennutzungsverhalten auch einmal kritisch zu reflektieren.

Um es noch einmal verkürzt zu resümieren: der amtierende Präsident sagt während des Auftritts in einer satirischen Fake-News-Show, dass es sich gerade um eine seriöse Nachrichtensendung handele, dass Auftritte in humorvoll gerahmten Sendungen grundsätzlich unter der Würde des Amtes lägen und dass das Publikum aufgrund seiner mangelnden Bereitschaft, Informationssendungen zu rezipieren, eigentlich nur über solche Comedy-Formate erreichbar sei. Diese Sequenz ist schon atemberaubend in ihrer Dichte und macht deutlich, welche Erkenntnispotentiale in der Satire stecken, ohne dass die positive Feel-Good-Stimmung darunter leiden müsste und ohne dass der selbstironische politische Akteur seine Chancen der Sympathiegewinnung dadurch schmälern würde.

Selbstironie erreicht hier ein virtuoses Ausmaß, indem politische Akteure direkt (und mit Rollentausch) als aktive Ensemblemitglieder einer Satiresendung auftreten, dort von professionellen Autoren und Redaktionen dramaturgisch effektvoll in Szene gesetzte Texte benutzen und so das Publikum im Saal wie vor den Bildschirmen begeistern. Das Ganze stellt sich als Win-Win-Situation für politische Akteure und Medienakteure dar und entfaltet doch auch Potentiale einer kritischen Sicht auf das Geschehen, in das moderne politische Medienkommunikation heute eingebettet ist.

Über die gesamte Sequenz hinweg trägt Obama sehr gekonnt und souverän vor, lässt sich auch durch das für ihn teilweise unberechenbare Lachen des Publikums nicht aus dem Konzept bringen und beweist hohe inszenatorische Professionalität. Die Zielgruppe, die er dabei zunächst in der TV-Ausstrahlung, dann aber vor allem über die Abrufe der Sendung im Internet erreicht (die Mean Tweets mit Obama wurden schon über 28 Millionen mal angeklickt, Stand 23.7.2015), sind junge, relativ gut gebildete Menschen, die insgesamt Satire- und Comedyformate als wichtigen Bestandteil der politischen Medienöffentlichkeit nutzen und goutieren.

Die Besonderheit eines solchen Auftretens wird sichtbar, wenn man sich einmal vorstellt, Angela Merkel würde einen ähnlichen Rollentausch vornehmen und beispielsweise anstelle von Oliver Welke die heute-show moderieren. Wie schwierig sich das Humorterrain in der politischen Kultur Deutschlands darstellt, lässt sich gut beobachten am Beispiel der Kampagne Peer Steinbrücks im Bundestagswahljahr 2013. 


\section{Peer Steinbrück: Ein »Wahlkampf mit Humor und guten Bildern «}

In Deutschland stellt sich die Situation, wie oben dargestellt, komplett anders dar. Humorverwendung durch politische Akteure ist traditionell etwas, das mitunter vorkommt, aber im Großen und Ganzen Seltenheitswert hat. Vor diesem Hintergrund war es bemerkenswert, dass SPD-Kanzlerkandidat Peer Steinbrück bei den Bundestagswahlen 2013 durch einen Wahlkampf mit "Humor und guten Bildern" punkten wollte. Kurz nach seiner offiziellen Nominierung als Spitzenkandidat der Sozialdemokraten kündigte er an, es werde keinen »lustlosen und langweiligen « Wahlkampf mehr geben (siehe Die Welt, 01.10.2012). Die Abgrenzung zielte vor allem auf den vorangegangenen Wahlkampf $2009 \mathrm{ab}$. Die Sozialdemokraten mussten damals aus einer großen Koalition heraus agieren, was erhebliche Probleme hinsichtlich der Profilierung gegenüber der Union generierte.

Insgesamt war die Kampagne 2009 von vielen als langweilig wahrgenommen worden, woraus 2013 ein starkes Bedürfnis nach mehr Lebendigkeit beim Wahlvolk wie bei professionellen Beobachtern resultierte.

Aus dieser Stimmung heraus schlug auch der ehemalige Bayerische Ministerpräsident und frühere Kanzlerkandidat Edmund Stoiber vor, in das rituell erstarrte TV-Duell der Kanzlerkandidaten anstelle eines weiteren seriösen Journalisten den Moderator Stefan Raab vom Privatsender ProSieben einzuladen. Stefan Raab, der in seiner Sendung tv total auch mehrfach Politikerinnen oder Politiker zu Gast hatte und mit einem neuen Format Absolute Mehrheit die politische Debattenshow für jüngere Zuschauer attraktiv zu machen suchte, konnte tatsächlich mit originellen Fragen das Duellgeschehen auflockern. ${ }^{3}$ Raab sorgte auch für die eingängigste Formulierung des Tages, als er den Kandidaten Peer Steinbrück für vage Äußerungen über seine spätere Rolle in einer allfälligen Neuauflage der großen Koalition kritisierte: »Das ist doch keine Haltung, zu sagen: Ich will nur gestalten, wenn ich >King of Kotelett « bin«. Der »King of Kotelett « - gemeint ist derjenige, der das Sagen hat -, eine Formulierung, die Raab von seinem Autor Christoph Schulte-Richtering geschrieben wurde, war in den folgenden Tagen in der Presseberichterstattung das zentrale Bonmot. ${ }^{4}$ Thorsten Faas resümierte später in einer empirischen Studie zur Rezeption des TV-Duells, dass 2013 das Duell vor allem von jüngeren Zuschauern positiv bewertet wurde und die Moderation des Entertainers Raab daran einen deutlichen Anteil hatte (Faas 2015: 308).

3 Zum TV-Duell 2013 siehe Claßen u.a. (2015)

4 Siehe etwa den Artikel von Carolin Gasteiger in der Süddeutschen, der die Formulierung direkt als Überschrift trägt (www.sueddeutsche.de/medien/stefan-raab-im-tv-duell-kingof-kotelett-1.17 60136), oder die Ausführungen von Antje Hildebrandt in der Welt (»King of Kotelett - Raab siegt mit Hartnäckigkeit«, www.welt.de/vermischtes/article119615507/King-ofKotelett-Raab-siegt-mit-Hartnaeckigkeit.html). Der Stern titelte, ebenfalls mit Hinweis auf den »King «: »Raab for Kanzler« (www.stern.de/politik/mdeutschland/tv-duell-raab-for-kanzler3904042.html). Zugriff auf die Seiten jeweils am 1.6.2016. 
Es ist in diesem Kontext kein Zufall, dass Kandidat Steinbrück in seiner Wahlkampagne bewusst ähnliche, d.h. humormodulierte und beim jugendlichen Publikum populäre Formate aufsuchte, um für sich Wählerstimmen zu generieren. Er kontaktierte jedoch nicht Stefan Raab, sondern ein anderes, sehr beliebtes Moderatorenduo des Jugendsenders ProSieben: Klaas Heufer-Umlauf und Joko Winterscheidt. Die waren schon länger mit ihrem Blödelformat Circus HalliGalli bei den jüngeren Zuschauern erfolgreich, so dass es durchaus vielversprechend erscheinen musste, dort aufzutreten.

Die Sendung wurde am 16. September 2013, also sechs Tage vor der Bundestagswahl, ausgestrahlt. Steinbrück gelang insgesamt ein guter Auftritt bei diesem Format, das ansonsten eher popkulturelle Showgrößen zu Gast hat und durch flache, ausgelassene Komik punktet. Ein Beispiel: Moderator Klaas fragt den Kandidaten: "Was glauben Sie, wo wird mehr getrunken: Im Wahlkampf oder beim Winterfest der Volksmusik?«. Steinbrück reagiert darauf nicht mit weiterem Blödeln, sondern wechselt in den ernsten Modus. Er habe einigen Leuten versprochen, bis zum Wahlkampf gar nichts mehr zu trinken, was ziemlich anstrengend sei, ihn aber einigermaßen fit halte. Steinbrück bleibt dann jedoch nicht ernst, was in einer Blödelsendung wie Circus HalliGalli leicht zu einem Image des Spielverderbers hätte führen können, sondern zeigt sich kurz danach wieder sympathisch, als er fragt: »Haben Sie was hier?«. Das wird von Joko und Klaas sofort bejaht und vom Publikum mit lautem Lachen belohnt. Es wird ein Bier hereingebracht, und der Kandidat kann ungeachtet seines Versprechens dem Publikum zuprosten, selbstironisch eine kleine Charakterschwäche zugeben, zeigen, dass er sich nicht zu ernst nimmt und dass er den grundlegenden Kommunikationsmodus des Formats verstanden hat. 
Abbildung 3: Peer Steinbrück bei Circus HalliGalli: Der Kanzlerkandidat mit einem Glas Bier, mit dem er kurz zuvor dem Publikum zugeprostet und seine kleine Charakterschwäche, nicht aufAlkohol völlig verzichten zu wollen, eingestanden hat.

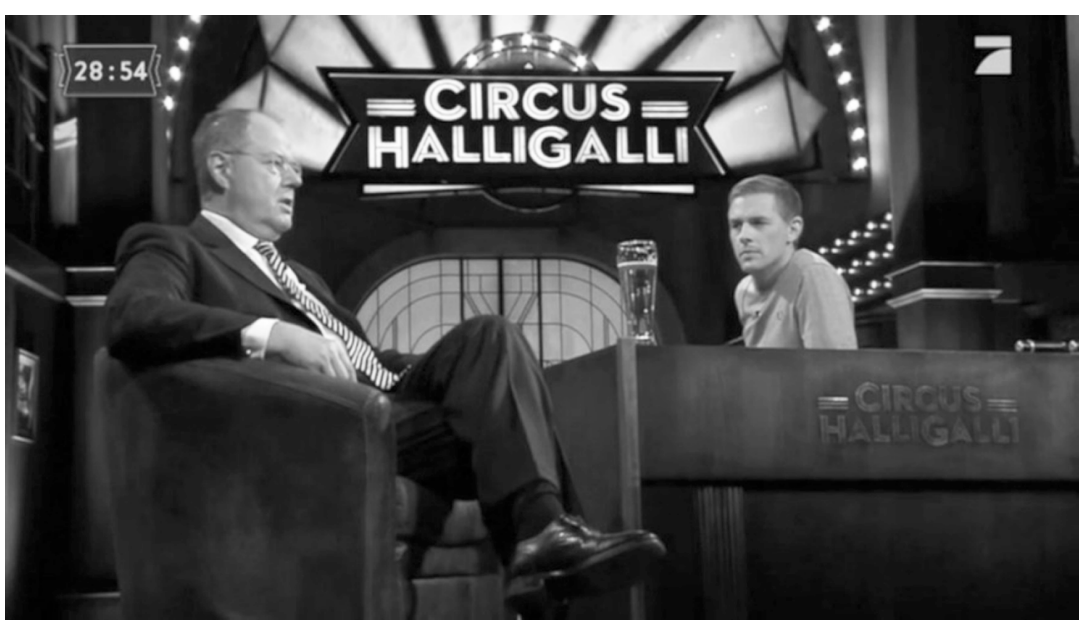

In der Tat ist Selbstironie ein zentrales Humorinstrument, und dies weiß Steinbrück, schon gleich zu Beginn zu nutzen. Als Moderator Klaas den lebhaften Beifall des Publikums kommentiert mit der Anmerkung, nicht jeder bekomme einen so warmen Applaus, erwidert der Gast trocken, dass die Zuschauer im Studio ihn vielleicht verwechselten. Auch die weiteren Interaktionen zeigten einen lockeren, selbstironischen und schlagfertigen Gast. So lautete eine Frage der Moderatoren, ob Steinbrück eigentlich noch all die Bratwürste zählen könne, die er im Wahlkampf gegessen habe, weil dies so schön bodenständig wirke. Der Politiker erwiderte, dass die »diversen Pappbrötchen« das eigentliche Problem darstellten, so auch die, welche er vor der Sendung von ProSieben gereicht bekommen habe. Das Publikum lachte und spendete wieder Beifall. Steinbrück hatte die kleine Konfrontation mit dem Hinweis auf >Bodenständigkeit ‘ gut pariert und eine eigene Pointe lanciert, die beim Publikum gut ankam.

Vor allem gelang es Steinbrück, sich in den Kontext einzupassen, ohne doch anbiedernd zu wirken. Das zeigt folgende Dialogsequenz:

Joko: »]etzt mal für die Kids da draußen - wer ist cooler: Obama oder Putin?«Steinbrück: »Keiner von beiden. «(Lachen und Applaus) Joko: »Dann lassen Sie es mich anders formulieren: Wer hat mehr Swag?«Steinbrück: »Da muss ich passen, weil ich nicht weiß, was das ist.«Joko und Klaas (ausrufend): »Epic Fail!«Steinbrück, ab- 
schließend: »Lieber gleich zugeben als rumschwafeln.«(Das Publikum lacht wohlwollend). ${ }^{5}$

Herausgefordert durch jugendsprachliches Vokabular, gibt der Kandidat schnell $\mathrm{zu}$, nicht wirklich mitreden zu können. Diese Klarheit kommt zumindest beim Studiopublikum gut an.

In den allgemeinen Ausführungen über Funktionen von Humor und Komik in der politischen Kommunikation wurde betont, dass die komische Modulation immer auch ein kommunikatives Glatteis bereitet, weil die anvisierten positiven Effekte ins genaue Gegenteil umschlagen können. Das gilt insbesondere für den Einsatz von Ironie. Ironie muss vom Publikum verstanden werden, wenn sie Sympathie generieren soll. Gelingt das nicht, kann es zum kommunikativen Totalschaden kommen. Das passierte dem Kandidaten Steinbrück, als er in der Endphase seiner Kampagne mit einer umstrittenen Fotografie viele Sympathien bei den Wählern einbüßte.

Das SZ-Magazin hatte auf seiner Titelseite am 13. September 2013 ein Foto veröffentlicht, auf dem Steinbrück demonstrativ den Mittelfinger in die Kamera zeigt. Das Bild war für die Reihe Interviews ohne Worte produziert worden. Hier sollten prominente Mitbürger ausschließlich mimisch, gestisch und teilweise mit Einsatz von Requisiten auf die im Interview gestellten Fragen antworten. Ist schon sprachliche Ironie riskant, so stellt sich die Situation bei rein visuell inszenierter Ironie noch deutlich gefährlicher dar.

Steinbrücks visuelle Ironie jedenfalls wurde von vielen nicht verstanden oder aber schlicht als misslungen bewertet. Eine typische Bewertung lieferte beispielsweise die Rheinische Post »So ein Foto wird ein Spitzenpolitiker im Leben nicht mehr los «. ${ }^{6}$ Der größte Teil der Pressebeobachter sah die PR-Aktion des Spitzenkandidaten als misslungen und in jedem Fall so kurz vor dem Wahltermin als viel zu riskant an. Steinbrück habe sich zwar wirkungsvoll von der Merkel-Raute ${ }^{7}$ und der damit assoziierten Unverbindlichkeit abgegrenzt, aber dazu eine Geste benutzt, die für einen Kanzlerkandidaten nicht akzeptabel sei. Während also Barack Obama einen Ironieeinsatz bis hin zum Rollentausch zwischen Politiker und Komiker mit großem Erfolg darbieten konnte, führte ein mutiger visueller Ironiegebrauch beim deutschen Kanzlerkandidaten zum genauen Gegenteil. Kommunikatives Glatteis

5 Zur Erklärung für ältere Leserinnen und Leser: mit »Epic Fail« ist eine schwerwiegende Fehlleistung bezeichnet, und »Swag«steht für eine besonders coole, attraktive Ausstrahlung.

6 Einen kleinen Überblick zu den Reaktionen gibt auch folgender Artikel aus der Zeit: www.zeit.de/ politik/deutschland/2013-09/steinbrueck-mittelfinger-stinkefinger, Zugriff am 1.6.2016.

7 So der Focus am 13.09.2013, www.focus.de/politik/deutschland/bundestagswahl-2013/ eindeutige-geste-peer-steinbrueck-zeigt-seinen-kritikern-den-stinkefinger_aid_1099309.html, Zugriff am 4.6.2016. 
stellt die Ironie vor allem in der deutschen politischen Kultur dar, wo man von einem Repräsentanten des Volkes eher staatstragenden Ernst, vielleicht ein wenig wohldosierte Selbstorinie, aber niemals ausgelassene Spaßexperimente erwartet.

Abbildung 4: Peer Steinbrück auf der Titelseite des Süddeutsche Zeitung Magazins.

\section{Nemmer 39 | 13. Scpecmber 2013 \\ Siiddeutsche Zeitung Magazin}

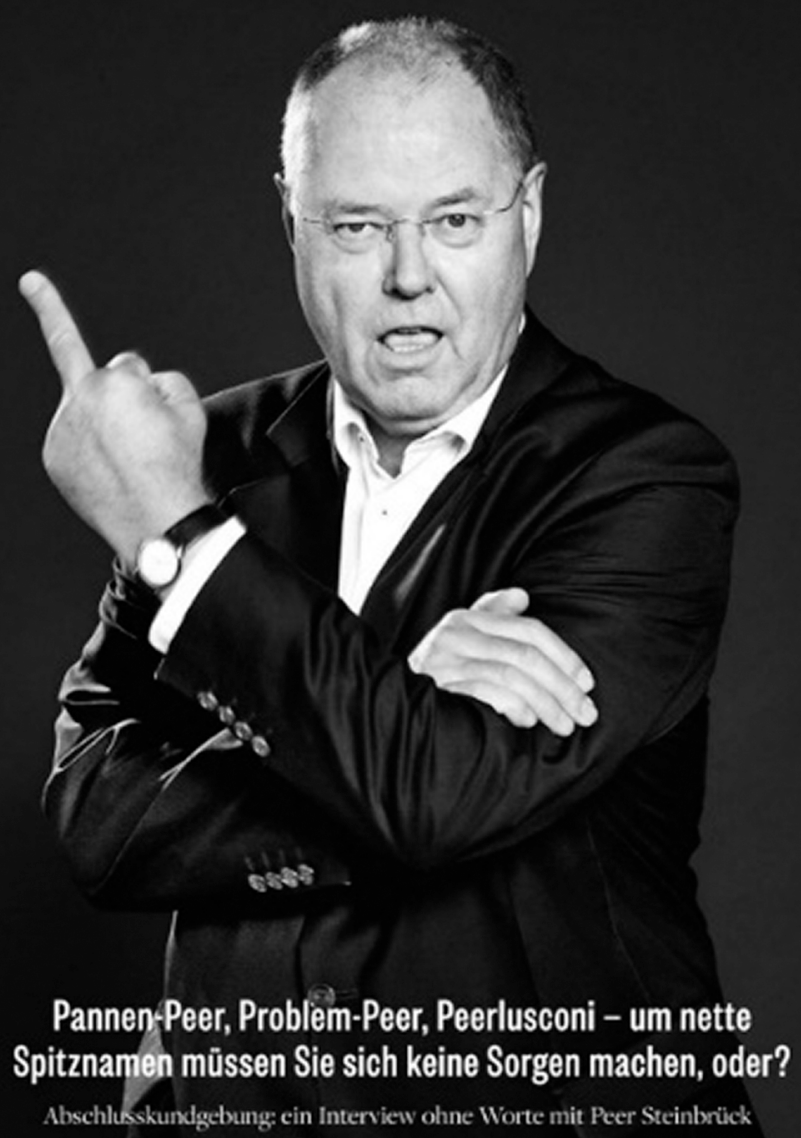




\section{Fazit}

Wie sehr sich die politischen Kulturen Deutschlands und der Vereinigten Staaten im Hinblick auf die komische Modulation politischer Kommunikation unterscheiden, wurde aus der Sichtung der Forschungsliteratur wie aus den beiden kleinen Fallstudien gleichermaßen deutlich. Während Barack Obama die Normalitätserwartungen in der US-amerikanischen politischen Kultur bediente und bei seinen Auftritten ausgiebig von der komischen Modulation Gebrauch machte, erlitt Peer Steinbrück mit seinem Humorwahlkampf 2013 doch deutlich Schiffbruch. Seine Ironie wurde nicht verstanden, er erlitt Seriositätsverluste, und sein Ausflug in die jugendliche Spaßkultur von Joko und Klaas brachte keine entscheidenden Gewinne in seiner Kampagne.

Und während der letzte Präsidentschaftswahlkampf zwischen Donald Trump und Hillary Clinton 2016 dadurch gekennzeichnet war, dass beide Kandidaten gezielt Satire- und Comedyformate aufsuchten, um dort zu punkten und für ihre Politik zu werben, zeigte der Bundestagswahlkampf 2017 eine auffällige Humorabsenz. Satirische Talkformate spielten, anders als im Humorwahlkampf 2013, nahezu keine Rolle. Komisch modulierte Politikerauftritte bildeten die große Ausnahme in einem insgesamt sehr unspektakulären, für viele Beobachter von großer Langweile geprägten Wahlkampf.

Die hier formulierten vergleichenden Beobachtungen müssten natürlich systematisch weitergeführt werden, um seriöse und belastbare wissenschaftliche Befunde zu ermöglichen. Vor allem eine Ausweitung auf weitere politische Kulturen, etwa die Großbritanniens und Frankreichs, verspricht durchaus interessante Erkenntnisse in einer Thematik, die bislang fast vollständig aus dem Fokus der politischen Kulturforschung ausgegrenzt blieb.

\section{Literatur}

Baym, Geoffrey 2009: Stephen Colbert's Parody of the Postmodern. In: Jonathan Gray u.a. (Hg.), Satire TV. New York, London, S. 124-144.

Behrmann, Sven 2002: Politische Satire im deutschen und französischen Rundfunk. Würzburg.

Brummack, Jürgen 1971: Zu Begriff und Theorie der Satire. In: Deutsche Vierteljahresschrift für Literaturwissenschaft und Geistesgeschichte 45, S. 275-377.

Budzinski, Klaus/Hippen, Reinhard 1996: Metzler Kabarett Lexikon. Stuttgart.

Claßen, Ralf u.a. 2015: Das TV-Duell. In: Oliver Strijbis, Kai-Uwe Schnapp (Hg.), Aktivierung und Überzeugung im Bundestagswahlkampf 2013. Wiesbaden, S. 159-176. 
Cooper, Anthony Ashley, Third Earl of Shaftesbury 1709: Sensus Communis. Ein Versuch über die Freiheit von Witz und Laune [1709]. Zweisprachige Ausgabe englisch/deutsch, herausgegeben, übersetzt und kommentiert von Wolfram Benda u.a., Stuttgart 1992.

Dagnes, Alison 2012: A Conservative Walks Into a Bar. The Politics of Political Humor. Basingstoke.

Dörner, Andreas 2001: Politainment. Politik in der medialen Erlebnisgesellschaft. Frankfurt a.M.

Dörner, Andreas 2003: Politische Kulturforschung. In: Herfried Münkler (Hg.), Politikwissenschaft. Ein Grundkurs. Reinbek bei Hamburg, S. 587-619.

Dörner, Andreas 2006: Politische Unterhaltung zwischen Inszenierung und Kontingenz. Fernsehtalk als Kampfarena am Beispiel der Harald-Schmidt-Show. In: Brigitte Frizzoni, Ingrid Tomkowiak (Hg.), Unterhaltung. Konzepte - Formen - Wirkungen. Zürich, S. 107-130.

Dörner, Andreas/Vogt, Ludgera 2016: Politiker im Satireformat: Aspekte der Selbstund Fremdinszenierung politischer Akteure in der »heute-show«. In: Zeitschrift für Parlamentsfragen 47, Heft 1, S. 195-211.

Dörner, Andreas/Vogt, Ludgera (Hg.) 2017: Wahlkampf mit Humor und Komik. Selbst- und Fremdinszenierung politischer Akteure in Satiretalks des deutschen Fernsehens. Wiesbaden.

Eco, Umberto 1985: Der Name der Rose. Roman. München.

Fleischer, Michael 1989: Eine Theorie des Kabaretts. Versuch einer Gattungsbeschreibung. Bochum.

Fleischer, Michael 2003: Kabarett. In: Hans-Otto Hügel (Hg.), Handbuch populäre Kultur, Begriffe, Theorien und Diskussionen. Stuttgart, S. 274-278.

Freud, Sigmund 1905/1986: Der Witz und seine Beziehung zum Unbewussten. Frankfurt a.M.

Gäbler, Bernd 2016: Quatsch oder Aufklärung? Witz und Politik in heute show und Co. Frankfurt a.M.

Glodek, Tobias u.a. (Hg.) 2007: Politisches Kabarett und Satire. Berlin.

Goffman, Erving 1977: Rahmen-Analyse. Ein Versuch über die Organisation von Alltagserfahrungen. Frankfurt a.M.

Halper, Donna L. 2009: Icons of talk. The Media Mouths that Changed America. Westport, CT.

Harrington, Stephen 2011: The Uses of Satire: Unorthodox News, Cultural Chaos and the Interrogation of Power. In: Journalism: Theory, Practice and Criticism 13, H. 1, S. 38-52.

Harris, Leon 1972: Humor in der Politik. Boppard/Rhein.

Henningsen, Jürgen 1967: Theorie des Kabaretts. Ratingen. 
Hoffman, Lindsay A./Young, Dannagal G. 2011: Satire, Punch Lines, and the Nightly News: Untangling Media Effects on Political Participation. In: Communication Research Reports 28, H. 2, S. 159-168.

Hoinle, Markus 2003: Ernst ist das Leben, heiter die Politik. Lachen und Karneval als Wesensmerkmale des Politischen. In: Aus Politik und Zeitgeschichte B 53 2003. S. 3-11.

Holbert, Robert Lance (Hg.) 2015: Entertainment Media and Politics. Advances in Effect-Based Research. New York.

Jones, Jeffrey P. 2005: Entertaining Politics. New Political Television and Civic Culture. Lanham.

Jones, Jeffrey. P. 2010: Entertaining Politics. Satiric television and political engagement. Lanham.

Keding, Karin/Struppert, Anika 2006: Ethno-Comedy im deutschen Fernsehen Inhaltsanalyse und Rezipientenbefragung $\mathrm{zu} »$ Was guckst du ?!«. Berlin.

Kleinen-von Königslöw, Katharina/Keel, Guido 2012: Localizing The Daily Show: The heute show in Germany. In: Popular Communication 10, S. 66-79.

Kleinen-von Königslöw, Katharina 2014: Politischer Humor in medialen Unterhaltungsformaten. In: Marco Dohle/Gerhard Vowe (Hg.), Politische Unterhaltung - Unterhaltende Politik, Forschung zu Medieninhalten, Medienrezeption und Medienwirkungen. Köln, S. 163-191.

Knop, Karin 2007: Comedy in Serie. Medienwissenschaftliche Perspektiven auf ein TV-Format. Bielefeld.

Kosthorst, Daniel 2010: »In lustiger Gesellschaft?« Politischer Humor als Spiegel der Zeit. In: Daniel Kosthorst, Stiftung Haus der Geschichte der Bundesrepublik Deutschland (Hg.), Spass beiseite, Humor und Politik in Deutschland. Leipzig, S. 11-23.

Kotthoff, Helga u.a. 2013: Komik (in) der Migrationsgesellschaft. Konstanz.

Tryon, Chuck 2016: Political TV. New York, London.

Leontiy, Halyna (Hg.) 2017: (Un)Komische Wirklichkeiten. Komik und Satire in (Post-)Migrations- und Kulturkontexten. Wiesbaden.

Lyttle, John 2001: The effectiveness of humor in persuasion: The case of business ethics training. In: Journal of General Psychology, 128, S. 206-216.

Morreall, John 1983: Taking Laughter Seriously. Albany.

Nagel, Armin 2003: Comedy. In: Hügel, Hans-Otto (Hg.), Handbuch populäre Kultur. Begriffe, Theorien und Diskussionen. Stuttgart, S. 138-142.

Nitsch, Cordula/Lichtenstein, Dennis 2013: Politik mal anders: Die Politikdarstellung in »Harald Schmidt« im Kontrast zur »Tagesschau«. In: Publizistik 58, S. 389-407.

Parkin, Michael 2014: Talk Show Campaigns. Presidential Candidates on Daytime and Late Night Television. Hoboken. 
Parsi, Kayhan 2011: The Political Satirist as Public Intellectual: The Case of Jon Stewart. In: American Journal of Bioethics 11 2011, H. 12. S. 3-6.

Peter, Barbara 2015: Satire in journalistischer Mission. Studie zu den journalistischen Leistungen von TV-Kabarettisten als Interviewer. Dissertation. Fribourg.

Peterson, Russell L. 2008: Strange Bedfellows. How Late-Night Comedy Turns Democracy into a Joke. New Brunswick u.a.

Ratzmann, Katharina u.a. 2016: Politiker- statt Politikverdrossenheit? Wie die Nutzung der heute-show die politischen Einstellungen von jungen Erwachsenen beeinflussen kann. In: Miriam Czichon u.a. (Hg.), Rezeption und Wirkung fiktionaler Medieninhalte, Baden-Baden, S. 211-238.

Rohe, Karl 1994: Politik. Begriffe und Wirklichkeiten. 2. Aufl. Stuttgart u.a.

Strasser, Hermann/Graf, Achim 2000: Schmidteinander ins 21. Jahrhundert. Auf dem Weg in die Spaß- und Spottgesellschaft?. In: Aus Politik und Zeitgeschichte, H. B12, S. 7-16.

Strasser, Hermann/Graf, Achim 2004: Kabarett nach dem Kabarett. Politik und Satire in der Spaßgesellschaft. In: Jörg-Uwe Nieland, Klaus Kamps (Hg.), Politikdarstellung und Unterhaltungskultur, Zum Wandel der politischen Kommunikation. Köln, S. 73-92.

Timberg, Bernard M. 2002: Television Talk. A History of the TV Talk Show. Austin, TX.

Tsakona, Villy/Popa, Diana Elena (Hg.) 2011: Studies in Political Humour. In between Political Critique and Public Entertainment. Amsterdam, Philadelphia.

Vogel, Benedikt 1993: Fiktionskulisse. Poetik und Geschichte des Kabaretts. Paderborn.

Wellstein, Benjamin 2007: Kabarett vs. Comedy. Welche Unterschiede machen den Unterschied?. In: Tobias Glodek u.a. (Hg.), Politisches Kabarett und Satire, Berlin, S. 157-167.

Yarwood, Dean L. 2004: When congress makes a joke. Congressional humor then and now. Lanham u.a.

\section{Abbildungsverzeichnis}

Abb. 1: Screenshot aus der Rubrik Mean Tweets bei Jimmy Kimmel live am 13. März 2015; abrufbar über https://www.youtube.com/watch?v=RDocnbkHjhI, TC: 1:18.

Abb. 2: Screenshot aus der Sendung The Colbert Report am 9. Dezember 2014, TC 1:57.

Abb. 3: Screenshot aus der Sendung Circus HalliGalli am 16. September 2013, TC 28:54.

Abb. 4: Titelseite des Süddeutsche Zeitung Magazins vom 15. September 2013. 



\section{Politische TV-Serien und Politische Kultur Ein Forschungsprogramm und Betrachtungen zu einem deutschen Sonderweg}

Andreas Dörner

\section{Einleitung: fiktive und reale Politikerinnen und Politiker}

Politische Fernsehserien erfreuen sich derzeit weltweit einer besonderen Beliebtheit. Nicht nur konventionelle Rundfunkanbieter, sondern gerade auch die Produzenten nicht-linearen Fernsehens auf Internetplattformen wie Netflix haben viele erfolgreiche Politserien im Angebot. Die Serien haben eine solche Medienpräsenz gewonnen, dass auch reale Politikerinnen und Politiker sich genötigt fühlen, auf ihre fiktionalen Konkurrenten einzugehen. So zeigte sich der amtierende USPräsident Barack Obama bei einem Meeting im Dezember 2013 begeistert von der im gleichen Jahr gestarteten Serie House of Cards. Er äußerte große Bewunderung für die rücksichtslose Effizienz, mit der Frank Underwood die politischen Dinge in Washington bewege. "This guy's getting a lot of stuff done « hieß es mit Blick auf die Serienfigur, die in der ersten Staffel der Serie als Mehrheitsführer (»Majority Whip«) im Kongress die Strippen zieht. In eine ähnliche Richtung ging eine Äußerung von Kevin McCarthy, der seit 2011 als realer Mehrheitsführer der Republikaner im Kongress agierte. McCarthy sagte mit Bezug auf den fiktiven Underwood: »If I could kill just one member of Congress I would never have to worry about another vote. ${ }^{1}$ Er könne also seinen Job auch effektiver machen, wenn ihm vergleichbare Mittel wie Underwood zur Verfügung stünden.

In den Äußerungen der realen politischen Akteure wird ein großes Maß an Anerkennung für die fiktive Figur sichtbar. Das erstaunt schon etwas, wenn man bedenkt, wie Frank Underwood Politik macht: Er lügt, er betrügt, er intrigiert und scheut auch vor Mord nicht zurück, um an die Macht zu kommen und diese zu erhalten. Warum dann trotzdem das Lob? Natürlich sind derartige Äußerungen ironisch gerahmt; das Publikum weiß, dass Obama sich nicht wirklich am Vorbild des skrupellosen Serienhelden orientieren wird. Die ironischen Bezugnahmen auf

1 Vgl. dazu den Artikel auf: www.politico.com/blogs/politico-now/2014/02/spacey-house-of-cardsnot-far-from-reality-183420, Zugriff am 23.02.2017. 
die politische Fernsehwelt dienen als Mittel der Imagebildung - nicht in der Weise, dass man Rückschlüsse von der fiktiven Figur auf sich selbst als Politikerin oder Politiker nahelegen will, sondern so, dass man sich in der Ironie als ein humorvoller Mensch inszenieren kann, der >coole Serien kennt und sich in lustiger Form mit anderen Zuschauern in eine unterhaltsame Kommunikation darüber begibt. Man gehört dazu, baut Distanz zum Wahlvolk ab und gelangt so auf angenehme Art in die Schlagzeilen. Das machen gerade die Äußerungen von McCarthy gut deutlich. Der Republikaner erzählte öffentlich, er habe sich erst an dem Punkt dazu bereitgefunden, den Schauspieler Kevin Spacey bei der Vorbereitung auf dessen Rolle zu unterstützen, als er erfahren hatte, dass die Figur Underwood Demokrat sei. So konnte sich der Politiker als selbstironisch-sympathischer Zeitgenosse inszenieren und punktete bei den Wählern.

Interessant ist vor diesem Hintergrund, wie deutsche Politikerinnen und Politiker die Serie kommentieren. Hierzu hat die Tageszeitung Die Welt vor einiger Zeit eine kleine Umfrage durchgeführt und die Äußerungen der echten Polit-Akteure fallen in Deutschland deutlich anders aus als die der amerikanischen Kollegen. ${ }^{2}$ Die meisten betonen eine starke Diskrepanz zwischen der Serienrealität und der außermedialen Realität, in der wirklich Politik gemacht werde. So sagt etwa Ralf Stegner, Stellvertretender SPD-Bundesvorsitzender: »Für mich als Politiker ist House of Cards genauso ein Pflichtprogramm, wie der Tatort für einen Polizisten - völlig unrealistisch, aber meistens sehr unterhaltsam.« Und Christine Lambrecht, die als Parlamentarische Geschäftsführerin der SPD-Bundestagsfraktion einen vergleichbaren Job wie Underwood macht, argumentiert in eine ähnliche Richtung:

»Die Serie lebt von einer filmischen Überspitzung, und genau das ist es, was mich so fasziniert. Die Realität des politischen Alltags in Deutschland spiegelt die Serie - zum Clück - nicht wider, auch wenn es immer wieder Bezüge zu politischen und parlamentarischen Abläufen gibt. Trotz meiner Faszination für die Serie bin ich fest überzeugt, dass ein solches Agieren auf der Grundlage von persönlichen Schwächen und unter Einbeziehung des familiären Umfelds in Deutschland nicht möglich ist.«

Ähnlich äußern sich auch der FDP-Bundesvorsitzende Christian Lindner und die Vertreterin der Linken Katja Kipping. Während die amerikanischen Politikerinnebn und Politiker mit Ironie und Augenzwinkern die Parallelen zwischen Realität und Fiktion betonen, weisen die deutschen Akteurinnen und Akteure nüchtern (und humorfrei) auf die Unterschiede hin, als hätten sie Angst, deutsche Zuschauer könnten da etwas verwechseln.

2 Alle im Folgenden angeführten Äußerungen sind zitiert nach: https://www.welt.de/politik/ deutschland/gallery137845657/Darum-fasziniert-House-of-Cards-unsere-Politiker.html, letzter Zugriff am 10.03.2017. 
Etwas differenzierter formuliert Renate Künast von den Grünen, die »einen Funken Wahrheit [...] über den politischen Betrieb« in der Serie entdeckt. Den radikalsten Kommentar in dieser Richtung formuliert schließlich ihr Parteifreund, der frühere Bundesminister Jürgen Trittin von den Grünen:

»House of Cards zeigt einfach, wie Politik funktioniert - bis hin zu den Lehrsätzen: >All politics is local und >Politics mainly is muddling through<. Das kann man da prima lernen. Neben der heute-show ist House of Cards die gelungenste Form der Staatsbürgerkunde.«

Hier wird klar und auch ganz unironisch gesagt, dass das Intrigenspiel des Frank Underwood mehr mit der politischen Alltagsrealität hierzulande zu tun habe, als es sich so manche Bürgerin und mancher Bürger vorstellt. Erscheint Jürgen Trittin die Serie nun deshalb so realistisch, weil er selbst ein moralfreier Machtpolitiker wie Frank Underwood ist? Das wäre wohl eine vorschnelle Schlussfolgerung. Aber das Image des früher oft sehr moralistisch auftretenden Grünen wird hier durchaus auf besondere Weise tangiert.

Nun ist die Bezugnahme echter politischer Akteure auf fiktive Polit-Figuren ein spezieller Fall, der zuweilen vorkommt, jedoch nicht besonders häufig ist. Und er ist dennoch Teil eines Spiels, in dem fiktionale Konstruktionen politischer Akteure durch Serien und Reihen das öffentliche Image von Politik und Politikern prägen. Zugleich ist er Teil deutungskultureller Diskurse in den politischen Kulturen der westlichen Welt. Politserien liefern Anlässe, um über Politik und politische Akteure öffentlich zu reflektieren.

Der vorliegende Beitrag möchte den Zusammenhang von politischen TV-Serien und politischer Kultur etwas genauer in den Blick nehmen. Politserien erscheinen hier als Bestandteil des politischen Imaginären: Als Bestandteil symbolisch verdichteter politischer Vorstellungswelten, anhand derer die Bürgerinnen und Bürger eines Landes Politik wahrnehmen und bewerten. Um den Zusammenhang von politischer Kultur und Politserien zu beleuchten, soll die Argumentation folgenden Gang nehmen: Der erste Teil beschreibt, was eine Politserie überhaupt ist und wie sich ihr Verhältnis zur politischen Realität gestaltet. Dabei erfolgt auch eine typologische Betrachtung der fiktionalen Konstruktionen des Politischen. Danach wird der Ort politischer Serien in der politischen Kultur bestimmt und ein konkretes Forschungsprogramm vorgestellt, in dem Politserien als Ausdrucksformen politischer Kultur analysiert werden können. Abschließend wird der deutsche Sonderweg im Bereich der Politserien betrachtet, der insbesondere bei vergleichenden Analysen deutlich wird. Dabei stellt sich die Frage nach politisch-kulturellen Ursachen für die spezifische Konstellation hierzulande. ${ }^{3}$

3 Grundsätzlich ist das Verhältnis von Medien und politischer Kultur als ein Wechselverhältnis zu denken: Zum einen bilden politisch-kulturelle Traditionen und Selbstverständlichkeiten ei- 


\section{Politserien als fiktionale Konstruktion des Politischen}

Eine Politserie ist eine in Episoden und Staffeln unterteilte, fiktionale und audiovisuelle Erzählung über politische Wirklichkeiten. ${ }^{4}$ Mit "politischen Wirklichkeiten« sind dabei zunächst politische Zusammenhänge im engeren Sinne gemeint: Geschehnisse, die mit politischen Institutionen und Akteuren wie Regierungen und Parlamenten, Präsidenten, Parteien und politischen Journalisten, Geheimdiensten und Spionage zu tun haben. Teilweise wird bei dem Begriff »Politserie« auch ein weiterer Politikbegriff zugrunde gelegt, sodass Serien, die sich mit Macht und Herrschaft sowie mit Kämpfen um Anerkennung befassen, ebenfalls einbezogen werden. In diesem Sinne kann dann auch etwa die Lindenstraße als Politserie bezeichnet werden.

Der Geltungsanspruch einer Serie ist ein anderer als der eines Berichts oder einer Reportage. Die Erzählung ist gerahmt wie eine Als-ob-Welt, vergleichbar einem Spiel, bei dem alle wissen, dass es eine zur Alltagswelt differente Wirklichkeit konstruiert (vgl. Stumm 1996: 147) . Und dennoch bleibt sie stets auf die Alltagswelt bezogen. Gerade dieser Rückbezug, der ständige Vergleich zwischen fiktionaler Welt und außermedialer Alltagswelt, begründet die Relevanz der erfundenen Wirklichkeit einer Serie. ${ }^{6}$

Entscheidend ist, dass Politserien keine Dokumentationen oder Teile der journalistischen Berichterstattung, sondern Unterhaltungsformate sind. Sie müssen bestimmten dramaturgischen Anforderungen gehorchen, wenn sie erfolgreich sein wollen. Das "starke und langsame Bohren von harten Brettern mit Leidenschaft und Augenmaß zugleich«, wie Max Weber das politische Handeln beschrieb (1919: 66), bringt vergleichsweise wenig Unterhaltungswert hervor. Daher müssen Seri-

ne wichtige Rahmenbedingung dafür, was in den Medien einer Cesellschaft erfolgreich produziert werden kann; zum anderen sind Medienprodukte an der Perpetuierung, Formung und somit auch Veränderung politischer Kulturen wesentlich beteiligt; vgl. dazu ausführlich schon Dörner (2000).

4 Zur Definition, Struktur und Funktionsweise der Politserie siehe grundlegend Dörner (2016). Zur Reflexion des aktuellen Serienbooms siehe die Analysen in Kiegeland et.al. (2014); grundlegende Perspektiven zur semantischen und ästhetischen Analyse politischer Serien finden sich in Frame (2014), Nitsch/Eilders (2014, 2015), Däwes et.al. (2015), Tryon (2016) und Kaklamanidou/Tally (2017).

5 Zur Funktionslogik des Spiels, das sich in vieler Hinsicht ähnlich wie die Fiktion verhält, siehe immer noch den Klassiker Homo ludens von Johan Huizinga (1956); Huizinga beschreibt die Andersartigkeit der Realität im Spiel als »Zauberzirkel«.

6 Zu dieser grundlegenden Logik der fiktionalen Unterhaltungswelten und ihrer Bezüge zur außermedialen Realität vgl. Luhmann (2010: 112). 
en verkürzen, verdichten, zuspitzen und beschleunigen ${ }^{7}$. Alles passiert schneller, einfacher, eindeutiger als in der alltäglichen Wirklichkeit, daher sind Serien orientierungsfreundlich. Sie wollen mit dramaturgischen Mitteln unterhaltsame Spannungsbögen oder amüsante Situationskomik produzieren - und sie dürfen doch bei aller Zuspitzung den Kontakt zur außermedialen Wirklichkeit nicht völlig verlieren, sonst erscheinen sie uns belanglos. Die richtige Balance zwischen Unterhaltungswert und Realitätsbezug herzustellen, das ist der Drahtseilakt, den politische Serien leisten müssen, um Erfolg zu haben.

Warum sind die Serien nicht nur in den USA, sondern auch in vielen europäischen Ländern so erfolgreich? Für das Publikum besteht die große Faszination politischer Serien in dem Versprechen, einen sonst in der medialen Berichterstattung unmöglichen Blick auf die Hinterbühne ${ }^{8}$ des politischen Betriebs erheischen zu können. Die Zuschauer sind dabei, wenn geheime Absprachen getroffen und Intrigen geschmiedet werden. Sie blicken den Protagonisten bei der politischen Arbeit über die Schulter. Sie erfahren sogar die geheimsten Gedanken der Akteure, wenn diese mit sich selbst sprechen, mit der Ehefrau intim eine Zigarette am Fenster teilen oder sich in vertrauensvollem Ton direkt an das Publikum wenden. Mit solchen "asides" produziert etwa die amerikanische Serie House of Cards eine Komplizenschaft zwischen dem skrupellosen Antihelden Frank Underwood und den Zuschauern, die durch die direkte Ansprache immer wieder ins gemeinsame Boot geholt werden.

Dabei wird den fiktionalen Welten eine besondere Authentizität dadurch verliehen, dass immer wieder Bezüge zur außermedialen Wirklichkeit eingebaut werden. Die Serien erhalten auf diese Weise Realitätsindikatoren. Das kann dadurch geschehen, dass reale politische Akteure in Gastrollen auftreten. So agiert beispielsweise die frühere US-Außenministerin Madeleine Albright in Staffel 2 der Serie Madam Secretary und belehrt das Publikum mit der feministischen Weisheit: »There is plenty of room in the world for mediocre men. There is no room for mediocre women. « Noch häufiger thematisieren Serien Geschehnisse, die das Publikum aus der außermedialen Realität kennt.

Oder aber die Figuren einer Serie sind komplett an echte Persönlichkeiten des öffentlichen Lebens angelehnt. So wurde die Hauptfigur Olivia Pope in der Serie Scandal nach dem Vorbild der realen Washingtoner Krisenberaterin Judy Smith konstruiert. Smith hatte früher in der Administration von George W. Bush gearbeitet, war in der Iran-Contra-Affäre Anfang der 1990er-Jahre ebenso tätig wie

7 Siehe hierzu auch die Arbeit von Sandra Nuy (2017), die systematisch den Stellenwert der zuspitzenden Dramaturgie für die fiktionale Konstruktion des Politischen am Beispiel von politischen Spielfilmen untersucht.

8 Zur Begrifflichkeit von Vorder- und Hinterbühne vgl. den Klassiker von Erving Goffman (2008). 
später bei der Schadensbegrenzung für Bill Clinton im Sexskandal um die Praktikantin Monica Lewinsky. Judy Smith gilt seit Jahren als einer der prominentesten Troubleshooter im politischen und wirtschaftlichen Leben der USA.

Wenn Smith dann auch noch im Produktionsteam der Serie als Co-Producerin tätig ist und die Autoren mit ihrem Praxiswissen berät, ist eine direkte Verbindung zwischen Fiktion und politischer Alltagswelt hergestellt. Eine solche Einbindung von Fachleuten und Insidern ist in der Serienproduktion durchaus an der Tagesordnung. Michael Dobbs, Autor der Buchvorlage für die britische wie für die amerikanische House of Cards-Serie, war Berater von Margaret Thatcher und arbeitete später als Stabschef der Britischen Regierung. Beau Willimon, Drehbuchautor, Produzent und Showrunner der amerikanischen House of Cards-Version war im Jahr 2000 an Hillary Clintons Kampagne für ihre Bewerbung als Senatorin des Staates New York beteiligt. Und auch die ZDF-Produktion Kanzleramt (2005) nahm bei den Drehbüchern die Dienste von Martin E. Süskind in Anspruch, der als politischer Redakteur unter anderem bei der Süddeutschen Zeitung, vor allem aber als Redenschreiber von Willy Brandt bekannt geworden war.

Welches Bild entwerfen Politserien nun von den politischen Akteuren und der von ihnen betriebenen Politik? Zwei spezifische Typen sind zunächst als antipodische Formen des Politischen in den Serien erkennbar: Idealpolitikerinnen und -politiker und Machtpolitikerinnen und -politiker.

Idealpolitikerinnen und -politiker, wie sie etwa in The West Wing, später auch bei Commander in Chief oder auch im deutschen Kanzleramt konstruiert wurden, verfolgen gute Ziele wie Gerechtigkeit, Freiheit oder Gemeinwohl und setzen dabei gute, d.h. legitime und legale Mittel zur Erreichung dieser Ziele ein. Die Protagonisten sind in dieser Welt umsichtig und moralisch integer, vor allem sind sie zumeist altruistisch motiviert. Sie kämpfen für die gute Sache, nicht für eigene Vorteile oder Karrieresprünge. Die Serien definieren hier einen utopischen Raum, der als normative Messlatte, als positives Gegenbild zur außermedialen politischen Realität, fungieren kann.

Auf der genau entgegengesetzten Seite befindet sich der Typus der Machtpolitikerin oder des Machtpolitikers. Machtpolitik definiert politisches Handeln als das Streben nach Macht. Diese wird jedoch nicht als Mittel zur Realisierung politischer Inhalte und Werte betrachtet, sondern unmittelbar als Selbstzweck: Macht wird eingesetzt zur Steigerung von Macht. Zugleich dient der Machterwerb als Mittel zur Steigerung von Anerkennung und Selbstwertgefühl. Ziel der Machtpolitik ist letztlich, das Ego der Akteure zu stärken. Machtpolitikerinnen und -politiker mag man nicht, sondern man respektiert und fürchtet sie. Frank Underwood ist ein Musterbeispiel des Machtbesessenen, der alle Normen und Werte ausblendet, um seine Ziele $\mathrm{zu}$ erreichen. Ihm geht es nicht um politische Inhalte, sondern um das Gestaltungsmedium selbst, das ihn wie eine Droge nach immer mehr verlangen lässt. 
Eine Figur wie Frank Underwood wiederum erfährt eine komische Spiegelung in satirischen Politserien wie Veep, in der die groteske Unfähigkeit einer Politikerin vorgeführt wird, die das Amt der Vizepräsidentin als Abstellgleis erfährt und nahezu alles dafür tut, wieder mehr in die Nähe wirklicher Macht zu kommen. Auch eine solche Akteurin hat Machterwerb und Machterhalt im Sinn. Auch bei ihr stellt sich das Mittel häufig als Zweck des Ganzen dar. Im Unterschied zum Königsdrama à la House of Cards geben die seriellen Komödien die Machtpolitik jedoch dem Gelächter des Publikums preis. Ihre Protagonisten sind lustig im ständigen Scheitern, sie denunzieren das eigene Tun durch Unfähigkeit und übertriebene Eitelkeit.

Zwischen Idealpolitik und Machtpolitik ist der Typus der Realpolitikerin oder des -politikers verortet. Realpolitikerinnen und -politiker deklarieren zwar gute Ziele, sehen aber, dass zu deren Erreichung mitunter moralisch fragwürdige Handlungsweisen erforderlich sind. Realpolitisch agieren beispielsweise die Hauptfiguren in Scandal, wenn sie den Präsidenten durch einen Wahlbetrug an die Macht bringen, dies aber in dem Glauben tun, dadurch tatsächlich den besseren Kandidaten zu fördern. Gerade Scandal zeigt aber auch sehr einprägsam, wie Realpolitik immer wieder in die Gefahr gerät, in reine Machtpolitik überzugehen. Wenn in der zweiten Staffel der Serie beispielsweise US-Präsident Fitzgerald Grant einer Verfassungsrichterin und Weggefährtin im Krankenhaus mit letalen Folgen die Luft abdreht, dann ist das ein politischer Mord mit dem Ziel, an der Macht zu bleiben. Und Scandal stellt auch moralische Fragen, wenn die Serie ihre Protagonisten immer wieder Folter einsetzen lässt, um die Wahrheit ans Licht zu bringen: Ist für den guten Zweck wirklich jedes Mittel erlaubt oder gibt es Grenzen?

Gerade angesichts der jüngst von Donald Trump geäußerten Sympathie für das Waterboarding ist ein solches fiktionales Szenario von höchster Aktualität und lädt dazu ein, über Möglichkeiten und Grenzen des Gewalteinsatzes in der Politik, insbesondere der Folter, zu reflektieren und sich damit auch Unterschiede in den politischen Kulturen der USA und der europäischen Demokratien klar zu machen. Die Drastik und die Häufigkeit, mit der Folterszenen in Scandal inszeniert sind, weist über einen realistischen Rahmen der Serie doch deutlich hinaus. Kaum ein Zuschauer wird ein solches, vor allem zu Unterhaltungszwecken zugespitztes Szenario für ein Abbild US-amerikanischer Alltagsrealitäten halten. Insofern opfert die Serie hier den Realismus zugunsten der dramaturgischen Erfordernisse einer Unterhaltungsproduktion.

Insgesamt jedoch scheinen realpolitische Typen in Politserien einen besonders engen Bezug zur außermedialen Wirklichkeit herzustellen, da sie ein simples Schwarz und Weiß vermeiden und den Preis für Machterwerb und politische Gestaltung aufzeigen. 


\section{Politserien und politische Kultur}

Die politische Kulturforschung ist seit ihren Anfängen in den 1950er-Jahren primär auf eine standardisierte, quantitative Methodik ausgerichtet (zum Überblick siehe Dörner 2003). Erst in den 1970er-Jahren beginnen einzelne Forscher wie Lowell Dittmer die Engführungen einer solchen Methodik aus der Perspektive eines kulturalistischen Wissenschaftsverständnisses zu kritisieren und Alternativen aufzuzeigen. Aaron Wildavsky in den USA und Karl Rohe in Deutschland haben solche Kritik zu einem Verständnis qualitativer politischer Kulturforschung ausgebaut (vgl. Rohe 1994: 162-167; Pesch 2000: 84-86; Pickel/Pickel 2006: 106-110), das dann auch theoretisch-methodologische Schnittstellen für eine Verknüpfung mit interpretativer Medienforschung eröffnete (Dörner 2003; Schwelling 2004: 21). Wenn mit politischer Kultur nicht nur quantitativ messbare Einstellungen, sondern auch qualitativ zu rekonstruierende Vorstellungen und symbolisch verdichtete Weltbilder inklusive der damit verbundenen Wertvorstellungen gemeint sind, dann ist es nur plausibel, methodisch den Weg über entsprechende symbolische Formen zu wählen: bildende Kunst, Denkmäler und Architektur, Musik, Literatur und Theater; schließlich vor allem Bewegtbildformate vom Kinofilm bis zur TV-Serie. ${ }^{9} \mathrm{Al}$ lerdings sind bislang nur wenige Arbeiten erschienen, die eine solche Perspektive systematisch weiterverfolgen (vgl. etwa Dörner 2000 und zuletzt Nuy 2017).

Interessant erscheint für den vorliegenden Zusammenhang vor allem eine Verbindung der qualitativen politischen Kulturforschung mit dem Begriff des »politischen Imaginären«, da hier die Dimension der Vorstellungen und symbolisch verdichteten Weltbilder akzentuiert werden kann im Hinblick auf Fiktionen, auf »Als-ob-Welten« (Vaihinger 1913), die kontrafaktisch und dennoch relevant für die politische Realität sind. Ungeachtet dessen, dass der Begriff des politischen Imaginären schillernd, vielfältig und schwer empirisch herunter $\mathrm{zu}$ brechen ist ${ }^{10}$, kann er gerade im Hinblick auf die fiktionalen politischen Welten von Fernsehserien doch verdeutlichen, dass solche fiktionalen Erzählungen das öffentliche Bild von Politik mitprägen und daher politisch-kulturell relevant sind.

Paula Diehl (2015; Diehl/Steilen 2016) hat im Kontext dieser Diskussion zudem darauf hingewiesen, dass es sinnvoll ist, auch den Begriff der Repräsentation kulturalistisch neu zu reflektieren. Vor diesem Hintergrund lassen sich dann die fik-

9 Hierzu grundlegend Dörner (2003). Eine wichtige Perspektive ist etwa die zur »visuellen Politik« (vgl. Drechsel 2009) sowie die Veröffentlichungen des Arbeitskreises Visuelle Politik, der maßgeblich von Wilhelm Hofmann gegründet und vorangetrieben wurde, beginnend mit Hofmann (1998). Zum Zusammenhang von politischer Kultur und Populärkultur siehe neben den einschlägigen Arbeiten der British Cultural Studies (vgl. Hepp 2010) vor allem Street (1997), van Zoonen (2005) und Baringhorst (2012).

10 Zur neueren Diskussion über das politische Imaginäre siehe u.a. Doll/Kohns (2014), Diehl (2015: 8off.) und Trautmann (2017). 
tionalen Figuren, die in Politserien agieren, in mehrfacher Hinsicht als politische Repräsentanten verstehen: als Repräsentanten real existierender politischer Akteurinnen und Akteure, die sich mit dem fiktionalen Bild von Politikerinnen und Politikern aus Serien auseinandersetzen müssen; aber auch als politische Repräsentantinnen und Repräsentanten der Bürger und Zuschauer, die ständig einen Abgleich zwischen realen und fiktiven Politikern vornehmen können.

In der Logik der politischen Repräsentation spielt zudem die Körperlichkeit der Akteurinnen und Akteure als Repräsentanten eine zentrale Rolle (Diehl 2015: 259). Gerade diese Dimension des Körpers gewinnt im audiovisuellen Text von TVSerien eine besondere Anschaulichkeit. Politische Serien definieren damit einen besonders relevanten imaginären Raum innerhalb der politischen Kultur, in dem Bilder des Politischen, Wahrnehmungen und Erwartungen an politische Akteure verhandelt werden können.

Die Diskussion über das politische Imaginäre lässt sichtbar werden, dass Imaginationen oft auch eine ästhetische Gestaltung erfahren haben (vgl. die Beiträge in Doll/Kohns 2016). Diese ästhetische Dimension der symbolischen Formen ist in der politischen Kulturforschung bislang weitgehend ausgeblendet geblieben. Dies ist umso erstaunlicher, als gerade Karl Rohe als einer der >Gründerväter < des kulturalistischen Konzeptes von politischer Kultur betont hat, dass politische Kulturen auch eine Ausdrucksseite haben, dass der politische Sinn immer auch sinnfällig werden muss (Rohe 1994: 174) und Politik auch unter Aspekten von Schönheit und passender Form betrachtet werde könne. ${ }^{11}$ Insbesondere die Verbindung von Bewegtbildästhetik und politisch-kulturellen Vorstellungswelten ist bislang nicht genauer betrachtet worden - was auch daran liegen mag, dass die erforderliche interdisziplinäre Kompetenz einer Verbindung von sozial- und medienwissenschaftlichen Methoden selten vorzufinden ist.

\section{Forschungsprogramm}

An dieser Stelle kann eine Art Forschungsprogramm für die Analyse von Politserien als Bestandteil politischer Kulturen skizziert werden. ${ }^{12}$ Eine solche Forschung hat

11 Vgl. Rohe (1996); siehe dazu auch, anschließend an Rohes Überlegungen, die Ausführungen zur politischen Ästhetik als »Charismagenerator « bei Dörner (1995: 68), die systematisierenden Perspektiven zum Zusammenhang von politischer Kultur und Medienästhetik bei Dörner (2003a) und die Beiträge in Vorländer (2003).

12 Eigene Vorarbeiten für die Umsetzung eines solchen Forschungsprogramms finden sich in Dörner $(1998,1999,2000)$. Thematisiert wurde hier der Beitrag von populären Kinofilmen und Fernsehproduktionen bei der Inszenierung, Aufführung und Neuinterpretation von Traditionsbeständen der US-amerikanischen politischen Kultur. Dabei wurde auch das Potential eines medienanalytischen Ansatzes für die qualitative politische Kulturforschung diskutiert und als me- 
grundsätzlich das Zusammenspiel von semantischer Dimension und ästhetischen Gestaltungsmitteln zu erfassen. Dabei ist zu klären, wie jeweils die fiktionale politische Welt funktioniert, welche kollektiven und individuellen Akteure sie gestalten, welche Institutionen relevant sind und welche politischen Handlungsformen mit Einsatz welcher Ressourcen und Instrumente beobachtet werden können. Auf der ästhetischen Ebene ist zu untersuchen, welche Erzählformen und Dramaturgien zum Einsatz kommen, wie die Figurenkonstruktion erfolgt, welche Formen der Bild- und Tongestaltung zum Einsatz kommen und welchen Einfluss all diese Gestaltungsmittel auf die Konstruktion des Politischen ausüben. Die rekonstruierten Muster der politischen Semantik und der politischen Ästhetik sind dann jeweils in der politischen Kultur der Gesellschaft, in der die Serien produziert und rezipiert werden, zu kontextualisieren. Hier geht es zum einen darum zu klären, inwieweit die Serien Vorstellungsmuster, Wahrnehmungsmuster und Werte der politischen Kultur aufgreifen und ggf. modifiziert verarbeiten; inwieweit die produzierenden Akteure der Serien gezielt deutungskulturelle Praxis betreiben, indem sie den Serien intendiert bestimmte politisch-kulturelle Muster einschreiben; und inwiefern Rezipienten politischer Serien bei der Aneignung der audiovisuellen Texte soziokulturelle Dispositionen der jeweiligen politischen Kultur einbringen.

Derartige Analysen können einer Differenzierung der drei Dimensionen Produktion, Text und Rezeption folgen, wie sie sich in anderen, bereits erfolgreich durchgeführten ethnografisch eingebetteten Videoanalysen bewährt hat. ${ }^{13}$

Produktion: Hier geht es darum, zunächst einmal Produktionsabläufe zu rekonstruieren im Sinne der »Production Studies« (Mayer et.al. 2009, Banks u.a. 2016) und des Ansatzes der »Production Culture« (Caldwell 2008). Dabei ist insbesondere zu klären, in welcher Weise und woher bei der Produktion Wissen über Abläufe des realen politischen Prozesses eingeholt wird. Werden systematisch auch politische Experten bei der Erstellung von Storylines, Figurenkonzeptionen und den konkreten Drehbüchern herangezogen und welche sind dies im Einzelnen? In welchen Interaktionskonstellationen werden die Grundlinien der politischen Welt,

thodischer Zugang entwickelt (Dörner 1998a, 2003, 2006). Später wurde zu diesem Themenbereich in Zusammenarbeit mit der Bundeszentrale für politische Bildung ein Sammelband zu aktuellen Phänomenen der politischen Unterhaltungskultur in Deutschland veröffentlicht (Dörner/Vogt 2012).

13 In zwei von der DFG finanzierten empirischen Projekten wurde das Forschungsdesign einer auf Produktion, Text und Rezeption fokussierten, ethnografisch eingebetteten Videoanalyse entwickelt, verfeinert und anhand von zwei populärkulturellen Cegenstandsbereichen erfolgreich erprobt: Das erste Projekt befasste sich mit der Selbst- und Fremdpräsentation von politischen Akteuren in Personality-Talkshows des deutschen Fernsehens (siehe dazu Dörner et.al. 2015), das zweite Projekt thematisierte den Wahlkampfeinsatz von Humor und Komik im Kontext satirischer Talkformate im Bundestagswahlkampf 2013 (siehe dazu Dörner/Vogt 2017 sowie zur Darstellung der Methodik im Besonderen Dörner/Vogt 2015). 
die den Handlungsraum einer Serie definieren, festgelegt? Weiterhin kann untersucht werden, mit welchen Motiven, Zielen und mit welchem Selbstverständnis die zentralen Akteure des Produktionsprozesses, nämlich Produzenten, Showrunner, Autoren und ggf. Berater, agieren. Verstehen sie sich beispielsweise primär als Unterhaltungsproduzenten, denen die jeweiligen fiktionalen politischen Welten nur ein geeignetes Setting für die Produktion von Spannung oder Komik bedeuten, oder verfolgen sie mit ihren Produktionen so etwas wie ein deutungskulturelles Projekt mit entsprechenden politischen Bildungsabsichten? Welche Rolle spielt für die Akteure die Feldbeobachtung in dem Sinne, dass Vorläufer- und Konkurrenzproduktionen beachtet, als Vorbilder, Anregungsquelle oder Kontrastfolien genutzt werden? Bilden sich auf diese Weise spezifische Kulturen politischer Serien mit eigenen Traditionslinien heraus?

Text: Die audiovisuellen Texte der Serien können erstens daraufhin analysiert werden, welche Bilder der Politik (politischer Prozess, politische Inhalte und Themen, politische Institutionen, Normen und Werte) und der politischen Akteure (Figurenkonstruktion im Hinblick auf soziale Merkmale, Charakterzüge, Handlungsformen, Bindung des Handelns an Normen und Werte) sie konstruieren. Dabei ist auch zu untersuchen, welche Bezüge die konstruierten fiktionalen Welten der Serien zur nichtfiktionalen, außermedialen Realität aufweisen: werden reale Ereignisse und Prozesse verarbeitet, treten sechte politische Akteure auf oder sind klare Bezüge zwischen fiktionalen Figuren und realen Personen erkennbar? Wie werden reale politische Institutionen, beispielsweise Parlamente oder Regierungskabinette, dargestellt? Inwieweit bietet der Text den Zuschauern jeweils Einblicke in die politische Hinterbühne, wie sind diese Hinterbühnen beschaffen, wie ist die innerdiegetische Diskrepanz zwischen politischer Vorder- und Hinterbühne jeweils konstruiert? Agieren die politischen Akteure primär aufrichtig oder agieren sie mit Täuschungen im Sinne Goffmans (2008); sind sie vielleicht sogar mit einer zynischen Haltung unterwegs?

Diese semantischen Dimensionen der Konstruktionen des Politischen können zweitens jeweils in Relation gesetzt werden zu den Formen der ästhetischen Gestaltung der Serien. Wie wirken sich Besonderheiten der Erzählweise oder der Figurenkonstruktion auf die entworfenen Politik- und Politikerbilder aus? Finden beispielsweise eher Gestaltungsmittel konventionellen Erzählens Anwendung oder aber Mittel narrativer Komplexität, wie sie im Kontext der Debatte über Quality TV erörtert werden, und führen diese unterschiedlichen Gestaltungsmittel auch zu unterschiedlich angelegten Konstruktionen politischer Abläufe? Wie wirkt sich ein Stilmittel wie das Durchbrechen der vierten Wand ${ }^{14}$ in House of Cards auf die im

14 Dieses Stilmittel findet im Theater und auch in den audiovisuellen Bewegtbild-Medien Verwendung. Gemeint ist, dass die üblicherweise vorhandene Abgrenzung der in sich abgeschlossenen fiktionalen Welt eines Films, einer Serie oder eines Theaterstücks aufgebrochen wird, indem die 
Text angelegten Zuschauerrollen aus (Distanz oder Komplizenschaft)? Finden Formen von Komik, Ironie und Satire Anwendung und was ist die spezifische Funktion solcher Elemente im audiovisuellen Serientext? Werden die fiktionalen Figuren moralisch eindeutig oder komplex-ambivalent gezeichnet, und wie wirkt sich das auf das Bild politischer Akteure in der Serie aus? Im Vergleich verschiedener Serienwelten soll untersucht werden, inwiefern es intertextuelle Ähnlichkeiten und Bezüge zwischen den Serien oder sogar so etwas wie gemeinsame Diskursräume gibt.

Drittens sollen die Konstruktionen des Politischen in den audiovisuellen Serientexten in Beziehung gesetzt werden $\mathrm{zu}$ den politischen Kulturen, in denen sie jeweils kontextualisiert sind. Lassen sich hier jeweils Bezüge erkennen, d.h. haben die Texte politisch-kulturelle Traditionsbestände sowie aktuelle Vorstellungsund Wertmuster aufgenommen und verarbeitet? Da Serien zunächst einmal Unterhaltungsprodukte sind, die ein möglichst großes Publikum finden sollen, ist zu vermuten, dass sie in ihrer Politikkonstruktion bewusst an vorhandenen Selbstverständlichkeiten und vertrauten Bildern einer politischen Kultur anknüpfen, um das Publikum sabzuholen . Werden solche Selbstverständlichkeiten jeweils bestätigt oder irritiert? So ist beispielsweise bei einer populären und kommerziell erfolgreichen Produktion wie The West Wing unverkennbar, dass hier zentrale Muster der republikanischen Tradition in der US-amerikanischen politischen Kultur zugrunde liegen. Die Figuren agieren altruistisch und gemeinwohlorientiert, sie stellen private Belange konsequent hinter ihre öffentlichen Aufgaben zurück und verstehen die aktive Teilhabe am politischen Prozess als Voraussetzung einer gelingenden menschlichen Existenz (zu den Traditionslinien der amerikanischen politischen Kultur siehe Bellah et.al. 1987: 52). In der Gegenbewegung lässt sich die politische Welt einer Serie wie House of Cards verstehen als radikale Interpretation des utilitaristischen Individualismus, wenn die Hauptfigur Frank Underwood ihren individuellen Nutzen maximiert, indem sie das politische Handeln (bis hin zum Aufbau sexueller Beziehungen mit Journalistinnen) vollkommen dem Erwerb und Erhalt persönlicher Macht und Anerkennung unterordnet. Im deutschen Kontext könnte die starke Ausrichtung der politischen Medienkultur auf Kriminalserien und - reihen als Hinweis auf die Wirksamkeit der traditionellen Staatsorientierung in der politischen Kultur gelesen werden, da die Polizei schließlich als eines der wichtigsten, ordnungsstiftenden Exekutivorgane des Staates fungiert (vgl. Rohe 1994: 171; dazu unten mehr).

Rezeption: In der Rezeptionsdimension kann untersucht werden, auf welche Weise Zuschauer sich das in den audiovisuellen Texten konstruierte Politikbild

Figuren sich direkt an die Zuschauer wenden. Ein bekanntes Beispiel ist die Adressierung des Publikums im epischen Theater Brechts. In House of Cards wendet sich die Figur Frank Underwood immer wieder an die Zuschauer, um das Geschehen zu kommentieren. 
aneignen. Welche Elemente aus dem vielfältigen Potential der Texte werden tatsächlich in der Rezeption ausgewählt, welche Vorstellungs- und Wertmuster aktualisiert, wie positionieren sich real existierende Zuschauer zu dem Gesehenen? Werden die fiktionalen Welten als realistisch, utopisch oder dystopisch bewertet? Werden die fiktionalen politischen Akteure als getreue Abbilder politischer Wirklichkeit, als positive Vorbilder und Modelle oder als kritisch zugespitzte Gegenbilder wahrgenommen? Realisieren die Rezipienten eher den Unterhaltungswert des populären Formats oder aber das politische Potential eines deutungskulturellen Beitrags? Welche je eigenen, politisch-kulturell geprägten Wert- und Vorstellungsmuster bringen die Zuschauer in den Aneignungsprozess jeweils ein? Wie unterscheiden sich verschiedene Rezipientengruppen in ihrer Rezeption? Wie wirkt sich ein interkulturell angelegter Rezeptionsprozess aus, wenn beispielsweise deutsche Zuschauer eine britische oder US-amerikanische Serie rezipieren und dabei Politik in einem komplett anderen politischen Systemkontext beobachten können? Wie werden die politischen Weltkonstruktionen, die auf ein anderes politisches System bezogen sind, in den eigenen Kontext übersetzt? Wie wird in der öffentlichen Anschlusskommunikation über die Serien kommuniziert, d.h. in welcher Weise werden die Serien im Feuilleton überregionaler Tageszeitungen und Magazine kommentiert, wird vielleicht auch jenseits dieser Ressorts in der Kommentierung politischer Prozesse oder durch realexistierende politische Akteure auf die Serien eingegangen?

Vor diesem Hintergrund ergeben sich folgende leitende Forschungsfragen:

- Welche Ziele verfolgen die produzierenden Medienakteure mit den Serien, welche Motive und Interessen liegen den Produktionen zugrunde?

- Wie sind die fiktionalen politischen Welten in den Serien konstruiert, welche individuellen und kollektiven Akteure sowie Institutionen prägen das dortige politische Geschehen?

- Welche Formen des politischen Handelns sind beobachtbar und inwieweit sind diese jeweils an eine politische Ethik gekoppelt?

- Wie ist das Verhältnis zwischen innertextueller und außertextueller politischer Realität beschaffen? Sind die Texte eher als >realistisches $<$ Abbild gegebener Verhältnisse oder eher als kritisches Gegenbild zu verstehen? In welcher Weise prägen die Texte das öffentliche Bild von Politik und den an Politik jeweils gerichteten Erwartungshorizont in der Gesellschaft?

- Wie sind politische Semantik und politische Ästhetik, wie sind Inhalte und ästhetische Formung bei der Konstruktion des Politischen aufeinander bezogen?

- Wie sind diese fiktionalen Konstruktionen jeweils bezogen auf ihre politisch-kulturellen Kontexte? Inwieweit sind sie jeweils zu verstehen als affirmative oder kritische Projekte deutungskultureller Eliten? Inwieweit gehen sie auf soziokulturelle Normalitäten der jeweiligen politischen Kultur ein? Und 
in welcher Weise bringen Rezipienten bei ihrer Aneignung politisch-kulturell bedingte Dispositionen ein?

- Wie gehen Rezipienten mit den ihnen dargebotenen Politikkonstruktionen selektiv um, welche Aspekte der fiktionalen politischen Welten werden wie wahrgenommen und kommentiert bzw. bewertet?

Insgesamt kann ein solches Forschungsprogramm wichtige Erkenntnisse zur Rolle fiktionaler Politserien für die politischen Kulturen moderner Gegenwartsgesellschaften erbringen.

\section{Der deutsche Sonderweg}

Auch in Deutschland werden politische TV-Serien produziert, in denen politische Akteure nach dem Muster der oben vorgestellten Typologie agieren. So ist die Welt in der ZDF-Produktion Kanzleramt (2005) sehr deutlich am Vorbild von The West Wing orientiert.

Der deutsche Bundeskanzler Weyer ersetzt den amerikanischen Präsidenten Bartlet. Der Kanzler und sein Stab sind wie in der US-Serie als engagiert, integer und altruistisch motiviert gezeichnet, und auch hier im politischen Berlin machen kleine menschliche Schwächen das Personal nahbarer und sympathischer.

Das Muster des Machtpolitikers findet sich in der deutschen Serienlandschaft eher im Comedy-Bereich. Ein deutscher Frank Underwood ist hier noch nicht in Sicht. In der Comedy aber agieren lustig scheiternde Machtpolitiker, beispielsweise in der ZDF-Produktion Eichwald, MdB (4 Folgen in einer Staffel, 2014) oder in Allein unter Bauern (Sat. 1, 10 Folgen in einer Staffel, 2006/07), wo gezeigt wird, wie ein abgehalfterter Bundespolitiker sich in der Brandenburgischen Provinz an einem Karriereneustart versucht.

Ein Beispiel für das realpolitische Muster bietet die ARD-Produktion Die Stadt und die Macht im Jahr 2016. Die sechsteilige Miniserie erzählt in der Art eines Entwicklungsromans die Geschichte der Desillusionierung einer jungen Politikerin, die ihren Wahlkampf für das Amt des Regierenden Bürgermeisters in Berlin schließlich mit schmutzigen Methoden, mit Lügen, Intrigen und Wählerbetrug vorantreibt.

Wenn man an dieser Stelle einmal einen Blick auf die genannten deutschen Serienbeispiele wirft, dann ergibt sich ein interessanter Befund: All diese durchaus ambitionierten Produktionen sind beim deutschen Publikum durchgefallen oder wurden kaum zur Kenntnis genommen. So startete Kanzleramt 2005, stimuliert durch aufwändiges Marketing des Senders, zunächst mit guten Quoten, die dann jedoch schnell absackten, sodass die letzten Folgen am Ende im Doppelpack geradezu >verramscht< wurden. Mit vielen Hoffnungen hatte die ARD 2016 ihre Minise- 
rie Die Stadt und die Macht ins Rennen geschickt, um dann ernüchtert festzustellen, dass trotz bestem Sendeplatz weniger als 3 Millionen Zuschauer das Schicksal der Heldin Susanne Kröhmer verfolgen wollten. Sat. 1 hatte für seine politische Comedyserie Allein unter Bauern mit Marc Terjung einen Erfolgsautor verpflichtet, der dem Sender mit der Anwaltsserie Edel \& Starck zuvor beste Quoten und Kritiken gebracht hatte. Die Polit-Serie jedoch scheiterte und wurde nach der ersten Staffel eingestellt. Selbst Dieter Wedel, Urgestein der gehobenen deutschen Fernsehunterhaltung, hatte 2002 angesichts des ausbleibenden Erfolgs seines Sechsteilers Die Affäre Semmeling verzweifelt ausgerufen: "So einen riesigen Absturz habe ich noch nie erlebt. Eine so niedrige Quote hatte ich noch nie. $\ll^{15}$

Politserien in Deutschland - das scheint beim großen Publikum nicht zu funktionieren. Der Stromberg-Autor Ralf Husmann, ein Fernsehprofi, der mittlerweile auch Tatort-Drehbücher für den MDR verfasst, hat es einmal auf die Formel gebracht: »Politik mag der deutsche Zuschauer nicht [...]. Alle Sachen, die man in Berlin toll findet, will in Bielefeld kein Mensch sehen. ${ }^{16}$ Interessanterweise trifft das auch für die internationalen Produktionen wie House of Cards, Scandal oder Borgen zu, die hierzulande zwar viel Lob und Aufmerksamkeit vom Feuilleton erhielten, jedoch niemals gute Quoten. ${ }^{17}$

Was sind die Gründe dafür? Sind die Deutschen etwa noch immer politisch-kulturell geprägt von einer Disposition des Unpolitischen, wie sie im Elitendiskurs von Thomas Mann (1918) und anderen beschworen wurde und wo das Politische als etwas Oberflächliches, Geistfernes stilisiert wird? Diese in den Kontext des Ersten Weltkriegs gehörende Polemik gegen die westlichen Demokratien hat eine Tradition begründet, in der man in Deutschland politisches Handeln oft als etwas Niederes, als ein sschmutziges Geschäft، angesehen hat.

Damit korrespondiert eine politisch-kulturelle Disposition, der zufolge eine sgute Politik jeweils über dem Parteienstreit angesiedelt ist und quasi von einer übergeordneten Instanz oder Warte aus betrieben wird. Der Politik im alltagsweltlichen Sinn, als Kampf um Interessen und Standpunkte, haftet demgegenüber immer etwas Minderwertiges an. Diese Tradition des Apolitischen setzte sich noch sehr viel später in Deutschland fort in einer politischen Kultur nach dem Zweiten Weltkrieg, die zwar in einem demokratischen politischen System positioniert war, das politische Handeln jedoch noch immer nicht als Selbstverständlichkeit für alle Bürger definiert hat. Die amerikanischen Politikwissenschaftler Gabriel Almond und Sydney Verba (1963) haben in ausführlichen empirischen Studien am Ende der

15 Siehe www.tagesspiegel.de/medien/die-niedrigste-quote-seit-es-wedel-gibt/281806.html, Zugriff am 10.03.2017.

16 Siehe www.sueddeutsche.de/medien/politische-tv-serien-das-geht-in-deutschland-nicht-1. 1937707, Zugriff am 10.03.2017.

17 Classen (2010: 289) formuliert einen ähnlichen Befund auch für den deutschen Film. 
1950er-Jahre noch einen entsprechenden Mangel an Bürgerkultur für Westdeutschland konstatiert, da sie eher den Typus der Untertanenkultur vorfanden. Die westdeutsche Bevölkerung war zwar an einer effizienten Verwaltung mit gutem politischem Output interessiert, jedoch nicht an einer eigenen Beteiligung am politischen Prozess. Eine solche Vorstellungswelt bezüglich der Politik würde durchaus erklären, warum die Menschen an Politik in Unterhaltungsformaten, etwa in politischen Serien, kaum interessiert sind. Tatsächlich kommt der Historiker Christoph Classen in einer Analyse von Filmen der Nachkriegszeit auch zu dem Schluss, dass gute Politik sich dort jeweils dadurch definierte, dass sie Abstand vom politischen Betrieb hielt (Classen 2010: 293).

Allerdings hat sich seit den 1950er- und 1960er-Jahren in der politischen Kultur Deutschlands doch ein breiter Wandel vollzogen (vgl. schon Rohe/Dörner 1990). In den 1970er-Jahren kam es zu einer Partizipationsrevolution, in deren Gefolge neue soziale Bewegungen entstanden und sich auch nachhaltige Veränderungen im Parteiensystem vollzogen. Die Bereitschaft, sich mit konventionellen, zunehmend auch mit unkonventionellen Methoden am politischen Prozess zu beteiligen, stieg vor allem bei den jüngeren Generationen deutlich an. Politik war nicht länger nur etwas, was für die Bürgerinnen und Bürger gemacht wurde, sondern auch durch die Bürgerinnen und Bürger. Mit der zunehmenden Partizipationsbereitschaft ging dann auch ein gestiegenes Misstrauen gegen die politischen Eliten und das Establishment einher. In dieser Perspektive ist die sgute Politik immer eine Politik gegen >die da oben`, gegen etablierte Akteure und Institutionen. Es ist interessant, dass diese kritische Sichtweise durchaus historische Kontinuitäten aufweist:

»Das Stereotyp der ıschmutzigen Politikı als einer Sphäre, die im Wesentlichen von Eigeninteressen und Taktik der Beteiligten geprägt sei, die sich durch Intrigen auszeichne und an der jede Form von Idealismus scheitern müsse, knüpft nahtlos an Perzeptionen des Parlamentarismus in der Weimarer Republik an« (Classen 2010: 300).

Die kritische Perspektive gegen »die da oben« ist auch diejenige, die häufig im politisch dimensionierten deutschen Fernsehkrimi ausgespielt wird, etwa in den sozial- und politikkritischen Tatort-Folgen oder in der ZDF-Reihe Unter Verdacht. Die guten Staatsdiener sind hier oft diejenigen, die sich nicht nach der Dienstvorschrift und den Anweisungen ihrer Vorgesetzten richten, sondern die nach eigenen moralischen Grundsätzen handeln und dabei auch mal zivilen Ungehorsam gegen die Amtshierarchie durchsetzen.

Solche Perspektiven kommen beim deutschen Publikum durchaus gut an, während das affirmative Bild, das in Kanzleramt gezeichnet wird, kaum Anklang findet. Polit-Märchen, denen zufolge man dem etablierten politischen Personal völlig vertrauen kann und am Ende des Tages immer wieder alles gut wird, möchte der 
deutsche Zuschauer nicht sehen. Kritische Helden jedoch, die den Eliten misstrauen und der Gerechtigkeit auch gegen Widerstände der Obrigkeit zum Durchbruch verhelfen, finden durchaus breite Zustimmung.

Interessanterweise begann ja mit dem Ende der 1970er-Jahre, als die Partizipationsrevolution sich in Deutschland durchgesetzt hatte und die Friedens- und AntiAKW-Bewegungen sich mit lauter Stimme zu Wort meldeten, der Aufstieg von Ermittlertypen wie Horst Schimanski im WDR-Tatort. Anders als frühere Kriminalbeamte wie Der Kommissar Keller oder Stefan Derrick zeigte Schimanski seit 1981 schon im äußeren Erscheinungsbild rebellische Distanz gegenüber der Amtshierarchie, die sich dann in eigenwilligen Ermittlungsmethoden und Entscheidungen fortsetzte. Politik im Unterhaltungsformat könnte in Deutschland also am ehesten noch da erfolgreich möglich sein, wo eine kritische Perspektive vorherrscht.

Gegenwärtig erleben Krimis in Deutschland einen beispiellosen Boom, der sich nicht nur in den hervorragenden Quoten und Marktanteilen der Tatort-Reihe in der ARD widerspiegelt, sondern auch in den zahllosen Serien, die mittlerweile das Vorabendprogramm im Ersten (etwa die Serien unter dem Formatdach Heiter bis tödlich) und beim ZDF (etwa mit den zahlreichen SoKos) prägen. ${ }^{18}$ Fernsehkrimis erfreuen sich einer ungebrochenen Popularität. Die Geschichten von der Störung der gesellschaftlichen Ordnung durch ein Kapitalverbrechen, das dann jeweils durch Kriminalbeamte als Vertreter der Staatsmacht aufgeklärt wird, wollen noch immer die meisten Zuschauer sehen. Tatort-Produktionen sind hinter den großen Sportevents im deutschen TV-Programm jeweils die reichweitenstärksten Sendungen (so 2016 eine Münsteraner Folge mit 13,31 Mio. Zuschauern). Wenn der deutsche Zuschauer also politische Akteure und Handlungen sehen will, dann am liebsten im Format des Kriminalfilms.

Welches Bild aber zeichnet der deutsche Fernsehkrimi von politischen Akteuren? Die meisten fiktionalen Politikerinnen und Politiker sind hier Vertreter des Typus Machtpolitiker, teilweise auch Realpolitiker mit einem Hang zum Werteverlust. Politikerinnen und Politiker im deutschen Fernsehkrimi, das sind »die da oben«, die sich um Karrieren und Einkünfte, um Seilschaften und Spezis kümmern, aber nicht um das Gemeinwohl. Es sind korrupte Akteure, die auf den Hinterbühnen der Macht ihre Deals mit anderen Politikerinnen und Politikern oder auch mit Wirtschaftsbossen aushandeln und dabei im Zweifelsfall über Leichen gehen - ein sehr negatives Bild, das geradezu Trostlosigkeit verbreiten würde, wenn es nicht auf der anderen Seite couragierte Aufklärerinnen und Aufklärer gäbe, die den düsteren Polit-Gestalten das Leben schwer machen. Die mutigen Kriminalbeamten, die Skandale in der Umwelt-, Wirtschafts- und Verteidigungspolitik aufdecken, bleiben zwar oft desillusioniert zurück und können die bestehenden Verhält-

18 Es handelt sich um Dachmarken verschiedener Serienproduktionen, die aufgrund thematischer oder stilistischer Ähnlichkeiten jedoch zu einem Korpus zusammengefasst werden können. 
nisse nicht grundsätzlich ändern. Aber sie zeigen, dass es sich gleichwohl lohnt, hartnäckig Widerstand zu leisten gegen die Machenschaften der Machtpolitikerinnen und -politiker. Sie stehen für das Gute im Angesicht des machtpolitischen Bösen und verhindern damit gleichsam, dass sich aus dem negativen Bild von politischen Akteuren bei den Zuschauern eine fundamental systemkritische Sicht entwickelt. Die Guten sind ebenso Teil des Systems wie die Bösen, daher wird das System insgesamt hier nicht in Frage gestellt.

Besonders scharf konturierte Beispiele für diese in der deutschen Medienkultur weitverbreitete Konstellation finden sich in der ZDF-Reihe Unter Verdacht. Hier spielt Senta Berger die Figur der Eva Prohacek, einer internen Ermittlerin im Polizeiapparat, die es immer wieder mit korrupten Politikern zu tun bekommt. Diese werden oft gedeckt durch Prohaceks Vorgesetzten Dr. Claus Reiter, der stets auf eigene Karrierevorteile bedacht ist und sich daher gern nach oben hin servil zeigt. Sichtbar wird jeweils das Spiel von bayerischen Amigo-Seilschaften mit Intrigen in der hohen Landespolitik ebenso wie mit Vorteilsnahmen in den Niederungen von Kommunalpolitikerinnen und -politikern und Behördenvorstehern (so etwa in den Folgen Betongold 2016 und Das Blut der Erde 2013). In der Episode Ein neues Leben (2006) kam es sogar zu einer großen Verschwörung von bayerischem Wirtschaftsministerium, Luftfahrtindustrie und US-amerikanischem Geheimdienst. Entscheidend aber ist: Eva Prohacek bleibt unbeirrt widerständig und lässt nicht locker, bis die skandalösen Zustände aufgedeckt sind.

Warum aber werden die politischen Akteure im Krimi so gern als machtbesessene Schurken konstruiert? Darauf lassen sich zwei plausible Teilantworten finden. Die eine lautet, dass die Mehrzahl der Drehbuchautorinnen und -autoren aus einer linksliberalen Weltanschauung heraus den kritischen Blick auf »die da oben« pflegt und als politische Stellungnahme in den öffentlichen Diskurs der Gesellschaft einbringen möchte. Ein Tatort mit einer Reichweite von 10 Millionen $\mathrm{Zu}$ schauern stellt ein einflussreiches Sprachrohr für politische Meinungen dar und das Publikum scheint diese Sicht der Politikerinnen und Politiker durchaus zu goutieren. Die zweite Teilantwort ist eher formal-ästhetisch dimensioniert: Ein Krimi braucht dramaturgisch nicht nur Täterinnenund Täter im Vordergrund, sondern auch Strippenzieherinnen und Strippenzieher im Hintergrund. Politiker, deren Hinterbühne der Erfahrungswelt der meisten Bürger und Zuschauer sehr fern liegt, bieten sich für solche Rollen geradezu an. Das Misstrauen gegen die Mächtigen wird also auch aus erzählerischen Gründen befeuert.

Mitunter fühlen sich politische Akteurinnen und Akteure dermaßen verzerrt dargestellt, dass sie Anlass sehen, öffentlich dagegen Protest zu erheben, so geschehen etwa aus Anlass der Tatort-Folge Der Inder (SWR), ausgestrahlt am 21. Juni 2015:

Das Drehbuch hatte hier die realen Geschehnisse um das Projekt Stuttgart 21 zum Hintergrund eines Kriminalfalles gemacht. Korrupte Politiker, die mit unse- 
riösen Investoren mauscheln, traten ebenso auf wie gewaltbereite »Wutbürger«. Real existierende politische Akteure, etwa der grüne Stuttgarter Oberbürgermeister Fritz Kuhn oder der CDU-Abgeordnete Georg Löffler nahmen unverzüglich in der Presse Stellung mit der Behauptung, das fiktionale Szenario habe mit der politischen Wirklichkeit nicht viel zu tun (Rhein-Neckar-Zeitung vom 23.06.2015). Spannend war dann, dass Zuschauer dies durchaus anders sahen. So hieß es in einem Online-Kommentar zu dem Artikel sarkastisch:

»Genau so läuft es doch: Die dargestellte Fiktion ist Realität. Finanzstrategen haben das alleinige Sagen und dadurch bestimmen auch sie allein, was letztendlich wie gemacht wird (und ob überhaupt), korrupte Politiker putschen sich gnadenlos an die Macht (um dadurch noch besser Pöstchen verteilen zu können), während sich das gedopte Volk das Ganze völlig wolkenumnebelt baff in der Röhre ankuckt und/oder ansonsten seine überschüssige Energie (falls überhaupt noch vorhanden) in völlig unnützen Straßendemos entlädt (weil ja schon längst alles beschlossene Sache ist); aber, schlussendlich geht ja alle Macht vom Volk aus, oder etwa nicht ... ?!«

Und ein anderer Leser schrieb zu den im Artikel zitierten Protesten der Politiker: »Unglaublich! Da läuft im TV ein Krimi und (getroffene?) Politiker aller Couleur fangen laut das Bellen an. ${ }^{19}$ Diese Äußerungen sind nicht repräsentativ. Aber sie zeigen dennoch, dass negative Politikbilder in fiktionalen Fernsehformaten so etwas wie Politikerverdrossenheit bei den Bürgern verstärken können.

\section{Fazit}

Politserien erscheinen als ein ebenso interessantes wie relevantes Thema für die politische Kulturforschung. Die TV-Produktionen sind einerseits Teil des politischen Imaginären und damit Ausdruck von politisch-kulturellen Vorstellungswelten in einer Gesellschaft. Andererseits sind die Serien auch Teil von deutungskulturellen Projekten, mit denen bestimmte Sichtweisen und Bewertungen des Politischen in attraktiver, unterhaltsamer Gestaltung in der Bevölkerung lanciert werden sollen. Ein typisches Beispiel für ein solches Unterfangen ist die erfolgreiche US-amerikanische Serie The West Wing, die ganz im Sinne der republikanischen Tradition der US-amerikanischen Kultur ein Bild von politischen Akteuren zeichnet, das durch Altruismus, Opferbereitschaft und den bedingungslosen Einsatz für eine gute Politik gekennzeichnet ist.

19 Siehe www.rnz.de/politik/suedwest_artikel,-Stuttgarter-S21-Tatort-Politiker-distanzieren-sich_arid,107144.html, Zugriff am 10.03.2017. 
Die politische Kulturforschung kann durch die Analyse von Politserien Einblicke in derartige deutungskulturelle Projekte bekommen, sie kann untersuchen, in welcher Form medial-fiktionale Konstruktionen des Politischen den öffentlichen Raum gestalten und damit auch die Perzeption politischer Realitäten durch die Bürgerinnen und Bürger mit beeinflussen. Und sie kann feststellen, wie sich die audiovisuellen Texte in ihrer spezifischen Ästhetik der Ausdrucksseite politischer Kulturen einpassen. Politischer Sinn, dies gilt auch und gerade für fiktionale Unterhaltungsproduktionen, muss jeweils sinnenfällig gestaltet werden, damit er evident erscheint und bei den Bürgern akzeptiert wird.

\section{Literatur}

Almond, Gabriel A./Verba, Sidney 1963: The Civic Culture. Political Attitudes and Democracy in Five Nations, Princeton, N.J.

Banks, Miranda u.a. (Hg.) 2016: Production Studies: The Sequel! Cultural Studies of Global Media Industries, New York, London.

Baringhorst, Sigrid 2012: Politik als populäre Kultur - populäre Kultur als Politik. Innovative Perspektiven der politischen Kulturforschung. In: Dietmar Schiller (Hg.): A change is gonna come: Popmusik und Politik, Münster, S. 27-41.

Bellah, Robert N. u.a. 1987: Gewohnheiten des Herzens. Individualismus und Gemeinsinn in der amerikanischen Gesellschaft, Köln.

Caldwell, John Thornton 2008: Production Culture. Industrial Reflexivity and Critical Practice in Film and Television, Durham, London.

Classen, Christoph 2010: Politik als Unterhaltung. Zur Medialisierung von Politik in populären Filmen und Fernsehserien. In: Klaus Arnold u.a. (Hg.), Von der Politisierung der Medien zur Medialisierung des Politischen? Zum Verhältnis von Medien, Öffentlichkeiten und Politik im 20. Jahrhundert, Leipzig, S. 287-306.

Däwes, Birgit/Ganser, Alexandra/Poppenhagen, Nicole (Hg.) 2015: Transgressive Television. Politics and Crime in 21st-Century American TV Series, Heidelberg. Diehl, Paula, 2015: Das Symbolische, das Imaginäre und die Demokratie. Eine Theorie politischer Repräsentation, Baden-Baden.

Diehl, Paula/Felix Steilen (Hg.) 2016: Politische Repräsentation und das Symbolische. Historische. Politische und soziologische Perspektiven, Wiesbaden.

Doll, Martin/Oliver Kohns (Hg.) 2016: Figurationen des Politischen. Band 1: Die Phänomenalität der Politik in der Gegenwart, Paderborn.

Dörner, Andreas 1995: Politischer Mythos und symbolische Politik. Sinnstiftung durch symbolische Formen am Beispiel des Hermannsmythos, Opladen.

Dörner, Andreas 1998: Medien als politische Identitätsgeneratoren. Zur Inszenierung des Republikanismus in der amerikanischen Medienkultur. In: Politische Vierteljahresschrift 39 (1998), S. 3-27. 
Dörner, Andreas 1998a: Das politische Imaginäre. Vom Nutzen der Filmanalyse für die Politische Kulturforschung. In: Wilhelm Hofmann (Hg.), Visuelle Politik, Filmpolitik und die visuelle Konstruktion des Politischen, Baden-Baden, S. 199-219.

Dörner, Andreas 1999: Politische Kulturforschung und Cultural Studies. In: Othmar Nikola Haberl/Tobias Korenke (Hg.), Politische Deutungskulturen, Festschrift für Karl Rohe, Baden-Baden, S. 93-110.

Dörner, Andreas 2000: Politische Kultur und Medienunterhaltung. Zur Inszenierung politischer Identitäten in der amerikanischen Film- und Fernsehwelt, Konstanz.

Dörner, Andreas 2003: Politische Kulturforschung. In: Herfried Münkler (Hg.), Politikwissenschaft, Ein Grundkurs, Reinbek bei Hamburg, S. 587-619.

Dörner, Andreas 2003a: Demokratie - Macht - Ästhetik. Zur Präsentation des Politischen in der Mediengesellschaft. In: Hans Vorländer (Hg.), Zur Ästhetik der Demokratie. Formen der politischen Selbstdarstellung, Stuttgart, S. 200-223.

Dörner, Andreas 2006: Political Culture and Media Culture: Constructing Political Identities in the US and Germany. In: William Uricchio/Susanne Kinnebrock (Hg.), Media Cultures, Heidelberg, S. 41-48.

Dörner, Andreas 2016: Politserien: Unterhaltsame Blicke auf die Hinterbühnen der Politik. In: Aus Politik und Zeitgeschichte 66, Heft 51, S. 4-11.

Dörner, Andreas et.al. 2015: Riskante Bühnen. Inszenierung und Kontingenz - Politikerauftritte in deutschen Personality-Talkshows, Wiesbaden.

Dörner, Andreas/Vogt, Ludgera 2015: Medienwissenschaft und Sozialwissenschaften: Ein Plädoyer für forschungspraktische Kooperation am Beispiel der ethnografisch eingebetteten Medienanalyse. In: Medienwissenschaft, Heft 2, S. 325-430.

Dörner, Andreas/Vogt Ludgera (Hg.) 2012: Unterhaltungsrepublik Deutschland. Medien, Politik und Entertainment, Bonn.

Dörner, Andreas/Vogt, Ludgera (Hg.) 2017: Wahlkampf mit Humor und Komik. Selbst- und Fremdinszenierung politischer Akteure in Satiretalks des deutschen Fernsehens, Wiesbaden.

Drechsel, Benjamin 2009: Trügerischer Augenschein? Hinweise zur Verflechtung von politischer Kultur und visueller Politik. In: Samuel Salzborn (Hg.), Politische Kultur. Forschungsstand und Forschungsperspektiven, Wiesbaden, S. 147-174.

Frame, Gregory 2014: The American President in Film and Television. Myth, Politics and Representation, Oxford u.a.

Goffman, Erving 2008: Wir alle spielen Theater. Die Selbstdarstellung im Alltag, 6. Aufl., München.

Hepp, Andreas, 2010: Cultural Studies und Medienanalyse. Eine Einführung, 3. Aufl. Wiesbaden. 
Hofmann, Wilhelm (Hg.) 1998: Visuelle Politik. Filmpolitik und die visuelle Konstruktion des Politischen, Baden-Baden.

Huizinga, Johan 1956: Homo ludens. Vom Ursprung der Kultur im Spiel, Hamburg. Kaklamanidou, Betty/Tally Margaret (Hg.) 2017: Politics and politicians in contemporary US television. Washington as fiction, London, New York.

Kiegeland, Julia/Klatt, Jöran/Rahlf, Katharina 2014: Politikserien. In: INDES - Zeitschrift für Politik und Gesellschaft (4).

Luhmann, Niklas 2010: Die Realität der Massenmedien, 4. Aufl., Wiesbaden.

Mann, Thomas 1918: Betrachtungen eines Unpolitischen, Berlin.

Mayer, Vicki et.al. (Hg.), 2009: Production Studies. Cultural Studies of Media Industries, New York, London.

Nitsch, Cordula/Christiane Eilders 2014: Die Repräsentation von Politik in fiktionaler Unterhaltung. Instrument, Anwendung und Befunde zur Systematisierung von Filmen und Fernsehserien. In: Studies in Communication: Media 3 (1), S. 120-143.

Nitsch, Cordula/Christiane Eilders 2015: Fictional Politics on TV. Comparing the Representations of Political Reality in U.S. Series The West Wing and the German Series Kanzleramt. In: Global Media Journal - German Edition (5.1), S. 1-19.

Nuy, Sandra 2017: Die Politik von Athenes Schild. Zur dramaturgischen Logik des Politischen im fiktionalen Film, Münster.

Pesch, Volker 2000: Handlungstheorie und politische Kultur, Wiesbaden.

Pickel, Susanne/Pickel, Gert 2006: Politische Kultur- und Demokratieforschung. Grundbegriffe, Theorien, Methoden. Eine Einführung, Wiesbaden.

Rohe, Karl 1994: Politik. Begriffe und Wirklichkeiten. 2. Aufl., Stuttgart u.a.

Rohe, Karl 1996: Politische Kultur: Zum Verständnis eines theoretischen Konzepts. In: Oscar Niedermeyer/Klaus von Beyme (Hg.), Politische Kultur in Ost- und Westdeutschland, Opladen, S. 1-21.

Rohe, Karl/Dörner, Andreas 1990: Von der Untertanenkultur zur »Partizipationskultur«? Kontinuität und Wandel der politischen Kultur in Deutschland. In: Politische Bildung 23, Heft 3 (= Themenheft Politische Kultur, hg. von Peter Haungs), S. 18-33.

Street, John 1997: Politics and popular culture, Philadelphia.

Stumm, Mascha-Maria 1996: Unterhaltungstheoreme bei Platon und Aristoteles. Eine Rückkehr zu den Ursprüngen der Diskussion um Funktionen und Wirkungen von Unterhaltung und der Versuch einer Auswertung fachfremder Literatur als Beitrag zur Klärung des kommunikationswissenschaftlichen Unterhaltungsbegriffes, Berlin.

Schwelling, Birgit (Hg.) 2004: Politikwissenschaft als Kulturwissenschaft. Theorien, Methoden, Problemstellungen, Wiesbaden. 
Trautmann, Felix (Hg.) 2017: Das politische Imaginäre. Freiheit und Gesetz V, Berlin.

Tryon, Chuck 2016: Political TV, New York, London.

Vaihinger, Hans 1913: Die Philosophie des Als Ob: System der theoretischen, praktischen und religiösen Fiktionen der Menschheit auf Grund eines idealistischen Positivismus, 2. Aufl., Berlin.

van Zoonen, Liesbet 2005: Entertaining the citizen. When politics and popular culture converge, Lanham Md. u.a.

Vorländer, Hans (Hg.) 2003: Zur Ästhetik der Demokratie. Formen der politischen Selbstdarstellung, Stuttgart.

Weber, Max 1919: Politik als Beruf, München, Leipzig.

\section{Online-Quellen}

www.politico.com/blogs/politico-now/2014/02/spacey-house-of-cards-not-far-

from-reality-183420 vom 16.02.2014.

https://www.welt.de/politik/deutschland/gallery137845657/Darum-fasziniert-

House-of-Cards-unsere-Politiker.html

www.tagesspiegel.de/medien/die-niedrigste-quote-seit-es-wedel-

gibt/281806.html vom 06.01.2002.

www.sueddeutsche.de/medien/politische-tv-serien-das-geht-in-

deutschland-nicht-1.1937707 vom 16.04.2014.

www.rnz.de/politik/suedwest_artikel,-Stuttgarter-S21-Tatort-

Politiker-distanzieren-sich-_arid,107144.html vom 23.06.2015.

\section{Filmverzeichnis}

Allein unter Bauern (Sat.1, 2006-2007)

Commander in Chief (ABC, 2005-2006)

Derrick (ZDF, 1974-1998)

Die Stadt und die Macht (Das Erste, 2016)

Edel \& Starck (Sat.1, 2002-2005)

Eichwald, MdB (ZDFneo, 2015)

Heiter bis tödlich (Das Erste, 2011-2015)

House of Cards (BBC, 1990)

House of Cards (Netflix, 2013-)

Kanzleramt (ZDF, 2005)

Der Kommissar (ZDF, 1968-1975) 
184 Andreas Dörner

Madam Secretary (CBS, 2014-)

Scandal (ABC, 2012-2018)

SOKO-Formate (ZDF, 1978-)

Stromberg (ProSieben, 2004-2012)

Tatort: Schimanski-Reihe (WDR, 1981-2013)

Tatort: Der Inder (2015) (D, R: Niki Stein)

The West Wing (NBC, 1999-2006)

Unter Verdacht (ARTE, 2002-) 


\section{Good Night, and Good Luck Über das Verhältnis von Narration, Film und Politik}

Sandra Nuy

\section{Politische Kulturforschung und Narration}

»Politische Kultur ist politischer Sinn, der auch sinnenfällig werden muss «, schrieb Karl Rohe 1990 in einem Aufsatz, der die Politische Kulturforschung nachhaltig geprägt hat (337). Folgt man dieser Begriffsbestimmung, ist es evident, dass Praktiken des Erzählens konstitutiv für die Ausbildung Politischer Kulturen sind. Schließlich verleihen Erzählungen Ereignissen, Orten, Personen und Symboliken Bedeutung: Mit Hilfe des Erzählens werden sich Kontingenz, Komplexität und Widersprüchlichkeit menschlicher Erfahrung in einer Weise angeeignet, dass sie überschaubar, verständlich und plausibel erscheinen. "Das Bedürfnis zu erzählen kann als anthropologische Konstante angesehen werden; auf Geschichten gründen sich die vielfältigen Formen der Vergemeinschaftung von Menschen« (Bergem 2014: 33).

Insofern Erzählen seit je her für Unterhaltung, Orientierung und Sinnstiftung sorgt, handelt es sich um ein Phänomen, das sich mit Roland Barthes' viel zitierter Bemerkung wie folgt zusammenfassen lässt: »Die Erzählung schert sich nicht um gute oder schlechte Literatur: sie ist international, transhistorisch, transkulturell und damit einfach da, so wie das Leben« (1988: 102). Unbeeindruckt von Fragen der Qualität, der Wahrheit, kulturell-historischer Verschiedenheit oder der medialen Form tritt die Narration als universelle kommunikative Variante der Verständigung auf. Erlebnisse werden im Gespräch erzählt, Erinnerungen und Ereignisse zu Erzählungen verdichtet, Sachverhalte jeder Art in Geschichten vermittelt, um Anschaulichkeit und Nachvollziehbarkeit zu erhöhen. Kurz: Erzählen kann mit einigem Recht als ein »kognitiver Modus der Selbst- und Wirklichkeitserfahrung sowie der kulturellen und sozialen Wirklichkeitskonstruktion« (Nünning 2013: 33) beschrieben werden.

Dieser Reichweite des Erzählens sind sich nicht allein die Literatur- und Kulturwissenschaften bewusst, auch in den Sozialwissenschaften macht sich seit ein paar Jahren ein sogenannter narrative turn bemerkbar (vgl. statt vieler: Hofmann et al. 2014). In der Beschäftigung der Politikwissenschaft mit der Erzählung lägen, so heißt es in einem Handbuch-Artikel, zwei Vorteile: zum einem könne »die 
performative Kraft des Fiktionalen damit sichtbar« gemacht werden und zum anderen eröffne sich ein »Blick auf liminale Kommunikationsräume, die in der Politik zunehmend an Bedeutung gewinnen « (Gadinger/Yildiz 2017: 158). Im Mittelpunkt der politikwissenschaftlichen Forschungsarbeiten, die sich dem narratologischen Paradigma zuwenden, steht zumeist die Frage, welche politischen Deutungsmuster durch Narrative repräsentiert werden und welche Rolle sie in politischen Handlungspraxen spielen. Einer disziplinären Logik folgend, die Desiderate des eigenen Faches konstatiert, hat die politologische Beschäftigung mit Narrationen als Teil einer diskursiven Konstruktion sozialer und politischer Realität gar den Entwurf einer "politikwissenschaftlichen Erzähltheorie« (Gadinger et al. 2014) hervorgebracht. Den Verfassern ging es dabei zunächst um eine Theoretisierung des Verhältnisses von Politik und Sprache, um eine Antwort auf die Frage zu finden, »in welcher Form und mithilfe welcher Sprachtechniken politische Akteure in die gesellschaftlichen Verhältnisse eigentlich intervenieren« (ebd.: 5). In der Zwischenzeit haben die Autoren ihr - im Übrigen: interdisziplinäres - Forschungsprogramm der Untersuchung politischer Narrative von der sprachlich vermittelten Erzählung auf die Medien der Audiovision ausgeweitet. Avisiert wird eine Filmpolitologie, in der sich Filmanalyse und Politikwissenschaft zu einem innovativen Forschungsfeld verbinden (vgl. Gadinger 2018: 307). Auch die folgenden Ausführungen sind in diesem Feld zu verorten.

\section{Spielarten des politischen Erzählens in den Medien der Audiovision}

Mit der Etablierung des Films im Mediengefüge des beginnenden 20. Jahrhunderts erweiterten und veränderten sich die Formen der Narration um die Spezifik des Erzählens in bewegten Bildern. Darauf wird noch einzugehen sein. Zunächst bleibt festzuhalten, dass sich der Spielfilm wie kaum ein anderes Medium eignet, politischen Sinn sinnenfällig werden zu lassen, kann doch der Film mit einem Wort von Robert Musil als »Möglichkeitssinn« einer Gesellschaft beschrieben werden, nämlich als die »Fähigkeit alles, was ebensogut sein könnte, zu denken und das, was ist, nicht wichtiger zu nehmen als das, was nicht ist « (1989: 16.). Anders ausgedrückt lassen sich kollektive Wissensbestände in der filmischen Narration ebenso ordnen wie speichern und >sinnenfällig` weitergeben. Damit einher geht eine Offerte von Deutungsangeboten, denn eine Erzählung hat nicht nur Ereignisse in eine Abfolge $\mathrm{zu}$ bringen, sondern muss überdies ein verstehbares Ganzes ergeben. Als intelligible Größen zielen Narrationen somit - wie eingangs schon erwähnt - auf die Vermittlung von Sinn. Gültigkeit, Legitimität, Angemessenheit und Reichweite dieser symbolisch generalisierten Sinnstiftung bedürfen jedoch der Aushandlung, so dass (filmische) Narrationen im Hinblick auf die Durchsetzung von Bedeutungszuweisungen in Konkurrenz zueinanderstehen. Da Narrationen im Aushandlungspro- 
zess kollektiv geteilter Wahrnehmungs-, Denk-, und Handlungsmuster ihren festen Platz einnehmen, sind sie per se politisch.

Insofern ist es nicht verwunderlich, dass eine Diskussion über das politische Potential des Films bereits in seiner Frühzeit einsetzte. Politische Poetiken des Films wurden ebenso thematisiert wie seine politische Instrumentalisierbarkeit. Neben den theoretischen Diskurs trat im Gefolge der russischen Oktoberrevolution von 1917 recht schnell auch die Praxis eines politischen Filmschaffens. Seither wird die Beziehung von Politik und Film historisch konstant vorzugsweise in dreierlei Hinsicht diskutiert: in Rede stehen das Politische des Films im Sinne einer Medienontologie, das Politische im Film als ästhetische Repräsentation sowie der politische Film als Medium der Persuasion.

Diese drei Betrachtungsperspektiven - das Politische des Films, das Politische im Film und der politische Film - schließen einander nicht aus, sondern können sich in variablen Anteilen überkreuzen. So hat etwa Andreas Dörner den fiktionalen Film als »Medium der Politischen Kultur« konturiert und zu Recht dessen Relevanz für das politisch Imaginäre betont (1998: 206). Dörners Überlegungen zielen dabei nicht allein auf den engen Bereich einer manifesten Darstellung des Politikbetriebs ab, vielmehr geht es ihm darum, die "Strukturen des Imaginären « zu bestimmen (ebd.: 210). Spezifisch narrative oder ästhetische Eigenschaften und Potentiale des Films sind dabei allerdings sekundär, Filme werden von ihm schlicht als »Daten« betrachtet, »aus denen sich interpretativ Rückschlüsse auf eine Politische Kultur oder Teilkultur ziehen lassen« (Dörner 1998: 203). Damit ergibt sich jedoch in der von Dörner angedachten "filmpolitologischen Hermeneutik « (1998: 209) ein blinder Fleck, den es zu beseitigen gilt. Als in dieser Hinsicht vielversprechend erscheint eine Theoretisierung des Verhältnisses von filmischer Narration und Politik aus einer dramaturgischen Perspektive heraus (vgl. dazu ausführlich Nuy 2017). Im Folgenden wird es daher zunächst darum gehen, Dramaturgie als Theorie und Methode zur Analyse der Narrativierung des Politischen ${ }^{1}$ vorzustellen. In einem zweiten Schritt sollen dann anhand von Medienfilmen Narrative einer politischen Öffentlichkeit betrachtet werden, um schließlich in einer Fallstudie

1 Der Aufsatz folgt der Differenzierung von Politik und dem Politischen gemäß den Begriffsverwendungen von Chantal Mouffe. Demnach lässt sich das Politische als antagonistische Konfliktkonstellation verstehen, wohingegen Politik die Praktiken, Diskurse und Institutionen zur Etablierung und Sicherung (staatlicher) Ordnung beschreibt, »die immer potenziell konfliktorisch sind, da sie von der Dimension des `Politischen ‘ affiziert sind« (Mouffe 2008: 103). Das Politische firmiert also als weiterer Begriff, der konfligierende Identitätsentwürfe, verschiedenste Machtbeziehungen und ideologische Deutungskämpfe zwischen Cruppen einschließt. In Anlehnung an Heidegger formuliert Mouffe die Differenz als Unterscheidung der >ontischen<- »die Praktiken der Politik im konventionellen Sinne« - von der >ontologischen Ebene, welche in der Frage nach dem Politischem »die Art und Weise betrifft, in der die Gesellschaft eingerichtet ist« (ebd.: 15). 
George Clooneys Film über die McCarthy-Ära in den USA, Good Night, and Good Luck (USA 2005), näher zu untersuchen.

\section{Konturen einer Dramaturgie politischer Erzählungen}

Als disziplinärer »Schnittstellenbegriff« verbindet Dramaturgie Aspekte des Ästhetischen, des Materialen und des Kommunikativen (Weber 2014: 11). Etymologisch betrachtet, setzt sich Dramaturgie - aus dem Altgriechischen stammend - aus den Worten ergon (Werk) und drân (handeln) bzw. das davon abgeleitete Substantiv drâma (Handlung) zusammen. Es geht also um die konzeptionelle Verfertigung einer dramatischen Handlung, die an ein Publikum adressiert ist. Gegenstand der Dramaturgie sind somit Prinzipien zur Komposition eines Handlungsverlaufs, der für eine szenische bzw. mediale Realisierung gedacht ist und Rezeption antizipiert. Ältestes Zeugnis eines dramaturgischen Regelwerkes ist die Poetik des Aristoteles, die im Übrigen bis heute in Drehbuch-Manualen herangezogen wird. Etabliert hat sich der Begriff im 18. Jahrhundert mit Lessings Hamburgischer Dramaturgie, einer Sammlung von Kritiken zu Aufführungen des Hamburgischen Nationaltheaters, die 1769 in Buchform veröffentlicht wurden. Was zunächst nur auf das Theater bezogen war, wurde mit Aufkommen der audio-visuellen Medien recht schnell auch auf eben diese angewendet.

Dramaturgische Strukturen wirken auf drei Ebenen als regulierendes Moment: erstens organisieren sie den Handlungsverlauf, zweitens ist es ihre Funktion, Aufmerksamkeit, Empathie und Wissen des Publikums anzuleiten und drittens etablieren sie Sinnhaftigkeit. Das Zusammenspiel dieser Komponenten eines dramaturgietheoretischen Modells ist mehrfach strukturiert: Zunächst einmal liegt ein Erzählschema vor, mit dessen Hilfe eine inferenzielle Bedeutungsgenerierung programmiert wird. Umsetzung und Gebrauch des Schemas variieren jedoch von Film zu Film, so dass die Dramaturgie eines einzelnen Films aus einer spezifischen Kombination von Anwendungen und Abweichungen besteht. Unterscheiden lässt sich außerdem zwischen a) einer eher funktionalen Dramaturgie der Bauformen und b) einer impliziten Dramaturgie, die an (politische) Wissenskontexte des Publikums anknüpft (vgl. Stutterheim 2014: 147). Die explizite bzw. funktionale Dramaturgie gibt die äußere Form der Handlungsarchitektur vor und hat Erzählmuster filmischen Erzählens ausgebildet, die sich paradigmatisch im populären Film finden lassen (vgl. dazu Eder 2007).

Demgegenüber hilft die implizite Dramaturgie bei der Veranschaulichung komplexer sowie abstrakter Sachverhalte und sorgt für eine emotionale Involvierung, was die damit verbundene Sinnstiftung besonders interessant für die Analyse politischer Bedeutung macht. Die implizite Dramaturgie arbeitet mit Referenzen, die auf unterschiedliche Weise an Wissenskontexte der Rezipien- 
ten anknüpfen, welche in politischer Hinsicht zum einen durch die umgebende politische Kultur als solche geprägt sind und zum anderen immer auch auf die politische Mythologie eines Gemeinwesens rekurrieren. Am Rande: Analytisch lassen sich die beiden Ebenen trennen, ihre Wirkung entfalten sie aber nur als integrative Gesamtheit.

Unter dem Begriff der diánoia ist die implizite Dramaturgie bereits bei Aristoteles auffindbar. Er benennt sechs wesentliche Bestandteile der Tragödie: mythos (Handlung), ethe (Figuren), lexis (Rede), diánoia (Gedanke, Absicht), ópsis (Anschauung, Szenerie) und melopoiía (Gesang, Musik). Eine politische Dramaturgie basiert in ihren implizierten Strukturen wesentlich auf der den Komponenten Handlung, Figur und Rede zugeordneten diánoia, obwohl oder gerade weil Aristoteles diese als »eher« der Rhetorik zugehörig betrachtet (Aristoteles 2012: 61). Ihre Funktion beschreibt er wie folgt:

»Zur Cedankenführung (diánoia, SN) gehört, was mit Hilfe von Worten zubereitet werden soll. Teile davon sind das Beweisen und Widerlegen und das Hervorrufen von Erregungszuständen, wie von Jammer oder Schaudern oder Zorn oder dergleichen mehr, ferner das Verfahren, einem Cegenstande größere oder geringere Bedeutung zu verleihen« (Aristoteles 2012: 61).

Dieses Konzept einer rhetorisch ausgerichteten `Gedankenführungくverweist auch auf die Techniken der Bedeutungszuweisung innerhalb der impliziten Dramaturgie, die Erzählungen politische Relevanz verleihen. Die narrative Verknüpfung einer Ereignisfolge verzichtet idealiter auf unnötige, langweilige oder störende Teile und widmet jenen Elementen, die geeigneter scheinen, die Aufmerksamkeit des Adressaten der Erzählung zu wecken und zu halten, ein vielfaches Mehr an Aufwand. Strukturen der Ellipse, der antithetischen Gegenüberstellung und der Steigerung erweisen sich als hilfreich für den Aufbau eines politischen Argumentationszusammenhangs. Dramaturgie zeigt sich in diesem Sinne als ein Strukturmuster für das Verhältnis aus Verschweigen und Betonen, Verbergen und Zeigen, Ausklammern und Ausschmücken. Genau genommen ist die Frage, was nicht erzählt wird, interessanter als die Frage, was erzählt wird. Erzählen ist immer auch Unterschlagung dessen, was verheimlicht, vertuscht oder vergessen werden soll (vgl. Müller-Funk 2002: 91). Auslassungen wie Ausgestaltungen steuern die Bedeutung des Erzählten; Verfahren der Steigerung, Abschwächung und Kontrastierung leiten durch die Herstellung von Zusammenhängen innerhalb der Erzählung ihr Verstehen an. In Verbindung mit der sprachlichen bzw. im Fall der Fiktion ästhetischen Verdichtung von Wirklichkeitserfahrungen, gerät Bedeutung somit zu einer der »formalen Komponenten« von (filmischen) Narrationen (vgl. Thompson 1995: 32). Im Anschluss an Kristin Thompson wird Bedeutung als ein System verstanden, "mit dem das Werk cues auf Denotationen und Konnotationen bereitstellt« (1995: 
33, Herv.i.O.). ${ }^{2}$ Als filminterne Hinweise erlauben cues die kognitive Konstruktion von Bedeutung, die dadurch unterstützt wird, dass sich der Zuschauer emotional engagiert und sein Alltagswissen und die Kenntnis anderer Filme in den Rezeptionsprozess einbringt.

Bedeutungskonstruktionen und Rezeptionsengagement werden dramaturgisch antizipiert und zusammen mit dem Handlungsverlauf organisiert. Letzterer erfordert zwingend den Widerstreit von Interessen, mithin den Konflikt als handlungstreibender Kraft - reiner Konsens ist nicht erzählbar, narrative Formen benötigen Dynamik, die sich aus der Kollision normativer Differenzen ergibt (vgl. Leschke 2017, passim). Für die Übersetzung von Politik als ordnungsgebender Praxis in narrative Strukturen ebenso wie für die Narrativierung des Politischen verstanden als machtbesetzter Antagonismus - bedeutet dies, dass Erzählbarkeit an eine Repräsentation normativer Konflikte gebunden ist. Vonnöten ist also eine moralische Codierung des Politischen. Diese findet innerhalb eines Koordinatensystems statt, welches anhand von historisch wie ideologisch variierenden Wertvorstellungen hinsichtlich des politisch >Guten >Schlechten stehen also aus einer Reihe kollidierender Moralnormen, die überdies auf einer Werteskala angeordnet und zumeist emotional besetzt sind. Wahrnehmbar werden diese Antagonismen und ihre jeweilige Bewertung durch ihre Verankerung eine entsprechende Merkmalsclusterung innerhalb des Figurenensembles.

Insofern handelt es sich bei Filmfiguren nicht um unabhängig handelnde Wesen, sondern um intentional gestaltete ästhetische Konstrukte. ${ }^{3}$ Figur wird hier als Bündel von Merkmalen im Sinne der Gesamtheit aller Informationen verstanden, die dem Rezipienten von Seiten des Films über eine Figur angeboten werden (vgl. Nuy 2017: 80; vgl. auch: Leschke 2017: 81). Die Bündelung der Merkmale zu einer Figur steht dabei nicht für sich alleine, sondern in Relation zu dem übrigen Personal. Merkmale einer Figur müssen daher immer im Zusammenhang mit dem gesamten Figurenensemble betrachtet werden. Zunächst einmal sind Merkmale per se auf Differenzen angewiesen, auf etwas nicht-identisches, anderes außerhalb ihrer selbst. Auch korrespondierende Merkmale benötigen die distinkte Abweichung,

2 Thompson nennt vier Ebenen der Bedeutung: referentiell, explizit, implizit und symptomatisch. Letztere entspricht einer politischen Lesart, wenn man nämlich von einer »nicht expliziten Ideologie eines Filmes spricht oder vom Film als Reflexion gesellschaftlicher Tendenzen« (1995: 33).

3 Der Begriff der Figur ist vielschichtig und hat unterschiedliche Beschreibungs- und Analysemodelle hervorgebracht. Grob lassen sich dabei an einem psychologischen Naturalismus orientierte Ansätze, die Figuren als fiktive Wesen beschreiben, von strukturalistischen und morphologischen Annahmen unterscheiden, die Figuren als Summe von Merkmalen definieren. An letzteren orientiert sich die hier vorgestellte Dramaturgie des Politischen. Diese beruht ferner auf der narratologischen Prämisse, dass Figuren durch die Kollision konfligierender Merkmale Handlung generieren (vgl. Nuy 2017). 
damit Unterscheidung möglich wird. Für die Entwicklung von Handlungsverläufen aus den Konflikten der Figuren sind Oppositionen unverzichtbar, Merkmale im Sinne handlungsmotivierender Eigenschaften oder normativer Zuschreibungen wie gut oder böse brauchen ihren jeweiligen Gegensatz, um sich als Differenz deutlich vom Umfeld aller vorhandenen Merkmale abzuheben, damit sie entsprechend wahrgenommen und verstanden werden können. Figuren als Bündel von Merkmalen sind dabei "nicht homogen, sondern zerlegbar, heterogen und damit offen für logische Widersprüche« (Vernet 2006: 17, Herv. i.O.). Das bedeutet auch, dass die einzelnen Merkmale sich gegenseitig relativieren können. Prinzipiell ist damit die Auswahl an Merkmalen einer Figur unendlich, doch bei der Darstellung historischer Personen oder solcher des Zeitgeschehens, in Fällen also, bei denen der Satz der Merkmale - einen entsprechenden Anspruch auf Wahrhaftigkeit vorausgesetzt - seine Entsprechung im realen Geschehen findet oder zumindest finden sollte, ist die Anzahl potentieller Merkmale stark eingeschränkt. Die Relation zwischen historischer Überlieferung und filmischer Merkmalsbündelung ist entsprechend ein gern genommener Gegenstand filmischer Kritik.

Um die Gefahr der Beliebigkeit einzudämmen, wird die Bündelung und >Programmierung، von Merkmalen entscheidend durch die Figurenkonzeption bestimmt, diese korreliert mit historischen, kulturellen und typologischen Variablen, ist also von sich verändernden Menschen- und Weltbildern ebenso abhängig wie von dem jeweils in Rede stehenden Genre.

Ohne Zweifel verfügen bereits die anthropologischen Vorstellungen, welche die Bündelung von Merkmalen zu einer Figur anleiten, über ein politisches Moment, sind ihnen doch historisch und kulturell variierende Subjektmodelle inhärent, die ihrerseits abhängig sind von der Kontextualisierung durch eine Politische Kultur. Für das Verstehen eines Films ist es nötig, dass die Figuren mit einem Koordinatensystem aus Werten und Normen verbunden sind, die der Zuschauer nachvollziehen kann. Vice versa beeinflussen die durch den fiktionalen Film vermittelten Vorstellungen von Merkmalsbündelungen, gerade dann wenn sie sich zu Stereotypen verdichten, wie Angehörige bestimmter Gruppen zu sein haben, die Vorstellungen der Rezipienten. Diese figurendramaturgische Logik soll nun am Beispiel von Medienfilmen weiter ausgeführt und konkretisiert werden.

\section{Medien der Politikdarstellung im Medium Film}

Die strukturalistisch-morphologische Betrachtung von Figuren schließt ein, dass auch Dinge über figurative Eigenschaften verfügen können (vgl. Leschke 2017: 129; s. auch Abb. auf S. 132). Damit werden Massenmedien zu potentiellen Figuren, die den Handlungsverlauf vorantreiben. Wenn Medien einen figurativen Status einnehmen, ergeben sich dramaturgische Strukturen in unterschiedlichen Kom- 
plexitätsgraden: von der einfachen Vermeldung einer Nachricht, die situationsverändernd wirkt, bis hin zu verschachtelten selbstreflexiven Diskursen über die Funktionen von Medien in politischen Systemen. Letztere sind von besonderem Interesse und Relevanz für die Analyse der Narrativierung des Politischen. Die historisch variierende Verbundenheit von Politik und Medien vermittelt sich vor allem im Genre des Medienfilms, in dem Medienunternehmen und Journalisten im Mittelpunkt der Handlung stehen.

Die Figur des Journalisten hat den Vorteil, dass sich normative Erwartungen an Medien personalisieren lassen und dadurch der Rezeption unmittelbarer zugänglich sind als abstrakte Zusammenhänge zwischen Medium, Rezipient und Gesellschaft. Doch auch die in Mediendispositiven eingeschriebenen Machtbeziehungen werden dramaturgisch funktionalisiert, wenn Medien handlungsfunktional in das Figurenensemble integriert sind und eine doppelte Aufgabe zu erfüllen haben: einerseits den Handlungsverlauf voranzutreiben und andererseits für die Wahrhaftigkeit der Erzählung zu bürgen. Fungieren Medien als Referenten des realen Geschehens, werden sie im Film situativ eingesetzt, strahlen in ihrer Wirkung aber auf den gesamten Handlungsverlauf aus und beglaubigen die Erzählung. Begrenzt ist die Zeugenschaft von Medien naturgemäß auf eine Erzählte Zeit der rezenten Vergangenheit, von der bereits audio-visuelle Aufnahmen existieren. Vor allem dann, wenn filmisches Reenactment von Zeitgeschichte intendiert ist, werden apparative Medien und ihre Inhalte als Zeugen herangezogen, um Realitätsnähe unter Beweis zu stellen und glaubwürdig zu wirken. Vor allem Fernsehnachrichten werden eingesetzt, damit deren sauthentische` Bilder für die Echtheit des Dargestellten bürgen. In der filmbildlichen Cadrage wird dann ein Fernsehgerät häufig so platziert, dass der Bildschirm deutlich zu erkennen ist und der Rezipient - meist in gleicher Blickachse wie die Figuren im Bild - mit Nachrichtenaufnahmen konfrontiert wird.

Vordergründig dient die Verwendung von Nachrichtenmaterial im Film der Orientierung der Rezipientinnen und des Rezipienten, so wird eine Zuordnung der erzählten Zeit vorgenommen und auch räumliche Koordinaten werden abgesteckt. Aufnahmen historischer oder amtierender Politikerinnen und Politiker knüpfen außerdem an die Lebenswirklichkeit und den Alltag der Rezipientinnen und Rezipienten an und sorgen somit für den Eindruck von Wahrheit und Authentizität des Dargestellten. Ausgehend von der - im Übrigen falschen - Erwartung, dass Nachrichtenbilder neutral darüber berichten, wie es >wirklich gewesen ist<, wird auf eine Eigenschaft visueller Medien vertraut, die Pierre Bourdieu als gefährlich beschreibt: Das Fernsehen könne erzeugen »was Literaturkritiker den effet du réel nennen, den Wirklichkeitseffekt: Es kann zeigen und dadurch erreichen, dass man glaubt, was man sieht« (1998, S. 27, Herv. i.O.).

Der Schauwert der scheinbaren Authentizität überlagert dabei die Problematik, dass der Film mit der Integration von Nachrichtenmedien eine Zweitverwer- 
tung von Bildern und Aussagen vornimmt, die die fernsehmedialen Auswahllogiken passiert haben und nachrichtenjournalistisch aufbereitet sind. Isoliert man diese Präsentation von Politik als Inszenierung in der Inszenierung, so ergibt sich eine Verschiebung der Ebene, auf der innerhalb der Narration Politisches verhandelt wird. Wenn Politik im Film durch Nachrichtenmedien gezeigt wird, geht es nicht länger um Politik im Sinne eines instrumentellen Entscheidungshandelns, sondern um Politikvermittlung, um die Darstellung politischen Handelns unter der Prämisse einer bestimmten Wirkungsabsicht oder um symbolische Politik per se. Trotzdem oder gerade deswegen ist die massenmediale Darstellung von Politik wesentlicher Teil der politischen Referenzlogik des Spielfilms. Aus diesem Befund erwächst die Frage, wie politische Öffentlichkeit ${ }^{4}$ innerhalb fiktionaler Handlungsverläufe dargestellt wird und welche Erzählmuster sich dabei identifizieren lassen.

Das Kommunikationssystem Öffentlichkeit - von Habermas auch als "Netzwerk für die Kommunikation von Inhalten und Stellungnahmen, also von Meinungen « (1994: 436; Herv. i.O.) beschrieben - fungiert in Demokratien als Leitbild und normative Zentralkategorie (vgl. Schicha 2000). Die Funktionalität von Öffentlichkeit erschöpft sich jedoch nicht in der Deliberation, vielmehr stellt sie auch die für eine demokratische Entscheidungsfindung notwendige Transparenz her. Politische Macht wird in der und durch die Öffentlichkeit abgesichert und legitimiert. Indem sie Kontrolle ausübt und Machtmissbrauch verhindert, begrenzt Öffentlichkeit Machtspielräume. Entsprechend haben die Massenmedien - folgt man Pippa Norris - vornehmlich drei Funktionen in der politischen Kommunikation: sie bieten ein Forum für pluralistische Debatten, treten als Kontrollinstanz auf (als swatchdogs) und dienen der Mobilisierung von Teilhabe (2000: 24). Diese hier nur grob skizzierten Ansprüche und Funktionszuweisungen an eine Medienöffentlichkeit sind selbst Teil öffentlicher Aushandlungsprozesse, daher soll im Folgenden aufgezeigt werden, wie sich der Spielfilm, genauer: der Medienfilm in diesen Diskurs einbringt. Der im Medienfilm zur Anschauung kommende >Möglichkeitssinn konturiert immer auch ein normatives Verhältnis von Massenmedien und Politik, welches auf außerfilmische Zusammenhänge verweist. $\mathrm{Zu}$ fragen ist folglich: Welche Muster einer diegetischen Medialisierung von Politik finden sich in Medienfilmen? Und wie verhält sich die in Szene gesetzte Öffentlichkeit zu lebensweltlichen Diskursen?

4 Unter politischer Öffentlichkeit wird hier ein durch Medien konstituiertes Kommunikationssystem verstanden, in dem über Angelegenheiten beraten und entschieden wird, die das Zusammenleben in einer Cesellschaft allgemeinverbindlich organisieren. Zugrunde liegt die Vorstellung einer massenmedial strukturierten Öffentlichkeit; die Rede ist also von einer Medienöffentlichkeit, die sich von einem professionellen Journalismus nicht trennen lässt. 


\section{Öffentlichkeit im Medienfilm}

Seit ihrem Auftreten in den dreißiger Jahren lässt sich an den Zeitungs-, Reporterund Medienfilmen eine »Geschichte der Öffentlichkeitsverständnisse des 20. Jahrhunderts«, ablesen (Wulff 2014: 257). Diese Mediengeschichte der Öffentlichkeit konstituiert sich nach Wulff anhand von vier Faktoren (vgl. 2014: 257): da ist zunächst die Darstellung journalistischer Selbstbilder, die einher geht mit einer Reflexion journalistischer Ethik. Zweitens kommen technische und organisatorische Veränderungen in der Medienlandschaft zur Geltung, drittens wird der soziale und kulturelle Einfluss journalistischer Arbeit aufgezeigt und viertens schließlich geht es um Machtkonstellationen politischer oder ökonomischer Art. In dieser Hinsicht leisten Medienfilme häufig auch eine Medienkritik.

Erzählmuster des Zeitungsfilms, der in den dreißiger Jahren einen Höhepunkt erlebte, stellten den Zeitungsjournalisten und seinen Berufsalltag in den Mittelpunkt. Unterschieden wird zwischen seriösem politischem Kommentar und einem Journalismus, der darauf ist, Skandale aufzudecken. Die Suche nach dem Verborgenen, das Enthüllen von Geheimnissen und Verschwörungen wird verknüpft mit einem Verständnis von Öffentlichkeit als einem Raum, der durch Wahrheit und Freiheit strukturiert ist. Und nicht nur das: im Sinne einer kommunikativen Ethik wird der Öffentlichkeit ein Anrecht auf Wahrheit zugeschrieben. Aufgegriffen wird das Konzept vom Journalisten als Aufklärer im Kino der Siebziger Jahre, als es zu einer neuen Welle an Reporterfilmen kommt, in denen Reporter investigativ tätig sind. Das bekannteste Beispiel ist hier sicherlich All The President's Men (USA 1976), Alan J. Pakulas Film über die Watergate-Affäre. Der deutsche Titel Die Unbestechlichen verweist schon darauf, dass Journalistenfiguren in dieser Phase häufig die Aufgabe zukommt, als integre, nicht-korrumpierbare Wächter der Wahrheit zu wirken.

Freilich gibt es auch den Gegenwurf des skrupellosen Reporters, der für eine gute Story über Leichen geht, wie etwa in Die verlorene Ehre der Katharina Blum (D 1975).

»Wenn im >edlen Journalisten das Bild einer Presse [...] aufscheint, die im Dienste einer demokratischen Öffentlichkeit steht, solchen Werten wie Wahrheit, Gerechtigkeit und Cleichberechtigung verpflichtet ist, so prangert die Figur des Sensationsreporters eine Medienlandschaft an, die diese Öffentlichkeitsaufgaben nicht wahrnimmt und die elementare ethische Bindungen journalistischer Arbeit mißachtet« (Wulff 2002: 8).

Gelegentlich wird Öffentlichkeit dann als entpolitisierte Unterhaltungsarena konzipiert, als ein Ort, an dem Sensationslüsternheit, Schaulust und Schadenfreude vorherrschen. 
Für das Kino seit den Neunziger Jahren konstatiert Wulff mit der Herausbildung des Medienfilms eine neue Perspektive auf Journalismus und Öffentlichkeit. Es ginge nicht mehr um journalistische Tugenden und um die soziale oder politische Verortung der Journalistenrolle, sondern um das Agieren von Personen im öffentlichen Raum der Medien (vgl. 2002: 12). Wenn die Manipulierbarkeit von Öffentlichkeit und die Inszenierbarkeit von Politik thematisiert werden, handelt es sich häufig um Satiren wie etwa Wag the Dog (USA 1997). Ein Film, in dem die USA einen fingierten Krieg gegen Albanien führen, der nur in den Medien stattfindet und von einem Hollywood-Produzenten spektakulär in Szene gesetzt wird, um von einem Missbrauchsvorwurf gegen den Präsidenten abzulenken. Wag the Dog ist auch ein Beispiel für die Reflexion der Macht moderner Medienkonzerne, Realität zu konstruieren, Informationen zu kontrollieren und zu steuern - und damit politische Macht zu sichern oder zu ermöglichen.

Während sich normative Erwartungen an Medien in der Figur des Produzenten oder Journalisten recht einfach personalisieren lassen, so sind in Machtkonstellationen eingebundene Medienkonzerne schwieriger zu narrativieren. Dass dennoch - wie oben ausgeführt - auch Medien figurativen Status annehmen können und damit Öffentlichkeitsnarrative generieren können, soll nun am Beispiel des Films Good Night, and Good Luck näher erläutert werden.

\section{Fallstudie: Good Night, and Good Luck}

Good Night, and Good Luck lautete der Satz, mit dem sich der legendäre Fernsehjournalist und Moderator Edward R. Murrow stets am Ende der Sendung von seinem Publikum verabschiedete. George Clooney hat ihn in seiner zweiten Regiearbeit porträtiert; der Gestus des in Schwarzweiß gedrehten Films ist durchaus ein dokumentarischer, stilistisch geprägt von einer »wunderschön verrauchten Jazzkneipen-Eleganz«, wie die Filmkritikerin Susan Vahabzadeh von der Süddeutschen Zeitung schreibt (2006). Die Handlungssequenzen sind unterbrochen - oder verbunden, wie man will - durch Auftritte der Jazzsängerin Dianne Reeves, so dass Musik und das unablässige Rauchen von Zigaretten Atmosphäre und Rhythmus der Narration bestimmen.

Good Night, and Good Luck ist als ein Kammerspiel im Studio des Fernsehsenders CBS inszeniert. Dies ist insofern bemerkenswert, als das Thema des Films die Kontrollfunktion medial vermittelter Öffentlichkeit ist. Es geht um die Recherchen des Nachrichtenmagazins See it now, die dazu beigetragen haben, dass die McCarthyÄra Mitte der Fünfziger Jahre ihr Ende fand. Dramaturgischer Ausgangspunkt im Film, der sogenannte Point of attack ist der Fall eines Air-Force-Soldaten, der aus dem Militärdienst entlassen wird, weil er sich geweigert hat, seine Schwester und seinen Vater zu denunzieren. Murrow und sein Produzent Fred Friendly schalten 
sich mit einem kleinen Team an Reportern in die Angelegenheit ein und intervenieren gegen das Vorgehen des von McCarthy geleiteten Senatsausschusses, der vermeintliche kommunistische Verschwörungen aufdecken sollte und dabei immer wieder gegen Bürgerrechte verstoßen hat. Trotz der Widerstände, die auf Seiten der Senderleitung und der Werbekunden auftreten, wird die Sendung über McCarthy ein großer Moment des Fernsehens und Murrow zum bewunderten Vorbild für Generationen von Journalisten.

Die Erzählung der Ereignisse in den Jahren 1953 und 1954 ist eingebettet in die Rahmenhandlung einer Preisverleihung 1958. Murrow nutzt seine Dankesrede zu einem Plädoyer für einen politischen Journalismus, der sich den Anfechtungen von Entertainisierung und Ökonomisierung entgegen stellt. Er skizziert das Fernsehen als moralische Instanz der Aufklärung - Aufgabe des Fernsehens müsse es sein, die Zuschauer zu belehren und $\mathrm{zu}$ inspirieren, da es ansonsten nicht mehr als ein Kasten mit Drähten und Leuchten sei.

Diese idealistische Konzeptionierung des Dispositivs Fernsehen geht erzählerisch einher mit einer Fokussierung auf die Produktionsbedingungen. Öffentlichkeit erscheint in erster Linie als journalistischer Arbeitszusammenhang. Murrow bildet als Protagonist das positive Zentrum einer Dramaturgie der Personalisierung. Die Narration folgt mit nur wenigen Ausnahmen seiner Erlebensperspektive und ist überdies in einer Form organisiert, die Andreas Dörner als den american monomyth bezeichnet hat (vgl. 1998a: 28-29). Gemeint ist eine wiederkehrende Grundstruktur politischer Erzählungen, nach der die gestörte Harmonie einer Gemeinschaft durch den selbstlosen Einsatz eines Helden wiederhergestellt wird. Im american monomyth werde, so Dörner, der zivilreligiöse Kern der amerikanischen politischen Kultur verhandelt: eine Orientierung an der Gemeinschaft, die mit einem utilitaristischen Individualismus eine an Spannungen reiche Synthese eingeht (vgl. ebd.). In diesem Fall bedeutet dies, dass Murrow Arbeitsplatz und Karriere zu riskieren bereit ist, um demokratische Grundwerte zu wahren. Good Night, and Good Luck kommt allerdings ohne die Melodramatik aus, die gewöhnlich an diesem Erzählschema klebt. Es geht vielmehr um eine Abfolge von Schlüsselsituationen, die aus heutiger Sicht zielgerichtet erscheint - ist doch der historische Verlierer in diesem Konflikt bekannt. So wirken die jeweils getroffenen Entscheidungen des Protagonisten und seiner Helfer sinnhaft und medienethisch richtig. Dieser Kohärenzeffekt beruht nicht allein auf dem narrativierten realen Geschehen, sondern auch darauf, dass hier eine mehrfach verschachtelte mis en abyme vorliegt. Die Dramaturgie des Films ist getrieben von einem Konflikt zwischen dem Wertesystem eines politischen Journalismus' und einer medialisierten Politik. Als Antagonist ist McCarthy nur in Originalaufnahmen zu sehen und zu hören, er wird nicht außerhalb seiner fernsehmedialen Selbstrepräsentation gezeigt. Dieser dramaturgische Kniff entspricht dem Vorgehen der See-it-now-Redaktion: Auch die Sendung, die den Senator demontierte, enthielt fast ausschließlich Aufnahmen von ihm selbst. 
$\mathrm{Zu}$ sehen und hören waren seine medial dokumentierten Anschuldigungen, Bezichtigungen und Zeugenbeschimpfungen. Die Methode, Gegner durch Verdächtigungen einzuschüchtern, hatte nun die entgegengesetzte Wirkung und sorgte für ein Umschlagen der öffentlichen Meinung (vgl. Michaelsen 2008).

In Good Night, and good Luck stehen sich also ein handelnder Protagonist und ein medial vermittelter Antagonist gegenüber, während das Dispositiv des Fernsehens die Authentizität der historischen Auseinandersetzung zwischen dem Fernsehjournalisten und dem Politiker bezeugt. Wenn ein politischer Akteur ausschließlich als mediale Imago präsentiert wird, fungiert nicht die Person sondern das Medium als Figur, sind doch die dispositiven Merkmale entscheidender als diejenigen des politischen Akteurs. Der Film zeigt in diesem Fall das Fernsehen, wie es Politiker zeigt. Oder noch präziser: Clooneys Film zeigt das Fernsehen der Fünfziger Jahre, wie es Fernsehaufnahmen eines Politikers für eigene Argumentationszusammenhänge benutzt. Dem Fernsehen im Fernsehen im Film kommt somit dramaturgisch eine doppelte Funktion zu: zum einen ist es handlungsfunktional in die Figurenkonstellation integriert und muss in der Position des Antagonisten den Handlungsverlauf vorantreiben, zum anderen soll es als Referent des historischen Geschehens für die Wahrhaftigkeit der Erzählung bürgen.

Insbesondere durch den Bildaufbau, der durch die prominente Platzierung von Bildschirmen, Kameras, Tongalgen und Mischpulten immer wieder die Medientechnik des Fernsehens aufruft, wird ein Gegensatz zwischen der Klaustrophobie des Studios als Arbeitsplatz der Journalisten und der medial ermöglichten Ubiquität von Informationen hergestellt. Journalisten und Technik präsentieren sich als Mittler zugunsten einer Watchdog-Öffentlichkeit, die politische Macht kontrolliert und Amtsmissbrauch zu beenden in der Lage ist. Dem Fernsehen wird dabei die Eigenschaft eines Protokollanten zugewiesen: die Aufnahmen dokumentieren, sie beweisen Fehlverhalten wie Unschuld und sie klären auf. Referenzpunkt für ein solcherart strukturiertes Dispositiv Öffentlichkeit ist eine kommunikative Ethik, die auf Freiheit, Wahrheit, Gerechtigkeit, Ehre und Loyalität gründet.

Nichts desto trotz werden Medien von Clooney als selbstreferenzielles System inszeniert, die Journalisten bleiben als Sender von Informationen unter sich, auch das Feedback der Empfänger bleibt intern: Die Reporter lesen sich Presserezensionen ihrer Sendungen vor. Ein Publikum außerhalb des Mediensystems taucht szenisch so gut wie nicht auf. Insofern ist der Konflikt zwischen Murrow und McCarthy als ein zeitversetztes Rededuell angelegt, das an einen abwesenden Dritten gerichtet ist. Dieser abwesende Dritte ist nun nicht nur das zeitgenössische Fernsehpublikum der Fünfziger, sondern auch und vor allem der Kinogänger des 21. Jahrhunderts.

Good Night, and Good Luck wurde bei einem Erscheinen als hoch politischer Film gefeiert. »Vielerorts wurde `Good Night, and Good Luck`gar als eine Art Schlüssel- 
ereignis angesehen: Hollywoods >Linke meldete sich nach Jahren selbst verordneter Unmündigkeit mit einem fulminanten Befreiungsschlag zurück« (Busche 2006)

Kaum eine Rezension, die nicht Parallelen gezogen hat zur Innenpolitik der USA und ihren Einschränkungen bürgerlicher Freiheiten im >Patriot Act « nach den Anschlägen vom 11. September. So schreibt etwa Cristina Nord in der taz anlässlich der Filmpremiere bei den Filmfestspielen in Venedig 2005:

»Zwar wird es an keiner Stelle ausgesprochen, doch `Cood Night, and Cood Luckı lässt keine Zweifel daran, dass das Panorama der Verdächtigung, der Willkür, der Denunziation und des Duckmäusertums, das der Film zeigt, in die Gegenwart hereinragt. An einer Stelle etwa sagt Murrow: >Wir können die Freiheit nicht außerhalb unseres Landes verteidigen und sie zu Hause mit Füßen treten.< Man müsste die Augen verschließen, um hierin kein Statement zur gegenwärtigen politischen Situation zu sehen« (Nord 2005).

Clooneys filmisches Plädoyer zielt auf die Pressefreiheit als demokratiekonstituierendem Moment. Zugleich appelliert er an die Medien, ihrer Verantwortung gegenüber der Gesellschaft gerecht zu werden. Die Haltung des Films beschreibt eine journalistische Ethik und eine politische Moral, die als vorbildhaft dargestellt werden. Murrow stellt nie in Frage, ob Kommunisten verfolgt werden sollen oder nicht, ihm geht es allein um die Methoden, die Vorverurteilungen ohne Beweise und die verletzten Grundrechte. Damit verkörpert die Figur zugleich einen politischen Journalismus, der als gesellschaftliche Institution der Wahrheit verpflichtet ist und politischer Machtwillkür als Kontrollinstanz gegenüber gestellt. Dass der in Good Night, and Good Luck formulierte Appell auch mehr als zwölf Jahre nach Erscheinen des Films nichts von seiner Aktualität eingebüßt hat, sei am Rande vermerkt.

\section{Schluss}

Als plurimediale narrative Praxis, so lässt sich bilanzieren, stellt der Spielfilm $\mathrm{Zu}$ sammenhänge her, die politischen Sinn sinnenfällig werden lassen. Mit Hilfe von Verfahren der Personalisierung, Polarisierung und Moralisierung kommt es zu einer Narrativierung des Politischen, die den Rezipienten bzw. die Rezipientin gleichermaßen kognitiv wie emotional adressiert. Zur Theoretisierung des Verhältnisses von Film, Narration und Politik wurde ein dramaturgisches Modell vorgeschlagen, das zugleich als methodisches Instrument fungieren kann. Dramaturgie als Zusammenspiel aus formaler Ordnung des Handlungsverlaufs, inszenatorischer Realisation und Wirkungskalkulation eignet sich in besonderer Weise, die Anatomie des Politischen im Film analytisch freizulegen. Der Politischen Kulturforschung kann der dramaturgische Ansatz eine neue interdisziplinäre Perspektive 
eröffnen, um Wechselwirkungen von filmischer Erzählung und Politischer Kultur zu erforschen. Der vorliegende Beitrag hat am Beispiel von Medienfilmen Narrative von Öffentlichkeit untersucht, um aufzuzeigen, wie Öffentlichkeit als normativ besetzte conditio sine qua non von Demokratie in eben dieser Öffentlichkeit verhandelt wird. Die (Teil-)Öffentlichkeit des Kinos konstituiert einen diskursiven Raum, der sich im Fall des analysierten Films Good Night, and Good Luck als Ort der Kritik und der Ermahnung darstellt, wenn er Politik wie Medien auf den Prüfstand stellt und sowohl Pressefreiheit als auch journalistische Verantwortung einfordert.

\section{Literatur}

Aristoteles 2012: Poetik. Übersetzt und herausgegeben von Manfred Fuhrmann, Stuttgart.

Barthes, Roland 1988: Das semiologische Abenteuer, Frankfurt a.M.

Bergem, Wolfgang 2014: Narrative Formen in Geschichtspolitik und Erinnerungskultur. In: Wilhelm Hofmann/Judith Renner/Katja Teich (Hg.), Narrative Formen der Politik, Wiesbaden, S. 31-48.

Bourdieu, Pierre 1998: Über das Fernsehen, Frankfurt a.M.

Busche, Andreas 2006: Rauchzeichen aus Hollywood. In: taz. die tageszeitung, 5.4.2006.

Dörner, Andreas 1998: Das politische Imaginäre. Vom Nutzen der Filmanalyse für die Politische Kulturforschung. In: Hofmann, Wilhelm (Hg.), Visuelle Politik. Filmpolitik und die visuelle Konstruktion des Politischen, Baden-Baden, S. 199-219.

Dörner, Andreas 1998a: Die Simpsons. Zivilreligion im Fernsehformat. In: medien praktisch, H. 2, S. 27-31.

Eder, Jens 2007: Dramaturgie des populären Films: Drehbuchpraxis und Filmtheorie, 3. Auflage, Hamburg.

Gadinger, Frank/Jarzebski, Sebastian/Yildiz, Taylan 2014: Politische Narrative. Konturen einer politikwissenschaftlichen Erzähltheorie. In: Dies. (Hg.): Politische Narrative. Konzepte - Analysen - Forschungspraxis, Wiesbaden, S. 3-38.

Gadinger, Frank/Yildiz, Taylan, 2017: Politik. In: Martínez, Matías (Hg.), Erzählen. Ein interdisziplinäres Handbuch, Stuttgart, S. 158-165.

Gadinger, Frank 2018: »Whatever it takes 24 und die Normalisierung des Ausnahmezustandes. In: Switek, Niko (Hg.), Politik in Fernsehserien. Analysen und Fallstudien zu House of Cards, Borgen \& Co., Bielefeld, S. 305-326.

Habermas, Jürgen 1994: Faktizität und Geltung. Beiträge zur Diskurstheorie des Rechts und des demokratischen Rechtsstaats, Darmstadt.

Hofmann, Wilhelm/Renner, Judith/Teich, Katja, (Hg.) 2014: Narrative Formen der Politik, Wiesbaden. 
Leschke, Rainer 2017: Die Formen massenmedialen Erzählens. Normative Strukturen und narrative Programme, Münster (im Druck).

Michaelsen, Hans-Jörg, 2008: Reporter Edward R. Murrow. Wahrheit als Waffe. In: Spiegel online, einestages, 25.04.2008, online unter: www.spiegel.de/einestages/reporter-edward-r-murrow-a-946825.html (22.11.17)

Mouffe, Chantal, 2008: Das demokratische Paradox, Wien.

Müller-Funk, Wolfgang 2002: Die Kultur und ihre Narrative. Eine Einführung, Wien, New York.

Musil, Robert 1989: Der Mann ohne Eigenschaften, hg. von Adolf Frisé, Hamburg.

Nord, Cristina 2005: Unter Verdacht, in: taz. die tageszeitung, 02.09.2005, online unter: www.taz.de/!551660/(22.11.17)

Norris, Pippa 2000: A Virtuous Circle. Political Communications in Postindustrial Societies. Cambridge.

Nünning, Ansgar 2013: Wie Erzählungen Kulturen erzeugen: Prämissen, Konzepte und Perspektiven für eine kulturwissenschaftliche Narratologie. In: Alexandra Strohmaier (Hg.), Kultur - Wissen - Narration. Perspektiven transdisziplinärer Erzählforschung für die Kulturwissenschaften, Bielefeld, S. 15-54.

Nuy, Sandra 2017: Die Politik von Athenes Schild. Zur dramaturgischen Logik des Politischen im fiktionalen Film, Münster.

Rohe, Karl 1990: Politische Kultur und ihre Analyse: Probleme und Perspektiven der politischen Kulturforschung. In: Historische Zeitschrift, Bd. 250, H. 2, S. 321-346.

Schicha, Christian 2000: Öffentlichkeit unter Medienbedingungen, Zur Diskrepanz zwischen normativen Konzepten und der Praxis der Politikberichterstattung. In: Ders./Carsten Brosda (Hg.), Medienethik zwischen Theorie und Praxis, Münster, S. 173-194.

Stutterheim, Kerstin 2014: Filmdramaturgie als >Geheimnis des Erzählensく. In: Christa Hasche/Eleonore Kalisch/Thomas Weber (Hg.), Der dramaturgische Blick, Potenziale und Modelle von Dramaturgie im Medienwandel, Berlin, S. 143-162.

Thompson, Kristin 1995: Neoformalistische Filmanalyse. Ein Ansatz, viele Methoden. In: montage/av. Jg. 4, H. 1, S. 23-62.

Vahabzadeh, Susan 2006: Im Kino: »Good Night, and Good Luck«. Der Kommunistenjägerbesieger. In: Süddeutsche Zeitung, Nr. 80, 05. April 2006. Online unter: www.sueddeutsche.de/kultur/im-kino-good-night-and-good-luck-derkommunistenjaegerbesieger-1.437074 (22.11.17).

Vernet, Marc, 2006: Die Figur im Film, in: montage/av Jg.15, H. 2, S. 11-44.

Weber, Thomas 2014: Vorwort. Dramaturgien im Medienwandel. In: Christa Hasche/Eleonore Kalisch/Thomas Weber (Hg.), Der dramaturgische Blick, Potenziale und Modelle von Dramaturgie im Medienwandel, Berlin, S. 9-13. 
Wulff, Hans J. 2002: Zeitungs-, Reporter-, Medienfilme: Journalismus und Medien im Film. In: Medien praktisch. Texte 5, S. 46-55. Zit. nach der Online-Fassung: www.derwulff.de/2-111 (22.11.17).

Wulff, Hans J. 2014: Zeitungs-Journalisten und populäre Konzeptionen des Zeitungswesens. Die Zeitung im Film. In: Kay Kirchmann/Jens Ruchatz (Hg.), Medienreflexion im Film. Ein Handbuch, Bielefeld, S. 257-278. 



\section{Alltagskulturelle Selbstdisziplinierung Über die Macht der Ohnmacht in Fernsehserien}

Samuel Salzborn

Politikwissenschaftliche Forschung kreist um die Frage der Macht- und Herrschaftsverhältnisse und die in diesen zum Ausdruck kommenden Beziehungsund Akteurskonstellationen. Dabei kann, einer Differenzierung von Hanna Fenichel Pitkin (1967) folgend, unterschieden werden zwischen power to (»Macht zu ...«) und power over (»Macht über ...«), also einem sozialen Phänomen, das gleichermaßen die individuelle wie die interaktive Dimension umfasst. Dieser Gedanke hat Gerhard Göhler (2004: 257f.) dazu veranlasst, transitive und intransitive Macht zu unterscheiden - transitive Macht als die nach außen gerichtete, die »den eigenen Willen auf andere überträgt «, und intransitive Macht als die nach innen gerichtete, die »in sich selbst, in der Gesellschaft erzeugt und aufrechterhalten wird «, so dass Selbst- und Fremdreferenz zum zentralen Anker werden, kontextualisiert mit der Potentialität und/oder Aktualität von Macht.

Zentral bei politischen und gesellschaftlichen Machtkonstellationen ist insofern stets eine Dimension, der auch die Hauptaufmerksamkeit der politischen Kulturforschung gilt: das Verhältnis von objektiven Strukturen und subjektiver Dimension des Politischen, also die Frage nach der sozialen Interdependenz und insofern faktisch nach der Kongruenz oder Inkongruenz von politischer und sozialer Ordnung, in denen das Verhältnis von objektiven und subjektiven Dimensionen vermittelt ist (vgl. Salzborn 2009). Die politischen und sozialen Orte, an denen diese Machtkonstellationen generiert und interagiert werden, sind mannigfaltig; einer dieser Orte mit einer massenwirksamen Relevanz sind Fernsehserien: Der machtanalytische Fokus liegt dabei, wie im Folgenden theoretisch noch argumentativ entwickelt wird, in einer Tendenz der optimierten Selbstüberwachung in der Alltagskultur, die ohne Gewalt erfolgt und dabei die Macht der Ohnmacht als leitendes Identifizierungsmotiv sichtbar werden lässt. Dieses Motiv wird im vorliegenden Beitrag in seinen Variationen anhand eines Vergleichs von drei Fernsehserien in den Blick genommen, die höchst different - und zugleich alle jeweils: höchst erfolgreich - sind: Benjamin Blümchen (Kiddinx, seit 1988), Game of Thrones (HBO, seit 2011) und American Dad (Fox/TBS, seit 2005). 
In der Ideengeschichte wurden Macht- und Herrschaftsverhältnisse in höchst unterschiedlichem Maße reflektiert (vgl. Anter 2012; Imbusch 2012), im vorliegenden Beitrag soll insofern eine Dimension in den Blick genommen werden, für die sich besonders psychoanalytische, kritisch-theoretische und strukturalistische Ansätze interessiert haben (vgl. Salzborn 2013): Wenn davon auszugehen ist, dass kein Handeln von politischen Akteuren und Akteurinnen interessenlos erfolgt, zugleich aber nicht selten - zuletzt vor allem immer wieder mit Blick auf die Anarchie in den internationalen Beziehungen betont - das Rationalitätsparadigma der Aufklärung jenseits von objektiven Interessenkonflikten in Entscheidungsprozessen suspendiert wird, also politische und soziale Akteure ihren objektiven Interessen zuwiderhandeln, stellt sich die Frage nach der Begründung für diese Diskrepanz. Einfacher gesagt: Warum entscheiden und/oder handeln Akteuren und Akteurinnen gegen ihre objektiven Interessen?

Man kann diese Frage durch Verweis auf die repressive und gewaltförmige Struktur von Herrschaftspraxis bzw. die Formierung von informellen Repressionsstrukturen beantworten, was für die Politik in autoritären Regimen eine hohe Plausibilität hat (vgl. Rensmann/Hagemann/Funke 2011) - zugleich aber für die Politik unter demokratischen Bedingungen als analytisches Instrumentarium nur sehr eingeschränkt tragfähig ist. Denn die gewaltförmige Repression ist in demokratischen Gesellschaften weiterhin die Ausnahme und damit die Abweichung, die gegen die Norm verstößt. Die Unterstellung struktureller Repression erfolgt insofern auch oft nur unter argumentativer Zuhilfenahme von Erklärungen, die eine latente Verschwörungsdimension beinhalten und insofern zwischen Ideologie und Lüge oszillieren. Dies kann man beispielsweise im Feld der surveillance studies sehen, die auf der einen Seite wichtige Einzelfallstudien erarbeiten (vgl. Zurawski 2007), zugleich aber aus diesen Einzelfällen Generalisierungen über die (so die Unterstellung: strukturell repressive) Funktionslogik von Sicherheitsarchitektur ableiten, die de facto nicht belegt, sondern stets nur intuitiv assoziiert werden. Auf diese Weise scheint dann aggressives, gewaltförmiges Verhalten in einer Institution wie der Polizei nicht mehr als individuelle, sondern als strukturelle Logik, die dazu dient, dass ein letztlich vergleichsweise einfaches Sozialmodell zur Erklärung von gesellschaftlichen Funktionslogiken herangezogen wird: Menschen agieren nicht gegen Ungleichheitsordnungen, weil sie Angst vor Repression hätten.

Dieses Analyseraster ist nicht nur zu einfach, sondern es ist auch ignorant gegenüber den Menschen als Subjekten, die für das, was sie (nicht) tun, stets in vollem Umfang verantwortlich sind und die man nicht durch eine - prinzipiell und grundsätzlich berechtigte - Kritik an der Struktur bürgerlicher Vergesellschaftung moralisch aus ihrer Verantwortung dafür entlassen darf, dass diese gesellschaftlichen und politischen Strukturen eben genau so eingerichtet sind, wie sie es sind. Wer Repression als analytischen Schlüssel für das Funktionieren von demokratischen Regimen versteht, bleibt insofern verfangen in einer Macht- und Herrschaftsana- 
lyse, die den Kontext der bürgerlichen Gesellschaft ausblendet - und vorsätzlich oder unbewusst ignoriert, dass diejenigen Individuen, die nicht Subjekte sein wol$l e n$, dies unter demokratischen Bedingungen höchst selbst zu verantworten haben.

$\mathrm{Zu}$ verstehen, dass es innerhalb von demokratischen Systemen nicht primär die repressive, sondern die internalisierte Dimension von Disziplinierung ist, die Menschen zu sozial angepassten und konformistisch agierenden Akteuren und Akteurinnen werden lässt und diese Internalisierung nicht zwingend im Einklang mit den strukturellen Vorgaben des politischen Systems stehen muss, sondern ganz im Gegenteil auch in (teilweiser oder grundsätzlicher) Opposition stehen kann, ist nicht nur wichtig, um zu begreifen, wie Prozesse der sozialen Entdemokratisierung funktionieren, sondern auch, um eine stärkere Sensibilisierung für alltagskulturelle Entwicklungen zu gewinnen, die scheinbar unpolitisch sind, aber gerade in ihrer Lüge der Politikabstinenz demokratische Freiheit und Partizipation untergraben. Denn Prozesse der Entdemokratisierung können ja nicht nur vom politischen System ausgehen, sondern auch und gerade von der politischen Kultur: der Wiederaufstieg rechtsextremer Parteien in Europa zeigt dies sehr deutlich, da deren Politik- und Gesellschaftsmodelle genuin auf eine Entdemokratisierung der jeweiligen staatlichen Ordnungen in Europa zielen, was seine Ursprünge eben nicht in den politischen Systemen hat, sondern in einer autoritären und/oder totalitären Veränderung in der politischen Kultur, bei der gegen die demokratische Ordnung opponiert wird (vgl. Salzborn 2017a, 2017b).

In diesem Beitrag soll ein theoretisches Konzept zum Verständnis dieser internalisierten Disziplinierung formuliert werden, das an einer alltagskulturellen Dimension in seiner realen Funktionalität und Wirkmächtigkeit entwickelt wird, das auf mindestens weitgehend, wenn nicht sogar ausnahmslos freiwilliger Rezeption basiert: eben in Fernsehserien. Insofern muss bei den Rezipienten und Rezipientinnen grundsätzlich von einer Identifizierungsdimension ausgegangen werden, die nicht bei allen identisch ist, die aber gleichwohl darauf verweist, dass sich in der Rezeption Formen von Identifizierung finden, die im Individuum faktisch Wunschhorizonte ansprechen und mobilisieren, auch wenn dies unbewusst geschieht und gerade darin die Ideologie der Ideologiefreiheit in der »symbolischen Repräsentation « als "symbolische Konstitution der Realität« (Ebrecht 2003: 67) so wirkmächtig sein kann, dass sie diese schleichend überformt und damit letztlich derealisiert.

Ein Beispiel: Wer zu Unterhaltungszwecken fernsieht, wird fast immer behaupten, dies sei kein politischer Akt; in der Sendungsauswahl, der Konsumdauer und der subjektiven Begeisterungsfähigkeit liegen jedoch mindestens subtil Entscheidungen für bestimmte Angebote, die jeweils spezifische Formen von sozialer und politischer Realität zeigen oder zu zeigen vorgeben, die unvermeidlich auch eine Entscheidung für den Glauben an ein Sozialmodell anbieten. Reality Shows wie Die Höhlen der Löwen (VOX, seit 2014) oder Gemany's Next Topmodel (ProSieben, seit 
2006) versprechen zum Beispiel die Hoffnung auf ein erfolgreiches bzw. glamouröses Leben mit nur punktueller Anstrengung und ohne besondere Mühe; dass die Kandidaten und Kandidatinnen in beiden Fällen hart arbeiten, findet auf der nicht-sichtbaren Hinterbühne der Shows statt - in einem Fall wirkt es so, als hätte jemand eine quasi naturwüchsige Idee und bekäme dafür »einfach« Geld (den ökonomischen Haken, der über die Firmenbeteiligungen generiert wird, übersieht man leicht). Im anderen Fall wird jemand (vermeintlich) reich und berühmt, »nur « weil sie schön ist; dass sich sowohl die erfolgreichen wie die erfolglosen Kandidaten und Kandidatinnen solcher Formate dann wieder in drittklassigen Shows wie dem Dschungelcamp (eigentlich: Ich bin ein Star - Holt mich hier raus!, RTL, seit 2004) verdingen müssen, die ihrerseits die gescheiterten Individuen zur Identifikation einladen und dabei suggerieren, dass man es doch zu etwas bringen könne, wenn man nur fies genug agiert und bereit ist, ekelige Dinge zu tun, wecken über Umwege erneut die Hoffnung, doch noch ohne großen Aufwand erfolgreich sein zu können.

Diese Beispiele appellieren an ein Gesellschaftsverständnis, das zum einen eine extreme Naturalisierungsdimension inkorporiert - am deutlichsten bei Germany's Next Topmodel, weil die Models in der Tat für ihre so verstandene Schönheit schlicht nichts können, sie ist ein Zufall der Natur und zugleich nur dadurch gesellschaftlich vermittelt, dass die Schönheitsideale sich wandeln (vgl. Hersey 1996; Hollander 1993) - und damit scheinbare Natürlichkeiten in den Mittelpunkt der Betrachtung rückt-, zum anderen aber auch suggeriert, dass eine kleine Gruppe von Menschen (gern inszeniert als Jury) über das Schicksal von Menschen nach letztlich intransparenten Kriterien entscheidet. Der Idealtypus dieser irrationalen und respektlosen Haltung ist Dieter Bohlen, der in der typischen Paradoxie aus Unterwerfung und Verehrung zugleich von zahlreichen Menschen fast schon wie ein externalisiertes Über-Ich verehrt wird.

So werden gesellschaftliche Bilder vermittelt, die das Soziale verblassen und Entscheidungsprozesse als intransparent und willkürlich erscheinen lassen, zumal wenn sie von einer allein durch Marktinteressen konstituierten Jury gefällt werden, die als unberechenbar inszenierte Gruppe agiert. Wer dieses Bild als realexistierende Gesellschaftsidee internalisiert, ist sehr schnell bei Phantasien, mit denen rechtsextreme Bewegungen arbeiten (vgl. Salzborn 2016) und die generell Formen von politischer Beteiligungsverweigerung generieren, da sie zugleich an Ohnmachts- wie Allmachtphantasien appellieren.

\section{Das Panoptikum der Unfreiheit}

War spätmittelalterliche Herrschaft klerikal monopolisiert und fielen dabei Herrschafts- und Deutungsmacht ineinander, wurde die mit der modernen 
Macht- und Herrschaftstheorie vollzogene systematische Differenzierung von Macht und Herrschaft (wie sie in ihrer Trennung am deutlichsten von Max Weber [1980] vorgenommen wurde) selbst zum Ausdruck einer Infragestellung dieser Monopolisierung, die besonders durch ihre mangelnde Legitimität gekennzeichnet war. Im Zentrum stand dabei genau jene Infragestellung, wie sie nachhaltig von Niccolò Machiavelli (vgl. Münkler 1984; Skinner 2008) und Thomas Hobbes (vgl. Kersting 2005; Münkler 1993) vollzogen wurde. Das Machtverständnis von Machiavelli und Hobbes war geprägt von einer anthropologischen Grundierung, d.h. beide hielten - wie viele andere Machttheoretiker und Machttheoretikerinnen nach ihnen - das Streben nach Macht und das Bedürfnis, selbst Macht zu haben und sie gegenüber anderen auszuüben, für eine anthropologische Funktion, einen »allgemeinen Trieb der gesamten Menschheit, der nur mit dem Tode endet« (Hobbes 1651: 75; Lev. I, 11). Das entscheidende Moment des mit Machiavelli und Hobbes begründeten modernen Machtverständnisses war die Emanzipation des Machtbegriffes von der Ethik verbunden mit der Annahme, dass »alle Menschen böse sind « und stets ihrer »bösen Gemütsart folgen, sobald sie Gelegenheit dazu haben« (Machiavelli 1531: 26; Disc. I, 3). Diese Auffassung begründet ein Verständnis von Macht, in dem die Frage von effizienter Machtausübung und politischer Herrschaft durch Macht nicht (mehr) an eine moralisch-ethische Implikation der Ziele dieses Machteinsatzes gebunden war. Zugleich wird der Machtbegriff damit aber auch humanisiert, d.h. aus dem Machtmonopol der göttlichen Schöpfungsordnung gelöst und als etwas dem Menschen Eigenes begriffen. Thomas Hobbes schreibt 1651 im Leviathan:

»Die größte menschliche Macht ist diejenige, welche aus der Macht sehr vieler Menschen zusammengesetzt ist, die durch Übereinstimmung zu einer einzigen natürlichen oder bürgerlichen Person vereint sind, der die ganze Macht dieser Menschen, die ihrem Willen unterworfen ist, zur Verfügung steht, wie z.B. die Macht eines Staates. (Hobbes 1651: 66; Lev. I, 10)

Macht kommt hier als Vehikel zur Emanzipation des Menschen als Subjekt zum Einsatz, die es einem mit intersubjektiver Orientierung handelnden Menschen ermöglicht, durch einen rationalen (Vertrags-)Schluss seinen Willen zu verwirklichen. Damit ist Macht an die Vorstellung sowohl des (freien) Willens wie des Handelns gebunden, verfügt also über eine soziale Dimension sowohl hinsichtlich ihrer Konstituierung wie ihrer (kollektiven) Interaktion. Der Bezug auf den Willen des Subjekts ist hier deshalb zentral, weil auf eine kognitive wie emotionale Dimension von Macht abgestellt wird, die zwar auf einem kognitiven Prozess (des Handelns) beruht, aber erst durch den Akt des Handelns in Übereinstimmung als verbindendes Surrogat von Macht als Macht generiert wird.

Der Machtbegriff und sein Herrschaftsbezug bei Machiavelli und Hobbes ist situativ - er bezieht sich auf eine konkrete, idealtypische Situation, ohne diese 
historisch zu kontextualisieren; er ist systematisch, nicht genetisch. Mit Karl Marx und Friedrich Engels bekommt das Machtverständnis eine historische Dimension, ja es wird deutlich, dass Macht eine zeitlich und räumlich relationale Kategorie des Sozialen ist, die abhängig von ihren politischen, sozialen und ökonomischen Kontexten variiert - sowohl was ihre Träger angeht wie auch mit Blick auf ihre Inhalte. Damit ist Macht auch als relative Beziehung ausdifferenziert - als Macht, die man hat oder die man nicht hat. Mit der Macht verbunden ist der Aspekt politischer und ökonomischer Herrschaft und zugleich, in dialektischer Reflexivität, der Ausschluss von dieser:

»Die Gedanken der herrschenden Klasse sind in jeder Epoche die herrschenden Gedanken, d.h. die Klasse, welche die herrschende materielle Macht der Gesellschaft ist, ist zugleich ihre herrschende geistige Macht. Die Klasse, die die Mittel zur materiellen Produktion zu ihrer Verfügung hat, disponiert damit zugleich über die Mittel zur geistigen Produktion, so daß ihr damit zugleich im Durchschnitt die Gedanken derer, denen die Mittel zur geistigen Produktion abgehen, unterworfen sind. Die herrschenden Gedanken sind weiter Nichts als der ideelle Ausdruck der herrschenden materiellen Verhältnisse, die als Gedanken gefaßten herrschenden materiellen Verhältnisse; also der Verhältnisse, die eben die eine Klasse zur herrschenden machen, also die Gedanken ihrer Herrschaft.« (Marx in: Marx/Engels 1845/46: 46)

Damit wird der Machtbegriff nicht nur relativ, sondern auch relational, als Ausdruck eines Zweckes und insofern instrumentell in seinem Charakter, nicht aber in seiner Erscheinung. Als Generalimplikation von Herrschaftsverhältnissen dient Macht insofern für Marx/Engels gleichermaßen in einem handfesten, materiellen und in einem symbolischen, geistigen Sinn, die miteinander verschränkt, aber eben doch nicht deckungsgleich sind, was eine Ausdifferenzierung des Machtverständnisses seinem Inhalt nach nahelegt.

Während das Machtverständnis von Marx/Engels ein historisches war und diesem Verständnis nach - genau wie bei Machiavelli oder Hobbes - Subjekte (wechselseitig) (inter-)agieren, also Macht eine konkrete Relationalität einer unterschiedlich intensiven Beziehung war, entwickelt Michel Foucault (vgl. Gutting 2005; Sarasin 2010) ein an die historisch-genetisch ausgerichteten Macht- und Herrschaftsverständnisse anschließendes Begriffsverständnis, das aber multidimensional formuliert wird. Foucault kontextualisiert Macht explizit in einem konkreten gesellschaftlichen Raum und an einem konkreten historischen Ort, betont damit also das Spannungs- und Variationsfeld von Machtphänomenen, die auch von ihm als soziales Verhältnis begriffen werden, das durch soziale und politische Kräfteverhältnisse geprägt ist: 
»Unter Macht, scheint mir, ist zunächst zu verstehen: die Vielfältigkeit von Kräfteverhältnissen, die ein Cebiet bevölkern und organisieren; das Spiel, das in unaufhörlichen Kämpfen und Auseinandersetzungen diese Kräfteverhältnisse verwandelt, verstärkt, verkehrt; die Stützen, die diese Kraftverhältnisse aneinander finden, indem sie sich zu Systemen verketten - oder die Verschiebungen und Widersprüche, die sie gegeneinander isolieren; und schließlich die Strategien, in denen sie zur Wirkung gelangen und deren große Linien und institutionelle Kristallisierungen sich in den Staatsapparaten, in der Cesetzgebung und in den gesellschaftlichen Hegemonien verkörpern.« (Foucault 1983: 93)

Foucaults Interesse gilt damit den strukturellen Dimensionen von Macht und Herrschaft, die er in Mikro-, Meso- und Makro-Strukturen ausdifferenziert: Auf der Mikroebene sind es die vielfältigen Kräfteverhältnisse, die auf einem Territorium lokalisiert sind, auf der als Spiel gefassten Mesoebene interagieren diese strukturellen Kräfteverhältnisse in Kämpfen und Auseinandersetzungen miteinander und verdichten sich systematisch, auf der Makroebene werden die Strategien der Konflikte konfiguriert und zugleich - eine Begriffsannäherung an Hobbes - in institutionellen Strukturen und Hegemonieverhältnissen verkörpert. Damit wird der Machtbegriff von Foucault multidimensional - in Genese und Wirkung, allerdings verschwinden die sozialen Akteure (und ihre Verantwortung und Verantwortlichkeit für ihr eigenes Handeln bzw. Nicht-Handeln) hinter der strukturellen Fassade von »Kräfteverhältnissen«.

Genau darin liegt auch die analytische Schwachstelle der Sozialkritik von Foucault, wie er sie in Überwachen und Strafen (1977) mit Blick auf das Gefängnis als sozialer Institution formuliert und auf den Begriff des Panoptismus gebracht hat: Foucault greift den Begriff des Panopticons/Panoptikums von Jeremy Bentham (1812) auf und er greift dessen mit dem Begriff verbundene architektonische Konzeption an, wenn er das Panoptikum nicht nur als Gebäudekomplex, sondern als »verallgemeinerungsfähiges Funktionsmodell« (Foucault 1977: 263) begreift, in dem sich Machtbeziehungen als soziales Beziehungsnetz entwickeln, in dem wechselseitige Kontrolle von Funktionen letztlich $\mathrm{zu}$ einer Internalisierung der Machtverhältnisses führen, bei der nicht mehr die reale Überwachung und die mit ihr verbundene Sanktion zum (Nicht-)Handeln motiviert, sondern die Verinnerlichung des Ordnungsprinzips der Überwachung als solchem zum selbstüberwachenden Panoptismus wird.

Die »panoptische Anlage« (ebd.: 257) ist eine architektonische Überwachungskonstruktion mit einem uneinsehbaren Turm in der Mitte. Dieser Turm macht ein ringförmiges, mit breiten Fenstern versehenes Gebäude überwachbar. Dieses ist lichtdurchflutet und in Zellen unterteilt. Auf diese Weise wird »die Macht sichtbar, aber uneinsehbar« (ebd.: 258) - durch den hohen Kontrollturm: 
»Das Panopticon ist eine Maschine zur Scheidung des Paares Sehen/Gesehenwerden: im Außenring wird man vollständig gesehen, ohne jemals zu sehen; im Zentralturm sieht man alles, ohne je gesehen zu werden.« (ebd.: 259)

Die damit konstituierte »fiktive Beziehung« - der Überwachte weiß nicht, ob und von wem er überwacht wird - als mechanische Basis der »wirklichen Unterwerfung « kann dabei auf »Gewaltmittel verzichten«, das Machtverhältnis wird internalisiert, der Überwachte spielt »gleichzeitig beide Rollen«, er wird »zum Prinzip seiner eigenen Unterwerfung« (ebd.: 260).

Foucaults Kritik richtet sich gegen die überwachende bzw. die Überwachung installierende Macht - und nicht gegen die Individuen, die als zur Reflexion fähige Subjekte ja dazu in der Lage wären, ihre repressiven Internalisierungsprozesse $\mathrm{zu}$ reflektieren, eben weil sie keine Automaten sind. Und genau hier müsste eine Erweiterung der Kritik des Panoptismus ansetzen, die die Akte der Internalisierung und Selbstdisziplinierung als Zentrum des Problems begreift und insofern an die Überlegungen von Sigmund Freuds Massenpsychologie und Ich-Analyse (1921) anschließt und das Hauptaugenmerk legt auf die Ersetzung des Über-Ichs des Individuums durch eine externe Autorität, die Adorno (1951: 416) als Externalisierung beschrieben hat.

In dem kulturellen Prozess der Internalisierung und Selbstdisziplinierung geht es unter demokratischen Bedingungen gerade um die freiwillige Unterwerfung unter das Prinzip des Panoptismus, um in der Allegorie zu bleiben, darum, dass der Insasse der Zelle und der des Kontrollturms identisch sind, dass das Paar Sehen/Gesehenwerden sich eben nicht akteursbezogen aufspaltet (was bei Foucault als theoretische Idee durchaus auch schon anklingt), sondern vielmehr einen Differenzierungsprozess im psychischen Apparat beschreibt, bei dem die Realüberwachung durch die Externalisierung des Über-Ichs ersetzt wird: der Selbstdisziplinierungsprozess geht soweit, dass er sich deshalb verselbstständigen kann, weil die sozialisatorischen Bedingungen in der bürgerlichen Gesellschaft dazu führen, dass ihre Totalität unter demokratischen Prämissen eben gerade nicht mehr repressiv sein muss, weil ein Großteil der Subjekte freiwillig die entsubjektivierten Reproduktionsbedingungen internalisiert und nach ihnen handelt. Denn diese Ich-Aufgabe bedeutet eben für das Individuum nicht nur die (Teil-)Aufgabe einer eigenen (potentiell selbstreflexiven) Persönlichkeit, sondern sie beinhaltet zugleich das Versprechen, an einem großen, machtvollen, einflussreichen und überragenden Kollektiv teilhaben zu dürfen (vgl. Clemenz 1998: 144ff.; Bohleber 1992: 139). Die freiwillige Unterwerfung erfolgt in der fiktiven und infantil-regressiven Hoffnung, dadurch die Chance zu bekommen, selbst als Teil eines homogenen Macht-Kollektivs andere unterwerfen zu können.

Die Selbstunterwerfung befriedigt dabei zugleich omnipotente Verschmelzungsund Größenphantasien, denn die Spezifik der Wirksamkeit dieser Macht- und 
Herrschaftsbeziehung liegt in den Momenten von Faszination und Identifizierung, wie sie Mario Erdheim beschrieben hat. Für Erdheim (1988: 373f.) sind es narzisstische »Größen- und Allmachtsphantasien«, die die soziale Wirksamkeit von Macht- und Herrschaftsbeziehungen begründen. Sie sind wechselseitiger Natur und erzeugen ein »Kraftfeld der Faszination«:

»Entweder man identifiziert sich mit den Herrschenden oder mit den Beherrschten. Im ersteren Fall werden die Crößen- und Allmachtsphantasien, im letzteren die Geschichte der Kränkungen, Erniedrigungen und Beleidigungen angesprochen und reaktiviert.« (ebd.: 374)

So entwickelt sich in Macht- und Herrschaftsbeziehungen eine Relation zwischen Herrschenden und Beherrschten, die gerade aufgrund ihrer Ambivalenz und Uneindeutigkeit stabil ist, ein "Legitimationsglaube« (ebd.: 376) als Basis für einen Konsens. An die Legimitation von Herrschaftsverhältnissen wird geglaubt, weil sie »die unannehmbare Realität verdrängen helfen, die Flucht in die Illusion erlauben und die Größen- und Allmachtsphantasien der Unterworfenen serlösen«.« (ebd.: 377) Erdheim betont damit das sozialintegrative Moment von Macht- und Herrschaftsbeziehungen, das jedoch paradoxerweise in seiner integrativen Funktion gerade auf sozial desintegrativen Momenten, respektive narzisstischen Kränkungen beruht.

Und hier liegt der analytische Schlüssel, um das Phänomen der internalisierten Disziplinierung fassbar machen zu können: Der Panoptismus funktioniert am Beginn des 21. Jahrhunderts als psychosozialer Mechanismus deshalb so perfektioniert, weil er die Überwachung ohne Gewalt, die Foucault als historische Entwicklungsstufe im Prozess der Disziplinierung beschrieben hat, in eine optimierte Selbstüberwachung ohne Gewalt verwandelt hat. In dieser ist die Macht der Ohnmacht omnipräsent geworden, weil die Subjekte sich selbst entsubjektivieren und damit der ohnmächtigen Rebellion zur Macht verhelfen.

\section{Die Macht der Ohnmacht in Fernsehserien}

\section{Die inszenierte Ohnmacht des besorgten Bürgers: Benjamin Blümchen}

Benjamin Blümchen war bereits als Kiosk- (später: Kiddinx-)Hörspielkassette (seit 1977) eine prägende Sozialisationsinstanz in bundesdeutschen Kinderzimmern, noch bevor er zum Zeichentrickfilmstar wurde - dem der Ruf anhaftet, in einer zunehmend beschleunigten und trivialisierten Welt des (Klein-)Kinderfernsehens noch etwas beschauliches und sinnstiftendes zu vermitteln. Die Erzählungen kreisen nahezu immer um denselben Plot, der sprechende Elefant ringt an der Seite 
seines nie alternden Freundes Otto, bisweilen mit Unterstützung seines Wärters Karl, des Zoodirektors Tierlieb und fast immer mit der der »rasenden Reporterin « Karla Kolumna um die Abwendung einer als ungerecht und moralisch falsch verstandenen Entwicklung in der fiktionalen Kleinstadt mit dem auf utopische Visionen anspielenden Namen Neustadt. Dabei geht es gleichermaßen um Alltagsprobleme wie Berufstätigkeit, Krankheit oder Wohnungssuche, oft aber (auch) um Fragen, die um die Themenfelder Umwelt-, Natur- und Tierschutz kreisen. Benjamin Blümchen stehen dabei der korrupte (und in so gut wie jeder Folge namenlose) Bürgermeister und sein schusseliger Assistent Pichler (dessen Namensgebung eine Anspielung auf das »Picheln«, also den übermäßigen Alkoholkonsum, sein dürfte) gegenüber, die bisweilen von Akteuren flankiert werden, die stets nur in einer Einzelfolge auftreten, also austauschbar dargestellt sind (mit Ausnahme des windigen Geschäftsmannes Schmeichler, der häufiger auftritt). Jede Folge endet mit einem Happy-End, bei dem oft auch die Antagonisten von Benjamin Blümchen wieder in den Sozialverbund Neustadts eingemeindet werden, in dem sie ihr Fehlverhalten einsehen oder sich entschuldigen (vgl. Strohmeier 2005). Auf den ersten Blick wirkt Benjamin Blümchen emanzipatorisch - vermittelt durch die Themenwahl; die gesamte Sozialkonstruktion ist aber die der inszenierten Ohnmacht, bei der Benjamin Blümchen letztlich agiert wie ein besorgter Bürger, also jemand, der seine eigene moralische Weltsicht allen anderen aufzwingen will, die Regeln demokratischer Partizipation verachtet und um jeden Preis sein egoistisches Partikularinteresse gegen alle anderen durchsetzt, während er gegen die zentralen Prinzipien der aufgeklärten Moderne rebelliert.

Denn während die Rezipienten und Rezipientinnen von Benjamin Blümchen vordergründig lernen, wie wichtig der Einsatz für Umwelt-, Natur- und Tierschutz sein soll, lernen sie auf der sozialstrukturellen Ebene zwei völlig andere Momente: die Aversion gegen die Demokratie und die Lobpreisung der Beendigung von Ohnmachtsgefühlen durch Überwältigung und Okkupation des Denkens. Denn Benjamin Blümchen handelt in der Darstellung immer moralisch richtig, auch wenn er sich über Gesetze und Regeln hinwegsetzt, die Reporterin Karla Kolumna ist der Inbegriff der Anti-Journalistin, da sie grundsätzlich parteiisch ist und jenseits von Fakten und Wahrheit ihre journalistische Tätigkeit generell dafür missbraucht, Benjamin Blümchen zu unterstützen, der in seinem Handeln - von wenigen Folgen abgesehen - durch niemanden legitimiert ist, sondern immer nur handelt, weil er selbst glaubt, recht zu haben:

»Karla Kolumna nutzt größtenteils ihre Freunde Benjamin und Otto als Quellen. Hauptsächlich berichtet sie über Abenteuer, die sie gemeinsam mit ihnen erlebt. Kollegen treten nicht in Erscheinung, nur ein Chef wird erwähnt. [...] Redaktionelle Zwänge gibt es keine, sie entscheidet, was gedruckt werden soll. [...] Neustadt 
scheint eine weitgehend rechtsfreie Zone zu sein, da Benjamin Bestrafungen für Verbrecher selbst festlegt.«(Dusny 2015: 33)

Karla Kolumna stellt damit de facto fast genau das Idealbild der Anhänger von Trump und Pegida dar - Journalismus ist bei ihr nicht Aufklärung (die oft und gerade auch gegen die gefühlte Volksmeinung geschehen muss), sondern getragen von einem geschlossenen Weltbild, in dem Widersprüche nicht vorkommen und beseelt von dem Gedanken, »dem Guten« zur Umsetzung zu verhelfen. Dieses "Gute« ist aber immer nichts anderes, als der Wille von Benjamin Blümchen, der absolut gesetzt wird:

»Diese Vereinfachung und Einseitigkeit kann bei den Kindern ein negatives Politikbild suggestiv verstärken und die Unbeliebtheit von >bösen > Politikerinnen und Politikern gegenüber sguten Repräsentanten des Volkes (Benjamin und seine Freunde) rechtfertigen, da Kinder sich gern mit ihren Helden identifizieren.« (Manzel 2012: 19)

Zugleich »lernt « man aber auch, dass eigentlich das Aufbegehren gegen die Mächtigen - verkörpert durch den Bürgermeister, der von seinem Assistenten Pichler stets unterwürfig als »Bürgermeister-Chef« angeredet wird, und der in der Erzählung quasi als Autokrat herrscht, der nie gewählt wurde und offenbar auch nie abgewählt werden kann, geschweige denn, dass es echte, also politische Opposition geben würde, und des Weiterenwe verkörpert durch stets als skrupellos gezeichnete, macht- und geldhungrige Akteure, die oft »von außen« nach Neustadt einfallen, was letztlich mit antisemitischen Macht- und Ohnmachtsfantasien spielt - sinnlos ist, weil man im realen Leben ja eben doch keinen sprechenden Elefanten zur Verfügung hat und auch, weil die Protestformen von Benjamin Blümchen, würde man sie in die Realwelt übertragen, scheitern müssen, weil er sich eben an keine Regeln hält, sondern immer nur glaubt, seine Sicht der Welt sei die einzig mögliche, was die ideellen und materiellen Widersprüche in der bürgerlichen Gesellschaft für Rezipienten und Rezipientinnen von Benjamin Blümchen unmöglich zu erlernen macht - bei denen zugleich der Glaube an das Konkrete (Benjamin Blümchen und seine Unterstützer und Unterstützerinnen) im Kampf gegen das Abstrakte (den namenlosen »Bürgermeister-Chef « und die von außerhalb Neustadts agierenden Dritten) motiviert wird.

Bemerkenswert ist dabei, dass Benjamin Blümchen als Figur, die in den späten 1970er Jahren entstand, sich dadurch, dass die gesellschaftlichen Umstände sich gewandelt haben, von einer im Anspruch emanzipatorischen Figur zum Sinnbild der Reaktion geworden ist (diesen gesellschaftlichen Wandel übersieht z.B. Emde 2016 vollkommen in seiner geradezu naiv-optimistischen Deutung von Benjamin Blümchen) - würde sich Benjamin Blümchen nicht für Umwelt- und Tierschutz einsetzen, er wäre der komplette Inbegriff der sich als besorgte Bürger etikettie- 
renden besorgten Rassisten von Pegida, AfD und Co. - als Verkörperung eines Weltbildes, in dem gegen die Idee der Repräsentation, die der Identität gesetzt wird, in der Aufklärung von Überwältigung abgelöst ist, und am Ende sich diejenigen, die den Konflikt verloren haben, auch noch der gemeinschaftlichen und homogenisierenden Eingemeindung durch Benjamin Blümchen unterwerfen müssen - die Vision ist eine der antidemokratischen, völkischen Gesinnungsdiktatur, nur eben: einer Öko-Gesinnungsdiktatur. So erlangt die Macht der Ohnmacht in Benjamin Blümchen zwei Facetten: Einerseits als die inszenierte Ohnmacht, in die sich Benjamin Blümchen immer hineinphantasiert und die zugleich, weil er keine adäquaten, sondern formal falsche Wege ihrer Beendigung aufzeigt, für seine Rezipienten und Rezipientinnen stets auch eine reale Ohnmachtserfahrung werden muss; andererseits führt eine Identifikation mit Benjamin Blümchen dazu, sich in der realen Ohnmacht einzurichten, da durch Benjamin Blümchen auch die Verbindung aus eigener narzisstischer Omnipotenzphantasie und der verinnerlichten Distanz zum politischen System (verbunden mit der Weigerung, auch nur zu verstehen, wie dieses funktioniert) zu einem Selbstbild aggressiver Passivität wird, das keiner äußeren Kontrolle mehr bedarf.

\section{Ohnmacht durch Identifizierung: Der Verlust aller Ordnung in Game of Thrones}

Die Handlung von Game of Thrones spielt auf den Inseln Westeros und Essos, wobei Westeros das zentrale Königreich ist, um dessen Thron in der Hauptstadt Königsmund (King's Landing) zahlreiche Adelshäuser kämpfen und sich in unzählige inter- und innerfamiliäre Intrigen verwickeln. Die große Erzählung von Game of Thrones ist der Kampf um Macht, der - gemahnend an Machiavelli - jenseits von Moral geführt wird, wobei im Laufe der Staffeln die Rolle der religiösen Legierung des Handelns zur Legitimation und Delegitimation zunehmend an Bedeutung gewinnt (vgl. Larrington 2016). Neben den aristokratisch aufgebauten Herrscherhäusern mit weitgehend extrem patriarchalen Strukturen, deren Herrschaftsräume geografisch aufgeteilt sind, fungieren unterschiedliche Religionen als cross cutting cleavages, wobei eine puritanische und totalitäre Religion sich auch aufschwingt (die »Spatzen«; Staffel 5 \& 6), die Gesamtherrschaft zu usurpieren. Während die mehr oder weniger rechtmäßige Thronerbin Daenerys Targaryen im Exil in Essos lebt und ihre Zeit damit fristet, eine Armee und Schiffe für den erneuten Angriff auf den Thron von Westeros zu organisieren, droht den in Westeros miteinander um den Thron, aber auch um partikulare Vorherrschaften (insbesondere um Schloss Winterfell und damit die Herrschaft über den Norden von Westeros) ringenden Adelshäusern noch eine potentiell vernichtende Bedrohung von den »weißen Wanderern«, von Untoten, die »jenseits der Mauer« - eines gigantischen Schutzwalls an der Nordgrenze von Westeros, der von einem Männerbund bewacht wird, der 
sich aus Kriminellen und wegen ihrer unehelichen Geburt Verstoßenen rekrutiert, die durch ihre lebenslange Mitgliedschaft bei der »Nachtwache« dem Tod entgehen können - darauf lauern, in Westeros einfallen und die Menschen ermorden zu können.

Auf rein phänomenologischer Ebene wird an der HBO-Erfolgsserie Game of Thrones eine - völlig zutreffende und richtige - Kritik formuliert, die insbesondere die brutalisierte Visualisierung von roher und von sexualisierter Gewalt problematisiert (vgl. Gjelsvik/Schubart 2016; Langley 2016). Gerade diese, aus erzählerischen Motiven in ihrer konkreten Darstellung nicht zu rechtfertigende und für das Sujet verzichtbare effektheischende Visualisierungsstrategie zeigt, warum eine antiintellektuelle Identifizierung mit Game of Thrones gerade auch von völkischer Seite möglich ist: es ist das Ineinanderfallen von abstrakt Erzähltem und konkret Gezeigtem als einer Facette von Game of Thrones, die den konkretistischen Barbaren ermöglicht, sich auf einer plumpen Ebene mit der Gewalt zu identifizieren.

Eine kritische Reflexion von Game of Thrones muss aber deutlich anders ansetzen. Denn allein die Facette der Integration des Hauses Graufreud (im Englischen noch grotesker: Greyjoy) zeigt, dass in die brutalisierte Sexualität zugleich auch eine so ernst erzählte Teilgeschichte verwoben ist, die eigentlich fast nur als Satire gelesen werden kann: die »Eisenmänner « leben auf einer vollkommen verdreckten und runtergekommenen (Eisen-)Insel; ihr Motto (»Wir säen nicht«) basiert auf der Ausplünderung ohne jede innovative oder kreative Dimension und ihr Glaube ist fokussiert auf den »ertrunkenen Gott«: »Was tot ist, kann niemals sterben« (vgl. Folge 2.3). Wenn man dann noch den Tonfall des zentralen Protagonisten Theon Graufreud hört, gemahnt die gesamte Konstruktion des Hauses Graufreud an eine Reminiszenz an Monty Python.

Insofern liegen viele Parallelfacetten im Game of Thrones-Universum, die erst in ihrer integrativen Dimension des Gesamtepos funktional verstanden werden können (vgl. Jacoby 2014; Langley 2016): Denn wie jede Fantasysaga wird auch diese um zahlreiche Heldinnen und Helden gewoben, die - aus höchst differenten Motiven dazu einladen, sich mit ihnen zu identifizieren oder sie, als Akt umgekehrter Identifizierung, zu verachten. Während das Verachten in Game of Thrones leicht fällt (z.B. Joffrey Baratheon, Walder Frey, Gregor Clegane, Ramsay Bolton), ist der eigentliche Kern der Erzählung von Game of Thrones der Verlust aller Ordnung - und damit die sich über die Staffeln hinweg immer wieder einstellende und weiter verfestigende Ohnmacht in der Identifizierung, die in der Hoffnung kulminiert, dass nicht noch ein auch nur ansatzweise sympathischer Charakter ermordet werden möge. Denn genau das passiert fortwährend: Alle Figuren, die aufgrund ihrer Überzeugungen und Haltungen in einem demokratischen Sinn zur Identifizierung angeboten und entwickelt werden, werden ermordet. Die Enthauptung von Eddard »Ned « Stark am Ende der Ersten Staffel ist so inszeniert, dass man bis zum Moment des fallenden Henkersschwertes immer noch davon überzeugt ist, dass ihn jemand retten 
werde (Folge 1.9) - etwa ein Bogenschütze oder ein Meuchelmörder, der den Henker umbringt. Es passiert nicht und die Figur, die als einziger Garant einer labilen, aber doch noch auf letzte Reste von Regeln verpflichteten Ordnung, inszeniert wurde, wird auf der Basis von Intrigen und Lügen mit dem Tode »bestraft«.

Bei den einzelnen Adelshäusern, die miteinander um den »Eisernen Thron« konkurrieren, obsiegen staffelweise stets die noch unsympathischeren; innerhalb der Adelshäuser setzen sich fast überall beständig die besonderen Widerlinge durch, die wenigen Charaktere, die langfristig noch Hoffnung verleihen könnten (v.a. Jon Schnee, Daenerys Targaryen, Arya Stark), müssen jeweils durch zutiefst entwürdigende und bestialisch erniedrigende Leidenswege gehen (vgl. Hansmann 2017), die sie so sehr in ihrer Individualität und Subjektivität brechen, dass man nach sechs Staffeln jeden Glauben verloren hat, es könne sich doch noch zum Guten wenden - droht doch der Einfall der »weißen Wanderer« von »jenseits der Mauer«, die als Außengrenze die labile Unordnung innerhalb des Königreiches Westeros aufrechterhält, bewacht vom Männerbund der »Nachtwache«. Gegen den Niedergang jeder Ordnung steht hier drohend nur noch der am Ende von Staffel 7 anvisierte Einfall der jenseits jeder Menschlichkeit agierenden »weißen Wanderer«, die nur vom Ziel der Vernichtung der Menschen getrieben sind.

Game of Thrones ist der Verlust aller Ordnung und die auf Dauer gestellte Ohnmacht, in der identifizierungswürdige Charaktere fortlaufend exekutiert werden, so dass zum Kern jedes Identifizierungsprozesses die Ohnmacht wird, sowie die Staffel für Staffel immer weiter getriebene Hoffnung, nach der am Ende bitte nicht auch noch die »weißen Wanderer« obsiegen mögen und die wenigen verbliebenen, noch rudimentären Vorstellungen von Freiheit und Gerechtigkeit verpflichteten Charaktere, nicht auch noch sterben und Westeros endgültig in Chaos und Vernichtung versinken möge. Die Identifizierung ist insofern letztlich eine depressive, ohnmächtige und hilflose, sich dem vermeintlich unaufhaltsamen Schicksal ergebende (was sich auch markant in den Dialogen »)enseits der Mauer « [Folge 7.6] andeutet, wenn vom Tod als dem ersten und dem letzten Feind die Rede ist) - und damit eine, die die Rezipienten und Rezipientinnen zurichtet für eine Realwelt, in der die Hoffnung fast auf den Hobbesschen Naturzustand reduziert wird, in der es nur noch zentral ist, zu überleben oder unter den Prämissen der Demokratie sich irgendwie durchzuschlagen. Ideen von Freiheit, Gleichheit oder gar Solidarität existieren in Game of Thrones nicht, es herrschen Verzweiflung und Ohnmacht in der Auslieferung an die totale Barbarei, wie sie - auch ohne die "weißen Wanderer « - bereits in der »Roten Hochzeit « (Folge 3.9 und 3.10) in aller Bestialität zelebriert wird. 


\section{Ohnmächtige Dummheit als Erfolgsprinzip: American Dad}

American Dad adressiert thematisch an Erwachsene, wird aber im Zeichentrickformat erzählt, so dass die formale Adaption bereits auf eine diffusere Zielgruppe verweist als Benjamin Blümchen oder Game of Thrones, die recht deutlich an Kinder oder Erwachsene als Zielgruppe gerichtet sind. Die Rezeptionsadressierung von American Dad ist hingegen offener, man kann diese Serie je nach (mangelndem) aufgrund ihres Darstellungsformats auch als für Kinder und Jugendliche geeignet (miss-)verstehen, so dass die um eine amerikanische Familie kreisende Erzählung auch als Familienserie rezipierbar ist, obgleich für Kinder thematisch überkomplex. American Dad ist fraglos auch als humoristische Erzählung interpretierbar, da gerade in den Überzeichnungen der Charaktere Potential für eine die Ambivalenzen und Widersprüchlichkeiten der modernen Gesellschaft kontextualisierende Rezeption liegt; allerdings ist diese einerseits intellektuell voraussetzungsvoll und, was wesentlicher ist, andererseits durch die Frage nach dem innerhalb der einzelnen Folgen jeweils für sein Handeln mit emotionalem Mehrwert ausgestatteten Figuren zwar eine Option, aber nicht die zentrale Linie der Erzählungen. Insofern liegen in American Dad auch sich überlappende Rezeptionsmöglichkeiten und die Serie ist in dieser Hinsicht weit weniger eindeutig und mit mehr Widersprüchen konzipiert als gerade Benjamin Blümchen. Das hinterlegte Sozialmodell bleibt hingegen trotz dieser Dimensionen unangetastet.

Vordergründig ist Familienvater Stan(ley) Smith die Hauptfigur der Serie: CIAAgent, in voller Überzeugung in patriarchalen und maskulinen Rollenvorstellungen verankert, der stets fürchtet, sein weicher und emotional verletzlich gezeichneter Sohn Steve könnte homosexuell sein und der seine Tochter Hayley, linksalternativökologisch orientiert, aber dabei trotzdem intellektuell eher limitiert gezeichnet, aufgrund ihrer weltanschaulichen Überzeugungen oder ihrer Affinität zu jungen Männern mit (Drogen-)Problemen auch schonmal an die CIA ausliefern würde. Stans amerikanischer Patriotismus steht über allem, allerdings wirkt dieser Patriotismus in einer naiven und aggressiven Weise - und nicht im Sinne einer Verteidigung von Freiheit und Gleichheit, sondern vaterländisch-emotional. Stans Frau Francine arrangiert sich mit ihrer Rolle als Hausfrau, mit der sie von Grund auf zufrieden ist, aber hier und da als punktuelle Infragestellerin fungiert, Einwände aber nur stets im Sinne einer familiären Identitätsrettung formuliert und nicht als gesellschaftskritische Alternative: Stan wird von ihr für sein Handeln gegen Sohn und Tochter nicht deshalb kritisiert, weil er damit antiemanzipatorischen Politikund Gesellschaftsmodellen folgt, sondern weil er damit die Familienidentität gefährden könnte.

Das Chaos in dieser pittoresken und grotesken Spießbürgeridylle generieren zwei nicht-menschliche Charaktere: Der sprechende Goldfisch Klaus (dessen Gehirn von der CIA versehentlich mit dem eines ostdeutschen Skispringers vertauscht 
wurde) und der Außerirdische Roger, der mit seiner Begeisterung für Alkohol, Zigaretten und Drogen faktisch den Antipoden zu Stan verkörpert. Anders als bei ALF (NBC, 1986-1990), dessen humoristische Intervention in die Familie Tanner in den 1980er Jahren ein umfangreiches Arsenal an Gesellschaftskritik inkorporierte, weil er sich stets auf die Seite der Schwachen schlug, sind der Goldfisch Klaus und der Außerirdische Roger aber reaktionär - beide zielen in ihrem Humor immer nur auf sich selbst, referenzieren nur ihre Situation und versuchen sie durch Degradierung und Unterwerfung anderer zu optimieren: Roger ist die gegenaufklärerische Antwort auf ALF, fast so, wie der Benjamin Blümchen der $2000 e r$ Jahre den Benjamin Blümchen der 1970er Jahre bekämpft.

Denn die faktische Hauptrolle, die Roger in American Dad zukommt, wird in der Erzählung zwar durch depressive und leidende Momente ausgefüllt, aber die visualisierte Flucht aus diesen Konstellationen ist nicht solidarisch, sondern egoistisch (so z.B. sein unterjochendes Auftreten in Folge 8.9, die von der Mitgliedschaft in einem Country Club handelt) - und getragen von derselben Überheblichkeit, in der die meisten Charaktere in der Serie agieren: Alle sind eingerichtet in ihrer Mittelmäßigkeit und Durchschnittlichkeit und halten sich zugleich für etwas Besseres. Misserfolge werden als Ergebnisse von Ungerechtigkeiten oder Verschwörungen gedeutet, sozialer und beruflicher Aufstieg folgt stets nur einer Selbstoptimierungsstrategie des eigenen Egos. Damit wird die ohnmächtige Dummheit zum Erfolgsprinzip verklärt: Das kleinbürgerliche Rebellentum, das nicht gegen Herrschaftsstrukturen opponiert, sondern die Unterwerfung und Unterordnung affirmiert und konformistisch rebellieren will - gegen die sozial Schwachen und Ausgegrenzten. Es ist der Entwurf einer Gesellschaft, die den eigenen Luxus auf Unterdrückung gründet und in der nichts mehr verhasst ist als Solidarität. Die infantile Regression, in die sich der Muskelprotz Stan immer wieder begibt, seine aufgrund mangelnder Intellektualität sich oft einstellende Hilflosigkeit wird charmant verklärt letztlich führen seine Affekte gegen gesellschaftliche Gleichheit und soziale Solidarität, ja auch gegen den von der patriarchalen Norm abweichenden Individualismus, wie ihn etwa die Freunde seiner Tochter oft verkörpern, aber stets zur Stabilisierung der familiären Identität und werden als Teil einer positiven Kollektividentität inszeniert, zu der auch Waffenwahn und Rassismus wie selbstverständlich dazugehören. Die infantilen Regressionen von Stan werden so zu einem Ordnungssurrogat, das die unterwürfige Anpassung bei gleichzeitiger Auslebung aggressiver Affekte gegen Schwache und Ausgegrenzte als erfolgreiches Sozialmodell verspricht, in dem die ohnmächtige Dummheit als Erfolgsprinzip versprochen wird. Dass für die Figur von Stan zentrale Moment der ohnmächtigen Dummheit wird aber auch, wenngleich -wie gesagt- teilweise ambivalent und mit humoristischer Brechung gezeichnet, bei den anderen Charakteren gleichermaßen inszeniert, die sich trotz kleinerer Rebellionen immer wieder in den repressiven Kosmos der bürgerlich-spießigen Kleinfamilie einfügen - selbst Roger durchbricht diese 
Logik nur selten, weil es ein Ver- und Entfremdungsmoment, wie es bei ALF etwa durch dessen Vorliebe für Katzen als Nahrungsmittel stets aufrechterhalten wurde, kaum gibt. Der Alkoholismus von Roger wirkt nur wie die halbierte Verdopplung der klassisch patriarchalen Familienrolle, die hier eben auf Stan und Roger aufgeteilt ist, und in der Roger insofern vor allem als alter ego funktioniert oder, psychoanalytisch gesprochen, wie das verdrängte Unbewusste von Stan (was ein Stückweit auch für den Goldfisch Klaus gilt, der in Francine verliebt ist und sie stalkt).

Die Einfügung in die Hierarchie der Gesellschaft und der patriarchalen Reproduktion in der Familie wird dabei in American Dad zu einem Erfolgsversprechen, bei dem jeder Ausbruchsversuch aus den Zwängen des Kleinbürgertums mit unwitzigen Witzen garniert wird und am Ende in der einsichtsvollen Rückkehr der Pseudo-Rebellen und Pseudo-Rebellinnen in die antiaufklärerische Ordnungsphantasie der Kleinfamilie mit Vorstadthaus mündet - soziale und politische Erfolge werden dabei in einer Mischung aus Naivität und Bildungsferne erzielt, die Anpassung zum obersten Prinzip von Glückseligkeit erklärt und eine Mentalität befördert, bei der die Rezipienten und Rezipientinnen sich durch ihre Selbstlimitierung und Selbstreglementierung dem Versprechen hingeben dürfen, am Ende an dem gemeinschaftlichen Idyll des sozialen Stillstandes teilhaben zu dürfen.

\section{Die verlorene Ordnung}

So different die Serien in Darstellungsformen, Erzählstrategien und Zielgruppenorientierungen sind, so sehr verbindet sie in ihren Identifizierungsangeboten das Moment, Facetten ohnmächtiger Rebellion zu idealisieren und damit als Projektionsflächen für regressive und deprimierte Phantasien von einer unmündigen Gesellschaft zu werden. Was die inszenierte Ohnmacht des besorgten Bürgers bei Benjamin Blümchen, die Ohnmacht durch Identifizierung im Verlust aller Ordnung in Game of Thrones und die ohnmächtige Dummheit als Erfolgsprinzip bei American Dad verbindet, ist, dass in diesem Meer der Ohnmachtsvariationen keine Inseln der aufgeklärten Hoffnung mehr aufscheinen, denn in dem Nebeneinander von deprimierenden Identifizierungsangeboten wird Wahrheit in Diskurs aufgelöst. Waren etwa die Kriminalserien wie Derrick, Der Alte oder Tatort bis in die späten 1990er Jahre hinein noch durchweg von dem Sujet durchdrungen, dass die unmissverständliche Wahrheit der Sieg des Rechts über das Unrecht, der Ordnung über die Anarchie, der Gerechtigkeit über die Willkür war - und zwar völlig gleichgültig, ob die Protagonisten wie bei Derrick selbst eine reaktionäre Ordnung verkörperten oder, wie epochemachend von Ben Becker und Ulrike Folkerts im Tatort »Tod im Hexler« inszeniert, gegen die Reaktion aufbegehrten und eine Ordnung von Freiheit errichten woll(t)en. Diese Ordnungsorientierung ist in den zeitgenössi- 
schen Identifizierungsangeboten zahlreicher TV-Serien genuin verloren: Maßstäbe und Leitbilder werden suspendiert zur willkürlichen Affirmation im Diskurs, der ein scheinbar gleichberechtigtes und damit faktisch beliebiges Nebeneinander von Varianten antiemanzipativer Gesellschaftsmodelle anbietet, die dadurch verbunden sind, dass sie »Geltungsansprüche« (Forst/Günther 2011: 15) unsichtbar machen. Die Wünsche und Sehnsüchte der Individuen, eben solche zu sein und als Subjekte dem eigenen Leben eine glückliche Wendung zu verleihen, werden dadurch, dass sie in den Identifizierungsangeboten gar nicht mehr vorkommen und obendrein noch die soziale Ordnung, die sie gewähren könnte (so ja das Versprechen der Vertragstheorie: Ordnung für Freiheit, Freiheit für Ordnung), inexistent ist oder sukzessive zerstört wird, zwar nicht aufgehoben, aber nivelliert in eine Formation der Selbstkontrolle, in der die Ohnmacht allmächtig wird und der soziale Reglementierungsapparat als Selbstüberwachung ohne Gewalt perfektioniert ist. Die traurige Wende dieses panoptischen Selbstregulierungsprozesses besteht darin, dass das in der strukturalistischen Kritik ursprünglich noch angelegte gesellschaftskritische Potential sich selbst dahinmeuchelt, da das poststrukturalistische Denken, in dem die Logik der hier diskutierten Serien funktioniert, jede Hoffnung auf Individualität und Subjektivität systematisch auslöscht. Es ist dies eine Wendung, die darin begründet liegt, dass die Kritik an den Strukturen vergaß, dass es stets die Akteure sind, die diese Strukturen produzieren und reproduzieren. Dabei geht es um die, wie Rahel Jaeggi (2014) sagt, »Kritik von Lebensformen«; bei dieser Kritik kann Jaeggi folgend keine Gleichrangigkeit subjektiver Lebensentwürfe angenommen werden, weil eben jede subjektive Variation als »kulturelle und soziale Reproduktion menschlichen Lebens « (ebd.: 21) bzw. als Zusammenhang von »Praktiken und Orientierungen und Ordnungen sozialen Verhaltens « (ebd.: 89) selbst auf soziale und politische Konflikte reagiert, sie aber eben auch zugleich wieder hervorbringt und damit reproduziert.

Weil sich die Subjekte an der Ordnung, die für ihre Unterwerfung verantwortlich ist, beteiligen und sie damit aufrechterhalten, muss eine Kritik der bürgerlichen Vergesellschaftung heute mehr denn je, will sie nicht in poststrukturalistische Zerstörung des Versprechens auf Individualität und Subjektivität umkippen und damit zur barbarischen Affirmation von völkischen und islamistischen Rackets werden, die eben jene Destruktionsabsicht mit dem Poststrukturalismus teilen, auf die Akteure fokussieren - eine Kritik der Gesellschaft wäre, geschärft durch eine Rejustierung der Überlegungen zum Panoptimus und dessen Transformationen am Beginn des 21. Jahrhunderts, demnach eine, die radikal an den Akteuren ansetzt, die sich weigern, ihr Potential zur Mündigkeit zu nutzen - womit Kant eine überraschende Aktualität erlangt in seiner Beschreibung von Aufklärung als den Ausgang aus der selbstverschuldeten Unmündigkeit. Es ist diese panoptische Selbstverschuldung durch die Akteure, die heute gesellschaftliche Emanzipation blockiert und torpediert. 


\section{Literatur}

Adorno, Theodor W. 1951: Freudian Theory and the Pattern of Fascist Propaganda. In: Ders.: Gesammelte Schriften, Bd. 8, Frankfurt 1997, S. 408-433.

Anter, Andreas 2012: Theorien der Macht zur Einführung, Hamburg.

Bentham, Jeremy 1812: Panopticon versus New South Wales, or >The panopticon penitentiary system and the penal colonization system compared, London.

Bohleber, Werner 1992: Nationalismus, Fremdenhaß und Antisemitismus, Psychoanalytische Überlegungen. In: Psyche, H. 8, S. 689-709.

Clemenz, Manfred 1998: Aspekte einer Theorie des aktuellen Rechtsextremismus in Deutschland. Eine sozialpsychologische Kritik. In: Hans-Dieter König (Hg.), Sozialpsychologie des Rechtsextremismus, Frankfurt, S. 126-176.

Dusny, Ramona 2015: Das Selbstverständnis von Journalisten in Kinderhörspielen am Beispiel von Karla Kolumna in der Hörspielreihe Benjamin Blümchen (unveröff. BA-Thesis), Hannover.

Ebrecht, Angelika 2003: Die Seele und die Normen. Zum Verhältnis von Psychoanalyse und Politik, Gießen.

Emde, Oliver 2016: Ziviler Ungehorsam im entpolitisierten Neustadt? Politische Partizipation bei »Benjamin Blümchen«. In: Ders./Lukas Möller/Andreas Wicke (Hg.), Von »Bibi Blocksberg« bis »TKKG«. Kinderhörspiele aus gesellschaftsund kulturwissenschaftlicher Perspektive, Opladen, S. 16-25.

Erdheim, Mario 1988: Die gesellschaftliche Produktion von Unbewußtheit. Eine Einführung in den ethnopsychoanalytischen Prozeß (EA 1984), 2. Aufl., Frankfurt.

Forst, Rainer/Günther, Klaus 2011: Die Herausbildung normativer Ordnungen. Zur Idee eines interdisziplinären Forschungsprogramms. In: Dies. (Hg.), Die Herausbildung normativer Ordnungen, Interdisziplinäre Perspektiven, Frankfurt/New York, S. 11-30.

Foucault, Michel 1977: Überwachen und Strafen. Die Geburt des Gefängnisses (frz. EA 1975 u.d.T. »Surveiller et punir. La naissance de la prison«), Frankfurt.

Foucault, Michel 1983: Der Wille zum Wissen. Sexualität und Wahrheit 1 (frz. EA 1976 u.d.T. »Histoire de la sexualité, 1: La volonté de savoir«), Frankfurt.

Freud, Sigmund 1921: Massenpsychologie und Ich-Analyse. In: Ders., Gesammelte Werke, Bd. XIII, Frankfurt 1999, S. 71-161.

Gjelsvik, Anne/Schubart, Rikke (Hg.) 2016: Women of Ice and Fire. Gender, Game of Thrones and Multiple Media Engagements, New York.

Göhler, Gerhard 2004: Macht. In: Ders./Mattias Iser/Ina Kerner (Hg.): Politische Theorie. 22 umkämpfte Begriffe zur Einführung, Wiesbaden, S. 244-261.

Gutting, Gary 2005: Foucault. A very short introduction, Oxford.

Hansmann, Silke 2017: Die Entwicklung weiblicher Figuren in Fantasy-Werken aus differenzfeministischer Perspektive am Vergleich von Éowyn in »Herr der Rin- 
ge« und Daenerys Targaryen in »Game of Thrones« (BA-Thesis, unveröff.), Göttingen.

Hersey, George L. 1996: The Evolution of Allure. Sexual Selection from the Medici Venus to the Incredible Hulk, Cambridge MA.

Hobbes, Thomas 1651: Leviathan oder Stoff, Form und Gewalt eines bürgerlichen und kirchlichen Staates (engl. EA u.d.T. »Leviathan or The Matter, Forme, and Power of a Commonwealth Ecclesiasticall and Civil«), herausgegeben und eingeleitet von Iring Fetscher, Neuwied/Berlin 1966.

Hollander, Anne 1993: Seeing Through Clothes, Berkeley.

Imbusch, Peter (Hg.) 2012: Macht und Herrschaft. Sozialwissenschaftliche Theorien und Konzeptionen, Wiesbaden.

Jacoby, Henry 2014: Die Philosophie bei >Game of Thrones<: Das Lied von Eis und Feuer: Macht, Moral, Intrigen, Weinheim.

Jaeggi, Rahel 2014: Kritik von Lebensformen, Berlin.

Kersting, Wolfgang 2005: Thomas Hobbes zur Einführung, 3. Aufl., Hamburg.

Kokoska, Tanja 2017: Sieben Gründe Dieter Bohlen gut zu finden In: Frankfurter Rundschau (FR7-Magazin) v. 22./23. April, S. 11.

Langley, Travis (Hg.) 2016: Game of Thrones Psychology. The Mind is Dark and Full of Terrors, New York.

Larrington, Carolyne 2016: Winter is Coming. Die mittelalterliche Welt von Game of Thrones, Darmstadt.

Machiavelli, Niccolò 1513: Il Principe/Der Fürst. Italienisch/Deutsch, übersetzt und hgg. von Philipp Rippel, Stuttgart 1986.

Manzel, Sabine 2012: Das Bürgermeisterbild bei Benjamin Blümchen. Mit politischen Fachkonzepten Klischees aufdecken. In: Weltwissen Sachunterricht, H. 4, S. 18-25.

Marx, Karl/Engels, Friedrich 1845/46: Die deutsche Ideologie. Kritik der neuesten deutschen Philosophie in ihren Repräsentanten Feuerbach, B. Bauer und Stirner, und des deutschen Sozialismus in seinen verschiedenen Propheten. In: MEW 3, S. 8-530.

Münkler, Herfried 1984: Machiavelli. Die Begründung des politischen Denkens der Neuzeit aus der Krise der Republik Florenz, Frankfurt.

Münkler, Herfried 1993: Thomas Hobbes, Frankfurt/New York.

Pitkin, Hanna Fenichel 1967: The Concept of Representation, Berkeley.

Rensmann, Lars/Hagemann, Steffen/Funke, Hajo 2011: Autoritarismus und Demokratie. Politische Theorie und Kultur in der globalen Moderne, Schwalbach/Ts.

Salzborn, Samuel (Hg.) 2009: Politische Kultur. Forschungsstand und Forschungsperspektiven, Frankfurt.

Salzborn, Samuel 2013: Sozialwissenschaften zur Einführung, Hamburg.

Salzborn, Samuel 2016: Vom rechten Wahn. »Lügenpresse«, »USrael«, »Die da oben« und »Überfremdung«. In: Mittelweg 36, H. 6, S. 76-96. 
Salzborn, Samuel 2017a: Angriff der Antidemokraten. Die völkische Rebellion der Neuen Rechten, Weinheim.

Salzborn, Samuel 2017b: Kampf der Ideen. Die Geschichte politischer Theorien im Kontext, 2. akt. Aufl., Baden-Baden.

Sarasin, Philipp 2010: Michel Foucault zur Einführung, 4. Aufl., Hamburg.

Skinner, Quentin 2008: Machiavelli zur Einführung, 5. Aufl., Hamburg.

Strohmeier, Gerd 2005: Politik bei Benjamin Blümchen und Bibi Blocksberg. In: Aus Politik und Zeitgeschichte, H. 41 v. 10.10., S. 7-15.

Weber, Max 1980: Wirtschaft und Gesellschaft. Grundriss der verstehenden Soziologie, 5. rev. Auflage bes. v. Johannes Winckelmann (EA: 1921), Tübingen.

Zurawski, Nils (Hg.) 2007: Surveillance Studies. Perspektiven eines Forschungsfeldes, Opladen. 

Kulturkämpfe und Identität 



\section{Die Identitäten des Populismus Die Politisierung der Kultur und der neue Kampf der Kulturen}

Jörn Knobloch

\section{Einleitung: Die zirkuläre Entdeckung der Kultur}

Die Kultur und deren Entdeckung für die politische Analyse der Gesellschaft nimmt in der Politikwissenschaft die Form eines Zirkels an. Einer Phase, in der die Selbstverständlichkeit der Kultur für die Politik und ihre wissenschaftliche Analyse zweifellos anerkannt wird, folgt ein Prozess des gegenseitigen Entfremdens, der von der Proklamation der absoluten Inkongruenz abgeschlossen wird. Dann folgt die gegenseitige »Wiederentdeckung « von Kultur und Politik mit der unvermeidlichen Wiedervereinigung beider Perspektiven. Dies hat natürlich Einfluss auf die Politische Kulturforschung, die je nach dem Status der Beziehung von Politik und Kultur vom Rand in das Zentrum und auch wieder zurück an den Rand politikwissenschaftlicher Forschung wandert. ${ }^{1}$ In jüngster Zeit erleben wir in Folge des »cultural turn« (Bachmann-Medick 2009) eine erneute Rückkehr der zuvor verdrängten Kultur in die Politikwissenschaft und insbesondere in die Politische Theorie (vgl. Knobloch 2016). Dabei ist jedoch zu beachten, dass diese Dynamik sowohl aus einer wissenschaftsinternen Nachfrage nach neuen Kategorien zur Erklärung politischer Phänomene bzw. zum Verständnis sozialer Strukturen als auch aus der wissenschaftsexternen Genese neuer Begriffe des gesellschaftlichen Selbstverständnisses herrührt. Beides interagiert, dennoch war der >cultural turn ‘ hauptsächlich das Ergebnis eines rein wissenschaftlichen Diskurses zu einem Paradigmenwechsel. Indes gewinnt augenblicklich die Kultur an neuer Aufmerksamkeit, weil sie nunmehr

1 Eine erste Verschiebung kann mit der Entstehung des neuzeitlichen Rationalismus konstatiert werden. Insbesondere Webers Differenzierung von wertenden und objektiven Aussagen und die Vorstellung von einer nicht wertenden Sozialwissenschaft haben die Kultur an den Rand gedrängt. Mit dem Ende des Zweiten Weltkrieges gab es gleich zwei Ansätze, den der politischen Kultur von Almond und Verba sowie die neue Wissenschaft der Politik von Voegelin, Kultur wieder zum Gegenstand und zur Perspektive der Politikwissenschaft zu machen (vgl. Schwelling 2004). 
gesellschaftlich politisiert wird. Hat der >cultural turn « die Kategorie der Kultur der Politikwissenschaft noch rein theoretisch einer gegenüber externen Angeboten offenen Theorie- und Konzeptdiskussion aufgedrängt, ${ }^{2}$ wird die Kultur jetzt selber politisch. Demzufolge handelt sich auch nicht um eine empirische Herausforderung im Sinne einer Erschließung von Kultur als Variable zur Erklärung unterschiedlicher Herrschaftsaffinitäten von Ländern, auf die einst Almond und Verba reagiert haben. Dieses Mal erfolgt die Rückkehr der Kultur als polarisierender Begriff innerhalb eines politischen Konflikts der liberalen Demokratien. Sowohl die Positionierungen in den Diskussionen über den neuen Populismus als auch die Auseinandersetzungen um Migration und der Streit über den Umgang mit dem radikalen Islam argumentieren mit dem Kulturbegriff, der Differenzierungen ermöglicht. Indem die Kultur wieder zum Motor und Fixpunkt politischer und sozialer Konflikte wird, gerät auch die These Huntingtons zum Clash der Kulturen erneut in den Fokus. ${ }^{3}$ Es wird aber klar, dass die aktuelle Polarisierung in den Konflikten nicht nur, wie es Huntington noch angenommen hat, zwischen den großen Zivilisationen stattfindet, sondern die westlichen Gesellschaften intern spaltet. Damit gewinnt die politikwissenschaftliche Kulturforschung einen völlig neuen Status, der sie konzeptuell herausfordert.

Im Folgenden möchte ich auf diese Herausforderung reagieren und den neuen Kampf der Kulturen aus der Perspektive der politischen Kulturforschung aufklären. Die Herausforderung sehe ich insbesondere in dem ambivalenten Begriff der Kultur. Um ihn als theoretische und empirische Kategorie zu systematisieren und für die politische Analyse der Gegenwart fruchtbar zu machen, sind sukzessive seine Dimensionen zu entfalten. Ausgehend von der Aktualität des neuen Populismus werde ich zunächst diesen Ansatz kritisch diskutieren und zeigen, dass es notwendig ist, auf die dahinterstehenden Spannungen zwischen unterschiedlichen normativen Leitideen einzugehen. Anschließend identifiziere ich zwei divergierende identitätspolitische Ansätze als diese polarisierenden Leitideen. Die Ursache für deren Divergenz ist ein differenziertes Verständnis von Kultur, welches einerseits als Leitidee fungiert, andererseits aber schon einen Ordnungsbegriff darstellt. Daraus entwickelt sich der neue Kulturkampf, der zu einem bestimmenden Konflikt der Gegenwart avanciert. Im letzten Teil werde ich den Beitrag der politischen Kulturforschung für die Aufklärung und Entschärfung dieses Konfliktes erläutern. Dafür ist es aber unabdingbar, dass detailliert zwischen den angewandten Kulturbegriffen unterschieden wird, um deren scheinbare Inkommensurabilität zu über-

2 Welche die scheinbar theoriearme Politische Theorie begierig aufzunehmen bereit ist (vgl. Göhler et al. 2009: 398f.).

3 Mit dem Erscheinen des Aufsatzes bzw. des Buchs von Huntington (1996) begann eine rege Diskussion über die ihm unterstellte normative Intention des Ansatzes. Das analytische Potential stand hier aber weniger im Fokus der Auseinandersetzung. 
winden. Genau in dieser Leistung, so die grundlegende These dieses Beitrages, liegt die Aufgabe für die politische Kulturforschung der Gegenwart, die von keiner anderen politikwissenschaftlichen Subdisziplin übernommen werden kann.

\section{Die Popularität des Populismus}

Durch die jüngsten politischen Ereignisse in den etablierten liberalen Demokratien ist der Populismus von einer Krisenranderscheinung zu einer der zentralen Herausforderungen der politischen Stabilität geworden (vgl. Priester 2007, 2012; Knöbl 2016; Inglehart/Norris 2016; Moffitt 2016; Müller 2016; Decker/Lewandowski 2017; Sauer 2017). Es ist vor allem das Infragestellen stabiler Erwartungen, die für Aufregung sorgen. Die Wahl Trumps, die Ablehnung des EU-Integrationsprozesses in Großbritannien, die Aufkündigung des Konsenses zur Flüchtlingspolitik in der EU, die Erfolge von Parteien in Europa, die sich bewusst von den Inhalten und Positionen der Mitte distanzieren usw. projizieren das Moment der Instabilität und die Möglichkeiten undemokratischer Entwicklungen in die traditionellen liberalen Ruhepole. Ein Merkmal dieser Veränderungen ist die neue Spaltung des Demos, wobei die Spaltung nicht mehr mit den klassischen Cleavages umrissen und erklärt werden kann. Und solange die neuen Konfliktlinien nicht deutlicher beschrieben werden, was eine Nivellierung der bisher gültigen Strukturierung der politischen Ordnung bedeutet, wird als Verlegenheitslösung zur Interpretation das Konzept des Populismus angewendet. Dieses konstatiert eine Spaltung zwischen dem Volk und den Eliten auf der Basis einer sehr einfachen Ideologie (Mudde 2007: 23). Fast unmerklich hat dieser konzeptuelle Zugriff auf die Wirklichkeit erfolgreich ältere Debatten zur Postdemokratie oder zur Krise der Demokratie verdrängt. ${ }^{4}$ Infolgedessen stellt sich die Frage nach den Gründen dieser Verdrängung und also nach der Popularität des Populismus.

In der jüngsten politikwissenschaftlichen Instrumentalisierung des Populismus lassen sich zwei Strategien erkennen: Während die erste Strategie die Existenz des Populismus skandalisiert, stellt die zweite Strategie sein Auftreten als Novum heraus, wobei beide zu einer »Exotisierung des Populismus (Knöbl 2016: 8) beitragen. Die Skandalisierung erfolgt durch die normative Differenzierung zwischen einem demokratischen Normalzustand und einem Populismus, der an diesem quasi natürlichen Zustand rüttelt und ein Tabu bricht. Der Populismus dient zur normativen Positionsbestimmung, um zwischen Demokratie und Nicht-Demokratie zu unterscheiden. Dies wird besonders in dem jüngst stark beachteten Essay von

4 In der öffentlichen Debatte wird der Populismus häufig rein polemisch verwendet. Im wissenschaftlichen Diskurs wird er explanativ, analytisch oder diagnostisch genutzt (Jörke/Selk 2017: 10). 
Müller deutlich, der moralisierend den Antipluralismus der Populisten als dessen antidemokratisches Grundmerkmal identifiziert (Müller 2016: 44). Wird für diese Unterscheidung im internationalen Kontext allgemein der Begriff der Autokratie herangezogen, fungiert der Begriff des Populismus als Differenzierung nach innen. Ein solches »Flüchten ins Normative« (Jörke 2016: 205) trägt einzig dazu bei, mit dem Label des Populismus Akteure als Nicht-Demokraten zu demaskieren. Somit knüpft die Populismusdiagnose methodisch an das Extremismuskonzept an, welches zur Bezeichnung kritischer Randbewegungen in einer traditionell durch eine Links-Rechts-Struktur geprägten politischen Kultur angewendet wird. ${ }^{5}$

Neben diesem normativ begründeten Differenzierungsangebot wird der Populismus auch für die Behauptung instrumentalisiert, dass es sich in seiner jüngsten Form um einen neuen Zustand handelt (vgl. Knöbl 2016: 8; Sauer 2017: 2). Auf der einen Seite kann damit der Anspruch auf eine notwendige Auseinandersetzung mit etwas Neuem begründet werden, was als Strategie einer um Aufmerksamkeit bemühten Sozialwissenschaft durchaus legitim ist. Auf der anderen Seite wird so aber auch ein Bruch in der historischen Reflexion in Kauf genommen, um, so ist zu vermuten, die in der Geschichte des Populismuskonzeptes klar zutage tretenden konzeptuellen Mängel auszublenden. Zwei davon sind für die Analyse besonders problematisch: (1.) Die empirische Heterogenität der behandelten Phänomene und (2.) die schwache Basis der Kategorisierung.

(1.) Die als populistisch behandelten Phänomene sind nicht nur historisch weitreichend, sondern auch geographisch weit gestreut. Historische Startpunkte des Populismus und seiner wissenschaftlichen Diskussion waren agrarische Bewegungen im Süden und dem Mittleren Westen der USA, die am Ende des 19. Jahrhunderts zur Formierung der »People's Party« geführt haben, wie auch die russische Bewegung der "narodnichestvo«, einer Gruppe von Intellektuellen, die zwischen 1860 und 1870 versuchten, aus den Bauern ein revolutionäres Subjekt zu formen (Moffitt 2016: 12). In den 1950er Jahren wurde das Konzept geöffnet und zur Bezeichnung der McCarthy-Ära herangezogen, wobei bereits eine Differenzierung des Konzeptes und damit seine konzeptuelle Auffächerung sich vollzog, indem der Populismus als ideologisches wie auch extremistisches Phänomen definiert wurde (Moffitt 2016: 13). Indes sind beide schon als normative Abweichungen vom "Idealbild einer liberalen Demokratie« behandelt worden (Knöbl 2016: 11). Später wird der Populismus als ein spezifisch lateinamerikanisches Herrschaftsphänomen analysiert, bei dem unter der Führung von charismatischen politischen Führern »multiclass urban alliances« geformt werden (Moffitt 2016: 13f.), die Modernisierungs-

5 Doch auch wenn beide Konzepte die gleiche Methode der Differenzierung bestimmter politischer Akteure teilen, sind sie konzeptuell verschieden und können nicht vermischt werden. Gleichzeitig provoziert die Popularität des Populismus die Extremismusforscher dazu, ihren Ansatz zu verteidigen (Jesse/Panreck 2017). 
und Wohlfahrtsversprechen formulieren. Daraus entfaltet sich ein ganzes System von spezifischen Herrschaftstechniken, die unter dem Begriff des Populismus vergleichend zwischen Argentinien (Peron), Mexiko (Cárdenas), Brasilien (Vargas) und Ekuador (Ibarra) analysiert werden konnten (Moffitt 2016: 14). Infolgedessen gelang es, die Besonderheiten der Herrschaftstechniken theoretisch neu einzuordnen und von westlichen bzw. europäischen Herrschaftssystemen wie dem Faschismus zu differenzieren (Knöbl 2016: 15; vgl. Priester 2012a: 221). In einer weiteren Spielart kommt der Populismus in die etablierten Demokratien zurück, nun aber als »Form des diskursiv-politischen Kampfes« (Knöbl 2016: 23) positiviert und theoretisch abstrahiert. Der Ansatz von Laclau gibt der Diskussion eine neue, wenn auch umstrittene Richtung (vgl. Straßenberger 2016). Unabhängig von der Nachvollziehbarkeit der Kritik kann hier zumindest gezeigt werden, dass das Konzept des Populismus durch die radikal-pluralistische Demokratietheorie über die rein analytische Ebene hinaus auf die kritisch-emanzipatorische Ebene ausgedehnt wird. Gleichzeitig wird es, und das ist der interessantere Aspekt, nicht mehr als Abweichung demokratischer Praxis interpretiert und mit der normativen Demokratietheorie vereint. Das Ergebnis dieser Ausdehnung ist die potentielle Grenzenlosigkeit für die Anwendung des Populismuskonzepts in der Gegenwart. Nach wie vor wird der Begriff des Populismus zur Charakterisierung latein- und südamerikanischer politischer Bewegungen und ihrer Herrschaftstechniken herangezogen wie für die aktuell sich im Abwind befindliche »rosa Welle populistischer Palaststürmer« (Müller 2016:10) in Venezuela, Brasilien und Argentinien. Kurz zuvor wurden noch diejenigen politischen Akteure als neopopulistisch bezeichnet, die wie Fujimori, Menem und Collor in der gleichen Region versucht haben, radikal neoliberale Strategien umzusetzen (Moffitt 2016: 17). Weiterhin wird das Konzept auf rechte Bewegungen und Parteien in Westeuropa angewendet (Berezin 2009; Wodak 2015) wie auch zur Deskription neuer post-postsozialistischer Parteien in Ost- und Mitteleuropa (Bugaric 2008). Schließlich werden auch Legitimationsstrategien in autoritären Regimen als populistisch identifiziert (Robinson/Milne 2017). Diese inflationäre Anwendung, mittlerweile gibt es sogar den »Medienpopulismus« (Priester 2012: 35), hat ihre Kosten und reduziert den analytischen Wert des Populismus auf ein Minimum. Konzeptuell markiert der Populismus zwar etwas, erklären kann er hingegen nichts, was seiner Popularität aber nicht zu schaden scheint - "populism matters« (Moffitt 2016: 11).

(2.) Mit der Karriere des Populismuskonzeptes begann auch das »conceptual stretching (Moffitt 2016: 13-16). So attraktiv es ist, den Populismus als Figur in einem weiten Kontext anzuwenden, so schwierig ist es, diese Differenzierung zu vereinheitlichen und klar zu benennen, was der konzeptuelle Kern des Populismus ist. Immer wieder gab es Versuche, diesen zu bestimmen, doch letztlich läuft die Theoriebildung hier den empirischen Anwendungen stets hinterher. Dementsprechend konsequent erscheint es, wenn in der Forschung derzeit nicht mehr versucht 
wird, eine Definition oder eine Theorie des Populismus zu entwickeln. Stattdessen wird zum Beispiel eine rein empirische Bildung eines Typs des Populismus vorgenommen (vgl. Priester 2012: 32-38). Doch auch mit dieser Vermeidungsstrategie kann man sich der Unschärfe des Begriffs nicht entziehen. So existieren mehrere, teils divergente Strategien zur Typologisierung des Populismus. Die eine Gruppe sucht die Konstruktion des Typs durch die Summierung bestimmter, empirisch beschreibbarer Merkmale. Die anderen gehen von einem »Prototyp« des Populismus aus, dem sukzessive weitere Fälle zugeordnet werden, welche dann zur Ausweitung des Prototyps beitragen. Beide Strategien kämpfen mit Schwierigkeiten: Die erste kann nicht mehr genau differenzieren, was "Substanz« oder »Akzidenz« ist (Priester 2012: 38), weil das definitorische Minimum bewusst im Dunkeln gelassen wird. Dies hat den Vorteil, dass programmatisch empirische Phänomene diesem Typ (und keinem anderen) hinzugefügt werden können. Im Gegensatz dazu sucht die andere Strategie durch die Bestimmung eines Urtyps nicht nach einer Differenzierung von Substanz und Akzidenz, denn hier ist klar, dass alle untypischen Fälle dem Idealbild allenfalls ähnlich sein können. Allein dies genügt, um der Kategorie des Populismus zugeordnet zu werden. Doch auch hier kommt über die Begründung der Definition des Urtyps das Problem der fehlenden Schärfe des Phänomens wieder ins Spiel, denn die auch rein analytische Bestimmung des ersten Populismus ist umstritten. Zudem ist man mit einem Zuordnungsdilemma konfrontiert, da Populisten sich selbst meist nicht als solche wahrnehmen (Priester 2012: 39). Ob der Typ durch die Aufsummierung von Merkmalen oder den Vergleich und Gruppierung von Fällen konstruiert wird, beide Strategien spielen die unscharfe Kategorisierung des Populismus idiographisch bzw. nomothetisch durch, ohne die Leistung einer klaren Theorie kompensieren zu können.

Infolgedessen kann der Versuch der rein empirischen Typenbildung nicht zur konzeptuellen Abgrenzung des Populismus beitragen. Es braucht eine Theorie des Populismus, doch die darf epistemologisch nicht weiter darauf hoffen, dass sie die eine, nicht existierende Definition des Populismus findet (Moffitt 2016: 27). Bleibt somit zunächst die Akzentuierung des Pluralismus und die Aufzählung unterschiedlicher Ansätze zur theoretischen Bestimmung des Populismus: Priester identifiziert drei Ansätze, die den Populismus als Ideologie, als Strategie zum Machterwerb und als Diskuspraxis definieren (2012: 40-48). Ähnlich differenziert Moffitt vier Ansätze: Populismus als Ideologie, als Strategie, als Diskurs und als politische Logik (2016: 17-25). Im Angesicht dieser Vielfalt und im Wissen, dass es die eine starke theoretische Definition von Populismus nicht gibt, ist das Ausweichen in weiche, unscharfe Kategorien naheliegend. Beispiele für solche unscharfen Kategorisierungen des Populismus sind:

- Populismus als Stil, "which is defined as the repertoires of embodied, symbolically mediated performance made to audiences that are used to create and 
navigate the field of power that comprise the political, stretching from domain of government through the everyday life« (Moffitt 2016: 29);

- Populismus als elitenkritische Mentalitäten der mittleren und unteren sozialen Segmente, die einem bestimmten Phasenmodell folgend sich zu einem politischen Akteur formieren können (Priester 2012: 95); oder

- Populismus als diskursiver Frame, der die soziale Produktion von Einstellungen ermöglicht, welche die Identifikation einer problematischen Realität erlaubt, in der korrupte Eliten die Souveränität des Volkes untergraben (Aslanidis 2016: 99).

Diese Ansätze vertagen das Problem der kategorialen Schwäche des Populismus und sehen den Ausweg in einer pragmatischen, konzeptuell eher offenen Theoriebildung des Populismus, der die Beliebigkeit seiner Ein- und Abgrenzung durch die Dichte seiner empirischen Anwendung zu kompensieren vermag. Beide behandelten Einwände machen das konzeptuelle Dilemma des Populismus deutlich. Kategorial ist er hervorragend zur normativen Abgrenzung politischer Akteure geeignet. Gleichzeitig kann er aufgrund seiner unscharfen konzeptuellen Basis wie auch seiner »heterogenen Faktizitäten« (Knöbl 2016: 35) nicht darüber aufklären, was genau Populismus ist und woher er kommt (vgl. Priester 2016: 218). Die Popularität des Populismus ist nicht die Folge seines heuristischen Potentials, sondern seiner Differenzierungsfähigkeit, die zunächst ein Rätsel bleibt.

\section{Identität als normative Leitidee}

Die Kritik des Populismus soll nicht bei der Hypostasierung seiner Unschärfe stehen bleiben. Auch wenn die Theoretisierung des Phänomens gescheitert ist (Knöbl 2016: 34), muss dennoch überlegt werden, ob nicht die Gründe seiner Popularität Erkenntnisse zur Aufklärung bereithalten. Dabei ist zu beachten, dass man erstens das Populismuskonzept nicht normativ auflädt und zweitens seine Anwendung auf wenige, kontextuell ähnliche Fälle reduziert (Knöbl 2016: 35). Infolgedessen nehme ich den Populismus nicht als abstraktes und damit weltweites moralisches Differenzierungsinstrumentarium ernst, sondern ziehe ihn allein zur Deskription der politischen Veränderungen in den liberalen westlichen Demokratien heran, ohne seine Normativität $\mathrm{zu}$ vernachlässigen. Es ist also auch wenig hilfreich, den Populismus exklusiv in anderen, zum Beispiel rein soziologischen Kategorien umzudeuten. Dazu gehört unter anderem auch der Ansatz, die im Populismus behandelten Konflikte in den westlichen liberalen Gesellschaften mit dem sozialen Abstieg großer gesellschaftlicher Gruppen zu Hauptursache seiner Existenz zu erklären (Baumel 2015: 116-118; Inglehart/Norris 2016: 10). Natürlich ist dieser Befund partiell richtig und konzeptuell für die soziologische Erfassung des Populismus not- 
wendig, doch verführt er aus politikwissenschaftlicher Perspektive zur Reduktion des Phänomens auf soziale Ungleichheiten, die mit Umverteilung behoben werden können. Des Weiteren ist diese Neuerzählung des Phänomens in Kategorien der sozialen Ungleichheit nicht in der Lage, den Erfolg populistischer Parteien in den egalitären Wohlfahrtsstaaten Nordeuropas zu erklären (Inglehart/Norris 2016: 12). Nochmal, die sozialen Veränderungen in der Sozialstruktur der liberalen Demokratien West- und Mitteleuropas haben sicher zur Dynamisierung des Populismus beigetragen, doch ausgelöst haben sie ihn nicht. Die linke Forderung nach einem Zurück zur sozialen Frage zeigt indes, dass dieser politische Konflikt sich nicht mehr an sozialen Fragestellungen entzündet, sonst würden die Abgehängten und Verängstigten genau dieses Problem politisieren. Stattdessen kristallisiert sich der populistische Protest an anderen, noch aufzudeckenden Differenzen.

Eine methodisch kontrollierte Aufklärung aus der Perspektive der Politischen Theorie setzt hierzu an der Normativität des Populismus an und fragt nach den Gründen seiner Fähigkeit zur Differenzierung und Polarisierung. So geraten normative Leitideen in den Fokus, welche in politischen Auseinandersetzungen zur Polarisierung und zur gegenseitigen Abgrenzung genutzt werden. Normative Leitideen präsentieren begrifflich grundlegende Ziele, Werte und Prinzipien von politischen Ordnungen, die dazu dienen, kritisch über die Legitimation solcher Ordnungen zu reflektieren (Göhler et al. 2009: 375). Dergestalt haben sie Kraft genug, in Konflikten zwischen eigenen und anderen Einstellungen zu differenzieren und die Positionierung $\mathrm{zu}$ polarisieren.

\section{Die Identität des Anti-Populismus}

Sucht man den Populismus als normative Leitidee zu verstehen, so fällt zunächst auf, dass der Populismus selbst keine ist. Dies klingt paradox, wurde doch oben von der normativen Kraft des Konzepts bei der Unterscheidung politischer Akteure gesprochen. Tatsächlich erlaubt der Populismus eine Differenzierung nach Populismus und Nichtpopulismus, jedoch ist er nicht in der Lage, die Kontingenz dieser Differenzierung durch normativ fixierte Ziele, Werte und Prinzipien aufzulösen. Der Mangel an normativem Inhalt zeigt sich in der Konzeptualisierung des Populismus als »thin-centered ideology« (Priester 2012: 40; Aslanidis 2016; Moffitt 2016: 18). Eine starke Ideologie ist ein geschlossenes sowie komplexes, von einer relativ homogenen Gruppe entwickeltes Produkt, dessen Eigenschaften eine substantielle innere Integration, ein dichter, mit politischen Konzepten verbundener Kern, die Fähigkeit zur Präsentation politischer Konzepte und Positionen, exklusive Antworten auf politische Fragen der Gesellschaft und weitreichende ideelle Ambitionen sind (Aslanidis 2016: 90). Der Populismus als allenfalls schwache Ideologie verfügt über keine dieser Eigenschaften (Aslanidis 2016: 90). 
Dass der Populismus trotzdem normativ aufgeladene Differenzierungen ermöglicht, beruht zum einen auf seiner methodisch rein negativen Anwendung. Es geht um die Markierung der Populisten als negativem Antipol zu den Nichtpopulisten, nicht um deren Aufklärung. Zum anderen operiert diese Abgrenzung, wie oben gezeigt, nicht politisch, sondern moralisch. Die Annahme einer universell gültigen moralischen Norm sorgt für die unterkomplexe Hierarchisierung der Differenz von Populisten und Nichtpopulisten. Diese instrumentelle Stärke kann der Populismus aber nur ausspielen, da er an eine andere normative Leitidee anschließt, die im wissenschaftlichen Diskurs zum Populismus selber nicht thematisiert wird. Nicht die Idee des Populismus konstruiert die Populisten, weil sie bloß die in der Wirklichkeit vorgefundenen politischen Konflikte mit ihren eigenen Labeln versieht. Die so durch den Populismus adaptierten Konflikte entzünden sich jedoch an anderen antagonistischen politischen Ideen. Ein Hinweis auf diese Ideen findet sich in der Diskussion zum Ausgang der ebenfalls als populistisch interpretierten amerikanischen Präsidentschaftswahl 2016. In seiner Analyse identifiziert der amerikanische Politologe Mark Lilla das Ende des »identity liberalism« als für die Wahlentscheidung verantwortlichen Kernkonflikt (Lilla 2016). ${ }^{6}$ Er sieht den Fehler der Kampagne der Demokraten in der Exklusion bestimmter sozialer Gruppen. Indem diese durch eine progressive liberale Identitätspolitik immer neue Minderheiten identifiziert, politisiert und emanzipiert haben, verloren sie die Teile des Demos aus den Augen, die eben nicht in dieses Raster passen (Lilla 2017: 9). Mit der Absicht, Gutes zu tun und für mehr zumindest legale Gleichheit zu kämpfen, hat die Identitätspolitik sich hoffnungslos auf die Diversität fokussiert. Doch produziert die Suche nach Unterschieden auf der politischen Ebene nur Unterschiede. »But the fixation on diversity in our schools and in the press has produced a generation of liberals and progressives narcissistically unaware of conditions outside their self-defined groups, and indifferent to task of reaching out to Americans in every walk of life« (Lilla 2016). Eine Politik, die sich allein auf Diversität fixiert, verliert die Fähigkeit, Einheit zu stiften. "National Politics in healthy periods is not about difference, it is about commonality« (Lilla 2016). Und selbst für einen individualistisch orientierten Liberalismus ist ein Konzept des >wir< notwendig (Lilla 2017: 14).

Lillas Kritik an der Identitätspolitik, die ihrerseits Anlass für Kritik und Gegenkritik bietet (Brahm 2017), verweist auf den Begriff der Identität und dessen normative Aufladung. Dies ist das Ergebnis eines sich selbst als aufgeklärt und links verortenden rein theoretischen Diskurses. Dessen Movens liegt in der Verknüpfung eines moralisch legitimierten Kampfes um Anerkennung mit der Identität des Subjekts (Calhoun 1995; Honneth/Fraser 2003). Basierend auf einer konstruktivistischen Epistemologie des Sozialen infolge des >cultural turns (Calhoun 1995: 198ff.) wird die aktive Re-Konstruktion von Identitäten zum Ziel politischer Aufklärung

6 Seine Überlegungen hat Lilla zwischenzeitlich zu einer Monographie zusammengefasst (2017). 
gemacht und als Aufgabe politischer Kämpfe unabhängig von der sozialen Frage definiert (Fraser 2003: 86). Freilich wird die soziale Frage nicht gänzlich ignoriert, doch die kategoriale Trennung beider Bereiche und die situative Fokussierung auf eines der beiden Felder erlauben der Kritik, jeweils partikulare Forderungen zu formulieren. Damit entwickelt sich sukzessive der Kampf um Anerkennung von Identitäten als eigenständiges und umfassendes Handlungsfeld für die Identitätspolitik. »Identity politics have more generally been basic to a whole range of movements that sought to use the public sphere to challenge existing arrangements or bring forward new possibilities in religion, sexual relations, the human relation to nature, community life, work and economics, and a host of other dimensions of sociale life« (Callhoun 1995: 215). Dabei geht es sowohl um Anerkennung für einen rechtlichen Schutz der Identitäten als auch vermehrt um die Anerkennung im Sinne einer »Wertschätzung« der verschiedenen Identitäten (Honneth 2003: 198).

Indes provoziert die Entdeckung, theoretische Auslotung und Normativierung der Identität als politisches Projekt Kritik aus zwei Richtungen: ${ }^{7}$ Eine erste Kritik setzt an der theoretischen Begründung an und moniert zum einen die konzeptuelle Trennung von sozialer und kultureller Anerkennung sowie zum anderen die Stringenz der gerechtigkeitstheoretischen Begründung der Identitätspolitik (Honneth 2003: 191ff.). Eine andere Kritik setzt an der Relevanz des emanzipatorischen Anspruchs an. Hier ist, obschon ihrer richtigen Voraussage, die Kritik von Richard Rorty über das Selbstverständnis der amerikanischen Linken zu nennen (1999). Rorty zeichnet kritisch die epistemologische Wende der linken Theoriebildung nach, bei der in der Analyse der Gesellschaft die materialistische Makroperspektive durch eine psychologische Mikroperspektive ersetzt wird (vgl. Lilla 2017: 74-78). Durch die »teilweise Ersetzung von Marx durch Freud als Gesellschaftstheoretiker wurde der Sadismus anstelle der Selbstsucht zum Hauptangriffsziel der Linken« (Rorty 1999: 74). Aus diesem epistemologischen Wandel entsteht die »Politik der Differenz« bzw. »die Politik der Identität« als Aktionsfeld einer linken Bewegung, die sich mehr mit dem »Stigma als mit dem Geld, mehr mit tiefliegenden und verborgenen psychosexuellen Motiven als mit prosaischer und offensichtlicher Habsucht « beschäftigt (Rorty 1999: 75). Die sich so verstehende »kulturelle Linke« verliert nach Rorty die alten Verbündeten wie die Gewerkschaften und verlagert ihr Aktionsfeld auf akademische Auseinandersetzungen über die Chancen und Notwendigkeiten einer eher pädagogischen, quasi unbegrenzten Vermittlung der Anerkennung von Anderssein, der jedoch ein Begriff nationaler Politik abhandenkommt. So verliert sie auch das Verständnis für den praktischen Alltag der nichtakademischen Schich-

7 Ich konzentriere mich hier auf die programmatische Kritik, welche nicht fundamental an der konstruktivistischen Basis, dem kritischen Habitus oder dem emanzipatorischen Ziel der Identitätspolitik ansetzt und derart ihre Legitimität grundsätzlich zur Disposition stellt. 
ten, redet lieber abstrakt vom System, statt konkrete Schritte für den gesellschaftlichen Wandel zu beraten (Rorty 1999: 100).

Unabhängig von ihrer Überzeugungskraft zeichnen die Kritiken ein genaues Bild der Identität der Identitätspolitik. Als spezifisches Identitätsverständnis ist sie das Ergebnis eines emanzipatorischen Diskurses, der mit einer konstruktivistischen Epistemologie die Mikrobedingungen von Anerkennung aufklären möchte. Die sich daraus bildende Perspektive der kulturalistischen Linken operiert mit einem theoretischen Identitätsbegriff, der für eine gewünschte gesellschaftliche Transformation politisiert wird, wobei die sich daraus ergebende Polarisierung von Identitätsfragen eine Ursache des populistischen Phänomens ist. Eine weitere Ursache ergibt sich aus alternativen Identitätsverständnissen.

\section{Die identitäre Identität}

Eine von der emanzipatorischen Identitätspolitik der kulturalistischen Linken abweichende Reaktion auf die Politisierung des Identitätsbegriffs kann in der Neuen Rechten identifiziert werden. ${ }^{8}$ Ausgangspunkt der Karriere des dort entwickelten identitären Identitätsbegriffs ist die kulturelle Subversion der Neuen Rechten, die sukzessive von den Linken dominierte Themen und Darstellungspraktiken übernehmen (Wagner 2008). Dabei kommt es zu einer ästhetischen und intellektuellen Aufladung der kollektiven Identität (Hentges/Kökgiran/Nottbohm 2014). Im Anschluss an Henning Eichberg wird Identität als scheinbar neutrales Distinktionsangebot entwickelt. »Identität konstituiert sich zugleich aufgrund von Unterscheidung, von Einsicht in das andere, das Fremde und das Eigentümliche« (Hentges/Kökgiran/Nottbohm 2014: 2). So lässt sich aus der Identität nur eine neutral wirkende Differenzierung ableiten, gegen eine Hierarchisierung wehrt sich der objektive Begriff scheinbar. Diese proklamierte Eigenschaft der Identität reagiert auf den emanzipativen Identitätsbegriff, wie Alain de Benoist, einer der intellektuellen Vertreter der Neuen Rechten, begründet. Er moniert die »Erosion der Unterschiede« die mit dem Phänomen der Globalisierung ihren Höhepunkt erreicht hat, wobei dadurch zwar »Kulturen homogenisiert « werden, jedoch auch neue Brüche entstehen, welche die Frage nach der Identität aufwerfen (Benoist 2008: 28f.). Doch positioniert er keinen vollkommen gegenläufigen Begriff der Identität, welcher statt von der Konstruktion von einer Unhintergehbarkeit der Identität ausgeht. Einem solchen essentialistischen Identitätsverständnis weist Benoist eine pathologische Qualität zu, welche der Homogenisierung der Globalisierung eine Homogenität auf einer niederen Ebene gegenüberstellt (ebd.: 95). »Indes ist Identität kei-

8 Ich will mich hier nicht mit der politischen Bewegung als Ganzes auseinandersetzen, sondern mich auf die Spezifik der normativen Leitidee der Identität konzentrieren. Zur Bewegung, Geschichte und ideologischen Grundlagen der Neuen Rechten vgl. Maaß (2014). 
ne Essenz oder statische Realität. Sie ist eine Substanz, eine dynamische Realität, und aufgrund dessen bilde sie ein Repertoire von Rollen « (ebd.: 68). Identität kann nicht als homogen, beständig oder einheitlich verstanden werden, sondern als eine in ihrer Dynamik und Dialektik ständig im Wandel begriffene Differenz. Benoist versteht Identität dialogisch, also als Medium, welches Kommunikation zwischen den Identitätskollektiven erst ermöglicht. Er spricht sich gegen eine Verabsolutierung und damit Idealisierung der eigenen Identität aus (ebd.: 94). Stattdessen plädiert er für die Kommunikation zwischen den Identitätsträgern und favorisiert einen Austausch mit dem Ziel, gegenseitig die Unterschiede versteh- und akzeptierbar zu machen (ebd.: 97).

Diese Kommunikation zwischen den Identitäten unterliegt in der Gegenwart einer Herausforderung, die zugleich die gesellschaftliche Kritik von Benoist umreist. Dabei verknüpft er zwei Ebenen: die fundamentale Ebene der Konstruktion des Selbst unter den Bedingungen des Liberalismus und die der Expansion dieses Ansatzes im Rahmen der Globalisierung. Die erste Ebene bezieht sich auf die rigorose Verknüpfung mit dem Einzelnen, der sich von seinen Beziehungen befreien muss, weil sie nicht nur seine Freiheit beschränken, sondern ihn a priori als nicht $\mathrm{zu}$ seinem Ich gehörend belasten (ebd.: 22). Erst ein so seines »Herkunftskontextes « entkleidetes Individuum kann sich als Gleicher und Gleichen vorbehaltlos »in den gerade entstehenden Markt eingliedern« (ebd.). Die Identität verschiebt sich im modernen Liberalismus in den Bereich einer institutionalisierten Sphäre der Unterschiedslosigkeit, in der die einzelnen Individuen sich ausschließlich als Individuen zwischen Individuen stetig neu erfinden müssen. Benoist kritisiert hier den Status des Marktes, der im liberalen Kapitalismus der exklusive Koordinationsmechanismus für die Selbstwerdung des Einzelnen wird und die Ausbildung eines für den Markt empfänglichen »freischwebenden Subjekts« befördert (ebd.: 104). Da der Markt und der liberale Individualismus die dominierenden Ideen und Motoren der Globalisierung sind, damit komme ich zum zweiten Kritikpunkt von Benoist, expandiert das Muster einer rein individuellen und rein marktkonstituierten Identität weltweit und es kommt zur erwähnten Erosion der Unterschiede und der kollektiven Identitäten. Gleichzeitig wird die Suche nach neuen Identitätsgemeinschaften jenseits nationaler Identitäten immer schwieriger. Daraus ergibt sich eine Situation der gegenseitigen Verstärkung von globaler Homogenisierung individueller Identitäten und einer Identitätskrise des Individuums bei seiner Suche nach Orientierung (ebd.: 29). Die identitäre Identität normativiert den Stellenwert der Identität als solche, obwohl sie ihr den essenziellen Charakter abspricht, indem sie die Identität zur Grundvoraussetzung eines interpersonalen und interkulturellen Dialogs macht. In dieser Lesart verhindert der Verlust bzw. die Pathologie von Identitäten, dass der Einzelne, jenseits seiner Identität als Konsument, eine weitergehende Selbstreflexion und -verortung vornehmen kann. 
Die intellektuelle Aufwertung der Identität zu einem zentralen Begriff der Neuen Rechten bricht mit ihren älteren Ansätzen der Nation, des Patriotismus und Nationalismus. Als Begriff kann die Identität mit unterschiedlichen Adjektiven verbunden werden, wodurch die im rechten Denken bis dahin dominierenden klassischen Hierarchisierungen von Gemeinschafts- und Gruppenzugehörigkeiten semantisch wie auch kategorisch relativiert werden sollen. Die identitäre Identität muss begrifflich, so der Wunsch der Vordenker der Neuen Rechten, eine Positionierung jenseits der tradierten Rechts-Links-Differenzierung ermöglichen, was später durch die Identitäre Bewegung aufgenommen und in Abgrenzung zu anderen rechten Gruppierungen als Selbstverortung in der politischen Mitte proklamiert wird (Hentges/Kökgiran/Nottbohm 2014: 19).

\section{Die Einheit der Differenz}

Es handelt sich um zwei Ansätze von Identität, die als normative Leitideen genau die Polarisierung strukturieren, die durch das Populismuskonzept beschrieben wird. Nicht die dünne Idee des Populismus treibt die Menschen in divergente politische Einstellungen, sondern das antagonistische Verständnis von Identität. Doch warum gelingt gerade dem Identitätsbegriff diese normativ aufgeladene Differenzierung? Dies führt methodisch über die bloße Registrierung eines neuen Cleavage und die Messung seiner empirischen Validität hinaus zur Ebene der Konstruktion des Begriffs. Zunächst fällt auf, dass beide Ansätze den konstruktiven Charakter der Identität betonen und sich damit erstens von einem statischen Verständnis von Identität wie auch zweitens von einem essenziellen Begriff distanzieren. Dies ist die Einheit ihrer Konstruktion. Benoist weist explizit auf den dialogischen, seiner Meinung nach produktiven Charakter der Identität hin. Zwar transzendiert Identität hier immer noch die Einheit der Gemeinschaft, doch greift sie dafür nicht mehr auf einfache, mit Blut und Abstammung arbeitende Identitätskonstruktionen zurück. Dieses offen zur Schau getragene dynamische und konstruktive Verständnis findet sich auch bei der aufgeklärten Identitätspolitik der Linken. Hier folgt die Konstruktion der post-strukturalistischen Kulturwissenschaft, die das Fremde als epistemologische Herausforderung begreift, welches es in seiner eigenen konstruierten Wirklichkeit unvoreingenommen aufzuklären gilt. Der wesentliche Unterschied beider Ansätze liegt nicht in der Erkenntnis, dass es unterschiedliche Identitäten und ihre Wirklichkeiten gibt, sondern in der Frage, was aus dem Pluralismus der Identitäten folgt? Die identitäre Identitätskonstruktion geht von der Unüberwindlichkeit der Differenzen zwischen den Identitäten aus. Ihr Ethnopluralismus transzendiert die Unterschiedlichkeit und macht sie zur ontologischen Größe der Ökumene. Hingegen nimmt die Identitätspolitik die Differenz zum Anlass, um eine neue Identität zu proklamieren. Sie manifestiert den Unterschied, um ihn in der Figur des Hybriden produktiv in einer neuen (besseren) Form aufzulösen. Glei- 
chen sich beide Zugriffe in der Akzeptanz der Kontingenz der Identitäten, also der Idee, dass kollektive Identitäten nicht essenziell konstruiert werden, versteht die identitäre Identität diesen Prozess als abgeschlossen. Die Identitätspolitik indes sucht durch eine weitere Konstruktion der Identität als synthetische Neukonstruktion bestehender Identitäten diese für das Ziel einer wie auch immer gerechteren Gesellschaft einzuspannen. Vollendete und permanente Identitätskonstruktionen stehen sich hier in der politischen Auseinandersetzung scheinbar unversöhnlich gegenüber.

Einerseits stimmen beide Zugriffe in der Akzentuierung der Konstruktion politischer Begriffe überein, indem sie einen essenziellen Status der Identität ablehnen. Dies sollte man ernst nehmen und nicht der Versuchung erliegen, dem identitären Identitätsbegriff obschon seiner Annahme der abgeschlossenen Konstruktion doch wieder einen Essenzialismus zu unterstellen. Die Einheit der Differenz beider Identitätskonstruktionen ist die theoretische Einsicht, dass die Kollektivsymbole zur Inklusion und Exklusion konstruiert und damit kontingent sind. Identitätspolitik versucht ihrerseits das Kontingenzverständnis soweit bewusst zu machen, um es in der alltäglichen Interaktion stetig mitzudenken bzw. mit zu kommunizieren. So wird auf einer theoretischen Ebene die Möglichkeit ausgeschlossen, dass einmal gesetzte Differenzierungen der Kontingenzperspektive entzogen werden. In der Absicht, das Machtpotential solcher Institutionalisierungen stetig kritisch zu befragen, wird in der emanzipativen Konzeptualisierung von Identität diese in einen immer wieder neu zu vollziehenden Prozess des Setzens und Auflösens von Humandifferenzierungen aufgelöst (Hirschauer 2014: 182). Dies ist in einem wissenschaftlichen Diskurs theoretischer Begriffe methodisch machbar und sinnvoll, um blinde Flecken zu identifizieren und kontingente Annahmen zu problematisieren. Im Alltag bricht solch ein Ansatz mit tradierten Formen der Kontingenzunterbrechung zur Legitimation sozialer Strukturen. Andererseits sucht der identitäre Identitätsbegriff, obwohl auch er die Kontingenz der Identitäten versteht, nicht die Radikalisierung des Kontingenzdenkens, sondern knüpft an ein nichttheoretisches Identitätsverständnis an. Da Gesellschaften keinesfalls auf ihre wissenschaftliche Auslegung warten und selbst Symbole ihres Selbstverständnisses kreieren, sieht sich die Politikwissenschaft in erster Linie mit diesen ihr vorausgehenden und nicht genuin theoretischen Symbolen und Theorien konfrontiert (Voegelin 2004: 43f.). Wenn diese nichttheoretischen Begriffe als normative Leitideen Diskurse initiieren und strukturieren, werden sie zum Objekt politikwissenschaftlicher Reflexion, wodurch sie auch zu theoretischen Begriffen verdichtet werden (Voegelin 2004: 43f.). Hierin liegt der Unterschied beider Identitätskonzepte. Die identitäre Identität vollzieht zwar theoretisch den konstruktiven Status von Identitäten nach, hält sich somit an die Kontingenzperspektive, legitimiert aber im Unterschied zum emanzipativen Identitätskonzept Kontingenzunterbrechungen durch nichttheore- 
tische Identitätsbegriffe und damit auch stabile Humandifferenzierungen. Dies ist der differente Zugang in der einheitlichen Konzeptualisierung von Identität.

\section{Die Politisierung der Kultur}

Der Vergleich beider Leitideen von Identität macht deutlich, dass die Ursache der unterschiedlichen Behandlung von Identität nicht im Begriff selber begründet ist. Identität ist kein Ordnungsbegriff im Sinne einer grundlegenden Strukturierung, die empirische Zugriffe ordnet (Göhler u.a. 2009: 375). Sucht man nach der grundlegenden Strukturierung für die zwei Identitätsbegriffe, muss deren Einheit fokussiert werden. Die Vorstellung einer kontingenten Konstruktion zeigt, dass die Einheit das kulturalistische Verständnis des Sozialen ist (vgl. Knobloch 2016). Analytisch stellt sich damit die Frage nach der Differenz der Differenzierungen, folglich nach deren Sinnhaftigkeit, was den kulturellen Kontext betrifft (Hirschauer 2014: 170).

\section{Die unterschiedliche Kulturalisierung der Gegenwart}

In seiner Reflexion der jüngsten populistischen Polarisierungen verweist der Kultursoziologe Andreas Reckwitz auf Veränderungen der kulturellen Lebensformen (2016; 2016a). Hierbei manifestieren sich zwei konträre Auffassungen über den allgemeinen Stellenwert der Kultur in der Spätmoderne (Reckwitz 2016: 1). Interessanterweise entspringen beide Auffassungen einem der Rationalisierung der Moderne entgegenlaufenden Trend der Kulturalisierung des Sozialen. Dieser setzt sich für die Wiederaufwertung von kulturell relevanten Objekten, Subjekten, Praktiken, Orten ein und stellt sich gegen die Profanisierung, Rationalisierung und Entzauberung der Welt (ebd.: 3). Die erste Auffassung opponiert mit einer expansiven Ästhetisierung der Lebensstile dagegen. Kultur wird zur Hyperkultur, in der "alles in höchst variabler Weise kulturell wertvoll werden kann« (ebd.: 4). Hierzu treffen Objekte mit Subjekten zusammen, die jene Objekte mit dem Wunsch nach Selbstverwirklichung nachfragen. »Kultur findet in dieser Konstellation immer auf kulturellen Märkten statt, in denen kulturelle Güter miteinander im Wettbewerb stehen« (ebd.: 4). Derart ökonomisiert wird Kultur zu einem Set von Gütern, die von Subjekten der mondänen Mittelschichten, die ihre eigene Identität als Patchwork kultureller Güter begreifen, angeeignet werden. Kultur ist ein subjektives Produkt aus der individuellen Kombination unterschiedlicher Praktiken und Artefakte. Als Hyperkultur zieht sie ihre Normativität aus der Möglichkeit, die Optionen auf Kombination kultureller Güter stetig zu vergrößern. »Diese Hyperkultur hat sich mit der Globalisierung verbreitet, im alten Westen und anderswo« und als Selbstverwirklichungsmodell umfasst sie auch den 
»Wettbewerb der spirituellen Praktiken und Glaubensgemeinschaften um das religiös bedürftige Individuum, das temporäre Engagement in politischen Initiativen und die Auswahl zwischen Bildungsangeboten auf dem Markt der Schulen, die sich der globalen akademischen Mittelklasse darbieten« (Reckwitz 2016a).

Dafür artikuliert die emanzipative Identitätsvorstellung das normative Programm, indem sie das Agieren auf kulturellen Märkten nicht als bloßen Narzissmus, sondern als Prozess der individuellen Emanzipation deklariert.

Demgegenüber sieht die zweite Auffassung in der Kultur kein »unendliches Spiel der Differenzen « auf einem offenen Markt (ebd.: 6). Stattdessen wird die Kultur als Antagonismus zwischen Innen und Außen, zwischen Wertvollem und Wertlosen gebraucht.

»Der Prozess der Valorisierung verläuft also nicht dynamisch und mobil, sondern arbeitet vielmehr daran, die Eindeutigkeit der wertvollen Güter - der Claubenssätze, Symbole, der nationalen Geschichte, der Leidensgeschichte einer Herkunftsgemeinschaft - nach innen aufrechtzuerhalten und zugleich nach außen eine konsequente Devalorisierung zu betreiben« (Reckwitz 2016: 6).

Reckwitz sieht hier eine stringente Praxis der Devalorisierung nach außen, denn die Kultur wird nun zur Hierarchisierung des Eigenen und des Fremden genutzt. Kultur baut einen Antagonismus auf und schreibt diesen immer einem Kollektiv $\mathrm{zu}$, welches hier auf seine Tradition zurückgreifen soll. Im Gegensatz zum individualistisch organisierten und auf das beständig Neue eines Kreativitätsdispositivs hin orientierten Kulturverständnis der Hyperkultur will dieses kollektiv organisierte Kulturalisierungsregime das Bewährte bewahren und durch die Orientierung an der historischen Narration die Kultur essenzialisieren. Damit trägt die Kultur zu einer »Schließung der Kontingenz« bei:

»Zum einen führt der zentrale Antagonismus zwischen Innen und Außen dazu, dass die Valorisierung der Güter nach innen nicht mobilisiert, sondern stabil gehalten werden soll. Zum anderen verringert und verengt die Orientierung am Kollektiv als Bezugseinheit der Kultur die Verhaltensspielräume für die Individuen.« (Reckwitz 2016: 6).

Dies ist das Fundament für den identitären Identitätsbegriff, der einerseits Teil einer kollektiv geteilten Kultur ist, folglich kollektiv konstruiert wird und somit kollektive Geltung beansprucht. Andererseits zieht die Leitidee eine normative Grenze zwischen den Kollektiven, weil sie die Geltung auf eine Kultur beschränkt, also nicht versucht, die nichttheoretischen Identitätskonzepte zu dekonstruieren. 


\section{Ein neuer Kampf der Kulturen?}

Der Antagonismus beider Auffassungen kann sich wegen seiner Unlösbarkeit zu einem fundamentalen Konflikt hochschaukeln. ${ }^{9}$ Auch in einer Demokratie können nicht alle Konflikte durch die Verteilung des Sozialprodukts zwischen verschiedenen Gruppen gelöst werden (Hirschman 2013: 356). Im Gegensatz zu Verteilungskonflikten, in denen es um ein Mehr-oder-weniger geht, provozieren diese Entweder-oder-Konflikte keine produktiven Lösungen, die den Zusammenhalt der Gesellschaft stärken, vielmehr fordern sie die grundlegende Einheit der Gesellschaft heraus. Unterschiedliche Kulturalisierungsregimes kreisen um divergente Ordnungsbegriffe der Kultur, die normativ Wahrheit beanspruchen. Treffen diese aufeinander, entwickelt sich ein neuer Kampf der Kulturen, der sich von Huntingtons Vorstellung einer Auseinandersetzung zwischen religiösen Zivilisationen löst und den Kampf auf die Innenseite der Kultur zieht. Dennoch lässt sich die neue Auseinandersetzung mit dem klassischen Konzept des Kulturkampfes definieren: »Wenn man Kultur als die Gesamtheit der typischen Lebensformen einer Bevölkerung, einschließlich der sie tragenden Geistesverfassung und Werteinstellungen, begreift « und diese im Kontakt mit anderen Kulturen um Geltung konkurriert, dann handelt es sich um einen Kulturkonflikt, oder politisch gesprochen, »um einen Kulturkampf « (Senghaas 1995: 198). Die Grenze zwischen den Kulturen wird aber nicht ausschließlich nach außen gezogen, sondern auch nach innen, als Gegensatz zwischen Kulturalisierungsregimes, wodurch es mehr zu einem Kampf um Kultur wird. Doch welche Szenarien kann ein interner Kulturkampf haben? Reckwitz entwirft hierzu eine Heuristik, die als Orientierung dienen kann (2016: 7):

- Vermittelnde Strategie: Anhänger beider Kulturalisierungsparadigmen versuchen, die jeweils anderen Standpunkte insoweit zu akzeptieren, dass sie der eigenen Perspektive beigestellt wird. Aus der Perspektive der Hyperkultur würde das bedeuten, dass sie den Kulturessenzialismus als weitere mögliche Optionen der Selbstverwirklichung versteht. Diese kann respektiert und toleriert werden, um sie einem freien Spiel der Kräfte zu überlassen, wie es etwa der Multikulturalismus gefordert hat. Hingegen würde die vermittelnde Strategie aus Sicht der Kulturessenzialisten so aufgefasst werden, dass die ihnen fremden pragmatischen Ästhetisierer eine Form partikularer Identitäten sind, die keinen Anspruch auf Universalität besitzen. Und solange diese nicht versuchen, den Status quo der Partikularität aufzugeben, kann man sich nebeneinander arrangieren. Diese Auffassung mündet in einer Kulturkreistheorie, die um friedliche Koexistenz bemüht ist.

9 Die Verbindung mit dieser Figur von Hirschman macht Honneth in seiner Replik auf Frasers Diskussion zur Identitätspolitik (Honneth 2003). 
- Konfrontative Strategie: In der Konfrontation zwischen den Kulturalisierungsregimes wird der Antagonismus soweit radikalisiert, dass mit ihm politische Freund-Feind-Differenzierungen legitimiert werden können, die dann, je nach Perspektive des Kulturalisierungsregimes, zu Abwertungen wie der Antizipation von Feinden der offenen Gesellschaft oder dem Dekadenzvorwurf gegenüber dem Westen führen. Diese Frontbildung ist der erste Schritt zu einem heißen globalen Kampf der Kulturen, der quer zu den Huntingtonschen Kulturen mitten in den Gesellschaften ausgefochten wird.

Beide Strategien entwerfen Szenarios, die auch als Blaupause für die Reaktion auf den Populismus dienen. Sie machen auf jeden Fall deutlich, dass die Annahme, populistische Einstellungen auf die Zustimmungen zu Parteien reduzieren zu können und man folglich nur warten müsse, bis diese Zustimmungen sich wieder änderten, falsch ist. Es muss akzeptiert werden, dass der dem Populismus zugrundeliegende Kampf um Kultur allenfalls durch Vermittlung zivilisiert, jedoch nicht gelöst werden kann.

\section{Herausforderungen für die Politische Kulturforschung}

Nachdem die Analyse der Ursachen des Populismus zwei differente Kulturverständnisse aufgedeckt hat, soll zum Schluss auf die Folgen für die politikwissenschaftliche Reflexion eingegangen werden. Die Inthronisierung der Kultur als Konfliktfeld moderner Gesellschaften zwingt die politikwissenschaftliche Analyse auf das Feld der politischen Kulturforschung. Als spezifische Disziplin klärt sie die Bedingungen für die Kategorisierung auf und gibt Aufschluss über die Unterschiede zwischen den Unterscheidern. Doch in Abgrenzung zur kultursoziologischen Perspektive von Reckwitz verknüpft die politische Kulturforschung diese Frage mit der Normativität der Differenzierungen. Es ist demzufolge notwendig, dass sie eine Distanz zur Kategorie der Kultur behält, also bei der Kulturalisierung des Politischen das Politische mit einem Eigenwert versieht (Knobloch 2016). Im Mittelpunkt steht der ambivalente Begriff der Kultur mit seinen spannungsreichen zwei Dimensionen: dem Gegensatz von theoretischen und nicht theoretischen Begriffen sowie der Differenz von Ordnungsbegriffen und normativen Leitideen. Entsprechend dieser Dimensionen muss die politische Kulturforschung darlegen, ob und wie sich, erstens, im neuen Kulturkampf theoretische oder nichttheoretische Kulturverständnisse gegenüberstehen und inwieweit, zweitens, Kulturen hier als normative Leitideen oder fundamentale Ordnungsbegriffe aufeinanderprallen. Die Aufgabe der politischen Kulturforschung liegt somit in der Verknüpfung der Analyse des Kampfes der Kulturen mit einer systematischen Aufklärung der Spannungen des Kulturbegriffs, um einen sinnvollen Kulturbegriff zur norma- 
tiven Evaluation des Konflikts zu erarbeiten. Zum Abschluss möchte ich vier Überlegungen skizzieren, welche die mögliche Richtung der weiteren Reflexion umreißen.

- Die weitere Aufklärung der Kulturen des Kulturkampfes muss präzise die Differenzen beider Ansätze systematisieren. Es fällt auf, dass die Kultur der Hyperkultur kein Ordnungsbegriff ist, ganz im Unterschied zum essenziellen Kulturbegriff, der die identitäre Identität mit Normativität versorgt. Infolge der Systematisierung der Differenzen kann anschließend ein gemeinsamer Nenner der Ordnungsbegriffe gefunden werden. Vermutlich kommt man so zu einem Individualismus, der in dieser Form auch philosophisch kritisiert werden kann. Womöglich lässt sich zwischen dem Kollektivismus der essenziellen Kultur und dem deskriptiven Individualismus der Hyperkultur, die den Einzelnen ohne überindividuelle Verweise manifestiert, eine vermittelnde Ebene eines normativen Individualismus definieren (Hastedt 1998), um ein gegenseitiges Verständnis beider Seiten zu ermöglichen.

- Auf der Ebene der Auseinandersetzungen zwischen den Kulturalisierungsregimes und damit den verschiedenen Identitätskonstruktionen ist aus kritischer Distanz zu prüfen, ob es eine sinnvolle Unterstützung der vermittelnden Strategie gibt. Dazu müssten die Gemeinsamkeiten beider Seiten systematisiert werden. Reckwitz selbst sieht die Einheit der Differenz in dem Bemühen beider Seiten begründet, durch die Kulturalisierung sich dem Diktat der Rationalisierung und damit der Profanierung des Sozialen entgegenzustemmen. Indem beide Ansätze die Kultur einspannen, um Dingen jenseits der formalisierten Sachlogik einen Wert zuzuweisen, werten sie das Gesellschaftliche wieder auf. Nach Reckwitz präsentieren beide Strömungen einen Reflex auf die Rationalisierungstendenzen der organisierten Moderne. Wenn diese Gemeinsamkeit stärker in den Blick genommen würde, könnte sich unter Umständen die Ursache für die Kulturalisierung im Kampf der Kulturen politisieren lassen, was zu einer Zivilisierung des Kampfes beitragen würde.

- Ausgehend von der Einheit der Differenz rückt die Politische Kulturforschung in den Mittelpunkt aktueller Gesellschaftsreflexionen. Ihr Forschungsgegenstand, als spezifische Perspektive auf die Politik der Gesellschaft, ist kein Randthema mehr, sondern der aktuell wichtigste Cleavage innerhalb und zwischen den Gesellschaften. Wenn es der Politischen Kulturforschung nun gelingt, ihre Erkenntnisse zur Kultur und der Kulturalisierung erfolgreich nach außen zu kommunizieren, kann sie dazu beitragen, die Ratlosigkeit einer zutiefst auf Rationalisierung und Formalisierung setzenden Politikwissenschaft in einer Krise des Rationalisierungsregimes der Moderne zu beenden.

- Die Politische Kulturforschung darf aber nicht vergessen, dass sie eine spezifisch politikwissenschaftliche Perspektive auf das Problem des Kulturkampfes 
hat. Dies differenziert sie auch von den kulturwissenschaftlichen (und kultursoziologischen) Ansätzen. Dementsprechend hat sie die normativen Verweisungen systematisch zu behandeln. Das kann sie aber nur, wenn sie auf die politische Konstruktion der Kultur hinweist. Die Frage, welches Kulturalisierungsregime gilt oder nicht, mündet in die Frage der Legitimität der Kultur. Doch kann Kultur legitim sein bzw. kann Kultur einen Anspruch auf Wahrheit besitzen? Natürlich: »Über Kultur zu reden heißt [...], nach Identität, Sinn und prägender Ordnung einer Gemeinschaft von Menschen zu fragen« (di Fabio 2005: 20). Folglich ist die Kultur immer auch eine politische Konstruktion.

\section{Literatur}

Aslanidis, Paris 2016: Is Populism an Ideology? A Refutation and a New Perspective. In: Political Studies, Heft 1, S. 88-104.

Baumel, Laurent 2015: Populismus als Hilferuf. In: Ernst Hillebrand (Hg.), Rechtspopulismus in Europa, Gefahr für die Demokratie?, Bonn, S. 115-120.

Benoist, Alain de 2008: Wir und die anderen, Berlin.

Berezin, Mabel 2009: Illiberal Politics in Neoliberal Times. Culture, Security and Populism in the New Europe, Cambridge.

Brahm, Gabriel Noah 2017: Killing the Messenger: Mark Lilla's »End of Identity Liberalism« and its Critics. In: Society, Heft 4, S. 326-330.

Bugaric, Bojan 2008: Populism, Liberal Democracy, and the Rule of Law in Central and Eastern Europe. In: Communist and Post-Communist Studies, Heft 2, S. 191-203.

Calhoun, Craig 1995: Critical Social Theory. Culture, History, and the Challenge of Difference, Cambridge Mass.

Decker, Frank/Lewandowsky, Marc 2017: Rechtspopulismus in Europa: Erscheinungsformen, Ursachen und Gegenstrategien. In: Zeitschrift für Politik, Heft 1, S. 21-38.

Di Fabio, Udo, 2005: Die Kultur der Freiheit, München.

Fraser, Nancy 2003: Soziale Gerechtigkeit im Zeitalter der Identitätspolitik. Umverteilung, Anerkennung und Beteiligung. In: Nancy Fraser/Axel Honneth: Umverteilung oder Anerkennnung? Eine politisch-philosophische Kontroverse, Frankfurt a.M., S. 13-128.

Fraser, Nancy/Honneth, Axel 2003: Umverteilung oder Anerkennung? Eine politisch-philosophische Kontroverse, Frankfurt a.M.

Göhler, Gerhard/Iser, Matthias/Kerner, Isa 2009: Entwicklungslinien der Politischen Theorie in Deutschland seit 1945. In: Politische Vierteljahresschrift, Heft 3, S. 372-407. 
Hastedt, Heiner, 1998: Der Wert des Einzelnen. Eine Verteidigung des Individualismus, Frankfurt a.M.

Hentges Gudrun/Kökgiran, Gürcan/Nottbohm, Kristina 2014: Die identitäre Bewegung Deutschland (IBD) - Bewegung oder virtuelles Phänomen. In: Forschungsjournal Soziale Bewegung - Plus, S. 1-26, (URL: http:// forschungsjournal.de/fjsb-plus, abgerufen am 8.12.2017).

Hirschauer, Stefan 2014: Un/doing Differences. Die Kontingenz sozialer Zugehörigkeiten. In: Zeitschrift für Soziologie, Heft 3, S. 170-191.

Hirschman, Albert O. 2013: Social Conflicts as Pillars of Democratic Market Society. In: Ders., The Essential Hirschman, Princeton, S. 345-362.

Hoffitt, Benjamin 2016: The Global Rise of Populism. Performance, Political Style, and Representation, Stanford.

Honneth, Axel 2003: Umverteilung als Anerkennung. Eine Erwiderung auf Nancy Fraser. In: Nancy Fraser/Axel Honneth: Umverteilung oder Anerkennnung? Eine politisch-philosophische Kontroverse, Frankfurt a.M., S. 129-224.

Huntington, Samuel P. 1996: Kampf der Kulturen. Die Neugestaltung der Weltpolitik im 21. Jahrhundert, München.

Inglehart, Ronald F./Norris, Pippa 2016: Trump, Brexit, and the Rise of Populism, RWP16-026, HKS Faculty Research Working Papers, S. 1-52, https:// research.hks.harvard.edu/publications/workingpapers/Index.aspx (abgerufen am 29.11.2017).

Jesse, Eckhard/Panreck, Isabelle-Christine 2017: Populismus und Extremismus. Terminologische Abgrenzung - das Beispiel AfD. In: Zeitschrift für Politik, Heft 1, S. 59-76.

Jörke, Dirk 2016: Moralismus ist zu wenig. Kommentar zu Jan-Werner Müllers Essay »Was ist Populismus?«. In: Zeitschrift für Politische Theorie, Heft 2, S. 203-208.

Jörke, Dirk/Selk, Veith 2017: Theorien des Populismus zur Einführung, Hamburg. Knobloch, Jörn 2016: Die politische Konstruktion der Kultur - Das Politische zwischen Offenheit, Erfahrung und Konstruktion. In: Wilhelm Hoffmann (Hg.), Die andere Seite der Politik, Theorien der kulturellen Konstruktion des Politischen, Wiesbaden, S. 113-136.

Knöbl, Wolfgang 2016: Über alte und neue Gespenster. Historisch-systematische Anmerkungen zum Populismus. In: Mittelweg 36, Heft 6, S. 8-35.

Lilla, Mark, 2016: »The End of Identity Liberalism«, in: New York Times, 18.11.2016, https://www.nytimes.com/2016/11/20/opinion/sunday/the-end-of-identityliberalism.html?_r=o (abgerufen am 29.11.2017).

Lilla, Mark 2017: The Once and Future Liberal: After Identity Politics, New York. Maaß, Sebastian 2014: Die Geschichte der Neuen Rechten in der Bundesrepublik Deutschland, Kiel.

Müller, Jan-Werner 2016: Was ist Populismus. Ein Essay, Berlin. 
Mudde, Cas 2007: Populist Radical Right Parties in Europe, Cambridge und New York.

Priester, Karin 2007: Populismus. Historische und aktuelle Erscheinungsformen, Frankfurt a.M. und New York.

Priester, Karin 2012: Rechter und linker Populismus. Annäherung an ein Chamäleon, Frankfurt a.M. und New York.

Priester, Karin 2012a: Populismus und Faschismus in Europa - Wahlverwandtschaft oder Mesalliance?. In: Totalitarismus und Demokratie, Heft 9, S. 213-234.

Priester, Karin 2016: Populismus und kein Ende. Müller belebt die Debatte, aber bereichert er sie auch? Kommentar zu Jan-Werner Müllers Essay »Was ist Populismus?«. In: Zeitschrift für Politische Theorie, Heft 2, S. 209-219.

Reckwitz, Andreas 2016: Zwischen Hyperkultur und Kulturessenzialismus. In: soziopolis, www.soziopolis.de/beobachten/kultur/artikel/zwischen-hyperkulturund-kulturessenzialismus/ (abgerufen am 13.12.2017), S. 1-10.

Reckwitz, Andreas 2016a: Alles so schön hyper. Alle Welt streitet nach den USWahlen plötzlich über Diversität und Identität. Freundliche Ignoranz hilft da nicht weiter. In: Die Zeit, Heft Nr. 51.

Robinson, Neil/Milne, Sarah 2017: Populism and Political Development in Hybrid Regimes: Russia and the Development of Official Populism. In: International Political Science Review, Heft 4, S. 412-425.

Rorty, Richard 1999: Stolz auf unser Land. Die amerikanische Linke und der Patriotismus, Frankfurt a.M.

Sauer, Birgit 2017: Gesellschaftstheoretische Überlegungen zum europäischen Rechtspopulismus. Zum Erklärungspotential der Kategorie Geschlecht. In: Politische Vierteljahresschrift, Heft 1, S. 1-20.

Schwelling, Birgit 2004: Der kulturelle Blick auf politische Phänomene. Theorien, Methoden, Problemstellungen. In: Dies. (Hg.), Politikwissenschaft als Kulturwissenschaft. Theorien - Methoden - Forschungsperspektiven, Wiesbaden, S. 11-29.

Senghaas, Dieter 1995: Die Wirklichkeit der Kulturkämpfe. In: Leviathan, Heft 2, S. 197-212.

Straßenberger, Grit 2016: Linkspopulismus als Gegengift? Zur Kritik der radikal-pluralistischen Demokratietheorie. In: Mittelweg 36, Heft 6, S. 36-55.

Voegelin, Eric, 2004:. In: Peter J. von Opitz (Hg.), Die Neue Wissenschaft der Politik, Eine Einführung, München.

Wagner, Bernd 2008: Neuer Rechtsextremismus und >kulturelle Subversion Forschungsjournal NSB, Heft 4, S. 6-16.

Wodak, Ruth 2015: The Politics of Fear. What Right-Wing Populist Discourses Mean, London u.a. 


\section{IIdentität ‘ in politischer Kultur, Demokratietheorie und der Identitären Bewegung}

Wolfgang Bergem

\section{Einleitung: Das Interesse an Fragen der Identität}

Ein zentraler Strang in dem seit einigen Jahren wieder stärkeren Interesse an politischer Kultur ist ein Bündel von Fragen nach Identität. Diese Fragen zielen nicht nur auf das Ich eines Individuums, sondern auf die Beziehung zwischen dem Eigenen und dem Anderen, auf das Verhältnis zwischen der Gruppe, der man sich zugehörig fühlt, und dem, was man als fremd empfindet. Dieses offenkundig gestiegene Bedürfnis nach Selbstverortung, Selbstverständigung und Selbstvergewisserung speist sich aus verschiedenen subjektiven Erfahrungen von Verunsicherung und Orientierungsverlust, aus Infragestellungen tradierter Orientierungsrahmen und gewohnter Sinngebungen. Dass Identität als etwas zu Rekonstruierendes oder auch neu zu Konfigurierendes empfunden wird, kann als Krisenindikator gedeutet werden, denn Identität tritt generell nur als Problem in Erscheinung, als eine Aufgabe oder als Postulat. Sie ist somit nie »zu einem Problem `geworden ; sie konnte überhaupt nur als Problem existieren, sie war von Geburt an ein >Problem`, wurde als Problem geboren« (Bauman 1997: 134, Hervorh. i. O.). Identität dient heute als Schlüsselbegriff für das Verständnis eines Verlangens nach Integration, Kohärenz und Sinn, einer Sehnsucht geradezu nach Sicherheit, Heimat und Verwurzelung. Wenn Zygmunt Bauman in den 1990er Jahren das »moderne >Problem der Identität « darin erkannt hat, »eine Identität $\mathrm{zu}$ konstruieren und sie fest und stabil zu halten«, und das »postmoderne >Problem der Identität« " vor allem darin, »die Festlegung zu vermeiden und sich die Optionen offenzuhalten« (Bauman 1997: 133, Hervorh. i. O.), kann das Problem der Identität mehr als zwanzig Jahre nach dieser postmodernen Diagnose darin ausgemacht werden, nach aller Vermeidung von Festlegung und Bindung und dem Offenhalten aller Optionen in Identitätsfragen zumindest ein Stück Boden unter den Füßen wiederzugewinnen oder als Restbestand zu erhalten.

Ihre Verbindung mit Sicherheitsbedürfnissen macht Fragen der Identität in Zeiten, die als unsicher und krisenhaft wahrgenommen werden - unabhängig davon, inwieweit sie das faktisch sind -, politisch prekär. Denn diese Fragen beziehen 
sich nicht nur auf ein personales Selbst in der Spannung von sameness und selfhood (in der Konstellation, »the same with it self and no other « [Locke 1987:328] zu sein) und in der Differenz zwischen den mit den beiden Fragen »qui suis-je? « und »que suis-je? « (Ricœur 1990: 147) charakterisierten Formen von Ipse-Identität und IdemIdentität, sondern sie zielen außer auf »Who am I?« auch auf »Where do I belong?« (Lynd 2001: 13). Sie problematisieren also nicht nur, wer und was jemand sei, sondern auch, wohin jemand gehöre, zu welchen Gruppen von Menschen er oder sie Zugehörigkeit empfinde. Die Fragen nach Identität fokussieren nicht allein die intrapersonalen Aspekte eines Individuums, sondern ebenso dessen soziale Realität. Sie beziehen sich damit auf gesellschaftliche Gruppen, auf wie auch immer generationell, geschlechtlich, beruflich, sozio-kulturell, religiös, ethnisch, national, regional oder etwa auch durch Bindungen an Fußballvereine abgrenzbare Kollektive. Indem lieb gewordene Gewissheiten über die Welt der Politik - internationale Bündnisse und supranationale Integration, das Parteiensystem und das Wahlverhalten wie die Modi politischen Entscheidens und der Stil politischer Kommunikation - derzeit zunehmend als flüchtig erlebt werden und kulturelle, zum Beispiel religiöse Verankerungen lose geworden sind, steigt der Bedarf an individueller Sicherung durch Gruppenzugehörigkeiten, die dort, wo sie rar oder dünn geworden sind, nunmehr emphatisch aufgebaut oder mit Verve revitalisiert werden, teilweise auch symbolisch inszeniert und expressiv zelebriert werden.

Fragen nach Identität (vgl. Bergem 2005: 36-133) werden nicht nur vom Einzelnen gestellt und zu beantworten versucht, sondern auch von dessen sozialer Umwelt. Die Selbsterkenntnis eines Individuums erfolgt daher nicht unabhängig von der Zuerkennung bestimmter Eigenschaften und der Anerkennung durch andere. So wie Selbstidentifikation auf Mitteilung, auf Spiegelung und Wahrnehmung durch andere zielt, verarbeitet sie stets auch Fremdzuschreibungen von Merkmalen und ist daher ein sozialer Prozess. Personale Identität ist daher erstens die von Faktoren, die wie Ort und Zeitpunkt der Geburt der optionalen Verfügbarkeit des Einzelnen entzogen sind, gegebene, zweitens die gegenüber dem Gestaltungswillen des Individuums offene, im Verlauf eines Lebens erworbene und stets neu $\mathrm{zu}$ erwerbende und drittens die von anderen Menschen zugeschriebene und damit dem Subjekt potentiell eingeschriebene Identität. Je mehr die gegenwärtig zunehmenden Kontingenzerfahrungen Identität kompensatorisch zum Gegenstand der Reflexion machen, umso weniger wird die Selbstverständlichkeit ihrer Gegebenheit, die anders als in der Vormoderne nicht mehr durch geschlossene Weltbilder, Transzendenzbezug und soziale Milieus bereitgestellt wird, anerkannt. Je mehr jedoch die Individualität von Einzelnen - etwa durch Prozesse kultureller Globalisierung oder durch die Entwicklung der Genforschung, die kurz davor oder auch bereits im Begriff ist, den Menschen über die Schwelle zum Zeitalter seiner technischen Reproduzierbarkeit zu rücken - als von Anonymität bzw. biotechnologischer Verfügbarkeit bedroht wahrgenommen wird, desto stärker fällt das Verlangen aus, 
das Selbst als unverwechselbar zu erleben und Personalität in Identität zu begründen.

Die Beziehung zwischen der ersten und zweiten bzw. je nach Adressatin oder Adressat in der gegebenen Kommunikationssituation zwischen der ersten und dritten Person Singular wie Plural, in der sich personale bzw. kollektive Identität ausprägt, ist ein Verhältnis der Reziprozität von Integration nach innen und Distinktion nach außen. Ihre Ausprägung erfolgt in der wechselseitigen Bezüglichkeit von Orientierungen an Homogenität oder zumindest einer kohärenten Einheit im Inneren und Markierungen von Differenz und Abgrenzung zur Umgebung. Der positiv identifizierenden, in einem Bestand eigener Werte oder Merkmale fundierten Integration steht als Komplement die ex negativo identifizierende Distinktion gegenüber. So wie sich im distinktiven Modus personale Identität durch die Definition der Beziehung zwischen der eigenen Person und anderen Individuen ausbildet, wird kollektive Identität durch die Bestimmung des Verhältnisses zwischen der jeweils zu identifizierenden Eigengruppe und den realen oder erfundenen Kennzeichen einer oder mehrerer Fremdgruppen konstruiert, durch das Benennen von Unterschieden $\mathrm{zu}$ ihnen. Sowohl innere Homogenisierung in Form eines Herausstellens von Übereinstimmung oder Ähnlichkeit zwischen Mitgliedern eines Kollektivs im Rekurs auf Gemeinsamkeiten ethnischer Art in Abstammung, Geschichte oder Religion oder demotischer Art in Willensbekundungen, Wertüberzeugungen oder politischen Zielsetzungen auf der einen Seite als auch Grenzziehungen zu den Mitgliedern anderer Kollektive auf der anderen Seite sind konstitutiv für die Entstehung und den Erhalt kollektiver Identitäten.

Im Unterschied zur Verwendung des Identitätsbegriffs in der Philosophie, die objektive Identität als ontisches Prädikat ins Zentrum stellt (vgl. z.B. Hegel 1991: 123-150), und auch zum Identitätsverständnis der Psychologie, der, wie vor allem bei Erikson (vgl. 2013: 123-212), Ich-Identität als eine in der Adoleszenz zu erringende Entwicklungsstufe des Individuums gilt, bezieht die sozialwissenschaftliche Rezeption des Begriffs der Identität ihren spezifisch sozialwissenschaftlichen Sinn erst dadurch, dass die Vorstellung, ein Individuum habe eine Identität, auf Gruppen oder ganze Gesellschaften übertragen wird. Dadurch, dass von einer vorausgesetzten personalen Identität auf die Existenz einer kollektiven Identität geschlossen wird, bezeichnet der Begriff der kollektiven Identität in doppelter Weise Gemeinsamkeit und Unterscheidung. Er fokussiert, was Individuen mit bestimmten anderen Individuen verbindet und gleichzeitig von anderen Individuen trennt. Die Übertragung der Vorstellung von Übereinstimmung, Gleichheit und Einheit von einem Individuum auf eine Gruppe von Individuen stellt eine Figur uneigentlichen Sprechens dar, eine Metapher. Mit dieser Metaphorisierung wird auch der Doppelcharakter eines individuellen Sich-Identifizierens, das die Feststellung von Gleichheit oder auch nur Ähnlichkeit mit anderen Personen ebenso bedeuten kann 
wie die Feststellung der Unterschiedlichkeit von anderen, und damit die Ambiguität des personalen Identitätsbegriffs auf die Ebene des Kollektivs transportiert.

Im Kontext kollektiver Identitäten bezeichnet der Identitätsbegriff nicht mehr, wie im primordialen Fall personaler Identität, dass eine Person mit sich selbst, mit ihrem Wesen, ihrem Ich oder Selbst oder mit sich selbst über Zeit hinweg identisch ist. Der Begriff hebt hier vielmehr darauf ab, dass eine Person im Bezug auf bestimmte Aspekte mit anderen Personen übereinstimmt oder auch nur Übereinstimmung empfindet und insoweit mit diesen >identisch ist nete Identität einer Gruppe kann auch bei Wechsel der Gruppenmitglieder relativ konstant bleiben, was durch gruppenspezifische Prozesse der Sozialisation und Enkulturation ermöglicht wird. Konkurrierend oder auch komplementär wirksame Kategorien wie geschlechtliche und generationelle, berufliche und soziale, religiöse und weltanschauliche, ethnische, nationale, sub- und supranationale Zugehörigkeiten oder auch Bindungen an politische und soziale Bewegungen und Organisationen stellen dem Einzelnen in der Gegenwart verschiedene, mehr oder weniger wahlweise verfügbare Referenzrahmen für komplexe und situativ variable Prozesse der Identifikation bereit. Damit erzeugen sie einen generellen Mischungs- bzw. Fragmentierungscharakter der Vergemeinschaftungserlebnisse des Subjekts und fördern einen Typus fluider und prozesshafter Identität.

Die in einem Referenzrahmen wie etwa der Nation ausgeformte Identität ist dann nur noch eine Facette einer zunehmend multiplen Identität; sie ist nicht mehr, aber auch nicht weniger als ein Aspekt mit individuell verschiedenem Wirkungspotential auf die Identität einer Person. In deutlichem Unterschied zu fundamentalistischen Auffassungen, die in personaler und kollektiver Identität ein durch Abstammung und Herkunft fest verbürgtes, statisches und unentrinnbares Erbe erkennen, gilt aus westlich-liberaler Sicht die Identität von Individuen und Kollektiven als plurales, dynamisches und entsprechend offenes Gebilde. Inwieweit diese Diversifikation von Gemeinschaftserfahrungen in den gegenwärtigen Gesellschaften des Westens nun als produktive Mischung und willkommene Chance gesellschaftlicher und kultureller Entwicklung begrüßt oder als Verlust von Mitte und Einheit und schädliche Fragmentierung beklagt und politisch befehdet wird, ist eine Frage der politischen Kultur.

\section{IIdentität in Konzepten politischer Kultur}

In ihrer ersten systematischen Konzeptualisierung durch Gabriel A. Almond und Sidney Verba (1963) behandelte die politische Kulturforschung Aspekte kollektiver Identität nur am Rande. In der Civic-Culture-Studie vom Anfang der 1960er Jahre "a bold and incautious book«, wie Verba in einer späteren Retrospektive auf dieses Alte Testament der politischen Kulturforschung zutreffend urteilte, wobei er 
auch klar machte, dass wenn dieses Buch vorsichtiger, weniger »foolhardy", gewesen wäre, »there would be [...] less to criticize, but also less to remember that was worth criticizing (1980: 394f. und 409) - standen Orientierungen gegenüber politischen Objekten im Zentrum: »The political culture of a nation is the particular distribution of patterns of orientation toward political objects among the members of the nation « (Almond/Verba 1963: 14f.). Es ging vor allem um die verstandesmäßigen, emotionalen und wertenden Haltungen gegenüber dem politischen System in seiner Ausdifferenzierung in das System als Ganzes, seine Input- und seine Output-Strukturen sowie die Rolle der eigenen Person im politischen Prozess (vgl. ebd.: 15-17).

Die jeweils rund 1.000 Befragten in den fünf Untersuchungsländern USA, Großbritannien, Bundesrepublik Deutschland, Italien und Mexiko wurden aber auch gefragt, auf was sie in ihrem Land am meisten stolz seien. Bei dieser Frage, die in Richtung kollektiver Identifikation weist, führten die interviewten Westdeutschen der Ära Adenauer nicht wie die US-Amerikaner und die Briten die politischen Institutionen an (die in deren politischen Systemen in lange währenden Traditionen von Verfassung, Rechtsstaat und Parlamentarismus verankert sind), sondern nannten am häufigsten die Charaktereigenschaften der Bevölkerung und das ökonomische System der Bundesrepublik (in dessen Rahmen die Westdeutschen einer verbreiteten Sicht zufolge Nachkriegsboom und Wirtschaftswunder erzielen konnten) (vgl. ebd.: 102). Die Akzeptanz des politischen Systems war demnach vor allem durch wirtschaftlichen Erfolg begründet; dieser Pragmatismus einer Gesellschaft, die nur wenige Jahre nach dem Ende der NS-Diktatur noch skeptisch gegenüber allen hochgesteckten Erwartungen an die Politik gestimmt war, erinnert an die Zeit nach 1848, in der die Enttäuschung über das Scheitern der im Vormärz politisch artikulierten liberalen, nationalen und demokratischen Hoffnungen für einen Großteil des Bürgertums den Anlass darstellte, sich von der Politik ab- und stärker der Wirtschaft zuzuwenden, zumal die bereits vor der 48er Revolution beschleunigte Industrialisierung das Interesse an ökonomischer Leistung begünstigte (vgl. Bergem 1993: 78-80).

Die Frage nach dem Verhältnis gegenüber dem eigenen Staat benennt ein $\mathrm{Ob}-$ jekt politischer Identifikation und gibt daher Auskunft über politische Identität. Die Orientierung der Bundesbürger gegenüber dem neuen demokratischen System wurde in der Civic-Culture-Studie mit den Begriffen "political detachment« und »subject competence « als distanziert und sehr pragmatisch, als fast zynisch beschrieben (Almond/Verba 1963: 428-439; vgl. auch Verba 1965b: 140-146, 153 und 162). Das in Umfragen ermittelte Bekenntnis der Westdeutschen zur Demokratie wurde von der amerikanischen politischen Kulturforschung noch bis in die 1960er Jahre hinein als »lip service« (Verba 1965b: 137) interpretiert. Dieser äußerlich bleibende und als übertrieben eingeschätzte Pragmatismus gab den Forschern zu Misstrauen Anlass, da er die innerliche Bindung an das politische System vermissen lasse, die 
eine stabile Demokratie benötige. Die zurückgenommene, nüchterne, teilweise zynische Haltung der Westdeutschen der Nachkriegszeit gegenüber allem Politischen war zum einen Ausdruck einer noch erkennbaren Kontinuität von autoritären, unpolitischen, etatistischen und eskapistischen Traditionen politischer Kultur (vgl. Bergem 2013: 121-125), zum anderen ist sie vor dem Hintergrund der Erfahrung der Überpolitisierung in der nationalsozialistischen Diktatur zu erklären. Darüber hinaus kann mit Klaus von Beyme gefragt werden, »ob die deutschen Zyniker nicht weitgehend bloß einen in der ganzen westlichen Welt vorherrschenden Zynismus in bezug auf die nationale Identität Deutschlands nachvollzogen«, denn die von dem deutschen Politikwissenschaftler wahrgenommene

»Inkonsequenz der Haltung der westlichen Nationen, die Teilung von Nationen für ein Unrecht zu halten, sie gleichzeitig aber aus Gründen der Sicherheit und Bequemlichkeit [...] in Deutschland verhältnismäßig positiv zu bewerten, [könne] bei den Betroffenen eigentlich nur Zynismus und Apathie in dieser Frage hervorrufen« (von Beyme 1987: 8of.).

Insgesamt stehen Einstellungen im Zentrum der von Almond und Verba begründeten Forschungstradition zur politischen Kultur und nicht Vorstellungen, auf die Karl Rohe mit der analytischen Unterscheidung von politischer Sozialkultur und politischer Deutungskultur den Akzent verlagern hat (1987: 41-44; 1990: 340f.). Vorstellungen sind auf einer tieferen Ebene politischer Kultur angesiedelt und liegen näher bei Fragen der Identität als Einstellungen. Die gesellschaftlich verankerten Vorstellungsmuster der politischen Soziokultur ergeben sich demnach aus überlieferter Tradition, eigenen Erfahrungen sowie den wahrgenommenen Sinn- und Deutungsangeboten und bilden in ihrer spezifischen Selektivität den Rahmen für politisches Handeln. Die politische Deutungskultur hingegen umfasst die politischen Ideen und Interpretationsangebote, die als das "politische Design" eines Herrschaftssystems affektive Bindungen herstellen. Funktional differenziert Rohe zwischen politischer Kultur als politischer Lebensweise, in der die fundamentalen politischen Vorstellungen gleichsam als ungeschriebene Verfassung einer Gesellschaft die politischen Beziehungen konditionieren, und politischer Kultur als politischem Weltbild, in dem Bilder, Theorien und Ideologien von politischer Organisation entworfen werden (1987: 44-46). In Rohes Verständnis von politischer Kultur als politischem »Sinn, der auch sinnenfällig werden muß« (1990: 337), wird symbolische Politik nicht in pauschaler Ideologiekritik als bloße Ersatzpolitik abgetan; vielmehr erhält sie als affektive und ästhetische Ausdrucksdimension politischer Kultur einen ähnlichen konstitutiven Stellenwert wie deren kognitive und normative Inhaltsdimension.

Es war vor allem die kulturalistische Kritik am Konzept der politischen Kultur, die mit diesem Begriff auch Aspekte kollektiver Identität einschloss. Auf Grundlage einer Kritik an einem verkürzten Kulturbegriff und in einer auch historischen Di- 
mensionierung des Konzeptes sollte politische Kultur nicht mehr bloß eine spezifische Aggregation von Einstellungen der Bürger gegenüber dem politischen System erfassen, sondern auch Sprache, Symbole, Erinnerung, Rituale, Mythen und andere Narrationen oder auch Architektur und weitere visuelle Repräsentationen als politisch-kulturelle Manifestationen ins Auge fassen (vgl. etwa Dörner 2003: 593-605; Greiffenhagen 2009: 20-27; Pickel/Pickel 2006: 106-111 und 123-132; Schuppert 2008: 57-80 und 589-655; Schwelling 2001). Methodisch trennen sich die Wege der politischen Kulturforschung damit in den etablierten empirisch-analytischen und einen mittlerweile breit ausgebauten hermeneutisch-interpretativen. Auch als Untersuchungsobjekte dieses vergrößerten methodischen Instrumentariums konnten Formationen kollektiver Identität $\mathrm{zu}$ einer relevanten Dimension politischer Kultur werden. Das bezog und bezieht sich keineswegs bloß auf nationale Identität: In Deutschland beispielsweise wird die im 19. Jahrhundert dominant gewordene nationale Identifikation bei aller ihr eigenen Persistenz heute ausbalanciert und teilweise auch kontrapunktiert von postnationalen Orientierungen an universalistischen Prinzipien, partialnationalen Selbstdefinitionen in West- oder Ostdeutschland, subnationalen Beheimatungen in der Region, binationalen Hybridbildungen von Identität mit Bindungen an die Herkunftsländer von Migranten sowie von supranationalem Zugehörigkeitsempfinden zu Europa oder zur Europäischen Union.

In David Eastons systemtheoretischem Konzept politischer Unterstützung gilt »diffuse support for the political community« im Sinne einer Identifikation mit der politischen Gemeinschaft, die neben den personalen Mitgliedern eines politischen Systems auch deren grundlegende Wertemuster und einen darauf gegründeten Gemeinschaftssinn umfasst, noch vor der bedingungslosen Unterstützung des Regierungssystems und der politischen Herrschaftsträger als wichtige Quelle für Legitimität und eine auch bei Systemstress fortbestehende Stabilität eines politischen Systems (vgl. 1965: 247-340). Mit seiner Differenzierung einerseits der Objekte politischer Unterstützung in die politische Gemeinschaft, die politische Ordnung und die Herrschaftsträger einschließlich ihrer politischen Outputs und andererseits der Typen politischer Unterstützung in eine spezifische, auf instrumentelle Abwägungen individueller Vor- und Nachteile beruhende, kurzfristige und potentiell volatile Unterstützung und eine so generalisierte wie diffuse, in bestimmten Werten oder auch affektiv verankerte und dauerhaft stabile Unterstützung hat Easton nicht nur der politische Kulturforschung eine stärkere Systematisierung bereitgestellt, sondern auch deren Perspektive auf die politische Identifikation der Bürgerinnen und Bürger gerichtet.

Die heute in der politischen Kulturforschung gängige Verwendung des Identitätsbegriffs zur Kennzeichnung eines offen bleibenden Resultats von Selbstund Fremdzuschreibungen von Eigenschaften verschieden abgrenzbarer Großgruppen, das sich als Ergebnis sozialer Interaktion und Kommunikation aus den reziproken Prozessen der Integration einer Gruppe und ihrer Abgrenzung 
gegen andere herstellt, geht auf eine begriffliche und konzeptuelle Anleihe aus der Sozialpsychologie zurück. Diese sozialpsychologisch orientierte Bedeutung des Begriffs unterscheidet zwischen den beiden Dimensionen personaler und kollektiver Identität und zielt auf das Selbstverständnis eines Individuums, einer Gruppe oder einer Gesellschaft, dessen Stabilität und Kontinuität auf Integration, Kohäsion und Konsens basieren, dessen Dynamik und Pluralität hingegen von Konflikten, Widersprüchen und Dissens motiviert werden. Diese Identifikationen beziehen sich auf die Vergangenheit in Form eines kollektiven Gedächtnisses, das die Erinnerungen an gemeinsam erlebte oder auch erlittene Ereignisse rekonstruiert und tradiert, auf die Gegenwart in Form eines Orientierungsrahmens bei der aktuellen Wahrnehmung und Ordnung von Informationen zur Herstellung von Sinn sowie auf die Zukunft in Form antizipierender Projektionen möglicher Entwicklungen. Während der weiter gefasste Begriff der kulturellen Identität die symbolisch codierten, inszenierten und ritualisierten Repräsentationen sozialer Inklusion und Exklusion bezeichnet, ist der engere Terminus der politischen Identität an der Legitimation von Herrschaft orientiert und damit auf die Unterstützungsbereitschaft von Menschen gegenüber ihrem politischen System gerichtet. "Participation tends to produce responsibility« (Verba 1965a: 557): Die mit politischer Identität verknüpfte Akzeptanz eines politischen Systems und das entwickelte Gefühl von Verantwortlichkeit für das Gemeinwesen werden vermittelt über die Chancen zu politischer Beteiligung und Einflussnahme der Bürgerinnen und Bürger - in Demokratien.

\section{IIdentitätı in der Demokratietheorie}

So wie Identität zum einen als gesellschaftlich verankertes und kulturell tradiertes Merkmal eines Kollektivs zu den Stabilitäts- und Persistenzbedingungen politischer Systeme gehört und zum anderen als Gegenstand einer bestimmten policy, der Identitätspolitik, auf das Verhältnis zwischen Integration und Diskriminierung von Minderheiten zielt, so fokussiert Identität schließlich als Prädikat der Demokratietheorie die Frage nach politischer Performanz im Blick auf die Realisierung des Prinzips der Volkssouveränität. Die Demokratietheorie kennt den Begriff der Identität im Zusammenhang der identitären Demokratie. Dieses (einzig) genuin politikwissenschaftliche Verständnis von Identität ist in der politischen Ideengeschichte der Demokratie verwurzelt und bezeichnet die Gleichheit oder Übereinstimmung von Herrschern und Beherrschten zur Gewährleistung direkt ausgeübter Volkssouveränität.

Zwei politische Denker vor allem, ein Utopist und ein Staatsrechtslehrer, stehen als Paten für die identitäre Demokratielehre: Jean-Jacques Rousseau und Carl Schmitt. In Rousseaus Contrat social von 1762 gilt die Identität zwischen Regieren- 
den und Regierten als Hauptmerkmal einer guten politischen Ordnung. Die von ihm unterstellte Identität zwischen natürlicher Sittlichkeit und praktischer Vernunft bedingt die Identität zwischen dem Willen des Einzelnen und dem der Gemeinschaft, damit die Identität zwischen Volk und Gesetzgeber sowie die zwischen Gesellschaft und Staat, wobei diese Identitäten bei ihm in der Idee der absoluten Volkssouveränität inkarniert, in der Konstruktion des Gemeinwillens konkretisiert und im Denkbild des politischen Körpers metaphorisiert werden. Wenn die gesamte Bürgerschaft zur Durchsetzung eines apriorischen Gemeinwillens die legislative Gewalt unmittelbar und die exekutiven Kompetenzen im Sinne administrativer Funktionen durch einen eng an die Ausführung der Gesetze gebundenen Verwaltungskörper ausübe, so Rousseau im Gesellschaftsvertrag, seien Sicherheit und Freiheit gleichermaßen gewährleistet (vgl. 1981: 288-290 und 349 und 352). Als Voraussetzung der volonté générale dürfe die unveräußerliche und unteilbare Souveränität des Volkes auf keinen Fall, wie in der Idee politischer Repräsentation vorgesehen, für die Gesetzgebung delegiert und von Mandatsträgern vergegenwärtigt werden.

In seiner demokratietheoretischen Bedeutung taucht der Begriff Identität bei Rousseaus nicht auf; im Contrat social ist im Kapitel über die Zivilreligion nur von »'identité des Dieux« die Rede (Rousseau 1964: 460). Das heißt aber nicht - wie Lutz Niethammer meint (vgl. 2000: 85 und 92) -, dass die zu Rousseau führende Spur der identitären Demokratietheorie erst später von Carl Schmitt gelegt worden sei, obwohl Rousseau eigentlich hierfür nicht in Anspruch genommen werden dürfe. Unbestreitbar entwickelt Carl Schmitt auf der Suche nach einem attraktiven Fahnenwort seinen Identitätsbegriff, mit dem er in diesem Zusammenhang auf Homogenität und Einheit des Volkes zielt, im expliziten Bezug auf Rousseau. In seiner Parlamentarismus-Schrift von 1923 weist er die Ausführungen Rousseaus im Contrat social als »für das demokratische Denken fundamental« sowie »einer alten Tradition« entsprechend aus; er postuliert als »Kern des demokratischen Prinzips [...] die Behauptung einer Identität von Gesetz und Volkswillen« und führt zur Definition der Demokratie »eine Reihe von Identitäten« an:

»In diese Reihe gehören: Identität von Regierenden und Regierten, Herrscher und Beherrschten, Identität von Subjekt und Objekt staatlicher Autorität, Identität des Volkes mit seiner Repräsentation im Parlament, Identität von Staat und jeweilig abstimmendem Volk, Identität von Staat und Cesetz, letztlich Identität des Quantitativen (ziffernmäßige Mehrheit oder Einstimmigkeit) mit dem Qualitativen (Richtigkeit des Gesetzes). Alle solchen Identitäten sind aber nicht handgreifliche Wirklichkeit, sondern beruhen auf einer Anerkennung der Identität« (Schmitt 1969: 34-36).

In seiner Verfassungslehre von 1928 wird Schmitt noch deutlicher, indem er Demokratie explizit als »Identität von Herrscher und Beherrschten, Regierenden und Re- 
gierten, Befehlenden und Gehorchenden« definiert, in »der substanziellen Gleichheit [...] die wesentliche Voraussetzung der Demokratie« ausmacht und postuliert:

»In der reinen Demokratie gibt es nur die Identität des wirklich anwesenden Volkes mit sich selbst, also keine Repräsentation. Mit dem Wort ।Identität « ist das Existenzielle der politischen Einheit des Volkes bezeichnet zum Unterschied von irgendwelchen normativen, schematischen oder fiktiven Cleichheiten. Demokratie setzt im Ganzen und in jeder Einzelheit ihrer politischen Existenz ein in sich gleichartiges Volk voraus, das den Willen zur politischen Existenz hat« (Schmitt 1965: 234f.)

Bei Rousseau kommt das so explizit nicht vor. Er kennt weder die existentialistische Fundierung noch den völkischen Einschlag dieses gemeinschaftlich-identitären Demokratiebegriffs. Dennoch ist nicht zu übersehen, dass Rousseau, ohne das im 18. Jahrhundert erst wenig übliche Wort l'identité zu verwenden, in seiner Theorie der Volkssouveränität mit der Vorstellung von Übereinstimmung und Gleichheit oder auch Wesenseinheit argumentiert, so dass er dabei implizit eine Identitätslehre der Demokratie entwickelt. In seinem 1755 erschienen Discours sur l'origine et les fondemens de l'inégalité parmi les hommes beschreibt Rousseau ein Land, in dem er gerne geboren worden wäre; in diesem Idealstaat haben der Souverän und das Volk »un seul et même intérêt«; da diese Interessenidentität nicht möglich sei außer wenn Volk und Souverän »une même personne«, also personell identisch, seien -, wünscht sich Rousseau, als realistischere Wahl also, unter einem "gouvernement démocratique, sagement tempéré« geboren worden zu sein (1964: 112). Rousseaus Favorisierung einer durch Weisheit gemäßigten demokratischen Regierung bei seiner fiktiven, aber die Bedingungen der Wirklichkeit anerkennenden Wahl des eigenen Geburtsortes erschwert es, der These von einer »reservierte[n] Einstellung zur Demokratie« bei Rousseau (Schmidt 2010: 91) oder der von einem »demokratieskeptischen Rousseau« (Niethammer 2000: 94) zu folgen.

Im Contrat social identifiziert Rousseau die Herrschenden mit den Beherrschten dadurch, dass er im Gesellschaftsvertrag, der eine »wechselseitige Verpflichtung von Öffentlichkeit und Einzelwesen" enthalte, jeden Einzelnen, »indem er gewissermaßen einen Vertrag mit sich selbst schließt, [als] auf doppelte Weise verpflichtet [ansieht], nämlich als Glied des Souveräns gegenüber den einzelnen und als Glied des Staates gegenüber dem Souverän« (1981: 282). Das demokratietheoretische Komplement zur Identität, die Repräsentation, lehnt Rousseau in dieser Abhandlung nachdrücklich ab:

»Die Souveränität kann nicht repräsentiert werden, und zwar aus demselben Grund, aus dem sie nicht veräußert werden kann; sie besteht wesentlich im Cemeinwillen, und der Willen läßt sich mitnichten vertreten: er ist er selbst, oder aber er ist ein anderer; einen Mittelweg gibt es nicht. Die Abgeordneten 
des Volkes sind also nicht seine Repräsentanten, noch können sie dies sein; sie sind nur seine Beauftragten und können nichts endgültig beschließen. Jedes Cesetz, welches das Volk nicht selbst bestätigt hat, ist nichtig; es ist kein Cesetz. ... [S]obald ein Volk sich Repräsentanten gibt, ist es nicht mehr frei; es ist nicht mehr« (1981: 350 und 352).

Demnach kann keine Rede davon sein, dass bei Rousseau die Identität bzw. die Identifizierung der Regierenden mit den Regierten als Vorstellung nicht vorkomme. Damit ist auch die Ansicht, Carl Schmitt habe zur Aufwertung seines Leitbegriffs Identität Rousseau eine demokratietheoretische Identitätslehre untergeschoben, die der Schweizer Wahlfranzose gar nicht vertreten habe, nicht zu vertreten und Niethammers (2000: 120) Präsentation des Verständnisses von Demokratie als Identität von Regierenden und Regierten als einer Erfindung von Carl Schmitt nicht haltbar. So wenig wie Niethammers Annahme einer identitätstheoretischen Usurpation Rousseaus durch Schmitt zutrifft, wird jedoch auch Ernst Fraenkels simplifizierende Identifikation in seinen Ausführungen über »vulgärdemokratisches Denken«, Schmitts Anti-Pluralismus sei »echter Jean Jacques Rousseau « (sic, Fraenkel 1991: 307), dem irisierenden CEuvre des vielseitigen Autodidakten und über die Aufklärung hinausweisenden staatsphilosophischen Aufklärers gerecht.

Zwar erkennen sowohl Rousseau als auch Carl Schmitt das Wesen der Demokratie in der Identität zwischen Regierenden und Regierten; jedoch zeigt sich im Zustandekommen dieser Identität der entscheidende Unterschied zwischen den identitären Demokratietheorien der beiden Denker: Während die Identität zwischen Herrschenden und Beherrschten bei Rousseau endogen entsteht, insoweit sie in der Übereinstimmung zwischen dem Willen des Einzelnen und dem Willen der Gemeinschaft fundiert ist, wird die demokratische Identität bei Schmitt exogen generiert. Sie stellt sich erst mit einer Homogenität des Volkes ein, die in erster Linie auf der Außenabgrenzung gegenüber einem gemeinsamen Feind beruht (vgl. Schmitt 1963: 27): Zur »wirkliche[n] Demokratie«, die im Gegensatz zu Parlamentarismus und Liberalismus stünde und keineswegs im Gegensatz zur Diktatur, gehört laut Schmitt »notwendig erstens Homogenität und zweitens - nötigenfalls - die Ausscheidung oder Vernichtung des Heterogenen « (1969: 13f. und 41). Nicht politische und rechtliche Gleichheit, sondern »Artgleichheit « (Schmitt 1933: 42) ist im »anthropologischen « und "politischen Pessimismus (Klünder 2017: 109 und 90) des Carl Schmitt die Bedingung für Demokratie. Rousseaus identitäre Demokratielehre betont Integration, Schmitts Vorstellung von Demokratie zielt hingegen vor allem auf Distinktion.

Die von Rousseau begründete demokratietheoretische Bedeutung des Identitätsbegriffs als Gleichheit oder Übereinstimmung zwischen Regierenden und Regierten prägt die Verwendung des Terminus mit und stellt für die Politikwissen- 
schaft nicht bloß einen von der heute dominierenden sozialpsychologischen Bedeutung als >kollektive Identität abgesonderten semantischen Nebenaspekt dar. Was auf den ersten Blick als bestenfalls geistesgeschichtlich interessant erscheinen mag, führt vielmehr ins Zentrum der politologischen Dimension von Identität, in der das Verhältnis zwischen zoon politikon und polis zu erkunden ist, in der zur Analyse ansteht, wie Interessen, Konflikt und Konsens, Herrschaft und Macht von Prozessen der Identitätsbildung beeinflusst werden und diese beeinflussen. Die Verknüpfung von Identität mit Konflikt und Macht wird nicht zuletzt in einer aktuell virulenten Verwendung des Begriffs der Identität erkennbar: Carl Schmitts demokratietheoretische Homogenitätsvorstellung grundiert das Identitätsverständnis der sogenannten >Identitären .

\section{ıIdentitätı in der Identitären Bewegung}

Mit einem besonderen Akzent konnte das demokratietheoretische Prädikat der Identität zum zentralen Referenzpunkt in einem aktuellen Phänomen in real existierenden Demokratien werden: Im Folgenden ist die Rede von der Begriffsverwendung bei den Identitären, in der Identitären Generation oder auch der Identitären Bewegung. Diese völkisch und ethnopluralistisch orientierte Bewegung entstand nicht zufällig - in Frankreich. Dort hatte die Nouvelle Droite mit ihrem Wortführer Alain de Benoist und dem Thinktank Groupement de Recherche et d'Études pour la Civilisation Européenne (GRECE), den de Benoist mit Dominique Venner und weiteren Aktivisten aus dem Milieu nationalistischer und neofaschistischer Studentenorganisationen 1968 gegründet hatte, dem rechtsextremen Spektrum eine intellektuelle Fundierung mit theoretischen Anleihen bei den Autoren der Konservativen Revolution der Weimarer Republik bereitgestellt, als sich nach der Jahrtausendwende die Identitären formierten.

Nachdem in der Folge eines gescheiterten Mordanschlags auf Präsident Jacques Chirac auf den Champs-Élysées am 14. Juli 2002 die rechtsextreme Gruppierung Unité Radicale verboten worden war, gründeten Fabrice Robert und Philippe Vardon mit anderen 2003 den regional und europäisch orientierten Bloc Identitaire mit der anfangs als dessen Jugendorganisation firmierenden Formation Jeunesse Identitaire, die ab 2005 als eigenständige Gruppierung aufgetreten ist, bevor sich 2012 die Génération Identitaire als Nachfolgerin etablierte. Damit kann sich der seit 2009 auch als politische Partei - in Konkurrenz zum damaligen Front National - zu Wahlen antretende Bloc Identitaire der politischen und strafrechtlichen Verantwortung für die von der Génération Identitaire verübten Gesetzesverstöße wie die Besetzung des Daches einer Moschee oder das Eindringen in Büros des Parti Socialiste entziehen. Nachdem sich die Génération Identitaire 2012 einer größeren Öffentlichkeit bekannt gemacht hatte, indem 30-40 ihrer Aktivisten das Dach der sich im Bau 
befindlichen Moschee von Poitiers besetzt und Banner mit der Jahreszahl 732 und dem Lambda-Symbol entrollt hatten, wurde sie zum Vorbild für die Gründung anderer identitärer Gruppen in Europa. Der in schwarz-gelber Farbgebung präsentierte griechische Buchstabe Lambda wurde fortan zum gemeinsam verwendeten Logo aller identitären Gruppierungen; entlehnt wurde dieses Symbol einer blutrünstigen Hollywood-Produktion aus dem Jahr 2006, der Comic-Verfilmung 300, die den Kampf der Spartaner und ihrer griechischen Bündnispartner gegen die übermächtigen Perser in der Schlacht bei den Thermopylen im frühen 5. Jahrhundert v. Chr. thematisiert. Ganz unabhängig von den historischen Realitäten gelten zum Teil bereits in dem Film und vor allem in der Rezeption der Identitären die Spartaner als Vertreter der europäischen Zivilisation und Demokratie gegenüber den nicht-europäischen barbarischen Horden der persischen Eindringlinge. Mit der Jahreszahl 732 wurde bei der Moscheedach-Besetzung auf die Schlacht von Poitiers und Tours verwiesen, in der die Franken unter Führung von Karl Martell die aus Spanien vorrückenden muslimischen Araber militärisch geschlagen haben (vgl. Bruns/Glösel/Strobl 2016: 72-83; Camus 2017: 233-241; Speit 2018c: 42-51). Mit dieser unter Identitären als Startschuss für eine neue Bewegung gefeierten Aktion konnte zum einen auf der ideologischen Ebene die islamfeindliche Haltung der Identitären in Szene gesetzt werden, zum anderen konnte auf der performativen Ebene ein bislang der politischen Linken zugeschriebener aktivistischer Habitus mit rechtsextremen Inhalten verknüpft werden.

Eine breite Aufmerksamkeit durch eine in den Sozialen Netzwerken und auf einschlägigen Internetseiten virale Verbreitung erzielte 2012 auch das Video Déclaration de guerre auf der Website der Génération Identitaire. Die Aussagen in dem zweieinhalbminütigen Clip, die von Jugendlichen in schwarz-weißen Nahaufnahmen und von pathetischer Musik untermalt präsentiert werden, vermischen Klagen über soziale Missstände und eine Dominanz von 68ern mit ethnopluralistischen und rassistischen Ideologemen; in diesen Aussagen heißt es unter anderem:

»Wir sind die Ceneration der ethnischen Spaltung, des totalen Scheiterns des Zusammenlebens und der erzwungenen Mischung der Rassen. Wir sind die doppelt bestrafte Ceneration: Dazu verdammt in ein Sozialsystem einzuzahlen, das so großzügig zu Fremden ist, daß es für die eigenen Leute nicht mehr reicht. Unsere Ceneration ist das Opfer der 68er, die sich selbst befreien wollten von Tradition, von Wissen und authoritärer [sic!] Erziehung. Aber sie haben es nur geschafft, sich von ihrer Verantwortung zu befreien. [...] Wir glauben nicht mehr, daß >Khader unser Bruder sein kann, wir haben aufgehört an ein >Clobales Dorf< und die >Familie aller Menschen zu glauben. [...] Unser Erbe ist unser Land, unser Blut, unsere Identität. [...] Wir erleben 25 \% Arbeitslosigkeit, Sozialschuld, Kollaps von Multikulti und eine Explosion des gegen Weiße gerichteten Rassismus. [...] Claubt 
nicht dies ist nur ein Manifest. Es ist eine Kriegserklärung. Ihr seid von gestern, wir sind von morgen. Wir sind Ceneration Identitaire. « ${ }^{1}$

Nach den Impulsen von Poitiers und des Videoclips der Génération Identitaire gründeten sich im Herbst 2012 die ersten identitären Gruppierungen in Deutschland; weitere Ableger der französischen Identitären entstanden in Österreich, Italien, der Schweiz, Schweden, Norwegen, Dänemark, Tschechien und Ungarn. Die Gruppen inszenieren sich nationsübergreifend als neue europäische Jugendbewegung, als metapolitische Kraft und als hippe Gegenkultur mit dem Ziel einer »Kulturrevolution von rechts« (de Benoist 1985b), wie in dem französischen Videoclip angeklungen als Gegenrevolte zu 1968 und in jedem Fall als Avantgarde eines neuen Zeitgeists (vgl. auch den Beitrag von Paula Diehl in diesem Band). Teilweise üben die Identitären eine Scharnierfunktion zwischen völkischem Rechtsextremismus und demokratischem Konservatismus oder auch zwischen verschiedenen Milieus der neurechten Szene aus; in Deutschland tauchen ihre Fahnen gelegentlich bei AfD- und Pegida-Demonstrationen auf. Sie verknüpfen ihre Aktionen online und offline, haben sich in den Sozialen Medien und bei öffentlichkeitswirksamen Aktionen wie Flashmobs, Tanz-, Masken- und Hardbass-Kurzauftritten in der Szene einen Namen als Kommunikations- und Spaßguerilla gemacht. So störte im Oktober 2012 eine kleine Gruppe von Aktivistinnen und Aktivisten die Eröffnung der »Interkulturellen Woche« in der Frankfurter Stadtbibliothek durch einen kurzen Auftritt mit Guy Fawkes- und Scream-Masken, begleitet von Hardbass-Musik und Schildern mit dem Lambda-Zeichen, dem Kürzel »IBD« für Identitäre Bewegung Deutschland oder auch dem Spruch »Multikulti wegbassen« (vgl. Bruns/Glösel/Strobl 2016:83). Ähnlich wie in Poitiers war eine Aktion in Berlin im Sommer 2016 eine Adaption der politischen Aktionsformen der Sozialen Bewegungen, als es einer kleinen Gruppe von Identitären gelungen war, über dem Brandenburger Tor das gelb-schwarze Lambda-Zeichen der Identitären Bewegung wehen zu lassen und an dem symbolträchtigen Bauwerk ein Transparent mit der Aufschrift »Sichere Grenzen - sichere Zukunft« anzubringen (vgl. Speit 2018d: 19). In einer bundesweit koordinierten Aktion haben Mitglieder der Identitären Bewegung Mitte Januar 2019 Gebäude von Parteien und Redaktionen mit Plakaten mit der Frage »Wann problematisieren Sie linke Gewalt? « und dem Lambda-Zeichen beklebt sowie Pflastersteine und Kanthölzer als Symbolisierungen dieser Gewalt vor ihnen abgelegt. Im Ziel standen die Berliner Parteizentrale der SPD sowie Büros der Grünen und Linken in Bayern und Niedersachsen ebenso wie das ARD-Hauptstadtstudio, das Redaktionsgebäude der taz, die Spiegel-Zentrale in Hamburg und das Bürogebäude

1 URL: https://www.youtube.com/watch?v=TUyTyg6XnsA; Übersetzung der deutschen Untertitelung. 
der Frankfurter Rundschau in Frankfurt a.M. Auf ihrer Website benannte die Identitäre Bewegung als Ziel der Aktion: »Die Schreibtischtäter benennen - Protest gegen Linke Gewalt«. ${ }^{2}$

Der mit dem Zeichen der Spartaner, die den Buchstaben Lambda für Lakedaimonier, ein Synonym für Spartaner, auf ihre Kriegsschilder gemalt hatten, hergestellte Bezug auf den Kampf der Griechen gegen die Invasion des Persischen Reichs verweist auf eine zentrale Programmatik der Identitären: die kulturrassistisch begründete Fremdenfeindlichkeit, das Schüren von Angst vor Überfremdung mit der zentralen Denkfigur der angeblichen Gefahr eines »Großen Austauschs« durch »ungebremste Masseneinwanderung und die daraus resultierende Islamisierung «. ${ }^{3}$ Gegenwärtig werde, so die Wahrnehmung der Identitären, die europäische Bevölkerung durch Migration gegen eine nichteuropäische ausgetauscht, die vor allem aus Kriminellen und Sozialleistungsbetrügern bestehe und gegen die das Volk Widerstand leisten müsse. Mit dem Konzept des Ethnopluralismus vertritt die Identitäre Bewegung einen kulturellen Rassismus, der nicht mehr biologisch-genetisch, sondern kulturalistisch argumentiert. Die Fremdenfeindlichkeit der Identitären ist zum größten Teil weniger nationalistisch - wie bei der NPD, dem Rassemblement National und den meisten Parteien und Gruppierungen im gegenwärtigen Spektrum eines »autoritären Nationalradikalismus« (Heitmeyer 2018: 231-276 und 323-373) - als vielmehr regionalistisch und/oder europäisch begründet, zum Beispiel in der regionalistischen, proeuropäischen und identitären Partei Alsace d'abord! ${ }^{4}$ Für die Einstellungen zu Migration und die Integration der Zugewanderten ist der ausschließende Charakter rechtspopulistischer »Grenzpolitik« (Wodak 2016: 54) entscheidend und hingegen zweitrangig, ob die Fremdenfeindlichkeit nationalistisch, regionalistisch oder mit europäischen Bezügen begründet wird.

In der Tradition der in Deutschland aus der Konservativen Revolution bekannten Kulturkampfvorstellungen zielt die Identitäre Bewegung darauf, den Begriff des Volkes ethnokulturell und völkisch sowie den Begriff der Identität kulturalistisch zu besetzen. Die Identitäre Bewegung Deutschland plädiert auf einem ihrer Plakate, in Abgrenzung vom rechten wie vom linken Lager bzw. in ihrer Diktion in Abgrenzung von »Nazis« und von »Multikultis«, nicht nur für »die ethnokulturelle Identität, als dynamische Synthese aus ethnischem und kulturellem Erbe«, sondern auch für »die direkte Demokratie, Meinungsvielfalt, und die Freiheit des Einzelnen im Rahmen seiner Gemeinschaft« (zit.n. Hentges/Kökgiran/Nottbohm

2 URL: https://www.identitaere-bewegung.de/presse/die-schreibtischtaeter-benennen-protestgegen-linke-gewalt/.

3 URL: https://www.identitaere-bewegung.de/kampagnen/grosser-austausch/.

4 URL: www.alsacedabord.org. 
2014: 18). Der aus der Konservativen Revolution bekannten Verachtung der liberalen und parlamentarischen Demokratie entspricht die Forderung nach identitärer und direkter Demokratie, um einem `wahren Volkswillen $>$ zum Ausdruck zu verhelfen. Man könnte diese spezifische Forderung nach Demokratie als instrumentelles Demokratiebekenntnis abtun - so wie die Identitären in Deutschland behaupten, weder rechts noch links, sondern identitär zu sein, und im Titel einer programmatischen Schrift für sich postulieren: »100\% Identität - 0 \% Rassismus« (zit.n. Speit 2018b: 71). Die Frage, ob dieses Bekenntnis zur direkten Demokratie instrumentell-extrinsisch oder doch aus der Sache selbst, intrinsisch begründet ist, kann mit einem Blick in die Schriften der Vordenker der Identitären Bewegung geklärt werden. Man muss gar nicht auf Guillaume Faye als Stichwortgeber der extremen Rechten und des Bloc identitaire in Frankreich hinweisen, der lange Zeit als Mitstreiter von Alain de Benoist in der Nouvelle Droite gewirkt hat, bis es 1986 zur Spaltung von GRECE kam und Faye sich dem neuheidnisch-antichristlichen Lager zuwandte sowie auch den Begriff der »identitären Bewegung« geprägt hat (vgl. Bruns/Glösel/Strobl 2016: 75), um zu erkennen, das die Identitären ihre Konzeption des Ethnopluralismus ganz deutlich von Alain de Benoist übernommen haben.

Dieser theoretische Kopf der Neuen Rechte hat eine Vorstellung von einer organischen Demokratie, einer »véritable démocratie« entworfen, die dem Volk als einem »organisme collectif « und einer »entité organique« angemessen sei und die nicht im liberalen, gewaltenteilenden Staat, sondern nur in einem Volksstaat, einem »Etat du peuple« realisiert werden könne; in dieser Form von Demokratie begründe erst die Volkszugehörigkeit Freiheit und politische Rechte der Bürger (de Benoist 1985a: 81, 86 und 32). Die Anleihen bei Carl Schmitt sind im Werk von de Benoist, der nach einer Bio-Bibliographie zu Ernst Jünger (de Benoist 1997) auch eine internationale Bibliographie zu Schmitt (de Benoist 2010) veröffentlicht hat, nicht zu übersehen: In Vu de droite belegt er seine Anthologie zeitgenössischer Ideen mit mehrfach mit Zitaten von Carl Schmitt; im Kapitel über La notion de politique werden dessen wichtigste Gedanken dargelegt (de Benoist 1978: 216-218). In Les idées à l'endroit bezieht de Benoist für seine Idee des ordre von dem deutschen Staatsrechtslehrer die Vorstellung eines notwendigen Primats des Politischen für alle Formen menschlicher Aktivität (vgl. 1979: 110). Schmitts Begriff des Politischen ist kaum zu übersehen, wenn Alain de Benoist die »désignation de l'ennemi principal« als »geste >politique élémentaire« und »acte de courage élémentaire« ausweist (1982: 33; vgl. auch 2001: 128). In einer an Schmitts Freund-Feind-Theorie geschulten »Bestimmung des Gegners« gilt ihm als »Hauptfeind [...] der bürgerliche Liberalismus und der atlantisch-amerikanische >Westen« (1985b: 131 und 133). Einen von Carl Schmitt geprägten Begriff greift de Benoist in dem Manifest Die Nouvelle Droite des Jahres 2000 auf, indem er seine Ansichten über die Welt als »Pluriversum« darlegt, und verwendet einen Buchtitel Schmitts, wenn er der These vom Ende der 
Geschichte seine Wahrnehmung vom »Zutagetreten eines neuen >Nomos der Erde« entgegensetzt (1999: 36f.).

In seinem Plädoyer Vers une démocratie organique greift de Benoist neben Demokratievorstellungen von Arthur Moeller van den Bruck und Edgar Julius Jung auch Carl Schmitts Empfehlung qualitativer und nicht mehr bloß quantitativer Zustimmungsverfahren auf, um eine direkte, identitär-demokratische Verbindung zwischen Regierenden und Regierten herzustellen und mit der gegenseitigen Identifikation von Volk und Entscheidungsträgern die Idee einer »démocratie incarnée« zu verwirklichen (1985a: 79), eine Idee, die die Identitären später begrifflich zur Idee der "patrie charnelle« verschärft haben, die mit der Vorstellung einer abstammungsbasierten, regionalen und daher "natürlichen« Zugehörigkeit der autochthonen Bevölkerung der modernen Idee des souveränen Nationalstaats entgegensetzt wird (Camus 2017: 233). Nach der Devise "suum cuique« behauptet de Benoist (1985b: 15) mit seiner Konzeption des Ethnopluralismus eine nur sich selbst genügende Einzigartigkeit der ethnisch bestimmten Kultur jedes Volkes. Er folgert daraus die kulturelle Unvereinbarkeit der Ethnien und fordert deren weltweite Segregation nach territorialen Aspekten. Diese Vorstellung von einer zu konservierenden Heterogenität in sich homogen bleibender Völker greift Schmitts auf den »Pluralismus der Staatenwelt« gemünzte Annahme auf, die politische Welt sei »ein Pluriversum, kein Universum« (1963: 54), und zielt im Grunde auf eine Globalisierung von Apartheid. Mit dem Konzept des Ethnopluralismus ficht die Nouvelle Droite und in ihrer Nachfolge auch die Identitäre Bewegung einerseits gegen ethnische Mischungen und kulturelle Fremdbestimmung, andererseits gegen universalistische Ideen und suprastaatliche Menschenrechte. Konsequenterweise denunziert de Benoist die Idee universell gültiger Menschenrechte als naturalistische »Ideologie«, die eine »echte Phobie vor der Gemeinschaft« offenbare, als »nicht stichhaltig[e]« monotheistische »Zwangsvorstellung vom Gleichen« und »Religion des ausgehenden 20. Jahrhunderts (1988: 43f., 48 und 71). Vor allem diese gegen den liberalen Universalismus individueller Menschenrechte gerichtete Frontstellung mit ihrer Behauptung einer absoluten Vorrangstellung der Gemeinschaft gegenüber dem Einzelnen verbindet die im Kern anti-aufklärerischen Positionen der Nouvelle Droite und der Identitären mit denen Carl Schmitts.

Alain de Benoist hat mit seinen theoretischen Anleihen bei Carl Schmitt, die in seiner neueren Publikation über Wir und die anderen (de Benoist 2008) kaum mehr explizit gemacht werden, den - neben oder auch vor Rousseau - wichtigsten Theoretiker der identitären Demokratietheorie ins Zentrum seiner Vorstellungen vom Politischen, von Demokratie und ethno-kultureller Identität gestellt. Diese zentrale Bezugnahme auf das Denken des deutschen Staatsrechtslehrers macht Schmitt auch für die gegenwärtige Identitäre Bewegung, für die die Nouvelle Droite »die maßgebliche Wegbereiterin« (François 2017: 209) war, zu einem auf einem Umweg vermittelten Referenzautor. Das stützt die These, dass das Bekenntnis der 
Identitären zur direkten Demokratie weniger instrumentell motiviert als vielmehr ideengeschichtlich konzis begründet ist. Die der Idee der identitären Demokratie folgende Bezugnahme auf Identität findet sich aktuell nicht nur bei den Identitären, sondern auch in der politischen Praxis demokratischer politischer Systeme.

\section{Coda: IIdentitätı in Legitimationskontexten demokratischer politischer Systeme}

Unbestritten sind die rechtsstaatlich verfassten parlamentarischen und präsidentiellen Demokratien westlicher politischer Systeme viel stärker mit dem Prinzip der Repräsentation verbunden als mit dem der Identität. Dennoch findet sich die Denkfigur einer Gleichheit oder Übereinstimmung zwischen politischen Akteuren und >dem Volkı gelegentlich, wenn es darum geht, die eigene Programmatik, den eigenen Protest, die eigene Kandidatur für ein Amt als idealen Schritt zur Erzielung von Gemeinwohl darzustellen. Dieser Anspruch, eins mit dem Volk zu sein, findet sich, wenn zum Beispiel die rechtspopulistische und fremdenfeindliche Organisation Pegida schriftlich oder vokal die Parole »Wir sind das Volk« verbreitet. Das ist, zumal wenn es in Ostdeutschland geschieht, die Beanspruchung einer starken historischen Parallele, da es direkt auf Legitimität, Erlebnis und Erbe der friedlichen Revolution vom Herbst 1989 verweist. Die Anmaßung, als Minderheit so zu tun, als spräche man »im Namen einer Mehrheit «, ist nicht nur »eine historische Fälschung«, sondern geradezu eine »dreiste Lüge« (Prosinger 2015). Identitäre Legitimationsstrategien sind auch mitunter bei der Beanspruchung und Besetzung staatlicher Führungsämter vorzufinden. In Frankreich ist das - wohl auch aufgrund eines bis heute wirksamen Einflusses Rousseaus - beim Verständnis des Amtes des direkt vom Volk gewählten Präsidenten der Republik, von dem man zumindest erwartet, dass er die grandeur der Nation verkörpere, auch wenn in der Lehrbuchversion der repräsentativen Demokratie »Verkörperung als totalitäre Versuchung« (Diehl 2018: 28) nicht mehr akzeptabel ist, spürbarer als in Deutschland, wo niemand ernsthaft bezweifelt, dass der Bundespräsident oder auch die Bundeskanzlerin bei aller symbolischen Funktion dem Gesetz unterworfen ist wie jeder andere Bürger auch und nicht als Repräsentant des Volkes wenigstens ein Stückchen über dem Gesetz steht. Daher beschränken sich identitäre Elemente in der Kommunikation über die Besetzung staatlicher Führungspositionen in der Bundesrepublik bestenfalls auf die Vermittlung des Gefühls, in Wahlkämpfen sei der Kandidat seiner von uns oder die Kandidatin seine von uns - vor der letzten Bundestagswahl in typisch sozialdemokratischem Gepränge wieder aufgeführt im Wahlkampf von Martin Schulz.

Im Zusammenhang von identitär-demokratischen Begründungen politischer Legitimation ist ein Blick auf die USA erhellend: Neben starken ideengeschicht- 
lichen Anleihen bei Locke und Montesquieu, also einem anti-rousseauistischen Grundzug, in der Ausgestaltung des Systems der checks and balances findet sich dort nämlich auch die - formal indirekt vollzogene - Wahl des Präsidenten als des politischen Führers und Staatsoberhaupts durch das Volk. Für das Verständnis des Verhältnisses zwischen Präsident und Volk war die Inaugurationsrede von Präsident Donald Trump vom Januar 2017 sehr aufschlussreich. Die zentrale Formulierung in dieser Rede im Kontext einer ziemlich rücksichtslos vorgebrachten Kritik am Establishment der Vorgängerregierungen, deren Mitglieder sich auf Kosten des Volkes bereichert und vielfache Not und Elend hinterlassen hätten, lautet: »[...] heute übergeben wir die Macht nicht nur von einer Regierung an die andere oder von einer Partei an die andere, sondern wir nehmen die Macht von Washington D.C. und geben sie an euch, das Volk, zurück. $\ll^{5}$

Der neugewählte Präsident spricht in dieser Rede vom 20. Januar 2017 als dem Tag, "an dem das Volk wieder zu den Herrschern dieser Nation wurde.« Offenkundig wurde an diesem Januartag jedoch nicht das amerikanische Volk, sondern Donald Trump in sein Amt eingeführt. Das amerikanische Volk hat ihn am 8. November 2016 als Ausdruck der repräsentativen Demokratie und im Rahmen des Wahlsystems der USA zum Präsidenten gewählt, war aber am 20. Januar gar nicht anwesend, um irgendetwas, und wenn es »die Macht von Washington D.C.« wäre, in Empfang nehmen zu können. Die Idee, das Volk müsse vor dem Kapitol anwesend gewesen sein, hat womöglich Trumps Pressesprecher Sean Spicer dazu veranlasst, die Zahlen über die Besucher bei diesem Ereignis deutlich überhöht anzusetzen, was wiederum Trumps Beraterin Kellyanne Conway veranlasst hat, den Begriff alternative facts in die politische Kommunikation einzuführen. Der Wahlsieger hat Macht in Empfang genommen, insoweit die Verfassung ihm als dem gewählten Präsidenten bestimmte Herrschaftsbefugnisse zuweist. Trumps Formulierung bezieht ihren Sinn demnach nur aus der Voraussetzung, dass der Präsident und das Volk identisch sind. Aus "We, the people of the United States", dem sich in der US-Verfassung konsitutierenden Volk, wird bei Donald Trump: »I, the people«. Das ist die Denkfigur der identitären Demokratie. Das identitäre Amtsverständnis impliziert bei Trump, dass er sich als das Volk verkörpernder Präsident, als mit dem Volk gleichsam identischer Präsident tendenziell außerhalb des in den USA bekannten Systems von Kontrollmechanismen und bremsenden sowie machthemmenden Gegengewichten, auch in Form der Presse als einer `vierten Gewalt , sieht. Trumps Empörung über die Berichterstattung zu seiner Person und Amtsführung sowie seine wüsten Beschimpfungen von Journalisten sind demnach nicht (nur) Ausdruck narzisstischer Kränkung, sondern Konsequenz seines Selbstverständnisses nicht nur als Repräsentant, sondern als Verkörperung des amerikanischen Vol-

5 URL: www.zeit.de/politik/ausland/2017-01/rede-amtsantritt-donald-trump-inauguration-komplett/komplettansicht. 
kes. Wer nun Trump kritisiert, wird zum Gegner der Demokratie, und wer den mit dem Volk identischen Präsidenten kritisiert, wird zum Feind des Volkes. Mit dieser Denkfigur aus der identitären Demokratietheorie wird das zuweilen grotesk wirkende manichäische Weltbild des US-Präsidenten zumindest nachvollziehbar.

In der Zusammenschau wird deutlich, dass Begriff und Thematik der Identität unter anderem als Gegenstand von Konzepten der politischen Kultur, als grundlegende Vorstellung einer bestimmten Demokratietheorie, als zentrales Thema einer gegenwärtig in Europa zunehmend einflussreichen Bewegung und als Legitmitätsgenerator in der politischen Praxis demokratischer Staaten einen prominenten Ort nicht nur in der politischen Kulturforschung, sondern in der Politikwissenschaft insgesamt beanspruchen kann. Nicht allein, aber primär Aufgabe der politischen Kulturforschung kann es dabei sein, die mit dem »absolut undefinierbar[en]« (Husserl 1984: 118) Begriff der Identität bezeichneten Phänomene zu identifizieren, $\mathrm{zu}$ analysieren und $\mathrm{zu}$ verstehen.

\section{Literatur}

Almond, Gabriel A./Sidney Verba, 1963: The Civic Culture. Political Attitudes and Democracy in Five Nations, Princeton, New Jersey.

Bauman, Zygmunt, 1997: Flaneure, Spieler und Touristen. Essays zu postmodernen Lebensformen. Aus dem Englischen von Martin Suhr (englische Originalausgabe 1995), Hamburg.

de Benoist, Alain, 1978: Vu de droite. Anthologie critique des idées contemporaines, 4. Auflage, Paris.

de Benoist, Alain, 1979: Les idées à l'endroit, Paris.

de Benoist, Alain, 1982: Orientations pour des années décisives, Paris.

de Benoist, Alain, 1985a: Démocratie: Le Problème, Paris.

de Benoist, Alain, 1985b: Kulturrevolution von rechts. Gramsci und die Nouvelle Droite, übersetzt von Charlotte Adelung, Vorwort von Armin Mohler, Krefeld. de Benoist, Alain, 1988: Die Religion der Menschenrechte. In: Krebs, Pierre (Hg.): Mut zur Identität. Alternativen zum Prinzip der Gleichheit, Struckum, S. 41-73. de Benoist, Alain, 1997: Ernst Jünger. Une Bio-Bibliographie, Paris.

de Benoist, Alain, 1999: Aufstand der Kulturen. Europäisches Manifest für das 21. Jahrhundert, Berlin.

de Benoist, Alain, 2001: Schöne vernetzte Welt. Eine Antwort auf die Globalisierung, Tübingen u.a.

de Benoist, Alain, 2008: Wir und die anderen, Berlin.

de Benoist, Alain, 2010: Carl Schmitt. Internationale Bibliographie der Primär- und Sekundärliteratur, Graz. 
Bergem, Wolfgang 1993: Tradition und Transformation. Eine vergleichende Untersuchung zur politischen Kultur in Deutschland. Mit einem Vorwort von Kurt Sontheimer, Opladen.

Bergem, Wolfgang, 2005: Identitätsformationen in Deutschland, Wiesbaden.

Bergem, Wolfgang, 2013: Zivilcourage in der Bundesrepublik Deutschland. Zum Wandel einer politischen Kultur, in: Jahrbuch zur Liberalismus-Forschung, Bd. 25, Baden-Baden, S. 117-134.

von Beyme, Klaus, 1987: Politische Kultur, in: Ders./Czempiel, Ernst-Otto/Graf Kielmannsegg, Peter/Schmoock, Peter (Hg.): Politikwissenschaft. Eine Grundlegung, Bd. 2: Der demokratische Verfassungsstaat, Stuttgart u.a., S. 70-86.

Bruns, Julian/Glösel, Kathrin/Strobl, Natascha 2016: Die Identitären. Handbuch zur Jugendbewegung der Neuen Rechten in Europa, 2. Auflage, Münster.

Camus, Jean-Yves, 2017: Die Identitäre Bewegung oder die Konstruktion eines Mythos europäischer Ursprünge. In: Hentges, Gudrun/Nottbohm, Kristina/Platzer, Hans-Wolfgang (Hg.): Europäische Identität in der Krise? Europäische Identitätsforschung und Rechtspopulismusforschung im Dialog, Wiesbaden, S. 233-247.

Diehl, Paula, 2018: Die Symbolisierung des Volkes in der Demokratie: Eine ikonografische Spurensuche. In: Huhnholz, Sebastian/Hausteiner, Eva Marlene (Hg.): Politische Ikonographie und Differenzrepräsentation (Leviathan Sonderband 34), Baden-Baden, S. 23-47.

Dörner, Andreas, 2003: Politische Kulturforschung. In: Herfried Münkler (Hg.): Politikwissenschaft. Ein Grundkurs, Reinbek bei Hamburg, S. 587-619.

Easton, David, 1965: A Systems Analysis of Political Life, New York u.a.

Erikson, Erik H., 2013: Identität und Lebenszyklus. Drei Aufsätze. Übersetzt von Käte Hügel (englische Erstausgabe 1959), 26. Auflage, Berlin.

Fraenkel, Ernst, 1991: Deutschland und die westlichen Demokratien. Erweiterte Ausgabe (1991). Mit einem Nachwort über Leben und Werk Ernst Fraenkels, hg. von Alexander v. Brünneck (Erstausgabe 1964), Frankfurt a.M.

François, Stéphane, 2017: Die Nouvelle Droite und der Nationalsozialismus. Zur Wiederaufnahme einer historiographischen Debatte. In: Hentges, Gudrun/Nottbohm, Kristina/Platzer, Hans-Wolfgang (Hg.): Europäische Identität in der Krise? Europäische Identitätsforschung und Rechtspopulismusforschung im Dialog, Wiesbaden, S. 209-231.

Greiffenhagen, Sylvia, 2009: Theorie(n) der Politischen Kultur. In: Samuel Salzborn (Hg.): Politische Kultur. Forschungsstand und Forschungsperspektiven, Frankfurt a.M., S. 11-29.

Hegel, Georg Wilhelm Friedrich, 1991: Enzyklopädie der philosophischen Wissenschaften im Grundrisse (Fassung von 1830), hg. von Friedhelm Nicolin und Otto Pöggeler, 8. Auflage, Hamburg. 
Heitmeyer, Wilhelm, 2018: Autoritäre Versuchungen. Signaturen der Bedrohung 1, Berlin.

Hentges, Gudrun/Gürcan Kökgiran/Kristina Nottbohm, 2014: Die Identitäre Bewegung Deutschland (IBD) - Bewegung oder virtuelles Phänomen? In: Forschungsjournal Soziale Bewegung - Plus, Supplement zu Heft 3, S. 1-26 (URL: http://forschungsjournal.de/fjsb-plus).

Husserl, Edmund, 1984: Logische Untersuchungen, Bd. 2,1: Untersuchungen zur Phänomenologie und Theorie der Erkenntnis. Text der 1. und der 2. Auflage ergänzt durch Annotationen und Beiblätter aus dem Handexemplar (Erstausgabe 1901). In: Gesammelte Werke, Bd. 19/1, hg. von Ursula Panzer, Den Haag u.a.

Klünder, Jan-Paul, 2017: Politischer Pessimismus. Negative Weltkonstruktion und politische Handlung(un)möglichkeit bei Carl Schmitt, Michel Foucault und Giorgio Agamben, Bielefeld.

Locke, John, 1987: An Essay Concerning Human Understanding (Erstausgabe 1690), hg. von Peter H. Nidditch, Oxford.

Lynd, Helen Merrell, 2001: On Shame and the Search for Identity (Erstausgabe 1958), London.

Niethammer, Lutz, 2000: Kollektive Identität. Heimliche Quellen einer unheimlichen Konjunktur, unter Mitarbeit von Axel Doßmann, Reinbek bei Hamburg.

Pickel, Susanne/Pickel, Gert 2006: Politische Kultur- und Demokratieforschung. Grundbegriffe, Theorien, Methoden. Eine Einführung, Wiesbaden.

Prosinger, Wolfgang, 2015: Pegida ist nicht das Volk, in: Der Tagesspiegel, 19.01.2015 (URL: https://www.tagesspiegel.de/politik/vereinnahmung-derparole-wir-sind-das-volk-pegida-ist-nicht-das-volk/11250492.html).

Ricœur, Paul, 1990: Soi-même comme un autre, Paris.

Rohe, Karl, 1987: Politische Kultur und der kulturelle Aspekt von politischer Wirklichkeit, Konzeptionelle und typologische Überlegungen $\mathrm{zu}$ Gegenstand und Fragestellung Politischer Kultur-Forschung. In: Berg-Schlosser, Dirk/Schissler, Jakob (Hg.): Politische Kultur in Deutschland. Bilanz und Perspektiven der Forschung, Opladen, S. 39-48.

Rohe, Karl, 1990: Politische Kultur und ihre Analyse. Probleme und Perspektiven der politischen Kulturforschung. In: Historische Zeitschrift, Heft 2, S. 321-346. Rousseau, Jean-Jacques, 1964: Euvres complètes, Bd. 3: Du Contrat social. Écrits politiques, hg. von Bernard Gagnebin und Marcel Raymond, Paris.

Rousseau, Jean-Jacques, 1981: Sozialphilosophische und Politische Schriften, München.

Schmidt, Manfred G., 2010: Demokratietheorien. Eine Einführung (Erstausgabe 1995), 5. Auflage, Wiesbaden.

Schmitt, Carl, 1933: Staat, Bewegung, Volk. Die Dreigliederung der politischen Einheit, Hamburg. 
Schmitt, Carl, 1963: Der Begriff des Politischen. Text von 1932 mit einem Vorwort und drei Corollarien, Berlin.

Schmitt, Carl, 1965: Verfassungslehre (Erstausgabe 1928), 4. Auflage, Berlin.

Schmitt, Carl, 1969: Die geistesgeschichtliche Lage des heutigen Parlamentarismus (Erstausgabe 1923), 4. Auflage, Berlin.

Schuppert, Gunnar Folke, 2008: Politische Kultur, Baden-Baden.

Schwelling, Birgit, 2001: Politische Kulturforschung als kultureller Blick auf das Politische. Überlegungen zu einer Neuorientierung der Politischen Kulturforschung nach dem »cultural turn«. In: Zeitschrift für Politikwissenschaft, Heft 2, S. 601-629.

Speit, Andreas (Hg.), 2018a: Das Netzwerk der Identitären. Ideologie und Aktionen der Neuen Rechten, Berlin.

Speit, Andreas, 2018b: Avantgarde rückwärts. Die geistigen Grundlagen der Identitären Bewegung. In: Speit 2018a, S. 56-72.

Speit, Andreas, 2018c: Identitärer Aufbruch. Die Vorbilder und Vordenker aus Frankreich. In: Speit 2018a, S. 42-55.

Speit, Andreas, 2018d: Reaktionärer Klan. Die Entwicklung der Identitären Bewegung in Deutschland. In: Speit 2018a, S. 17-41.

Verba, Sidney, 1965a: Conclusion: Comparative Political Culture, in: Lucian W. Pye/Ders. (Hg.): Political Culture and Political Development, Princeton, New Jersey, S. 512-560.

Verba, Sidney, 1965b: Germany: The Remaking of Political Culture, in: Lucian W. Pye/Ders. (Hg.): Political Culture and Political Development, Princeton, New Jersey, S. 130-170.

Verba, Sidney, 1980: On Revisiting the Civic Culture: A Personal Postscript, in: Gabriel A. Almond/Ders. (Hg.): The Civic Culture Revisited, Boston und Toronto, S. 394-410.

Westle, Bettina/Oscar W. Gabriel (Hg.), 2009: Politische Kultur. Eine Einführung, Baden-Baden.

Wodak, Ruth, 2016: Politik mit der Angst, Zur Wirkung rechtspopulistischer Diskurse, Wien und Hamburg. 



\section{"Wir sind das Volk" \\ Zur Herausbildung eines Diskurses \\ der identitären Demokratie im Umfeld der Partei Alternative für Deutschland (AfD)}

Jasmin Siri / Marcel Lewandowsky

\section{Einleitung}

»Es könnte aber sein, daß uns in absehbarer Zeit Konflikte ganz anderer Art ins Haus stehen: ethnische Konflikte, religiöse Konflikte, Identitätskonflikte, Konflikte über nicht verhandlungsfähige Werte, Normen, Überzeugungen. Zahlreiche fundamentalistische Bewegungen, wie sie in den letzten Jahrzehnten unerwarteterweise wieder aufgelebt sind, belegen, daß es diese ernsten, nichttrivialen Konflikte nach wie vor gibt und daß es eine Illusion war, alle Konflikte politisch auf Interessenskonflikte reduzieren zu können.« (Luhmann 2002: 218f.).

In den europäischen Staaten und darüber hinaus scheint sich der Bedarf an partikularen Kollektivitätsbeschreibungen zu erhöhen. (Vgl. für viele Decker et al. 2012) Die Komplexität der Weltgesellschaft und die Entgrenzung traditionaler Vergemeinschaftung beantworten Rechtspopulisten mit einer Gegenerzählung der nationalen Vergemeinschaftung. Die Partei Alternative für Deutschland (AfD) und das sie umgebende aktivistische Milieu legen hiervon im politischen System der Bundesrepublik Deutschland Zeugnis ab. So hat sich die Partei in ihrer jungen Geschichte bisher von einer vor allem eurokritischen Partei in der Gründungsphase hin zu einer rechtspopulistischen Partei entwickelt.

Der Impetus und die Argumentationen aktueller rechtspopulistischer Aktivismen und Parteien in Europa widerspricht einer seit den 1990er Jahren sehr erfolgreichen Erzählung einer globalisierten Weltgesellschaft, welche aus der Globalisierung nicht nur auf persönliche Lebens- und Flexibilitätschancen schließt, sondern zugleich gemeinsame Wirtschaftsräume, gemeinsame Verantwortung für Konflikte und die Entwicklung einer Weltkultur annimmt, die das nationale Denken langfristig ersetzen werde. Damit eng verbunden ist eine Ideologie des Freihandels, des freien Kapitalflusses und der freien Märkte, die weithin als »alternativlos« darge- 
stellt wird. Dasselbe gilt auch für ein »Europa ohne Grenzen«, in dem auf die Sogwirkung von Freizügigkeit und die Betonung gemeinsamer Ziele und Werte gesetzt wird.

Soziologische Theorien der Weltgesellschaft haben gezeigt, dass die Ausdifferenzierung der Welt in Nationalstaaten faktisch an Plausibilität verloren hat, praktisch aber große Bedeutung für politische Kollektivbildung zeitigt (vgl. für viele Greve/Heintz 2005). Auch wird deutlich, dass die ökonomische Fassung der Weltgesellschaft »Gewinner« und »Verlierer« sowie ein neoliberales Narrativ der Globalisierung produziert, was Kritiken der Legitimität von »Rechts« wie »Links« hervorbringt. Und doch: Die jüngsten elektoralen und medialen Erfolge rechtspopulistischer Parteien und Bewegungen sind keiner Pfadabhängigkeit oder dialektischen Schleife geschuldet. Sie sind soziologisch wie politikwissenschaftlich erklärungswürdig, denn der Erfolg rechtspopulistischer Parteien spiegelt nach unserer Auffassung mehr wider als nur eine Verschiebung in Parteiensystemen oder eine Abwehrhaltung zum kosmopolitischen Europa Ulrich Beckscher Façon.

Es liegt aus unserer Sicht nahe, hier die Verbindung von Organisationen, Bewegungen und Medien genauer in den Blick zu nehmen und in wissenssoziologischer Absicht danach zu fragen, inwiefern ein spezifisches, in den sozialen Netzwerken und auf Demonstrationen (Dresden, Leipzig, Erfurt) sich artikulierendes, mithin heterogenes Milieu sichtbar wird, welches von der Ablehnung des »Mainstreams" getragen wird und skeptisch auf Formen und Folgen liberaler Demokratien, der Globalisierung sowie pluralistischer Ethiken blickt. Es ist wohl kein Zufall, dass »Pegida« und AfD zu ähnlichen Zeitpunkten auftauchen, zum Teil vergleichbare Anliegen formulieren, sich gleichsam gegen die etablierten Akteure der repräsentativen Demokratie wenden und Überschneidungen im Wähler- bzw. Sympathisantenmilieu aufweisen - allen strategischen Differenzen zum Trotz (Vorländer et al. 2016: 40ff.). Wir wollen argumentieren, dass sich in diesen Milieus ganz Unterschiedliches verbindet: Erstens: Eine Parteienkritik, die für die deutsche Geschichte nichts Neues ist, sondern sich mit der Gründung der ersten Parteien aktiviert und seither über das gesamte politische Spektrum hin aktualisiert (Siri 2012). Zweitens: Eine Kritik an etablierten Medien (und anderen Funktionseliten), denen vorgeworfen wird, sich zum Gehilfen der Regierung zu machen. Auch diese Kritik ist altbekannt. Drittens: Ein Plädoyer für Volkssouveränität abseits des Parlamentarismus und ein Eintreten für Volksabstimmungen über Globalisierungsfolgen (wie Migration). Und viertens nutzen Partei und Unterstützermilieu moderne Medien zur Herstellung eines Kollektivs abseits der Welt der Interaktion unter Anwesenden. In diesen Praktiken des Mediengebrauchs liegt ihr Erfolg ebenso begründet

1 Der Milieubegriff dient uns daher auch nicht als Strukturbegriff sondern als Suchbegriff, um in wissenssoziologischer Absicht die Besonderheiten des die AfD umgebenden politisch-kommunikativen Resonanzraumes zu ordnen. 
wie ihre Toleranz im Umgang mit programmatischen und ideologischen Abweichungen. Dabei ist zu beachten, dass der Einsatz des digitalen Mediums die altbekannten Argumente in eine neue Form bringt und spezifische Anschlussfähigkeiten generiert.

Es sind in Deutschland nicht nur die "Altparteien «, die als gewohntes Feindbild des rechtspopulistischen Diskurses "abgewrackt « gehören, sondern zunehmend auch mediale Akteure (»Lügenpresse«) oder (vermeintliche) gesellschaftliche Mehrheiten (»links-grün-versiffter Mainstream«), denen zum Teil mit offener Feindlichkeit, nicht selten mit einem verschwörungstheoretisch überformten Blick auf politische Prozesse begegnet wird. Dem pluralistischen Modell stellen Rechtspopulisten einen mehrheitsdezisionistischen Demokratieentwurf entgegen, dessen Kern von einem nativistischen Begriff von »Volkssouveränität« gebildet wird (Mudde 2007). Dabei ist auch zu beachten, dass es sich bei jüngeren rechtspopulistischen Bewegungen und Organisationen um international vernetzte und sich gegenseitig inspirierende Gefüge handelt, was zur Übernahme und Adaption politischer Strategien sowie einem internationalen Resonanzrahmen in den Sozialen Medien führt. Diese Parteien sind also nicht nur traditionalistische Wiedergänger einer totgeglaubten reaktionären Politik im Sinne eines oft konstatierten $>$ Backlashes<, sondern eben auch sehr modern und international.

Anhand einer theoriegeleiteten Interpretation quantitativer und qualitativer empirischer Daten wollen wir uns daher im Folgenden fragen, inwiefern sich in der Bundesrepublik ein solches neues Milieu in der AfD und in ihrer politischen und publizistischen Umwelt konstituiert und organisiert. Dabei richten wir den Blick nicht nur auf jene, die nachweislich eine Mitgliedschaft der Partei besitzen, sondern wollen, wie es sich bei der Entstehung einer neuen Partei stets anbietet, auch den aktivistischen Resonanzraum im Netz und auf Demonstrationen beachten. Hierzu verwenden wir theoretisch die Arbeiten Mannheims zur Differenzierung des Konservatismus (vgl. Mannheim 1984; Siri 2016) wie auch in empirischer Hinsicht Daten über die Sympathisantenstruktur der Partei und Eindrücken aus der Analyse von Social-Media-Kommunikationen.

Die politikwissenschaftliche Forschung hat bereits Fragen nach der Veränderung des Parteiensystems mit Blick auf die AfD ergründet. Auf den vorliegenden Studien, die die Einstellungen von Wählern und Sympathisanten (Berbuir et al. 2015; Wagner et al. 2015), die programmatische bzw. ideologische Verortung (Arzheimer 2015; Franzmann 2014; Häusler 2013; Jankowski et al. 2016; Lewandowsky et al. 2016) sowie die sie tragenden Netzwerke untersucht haben (Bebnowski 2015), bauen wir auf. Im zweiten Abschnitt werden wir bisherige Forschungsergebnisse im Hinblick auf unsere Fragestellung vorstellen und diskutieren. Wir werden außerdem die Geschichte der Partei AfD in den Blick nehmen, da die Entwicklung der Partei und ihrer Programmatik auch als Interaktion mit und Suche nach einer stabilen Milieufundierung interpretiert werden kann. Hierfür spricht 
unter anderem die Heterogenität der Aussagen zur Programmatik im Vorgang des Programmparteitages und das »Testen« besonders >skandalöser A Aussagen, zum Beispiel zur Migration durch prominente Vertreter und Vertreterinnen der Partei (Berbuir et al. 2015).

Angesichts der europaweit erstarkenden Parteien ähnlicher Prägung betrachten wir den deutschen Fall spezifisch, aber auch prototypisch: Mittels einer wissenssoziologisch-systemtheoretischen Analyse wollen wir danach fragen, wie sich neue rechtspopulistische Bewegungen in pluralisierten, transnationalen Öffentlichkeiten bilden und stabilisieren. Dabei spielen für uns Netzbewegungen, die als publizistisches Reservoir der jungen Partei bezeichnet werden können, eine wichtige Rolle im dritten Abschnitt des Aufsatzes. Theoretisch wollen wir auch die Grenze von Organisation und Massenmedien/Sozialen Medien in den Blick nehmen. Wir vermuten, dass in diesen Verhältnissen die Selbststabilisierungsleistungen neuer politischer Gruppierungen der Rechten zu suchen sind. Diese Erörterung folgt in Abschnitt 4. In einem Fazit (Abschnitt 5) werden wir abschließend die Erfolgsbedingungen identitärer ${ }^{2}$ Demokratie im Umfeld der Partei AfD und deren Abgrenzung zum Konservatismus diskutieren (Mannheim 1984, vgl. auch Mannheim 1980; Siri 2016). Dahinter steht die aus den Daten begründete Vermutung, dass die aktuellen Bewegungen des Rechtspopulismus nur zu verstehen sind, wenn neben sozialstrukturellen und elektoralen Daten auch ein Blick auf die Nutzung von Medien und medienevolutionäre Entwicklungen geworfen wird.

\section{Forschungsstand: Die Alternative für Deutschland und ihr aktivistischer Resonanzraum im Netz und auf Demonstrationen}

\section{Der Begriff des Populismus}

Der Begriff des Populismus wird aktuell in öffentlichen Debatten oft und nicht immer mit der erforderlichen begrifflichen Tiefenschärfe genutzt. Er löst sich von der wissenschaftlichen Diskussion, die bereits eine jahrzehntelange Geschichte hat, ab. »Das Problem des Rechtspopulismusbegriffs liegt darin, dass er nicht nur eine wissenschaftliche Analysekategorie darstellt, sondern zugleich als wertgeladener Kampfbegriff in der politischen Auseinandersetzung herhalten muss. (Decker 2015a: 221). Auch die Forschung hat lange Zeit um ein gemeinsames Begriffsverständnis gerungen. Es ging dabei zuvorderst um die Frage, ob sich ein solches

2 Der Begriff des Identitären bezeichnet dabei eine Strategie politischer Programmatik und Kommunikation, die Homogenität einer nationalen und kulturellen Großgruppe zu bezeichnen und gegen die Modernität einer pluralisierten Demokratie in Stellung zu bringen. 
Phänomen, das sich ja gerade durch seine Kontextabhängigkeit und Wandlungsfähigkeit auszeichnet, überhaupt definitorisch fassen lasse (Decker 2004: 21ff.). Inzwischen kann die Definition von Mudde $(2004,2007)$ als politikwissenschaftlicher state of the art gelten, nicht zuletzt auch deshalb, weil diese sich zur empirischen Arbeit heranziehen lässt (Siehe hierzu beispielsweise Pauwels 2011; Rooduijn/Pauwels 2011; Lewandowsky et al. 2016.). Nach Muddes »Kernkonzept « des Populismus (2004) besteht dieser im Appell an das »Volk«, das gegen die politischen Eliten mobilisiert werden soll. Das Volk wird dabei nicht in der Unterschiedlichkeit seiner Interessen, sozio-ökonomischen und -kulturellen Merkmale adressiert. Gemeint ist vielmehr eine vermeintlich schweigende Mehrheit, der ein einheitlicher politischer Wille unterstellt werden kann. Im Gegensatz dazu attestieren Populisten gewählten Vertretern, diesen Volkswillen in ihren Entscheidungen nicht zu berücksichtigen, sei es durch Unfähigkeit, Ignoranz oder böse Absicht. Politische Repräsentanten gelten nicht als Vollzugsorgan diverser Interessen innerhalb des Volkes, sondern als Hemmschuhe bei der Vollstreckung der volonté générale. Jenseits seiner ideologischen Zuordnung (grob als Links- oder Rechtspopulismus) wohnt dem Populismus daher ein anti-pluralistisches Element inne. Er richtet sich gegen politische Eliten und »das Establishment«, gegen den - vermeintlichen - medialen Mainstream sowie zumindest in der Konsequenz auch gegen rechtsstaatliche Arrangements, die den Willen der Mehrheit zugunsten des Minderheitenschutzes beschneiden. Der anti-elitäre Habitus richtet sich dabei nicht nur gegen das politische »Establishment« selbst, sondern weitet sich auf die Massenmedien aus. »Die Medien« spielen in populistischen Diskursen nicht die Rolle unabhängiger Berichterstatter, sondern bilden zusammen mit den politischen Eliten und Institutionen der repräsentativen Demokratie ein diffuses »Kartell«, dem gleichsam ein gemeinsamer Betrug am Volk, Lügen und gezielte Desinformation vorgeworfen werden.

Obwohl der Populismus nach dieser Definition zunächst nicht ideologisch konnotiert ist, ja sogar an verschiedene Weltanschauungen anschließen kann, ist der Großteil der populistischen Bewegungen im rechten politischen Spektrum verortet (Decker 2004: 29). Das überrascht nicht, geht mit der anti-pluralistischen Komponente doch auch zumindest eine implizite Befürwortung autoritärer Demokratiekonzeptionen einher, in denen eine den Volkswillen verkörpernde charismatische Führungspersönlichkeit die Bedürfnisse der vermeintlichen Mehrheit gegen alle Widerstände - also auch die der Jurisprudenz - durchsetzt. Gleichwohl finden wir originär populistische Parteien auch auf der Linken vor, etwa in Gestalt der griechischen Syriza. So ist der Angriff auf das politische Establishment auch insofern nicht unpolitisch, als die Eliten als Schuldige für vermeintlich verfehlte Politiken identifiziert werden: aus ordo-liberaler Perspektive die europäische Wirtschaftsund Finanzkrise, aus konservativer Sicht die Erosion traditioneller Lebensweisen (und die zunehmende Sichtbarkeit alternativer Konzepte) sowie »Masseneinwanderung« und »Islamisierung«. 
In der Praxis also ist Populismus ideologisch attribuiert. In seiner definitorischen Reinform als Appell an die unbedingte Souveränität des Volkes zu verstehen, rekurriert der Rechtspopulismus zugleich auf die kulturelle Identität des Volkes. Von Mudde als »Nativismus« bezeichnet, wird das Bedrohungsszenario gewissermaßen auf die Außengrenzen der Nation ausgeweitet: Unterstellt wird, dass die kulturelle Integrität des Volkes durch »Fremde« bedroht würde. Wer diese Fremdgruppen sind, hängt maßgeblich vom jeweiligen nationalen Kontext ab: Im Gegensatz zu geschlossenen rechtsextremen Ideologien bezieht sich der Rechtspopulismus auf die jeweilige nationale Mehrheitsethnie, nicht etwa auf einen paneuropäischen Volksbegriff. So richtet sich der westeuropäische Rechtspopulismus gegen den Islam, während sich osteuropäische Parteien im Zuge irredentistischer Staatskonzeptionen gegen nationale und regionale Minderheiten wenden (Pirro 2014).

\section{Die AfD: vom funktionalen Äquivalent zur rechtspopulistischen Alternative im Parteiensystem?}

Die Geschichte der AfD kann aus unserer Perspektive als Suchbewegung einer jungen, heterogenen Organisation nach einem Trägermilieu des Rechtspopulismus in Deutschland verstanden werden, als eine Suchbewegung nach moderner Kollektivität. Es ist daher notwendig, die Geschichte der Partei zumindest kurz zu adressieren, bevor wir in eine medien- und gesellschaftstheoretische Diskussion eintreten.

Die AfD wurde im Februar 2013 ins Leben gerufen. Die Gründung der AfD resultierte zunächst aus einer ungleich längeren Entwicklung im konservativen Spektrum, die mit dem bürgerlichen Protest gegen die Maastrichter Verträge ihren Ausgang nahm (Decker 2015b). Der euroskeptische Bund Freier Bürger (BFB) entstand als frühe Vorgängerpartei der AfD, konnte sich allerdings nicht im Parteiensystem etablieren. Auch die unmittelbare Vorgängerorganisation der AfD, die »Wahlalternative 2013«, nahm zunächst nicht die Form einer Partei an, sondern kandidierte bei der niedersächsischen Landtagswahl im Januar 2013 auf der Liste der Freien Wähler. Als diese den Einzug in das Landesparlament verpassten, brachte eine Gruppe liberaler Ökonomen um Bernd Lucke, Joachim Starbatty und Hans-Olaf Henkel die Gründung der AfD in Parteienform auf den Weg. Auch der ehemalige Leiter der hessischen Staatskanzlei Alexander Gauland befand sich unter den Gründungsmitgliedern.

In den ersten beiden Jahren offenbarte die AfD eine gewisse Doppelgesichtigkeit. Zwar zeigte sie ein klar populistisches Profil, indem sie die europäische Wirtschafts- und Finanzkrise ebenso wie das Demokratiedefizit der Europäischen Union dem Verschulden der politischen Eliten auf nationaler wie europäischer Ebene zuschrieb (Franzmann 2014). Allerdings fehlten ihr die für Rechtspopulis- 
ten typischen nativistischen Elemente in Gestalt von Islamfeindlichkeit oder anderen Formen der Xenophobie (Arzheimer 2015). Zugleich spielten Akteure der Partei (u.a. über Soziale Medien) vereinzelt Angriffe gegen den Islam oder Gender Mainstreaming, ohne dass dies Eingang in die offizielle Linie der Partei gefunden hätte. So war die AfD zunächst keine offen rechtspopulistische Partei, sondern im soziologischen Sinne ein »funktionales Äquivalent« für eine rechtspopulistische Partei in der BRD (Berbuir et al. 2015: 173).

Allerdings zeigen verschiedene Studien, dass die AfD von Beginn an bereits rechtspopulistische Merkmale aufwies, die lediglich nicht offen zutage traten. Zwei Beispiele seien hier angeführt. So kann Bebnowski (2015) deutlich machen, dass die Partei schon in der Gründungsphase nicht nur von ökonomischen, sondern auch von gesellschaftspolitischen Netzwerken getragen wurde, etwa der »Zivilen Koalition« unter Vorsitz der späteren Europaabgeordneten Beatrix von Storch. Gauland konstatierte bereits im Dezember 2013 öffentlich, dass sich in der AfD Menschen zusammengefunden hätten,

»für die eine Familie aus Vater Mutter und Kind besteht [und] die Zuwanderung besonders in unsere Sozialsysteme nicht automatisch als einen Cewinn ansehen und denen die Buntheit mancher Lebensformen für ein Land, in dem die Kinder fehlen, zu bunt erscheint« (zit. n. Berbuir et al. 2015: 177).

Das zeigt sich zweitens auch in dem Antreten der Partei zur Bundestagswahl 2013. Galt die AfD hier noch als euroskeptisch, aber nicht rechtspopulistisch, zeigt die Untersuchung der Kandidaten zur Bundestagswahl (Lewandowsky et al. 2016), dass unter diesen rechtspopulistische Einstellungsmerkmale bereits ausgeprägt waren.

Konzentrierte sich die AfD jedoch bis zur Europawahl 2014 auf das EuroThema, so rückten anlässlich der Landtagswahlen in Brandenburg, Sachsen und Thüringen im Herbst 2014 gesellschaftspolitische Fragen zunehmend in den Mittelpunkt der Kampagnen (Lewandowsky 2015: 127). Dabei plädierte die AfD etwa für ein Einwanderungsmodell nach kanadischem Vorbild. Zugleich bildete sich ein interner Konflikt zwischen einer um Bernd Lucke organisierten, medial häufig als "moderat « adressierten Gruppe und einem nationalkonservativen Personenkreis heraus, dem u.a. der thüringische Partei- und Fraktionsvorsitzende Björn Höcke angehörte. Die Spannungen entzündeten sich zum einen am Führungsanspruch und -stil Luckes, der formal lediglich Co-Sprecher neben Frauke Petry war und den alleinigen Vorsitz anstrebte, zum anderen am Verhältnis zur sächsischen Pegida-Bewegung. In einem Papier, das den Titel »Erfurter Resolution« trug, forderten die Unterzeichner - darunter Björn Höcke und der spätere sachsen-anhaltische Fraktionschef André Poggenburg - den Schulterschluss mit Pegida und eine insgesamt konservativere Ausrichtung der Partei. Im Gegenzug organisierte sich um Lucke ein Verein mit der Bezeichnung "Weckruf 2015", der sich gegen einen Rechtsruck der Partei zu stemmen suchte. 
Am 4. Juli 2015 löste Frauke Petry den bisherigen Vorsitzenden ab; ihr CoSprecher wurde der Ökonom und baden-württembergische Landesvorsitzende Jörg Meuthen. Nachdem sich eine Gruppe um Lucke, Starbatty, Hans-Olaf Henkel und Ulrike Trebesius von der AfD abgespalten und eine neue Partei unter dem Akronym ALFA (Allianz für Fortschritt und Aufbruch) gegründet hatte - die sich nach einem Rechtsstreit im November 2016 in LKR (Liberal-Konservative Reformer) umbenannte und an den Wahlurnen erfolglos blieb - konzentrierte sich die AfD auf gesellschaftspolitische Themen und bildete in der Folgezeit ein deutlich konservativeres Profil aus. Was sich zuvor lediglich bei Kandidaten und Sympathisanten gezeigt hatte, schlug sich nun auch im offiziellen Grundsatzprogramm nieder.

Auch wenn der innerparteiliche Richtungsstreit 2015 zugunsten der konservativen Faktionen entschieden wurde, entzünden sich Konflikte immer wieder an der zunehmend sichtbaren Radikalität einzelner Funktionäre und Mandatsträger. Der Antisemitismus-Streit um den baden-württembergischen Abgeordneten Wolfgang Gedeon, der zur Spaltung (und später zur Wiedervereinigung) der Landtagsfraktion führte, präsentiert dafür ebenso ein Beispiel wie die Bemühung des Bundesvorstandes, Björn Höcke aufgrund seiner Weimarer Rede, in der er das Berliner Holocaustdenkmal als »Mahnmal der Schande« bezeichnet hatte, aus der Partei auszuschließen. Die AfD wird also bis heute durch innerparteiliche Spannungen geprägt. Deutlich wird in der Gesamtschau auch, dass die programmatischen Änderungen nicht nur als Kämpfe zwischen ideologischer Abgrenzung von Gruppen innerhalb der Partei, sondern vielmehr als Suchbewegung nach einer stabilen Entsprechung im Elektorat verstanden werden können. Dabei spielen nicht nur sachlich-thematische Abgrenzungen eine Rolle, sondern es kommt auch eine affektuell-sozialpsychologische Dimension zum Tragen. Die Ansprache und Reaktionen des Unterstützungsmilieus via Social Media scheinen hierfür relevanter als Verhandlungsprozesse innerhalb der Partei zu sein, die oft sehr kurz ausfielen und vor allem das Abstimmen großer Programmblöcke per Akklamation beinhalteten.

\section{Gibt es ein rechtspopulistisches Trägermilieu? Suchbewegungen in Organisation, Bewegung und medialem Resonanzraum}

Unsere Fragestellung lautet also: Wie sieht das Trägermilieu rechtspopulistischer Politiken aus und wie verbinden sich Organisation, Medien und soziale Bewegung im spezifischen Fall des aktivistischen Milieus um die Alternative für Deutschland? Dies ist freilich eine große Frage und wir erwarten nicht, dass diese schnell ausdiskutiert oder anhand weniger Daten zu beantworten ist. Jedoch können wir an den bisher zur Verfügung stehenden Daten bereits zeigen, dass der aktuelle Rechtspopulismus sich von dem der 1990er Jahren in dem Sinne unterscheidet, 
dass neue Aktivismen und Freiheitsgrade in der ideologischen Diskussion deutlich werden, die (auch) medialen Effekten geschuldet sind. Es scheint, dass die rechtspopulistischen Filter-Bubbles eine eigentümliche Toleranz für Abweichung ebenso mit sich führen wie auch die Möglichkeit zur Ausblendung nicht oder zumindest nicht auf den ersten Blick wahrheitsfähiger Aussagen von Parteimitgliedern und Unterstützern. Diese medialen Verhandlungen abseits der Organisationswirklichkeit erleichtern die Konstitution von Kollektivität erheblich und damit ist es so interessant wie notwendig, nach den Gemeinsamkeiten eines auf den ersten Blick chaotischen und heterogenen Umfelds der AfD zu suchen.

Die Fragestellung lässt sich unseres Erachtens nur diskutieren, wenn unterschiedliche Datensorten einer theoretisch geschulten Interpretation zugänglich gemacht werden. ${ }^{3}$ Besonders geeignet scheint uns eine Verbindung wissenssoziologischer und systemtheoretischer Interpretation, da erstere die verschiedenen Dimensionen einer Kollektivitätsbildung und -stabilisierung in den Blick nimmt und zweitere sowohl Sach- als auch Zeit- und Sozialdimension des Politischen für eine soziologische Beobachtung offen hält, während die Parteienforschung häufig nur die sachlich-thematische Dimension der Programmatik und wenige, z.B. sozialstrukturelle Faktoren der Milieubildung untersucht.

\section{Untersuchungen des Wähler- und Unterstützermilieus}

Bei der Untersuchung des Wähler- und Unterstützermilieus rechtspopulistischer Parteien ist zum einen zwischen den sozio-kulturellen und sozio-ökonomischen Merkmalen, zum anderen zwischen unterschiedlichen ideologischen Verortungen sowie externen Faktoren zu unterscheiden, die die Unterstützung dieser Parteien begünstigen. Zugleich stellt sich die Frage, wie die Kommunikation zwischen Unterstützern und Partei sich praktisch vollzieht (siehe Abschnitt 4).

Eine klassische Vorstellung besteht darin, dass Rechtspopulisten Profiteure von Modernisierungsprozessen seien. Rechtspopulisten erhalten demnach klassischerweise Zulauf aus jenen sozialen Gruppen, die von den negativen Folgen ökonomischer Entwicklung stark betroffen sind. Die sogenannte »Modernisierungsverlie-

3 Bevor wir in die Analyse eintreten, gilt es, diese Daten und die Form der Interpretation kurz vorzustellen. Die Datengrundlage für unsere Überlegungen bilden die folgenden Arbeiten: Die Daten für die Wähler und Sympathisanten der AfD entnehmen wir Berbuir et al. 2015, Kroh/Fetz 2016 sowie Niedermayer/Hofrichter 2016; die Daten zu Wählern rechtspopulistischer und -extremer Parteien entstammen Arzheimer 2011 sowie Arzheimer/Carter 2006. Für die ideologische Verortung der Partei sowie deren Kandidaten wurden Arzheimer 2015 und Lewandowsky et al. 2016 konsultiert. Bei der Betrachtung des Parteienwettbewerbs rekurrieren wir aufWagner et al. 2015. Qualitative Daten sind entnommen dem Datenkumulus für ein Habilitationsprojekt an der LMU München zur »Emergenz des politischen Selbst. Die Politik der Vereinzelten und Probleme der Synchronisierung« von Jasmin Siri, vgl. z.B. Siri 2015, 2016. 
rerthese« (Spier 2006) fand in den letzten Jahren einigen Zuspruch, nicht zuletzt öffentlich, da beispielsweise immer wieder auf die hohen Anteile von Arbeitslosen an der Wählerschaft von Rechtspopulisten im internationalen Vergleich hingewiesen wurde. Rechtspopulisten schienen in einigen europäischen Ländern Sozialisten und Sozialdemokraten als Vertreterinnen der Arbeiterschaft abgelöst zu haben. Spier zeigt, »dass es einen inneren Zusammenhang zwischen krisenhaft verlaufenden Modernisierungsprozessen und der Unterstützung populistischer Parteien und Bewegungen gibt« (ebd.: 65): so lag der Anteil von Angehörigen des Mittelstandes (Handwerker, Ladenbesitzer), Arbeitern und Arbeitslosen in der Wählerschaft rechtspopulistischer Parteien zum Teil sehr hoch (ebd.: 54). Der »typische«Wähler der populistischen bzw. extremen Rechten ist demnach männlich, weiß und verfügt über einen niedrigen bis mittleren formalen Bildungsgrad (Arzheimer 2011). Auch unter den AfD-Wählern sind Arbeiter und Arbeitslose überdurchschnittlich hoch vertreten, wobei sich deren Anteil seit Gründung der Partei sukzessive erhöht hat, während der der anderen Gruppen relativ stabil geblieben ist (Kroh/Fetz 2016: 715) ${ }^{4}$. Das Problem der »Modernisierungsverliererthese« ist jedoch der kausale Mechanismus, den sie impliziert, so als ob mit einem bestimmten sozialen Status auch die Unterstützung bestimmter rechtspopulistischer Parteien einherginge. Jüngere Befunde legen eine differenziertere Sicht nahe, und zwar zum einen hinsichtlich der externen Faktoren, zum anderen bezüglich der individuellen Einstellungsmerkmale.

Erstens: Tatsächlich steigt die Wahrscheinlichkeit, Parteien des rechten Rands zu unterstützen, in der bereits erwähnten Gruppe junger, weißer Männer im industriellen Sektor (Arzheimer/Carter 2006: 439). Die Befunde können den Wahlerfolg dieser Parteien jedoch nicht in Gänze erklären. Vielmehr profitieren Arzheimer und Carter zufolge Rechtsparteien von systemischen Faktoren: Zum einen steigen ihre Wahlergebnisse, wenn sich zuvor eine konservative Partei des politischen Mainstreams selbst rechts positioniert hat, zum anderen dann, wenn große Koalitionen herrschen. Spies und Franzmann haben zeigen können, dass Rechtsparteien von der mangelnden ideologischen Polarisierung der mainstream parties profitieren, und zwar sowohl auf der ökonomischen als auch auf der kulturellen Konfliktlinie des politischen Wettbewerbs (Spies/Franzmann 2011). So passt es auch ins Bild, dass die AfD bei der ersten Bundestagswahl von den Wählern im politischen Spektrum nur unweit rechts der Union stehend verortet wurde, während gerade die CDU stark in Richtung Zentrum gerückt war (Wagner et al. 2015).

Zweitens: Verschiedene Studien haben sich der Frage angenommen, inwiefern bestimmte Einstellungsmerkmale und Themenorientierungen bei der Wahl rechtspopulistischer bis -extremer Parteien eine Rolle spielen. Sie schließen damit - zu-

4 Auf Basis von Umfragedaten kommen Niedermayer/Hofrichter 2016 zu einem gegenläufigen Schluss. 
mindest implizit - an die Überlegung an, dass es sich bei Rechtspopulisten um Protestparteien handle, die jenseits ihrer ideologischen Verortung Wählerpotentiale mobilisieren können. Demgemäß wäre also die Wahl rechtspopulistischer Parteien zum großen Teil nur der Tatsache geschuldet, dass es sich um eine Alternative zum Mainstream handelt, unabhängig von ihrer ideologischen Positionierung. Jedoch haben Akkerman und andere für die Niederlande demonstriert, dass die Wähler der rechtspopulistischen Partij voor de Vrijheid (PVV) durch anti-pluralistische und elitistische Haltungen gekennzeichnet sind (Akkerman et al. 2014). Andere Arbeiten zeigen zugleich, dass langfristige Einstellungen sowie Themenorientierung bei der Motivation, für Rechtspopulisten zu stimmen, eine gewichtige Rolle spielen.

Mit Blick auf die AfD konnte gezeigt werden, dass deren Sympathisanten keineswegs >ökonomisch prekäre` Protestwähler sind, sondern über einen mittleren bis hohen Bildungsgrad sowie über ein mittleres bis hohes Einkommen verfügen. Zugleich waren ökonomische Einstellungsmerkmale von denen der Sympathisanten anderer Parteien weniger verschieden als die ablehnende Haltungen gegenüber dem Islam sowie die Befürwortung traditioneller familiärer Ordnungen (Berbuir et al. 2015). Schwarzbözl und Fatke haben hohe Kongruenz zwischen der Programmatik der AfD und ihren Unterstützern herausgearbeitet, und zwar zum einen in der Ablehnung der politischen Eliten, zum anderen in kulturellen Fragestellungen. Die kulturelle Dimension politischer Auseinandersetzung bietet also für den Fall dieser Partei mehr Aussagekraft als die ökonomische Dimension. Hierfür spricht auch, dass qualitative Analysen der Social-Media-Kommunikation eine besondere Beachtung und Resonanz für Themen wie »(Anti-)Political Correctness« oder sog. »(Anti-)Gender « zeigen, mithin keine starken Auseinandersetzungen über Fragen der Wirtschaftspolitik, die die Partei für sich in der Gründungsphase als Kerngeschäft definierte. In sozialen Medien und Parteigliederungen werden darüber hinaus das >Home Schooling` (als Abwehr gegen die >sozialistische und >früh-sexualisierende Erziehung in Schulen) wie auch die `Zerschlagung des öffentlich-rechtlichen Rundfunks (`Staatsmedien`, >Lügenpresse`) diskutiert.

Auch in anderen Kontexten lässt sich ersehen, dass die ökonomische Situation weniger Aussagekraft für die Wahl einer rechtspopulistischen Partei hat als politisch-kulturelle Einstellungsmerkmale (Schwarzbözl/Fatke 2016). Erste Arbeiten, die sich mit der Wählerschaft Donald Trumps bei der jüngsten USPräsidentschaftswahl befassen, deuten an, dass die Unterstützung des rechten Kandidaten vielmehr von der Befürwortung von Rassismus und Sexismus abhängt als von der konkreten ökonomischen Situation der Wähler (Schaffner et al. 2017).

Kurzum profitiert der Rechtspopulismus zwar zu einem großen Teil innerhalb auch ökonomisch bestimmbarer Gruppen; hohe Wahlergebnisse sind aber vor allem auf die Aktivierung des Wählerpotentials durch im weiteren Sinne politische Faktoren, die kulturelle Konfliktlagen adressieren. Diese finden ihren Resonanz- 
und Testraum nicht zuletzt in Netzöffentlichkeiten, auf die wir nun eingehen wollen.

\section{"Post-Truthism «? Netzpolitiken als publizistischer Resonanzraum der Partei Alternative für Deutschland}

Das rechtspopulistische Elektorat ist also durchaus heterogen, ebenso heterogen sind ihre Unterstützungsstrukturen im Netzaktivismus. Durch die Eigenlogik der Netzkommunikation - hier fordert die Welt der Algorithmen, geordnet von ökonomischen Akteuren, denen es vor allem darum geht, dass wir uns lange im Medium aufhalten, geradezu dazu auf, sich nur in den »eigenen Kreisen « zu bewegen - wird es wahrscheinlicher, eher auf politisch Gleichgesinnte zu treffen als auf Gegner.

Blickt man auf Blogs und das publizistische Umfeld der AfD in den Sozialen Medien Twitter und Facebook, so wird deutlich, dass dieses Milieu sehr differente Ansätze verfolgt und sich besonders mittels einer Kritik der und Abgrenzung von Funktionseliten (dem »Mainstream«) beschreibt. Vielfach wird zum Beispiel Misstrauen gegenüber den etablierten Medien artikuliert, zur Vorsicht gegenüber diesen aufgerufen und das Engagement in Social Media nicht zuletzt damit begründet, dass die etablierten Medien als »Staatsmedien« kein Interesse an unabhängiger Berichterstattung hätten. Ebenso kritisch werden Wissenschaft und etablierte Politik gesehen.

Im Netz und auf Demonstrationen entstand so in den letzten Jahren eine (wenngleich heterogene) Gegenkultur mit eigenen Medien, eigener Sprache und eigenen identitätsstiftenden Geschichten. Und dies geschieht nicht nur auf Facebook oder Twitter. So lässt sich im Videomedium Youtube eine Vielzahl von selbstproduzierten bzw. -geschnittenen und kommentierten Videos finden, die Positionen der Partei AfD und ihres Umfeldes artikulieren bzw. in denen sich auf die Partei und ihre Protagonisten bezogen wird. Die Nutzerinnen und Nutzer formulieren einen Vertrauensverlust in jene Medien (»Lügenpresse«), denen sie eine $\mathrm{zu}$ große Nähe zur Regierung und den »Altparteien« vorwerfen und weisen dies unter anderem durch kritische Berichterstattungen über die AfD nach. Ein weiteres sich wiederholendes empirisches Moment dieses Vorwurfes ist die Berichterstattung über Migrationsbewegungen und Migrationsfolgen, insbesondere die positive Berichterstattung im Sinne des von der Bundeskanzlerin Angela Merkel geäußerten Diktums »Wir schaffen das«. Die Beiträge skandalisieren zudem eine Nicht-Berichterstattung über Kriminalität von Geflüchteten und Migranten. Bspw. kritisieren die User eine Abwehr etablierter Medien, über negative Folgen der Migration zu berichten, wie beispielsweise über die sexualisierten Übergriffe auf der Kölner Domplatte zum Jahreswechsel 2016. Und auch das Thema »Gender « wird wie oben schon kurz angeschnitten, als ein Beispiel für eine überbordende 
political correctness herangezogen, die oft Thema kritischer Auseinandersetzung ist (Siri 2015).

Anders als bei anderen bzw. älteren Parteien und Bewegungen fiel bei der Analyse besonders die Heterogenität und Toleranz der Unterstützer gegenüber »eigentlich« ideologisch nicht widerspruchsfrei vermittelbaren Argumenten auf. In und um die Partei AfD finden sich zahlreiche Blogs und Social-Media-Accounts, in denen das Personal und die Aktivitäten der Partei diskutiert und propagiert werden. Dabei handelt es sich weniger um eine durch die Partei instruierte als um eine vielfältige und ideologisch breite Bewegung, die sich - die zuvor genannte Toleranz oder auch Ignoranz als kommunikative Strategie nutzend - vor allem anhand von Querschnittsthemen und die Abwehr von als etabliert und diskursmächtig empfundenen Positionen der Linken und Liberalen definiert. Gemeinsame Themen rekrutieren sich primär aus kulturellen Kämpfen, die anhand der Ausarbeitung von Feindbildern Schließung finden. Wir können also hinsichtlich des Unterstützermilieus durchaus von einer Sammlungsbewegung sprechen, die Differenzen aushält, um Homogenität zu erzeugen. Relevant sind diese Formen der Kommunikation in Sozialen Medien, weil sie als ein Testraum für Themen rechtspopulistischer Themen ebenso genutzt werden wie als Zuträger von möglichen Skandalen und als publizistische Unterstützungsstruktur.

Es nimmt also nicht Wunder, dass sowohl Partei als auch Unterstützer in Sozialen Medien überdurchschnittlich stark aktiv sind. Das Abtesten von konsensfähigen Themen wird intentional wie intuitiv anhand der Beobachtung eines Resonanzraumes im Netz vollzogen, weniger als durch eine Beobachtung der etablierten Medien. Durch das Drehen eigener Videos, Podcasts und Blogs wird zudem eine publizistische Struktur für das Trägermilieu (nicht zuletzt aus diesem heraus) erzeugt.

In Sozialen Medien werden also Themen getestet und auch gesetzt, wie der Umgang der Partei mit Gender-Themen sehr deutlich gezeigt hat. So war das Thema »Anti-Gender« in Sozialen Medien Jahre vor einer programmatischen Umsetzung im Parteiprogramm bereits stark vertreten und Inhalt vieler Netzkampagnen der Partei und ihres publizistischen Umfeldes. Durch vielfältige Interaktionen zwischen Parteifunktionären und Sympathisantenmilieu wurde deutlich, dass das Thema, wenngleich es zunächst nicht auf der Agenda der Partei stand, starke Bindungswirkung und publizistische Wirkung in den Sozialen Medien erzeugen konnte und dementsprechend relevant wurde es für die spätere Selbstdarstellung der Partei (ebd.).

Zahlreiche Kommunikationen formulieren seither auf den alternativen Kanälen der Sozialen Medien ein "Wir schaffen das nicht«, welches mit Beispielen zum Beispiel über deviante Geflüchtete oder skandalisierbare Geschichten über islamischen Terrorismus und die Unterdrückung von Frauen im Islam - unterlegt wird. Dabei wird teilweise auf real nachvollziehbare Ereignisse verwiesen und die 
Form der Berichterstattung an Beispielen kritisiert. Zugleich finden sich aber auch viele Berichte, die im Sinne verschwörungstheoretischer Logik auf polizeilich nicht nachvollziehbare Verbrechen von Migranten an der >deutschen Bevölkerung< verweisen. Nicht stattgefundene - bzw. erfundene - Vergewaltigungen spielen hier eine große Rolle, ebenso Berichte über angebliche Plünderungen von Supermärkten. Die Gefährdung des Nahbereichs der Bevölkerung wird dabei als »Volksverrat « durch die etablierte Politik der »Altparteien« diskutiert. Derlei Phantasien ließen sich einerseits (und rein rational) leicht mittels Fakten widerlegen, finden aber (und so manifestiert sich ihre Persistenz) ihren Resonanzraum in einem Milieu, das es für sehr plausibel hält, dass derlei Vorgänge von einer herrschenden Clique aus Medien und Politik verdeckt und vertuscht werden sollen. Der Widerspruch gegen eine Verschwörungstheorie oder gegen >Fake News $<$, die dem rechtspopulistischen Narrativ zuträglich sind, macht einen Abbruch der Kommunikation oder eine Verlagerung dieser in ein Freund/Feind-Schema, das Gegenargumente aus sich heraus widerlegt, wahrscheinlich.

Es entsteht so eine Teilöffentlichkeit, in der der Widerspruch gegen einzelne Vorfälle entweder nicht geglaubt oder mittels Verweis auf einen weiteren Vorfall gekontert wird. Im zweiten Fall wird die Gegenargumentation kommunikativ nicht widerlegt, sondern anhand der Nennung weiterer Beispiele für die eigene These herausgefordert. Beide Formen ermöglichen hohe Stabilität und Kohärenz der Social-Media-Kommunikation des Unterstützermilieus. Sie ermöglichen eine starke Schließung und Abschottung des Milieus von dem, was als "Mainstream« gelesen wird.

\section{Das Netz als publizistischer Resonanzraum: Suchbewegungen der Kollektivität}

Wie können wir diese empirischen Hinweise nun theoretisch einordnen? Wie stabilisiert sich das rechtspopulistische Milieu? Und was bedeutet es, wenn Teile der Öffentlichkeit - also des Publikums - die Verfahren und die Entscheidungen nicht als kollektiv verbindlich ansehen und dem Parlamentarismus die Forderung nach Volksbefragungen entgegenrufen? Zur Bearbeitung dieser Fragen scheint es uns nötig zu sein, eine gesellschaftstheoretische Perspektive einzuführen, mit der die Grenzen von Organisation, Massenmedien und Sozialen Medien scharf gestellt werden, um die Stabilisierungsbedingungen des aktuellen Rechtspopulismus zu ergründen.

Herkömmliche soziologische Perspektiven auf politische Öffentlichkeiten >vor dem Computer gehen davon aus, dass Gatekeeper und Dominanz über relevante Themen ein notweniger Selektionsmechanismus sei, der von Massenmedien bereitgestellt werde (Habermas 1990; Luhmann 2009). 
Soziale Medien und andere Formen der computervermittelten Interaktion machen deutlich, dass diese Dominanz bröckelt und sich alternative Medienangebote für spezifische Publika herausbilden. Die Erfahrung gleichzeitiger und voneinander abweichender Publika - die voneinander kaum mehr wissen müssen und ggf. auch wollen - wird oft mit dem Effekt der Filter-Bubble oder "Echokammer ${ }^{5}$ beschrieben. Jeder Nutzer und jede Nutzerin findet eine spezifische Medienwirklichkeit vor, die von eigenen Entscheidungen der Mediennutzung abhängt und es wahrscheinlicher macht, zu spezifischen Themen spezifische Informationen algorithmisch dargereicht zu bekommen. Der Soziologie Dirk Baecker spricht im Zusammenhang mit der Erfindung des Computermediums sogar von dem Übergang in eine »nächste Gesellschaft« (Baecker 2007). Die Evolution des Computermediums stelle eine gesellschaftliche Katastrophe dar, welche die Logiken der funktionalen Differenzierung konterkariere.

Ausgehend von einer Theorie der Differenzierung sozialer Systeme lässt sich das politische System als ein Machtkreislauf von Politik, Publikum und Verwaltung begreifen, zumeist aufgehoben in einem nationalstaatlichen Gebilde (Luhmann 1981: 42ff.). Abseits normativer Erwägungen formuliert diese Theorie, dass die Ausdifferenzierung eines autonomen Systems für Politik, ihrer Verfahren und Institutionen, die »Bereithaltung der Kapazität zu kollektiv bindendem Entscheiden« (derslb. 2002: 84, Hervorh. i. Org.) zum Ziel hat. »In all diesen Fragen, die zu Kontroversen und Konflikten Anlass geben könnten, kommt es politisch letztlich auf die Durchsetzung der Entscheidung über Prämissen von Entscheidungen an«, so Luhmann schmittianisch (2002: 85). Das Erkenntnisinteresse unseres Aufsatzes und die empirischen Beispiele, die wir besprechen, betonen einen anderen Teil der Luhmannschen Funktionsformel: nämlich den der Herstellung von Kollektiven.

Armin Nassehi hat darauf hingewiesen, dass es in der modernen Gesellschaft um mehr gehe als die Bereithaltung von Entscheidungsfähigkeit und die Selektion politischen Personals. Vielmehr werde die Herstellung und Sichtbarmachung von Zurechenbarkeit - mithin: die Sichtbarmachung eines zurechenbaren Publikums - zur vornehmlichen Herausforderung für politische Kommunikationen in Demokratien. ${ }^{6}$ Moderne Demokratien schulen ihre Organisationen und Bewegungen vor allem in einem: in der Suche nach einem Publikum, welches nicht mehr vorausgesetzt werden kann. Eine Öffentlichkeit im Singular, ein vorausgesetztes und berechenbares Publikum gibt es nicht. Die Suchbewegungen nach einem kommunikativen Trägermilieu erklären sich genau hieraus.

»Denn das Medium, in dem solche Sichtbarkeit und Zurechenbarkeit hergestellt wird, sind unterstellte Kollektivitäten, für die Sichtbarkeit und Transparenz kol-

5 Sunstein 2001.

6 Nassehi 2003, 2006: 342ff. 
lektiv wirksamer Kausalitäten ebenso hergestellt wie diese dadurch erst erzeugt werden.«(Nassehi 2006: 345).

Das System des liberalen Konstitutionalismus, so Luhmann, rechne nicht mit Störquellen wie Kapitalismus oder, man möchte ergänzen, einem Computermedium, das die Idee einer Öffentlichkeit im Singular dekonstruiert und pluralisierte Öffentlichkeiten entstehen lässt.

Die Gesellschaft des 19. Jahrhunderts löste ihre Konflikte nicht zuletzt über die Erfindung der Organisation als Vertretungen von Mitgliederinteressen und Organisatorin von Karrieren, Arbeitsbedingungen und Biographien. Durch die Einführung des Computermediums und seiner pluralisierten Öffentlichkeiten ergibt sich ein von der Organisation kommunikativ getrennter (und sie damit weithin entlastender) Testraum für die politische Kommunikation wie auch ein Ort, an dem die Suche nach und die Konstruktion der Unterstützerschaft stattfinden kann. Hierin begründet sich die hohe Zahl der Provokationen, die rechtpopulistische Politiken in die Social-Media-Kommunikation einbringen. Denn im Spiel aus Provokation, Leugnung der Provokation und und Re-Iteration der Leugnung werden hier Themen der politischen Auseinandersetzung verdichtet und die Anschlussfähigkeit an ein heterogenes Publikum getestet (Siri 2016: 101). Dabei wird ein Sog zu Kulturargumenten deutlich, die sich für die politische Kommunikation in Social Media besonders gut zu eignen scheinen. Und es wird auch deutlich, dass Modelle des Konsenses oder der Deliberation für Teile des Elektorats weit weniger plausibel sind als ein Bezug auf identitär geformte und anti-pluralistische Kollektive, die sich vielmehr einer Auseinandersetzung stellen sollen als Konsens und den Interessensausgleich zu suchen.

Zugleich dekonstruiert das Computermedium endgültig das, was mit dem Beginn der Wahlforschung als »öffentliche Meinung (im Singular) diskutiert wurde. Öffentliche Meinung bezeichnet im Allgemeinen einen aus Daten abgeleiteten Mittelwert politischer Haltungen und Forderungen »des Volkes«, diese Beschreibung blieb natürlich auch `vor dem Computer « nicht unwidersprochen. So kritisierte Elisabeth Noelle-Neumann bereits in den 1980er Jahren, dass die Orientierung an einer scheinbaren Mehrheitsmeinung stark medial geprägt sei und in einer Schweigespirale resultieren könne (Noelle-Neumann 1989) und Pierre Bourdieu formulierte im Nachgang einer Kritik an Postulaten der Meinungsforschung, dass es die öffentliche Meinung nicht gebe (1993). Dies vorausgesetzt: Die Oberflächen und Algorithmen der Sozialen Medien haben die Konstruktion einer Mehrheitsmeinung weiter prekarisiert und bieten paradoxerweise identitären Kollektivitätskonstruktionen eine neue Heimat. ${ }^{7}$

7 Und dies übrigens nicht nur im rechten Spektrum, sondern auch in den vielen Formen der Identity Politics linker Bewegungen. 


\section{Fazit: "Wir sind das Volk»: \\ Zur Herausbildung eines Diskurses der identitären Demokratie im Umfeld der Partei Alternative für Deutschland (AfD)}

Für die AfD haben die vorangestellten Ausführungen das Folgende gezeigt: Sie ist keine Protestpartei in dem Sinne, dass sie allein die Enttäuschung ökonomischer Erwartungen - oder umgekehrt: die Angst vor sozialem Abstieg - artikuliert. Vielmehr profitiert die Partei von einer jetzt sichtbar werdenden populistischen Demokratieauffassung, die sich im Nachgang der Euro- und besonders im Zuge der Flüchtlingskrise Bahn bricht und sich im Hinblick auf die politische Kommunikation der Partei nicht zuletzt aus der Beherrschung Sozialer Medien erklärt. Ebenso wie Pegida (dort allerdings stark regional begrenzt) ist die AfD Nutznießerin und Produzentin einer diffusen identitären Demokratievorstellung, die sich durch dreierlei Merkmale auszeichnet: Erstens die Forderung nach der uneingeschränkten Ausführung des Volkswillens, zweitens das grundlegende Misstrauen gegenüber den Repräsentanten der repräsentativen Demokratie und des Rechtsstaats (Funktionseliten) sowie drittens die gefühlte Bedrohung der kulturellen Identität durch abgrenzbare Fremdgruppen, allen voran aktuell »der Islam« und Geflüchtete. Die Grenzen zwischen Populismus und autoritären Demokratiekonzeptionen erscheinen dabei fließend. Der Populismus des 21. Jahrhundert geriert sich in geradezu apokalyptischem Duktus als (einziger) Verteidiger der Demokratie - gleichgesetzt mit dem Majoritätsprinzip - gegen das Establishment und »Fremde«. Deutlich wird ebenfalls, dass das Konzept einer identitären Demokratie mit dem besonnenen Konservatismus der Nachkriegszeit in Konkurrenz tritt. Der Konservatismus z.B. Merkelscher Façon ist der identitären Konzeption fast noch verhasster als eine sozialdemokratische oder linke Programmatik. Mit Karl Mannheim kann dies als Re-Aktualisierung des revolutionären Konservatismus im Gegensatz zu einem auf Subsidiarität und Regionalität verpflichteten, traditionellen Altkonservatismus bezeichnet werden, verstehen sich aktuelle Rechtspopulisten doch als Kritiker der staatsaffinen und konsensorientierten konservativen Politiken der Nachkriegszeit. (Mannheim 1984; vgl. auch Mannheim 1980; Siri 2015). Hier wird ein Schisma im Konservatismus deutlich, welches für die BRD zuletzt in der Weimarer Republik festgestellt werden konnte. Doch auch wenn identitäre Elemente eine relevante Form moderner rechtspopulistischer Kommunikation darstellen: Die konkreten Themen der politischen Kommunikation sind ideologisch-programmatisch keinesfalls festgelegt, sondern werden in alternativen Öffentlichkeiten der Sozialen Medien verhandelt und von der Parteiorganisation "getestet« und aktuellen Entwicklungen rangepasst‘.

In den Filter-Bubbles der Sozialen Medien konstituiert sich das kommunikative Trägermilieu der Politiken des Rechtspopulismus. Das Netz ist dabei Möglichkeitsspielraum in dem Sinne, dass in etablierten Medien sanktionierte Botschaften 
hier (relativ) sanktionsfrei geäußert werden können. Zudem bringt das Netzmedium aufgrund der personalisierten Algorithmen Möglichkeiten der Isolierung von politischen Szenen mit sich, die es ermöglicht, relativ frei von der Erfahrung kognitiver Dissonanz und diskursiver Auseinandersetzung politische Haltungen in einem homogenen Diskussionszusammenhang zu vertreten. Dadurch werden positive und konfliktfreie Sozialisationserfahrungen in dem Milieu des Rechtspopulismus wahrscheinlicher.

Die in interaktionsnahen sozialen Gruppierungen wie Pegida, rechten Blogs und in Sozialen Medien wie Facebook und Twitter geäußerten Kritiken bilden im Sinne dieser neuen medialen Konfiguration politischer Öffentlichkeiten den Resonanzraum für die (Oppositions-)Politiken der AfD-Parteiorganisation. Sie bilden ein Publikum, zu dem die Partei vornehmlich spricht, einen Testraum für (Selbst)Skandalisierungen, die der Medienresonanz, dem Austesten von Konsenschancen wie auch der Identitätsbildung dienen. Die Selbststabilisierung des Milieus erfolgt also durch wechselseitige Beobachtungen und Bestätigung von Partei und Anhängern in Sozialen Medien und ersetzt damit zumindest potentiell die Interaktion in politischen Räumen abseits des Netzes. Die Unschärfe in der Argumentation, der Bezug auf Kultur und die Provokation eröffnen dabei hohe Toleranz für Abweichung. So lässt sich zum Beispiel erklären, dass die Teilnehmerschaft von Demonstrationen und die Kommentatorenschaft in Social Media über einen weiten range enttäuschter Konservativer und Liberaler, über Protestwähler, über fundamentalistische Christen und »Lebensschützer«, über Maskulinisten und Verschwörungstheoretiker bis hin zu Neonazis reicht. Konzepte identitärer Demokratie sprechen aus dieser Perspektive nicht für einen einfachen >Backlash<, sondern sind vielmehr eine sehr moderne Angelegenheit. Sie sind Resultat einer im Computermedium vermittelten Suchbewegung nach Kollektivität, die sich eben nicht ausschließlich an die Idee einer Nation oder eines Staatsvolkes gebunden sieht und programmatische Unschärfen ebenso aushält wie ein heterogenes Publikum.

\section{Literatur}

Akkerman, Agnes/Mudde, Cas/Zaslove, Andrej 2014: How Populist Are the People? Measuring Populist Attitudes in Voters. In: Comparative Political Studies, Heft 9, S. 1324-1353.

Arzheimer, Kai 2011: Electoral Sociology - Who Votes for the Extreme Right and Why - and When?. In: Uwe Backes/Patrick Moreau,(Hg.), The Extreme Right in Europe, Current Trends and Perspectives, Göttingen, S. 35-50.

Arzheimer, Kai 2015: The AfD: Finally a Successful Right-Wing Populist Eurosceptic Party for Germany?. In: West European Politics, Heft 3, S. 535-556. 
Arzheimer, Kai/Carter, Elisabeth 2006: Political opportunity structures and right-wing extremist party success. In: European Journal of Political Research, Heft 3, S. 419-443.

Baecker, Dirk 2007: Studien zur nächsten Gesellschaft, Frankfurt a.M.

Bebnowski, David 2015: Die Alternative für Deutschland. Aufstieg und gesellschaftliche Repräsentanz einer rechten populistischen Partei, Wiesbaden.

Berbuir, Nicole/Lewandowsky, Marcel/Siri, Jasmin 2015: The AfD and its Sympathisers: Finally a Right-Wing Populist Movement in Germany?. In: German Politics, Heft 2, S. 154-178.

Bourdieu, Pierre 1993: Die öffentliche Meinung gibt es nicht. In: Ders., Soziologische Fragen, Frankfurt a.M., S. 212-223.

Decker, Frank 2004: Der neue Rechtspopulismus, Opladen.

Decker, Frank 2015a: Parteiendemokratie im Wandel. Beiträge zu Theorie und Empirie, Baden-Baden.

Decker, Frank 2015b: Alternative für Deutschland und Pegida: Die Ankunft des neuen Rechtspopulismus in der Bundesrepublik. In: Frank Decker/Bernd Hennigsen/Kjetil A. Jakobsen (Hg.), Rechtspopulismus und Rechtsextremismus in Europa, Baden-Baden, S. 75-90.

Decker, Oliver/Kiess, Johannes/Brähler, Elmar 2012: Die Mitte im Umbruch. Rechtsextreme Einstellungen in Deutschland 2012. Bonn.

Franzmann, Simon T. 2014: Die Wahlprogrammatik der AfD in vergleichender Perspektive. In: Mitteilungen des Instituts für Parteienrecht und Parteienforschung, Heft 20, S. 115-124.

Greve, Jens/Heintz, Bettina 2005: Die >Entdeckung< der Weltgesellschaft. Entstehung und Grenzen der Weltgesellschaftstheorie. In: Zeitschrift für Soziologie, Sonderheft »Weltgesellschaft«, S. 89-119.

Habermas, Jürgen 1990: Strukturwandel der Öffentlichkeit, Neuauflage, Frankfurt a.M.

Häusler, Alexander 2013: Die »Alternative für Deutschland« - eine neue rechtspopulistische Partei? Materialien und Deutungen zur vertiefenden Auseinandersetzung. Düsseldorf.

Jankowski, Michael/Schneider, Sebastian/Tepe, Markus 2016: Ideological alternative? Analyzing Alternative für Deutschland candidates' ideal points via black box scaling. In: Party Politics (online first).

Kroh, Martin/Fetz, Karolina 2016: Das Profil der AfD-AnhängerInnen hat sich seit Gründung der Partei deutlich verändert, DIW-Wochenbericht, Heft 34, S. 711-719.

Lewandowsky, Marcel 2015: Eine rechtspopulistische Protestpartei? Die AfD in der öffentlichen und politikwissenschaftlichen Debatte. In: Zeitschrift für Politikwissenschaft, Heft 1, S. 121-135. 
Lewandowsky, Marcel/Giebler, Heiko/Wagner, Aiko, 2016: Rechtspopulismus in Deutschland: Eine empirische Einordnung der Parteien zur Bundestagswahl 2013 unter besonderer Berücksichtigung der AfD. In: Politische Vierteljahresschrift, Heft 2, S. 247-275.

Luhmann, Niklas 1981: Politische Theorie im Wohlfahrtsstaat. München und Wien. Luhmann, Niklas,2002: Die Politik der Gesellschaft, Frankfurt a.M.

Luhmann, Niklas 2009: Die Realität der Massenmedien, Wiesbaden.

Mannheim, Karl 1980: Strukturen des Denkens, Frankfurt a.M.

Mannheim, Karl 1984: Konservatismus. Ein Beitrag zur Soziologie des Wissens, Frankfurt a.M.

Mudde, Cas 2004: The Populist Zeitgeist. In: Government and Opposition. Heft 4, S. 541-563.

Mudde, Cas 2007: The Populist Radical Right in Europe, Cambridge.

Nassehi, Armin 2003: Politik des Staates oder Politik der Gesellschaft? Kollektivität als Problemformel des Politischen. In: Kai-Uwe Hellmann (Hg.), Das System der Politik, Opladen, S. 38-59.

Nassehi, Armin 2006: Der soziologische Diskurs der Moderne, Frankfurt a.M.

Niedermayer, Oskar/Hofrichter, Jürgen 2016: Die Wählerschaft der AfD: Wer ist sie, woher kommt sie und wie weit rechts steht sie? In Zeitschrift für Parlamentsfragen, Heft 2, S. 267-284.

Noelle-Neumann, Elisabeth 1989: Die Theorie der Schweigespirale als Instrument der Medienwirkungsforschung. In: Kölner Zeitschrift für Soziologie und Sozialpsychologie, Sonderheft »Massenkommunikation. Theorien, Methoden, Befunde«, S. 418-440.

Pauwels, Teun 2011: Measuring Populism: A Quantitative Text Analysis of Party Literature in Belgium. In: Journal of Elections, Public Opinion \& Parties, Heft 1, S. 97-119.

Pirro, Andrea L. 2014: Populist Radical Right Parties in Central and Eastern Europe: The Different Context and Issues of the Prophets of the Patria. In: Government and Opposition, Heft 4, S. 600-629.

Rooduijn, Matthijs/Pauwels, Teun 2011: Measuring Populism: Comparing Two Methods of Content Analysis. In: West European Politics, Heft 6, S. 1272-1283.

Schaffner, Brian F./Mac Williams, Matthew/Nteta, Natishe 2017: Explaining White Polarization in the 2016 Vote for President: The Sobering Role of Racism and Sexism. Paper prepared for presentation at the Conference on The U.S. Elections of 2016: Domestic and International Aspects. January 8-9, 2017, IDC Herzliya Campus.

Schwarzbözl, Tobias/Fatke, Matthias, 2016:Äußer Protesten nichts gewesen? Das politische Potenzial der AfD. In: Politische Vierteljahresschrift, Heft 2, S. 276-299.

Siri, Jasmin, 2012: Parteien. Zur Soziologie einer politischen Form, Wiesbaden. 
Siri, Jasmin, 2015: Rechter Protest? Zur Paradoxie konservativer Protestbewegungen. In: Sabine Hark/Paula-Irene Villa (Hg.), (Anti-)Genderismus. Sexualität und Geschlecht als Schauplätze aktueller politischer Auseinandersetzungen, Bielefeld, S. 239-255.

Siri, Jasmin 2016: Zur Aktualität von Karl Mannheims Analyse des politischen Konservatismus. In: Martin Endreß/Klaus Lichtblau/Stephan Moebius (Hg.), Zyklos 3. Jahrbuch für Theorie und Geschichte der Soziologie, Wiesbaden, S. 91-111.

Spier, Tim 2006: Populismus und Modernisierung. In: Frank Decker (Hg.), Populismus. Gefahr für die Demokratie oder nützliches Korrektiv? Wiesbaden, S. 33-58.

Spies, Dennis C./Franzmann, Simon 2011: A Two-Dimensional Approach to the Political Opportunity Structure of Extreme Right Parties in Western Europe. In: West European Politics, Heft 5, S. 1044-1069.

Sunstein, Caas, 2001: Echo Chambers: Bush v. Gore, Impeachment, and Beyond, Princeton.

Vorländer, Hans/Herold, Maik/Schäller, Steven 2016: PEGIDA. Entwicklung, Zusammensetzung und Deutung einer Empörungsbewegung, Wiesbaden.

Wagner, Aiko/Lewandowsky, Marcel/Giebler, Heiko, 2015: Alles neu, macht der Mai? Die AfD und die Europawahl 2014. In: Kaeding, Michael/Switek, Niko: Die Europawahl 2014. Spitzenkandidaten, Protestparteien, Nichtwähler, Wiesbaden, S. $137-148$. 



\section{Identitätserzählungen im Konflikt Der Wettstreit der Narrative im israelischen Kulturkampf}

Jan Christoph Suntrup

\section{Einleitung: Zur Notwendigkeit einer kulturwissenschaftlichen Vertiefung der politischen Kulturforschung ${ }^{1}$}

Die Politikwissenschaft, zumindest in ihrer deutschen Tradition, hat lange Zeit die reichhaltigen methodischen, theoretischen und epistemologischen Debatten im Zuge des cultural turn ignoriert und ist bis heute nicht selten der Illusion erlegen, bei >Kultur handele es sich im Wesentlichen um abfragbare Einstellungen von Bürgern und Eliten zu verschiedenen sozialen Objekten. Wird hingegen der Blick auf klassische oder aktuelle kultursoziologische Theorien gerichtet oder sogar auf die transdisziplinäre geistes- und sozialwissenschaftliche Kulturforschung insgesamt, wird das Defizit einer solch kognitivistisch-mentalistischen Engführung offensichtlich, die nichts über die Genese und Reproduktion von bestimmten subjektiven Orientierungen oder die kollektive Verbindlichkeit und empirische Legitimität von Vorstellungen auszusagen vermag, also deren prozessuale und performative Dimension. Eine neujustierte politische Kulturforschung sollte somit Symbole, Rituale und andere Praktiken, materielle Konstrukte und ähnliches adressieren, nicht um der vergänglichen Konjunktur der Proklamation immer neuer turns zu folgen, sondern um das über sehr lange Zeit gewachsene Analysepotential einer kulturwissenschaftlichen Perspektive zu nutzen und dadurch auch eingefahrene Deutungsmuster gewinnbringend $\mathrm{zu}$ irritieren.

In diesem Beitrag soll exemplarisch das Konzept des >Narrativs`im Vordergrund stehen, das erst allmählich Einzug in die Politikwissenschaft und viele Nachbardisziplinen erhält. Die Relevanz von Erzählungen für die Kulturforschung liegt darin, dass sich in ihnen »mentale Dispositionen [manifestieren], also kollektiv vorherrschende Denkweisen, Überzeugungen, Normen und Wissensordnungen, durch die eine Kultur beobachtbar wird« (Nünning 2013: 28). Nach einer kurzen

1 Dieser Beitrag greift in Teilen auf Auszüge aus dem Kapitel »Die narrative Dimension des Rechts« aus meinem Buch »Umkämpftes Recht. Zur mehrdimensionalen Analyse rechtskultureller Konflikte durch die politische Kulturforschung«, Frankfurt a.M.: Klostermann, 2018, zurück. 
Einordnung eines solches erzähltheoretischen Zugangs soll ein spezifischer Typ von Narrativen, nämlich derjenige öffentlicher identitätspolitischer Erzählungen, im Vordergrund stehen. Die Analyse des gegenwärtigen israelischen Kulturkampfes zwischen säkularen und religiösen Kräften profitiert nicht nur von einem narratologischen Ansatz, sondern vermag generelle Funktionen und Dynamiken politischer Narrative zu verdeutlichen.

\section{Narrative als politikwissenschaftliches Forschungsfeld}

»Wo immer sozial Bedeutsames verhandelt wird, ist das Erzählen im Spiel« - diese simple Einsicht, die Albrecht Koschorke (2012: 19) in seiner Allgemeinen Erzähltheorie formuliert, ist nur sehr langsam ins Bewusstsein der analytisch operierenden Politikwissenschaft gerückt. Als philosophischer und nicht nur literatur- und sprachwissenschaftlicher Terminus sind die >Erzählungen`im 20. Jahrhundert im Moment ihrer partiellen Verabschiedung zu großem Ruhm gekommen. Jean-François Lyotard konstatierte 1979 in seinem Bericht über La condition postmoderne (Lyotard 2005: 63-68) den finalen Legitimationsverlust der grands récits, der großen spekulativen und emanzipatorischen Theorien, welche die Moderne ausgezeichnet habe. $\mathrm{Zu}$ diesen sogenannten >Metaerzählungen zählte Lyotard an anderer Stelle

»progressive Emanzipation von Vernunft und Freiheit, progressive oder katastrophische Emanzipation der Arbeit (Quelle des entfremdeten Werts im Kapitalismus), Bereicherung der gesamten Menschheit durch den Fortschritt der kapitalistischen Techno-Wissenschaft und sogar, wenn man das Christentum selbst zur Moderne zählt (also im Gegensatz zum antiken Klassizismus), Heil der Kreaturen durch die Bekehrung der Seelen zur christischen (cristique) Erzählung von der Märtyrerliebe« (Lyotard 1987: 32).

Mit der Bezeichnung dieser wirkmächtigen Theorien als Erzählung wird ihr fiktionales Element betont und insbesondere auf eine gewisse narrative Struktur hingewiesen, weisen sie doch einen plot auf, der bestimmte Ereignisse und Aktionen miteinander verkettet - und zwar trotz ihrer augenscheinlichen Disparatheit auf analoge Weise. Lyotards Einschätzung, dass diese Art der teleologischen, eine notwendige Entwicklungsgeschichte postulierenden Theoriebildung vollständig an Überzeugungskraft eingebüßt habe, war dabei weniger resignativ als häufig unterstellt und alles andere als ein neokonservativer Abgesang auf das kritische Denken, dessen Weg sie doch im Gegenteil erst ebnen wollte (Suntrup 2010: 331-346). Viel bedeutsamer ist jedoch die paradigmatische Bedeutung, die von Lyotards Analysen ausging; und hier ist nicht nur das proklamierte Ende der großen Erzählungen von Belang, sondern auch die grundsätzliche »narratologische Wende in der Philosophie« (Müller-Funk 2008: 64), die er einleitete. In La condition postmoderne beschrieb 
Lyotard den Grundkonflikt der Wissenschaft mit narrativen Wissensformen traditionaler Gemeinschaften, wobei sich letztere nach szientistischen Kriterien in der Regel als nicht wahrheitsfähig, als bloße Fabeln erwiesen. Lyotard hielt einer solchen Dichotomie entgegen, dass die Wissenschaft zum Zwecke ihrer eigenen Legitimation selbst auf Narrationen angewiesen sei (Lyotard 2005: 7/51).

Jene Einsicht, dass die Erzählung nicht nur im Reservat der Literatur oder bei den oralen Wissenskulturen »traditionaler« Gemeinschaften anzutreffen ist, sondern weite Wissens- und Erkenntnisformen strukturierend durchzieht, hat dazu beigetragen, dass der »Begriff der Erzählung zu einer der transdisziplinär erfolgreichsten und expansionsfreudigsten literaturwissenschaftlichen Kategorien « (Koschorke 2012: 19) geworden ist. Diese Diffusion hat dabei eine auch nur annähernd verbindliche terminologische Verständigung bedeutend erschwert, die oft schon am unterschiedlichen Erkenntnisinteresse scheitert. Die klassische strukturalistische Narratologie, die sich - unter Rückgriff auf Erkenntnisse der Russischen Formalisten - seit den 1960er Jahren entwickelte, war auf die generelle Analyse der erzählerischen Strukturen fiktionaler Texte gerichtet, bildet also in dieser Hinsicht das genaue Gegenteil einer kulturwissenschaftlichen Analyse von Narrativen, die komparativ auf den spezifischen sozialen Kontext überwiegend nicht-fiktionaler Erzählungen blickt (Nünning 2009: 48f.). Mit diesen verschiedenen Perspektiven gehen wenig überraschend voneinander abweichende Kategorienapparate einher.

Die analytische Politikwissenschaft hat sich dabei, vor allem in Deutschland, erst in den letzten Jahren verstärkt narratologischen Ansätzen geöffnet (Gadinger/Jarzebski/Yildiz 2014b; Hofmann/Renner/Teich 2014), während der politischen Theorie der Gedanke der Narrativität schon lange vertraut ist, wie schon ein kursorischer Blick auf das westliche politische Denken der zweiten Hälfte des 20. Jahrhunderts zeigt. Für Hannah Arendt war nicht nur »das Bezugsgewebe menschlicher Angelegenheiten «, also der politische Zwischenraum zwischen den Menschen, wesentlich narrativ, nämlich durch Sprechen und Handeln geprägt (2007: 222-234), sondern auch das theoretische Nachdenken über Politik, das sie als "storytelling" verstand (Benhabib 1990: 186-191): als sinnstiftende Aktivität, welche die Lücke zwischen Vergangenheit und Zukunft stets neu zu betrachten habe (Arendt 2006: 947). Arendt, aber auch etwa Michael Walzer und Martha Nussbaum setzen sich für eine erfahrungsgesättigte Art der Theoriebildung in den Bereichen der Moral und der Politik ein, die auf in Narrationen tradierte Erfahrungen von Individuen und Kollektiven rekurriert (Straßenberger 2005). Insbesondere bei den nordamerikanischen kommunitaristischen Philosophen (zu denen ja auch Walzer zu zählen ist) wird dann das narrative Bezugsgewebe einer konkreten Gemeinschaft zum sinnstiftenden und identitätspolitischen Faktor; ob bei Charles Taylor, der den Menschen als »sich selbst interpretierendes Tier« (1975: 171) konzipiert, das im Rahmen eines »sozialen Imaginären « Gestalt annimmt, nämlich vor dem Hintergrund von Bildern, Geschichten und Legenden (Taylor 2004: 23); oder in der überaus mo- 
dernisierungskritischen Erzählung After Virtue von Alasdair MacIntyre, in der der Mensch als »story-telling animal« auftaucht:

»I can only answer the question What am I to do? « if I can answer the prior question >Of what story or stories do I find myself a part? [ [...] Deprive children of stories and you leave them unscripted, anxious stutterers in their actions as in their words. Hence there is no way to give us an understanding of any society, including our own, except through the stock of stories which constitute its initial dramatic resources.«(Maclntyre 2007: 216)

MacIntyre hat großen Wert darauf gelegt, die anthropologische Notwendigkeit der Sinnkonstitution durch das Erzählen von Geschichten nicht mit einem freien Selbstentwurf des Individuums, das sich seine eigene Geschichte kreiert, zu verwechseln - das ironische Spiel mit verschiedenen Geschichten und die Fluidität von sozialen Rollen und persönlichen Identitäten sind ihm vielmehr Symptome des konstatierten Tugendverlustes. Das Individuum ist immer Teil einer Gemeinschaftsgeschichte und bestenfalls deren Co-Autor (MacIntyre 2007: 213). Welche normativen Schlüsse in Bezug auf die je eigene Gemeinschaft aus diesen sozialanthropologischen Überlegungen abzuleiten sind, bleibt auch nach dem Abflauen der Debatte zwischen Kommunitaristen und Liberalen unentschieden; jedoch kann der narrative Gemeinschaftsbezug auf der sozialontologischen, also Subjekte sozialisierenden Ebene nicht ernsthaft bestritten werden. So ist die anthropologische Auszeichnung des Subjekts als »homo narrans « (Koschorke 2012: 9) durchweg plausibel: Der menschliche Weltbezug ist nicht nur sprachlich, sondern narrativ verfasst, um dem »Absolutismus der Wirklichkeit« (Blumenberg 1996: 9-39) zu begegnen. Dies bedeutet, »dass die Erzählung eine kulturelle Universalie bildet« (Koschorke 2012: 10) - mit der Folge, dass es kaum mehr möglich sein dürfte, das Narrative per se mit einer Schwundstufe von Rationalität in Verbindung zu setzen, wie es die einflussreiche platonische Trennung von >Mythos< und >Logos< suggerierte.

Erzählen als eine kulturelle Universalie zu verstehen, bedeutet nicht, dass jede Form der Sinnstiftung oder jede Form der Textualität schon narrativ strukturiert ist, wenn man nicht jegliche Differenz der Kommunikationsmodi einebnen will. Argumente lassen sich auch ohne plot, ohne die prozessuale Verknüpfung von Ereignissen, vorbringen. Das gilt selbst dann, wenn vermeintlich nüchtern und rein rational vorgetragene Gründe nicht selten versteckte narrative Züge oder Einbettungen aufweisen und umfassende politische Reden kaum ohne Erzählelemente zu denken sind, wobei deren Kraft durchaus in der Latenz liegen kann:

»Die wirksamsten Erzählungen sind nicht die manifesten, sondern die latenten, die selbstverständlich geworden sind und nur gelegentlich zelebriert zu werden brauchen. Erst im Kampf um Bedeutung, wie es der Alltag moderner Cesellschaften ist, treten die narrativen Grundmuster zutage« (Müller-Funk 2008: 14). 
Aber in einen "Pan-Narrativismus« (von Arnauld 2009: 47, in einem anderen Kontext) $\mathrm{zu}$ verfallen, wie es beispielsweise auch Ansätze machen, die jede Form der Theoriebildung als Erzählung verstehen ${ }^{2}$, ist kein konzeptueller Fortschritt.

Was macht nun die Funktion der universalen Kulturtechnik des Erzählens aus? Ihre Kraft transzendiert bei weitem die archaischen Motive des Vertreibens von Zeit und Furcht, die Hans Blumenberg zunächst in den Vordergrund gerückt hat (1996: 40). Das Motiv der Sinnstiftung geht deutlich darüber hinaus:

»Als steter Sinnsucher transformiert der Mensch die Unübersichtlichkeit seiner Erlebnisse und die mit ihnen verbundenen Kontingenzerfahrungen in den Prozessen einer narrativen Wahrnehmung und Ordnung zu Verständlichkeit, Plausibilität und Zielgerichtetheit. Was zuvor unverbunden und zufällig war oder so zu sein schien, wird in der narrativen Bearbeitung zu einer Geschichte aus einem Cuss das gilt für die erzählte Lebensgeschichte eines einzelnen wie für die Ceschichtserzählung eines Kollektivs.«(Bergem 2009: 205)

Diese Art der Kontingenzbewältigung ist sicherlich eine der wesentlichen kulturellen Funktionen von Erzählungen - wenn Albrecht Koschorke auch zu Recht darauf hinweist, dass Narrationen Sinn nicht nur konstituieren, sondern auch destruieren können (2012: 11). Geschichten lassen sich in Frage stellen, so dass Kontingenz nicht bewältigt, sondern sichtbar gemacht wird. Oder sie lassen sich umerzählen (Viehöver 2014: 73f.), was Fragen nach hegemonialem Sinn, Deutungsmacht und den »Kämpfe[n] um Artikulationschancen« (Gadinger/Jarzebski/Yildiz 2014a: 11) aufwirft. Während die Stärke mancher Erzählungen in ihrer Latenz liegt, kann die Bindungskraft anderer Narrationen erst aus der Dynamik von expliziter Rechtfertigung und Kritik erwachsen (ebd.: 10).

Eine generelle Eigenschaft von Narrationen ist es, dass es sich bei ihnen nicht um bloße Repräsentationen, um Nach-Erzählungen der sozialen Welt handelt; sie sind vielmehr wirklichkeitserzeugend und in diesem Sinn performativ (Nünning 2013: 40; Viehöfer 2014: 73). Zwar lassen sich Narrationen durchaus auf ihren fiktionalen Gehalt und ihre Übereinstimmung mit bestimmten Fakten abklopfen (Shenhav 2006). Ihr Realitätsstatus hängt aber nicht vom Grad der artikulierten Wahrheit $\mathrm{ab}$, sondern von ihrer Wirkmächtigkeit in Form sozialer Konsequenzen; und nicht

2 So zumindest tendenziell Margaret Somers (1994: 620), wenn sie von »conceptual narrativity« spricht, um darunter mit wissenschaftlichem Erklärungsanspruch auftretende Theorieerzählungen zu fassen. Dieser Begriff verdeckt, dass Theorien, auch wenn sie nicht selten erzählerische Elemente enthalten, in der Regel weder in dieser Erzählstruktur aufgehen, da sie eine Explikationskraft behaupten, die nicht mit der von Geschichten identisch ist, noch überhaupt konstitutiv auf Narrationen angewiesen sind. Allerdings ist es durchaus richtig, dass sich Theorien desto weniger dem Zwang zur Erzählstruktur völlig entziehen können, je umfassender ihr sozialtheoretischer Anspruch ist. 
nur Bruno Latour hat darauf hingewiesen, dass die Urfunktion der politischen Rede wesentlich darin besteht, ein Kollektiv, ein »Wir« ins Leben zu rufen und Gehorsam zu finden, nicht wahrheitsgetreu Informationen zu übertragen (2014: 22).

Im politischen Feld kommen diese verschiedenen Elemente von Narrationen ganz offensichtlich zum Tragen: Hier werden politische Ordnungen durch kleinere oder größere Erzählungen begründet, aber auch, mit ideologiekritischem Anspruch oder auf andere Weise, zu delegitimieren versucht. Gert Melville und Hans Vorländer sprechen hierbei von »Geltungsgeschichten« (Melville/Vorländer 2002): Traditionen und Zukunftsversionen werden narrativ konstruiert, um einer sozialen Grundordnung (Verfassungen, Institutionen etc.) Kontinuität und Legitimität zu verleihen. Nicht nur die politische Ideengeschichte ist voll von solchen Narrativen (man denke nur an den Kontraktualismus mit seiner narrativen Wegweisung vom Naturzustand in jenen der geordneten Gesellschaft), sondern sie stehen im Mittelpunkt von aktuellen Verfassungsgründungen, gesellschaftlichen Transformationen, politischen Projekten, Leitideen und generell von politischen Kämpfen um die gesellschaftliche Ordnung. Ganz unabhängig von der Aufrichtigkeit des Erzählers haben solche Narrationen stets, zumindest partiell, strategischen Charakter; dies erschließt sich nicht nur in neueren Untersuchungen, die politische Narrative im Rahmen einer Kommunikationsmacht-Perspektive untersuchen (Miskimmon/O'Loughlin/Roselle 2014), sondern lässt sich aus den Grundreflexionen der Rhetorik lernen (Llanque 2014: 12-17). Mit Erzählungen lässt sich um politische Gefolgschaft werben, lassen sich Machtansprüche artikulieren, Emotionen mobilisieren, imagined communities kreieren, die durch die Konstruktion von Identitäten bzw. die Hervorhebung von spezifischen Identitätsmerkmalen ein- und ausschließen. Dies geschieht im besten Fall, um ein notwendiges zumindest minimal solidarisches Zusammengehörigkeitsgefühl innerhalb einer sozialen Gruppe zu schaffen, ohne dass die Exkludierten Schaden nehmen; im schlimmsten Fall, um mit mythischen Erzählungen eine Freund-Feind-Dynamik auf blutige Weise zu initiieren, wie in Ruanda oder im zerfallenen Jugoslawien.

Sowohl die Funktionen als auch die Konfliktdynamik solcher Identitätsgeschichten werden im Fokus des folgenden Fallbeispiels stehen. Zuvor soll aber noch kurz der Begriff des Narrativs im Rahmen der politikwissenschaftlichen Erzählforschung konturiert werden, um einen konzeptuellen Zugang zur unterschiedlichen Relevanz identitätspolitischer Erzählungen zu gewinnen. Diese lassen sich nämlich im Hinblick auf den Grad ihrer Generalisierung und kulturellen Verbindlichkeit unterscheiden, was nicht zuletzt für die politische Kulturforschung unerlässlich ist. Hier lässt sich die Typologie von Albrecht Koschorke gewinnbringend aufgreifen, der die Vielzahl der individuellen, unsystematischen, polymorphen Geschichten (stories) von erzählerischen Generalisierungen abgrenzt, 
die er mit dem Terminus des »Narrativs« besetzt ${ }^{3}$ : »Einzelne Erzähltexte können außerordentlich verwickelten Bauplänen folgen; ihre kommunikative Verbreitung und soziale Verhandelbarkeit hängen jedoch davon ab, in welchem Maß sie dem Grundmuster eines gebräuchlichen Narrativs gehorchen - oder sich nach dessen Vorgaben fehldeuten lassen « (Koschorke 2012: 30f.). Narrative sind also Geschichten, die einem bestimmten Schema und Muster (so auch Müller-Funk 2008: 15) folgen und damit kulturelle Vorstellungen und Erwartungen einer Erzählgemeinschaft reflektieren, die dieser nicht stets bewusst sein müssen. Politische Narrative, insbesondere Identitätserzählungen, adressieren in der Regel ein größeres Publikum (oder mehrere Publika zugleich) und sind damit öffentlich.

In der sich hier anschließenden Fallstudie zur politischen Kultur Israels geht es um solche öffentlichen Narrative, deren identitätspolitischer Erfolg darin besteht, dass sie es vermögen, das Selbstverständnis einer Gruppe entscheidend zu prägen, und zwar wesentlich in Konkurrenz zu alternativen Erzählungen. Dabei soll es nicht um den Konflikt zwischen Israel und den Palästinensern gehen, sondern um einen genuinen Kulturkampf, um fundamental divergierende Ordnungsvisionen im Herzen der jüdischen Mehrheitsgesellschaft: »Verschiedene Narrative konkurrieren um den Charakter der israelischen Gesellschaft und ihre sozialen Grenzen wie die geographischen Grenzen des Staates« (Hagemann 2006: 163).

\section{Der Konflikt von Narrativen im israelischen Kulturkampf}

Israel ist ein in vielfacher Hinsicht multikulturell geprägter Staat. Schon ein flüchtiger Blick auf die israelische Gesellschaft zeigt ein wesentliches kulturelles Schisma, dessen politische Brisanz nicht zu übersehen ist: Mittlerweile macht der Anteil der arabischen Bevölkerung in Israel ein Fünftel (mit steigender Tendenz) aus, was in einem Staat, der sich explizit als jüdisch definiert, $\mathrm{zu}$ offensichtlichen Konflikten führt. So sind die arabischen Bürger in vielen Fragen rechtlich nicht den jüdischen Bürgern Israels gleichgestellt, werden bei nationalen Symbolen und Narrativen übergangen und sind vielfach in Parallelgesellschaften segregiert (Mautner 2011: 193-200).

3 Diese Terminologie weicht von der Terminologie der strukturalistischen Erzählanalyse etwa eines Cérard Cenette ab, in der mit story/histoire eine bestimmte bekannte Sequenz von Ereignissen bezeichnet wird, die jedoch im discours oder im récit auf unterschiedliche Art und Weise narrativ dargeboten werden kann (Fludernik 2009). Mit der Unterscheidung von $>$ Narrativ< und, allgemeiner, Erzählung/Narration soll hingegen der Grad der kulturellen Schematisierung und Ceneralisierung bezeichnet werden. Das bedeutet auch, dass sich Narrationen oft nicht eindeutig zuordnen lassen, sondern partiell Elemente eines gebräuchlichen Narrativs übernehmen können. 
Damit sind die kulturellen Konfliktlinien in Israel aber keinesfalls erschöpft, da sie vielmehr auch die jüdische Mehrheitsgesellschaft auf folgenreiche Weise durchziehen. Der Beobachtung einer zentrifugalen Entwicklung der israelischen Gesellschaft (Mautner 2011: 181-190) lässt sich wenig entgegenhalten. Die nicht zu übersehende Aufspaltung in mehrere ideologische Lager bedingt ein Ringen um die kulturelle Hegemonie, insbesondere die politische Ausrichtung des Landes mit seinen wesentlichen rechtsstaatlichen Institutionen. Der zur Bezeichnung dieser Konstellation vielfach bemühte Ausdruck des »Kulturkampfes« (Mautner 2011: 184f.; Segev 2010: 16) deutet dabei schon an, dass es sich hierbei um fundamental divergierende Ordnungsvisionen und Identitätsentwürfe handelt, die kaum unter einem overlapping consensus zusammengefasst werden können. Liberal-säkulare Kräfte und religiöse Fundamentalisten stehen einander als unversöhnliche Lager gegenüber, wobei letztere selbst keine homogene Gruppe bilden: Wie nun gezeigt werden soll, stimmen messianisch-religiöse Zionisten und ultraorthodoxe Juden zwar in ihrem Eintreten für die kategorische Geltung des jüdischen Rechts überein, nehmen aber konträre Positionen in Bezug auf den Staat Israel ein, selbst wenn es in jüngster Zeit zu Konvergenzen gekommen ist.

Diese skizzierten Konfliktlinien werden in der Organisation des Bildungswesens widergespiegelt, das sich bereits seit den 1920er Jahren durch eine hohe Fragmentierung auszeichnet (Segev 2010: 240f.). Während es nur wenige kulturell durchmischte Schulen gibt, haben alle genannten Gruppen, Staatlich-Säkulare, religiöse Zionisten, Ultraorthodoxe (die sich noch in aschkenasische und sephardische Juden gliedern) sowie Araber ihre eigenen Bildungseinrichtungen (Mautner 2011: 188). Dabei steigt gerade der Anteil der in arabischen und jüdisch-orthodoxen Institutionen sozialisierten Schüler schon aus demographischen Gründen kontinuierlich. 2013 wurden zum ersten Mal weniger als die Hälfte der Erstklässler in das staatlich-säkulare Schulsystem eingeschult (Brenner 2016: 228), was eine offensichtliche Herausforderung für die politische Kultur des Liberalismus darstellt und den Blick auf die Reproduktionsbedingungen fundamentaler Gesellschaftskonzeptionen lenkt.

Die Pluralität von Erziehungsinstitutionen verdeutlicht zudem, dass die verschiedenen Ordnungsentwürfe, die miteinander wetteifern, sich nicht, wie bei den multikulturellen Strukturen der meisten europäischen Staaten, als Herausforderung des liberalen Rechtsstaates durch minoritäre religiöse Gruppen verstehen lassen, da es nicht um einen Kampf um Anerkennung an der Peripherie des Staates geht - auf dem Spiel steht vielmehr die Gestaltung des Zentrums, der politischen und rechtlichen Leitlinien der israelischen Gesellschaft.

Die besondere Lage der Rechtsfortbildung unter überwiegend heteronomen politischen Strukturen barg für die Bestimmung der jüdischen Identität und Rechtsordnung nach der Wiedererlangung der staatlichen Souveränität 1948 eine besondere Brisanz. So wurde Israel in der Unabhängigkeitserklärung als jüdischer 
Staat definiert, zugleich aber an demokratische Strukturen und die Geltung der Menschenrechte gebunden, indem allen Bürgern »ohne Unterschied der Religion, der Rasse und des Geschlechts « die politische und soziale Gleichberechtigung zugesichert wurde (Ben Gurion 2010: 57); in den 1992 von der Knesset verabschiedeten Grundrechten ${ }^{4}$ wurde Israel dann explizit als zugleich »jüdischer und demokratischer Staat « definiert. Die narrative Rahmung des jungen Staates schwankte ohnehin zwischen Partikularismus und Universalismus sowie zwischen dem Wunsch nach Normalität und dem Herausstellen der eigenen exzeptionellen Geschichte. So betont die Unabhängigkeitserklärung das Streben, als gleichberechtigtes Mitglied der Nationenfamilie aufgenommen zu werden, während der charismatische Staatsgründer David Ben Gurion nicht nur in seinen Memoiren, sondern auch in vielen offiziellen Stellungnahmen die »universellen Inhalte« des eigenen Staatsprojekts und die durch Jahrtausende der Verfolgung errungene "geistige, sittliche und intellektuelle Überlegenheit« des jüdischen Volkes heraushob (2010: 17). Die damit implizierte Idee eines jüdischen Musterstaats ging dabei schon auf eine der Gründungsfiguren des Zionismus, Theodor Herzl, zurück, der in seiner Abhandlung Der Judenstaat und noch mehr in seinem utopischen Roman Altneuland das Ideal einer kosmopolitischen neuen Gesellschaft entwickelt hatte (Brenner 2016: 60-70).

Die Frage der Gewichtung religiöser und menschenrechtlicher Prinzipien wurde zunächst zugunsten des säkularen Rechtsstaats entschieden. Das positive Recht trug deutliche Züge einer Anglisierung, die die Folge des britischen Protektorats in Palästina in den drei Jahrzehnten zuvor war (Mautner: 2011: 35-38). Aber die modernistischen Tendenzen in der jüdischen Kultur gingen nicht allein auf diese Protektoratszeit zurück, sondern entstammten schon der Aufklärungsphilosophie und dem Modernisierungsprozess seit dem Ende des 18. Jahrhunderts, der viele europäische Juden zu einer Akkulturation gegenüber der nicht-jüdischen Mehrheitsgesellschaft veranlasst hatte (Hagemann 2006: 38-40).

Die Bewegung der religiösen Orthodoxie entstand in dieser Zeit als Suche nach einem Gegengewicht gegen die als bedrohlich empfundene Entwicklung der Modernisierung, Pluralisierung und Liberalisierung. Die Orthodoxen setzten dieser Entwicklung einen Lebensentwurf entgegen, der auf einer rigiden theonomen Grundlage basierte (Hagemann 2006: 40). Der Zionismus, der sich zum Ende des 19. Jahrhunderts herausbildete, stellte dann eine neue Kraft dar, die zum Teil durch messianische Vorstellungen, zum Teil durch säkulare Ideen geprägt war.

4 Da Israel über keine Verfassung verfügt, sind die grundlegenden Rechtstexte die Unabhängigkeitserklärung von 1948, die schrittweise zwischen 1958 und 1988 verabschiedeten Crundgesetze, die beiden Grundrechte von 1992, die sich auf die Menschenwürde und Freiheit sowie die Freiheit der Berufswahl beziehen, und das im Sommer 2018 verabschiedete, hochumstrittene Nationalstaatsgesetz. 


\section{Nationale Narrative nach der Staatsgründung: Republikanismus, Kulturgeschichte und »Neuer Jude»}

Aus dieser heterogenen Lage von konkurrierenden Gesellschaftsentwürfen gingen die Verfechter einer säkularen, modernen und westlich orientierten Kultur in der ersten Hälfte des 20. Jahrhunderts als Sieger hervor (Mautner 2011: 29), eine Ausrichtung, die auch die politische Kultur der ersten Jahrzehnte des Staates Israel prägte. Ob man diese Phase - angesichts des Ausschlusses des arabischen Teils der Bevölkerung, der durch das ethnisch-religiös definierte Konzept der Staatsbürgerschaft nicht Teil der Gemeinschaft und des Gesellschaftsvertrags war (Barzilai 2005: 60-66), und der vielfach nur schlecht kaschierten soziokulturellen Konflikte im neuen Staat (Segev 2010: 133-154) - wirklich als »republikanisch«, bestimmt durch den Glauben an ein gemeinsames Gutes, bezeichnen kann (so Mautner 2011: 186f.), ist zweifelhaft, will man nicht den staatlichen Gründungsmythos mit der gesellschaftlichen Realität verwechseln; ein solches republikanisches Narrativ wurde besonders von Ben Gurion gepflegt, der nicht nur in Rousseau'scher Manier an den Allgemeinen Willen der Bürgerinnen und Bürger appellierte, sondern auch an ein bürgerliches, von Toleranz und Respekt geprägtes Bewusstsein (mamlakhtiyut) (Ben Gurion 2000).

Fest steht jedoch, dass der in der Unabhängigkeitserklärung deklarierte jüdische Charakter des Staates primär in nationale Narrative eingebettet wurde, nicht in fundamentalistisch-religiöse, welche die Bindung der neuen rechtlichen und politischen Ordnung an die Halacha, das traditionelle jüdische Recht, vorgesehen hätten. Diese nationalstaatliche Codierung des Judentums ging in wesentlichen Punkten auf eine Weichenstellung des säkularen Zionismus zurück, für den das Jüdische nicht durch die absolute Geltung der Halacha, sondern durch die Kenntnis der jüdischen Geschichte und Tradition sowie durch das Hebräische als Nationalsprache bei gleichzeitiger enger Bindung an die Kultur der westlichen Moderne definiert war. Dieses Narrativ versuchte nicht, dem Judentum die Religion auszutreiben, aber es stand nicht die normative Verbindlichkeit religiöser Gebote im Mittelpunkt, sondern eine nationalistisch-kulturgeschichtliche Lesart der eigenen Tradition:

»The Zionist narrative emphasized the Biblical and second temple period rather than Rabbinical and medieval ones. It spoke about kings, heroes, poets and prophets rather than rabbis, philosophers and mystics, let alone Hallachik authorities; it told the stories of wars, political struggles and great cultural movements rather than about religious movements and writings. It was a story anchored in the Land of Israel rather than a story telling about exilic life in the Diaspora. It was a narrative based on scholarly historiographic and archaeological work rather than one 
based on traditional authoritative religious sources and mythical past-memory.« (Amir 2011: 22)

Dem religiösen Element kam dadurch eine eindeutig instrumentelle Funktion zu: »Als Legitimationsgrundlage für das zionistische Projekt spielte die Religion dennoch eine wesentliche Rolle: Sie diente als Bindeglied zwischen jüdischer Nation und biblischem Land, stellte wesentliche Symbole für den jüdischen Staat bereit und bestimmte die sozialen Grenzen des jüdischen Kollektivs. Doch diese Einbeziehung in die Definition des Staates sicherte der Religion keineswegs eine zentrale Rolle: Die Bibel wurde als historisches Dokument interpretiert, die Religion in den Dienst der Nation gestellt und der Staat (und nicht Gott) zum zentralen Bezugspunkt des Zionismus erkoren. Der israelische Nationalismus basierte einerseits auf dem allumfassenden, zentralisierenden Staat, andererseits auf dem Schmelztiegelkonzept, das alle Einwanderer nach dem Vorbild des neuen hebräischen Juden sozialisieren sollte.« (Hagemann 2006: 158)

Dieser »neue Jude« sollte die selbstbewusste Antithese zum Juden im Exil darstellen und ein Bürger sein, der als entschlossener Verteidiger des neuen, säkular-modernen Staates auftrat. In den lyrischen Worten Ben Gurions:

»Wagemutige und selbstbewußte Juden faßten von neuem Wurzel in der Erde der Heimat. [...] Ein neuer Abschnitt in der Geschichte des Volkes und des Landes begann, von Juden geprägt, die bereit waren, ihr eigenes und das Schicksal ihres Volkes in die Hand zu nehmen und Angriffen des Feindes die Stirn zu bieten « (Ben Gurion 2010: 15).

Auch diese Erzählung, die natürlich wie die gesamte Staatsgründung wesentlich durch die nationalsozialistische Vernichtung der Juden gerahmt wurde, war nicht ohne Vorläufer, sondern orientierte sich an dem um die Jahrhundertwende entstandenen zionistischen Motiv des "Muskeljuden « (Max Nordau), dessen geistig-moralischer Exzellenz nicht zuletzt aus politischen Gründen eine physische Veränderung beigesellt werden sollte. Dieses Konzept griff auch offensichtlich das antisemitische Stereotyp des »angeblich blassen, unsportlichen, in die Studierstube gezwängten >Talmudjuden o oder >Nervenjuden« (Brenner 2016: 79) auf. Das Idealbild des jüdischen Arbeiters und Kämpfers für den neuen Staat führte aber auch zur Geringschätzung einer Vielzahl von neuen Einwanderern (»Heimkehrern«), die diesem physischen Ideal nicht entsprachen (Segev 2010: 154-194).

Die Integrationskraft dieses vielschichtigen nationalen Narrativs war in den Gründungsjahren des Staates Israel immerhin so stark ausgeprägt, dass strengreligiöse Bewegungen nur eine marginale Rolle im politischen Prozess spielten. Gleichwohl wurde der Religion nicht nur eine symbolische Rolle im gesellschaftli- 
chen Leben eingeräumt. Um die Legitimation des neuen Staates auch bei religiösen Juden zu festigen, hatte David Ben Gurion bei der Staatsgründung die Einhaltung wichtiger religiöser Vorschriften ${ }^{5}$ im neuen Israel zugesichert, ohne die Politik theokratischen Strukturen zu unterwerfen. Zudem wurde den orthodoxen Juden ein gehöriger Spielraum der eigenen Lebensgestaltung eingeräumt (etwa, wie beschrieben, im Erziehungswesen oder in Fragen des Personenstandsrechts, das ausschließlich religiösem Recht unterworfen ist) und ihre partielle politische Einbindung angestrebt ${ }^{6}$ (Gordon 1989: 633). Allerdings war es bezeichnend für die grundsätzliche Spaltung zwischen dem religiösen und dem säkularen Lager, dass das Projekt einer Verfassung schließlich aufgegeben wurde; denn aus religiöser Sicht stellte die versuchte Knüpfung rechtlicher Grundsätze an säkular-demokratische und nicht göttliche Quellen einen inakzeptablen Schritt dar, wobei allerdings auch die lange regierende Arbeiterpartei aus pragmatischen Gründen keine Einschränkung ihres politischen Gestaltungsraums herbeiführen wollte (Hirschl 2001: 323; Segev 2010: 306).

\section{Das messianische Narrativ der Ultraorthodoxen: Exilanten im Heiligen Land}

Die ultraorthodoxen, charedischen Juden ${ }^{7}$ konnten sich in diesem nationalen Narrativ der Gründungsjahre aus mehreren Gründen nicht wiederfinden und standen dem jungen Staat aus prinzipiellen Motiven ablehnend gegenüber (obgleich sie sich aus pragmatischen Überlegungen in mancher Hinsicht mit ihm arrangieren mussten). Das in weiten Teilen weltliche Recht in Israel steht der eigenen fundamentalistischen Überzeugung entgegen, dass allein die Halacha (in einer bestimmten fundamentalistischen Interpretation, die die Tatsache der Deutung selbst verschlei$\mathrm{ert}^{8}$ ) normative Verbindlichkeit besitze und das rituelle Leben der Charedim so-

5 So wurde der Sabbath zum offiziellen wöchentlichen Ruhetag erklärt und in staatlichen Einrichtungen die Küche den jüdischen Bestimmungen unterworfen.

6 Cohen/Susser (2000: 17-37) sprechen für die ersten drei Jahrzehnte nach der Staatsgründung in Anknüpfung an Arend Lijphart - von einem »consociation model«, also einer Politik des Ausgleichs und des pragmatischen Austarierens gesellschaftlicher und politischer Konflikte.

7 Diese bilden keine homogene Gruppe, sondern bestehen aus verschiedenen Untergruppen, die trotz leichter politischer und theologischer Unterschiede doch eine große Zahl an gemeinsamen Merkmalen aufweisen. Weder der Begriff der $>$ Ultra-Orthodoxie $<$ noch derjenige des $>$ (c) haredim ( (hebräische Bezeichnung für die Juden, die vor dem Wort Cottes erzittern) dienen ihnen als Selbstbezeichnung, sondern vielmehr in den meisten Fällen der jiddische Ausdruck »erlicher Yidn « (wahrer, tugendhafter Jude) (Paine 1997: 267).

8 Denn darin besteht das Wesen fundamentalistischer Bewegungen. Im Judentum selbst zeigt schon die vielfältige, pluralistische Tradition der Textexegese, dass der Cedanke des einzig wahren Textes oder der absolut wahren Norm kulturell nicht konsensfähig ist, was jüdisch-fundamentalistischen Strömungen jedoch nicht im Weg steht (Hagemann 2006: 36f.). 
mit auch ganz der Verehrung des Wortes Gottes verpflichtet sei. Die kategorische Ablehnung der westlichen Moderne bringt insofern bereits ein fundamentalistisches Weltbild mit sich, das keinen Platz für Pluralismus oder die demokratische Generierung von Recht hat. Aber die Charedim lehnen den Staat noch aus tiefer gehenden Gründen $a b$, da sie im nationalistischen Zionismus, dessen Kampf die Staatsgründung erst ermöglicht hatte, einen eklatanten Widerspruch zur eschatologischen messianischen Erlösung sehen (Barzilai 2005: 218).

So erweisen sich zwei narrative Elemente als bestimmend für ihr Bild von Recht und Politik: Zum einen wird das jüdische Recht durch seinen göttlichen Offenbarungscharakter als einzig legitimes Recht anerkannt, zum anderen jede Form der aktiven Politik als profan, degeneriert und destruktiv abgelehnt. Dies hängt mit dem messianischen Narrativ zusammen, welches das passive Warten auf die Erlösung durch den Messias beinhaltet ${ }^{9}$. Die Juden als das auserwählte Volk stehen laut diesem Narrativ außerhalb von Geschichte und weltlichen Gesetzen und unterliegen einzig dem Willen der göttlichen Vorsehung. Ein aktives Eingreifen ins Weltgeschehen, ein politisches Engagement jeder Art, also nicht zuletzt der Zionismus, muss unter diesen Umständen als Häresie erscheinen.

Die Erlösung kann es nur als absolute, nicht als graduelle oder gar durch menschliches Streben erlangte geben - diese Erlösung besteht in der Einheit der heiligen Trinität von Volk Israel, biblischem Land Israel (Eretz Israel) und Thora (Hagemann 2006: 41f.). Folglich bestimmen die Charedim ihre Identität innerhalb des Staates Israel als »Exilanten im Heiligen Land« (Mautner 2011: 122). Sie haben eine Gegenkultur, wenn auch keine absolute Abschottung, am Rande der Gesellschaft, eine Art-Selbstghettoisierung, betrieben, um einen Schutzschild gegen die Missstände und Verführungen der modernen Welt zu errichten (Paine 1997: 268f.), selbst wenn sie (etwa im Gegensatz zu den amerikanischen Amish) das Instrument der modernen Technik akzeptieren (Mautner 2011: 122). Ihr überwiegender Gebrauch des Jiddischen stellt ein weiteres Abgrenzungsmerkmal dar, ebenso wie die generelle Orientierung ihrer täglichen Praktiken an einem (vermeintlich) traditionellen osteuropäischen Lebensstil (Glinert/Shilhav 1991: 59f.). Die Marginalisierung der Ultra-Orthodoxen drückt sich nicht zuletzt in ihrer Freistellung vom Armeedienst aus, deren Aufhebung seit Jahren Knesset und Oberstes Gericht beschäftigt und mehrfach militante Reaktionen hervorgerufen hat (Schmid 2017). Aufgrund der strukturellen Konfliktsituationen mit den Nachbarn Israels und den zahlreichen bereits ausgetragenen Kriegen wurden der Militärdienst und auch das Andenken an die Opfer sowie die Opferbereitschaft für das Kollektiv

9 Diese Vorstellung wird aus Jeremias 27, 22 abgeleitet, wo es heißt: „Nach Babel werden sie gebracht und dort bleiben sie bis zu dem Tag, an dem ich mich ihrer annehme - Spruch des Herrn - und sie wieder an diesen Ort heraufbringe« (vgl. hierzu Paine 1997: 270). 
essentieller Bestandteil der nationalen Symbolik und Erinnerungskultur, von der die Charedim sich selbst ausgeschlossen haben.

\section{Das Narrativ des fundamentalistischreligiösen Zionismus: Eine messianische Deutung des Staates}

Die andere relevante Version des jüdischen Fundamentalismus stellt jener Zweig der Zionisten dar, der als »messianischer Fundamentalismus« (Hagemann 2006) bezeichnet werden kann, der vom Großrabbiner Abraham Isaac Kook und seinem Sohn Zwi Jehuda Kook entwickelt wurde und sich in der Siedlerbewegung Gusch Emunim praktisch manifestierte. Mit den Charedim teilt diese Ideologie wesentliche Überzeugungen: das Ideal einer Einheit von israelischem Volk, Eretz Israel und Thora, also auch die Lebensführung auf der strengen Grundlage des jüdischen Rechts, ein deterministisches Geschichtsnarrativ, das einen Zustand der vollkommenen Erlösung durch den Messias als Befreiung des jüdischen Volkes aus dem Exil vorsieht und auch ein für fundamentalistische Bewegungen symptomatisches striktes Freund-Feind-Schema, in dem die Gegner Israels als gottlose Feinde erscheinen.

Dennoch stehen die messianischen Fundamentalisten in grundsätzlicher Konfrontation zu den ultra-orthodoxen Juden, da sie der Existenz eines souveränen, aber säkularen Staates Israel nicht prinzipiell ablehnend gegenüber stehen, sondern sie im Gegenteil in ein messianisches Narrativ einbetten. Dieses stellt die Staatsgründung nicht als Akt der Profanierung des Judentums dar, sondern als Hinweis auf den sich ankündigenden Erlösungsprozess:

»In der Auslegung des nationalreligiösen Zionismus stellen die zionistische Bewegung selbst und insbesondere der Staat Israel eine entscheidende Etappe in der Entfaltung des messianischen Plans dar. Der Messianismus wird also nicht als Antithese zur Realität verstanden, sondern zeige sich in der weltlichen Sphäre, in der Rückkehr nach Zion« (Hagemann 2006: 46).

Der Staat und seine Aktivitäten stehen in dieser Sicht also auf einem sakralen Grund.

Auch wenn der historische Determinismus keine Abkehr von diesem Weg der Erlösung vorsehen kann, unterliegt die Dynamik dieses Prozesses laut messianischem Fundamentalismus doch auch menschlichem Handeln, was dem Politischen (vor allem der theonomen Umprogrammierung der Gesellschaft und der religiös legitimierten Besiedlung der »heiligen« Gebiete) einen legitimen Platz einräumt. Der Beginn der messianischen Erlösung befreie somit die Juden von ihrem Schwur der Passivität (Hagemann 2006: 47-51). Selbst die säkularen Zionisten finden noch Platz in diesem Narrativ, indem sie zum unbewussten Gehilfen des Fortschritts in einem dialektischen kosmisch-göttlichen Geschehen werden: »Secular Zionists be- 
lieved that they were departing from religion and from sacred history; Kook argued that they were merely unaware of their role as loyal servants in the Divine plan for redemption « (Amir 2011: 31). So ließ sich dann auch die aktive Kooperation mit der säkularen Seite legitimieren, wobei die Koalition aber auf das Projekt der Eroberung der »heiligen« Gebiete beschränkt sein sollte, das nur der erste Schritt im messianischen Plan sei - der zweite sollte in der verbindlichen Durchsetzung der religiösen Gebote für alle Juden in allen Lebensbereichen bestehen (Hagemann 2011: 61f.).

Diese originelle politische Selbstbevollmächtigung der Fundamentalisten stieß nicht ohne Grund zu einem Zeitpunkt auf offene Ohren, als der säkular dominierte Status Quo der politischen Kultur Israels, der in den ersten Jahrzehnten nach der Staatsgründung vorgelegen hatte, zu bröckeln begann. Hatte lange Zeit ein säkularer Nationalismus dominiert, der sich politisch mit der Vorherrschaft des Arbeiterzionismus verband, markierte der Wahlsieg des rechtsgerichteten Likud-Blocks 1977 das Ende dieser Hegemonie, die sich schon in den Jahren zuvor als fragil erwiesen hatte (Mautner 2011: 99-127). Vor allem die Kriege von 1967 und 1973 hatten einen Wandel induziert. Der Sieg im Sechstagekrieg von 1967 kam einer »zweiten Staatsgründung (Brenner 2016: 165) gleich; Außenminister Abba Eban erklärte in der Knesset: »In sechs Gefechtstagen wurde ein neuer Staat Israel erschaffen" (zit.n. Segev 2009: 661). Im Gegenteil zu 1948 dominierten nun messianische Narrative im religiösen Feld, da Israel die Gebiete des biblischen Urlands, Judäa und Samaria, erobert hatte, was vielfach als Wunder und Zeichen der Erlösung gedeutet wurde (Brenner 2016: 166). Dem jüdisch-fundamentalistischen Zweig der Zionisten boten sich dadurch neue Optionen. Er versuchte, nicht mehr die profane Nation, sondern die Besiedlung und Verteidigung des Landes Eretz Israel ins Zentrum der israelischen politischen Kultur zu rücken, und zwar nicht nur auf einer symbolischen Ebene:

»Das Land ist [...] keineswegs nur Instrument der nationalen Renaissance, sondern besitzt einen intrinsischen Wert. Es steht nicht als Symbol für den messianischen Prozess und das Heilige, sondern ist in seiner physischen Substanz von religiöser Bedeutung: Jeder Ort, jeder Stein, jeder Baum ist heilig und erlösungsbedürftig.« (Hagemann 2011: 61)

Das biblische Land ist, einmal zurückgewonnen, politisch unantastbar und unverfügbar. Der Ruf nach einer die Symbolpolitik übersteigenden Sakralisierung des politischen und sozialen Lebens stieß durchaus auf offene Ohren, befand sich der säkulare Zionismus doch in einer Legitimationskrise. Der Verlauf des Jom-KippurKriegs 1973, als Israel in den ersten Tagen, überrascht von der syrisch-ägyptischen Offensive, empfindliche Gebietsverluste hinnehmen musste, die es gleichwohl später nach der Mobilisierung seiner Truppen kompensierte, hatte eine traumatische Wirkung auf die israelische Öffentlichkeit. Von der religiösen Rechten wurde die 
anfängliche Planlosigkeit nicht nur als Symbol der Unfähigkeit der verantwortlichen politischen und militärischen Elite gewertet, sondern sie wurde auch als Konsequenz des moralischen Verfalls der israelischen Gesellschaft dargestellt (Hagemann 2006: 68). Auf dieser Basis konnten die messianischen Fundamentalisten dann für ihr Projekt einer religiös legitimierten, expansiven Siedlungspolitik und einer normativen Umgestaltung der Gesellschaft nach der Halacha werben, die den Weg zurück zu Wahrheit und Tugend ebnen sollte.

Die außerparlamentarische Siedlerbewegung Gusch Emunim war wesentlicher Träger dieses Fundamentalismus und übte vermittelt über Teile der Nationalreligiösen Partei zeitweilig auch im politischen Feld selbst starken Einfluss aus. Dass es hinsichtlich der geographischen Grenzen Israels und damit der Siedlungspolitik eine (wie gesehen, selbst messianisch begründete) Interessenkonvergenz mit dem aufstrebenden säkularen, revisionistischen Ultra-Nationalismus (in erster Linie verkörpert in der Cherut-Partei Menachem Begins) gab, verstärkte diesen Einfluss, zumal letzterer religiös-messianische Motive übernahm und in den alltäglichen politischen Diskurs der gesellschaftlichen Mehrheit einführte (Hagemann 2006: 73-77). Dies gelang insofern problemlos als der säkulare Zionismus sich ja selbst stets religiöser Symbole und Texte bedient hatte, die er nationalistisch umgedeutet hatte. Nun wurden sie durch ihre religiöse Einbettung einer Re-Codierung unterzogen.

Das Identitätsnarrativ des >neuen Juden`, das der Gründung des Staates zugrunde lag, vermochte derweil seine Integrationskraft nicht mehr zu entfalten (Mautner 2011: 114), so dass die kulturelle Hegemonie neu verhandelt wurde. Hatten sich Teile der religiös-zionistischen Bewegung nach der Staatsgründung aus ihrer marginalisierten Position noch mit der herrschenden säkularen Elite und der politischen Kultur dieser Zeit zu arrangieren versucht, führte die Radikalisierung des Religiösen mit der expliziten Forderung nach der Ausrichtung von Staat und Gesellschaft nach der Halacha zu einem eindeutigen Konfrontationskurs zum Säkularismus (Mautner 2011: 119f.).

\section{Religiöser Fundamentalismus: Kräfteverschiebungen und Konvergenzen}

Der sich in dieser Phase radikalisierende Kulturkampf um die jüdische Identität hält bis heute mit wechselnden Kräfteverhältnissen an. Der Aufschwung der messianischen Fundamentalisten, die ideologisch bedingt eine aktivere politische Rolle einnehmen als die Charedim, ist seit den 1990er Jahren in einen Niedergang zugunsten der ultraorthodoxen Gruppen übergegangen. Ein wesentlicher Grund dafür besteht darin, dass zentrale politische Entscheidungen sich nicht bruchlos in das messianische Narrativ einfügen lassen. Seit Jahrzehnten ist die politische Realpolitik in Israel vom Primat der nationalen Sicherheit angetrieben worden, 
um gegen territoriale Zugeständnisse die Chance des Friedens zu erhöhen (was freilich nicht die konsequente Abkehr von einer expansiven Siedlungspolitik mit all ihren Problemen mit sich brachte). So wurden noch in der Amtszeit Menachem Begins israelische Siedlungen im Sinai geräumt und das Gebiet an Ägypten zurückgegeben. Im 1993 eingeläuteten und später nicht zuletzt durch die Ermordung von Ministerpräsident Rabin torpedierten Friedensprozess von Oslo wurde ein Grundabkommen zwischen der israelischen und der palästinensischen Seite beschlossen, das eine weitgehende Autonomie der Palästinenser mit der Aussicht auf einen eigenen Staat vorsah, zumindest aber eine Teilung des Landes. Die Räumung von Siedlungen im Gazastreifen und der Westbank, die ausgerechnet der als Kriegsheld und »Vater der Siedler« angesehene Ariel Scharon 2005 vollziehen ließ, kollidierte dann endgültig mit dem messianischen Narrativ der Siedler: Die freiwillige Aufgabe »heiligen« Landes passte nicht zum proklamierten irreversiblen Erlösungsprozess. Zudem hatte gerade der Oslo-Prozess die angekündigte religiöse Erneuerung des jüdischen Volkes als Chimäre erwiesen, da dieser als Vormarsch der säkularen, realistischen Friedenspolitik gedeutet werden musste (Hagemann 2006: 99-147).

Der einzige Ausweg bestand darin, diese politische Entwicklung als nur temporären Rückschritt zu interpretieren und die Krisenursache einmal mehr der säkularen Gesellschaft zuzuschreiben. Das Verhältnis zum Staat ist nun noch spannungsreicher als zuvor: Einerseits muss an dessen Heiligkeit festgehalten werden, andererseits wird aber dessen konkrete säkulare Erscheinungsform bekämpft. So hat die ideologische Krise eine mehrfache Radikalisierung der Messianisten bewirkt: Zum einen scheut der politische aktive Flügel nicht vor einer auch zur Gewalt gegen »Ungläubige« entschlossenen kategorischen Verteidigung der Siedlungen zurück, zum anderen setzt der halachische Flügel noch strikter als zuvor auf die »Besiedlung der Herzen«, also eine religiös-kulturelle Revolution, die sich ihren Weg nicht durch parteipolitischem, sondern außerparlamentarischen Einfluss bahnen soll (Hagemann 2011: 66-70).

Die Charedim stellt der Wandel der Siedlungspolitik vor keine ideologischen Probleme. Sie stehen dem Staat nach wie vor ablehnend (wenn auch aus Selbsterhaltungsgründen pragmatisch bis opportunistisch) gegenüber und richten ihren Aktivismus auf die eigene Gemeinschaft, zunehmend aber auch nach außen; ihr in den letzten Jahren vermehrt zu beobachtendes aggressiven Auftreten gegenüber nicht-orthodoxen Juden innerhalb des Gebiets ihrer Gemeinschaft, gerade in Jerusalem, belegt dies (Schmid 2017). Während die Ultra-Orthodoxen einerseits nach wie vor in einer gesellschaftlichen Parallelwelt mit eigenen Bildungsinstitutionen und Gerichten leben, nehmen sie andererseits aber seit dem Sieg des konservativen Lagers in der Parlamentswahl von 1977 und der zunehmenden religiösen Imprägnierung der politischen Kultur stärker am politischen Prozess und zum Teil 
auch an der Regierungsbildung teil, was sie in einen Widerspruch zu ihrem anti-zionistischen und anti-politischen Narrativ setzt (Mautner 2011: 122f.).

Stellte die Agudat Jisrael bis in die 1980er Jahre die parlamentarische Vertretung der überwiegenden Mehrheit der Ultra-Orthodoxen dar, kam es in der Folge zu einer Aufspaltung, die durch die Gründung der Partei der sephardischen UltraOrthodoxen, Schas, ausgelöst wurde. Schas hat seit ihrem erstmaligen Einzug in die Knesset 1984 beachtliche Erfolge bei den sozial häufig ausgegrenzten »orientalischen « orthodoxen Juden, also jenen, die aus den islamischen Regionen eingewandert sind, gefeiert. Animiert wird die Partei von einem ganz eigenen Opfernarrativ:

»In their countries of origin, so goes the tale, Sephardic Jews had lived pure and upright lives in the light of religion. When secular Zionism moved them to Israel, it relegated them to the margins of society, violated their dignity, robbed them of their religious and cultural legacy, and devastated their lives: the outcome was broken families, poverty, use of alcohol and drugs, crime, and prostitution. The way for Sephardim to rectify the grave wrongs that Zionism inflicted upon them is to return to a life of Torah and religious observance and to restore the ancestral traditions. Only thus can Sephardim regain their self-dignity, repair their family and human relationships, restore themselves to a proper moral and spiritual level, and attain economic prosperity.«(Mautner 2011: 124).

Schas hat sich von Anfang an als politische Bewegung verstanden, die nicht nur wie die aschkenasischen Ultra-Orthodoxen ihre staatlich garantierten Privilegien verteidigen möchte, sondern ihre Botschaft an die gesamte Gesellschaft richtet: Sie zielt auf die Einrichtung einer Theokratie, also eine Umprogrammierung des Staates nach sephardisch-halachischen Geboten, ab, aber auch auf die Überwindung der aschkenasischen Dominanz in der Gesellschaft, was Schas partiell zu einer Protestwählerpartei macht (Timm 2003: 98f.). Über ihre wiederholte Regierungsbeteiligung, aber auch darüber hinaus, übt Schas einen nicht $\mathrm{zu}$ vernachlässigenden politischen Einfluss aus.

Bei allen bestehenden Differenzen und vielfältig ausfallenden Konfliktlinien haben sich beide fundamentalistischen Bewegungen mittlerweile faktisch in ihren Zielen und Mitteln, vor allem aber auch in ihrem Feindbild (Palästinenser, generell Nicht-Juden und Säkulare) angenähert, indem sie eine Gesellschaft auf ethnisch-exkludierender Basis und nach Maßgabe der Halacha errichten möchten und sich damit gegen das alternative Narrativ eines säkularen, in Teilen kosmopolitischen Israels wenden, das sich an universellen Menschen- und Bürgerrechten orientiert (Hagemann 2006: 163f.). ${ }^{10}$

10 Anflüge einer für einen kulturellen Pluralismus offeneren Interpretation der eigenen jüdischen Identität waren vor allem in den $1990 e r$ Jahren erkennbar, ohne dass sich diese Identität mehr- 


\section{Das Narrativ des Obersten Gerichts und der Kampf ums Recht}

Dieser Kulturkonflikt äußert sich nicht zuletzt in einem Kampf um das richtige Recht. Das Oberste Gericht in Jerusalem, die höchste gerichtliche Instanz, versucht seit einiger Zeit die politische Schwäche des liberal-säkularen Lagers durch eigenen Aktivismus zu kompensieren. Es tut dies so nachdrücklich, dass Menachem Mautner nicht nur von einem Konflikt zwischen religiösem und staatlichem Recht spricht, sondern von einem »clash between religious fundamentalism and legal fundamentalism « (2011: 3; Herv. i. O.). Um diese Tendenz zur Verrechtlichung der israelischen Politik zu verstehen, die Mautner mit seiner Formulierung andeutet, muss die gewandelte Stellung des Obersten Gerichts innerhalb des politischen Systems Israels verstanden werden. Grundsätzlich kann daran erinnert werden, dass Obergerichte, insbesondere Verfassungsgerichte, über eine institutionelle Deutungsmacht nicht nur über rechtliche Fragen, sondern auch über das Politische verfügen. Gad Barzilai hat $\mathrm{zu}$ Recht von Institutionen als »carriers of narratives « (2005: 59) gesprochen und darauf hingewiesen, wie das Oberste Gericht in Israel seit Beginn identitätskonstituierende Kategorien der politischen Welt geprägt hat, in erster Linie bei der Entwicklung des Staatsbürgerkonzeptes.

Dennoch ist die Bedeutung des staatlichen Gerichtswesens lange hinter der Macht der Legislative zurückgeblieben, nicht zuletzt bedingt durch die fehlende Verfassung und die damit einhergehende Absenz einer Verfassungsgerichtsbarkeit. Dies änderte sich 1992 durch einen politischen Akt, der von Aharon Barak, damals Richter und später auch Präsident des Obersten Gerichts, als »constitutional revolution« (Barak 1993; Hirschl 2001) aufgefasst wurde. Nachdem das Vorhaben einer konstitutionellen Grundlegung nach der Staatsgründung aus den oben genannten Gründen gescheitert war, hatten sich die Parteien in der sogenannten Hariri Resolution auf das Modell einer kumulativen Verfassung geeignet, das eine schrittweise parlamentarische Verabschiedung von Gesetzen mit grundgesetzlichem Charakter vorsah (Hirschl 2001: 323). Während die zwischen 1958 und 1988 beschlossenen Grundgesetze sich im Wesentlichen auf die institutionelle Gestaltung des Staates bezogen, stellten die beiden Gesetze von 1992 eine folgenreiche Innovation dar: Zum ersten Mal wurden verfassungsähnliche Grundrechte statuiert, welche den Schutz der Menschenwürde, der Freiheit allgemein und der Freiheit der Berufswahl im Besonderen festhielten. Mit dieser neuen grundrechtlichen Garantie ging aber ein bedeutender Machttransfer zugunsten der Judikative einher, dem Obersten Gericht wurde nämlich die Kompetenz zur Normenkontrolle, also zur Gesetzesüberprüfung, zugesprochen, was im Gegenzug die Gestaltungsmacht der Legislative einschränkte. Dieser Machttransfer erschließt sich erst dann, wenn

heitlich durchgesetzt hätte, zumal durch die zweite Intifada ab 2000 der »nationale Imperativ« wieder die wichtigsten politisch-kulturellen Fragen überlagert (Timm 2003: 283-285). 
man ihn als Resultat der zuvor erfolgten kulturellen Wandlungsprozesse versteht. Die säkulare politische Elite, die fast ausschließlich aus bürgerlichen aschkenasischen Juden bestand, hatte in den ersten Jahrzehnten nach der Staatsgründung das politische Feld dominiert, ihre politische und kulturelle Hegemonie nun aber auf demokratischem Weg verloren. Diese Gruppe beabsichtigte nun, den Supreme Court zur Verteidigung der kulturellen Werte ihres Milieus zu ermächtigen (oder $\mathrm{zu}$ instrumentalisieren), was auf dem parlamentarischen Weg nicht mehr gewährleistet war (Hirschl 2001: 322f.).

Das Oberste Gericht gab nach dieser Ermächtigung seine frühere politische $\mathrm{Zu}$ rückhaltung auf und akzeptierte die Rolle des Verteidigers säkular-liberaler Werte; die Rechtfertigung dieser aktivistischen Rolle hat insbesondere Aharon Barak übernommen, indem er die Justiz als moralisches Bollwerk gegen eine stetig drohende Diktatur der Mehrheit und als didaktischen Vermittler demokratischer Werte darstellt (Barak 2008). Die Stärkung von Bürgerrechten, die im Zeichen der nationalen Sicherheit permanent bedroht sind, gehört genauso dazu wie eine tendenziell religionskritische Rechtsprechung, die ihren Höhepunkt in der erstmals 1998 deklarierten und fortan beständig angemahnten Aufhebung des Privilegs für ultra-orthodoxe Gruppen fand, ihnen zugehörige Studenten der Talmud-Hochschule Jeschiwa nicht zum Wehrdienst entsenden zu müssen (Hirschl 2001: 326-329). Dass sich diese Urteile nicht auf eine nüchterne Grundrechtsabwägung reduzieren lassen, sondern eine weitreichende identitätspolitische Funktion haben, hatte Barak schon 1993 offen eingestanden: »We must crystallize the modern self-understanding of Israeli society; in other words, its very identity« (Barak 1993: 84). Wie die Grundausrichtung dieser Identitätsbildung aussehen sollte, wurde deutlich, als er über den Charakter des Staates sprach:

»[T] he term »]ewish and Democratic does not contain a contradiction, but rather a completion, a complementing. [...] The State is Jewish, not in the religious sense, but in the sense that Jews have the right to immigrate here, and that their national experience is that of the State. [...] The fundamental values of Judaism - which we bequeathed to the whole world - are our basic values. I am referring to the values of love of humanity, sanctity of life, social justice, doing what is good and just, protecting human dignity, the rule of the law-maker, and other such eternal values.«(Barak 1993: 84)

Ganz wie zur Gründungszeit des Staates Israel versuchte die säkulare Elite, dem spezifisch Jüdischen des Staates eine nationale, nicht religiöse Färbung zu geben und zugleich das Narrativ weiterzuspinnen, dass mit diesem Staatsprojekt, anknüpfend an die grundlegenden eigenen Werte, eine universelle Idee verbunden sei. 


\section{Schluss}

Der Kulturkampf zwischen säkularen und religiös-fundamentalistischen Juden hat sich also zum Teil aus der politischen Arena ins juristische Feld verlagert, zuletzt auch vermehrt auf das Feld des Privatrechts, auf dem Ultra-Orthodoxe und fundamentalistische Zionisten durch die Etablierung einer Paralleljustiz versuchen, die rechtsstaatlichen Strukturen $\mathrm{zu}$ diskreditieren und auszuhöhlen (Hofri-Winogradow 2011). So wie der judicial activism des Obersten Gerichts durchaus Kritik im liberalen Lager provoziert (Mautner 2011: 170-180), so ist auch diese Strategie der Gegenseite durchaus der Kritik des eigenen Lagers ausgesetzt. Innerhalb des religiösen Judentums stoßen die Ambitionen der Fundamentalisten vermehrt auf Kritik. Die alleinige Zuständigkeit orthodoxer Rabbinatsgerichte für religiöse und viele zivilrechtliche Fragen wird von Strömungen des Reformjudentums und der >Modernen Orthodoxie als nicht mehr zu legitimierende einseitige Festlegung kritisiert. Diese Gruppen, die zum Lager der religiösen Zionisten gehören, sprechen sich stattdessen für einen weitgehenden religiösen Pluralismus, Toleranz und nicht zuletzt eine Gleichberechtigung von Frauen aus, die in der patriarchalisch-androkratischen Kultur der Orthodoxie völlig untergeordnet sind (Timm 2012: 138f.).

Da sich diese reformorientierten Bewegungen innerhalb des religiösen Narrativs bewegen und damit die alleinige Deutungshoheit der orthodoxen Richtung über religiöse Fragen angreifen, könnte von ihrem noch nicht abzusehenden Einfluss die zukünftige Gestaltung der israelischen politischen und rechtlichen Kultur in mancher Hinsicht abhängen:

»[I]n many ways the religious-Zionist group holds the key to Israel's future cultural character. Put differently, the struggle between religious fundamentalism and Modern Orthodoxy is not just an internal struggle over the soul of religiousZionism, but also a struggle over Israel's soul. If a majority of members of the religious-Zionist group insists on preserving Israel's current regime and political culture, Israel's liberal democracy will continue to exist and may even flourish. However, if the religious-Zionist group endorses more and more ultra-Orthodox, fundamentalist cultural elements and practices, Israel's liberal democracy is bound to find itself in great jeopardy.« (Mautner 2011: 207)

Es droht zwar auch weiterhin keine vollständige theokratische Umgestaltung des israelischen Staates. Aber sollte die fundamentalistische Seite, der die demographische Entwicklung in die Hände spielt, weiter an Einfluss gewinnen, besteht die Gefahr einer partiellen Aushöhlung des Rechtsstaates und einer strikteren Normierung der Alltagskultur. Der Kulturkampf zwischen Säkularen und Fundamentalisten hat in jedem Fall wenig Aussicht, gelöst zu werden, und er färbt auf die Selbstverständigungsdiskurse der gesamten Gesellschaft ab. Der Staat Israel muss 
weiterhin mit der Spannung leben, eine jüdische Demokratie sein zu wollen, für deren Definition kein Einheit stiftendes Narrativ und kein overlapping consensus in Sicht ist. Wie stark mittlerweile im politischen Diskurs die demokratischen und egalitären Elemente des israelischen Gründungsnarrativs in den Hintergrund gerückt sind, zeigt die - nach mehreren vergeblichen Anläufen in den Jahren zuvor hochumstrittene Verabschiedung eines neuen Grundgesetzes unter der rechtskonservativen Regierung Benjamin Netanjahus im Sommer 2018, das Israel als »nationale Heimstätte des jüdischen Volkes« festlegt. Maßgeblich animiert von nationalistisch-säkularen und nationalreligiösen Kräften der Koalition ist dadurch die jüdische Identität des Staates rechtlich kodifiziert worden (Lintl 2018). Während die genauen rechtlichen Konsequenzen des Grundgesetzes (das unter anderem den nationalen Wert des Siedlungsbaus betont und das Hebräische zur alleinigen Amtssprache deklariert) noch offen sind, ist dies eine symbolpolitische Rejustierung der staatlichen Identität, die sich ganz bewusst gegen die Deutungsansprüche des Obersten Gerichts wendet. So betonte Justizministerin Ayelet Shaked von der nationalreligiösen Partei HaBajit haJehudi (Jüdisches Heim) den Primat jüdischer Prinzipien gegenüber den Menschenrechten (Lintl 2018) und sprach gemeinsam mit Bildungsminister Naftali Bennett schon im Vorfeld des Nationalstaatsgesetzes vom umfassenden Plan, der Selbstermächtigung des Supreme Court eine »GegenRevolution« entgegenzusetzen (Bob/Harkov 2017).

Dieses Fallbeispiel führt also insgesamt vor Augen, wie eine in sich selbst plurale, religiös-fundamentalistische Gruppe einer säkular-bürgerrechtlichen Staatsversion entgegentritt - eine Konstellation, die seit längerer Zeit in einen Kulturkampf über soziale und geographische Grenzen, über den Primat staatlichen oder religiösen Rechts und generell über die jüdische Identität gemündet ist, einen Kampf, den man gewinnbringend mit einer narratologischen Analyse durchleuchten kann.

Über den besonderen Fall Israels hinaus wurden dabei essentielle Funktionselemente politischer Narrative deutlich: Prozesse der Sinnstiftung und Identitätsbildung, die jeweils mit eigenen Erzählungen von Vergangenheit und Zukunft operieren, aber auch Strategien der Legitimationsbeschaffung, Herrschaftsstabilisierung und Ordnungsbegründung sowie, was im Pluralismus der Erzählungen angelegt ist, die Kritik anderer Narrative. Die Erfolgschancen von Narrativen, so konnte zumindest angedeutet werden, hängt dabei vom Charisma und vom symbolischen Kapital führender Politiker wie Ben Gurion genauso ab wie von institutionellen Bedingungen der Interpretationshoheit: Die »revolutionäre« Ermächtigung des Obersten Gerichts zum wohl wichtigsten Interpreten der israelischen Politik wurde vor allem deshalb auf den Weg gebracht, um der nicht zuletzt demographisch bedingten und durch das plurale Bildungssystem reproduzierten Erosion des liberalen Narrativs ein Veto entgegenzusetzen - und diese Ermächtigung ist derzeit heftigerem politischen Gegenwind ausgesetzt als je zuvor. Der Kampf um 
die kulturelle Hegemonie ist keineswegs nur einer um die bessere Erzählung, sondern ganz wesentlich auch einer um die Chancen und die Durchsetzung von Deutungsmacht.

\section{Literatur}

Amir, Yehoyada 2011: Israel as a Jewish State - Religious and Secular Dimensions. The Challenges of Jewish Secularization. In: Irene Diegel/Christiane Tietz (Hg.), Die politische Aufgabe von Religion. Perspektiven der drei monotheistischen Religionen, Göttingen, S. 13-35.

Arendt, Hannah 2006: Elemente und Ursprünge totaler Herrschaft. Antisemitismus, Imperialismus, totale Herrschaft, München/Zürich.

Arendt, Hannah 2007: Vita activa oder Vom tätigen Leben, München/Zürich.

Arnauld, Andreas von 2009: Was war, was ist - und was sein soll. Erzählen im juristischen Diskurs. In: Christian Klein/Matías Martínez (Hg.), Wirklichkeitserzählungen. Felder, Formen und Funktionen nicht-literarischen Erzählens, Stuttgart/Weimar, S. 14-50.

Barak, Aharon 1993: A Constitutional Revolution: Israel's Basic Laws. In: Constitutional Forum, Heft 4, S. 83-84.

Barak, Aharon 2006: The Judge in a Democracy, Princeton.

Barzilai, Gad 2005: Communities and Law. Politics and Cultures of Legal Identities, Ann Arbor.

Ben Gurion, David 2000: The Eternity of Israel. In: Michael Walzer/Menachem Lorberbaum/Noam J. Zohar (Hg.): The Jewish Political Tradition. Band 1: Authority, New Haven/London, S. 490-497.

Ben Gurion, David 2010: Israel. Der Staatsgründer erinnert sich, Frankfurt a.M.

Benhabib, Seyla 1990: Hannah Arendt and the Redemptive Power of Narrative, in: Social Research, Heft 57(1), S. 167-196.

Bergem, Wolfgang 2009: Politische Kultur und Geschichte. In: Samuel Salzborn (Hg.), Politische Kultur. Forschungsstand und Perspektiven, Frankfurt a.M., S. 201-227.

Blumenberg, Hans 1996: Arbeit am Mythos, Frankfurt a.M.

Bob, Yonah Jeremy/Harkov, Lahav 2017: Bennett, Shaked >Counter-Revolution Against High Court Activism< Unveiled. In: The Jerusalem Post, 19. Dezember 2017, https://www.jpost.com/Israel-News/Bennett-Shaked-counterrevolution-against-High-Court-unveiled-518487.

Brenner, Michael 2016: Israel. Traum und Wirklichkeit des jüdischen Staates, München.

Cohen, Asher/Susser, Bernard 2000: Israel and the Politics of Jewish Identity, Baltimore. 
Fludernik, Monika, 2009: An Introduction to Narratology, Abingdon/New York. Gadinger, Frank/Jarzebski, Sebastian/Yildiz, Taylan (Hg.) 2014a: Politische Narrative. Konturen einer politikwissenschaftlichen Erzähltheorie. In: Dies. (Hg.), Politische Narrative, Konzepte - Analysen - Forschungspraxis, Wiesbaden, S. 3-38.

Gadinger, Frank/Jarzebski, Sebastian/Yildiz, Taylan (Hg.) 2014b: Politische Narrative. Konzepte - Analysen - Forschungspraxis, Wiesbaden.

Gordon, Carol 1989: Mutual Perceptions of Religious and Secular Jews in Israel. In: The Journal of Conflict Resolution, Heft 33(4), S. 632-651.

Glinert, Lewis/Shilhav, Yosseph 1991: Holy Land, Holy Language. A Study of an Ultraorthodox Jewish Ideology. In: Language in Society, Heft 20(1), 1991, S. 59-86.

Hagemann, Steffen 2006: Für Volk, Land und Thora. Ultra-Orthodoxie und messianischer Fundamentalismus im Vergleich, Berlin.

Hagemann, Steffen 2011: Messianischer Fundamentalismus in der Krise? Die religiöse Siedlerbewegung und ihr Verhältnis zum Staat Israel. In: Irene Diegel/Christiane Tietz (Hg.), Die politische Aufgabe von Religion, Perspektiven der drei monotheistischen Religionen, Göttingen, S. 55-75.

Hirschl, Ran 2001: The Political Origins of Judicial Empowerment through Constitutionalization: Lessons from Israel's Constitutional Revolution. In: Comparative Politics, Heft 33(3), S. 315-335.

Hofmann, Wilhelm/Renner, Judith/Teich, Katja (Hg.) 2014: Narrative Formen der Politik, Wiesbaden.

Hofri-Winogradow, Adam S. 2011: A Plurality of Discontent: Legal Pluralism, Religious Adjudication and the State. In: Journal of Law and Religion, Heft 26(1), S. 57-89.

Koschorke, Albrecht 2012: Wahrheit und Erfindung. Grundzüge einer Allgemeinen Erzähltheorie, Frankfurt a.M.

Latour, Bruno 2014: Sollten wir nicht mal über Politik reden?. In: Trivium, Heft 16. Lintl, Peter 2018: Israel kodifiziert den jüdischen Charakter des Staates. In: Stiftung Wissenschaft und Politik, Kurz gesagt, 19. Juli 2018, https:// www.swp-berlin.org/kurz-gesagt/2018/israel-kodifiziert-den-juedischencharakter-des-staates/.

Llanque, Marcus 2014: Metaphern, Metanarrative und Verbindlichkeitsnarrationen: Narrative in der Politischen Theorie. In: Hofmann, Wilhelm/Renner, Judith/Teich, Katja (Hg.), Narrative Formen der Politik, Wiesbaden, S. 7-29.

Lyotard, Jean-François 1987: Postmoderne für Kinder. Briefe aus den Jahren 1982-1985, Wien.

Lyotard, Jean-François 2005: La condition postmoderne. Rapport sur le savoir, Paris.

MacIntyre, Alasdair C. 2007: After Virtue. A Study in Moral Theory, Notre Dame. Mautner, Menachem 2011: Law and the Culture of Israel, Oxford/New York. 
Melville, Gert/Vorländer, Hans (Hg.) 2002: Geltungsgeschichten. Über die Stabilisierung und Legitimierung institutioneller Ordnungen, Köln/Weimar.

Miskimmon, Alister/O'Loughlin, Ben/Roselle, Laura 2014: Strategic Narratives. Communication Power and the New World Order, Abingdon.

Müller-Funk, Wolfgang 2008: Die Kultur und ihre Narrative. Eine Einführung, Wien.

Nünning, Ansgar, 2009: Surveying Contextualist and Cultural Narratologies: Towards an Outline of Approaches, Concepts and Potentials. In: Sandra Heinen/Roy Sommer (Hg.), Narratology in the Age of Cross-Disciplinary Narrative Research, Berlin, S. 48-70.

Nünning, Ansgar 2013: Wie Erzählungen Kulturen erzeugen. Prämissen, Konzepte und Perspektiven für eine kulturwissenschaftliche Narratologie. In: Alexandra Strohmaier (Hg.), Kultur - Wissen - Narration, Perspektiven transdisziplinärer Erzählforschung für die Kulturwissenschaften, Bielefeld, S. 15-53.

Paine, Andrew 1997: Religious Fundamentalism and Legal Systems: Methods and Rationales in the Fight to Control the Political Apparatus. In: Indiana Journal of Global Legal Studies, Heft 5(1), S. 263-296.

Schmid, Ulrich 2017: Israels expansive Ultraorthodoxie. In: Neue Zürcher Zeitung, 3. Februar 2017, S. 46.

Segev, Tom 2009: 1967. Israels zweite Geburt, München.

Segev, Tom 2010: Die ersten Israelis. Die Anfänge des jüdischen Staates, München. Shenhav, Shaul R. 2006: Political Narratives and Political Reality. In: International Political Science Review, Heft 27(3), S. 245-262.

Somers, Margaret R. 1994: The Narrative Constitution of Identity: A Relational and Network Approach. In: Theory and Society, Heft 23(5), S. 605-649.

Straßenberger, Grit, 2005: Über das Narrative in der politischen Theorie, Berlin.

Suntrup, Jan Christoph 2010: Formenwandel der französischen Intellektuellen. Eine Analyse ihrer gesellschaftlichen Debatten von der Libération bis zur Gegenwart, Münster/Berlin.

Taylor, Charles 1975: Erklärung und Interpretation in den Wissenschaften vom Menschen. Aufsätze, Frankfurt a.M.

Taylor, Charles 2004: Modern Social Imaginaries, Durham/London.

Timm, Angelika 2003: Israel - Gesellschaft im Wandel, Opladen.

Timm, Angelika 2012: Die Rolle jüdisch-orthodoxer Parteien in Israel. In: Bernd Oberdorfer/Peter Waldmann (Hg.), Machtfaktor Religion. Formen religiöser Einflussnahme auf Politik und Gesellschaft, Köln/Weimar, S. 127-141.

Viehöver, Willy 2014: Erzählungen im Feld der Politik, Politik durch Erzählungen. Überlegungen zur Rolle der Narrationen in den politischen Wissenschaften. In: Frank Gadinger/Sebastian Jarzebski/Taylan Yildiz (Hg.), Politische Narrative Konzepte - Analysen - Forschungspraxis, Wiesbaden, S. 67-91. 



\section{Autorinnen und Autoren}

Prof. Dr. Sigrid Baringhorst, Professorin für Politikwissenschaft an der Universität Siegen, Studium an der RWTH Aachen, Promotion an der WWU Münster, Habilitation an der JLU Gießen, Forschungsschwerpunkte: Neue Formen der politischen Partizipation und des zivilgesellschaftlichen Engagements, Politik im Netz, Vergleichende Migrations- und Integrationsforschung. Veröffentlichungen: Baringhorst, S./Yang,M./Voss, K./Villioth, L., 2017: Webzentrierte Hybridkampagnen Ausdruck postdemokratischer Protestpartizipation?, in: Protest in Bewegung. Zum Wandel von Bedingungen, Formen und Effekten politischen Protests, Leviathan, Sonderheft 31, Baden-Baden, S. 171-197; Baringhorst, Sigrid, 2012: Politik als populäre Kultur - populäre Kultur als Politik. Innovative Perspektiven der politischen Kulturforschung, in: Schiller, Dietmar (Hg.): »A Change Is Gonna Come«. Popmusik und Politik. Empirische Beiträge zu einer politikwissenschaftlichen Popmusikforschung. Berlin; Baringhorst, Sigrid, et.al., 2010: Unternehmenskritische Kampagnen im Zeichen digitaler Kommunikation, Wiesbaden; Baringhorst, Sigrid, et.al. (2009) (Hg.): Political Campaigning on the Web, Bielefeld; Baringhorst Sigrid, et.al. 2007, (Hg.): Politik mit dem Einkaufswagen. Unternehmen und Konsumenten als Bürger in der globalen Mediengesellschaft, Bielefeld. Baringhorst@politikwissenschaft.uni-siegen.de

Prof. Dr. Wolfgang Bergem, außerplanmäßiger Professor für Politikwissenschaft an der Universität Siegen, Studium in Saarbrücken, München und Berlin/DDR, Promotion an der Ludwig-Maximilians-Universität München, Habilitation an der Bergischen Universität Wuppertal, Sprecher des Arbeitskreises »Politik und Kultur« der DVPW. Forschungsschwerpunkte: Politische Kulturforschung, Politisches System der Bundesrepublik Deutschland, Vergleichende Politikwissenschaft. Veröffentlichungen u.a.: Die NS-Diktatur im deutschen Erinnerungsdiskurs (Hg.), Opladen 2003; Identitätsformationen in Deutschland, Wiesbaden 2005; Deutschland fiktiv. Die deutsche Einheit, Teilung und Vereinigung im Spiegel von Literatur und Film (Hg. mit Reinhard Wesel), Münster 2009; »Stunde Null« und »Achtundsechzig« als Gründungsmythen der deutschen Nachkriegsdemokratie, in: Yves Bizeul/Stephanie Wodianka (Hg.): Mythos und Tabula rasa, 
Bielefeld 2018; Volkserzählungen. Narrative des Volkes, Narrative über das Volk, in: Michael Müller/Jørn Precht (Hg.): Narrative des Populismus, Wiesbaden 2019. wolfgang.bergem@uni-siegen.de

Prof. Dr. Paula Diehl, Professorin für Politische Theorie, Ideengeschichte und Politische Kultur an der Christian-Albrecht-Universität zu Kiel. Sie war verantwortlich für den Bereich »Theorie, Geschichte und Kultur des Politischen« an der Universität Bielefeld sowie Dilthey Fellow und Nachwuchsgruppenleiterin an der Humboldt Universität zu Berlin und ist Sprecherin des Arbeitskreises »Politik und Kultur« der DVPW. Forschungsschwerpunkte Repräsentationstheorie, Politische Kultur, Medien und Politik. Diehl ist Autorin u.a. von Das Symbolische, das Imaginäre und die Demokratie. Eine Theorie politischer Repräsentation, Nomos Verlag und Mitherausgeberin von Politische Repräsentation und das Symbolische. Springer Verlag. Zuletzt erschienen: The Populist Twist. The Relationship Between the Leader and the People in Populism; In: Johannes Pollak/Dario Castiglione (ed.): Making Present. Theorizing the new Politics of Representation, University of Chicago Press sowie Rechtspopulismus und Massenmedien; in: Armin Flender, Volker M. Heins, Jo Reichertz, Jennifer Schellhöh (Hg.): Großerzählungen des Extremen. Transcript Verlag.e-Mail: paulaDiehl@gmx.com

Prof. Dr. Andreas Dörner, Professor für Medienwissenschaft an der PhilippsUniversität Marburg; Promotion an der Universität Essen, Habilitation an der Otto-von-Guericke-Universität Magdeburg; Forschungsschwerpunkte Politische Kommunikation, Fernsehen als politisches Medium, TV-Serien; Veröffentlichungen: Politischer Mythos und symbolische Politik. Der Hermannmythos: zur Entstehung des Nationalbewußtseins der Deutschen. Reinbek: Rowohlt 1996; Politische Kultur und Medienunterhaltung. Zur Inszenierung politischer Identitäten in der amerikanischen Film- und Fernsehwelt. Konstanz: UVK 2000; Politainment. Politik in der medialen Erlebnisgesellschaft. Frankfurt: Suhrkamp 2001 (edition suhrkamp, Bd. 2203); Unterhaltungsrepublik Deutschland. Medien, Politik und Entertainment. Bonn: Bundeszentrale für politische Bildung 2012 (hg. mit L. Vogt); Riskante Bühnen. Inszenierung und Kontingenz - Politikerauftritte in deutschen Personality-Talkshows. Wiesbaden: Springer-VS 2015 (mit L. Vogt, M. Bandtel, B. Porzelt); Wahlkampf mit Humor und Komik. (Hg. mit L. Vogt). Wiesbaden: Springer VS 2017. doerner@staff.uni-marburg.de

Dr. Bernadette Goldberger, MMag., Studium Politikwissenschaft und Geschichte in Wien, Paris und Madrid. 2011/2012 Forschungsaufenthalt am Instituto Gino Germani, Universität Buenos Aires. 2017 Promotion im Fach Politikwissenschaft mit einer Arbeit zu Fußball und symbolischen Inszenierungen des Popularen im argentinischen Kirchnerismus. Forschungsschwerpunkte Kollek- 
tive Identitäten, Rechtsextremismus, Populismus, Narrative Diskursanalyse. Veröffentlichungen: Populismus und kulturelle Symbolisierung (Arbeitstitel!). Weilerwist 2018: Velbrück (im Erscheinen). Fußball, »Argentinität« und Kirchnerismus. Symbolische Inszenierung politischer Identitäten im kirchneristischen Mediendiskurs zur Fußballweltmeisterschaft 2010. In: Journal für Entwicklungspolitik 2/2018 (im Erscheinen). »Rechtsextremismus und Fremdenfeindlichkeit. Gesellschaftstheoretische und sozialpsychologische Erklärungsfaktoren basaler Zugehörigkeitskonflikte.«Wien 2013: Wiener Verlag für Sozialforschung. bernadette.goldberger@univie.ac.at

PD Dr Jörn Knobloch, Privatdozent und akademischer Mitarbeiter am Lehrstuhl für Politische Theorie der Universität Potsdam, 2006 Promotion zum Dr. rer. pol. (summa cum laude), 2015 Habilitation und Erteilung der Lehrbefugnis für das Fach Politikwissenschaft, Forschungsschwerpunkte u.a.: Der neue Kampf der Kulturen, Demokratie und das Geheimnis, Die politische Produktion von Zukunft, Publikationen u.a.: Unschärferelationen - Konstruktionen der Differenz von Politik und Recht (Hg. Zusammen mit Thorsten Schlee), Reihe »Politologische Aufklärung konstruktivistische Perspektiven «, Springer VS, 2018, Demokratie und Geheimnis, in: Rüdiger Voigt (Hg.), Staatsgeheimnisse: Arkanpolitik im Wandel der Zeiten, Reihe: Staat-Souveränität-Nation, Wiesbaden: Springer VS, 2017, 205-224; Demokratie und summative politische Ordnung - Die praktische Möglichkeit demokratischer Herrschaft in nicht westlichen Gesellschaften, in: Sophia Schubert, Alexander Weiß (Hg.), Demokratie jenseits des Westens, PVS-Sonderheft 51, BadenBaden: Nomos, 2016, 225-244; Normativität und >Know-how Politischer Ordnung, Weilerswist: Velbrück, 2016. jokknob@uni-potsdam.de

Dr. Marcel Lewandowsky, wissenschaftlicher Mitarbeiter am Lehrstuhl für vergleichende Regierungslehre der Helmut-Schmidt-Universität/Universität der Bundeswehr Hamburg. Zuvor war er als Wissenschaftlicher Mitarbeiter an der Leuphana Universität Lüneburg tätig. 2012 schloss er an der Universität Bonn seine Dissertation zum Thema »Landtagswahlkämpfe« ab. Im Mittelpunkt seiner Forschung stehen populistische Parteien in Europa, Parteien und Parteiensysteme sowie Verwaltungsstrukturreformen auf der deutschen Länderebene. Veröffentlichungen: Rechtspopulismus in Europa: Erscheinungsformen, Ursachen und Gegenstrategien. Zeitschrift für Politik 64 (1) 2017, S. 21-38 (mit Frank Decker). Rechtspopulismus in Deutschland: Eine empirische Einordnung der Parteien zur Bundestagswahl 2013 unter besonderer Berücksichtigung der AfD. Politische Vierteljahresschrift 57 (2) 2016, S. 247-275 (mit Heiko Giebler und Aiko Wagner). lewandowsky@hsu-hh.de 
Prof. Dr. Hans J. Lietzmann, Lehrstuhl für Politikwissenschaft an der Bergischen Universität Wuppertal/Jean-Monnet-Professor for European Politics/Direktor des Instituts für Demokratie- und Partizipationsforschung [i:DPF]. Forschungsaufenthalte in Cambridge/MA (Harvard), New York u.a. Gastprofessuren in New Delhi (Jahawarla-Nehru-Univ.), Berlin (WZB). Sprecher des Arbeitskreises »Politik und Kultur« der DVPW. Forschungen zu Politischer Theorie, Verfassungs- \& Europapolitik, Politischer Kultur. Letzte Veröffentlichungen: »Postfaktisches Argumentieren«: Über ein politisches Narrativ des liberalen >juste milieu<. In: Rennhak/Martinez, Postfaktisches Erzählen? (im Erscheinen/2019).//Citizenship, Democracy and the Iconology of Politics. A Plea for an Iconological Turn in Democratic Theory. In: A. Björk/C. Wiesner u.a., Shaping Citizenship. London: Routledge. 2018, S. 55-70. Hans.J.Lietzmann@idpf.eu

PD Dr. habil. Sandra Nuy, Privatdozentin für Medienwissenschaft an der Universität Siegen. Forschungsschwerpunkte: Dramaturgien des Politischen; Mediengeschichte des Films; Erinnerungskulturen. Abschluss des Magister-Studiums der Germanistik, Soziologie und Politikwissenschaft mit einer Arbeit über Paul Kornfelds »Jud Süß« (1995 als Monographie veröffentlicht). Dissertation: Arthur Schnitzler ferngesehen. Ein Beitrag zur Geschichte des Theaters im Fernsehen der Bundesrepublik Deutschland (1999); Habilitation: Die Politik von Athenes Schild (2015). Aktuelle Publikation: Die Politik von Athenes Schild. Zur dramaturgischen Logik des Politischen im fiktionalen Film (2017). sandra.nuy@uni-siegen.de

Dr. Dennis Bastian Rudolf, wissenschaftlicher Mitarbeiter am Lehrstuhl für Politische Theorie und Ideengeschichte der Universität Rostock. Studium der Politikwissenschaft sowie der Neueren und Neuesten Geschichte an der Eberhard Karls Universität Tübingen. Externer Kollegiat am DFG-Graduiertenkolleg »Deutungsmacht - Religion und belief systems in Deutungsmachtkonflikten«. Promotion zur Analyse und zum Vergleich politischer Mythen in Demokratien und Autokratien. Forschungsschwerpunkte: Politische Kultur, politischer Mythos sowie Demokratieund Autokratietheorie. Aktuelle Veröffentlichung: Gibt es eine kulturelle Identität? (Hg.), zusammen mit Yves Bizeul, Baden-Baden: Nomos (2019, in Vorbereitung). dennis.rudolf@uni-rostock.de

Prof. Dr. Samuel Salzborn, Gastprofessor für Antisemitismusforschung am Zentrum für Antisemitismusforschung (ZfA) der Technischen Universität Berlin. Forschungsschwerpunkte: Politische Theorie und Gesellschaftstheorie; Politische Soziologie und Demokratieforschung. Letzte Buchveröffentlichungen u.a. Globaler Antisemitismus. Eine Spurensuche in den Abgründen der Moderne. Mit einem Vorwort von Josef Schuster, Weinheim 2018; Handbuch Politische Ideengeschichte. 
Zugänge - Methoden - Strömungen (als Hg.), Stuttgart 2018. salzborn@tu-berlin.de

Dr. Jasmin Siri, Post-Doc am Institut für Soziologie der Ludwig-MaximiliansUniversität München. Im Mittelpunkt ihrer Forschungen stehen politische Soziologie, Parteiensoziologie, soziologische Theorie, politische Theorie und qualitative empirische Politikforschung. Aktuell arbeitet sie insbesondere über die Folgen der Digitalisierung für das politische System, politische Organisationen und politische Kultur. Aktuelle Monografie: Siri, Jasmin (2018): Kampfzone Gender. Über die Politisierung wissenschaftlicher Expertise, Berlin. Weitere Publikationen und Hinweise auf aktuelle Forschung finden sich unter https://www.researchgate. net/profile/Jasmin_Siri.jasmin.siri@soziologie.uni-muenchen.de

PD Dr. Jan Christoph Suntrup forscht als Assoziierter Wissenschaftler am Käte Hamburger Kolleg »Recht als Kultur« und lehrt am Institut für Politische Wissenschaft und Soziologie der Universität Bonn, an der er im Sommer 2017 habilitiert wurde. Derzeit ist er Visiting Fellow am European Institute der London School of Economics. Seine Forschungsschwerpunkte liegen im Bereich der Demokratieund Verfassungstheorie, der Rechtsanalyse und der politischen Kulturforschung. Letzte Publikationen: Umkämpftes Recht. Zur mehrdimensionalen Analyse rechtskultureller Konflikte durch die politische Kulturforschung (Frankfurt a.M. 2018); The Symbolic Politics of the State of Exception: Images and Performances, in: Zeitschrift für Politikwissenschaft, Heft 28(4), 2018, S. 565-580; Constitutional Cultures in Comparative Perspective (hg. zusammen mit Werner Gephart, Frankfurt a.M. 2019 (i.E.).jan.suntrup@uni-bonn.de

Prof. Dr. Ludgera Vogt, Professorin für Allgemeine Soziologie, insbesondere Handlungs- und Interaktionstheorien an der Bergische Universität Wuppertal; Promotion an der Universität Regensburg, Habilitation an der TU Dortmund; Forschungsschwerpunkte Politische Soziologie, Kultur- und Mediensoziologie; Veröffentlichungen: Zur Logik der Ehre in der Gegenwartsgesellschaft. Differenzierung - Macht - Integration. Frankfurt a.M.: Suhrkamp 1997; Hauptwerke der Soziologie. Stuttgart: Kröner-Verlag 2000. (Hg. mit D. Kaesler). 2. Aufl. 2007; Das Kapital der Bürger. Theorie und Praxis zivilgesellschaftlichen Engagements. Frankfurt, New York: Campus 2005; Das Geflecht aktiver Bürger. >Kohlen « eine Stadtstudie zur Zivilgesellschaft im Ruhrgebiet. Wiesbaden: VS-Verlag für Sozialwissenschaften 2008 (mit. A. Dörner); Riskante Bühnen. Inszenierung und Kontingenz - Politikerauftritte in deutschen Personality-Talkshows. Wiesbaden: Springer-VS 2015 (mit A. Dörner, M. Bandtel, B. Porzelt); Wahlkampf mit Humor und Komik. (Hg. Mit A. Dörner). Wiesbaden: Springer VS 2017. lvogt@uni-wuppertal.de 
Dr. Katharina Witterhold, wissenschaftliche Mitarbeiterin an der Universität Siegen, Studium an der JLU Gießen, Promotion an der Universität Siegen, Forschungsschwerpunkte: Politische Soziologie, vor allem im Kontext von Konsum, Beteiligung und Digitalisierung. Dissertation: Politische Konsumentinnen im Social Web. Praktiken der Vermittlung zwischen Bürger- und Verbraucheridentität. (Erschienen 2017, Transcript Bielefeld) witterhold@politikwissenschaft.uni-siegen.de

Dr. Mundo Yang, wissenschaftlicher Mitarbeiter an der Universität Siegen, Studium an der Freien Universität Berlin, Promotion an der Universität Flensburg, Forschungsschwerpunkte: Neue Formen von Zivilgesellschaft und politischer Partizipation, Engagement im Internet, politischer Konsum, Engagement für Nachhaltigkeit, Medien und Öffentlichkeit. Veröffentlichungen: Baringhorst, S./Yang, M./Voss, K./Villioth, L., 2017: Webzentrierte Hybridkampagnen - Ausdruck postdemokratischer Protestpartizipation?, in: Protest in Bewegung. Zum Wandel von Bedingungen, Formen und Effekten politischen Protests, Leviathan, Sonderheft 31, Baden-Baden, S. 171-197; Yang, Mundo/Baringhorst, Sigrid (2017): Politischer Konsum im Netz als Ausdruck des Wandelns politischer Partizipation. In: Jaeger-Erben, Melanie/Rückert-John, Jana/Schäfer, Martina (Hg.): Soziale Innovationen für nachhaltigen Konsum: Wissenschaftliche Perspektiven, Strategien der Förderung und gelebte Praxis. Wiesbaden: Springer Fachmedien, S. 191-215. Mundo.yang@uni-siegen.de 


\section{Politikwissenschaft}

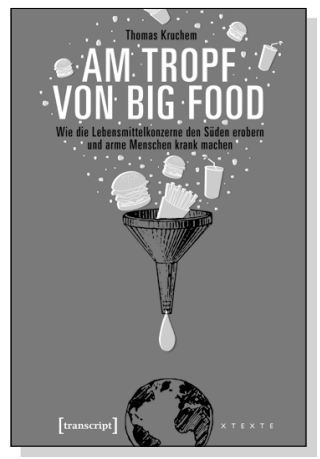

Thomas Kruchem

Am Tropf von Big Food

Wie die Lebensmittelkonzerne den Süden erobern

und arme Menschen krank machen

2017, 214 S., kart., zahlr. Abb.

$19,99 €(D E), 978-3-8376-3965-0$

E-Book: 16,99 € (DE), ISBN 978-3-8394-3965-4

EPUB: $16,99 €(D E)$, ISBN 978-3-7328-3965-0

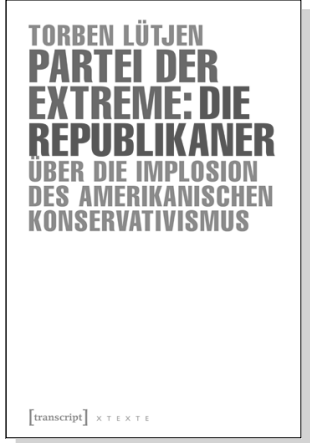

Torben Lütjen

Partei der Extreme: Die Republikaner

Über die Implosion des amerikanischen Konservativismus

2016, 148 S., kart.

$14,99 €(D E), 978-3-8376-3609-3$

E-Book: 12,99 € (DE), ISBN 978-3-8394-3609-7

EPUB: $12,99 €$ (DE), ISBN 978-3-7328-3609-3

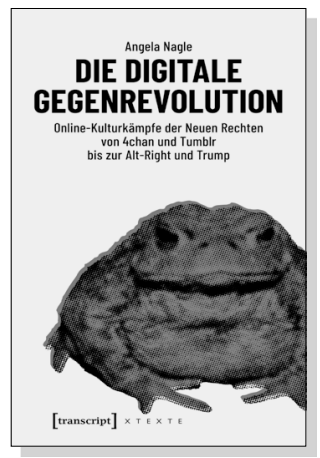

Angela Nagle

Die digitale Gegenrevolution

Online-Kulturkämpfe der Neuen Rechten von 4chan und Tumblr bis zur Alt-Right und Trump

2018,148 S., kart.

$19,99 €(D E), 978-3-8376-4397-8$

E-Book: $17,99 €(D E)$, ISBN 978-3-8394-4397-2

EPUB: $17,99 €$ (DE), ISBN 978-3-7328-4397-8 


\section{Politikwissenschaft}

Ines-Jacqueline Werkner Gerechter Frieden Das fortwährende Dilemma militärischer Gewalt

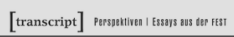
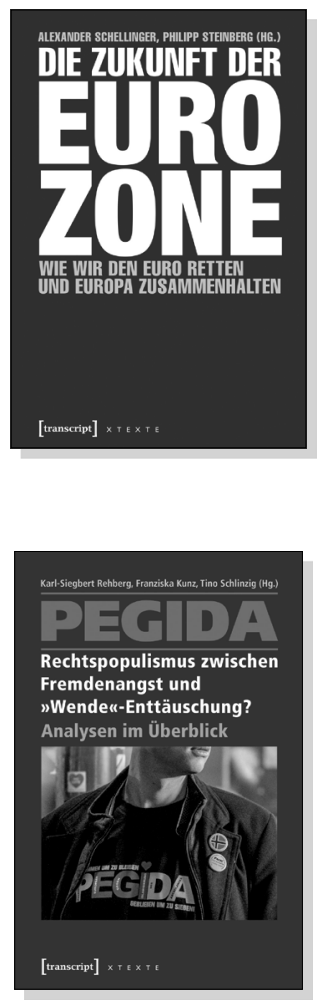

Ines-Jacqueline Werkner

\section{Gerechter Frieden}

Das fortwährende Dilemma militärischer Gewalt

2018,106 S., kart.

$14,99 €(D E), 978-3-8376-4074-8$

E-Book: 12,99 € (DE), ISBN 978-3-8394-4074-2

Alexander Schellinger, Philipp Steinberg (Hg.)

\section{Die Zukunft der Eurozone}

Wie wir den Euro retten und Europa zusammenhalten

2016, 222 S., kart.

$19,99 €(D E), 978-3-8376-3636-9$

E-Book: 17,99 € (DE), ISBN 978-3-8394-3636-3

EPUB: 17,99 € (DE), ISBN 978-3-7328-3636-9

Karl-Siegbert Rehberg, Franziska Kunz, Tino Schlinzig (Hg.)

\section{PEGIDA -}

\section{Rechtspopulismus zwischen Fremdenangst} und "Wende «-Enttäuschung?

Analysen im Überblick

2016, 384 S., kart.

29,99 € (DE), 978-3-8376-3658-1

E-Book: $26,99 €$ (DE), ISBN 978-3-8394-3658-5

EPUB: $26,99 €$ (DE), ISBN 978-3-7328-3658-1 